
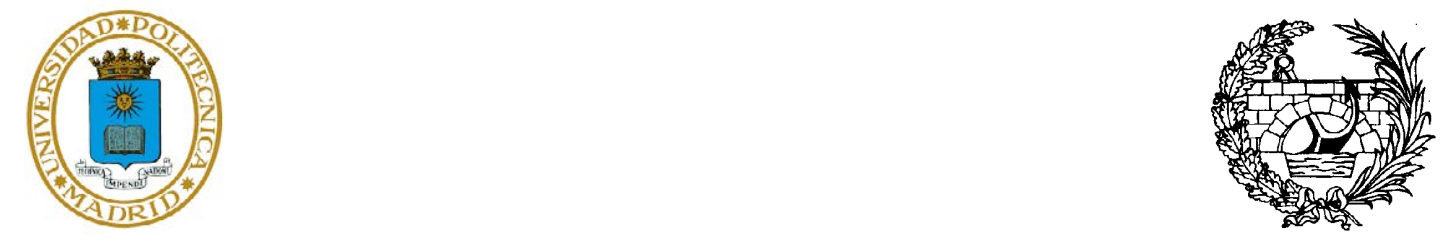

UNIVERSIDAD POLITECNICA DE MADRID

ESCUELA TECNICA SUPERIOR DE INGENIEROS DE CAMINOS CANALES Y

PUERTOS.

\title{
ANÁLISIS TEÓRICO DE LA VARIACIÓN ALEATORIA DE LOS ESFUERZOS DE MONTAJE DE LOS CABLES EN LOS PUENTES ATIRANTADOS
}

TESIS DOCTORAL

LUIS CARRILLO ALONSO

Ingeniero de Caminos, Canales y Puertos

Madrid, 2015 


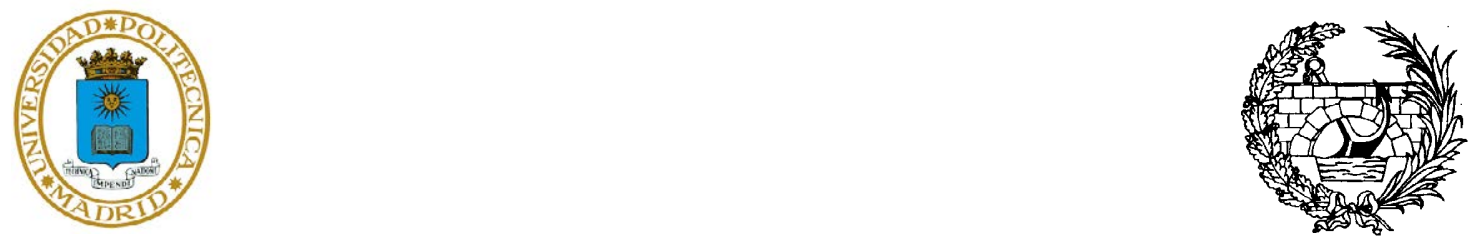

DEPARTAMENTO DE MECANICA DE LOS MEDIOS CONTINUOS Y TEORIA DE ESTRUCTURAS

ESCUELA TECNICA SUPERIOR DE INGENIEROS DE CAMINOS CANALES Y PUERTOS.

TESIS DOCTORAL

\title{
ANÁLISIS TEÓRICO DE LA VARIACIÓN ALEATORIA DE LOS ESFUERZOS DE MONTAJE DE LOS CABLES EN LOS PUENTES ATIRANTADOS
}

\author{
Autor: \\ Luis Carrillo Alonso \\ Ingeniero de Caminos, Canales y Puertos \\ Director: \\ Miguel Angel Astiz Suárez \\ Doctor Ingeniero de Caminos, Canales y Puertos
}

Madrid, 2015 

TESIS DOCTORAL

\section{ANÁLISIS TEÓRICO DE LA VARIACIÓN ALEATORIA DE LOS ESFUERZOS DE MONTAJE DE LOS CABLES EN LOS PUENTES ATIRANTADOS}

Autor:

Luis Carrillo Alonso

Director:

Miguel Angel Astiz Suárez

Tribunal nombrado por el Mgfco. y Excmo. Sr. Rector de la Universidad Politécnica de Madrid, el día de de 2015.

PRESIDENTE:

VOCAL:

VOCAL:

VOCAL:

VOCAL SECRETARIO:

Acuerda otorgarle la calificación de:

Madrid,

de

de 2015. 

a Rocío y Alejandra 



\section{AGRADECIMIENTOS}

Cuando han sido escritas estas líneas el fruto del trabajo desarrollado en los últimos años ha quedado ya plasmado en este texto y es ahora cuando debo mostrar mi agradecimiento a todos aquellos cuya contribución permitió que esta tesis llegara a buen puerto. A todos ellos, familiares, amigos, profesores y compañeros, les quiero dedicar estas líneas, y aunque alguno no quede citado expresamente, por no extender en demasía estos párrafos, quiero señalar que los contribuyentes al trabajo han sido numerosos y sus diferentes aportaciones han sido todas ellas, de una $u$ otra forma, imprescindibles.

En primer lugar quiero mostrar mi enorme gratitud a mi Director de Tesis D. Miguel Angel Astiz, a quien debo el origen de esta investigación, y cuya orientación y dedicación, dado su conocimiento y experiencia del problema planteado, ha hecho posible el resultado final que se refleja en este texto. Por otro parte quiero significar que la confianza que depositó en mi desde el comienzo del trabajo me ha servido siempre como estímulo fundamental para el desarrollo de esta tesis.

En segundo lugar quiero recordar aquí la contribución de mi padre, también Doctor Ingeniero de Caminos, al conjunto del trabajo, ya que sin su ayuda, sabiduría, apoyo y consejo, muchos de los estudios realizados no se podrían haber llevado a cabo en la forma en la que finalmente han quedado reflejados. Tanto su conocimiento del problema del cálculo de puentes, que me ha transmitido a lo largo de los años en los que ha desarrollado su carrera profesional, como su ejemplo como técnico, han sido una suerte poder disfrutarlos y son y serán una deuda difícilmente pagable.

También quiero recordar aquí a mis amigos ingenieros Alejandro, Iñigo y Juanjo, ya que cada uno aportó su correspondiente granito de arena, animándome a la realización de este trabajo. En especial quiero destacar que la tesis presentada por mi amigo Juanjo ha sido un referente que me ha permitido sacar varias ideas que he podido trasladar y aplicar a mi investigación.

Igualmente tengo que agradecer al servicio de Reprografía de Acciona Ingeniería el trabajo realizado para el maquetado y encuadernado de la tesis.

Para terminar quiero tener un recuerdo para todas aquellas personas a quien el desarrollo de este trabajo ha supuesto la pérdida de un tiempo en su compañía, en especial a Rocío y Alejandra, a mi madre María Teresa y mi hermano Santiago, a quienes no puede hacer otra cosa que prometerles la devolución en adelante del tiempo que les robé. 


\section{RESUMEN}

Los puentes atirantados son una de las tipologías estructurales con más desarrollo en los últimos años ya que cuentan con un ámbito de aplicación que se ha extendido en gran medida, llegando hasta el rango de luces de más de $1000 \mathrm{~m}$. Por otra parte, para el caso de luces medias, este tipo de puentes aporta unas características resistentes y formales que los hacen muy adecuados en gran número de situaciones.

Simultáneamente al importante número de realizaciones llevadas a cabo con esta tipología en los últimos años, se ha producido un gran desarrollo del conocimiento teórico de diferentes aspectos técnicos específicos de estos puentes, tanto a nivel de su comportamiento estructural como de la simulación y mejora de sus procesos constructivos. Estos desarrollos se han producido en gran parte gracias al avance en las capacidad de computación disponible hoy en día con los numerosos y cada vez más sofisticados programas comerciales de cálculo estructural, los cuales permiten la realización de análisis que hasta hace poco tiempo eran muy complicados de desarrollar.

Una de las principales características de este tipo de estructuras, además de su elevado hiperestatismo, es la importancia del proceso constructivo, y en concreto del proceso de tesado de los cables, a la hora de conseguir alcanzar una situación final con la estructura en servicio que cumpla los requisitos establecidos a priori. Por este motivo se han llevado a cabo bastantes investigaciones orientadas a la optimización de los axiles a aplicar a los cables en el momento de su colocación y en su situación final. Parte de estos análisis se han centrado en particular en la posible influencia de ciertos parámetros geométricos y mecánicos aleatorios sobre los esfuerzos provocados sobre la estructura.

Por otra parte, el propio comportamiento resistente de un tirante introduce ciertas incertidumbres en los formatos de seguridad asociados a los esfuerzos generados sobre ellos, en concreto en relación a la dependencia de estas fuerzas respecto de las cargas permanentes y a su consideración a nivel de coeficientes de seguridad asociados a dichas acciones.

En vistas de estos aspectos particulares se ha procedido a desarrollar la investigación que se expone a continuación, en la que se ha pretendido obtener un conocimiento adicional de algunos de estos aspectos para así poder contrastar la validez de las hipótesis que hoy en día son establecidas por la normativa vigente. 
De esta forma el planteamiento adoptado en esta tesis ha sido en primer lugar proponer un método novedoso de introducción de errores aleatorios sobre los esfuerzos o deformaciones de tesado de los cables, de tal manera que se pueda aplicar dicha técnica a distintas tipologías de puentes atirantados. Se pretende con ello conocer la influencia de dichos errores sobre el comportamiento de estas estructuras y valorar la magnitud de las desviaciones finalmente creadas respecto de la situación teórica.

Una vez conocida la implicación que supone la aleatoriedad aplicada en la colocación de los tirantes, tanto cualitativa como cuantitativamente, se ha procedido a realizar un análisis de las consecuencias respecto de los formatos de seguridad que de estos datos se deducen.

Señalar que esta tesis se ha centrado en el caso concreto de puentes atirantados construidos por voladizos sucesivos y con tableros de hormigón, dado el gran número de puentes ejecutados con estas características, y con el objetivo de concentrar el estudio realizado en una tipología particular, pero de gran aplicación. 


\section{$\underline{\text { ABSTRACT }}$}

Cable-stayed bridges represent one of the most developed structural typologies being used recently as it has a span range that can extend beyond $1000 \mathrm{~m}$. Furthermore, when it comes to bridges that span to a medium range we could say their shape and resistance are features that make this kind of bridge suitable for many different applications.

Moreover, due to this type of bridge being used newly more extensively it has allowed for a better understanding of the theoretical knowledge of the various technical aspects both at a structural and simulation level in order to improve the constructions process of this type of bridge. Knowledge and development of these bridges can be attributed to the increasing apprehension of I.T. skills and the development of more advanced computer software with regards to structural calculations. The latter can aide more demanding analysis that was previously difficult to ascertain

Defining features of this type of structure, besides its hyper static attributes, is the importance of the construction process, namely the cable tensioning process, which determines whether the requirements set out in the construction process will be met once the bridge is finished and traffic loads are applied. This is why much research has been conducted into the optimisation of the axial forces to be applied to the cables. Focus, of part of this research, goes into the possible influences of random geometrical and mechanical parametres on the forces applied to the structure.

We have therefore proceeded to develop research in which we have tried to gain more in depth knowledge which considers these aspects so that we can validate the hypothesis which are currently established in the regulations

Firstly, the purpose of this thesis is to provide a new method to introduce random errors on the forces or deformations of cable tensioning so that this technique can be extended to various models of cable-staying bridges.

It is important to highlight that this thesis has focused on cable-stayed bridges built through the balanced cantilever method and with concrete girders and considering there are many actual bridges with these characteristics the research has focused on a specific yet extensively applied method. 


\section{INDICE}

CAPITULO 1. INTRODUCCION. ......................................................................................... 1

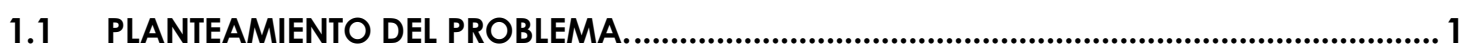

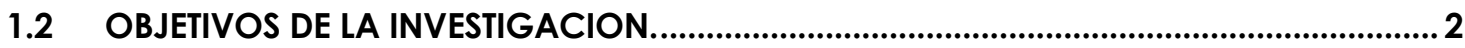

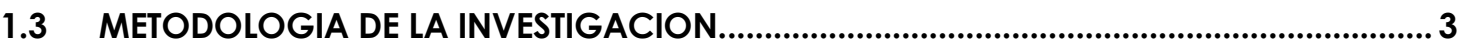

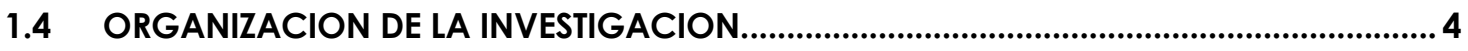

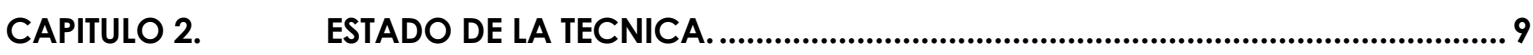

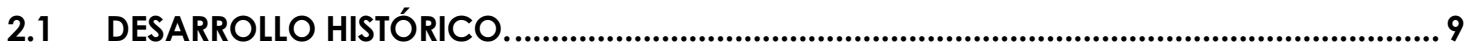

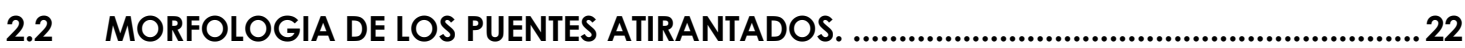

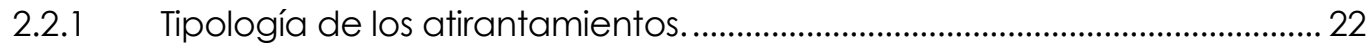

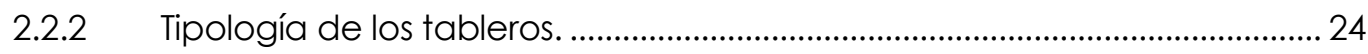

2.2.3 Tipología de pilonos y vinculación tablero-pilono........................................... 31

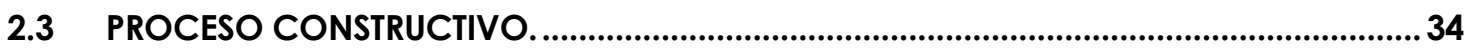

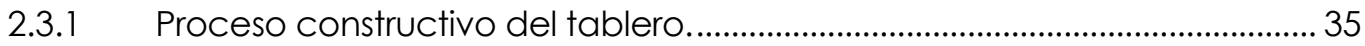

2.3.2 Métodos de puesta en carga de los tirantes................................................. 40

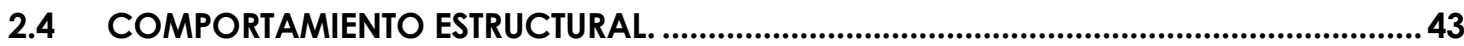

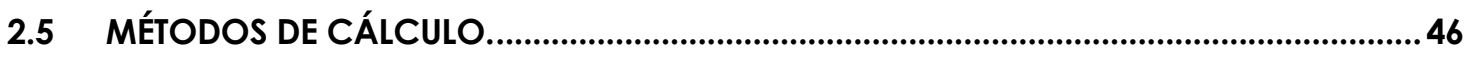

2.5.1 Situación final objetivo y axiles de montaje de los tirantes............................. 49

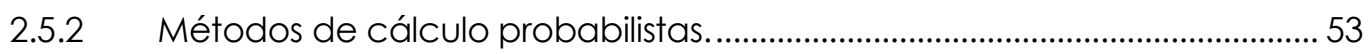

2.5.3 Efectos no lineales en los cálculos..................................................................... 56

CAPITULO 3. COMPORTAMIENTO ESTRUCTURAL DE UN TIRANTE. ........................................61

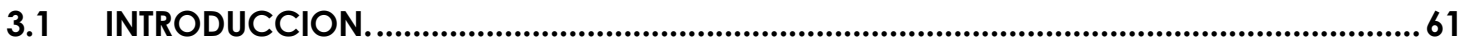

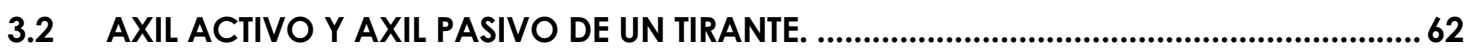

3.3 FACTORES QUE INFLUYEN EN EL AXIL ACTIVO Y PASIVO. ...............................................6

3.3.1 Variación del $\% F_{p} / F_{\text {tot }}$ en función de la separación entre anclajes. ........... 70

3.3.2 Variación del $\% \mathrm{~F}_{\mathrm{p}} / \mathrm{F}_{\text {tot }}$ en función de la longitud del tirante.......................... 71

3.3.3 Variación del $\% \mathrm{~F}_{\mathrm{p}} / \mathrm{F}_{\text {tot }}$ en función del ancho del tablero............................... 73

3.3.4 Variación del $\% \mathrm{~F}_{\mathrm{p}} / \mathrm{F}_{\text {tot }}$ en función de la carga muerta aplicada................. 75

3.3.5 Variación del $\% F_{p} / F_{\text {tot }}$ en función de la tensión generada en el tirante. .. 76

3.3.6 Variación del $\% \mathrm{~F}_{\mathrm{p}} / \mathrm{F}_{\text {tot }}$ en función de la rigidez del tablero............................. 77

3.3.7 Análisis de los resultados anteriores..................................................................... 79 


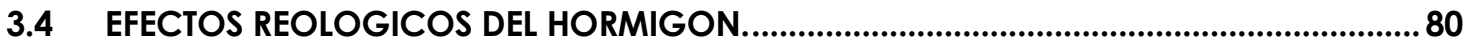

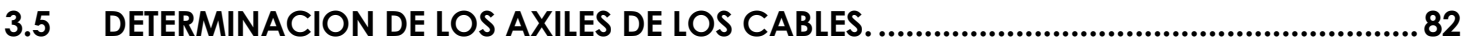

3.5.1 Cálculo de los axiles finales de los cables........................................................ 82

3.5.2 Cálculo de los axiles o deformaciones de montaje de los tirantes............ 84

3.5.3 Métodos de colocación de los tirantes............................................................. 85

CAPITULO 4. INFLUENCIA DEL METODO DE INSTALACION DE LOS CABLES..........................93

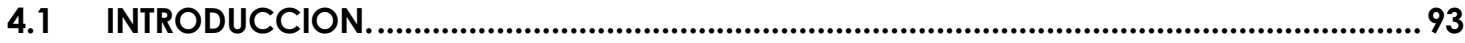

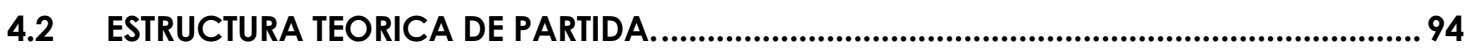

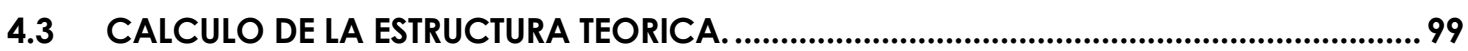

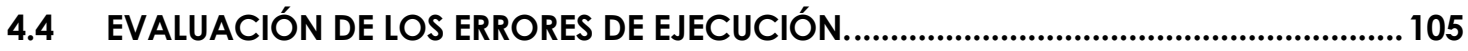

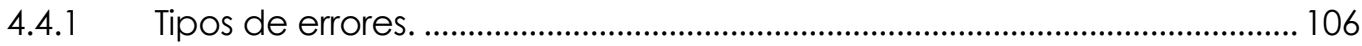

4.4.2 Algoritmo para la generación de muestras de valores aleatorios............ 107

4.4.3 Errores máximos admisibles................................................................................... 109

4.4.4 Verificación de la normalidad de las muestras............................................ 111

4.4.5 Desviación típica de las muestras de valores aleatorios. .............................. 113

4.4.6 Definición del tamaño de las muestras aleatorias. ........................................ 114

4.4.7 Validación del método de generación de aleatorios................................... 117

4.5 COMPARATIVA DE LOS MÉTODOS DE COLOCACIÓN DE LOS CABLES.........................120

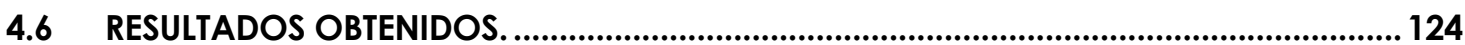

4.6.1 Definición de valor característico y de los esfuerzos e hipótesis a

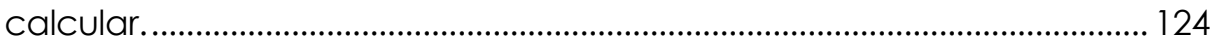

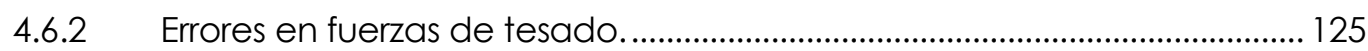

4.6.3 Errores en alargamientos de los cables........................................................... 132

4.6.4 Errores en la posición de los anclajes......................................................... 139

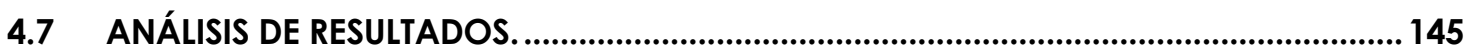

4.7.1 Resultados con errores en fuerzas de tesado.................................................. 145

4.7.2 Resultados con errores en deformaciones..................................................... 146

4.7.3 Resultados con errores en posición de anclajes............................................ 147

4.7.4 Comparación de resultados. ............................................................................. 148

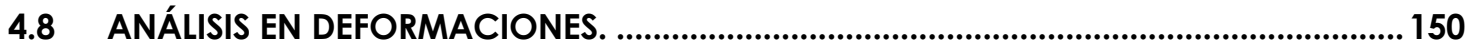

CAPITULO 5. INFLUENCIA DEL NUMERO DE CABLES DE LA ESTRUCTURA.............................157

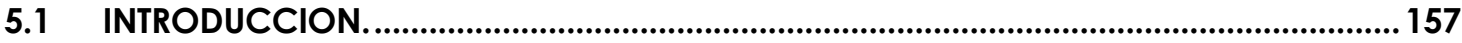

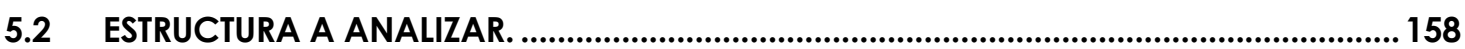

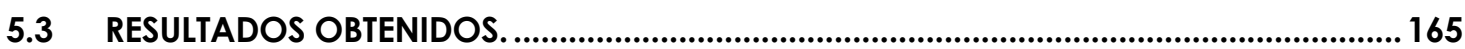

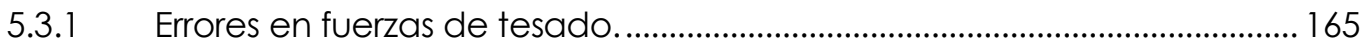

5.3.2 Errores en alargamientos de los cables......................................................... 172

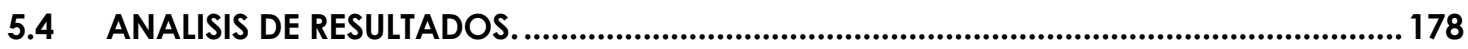

5.4.1 Resultados con errores en fuerzas. ................................................................ 178

5.4.2 Resultados con errores en deformaciones..................................................... 179

5.4.3 Comparación de resultados. .......................................................................... 180 
CAPITULO 6. INFLUENCIA DE LA LUZ DE LA ESTRUCTURA. .................................................... 181

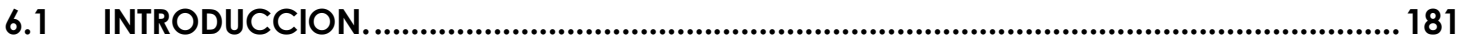

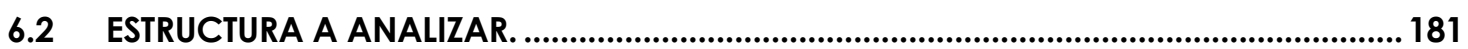

6.3 COMPARATIVA DEL METODO DE INSTALACION DE LOS CABLES..................................188

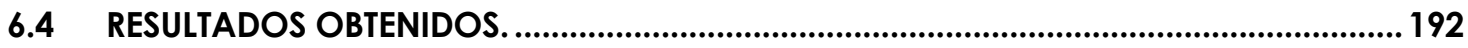

6.4.1 Errores en fuerzas de tesado......................................................................... 192

6.4.2 Errores en alargamientos de los cables....................................................... 199

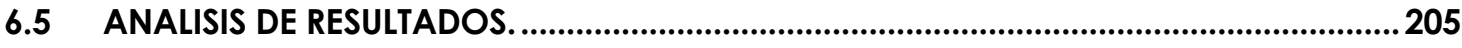

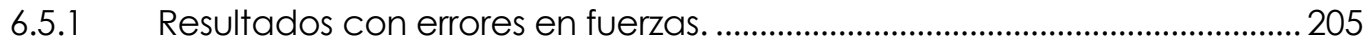

6.5.2 Resultados con errores en deformaciones.................................................... 206

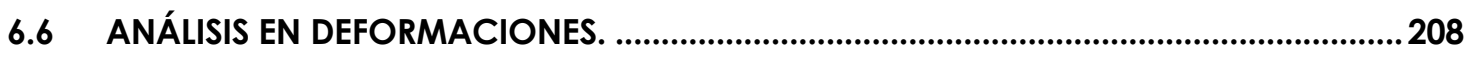

CAPITULO 7. INFLUENCIA DE LA RIGIDEZ DEL TABLERO

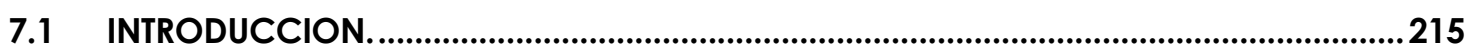

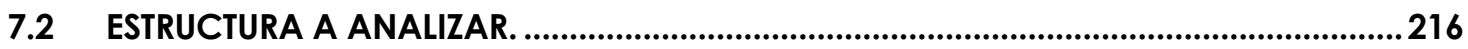

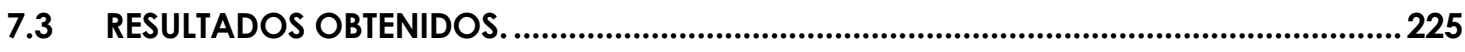

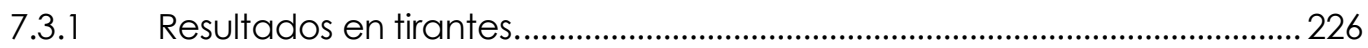

7.3.2 Comparativa de resultados en tirantes. ........................................................ 229

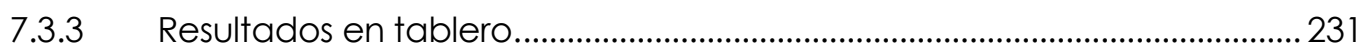

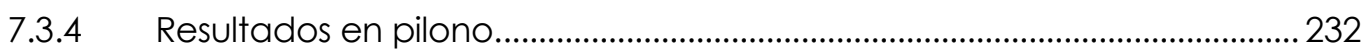

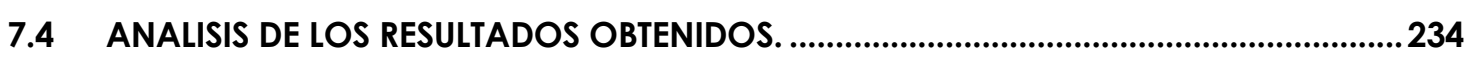

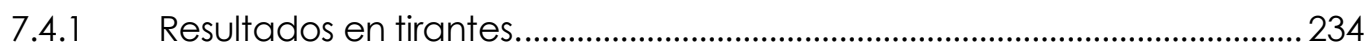

7.4.2 Comparación de resultados en tirantes.......................................................... 234

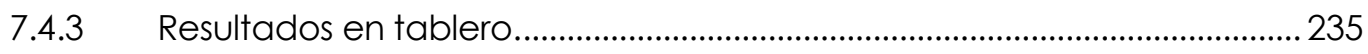

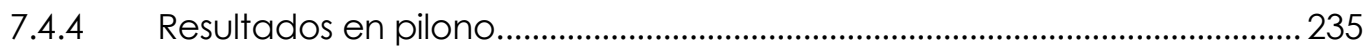

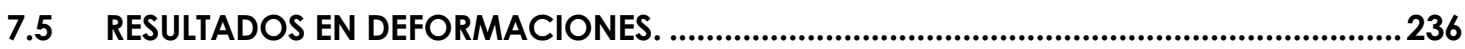

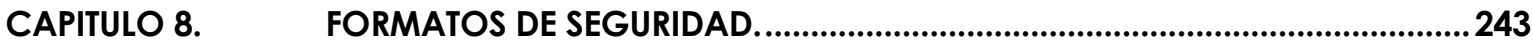

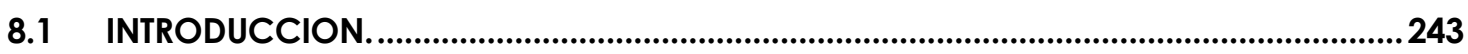

8.2 EFECTO DE LA PUESTA EN CARGA ERRONEA DE UN TIRANTE. .....................................244

8.3 EFECTO DE LA VARIABILIDAD DE LAS CARGAS PERMANENTES..................................246

8.4 CUANTIFICACION DE LOS FACTORES DEL AXIL DE UN TIRANTE. ....................................248

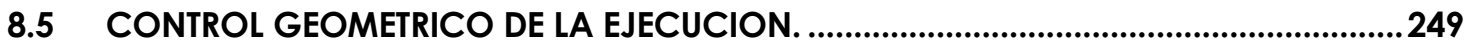

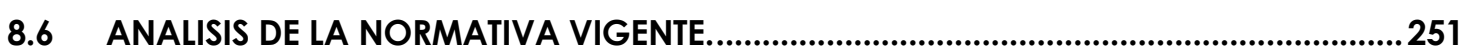

8.6.1 Instrucciones de acciones en puentes: IAP1 1 e IAPF07...............................251

8.6.2 Instrucción de hormigón estructural (EHEO8) y Código Modelo CEB-FIP

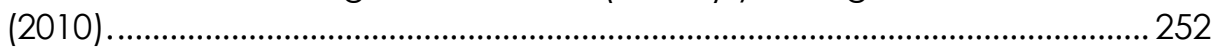

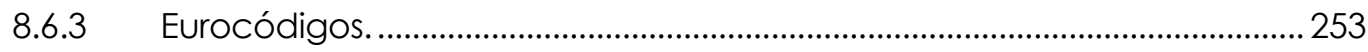

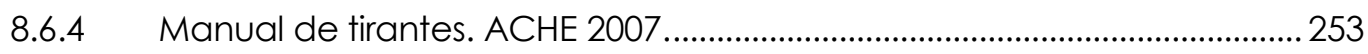

8.6.5 Haubans. Recommandation de la commission interministérielle de la

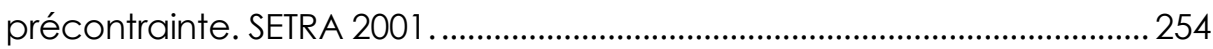




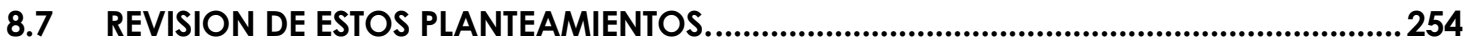

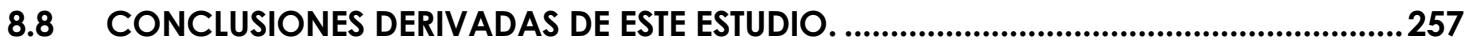

8.8.1 Cuantificación del efecto del esfuerzo de tesado de los cables. ........... 257

8.8.2 Determinación del axil pasivo y activo.......................................................... 258

8.8.3 Influencia del método de puesta en carga de los tirantes. ...................... 259

8.8.4 Validez del control geométrico del tablero..................................................... 261

8.8.5 Propuesta de formato de seguridad para el caso analizado.................... 261

CAPITULO 9. CONCLUSIONES Y FUTURAS LINEAS DE INVESTIGACION. ...............................263

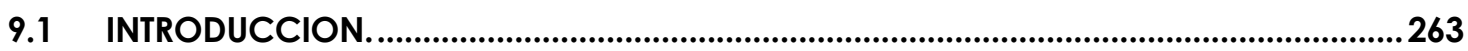

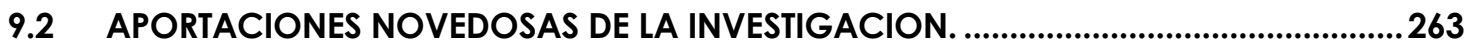

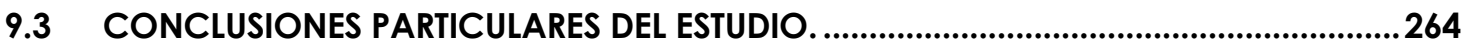

9.3.1 ANALISIS DE LOS FACTORES QUE COMPONEN EL AXIL DEL TIRANTE. ........ 265

9.3.2 ANALISIS DE LOS METODOS EN FUERZAS Y DEFORMACIONES..................... 266

9.3.3 ANALISIS DE LA INFLUENCIA DEL NUMERO DE CABLES ................................. 268

9.3.4 ANALISIS DE LA INFLUENCIA DE LA LUZ DE LA ESTRUCTURA........................269

9.3.5 ANALISIS DE LA INFLUENCIA DE LA INERCIA DEL TABLERO ........................... 270

9.3.6 ANALISIS DE LOS FORMATOS DE SEGURIDAD. ................................................. 271

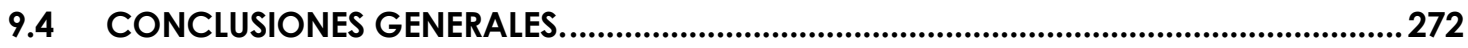

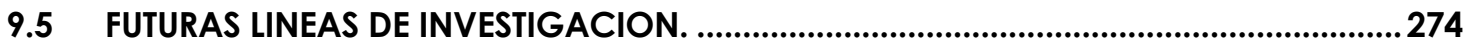

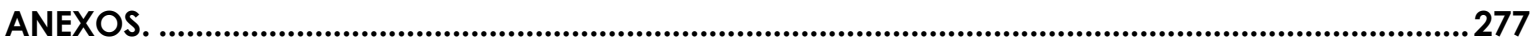

A.1. CARACTERISTICAS DE LOS MODELOS DE CALCULO EMPLEADOS. .............................279

A.2. ARCHIVOS LOGICOS PARA DEFINICION DEL PROCESO CONSTRUCTIVO...................289

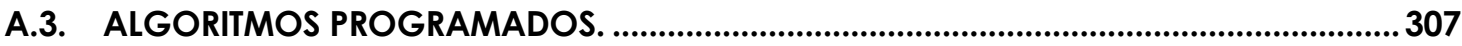

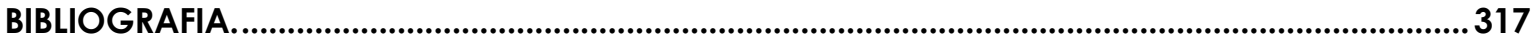




\section{CAPITULO 1.}

\section{INTRODUCCION.}

\subsection{PLANTEAMIENTO DEL PROBLEMA.}

El proceso constructivo de cualquier puente atirantado conlleva siempre una cierta complejidad e incertidumbre en la determinación de la forma de alcanzar la configuración geométrica definitiva, más aun si el puente se construye por avance mediante voladizos desde los pilonos. Es por ello que muchas de las investigaciones realizadas hasta la fecha sobre este tipo de estructuras se han focalizado en cómo determinar los axiles o deformaciones a aplicar en los tirantes, para poder conseguir alcanzar la configuración final geométrica con la que se quiere dejar el tablero en la situación de servicio.

Asociado a esos estudios teóricos, se ha desarrollado igualmente otro conjunto de investigaciones encaminadas a preparar tanto un procedimiento constructivo para la ejecución de este tipo de estructuras como herramientas para su control y seguimiento, de tal forma que se pueda conseguir realizar un control geométrico del tablero, que detecte las desviaciones respecto de la situación teórica, analice su causa e incluso pueda llegar a corregir los posibles errores encontrados.

Es a partir de este punto cuando se comienzan a encontrar diferencias entre las alternativas propuestas para ese control geométrico en el caso de los puentes con tablero metálico y de los puentes con tablero de hormigón, dado que sus procedimientos de ejecución difieren en gran medida. En concreto, en el caso de los tableros de hormigón, se podrán ir corrigiendo los errores geométricos detectados mediante el posicionado del carro de avance en cada fase del proceso constructivo, prefiriéndose en el caso de los tableros metálicos el ajuste de las tensiones de los tirantes para mitigar estos efectos. Ambos procedimientos de ajuste conllevan sus 
dificultades, además de generar ciertas incertidumbres a la hora de evaluar qué implicaciones estructurales tienen.

Por otro lado, el propio origen del esfuerzo existente sobre cada tirante al final del proceso constructivo genera ciertas incertidumbres en los formatos de seguridad a considerar en relación a esta acción, dado que parte de este esfuerzo depende directamente de la carga aplicada sobre el tirante en el instante de su colocación y parte de éste dependerá de las cargas permanentes, al actuar el tirante como elemento pasivo de la estructura y tomar el esfuerzo correspondiente a la aplicación de los pesos propios y cargas muertas.

El comienzo de este estudio nace así de la propuesta de mi Director de Tesis, D. Miguel Angel Astiz, que me planteó en su momento la realización de un estudio que evaluara tanto los problemas de ajuste geométrico en la ejecución del tablero de un puente atirantado, como las incertidumbres a nivel de formatos de seguridad que existen respecto de la acción del tesado de los tirantes. A partir de aquella propuesta se desarrolló y presentó en el año 2011, para la obtención del Diploma de Estudios Avanzados, un Trabajo de Investigación que adelantaba en cierto modo el desarrollo que se ha realizado en esta investigación.

\subsection{OBJETIVOS DE LA INVESTIGACION.}

A la vista de la anterior problemática específica de estas estructuras se ha decidido desarrollar una investigación encaminada a la obtención de dos objetivos fundamentales:

1) En primer lugar conocer la propia influencia de la aleatoriedad de la acción del tesado sobre la estructura, para de esta manera poder determinar en qué medida se puede alejar el estado real de una estructura de la situación de teórica de cálculo.

Este aspecto se analizará en función a su vez de:

- El método de puesta en carga de los tirantes; con el objetivo de conocer si existen consecuencias en la elección de un método $u$ otro de instalación de los cables, en relación al problema que se estudia. 
- Las características mecánicas y geométricas de la estructura; para conocer en qué medida ciertos parámetros influirán en este tipo de análisis.

Además este análisis se podrá realizar bien a nivel de esfuerzos bien a nivel de deformaciones, estando el primer enfoque dirigido a evaluar la modificación de los niveles de seguridad que se pueden producir en la estructura por la aleatoriedad del tesado de los tirantes, mientras que el segundo aspecto está relacionado con el propio control del proceso de ejecución, ya que a través de un control geométrico del tablero se puede indirectamente tener calibrados los efectos del tesado de los tirantes.

2) Una vez conocido y evaluado, cuantitativamente y cualitativamente, el efecto de la aplicación de procesos erróneos de colocación de cables, se puede proceder a valorar en qué medida es necesaria la aplicación de unos coeficientes de seguridad sobre estas acciones.

Estos coeficientes de seguridad serán función del distinto origen del esfuerzo generado en el tirante, por lo que adicionalmente se ha procedido a cuantificar en el peso de uno u otro factor sobre el esfuerzo total del tirante.

De esta forma, partiendo de un análisis de los efectos que se pueden producir en un caso real durante el montaje de los cables de los puentes atirantados, se puede llegar a deducir la influencia sobre los niveles de seguridad de la estructura y a su vez, a partir de dicho análisis, se puede valorar como afectar a los esfuerzos en los tirantes para mantener unos formatos de seguridad coherentes con el origen de dicho esfuerzo.

\subsection{METODOLOGIA DE LA INVESTIGACION.}

En un primer lugar, para poder avanzar hacia los objetivos planteados en esta investigación se ha profundizado en el comportamiento estructural de un tirante, estudiando los diferentes aspectos que influirán en la composición de su esfuerzo y la evolución de dicha acción a lo largo del proceso constructivo.

A continuación se ha procedido a elegir una serie de parámetros a estudiar al tener en cuenta la existencia de un proceso erróneo de montaje de los cables, es decir se han elegido una serie de factores que tendrán influencia en este aspecto y en función de 
ellos se han propuesto una serie de modelos de estructuras que representen puentes atirantados reales con esas características.

Decididas las estructuras a estudiar para realizar el estudio parámetrico anterior, se ha procedido a realizar su cálculo teórico, el cual servirá de referencia para los análisis realizados a continuación.

Es llegados a este punto cuando se ha procedido a plantear la simulación de la introducción de los errores aleatorios durante el montaje de los cables, aspecto este que es importante señalar su novedad, ya que, a día de hoy, no se han encontrado estudios específicos similares en la bibliografía consultada.

Señalar que para concretar el alcance del estudio a realizar, se ha procedido a desarrollar esta tesis para el caso concreto de puentes con tableros de hormigón y luces cortas o medias. Esta decisión se ha tomado basada en la multitud de ejemplos de puentes atirantados realizados hoy en día con esas características, lo que da cuenta de la idoneidad de este tipo de soluciones para esos rangos de luces. Esta decisión se justifica más aún dado que la aplicación en España de la tipología de puentes atirantados normalmente lo ha sido en el caso de puentes de luces cortas o medias y en muchos casos con tableros de hormigón.

Finalmente, a la vista de todo el desarrollo anterior, se procede a estudiar los formatos de seguridad de los tirantes y a proponer unas recomendaciones respecto de su tratamiento a nivel de coeficientes de seguridad.

\subsection{ORGANIZACION DE LA INVESTIGACION.}

Con toda esta perspectiva previa, se ha procedido a desarrollar el estudio que compone esta tesis, la cual se ha estructurado en 9 capítulos, incluyendo este de introducción, en cada uno de los cuales se ha analizado:

\section{- $\quad$ Capítulo 2 - Estado de la técnica.}

En este capítulo se procede a realizar una revisión de todos los planteamientos y estudios realizados hasta la fecha, en relación a los procesos constructivos de este tipo de estructuras. Se comienza así presentando una breve reseña histórica de la evolución de este tipo de estructuras, para seguidamente proceder a describir de 
forma esquemática las características del comportamiento estructural de estos puentes.

Por último, en este capítulo, se procede a realizar un desglose de los distintos métodos de cálculo que se propone aplicar a estas estructuras, señalándose de forma específica aquellos métodos que se han orientado a la evaluación e introducción de ciertos parámetros de forma no determinista o aleatoria.

\section{- Capítulo 3 - Comportamiento estructural de un tirante.}

En este capítulo, y como paso previo a los estudios a desarrollar a continuación, se procede a realizar un análisis del comportamiento estructural de un tirante en relación, por un lado a las componentes que conforman el esfuerzo axil que soporta y por otra a la influencia de los efectos reológicos del hormigón y a los posibles métodos para la colocación de los cables.

\section{- $\quad$ Capítulo 4 - Influencia del método de colocación de los cables.}

Se desarrolla en este capítulo el cuerpo principal de esta tesis, ya que en él se avanzan y detallan varios aspectos que posteriormente se volverán a utilizar en el resto de capítulos:

- En primer lugar se analiza la estructura de la que han surgido el resto de las calculadas en este estudio, obteniéndose y evaluándose los resultados del proceso de ejecución teórico.

- A continuación se procede a detallar el método desarrollado para la generación de los procesos de montaje de los cables que incorporen los posibles errores aleatorios que se considera que es posible que se generen en un caso real.

- Por último, se procede a aplicar dichos procesos erróneos siguiendo los dos métodos de instalación de los cables que se ha propuesto utilizar, el método en fuerzas y el método en deformaciones, analizándose los resultados obtenidos en esfuerzos en los propios tirantes, en el tablero y en el pilono, tanto en las situaciones de construcción como de servicio. 
Adicionalmente se han obtenido resultados en deformaciones, que permiten valorar qué implicación tiene el proceso erróneo de ejecución respecto de este aspecto particular.

- $\quad$ Capítulo 5 - Influencia del número de cables de la estructura.

Como ampliación del proceso iniciado en el capítulo anterior, en este caso se plantea la modificación del número de cables de la estructura para evaluar qué implicaciones tiene este parámetro, en lo relativo al efecto de la introducción de errores aleatorios de los cables. Así, se analiza una nueva estructura con la mitad de cables que la original y se aplican de nuevo los dos métodos de colocación de cables utilizados en el capítulo anterior, discutiéndose los resultados obtenidos.

\section{- Capítulo 6 - Influencia de la luz de la estructura.}

En este nuevo capítulo se continúa con el análisis paramétrico que permite valorar la influencia del proceso de montaje erróneo, esta vez en función de la luz de la estructura. El esquema del estudio realizado es similar al utilizado en el capítulo anterior, incorporando adicionalmente en éste una comparativa de los métodos de colocación de cables, en fuerzas o deformaciones, aplicado a las distintas estructuras analizadas hasta este punto de la investigación.

Se incluye en este caso también un estudio en deformaciones de los resultados obtenidos función de la aleatoriedad de los esfuerzos introducidos en los cables, de forma similar a lo desarrollado en el capítulo 4.

\section{- $\quad$ Capítulo 7 - Influencia de la rigidez del tablero.}

En este capítulo se analiza la última variable que se considera en el análisis paramétrico realizado en relación a los procesos erróneos de colocación de los cables que ha sido la inercia del tablero.

En primer lugar se ha realizado una comparativa de la influencia de este parámetro sobre el comportamiento teórico de la estructura, para a continuación proceder a aplicar de nuevo los errores en los cables, en este caso sólo utilizando el método de colocación de éstos mediante fuerzas. 
Adicionalmente, en este capítulo, se ha incluido también un análisis de las deformaciones verticales del tablero durante el proceso constructivo, similar a los desarrollados en los capítulos anteriores.

- Capítulo 8 - Formatos de seguridad.

En este nuevo capítulo se ha evaluado la implicación en los formatos de seguridad de los estudios realizados con anterioridad, comparando primeramente las propuestas actuales de la normativa vigente, para acto seguido plantear un análisis crítico de dichos planteamientos y añadir algunas consideraciones deducidas de todo el estudio anterior.

- Capítulo 9 - Conclusiones y futuras líneas de investigación.

Por último se finaliza este estudio proponiendo, tanto las conclusiones del trabajo desarrollado, como las futuras líneas de investigación que de este documento se podrían derivar. 


\section{CAPITULO 2.}

\section{ESTADO DE LA TECNICA.}

\subsection{DESARROLLO HISTÓRICO.}

Los puentes atirantados constituyen la tipología estructural con más desarrollo en la actualidad para puentes con un rango de las luces medias o grandes (más de $200 \mathrm{~m}$ ) debido a diferentes aspectos, tanto económicos, técnicos o incluso estéticos, lo que ha impulsado su aplicación en gran medida en los últimos años.

Aunque existen realizaciones anteriores a la segunda mitad del siglo XX [37] en los que el concepto de atirantamiento del tablero desde un pilono dispuesto por encima de él ya se venía proponiendo, como por ejemplo el Puente de Brooklyn en Nueva York (1869), el Albert Bridge en Londres (1873), el Puente Lézardrieux (1925) y el Puente DonziêreMondragon (1952) en Francia, y el Puente de Amposta (1919) y el Acueducto de Tempul de E.Torroja (1929) en España, es en los años 50 y en concreto en Alemania donde comienza el desarrollo moderno de esta tipología de puentes.

En dicho país, la necesidad de recuperar gran parte de las infraestructuras destruidas durante la Segunda Guerra Mundial, y la existencia de cauces fluviales de gran importancia, conformaron el clima adecuado para el surgimiento y desarrollo de esta tipología estructural. Es entonces cuando, a partir de los estudios teóricos del profesor F. Dischinger, se dispone del conocimiento conceptual del problema resistente y cuando se proyecta el primer puente moderno atirantado, curiosamente fuera de Alemania, que generalmente es considerado el Puente Stromsund en Suecia (1955 y 195 $\mathrm{m}$ de luz).

A continuación, es ya en la propia Alemania donde se procede a proyectar una serie muy importante de estructuras de este tipo. El ejemplo más claro son los numerosos puentes construidos sobre el Rhin, y en particular la importante colección desarrollada en la ciudad de Dusseldorf, donde se ejecutan los puentes Theodore Heuss (1957 con 260 
m de luz), Knie Bridge (1969 con 319 m de luz, Figura 1) y Oberkassel (1976 con 258 m de luz) proyectados por el equipo de F. Leonhardt y W.Andrä. Otros ejemplos iniciales de esta tipología son el Puente Severin en Colonia (1959 con 301 m de luz), el Puente de Kölhbrand en Hamburgo (1974 con 325 m de luz) y el Puente Flehe en Dusseldorf (1979 con 369 m de luz, Figura 2).

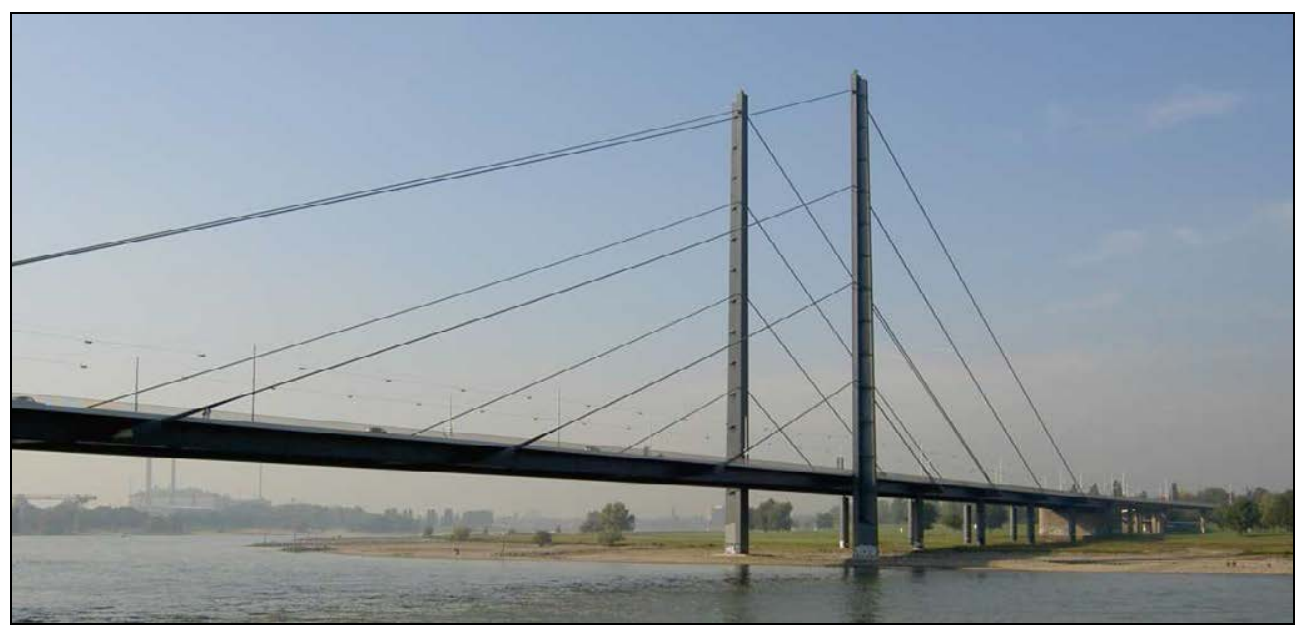

Figura 1. Knie Brucke $\left(L_{\max }=319 \mathrm{~m}\right)$. Dusseldorf. Alemania. 1969.

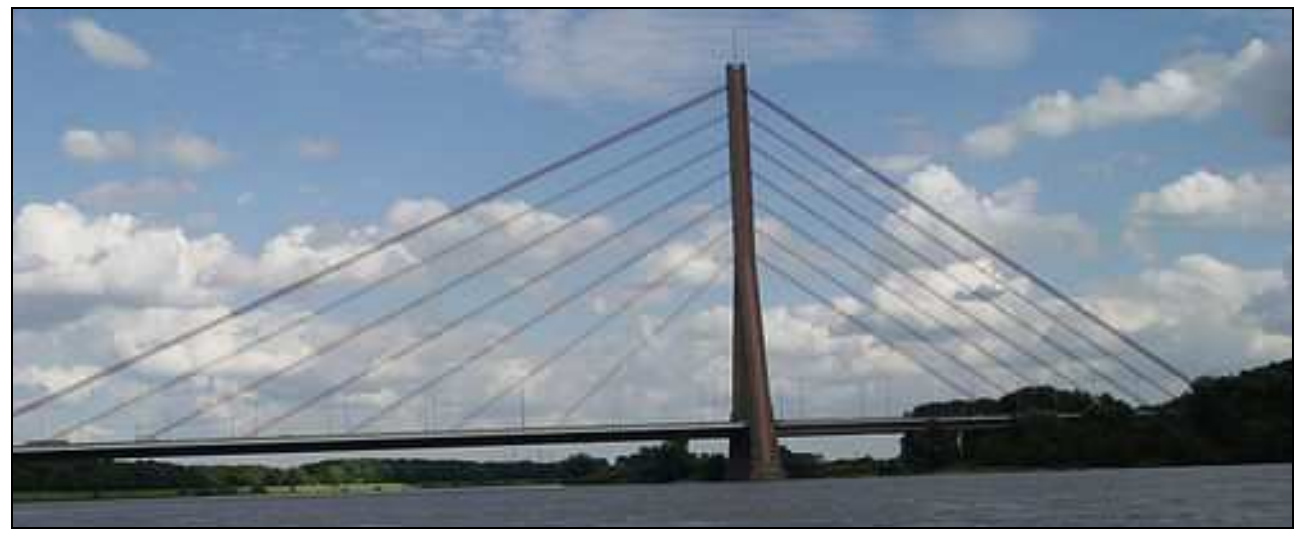

Figura 2. Puente Flehe sobre el Rhin $\left(L_{\max }=369 \mathrm{~m}\right)$. Dusseldorf. Alemania. 1979.

La característica fundamental de la mayoría de estas primeras estructuras es la presencia de atirantamientos con cables paralelos, en esquema tipo arpa, donde además la separación entre tirantes es muy grande, requiriéndose por lo tanto una gran rigidez en el tablero para soportar las importantes flexiones que se generan sobre este elemento.

El siguiente escalón en la evolución de estas tipologías se produjo a partir del diseño de H. Homberg del Puente Friedrich Ebert en Bonn (1967 con 260 m de luz, Figura 3) donde se introducen dos cambios sustanciales en la configuración de los tirantes: el primero de 
ellos es que se pasó a utilizar un gran número de tirantes de menores dimensiones dispuestos por lo tanto con separaciones entre anclajes más pequeñas, por lo que las flexiones en el tablero se reducían en gran medida y por lo tanto el canto de este elemento se podía reducir de forma significativa. El segundo aspecto es que en la zona cercana al pilono se eliminaba la presencia de tirantes para así conseguir un comportamiento de todos ellos más homogéneo, ya que los tirantes cercanos al pilono, al ser casi verticales y muy cortos, son mucho más eficaces que el resto. Este último aspecto del diseño es lo que pasó a denominarse de manera informal como "Homberg's window".

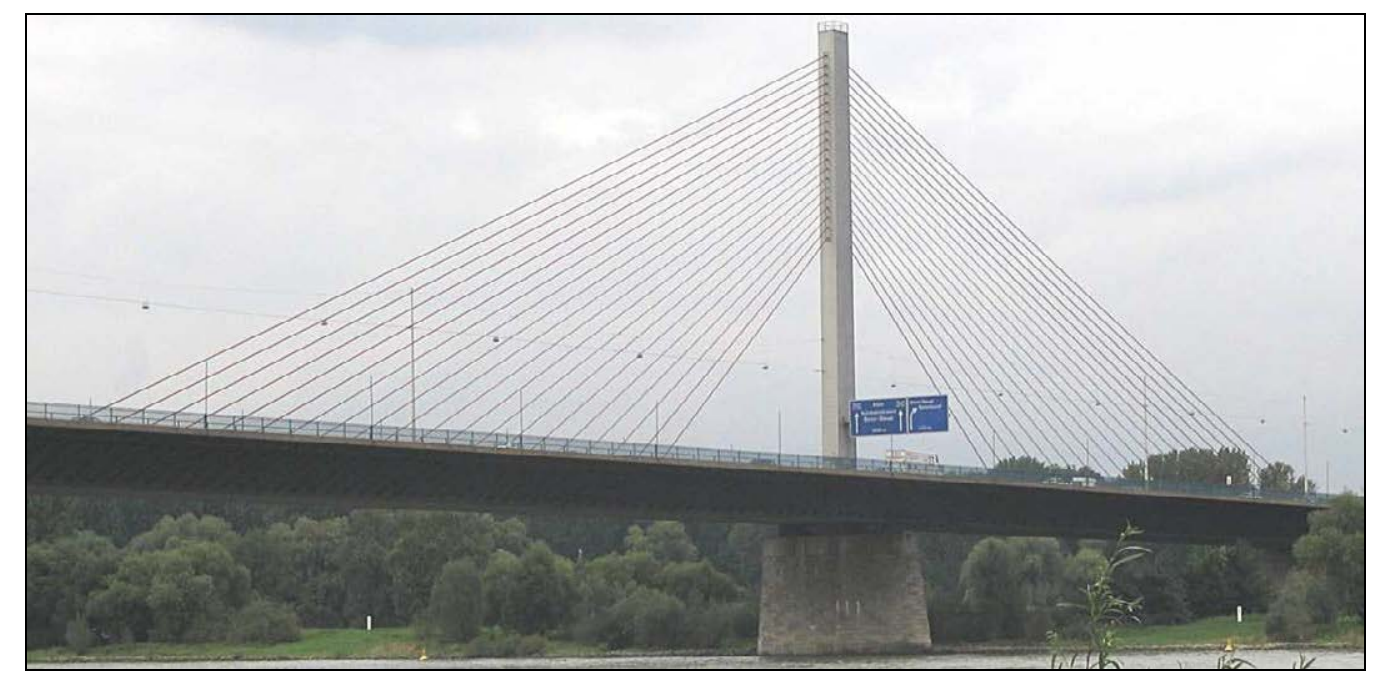

Figura 3. Puente Friedrich Ebert sobre el Rhin $\left(L_{\max }=260 \mathrm{~m}\right)$. Bonn. Alemania. 1967.

Una vez generalizado en Alemania el uso de esta tipología se comenzó a extender a otros países de Europa y a EEUU. Así, fuera de Alemania, algunos de los primeros ejemplos significativos pueden ser el Puente Zárate-Brazo Largo en Argentina (1977 con $330 \mathrm{~m}$ de luz) diseñado también por la oficina de Leonhardt\&Andrä a cargo de Reiner Saul y el Puente de Saint Nazaire en Francia (1975 con 404 m de luz, Figura 4).

Todos los anteriores ejemplos son puentes con tableros metálicos con diferentes tipologías, e incluso en algunos casos con pilonos también metálicos. 


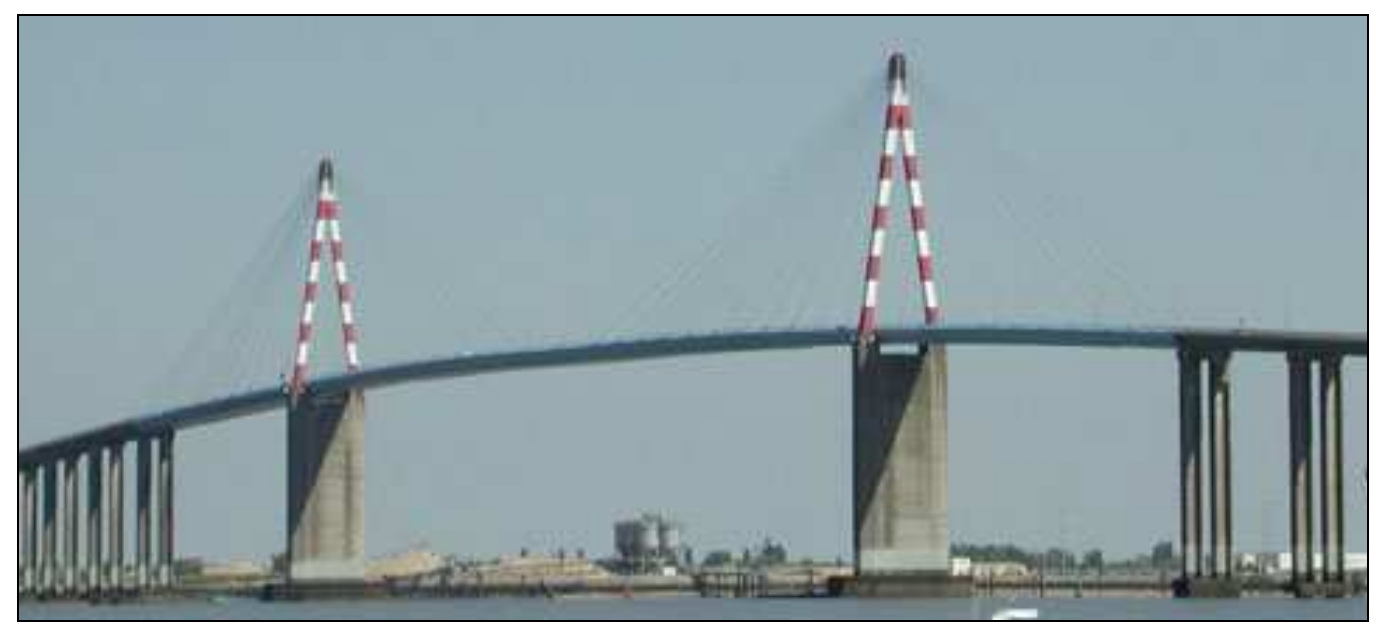

Figura 4. Puente sobre el estuario del Loira $\left(L_{\max }=404 \mathrm{~m}\right)$. Saint Nazaire. Francia. 1979.

Una de las primeras realizaciones con tablero de hormigón fue el Puente Hoescht en Alemania diseñado por U. Finsterwalder (1972 con 148 m de luz). Las realizaciones de R. Morandi son algo anteriores pero sus soluciones son muy particulares desde el punto de vista tipológico. En ellas se utilizaban cables de hormigón y pilonos con una configuración muy rígida, ya que eran los primeros ejemplos de puentes atirantados continuos. De todos ellos se puede destacar el Puente de Maracaibo en Venezuela (1962 con 6 vanos de $235 \mathrm{~m}$ de luz), el Viaducto de Polcevera en Génova (1964 con 2 vanos de 208 y 200 m de luz) y el Puente Wadi-Kuff en Libia con (1971 con 282 m de luz).

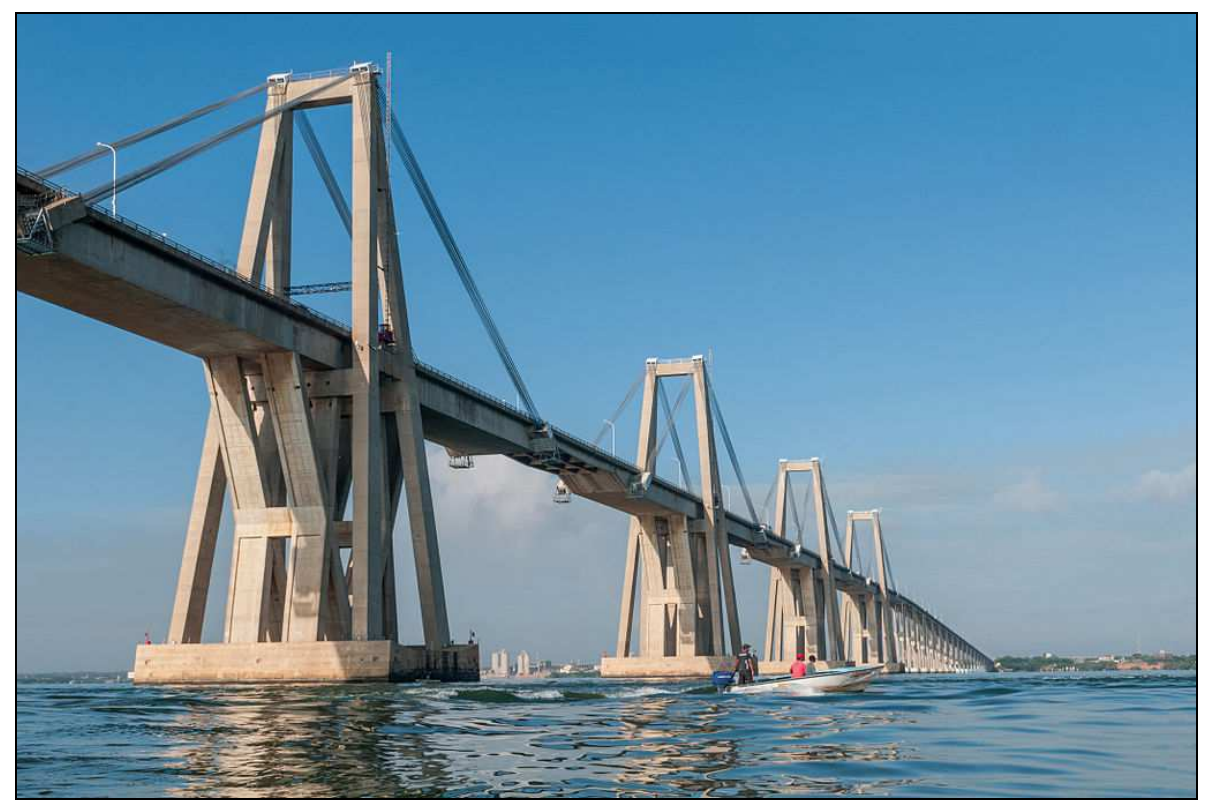

Figura 5. Puente sobre el Lago Maracaibo $\left(L_{\max }=5 \times 235 \mathrm{~m}\right)$. Venezuela. 1962. 
También como una de las primeras realizaciones de tableros de hormigón, se construye en Francia el Puente Brotonne sobre el Sena (1977 con 320 m de luz, Figura 6) diseñado por Jean Muller y su equipo, constituyendo una solución clásica de tablero en sección cajón y atirantamiento en un único plano.

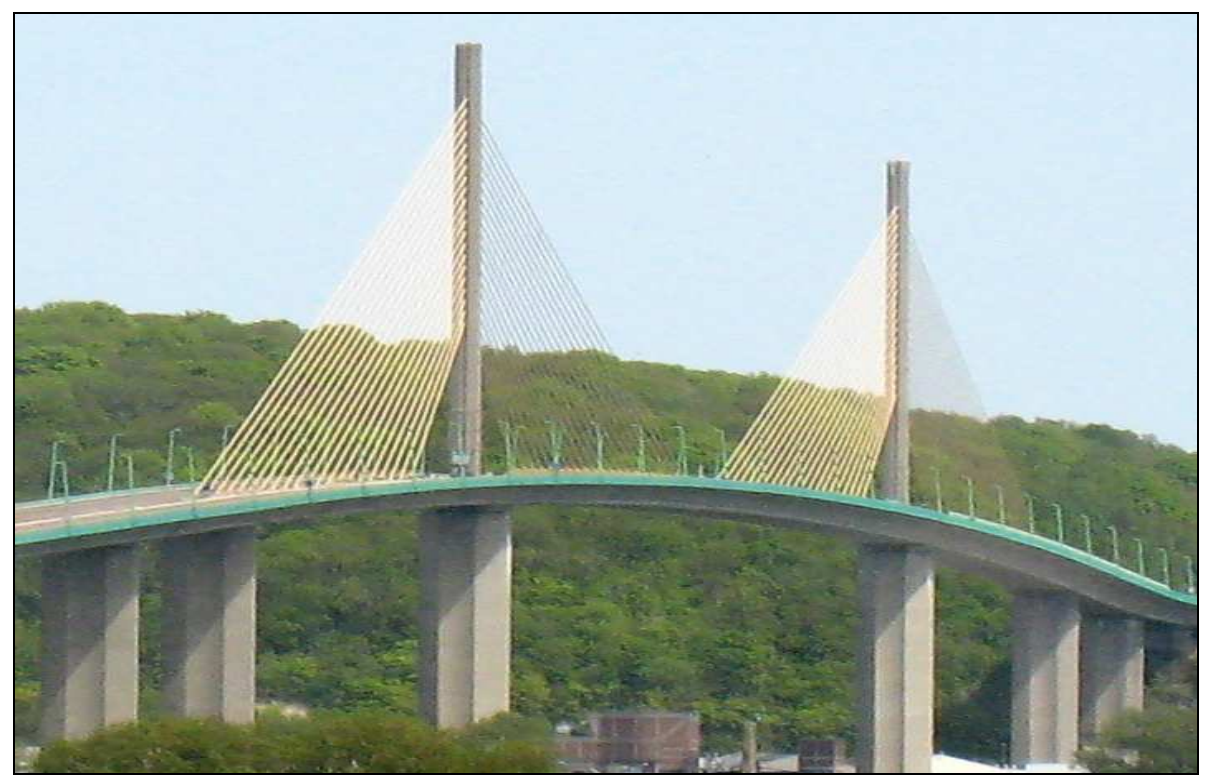

Figura 6. Puente Brotonne sobre el Sena $\left(L_{\max }=320 \mathrm{~m}\right)$. Francia. 1977.

En EEUU las primeras realizaciones de puentes atirantados son el Puente PascoKennewick (1978 con 300 m de luz), el Puente Sunshine Skyway (1982 con 366 m de luz) y el Puente East Huntington (1985 con 274 m de luz, Figura 7).

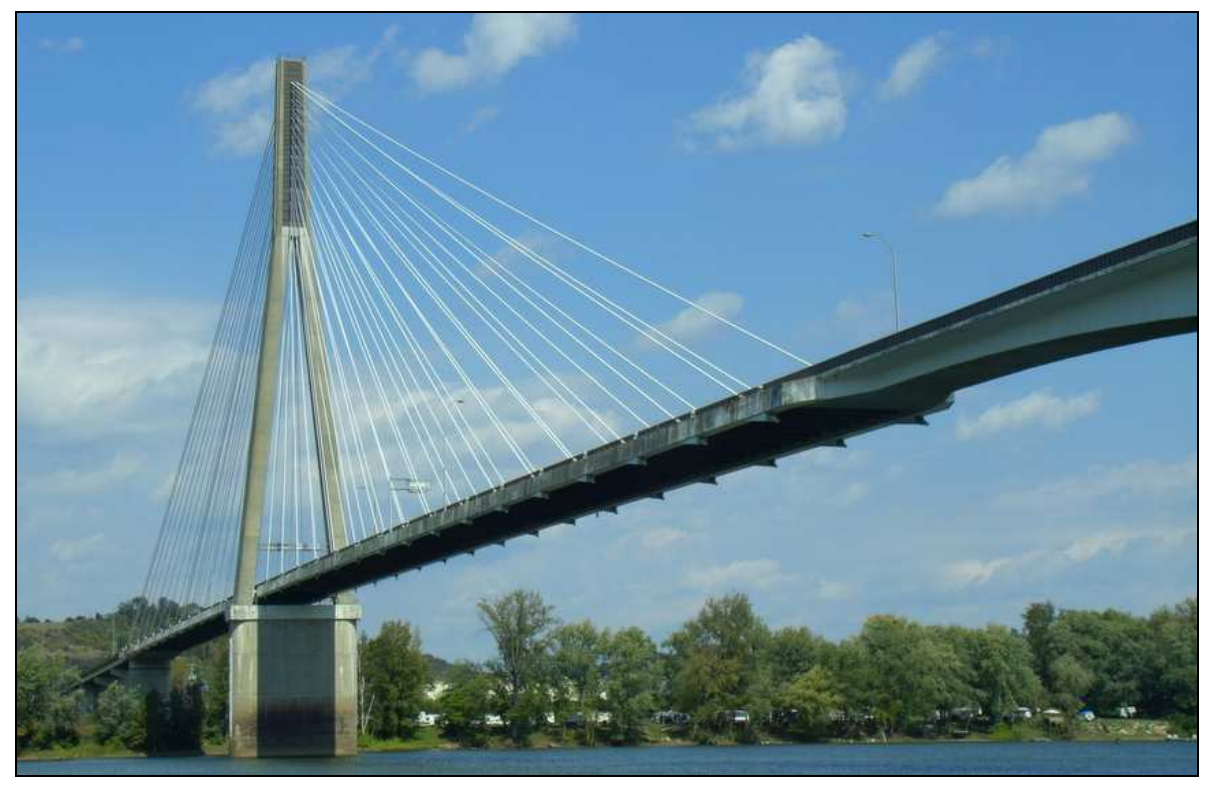

Figura 7. Puente East Huntington sobre el río Ohio $\left(L_{\max }=274 \mathrm{~m}\right)$. USA. 1985. 
Es en el caso de los tableros de hormigón, donde a partir de los años 80 del siglo XX se produce una evolución más significativa de esta tipología de estructuras. Así encontramos ejemplos singulares como el Puente Coatzacoalcos en México (1984 con $288 \mathrm{~m}$ de luz), Puente Posadas-Encarnación (1987 con 330 m de luz), Puente Helgeland en Norvega (1991 con 425 m de luz), el Puente Skarnsundet también en Noruega (1991 con 530 m de luz, Figura 8) o el Segundo puente sobre el Canal de Panamá (2004 con 420 m de luz).

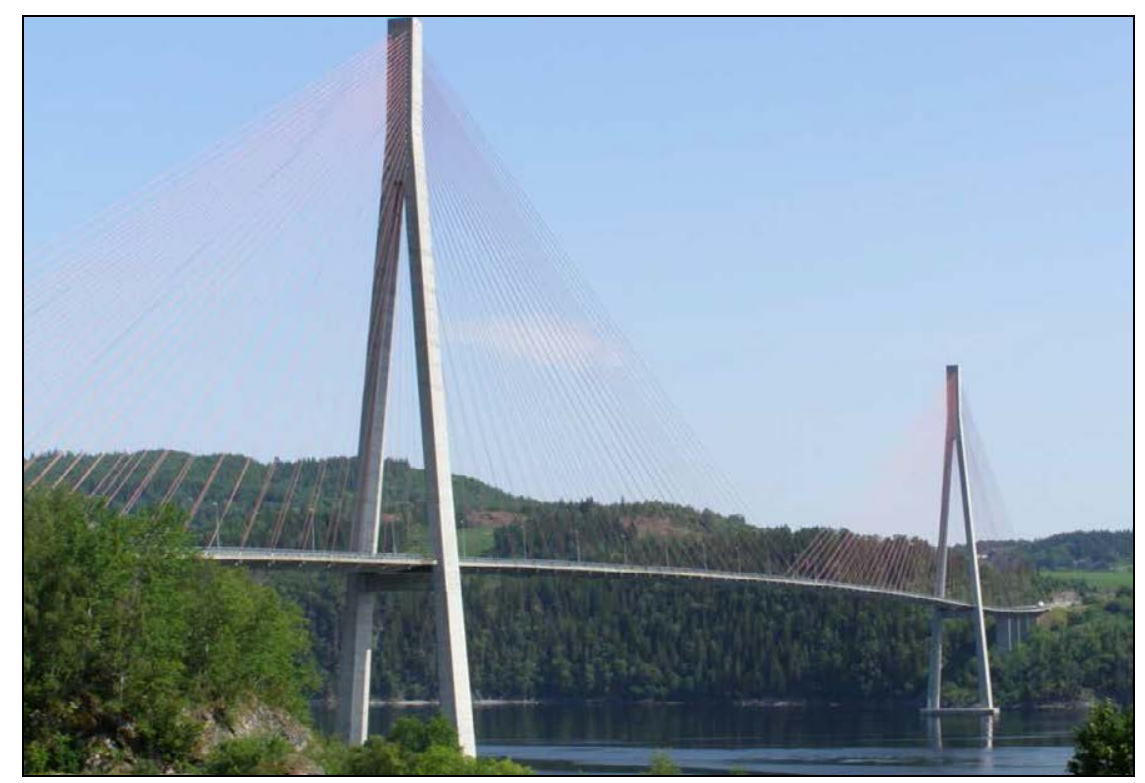

Figura 8. Puente Skarnsundet $\left(L_{\max }=530 \mathrm{~m}\right)$. Trondheim. Noruega. 1991.

Por otra parte el comienzo del uso de tipologías con tablero mixto se produce a partir del proyecto de la oficina de Schlaich\&Bergermann del Puente Hooghly en Calcuta (1992 con 457 m de luz, Figura 9). Hay que señalar que este puente se comenzó a construir en los años 70, pero numerosos contratiempos de todo tipo llevaron a su terminación en fecha tan tardía como la señalada. Así antes que la anterior estructura, aunque con diseño posterior, se terminó otro puente con tablero mixto como fue el Puente Annacis en Vancouver (1986 con $465 \mathrm{~m}$ de luz, Figura 10). Poco tiempo después que los anteriores ejemplos se terminó el Puente Yang Pu en China (1993 con 602 m de luz, Figura 11).

Este último puente marca la irrupción de China en la construcción de este tipo de estructuras, aspecto que resulta fundamental a partir de la primera década del siglo XXI. 


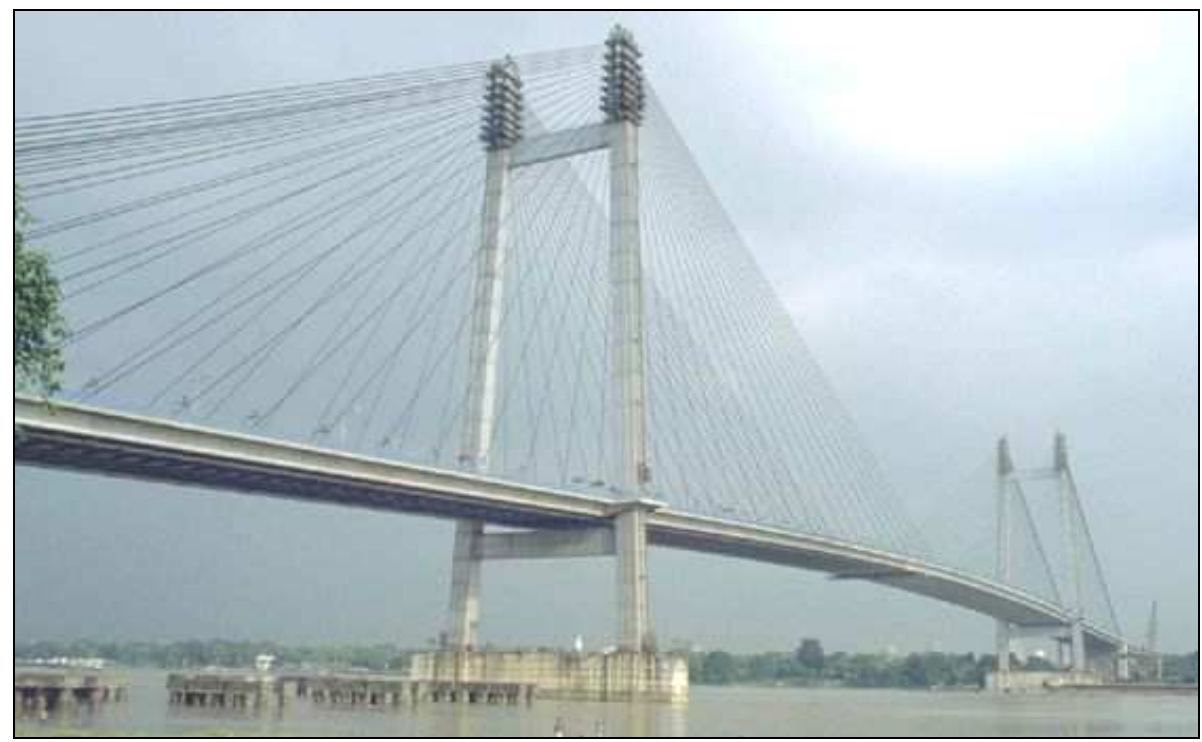

Figura 9. Puente Hoogly $\left(L_{\max }=457\right.$ m ). Calcuta. India. 1992.

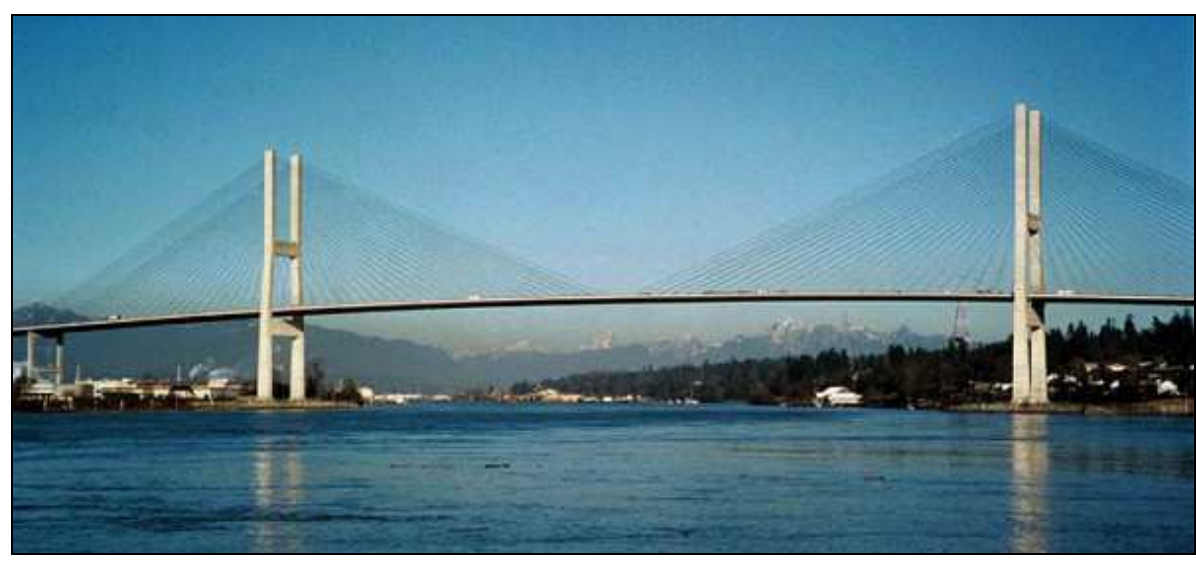

Figura 10. Puente Annacis ( $L_{\max }=465 \mathrm{~m}$ ). Vancouver. Canadá. 1986.

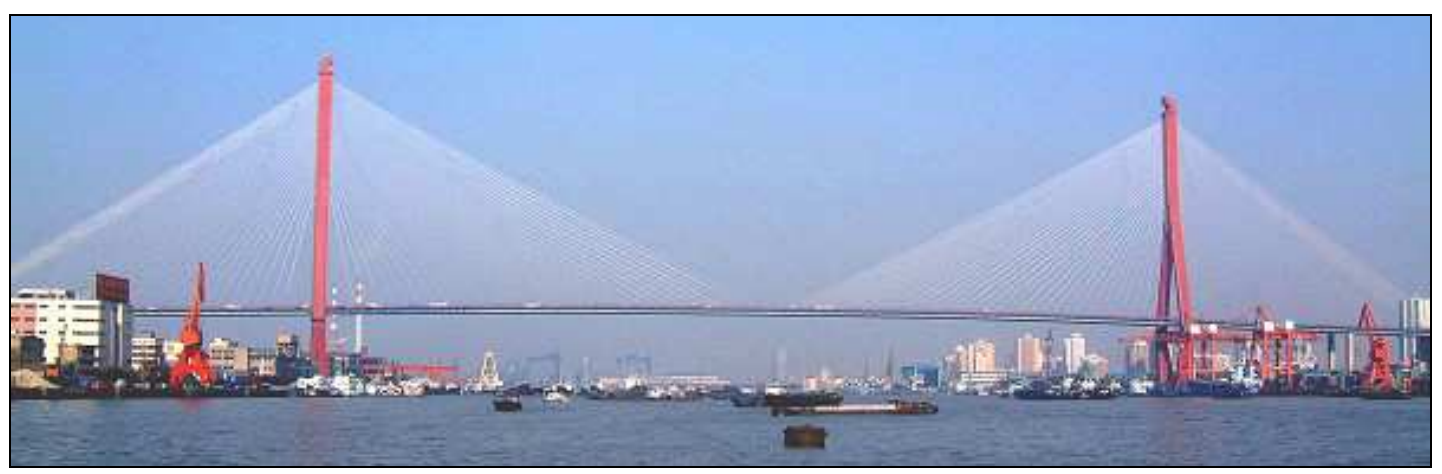

Figura 11. Puente Yang $P u\left(L_{\max }=602 \mathrm{~m}\right)$. Shangai. China. 1993.

El siguiente salto tecnológico en la construcción de Puentes atirantados se produce con la ejecución del Puente de Normandía diseñado por el equipo de M. Virlogeux (1995 con 856 m de luz, Figura 12) conformado por un tramo central metálico, siendo el resto 
del tablero de hormigón. Con esta estructura se comienza a vislumbrar que el rango de aplicabilidad de esta tipología se podía incrementar hasta el caso de luces de más de $1000 \mathrm{~m}$, rango que hasta ese momento era exclusivo de los puentes colgantes.

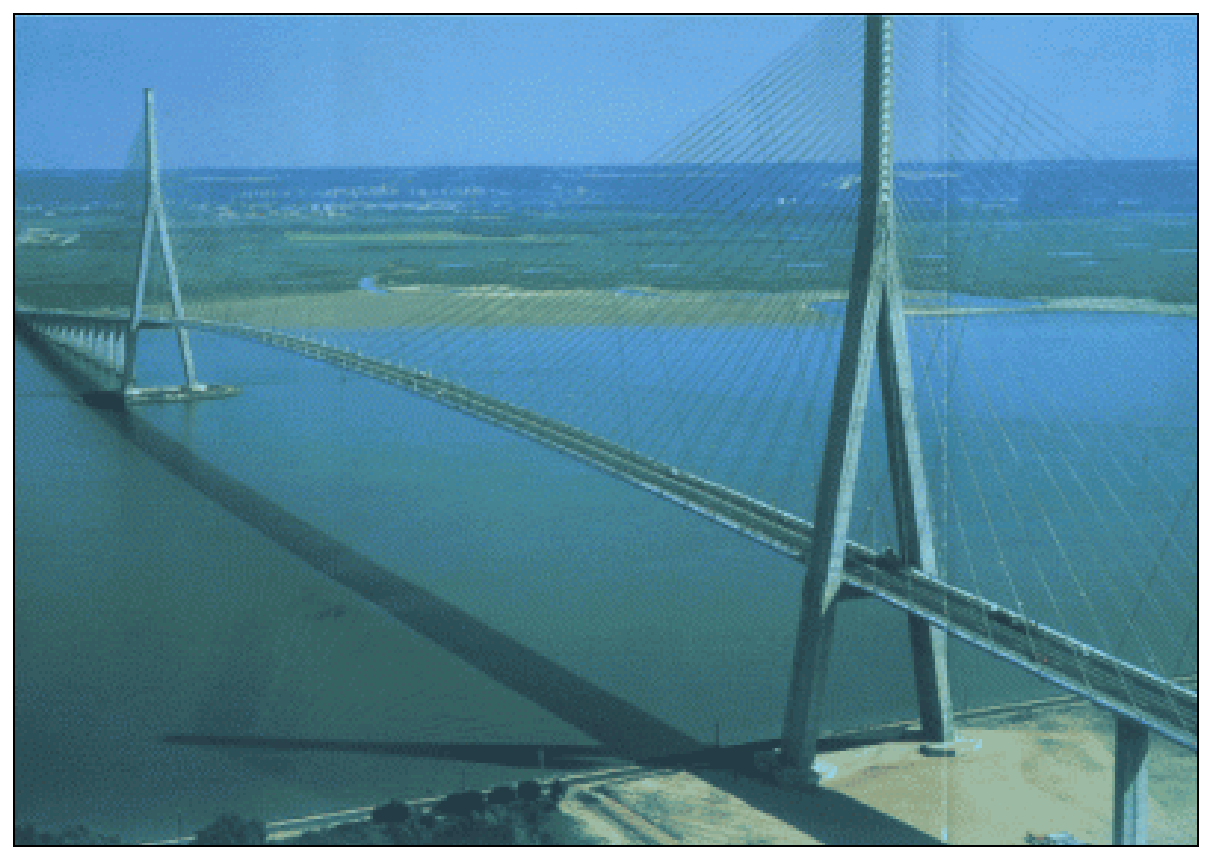

Figura 12. Puente de Normandía sobre el Sena $\left(L_{\max }=856 \mathrm{~m}\right)$. Francia. 1997.

Así, en las 2 últimas décadas, se pueden destacar algunos ejemplos singulares de puentes con tableros metálicos y mixtos metálicos como el Puente Kap Shui Mun en Hong Kong (1995 con 431 m de luz), el Puente del Oresund entre Dinamarca y Suecia (2000 con 490 m de luz, Figura 13) el Puente Rion-Antirion sobre el estrecho de Corinto en Grecia (2004 con 3 vanos de 560 m de luz, Figura 14) diseñado por la oficina de Jacques Combault, el Viaducto de Millau en Francia (con 6 vanos de $342 \mathrm{~m}$ de luz, Figura 15), diseñado por el equipo de M. Virlogeux, así como la numerosa serie de puentes construidos en China como el Puente E'dong sobre el rio Yangtse (2010 con 926 m de luz), el Puente JiuJiang también sobre el Yangtse (2013 con $818 \mathrm{~m}$ de luz) y el Puente Jingyue igualmente sobre el río Yangtse (2010 con $810 \mathrm{~m}$ de luz).

Es de destacar que, en la fecha actual, de los 20 puentes atirantados con mayor luz del mundo, 15 de ellos han sido construidos en China. 


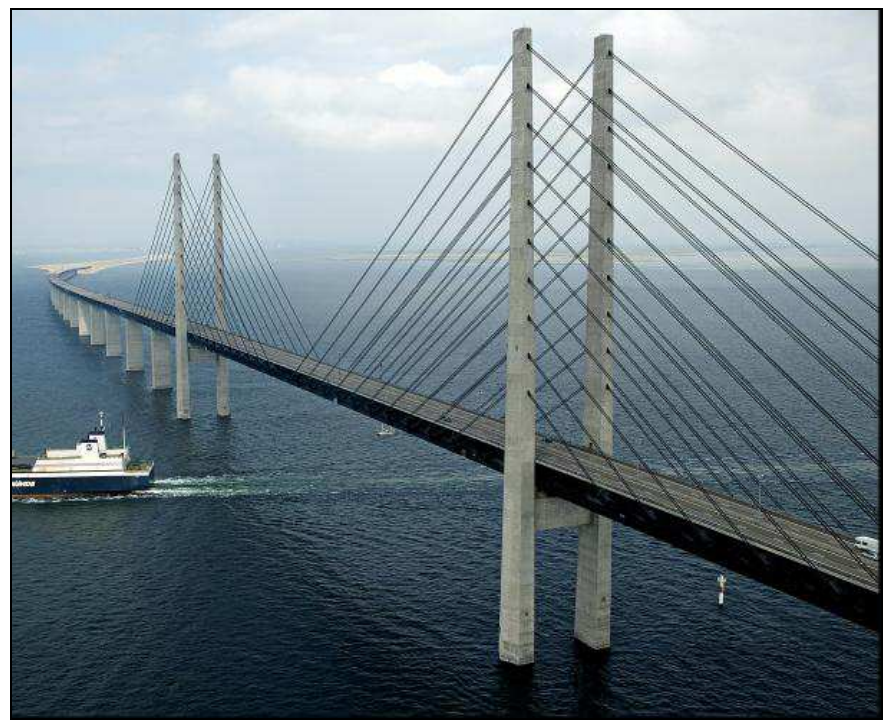

Figura 13. Puente Oresünd. ( $L_{\max }=490 \mathrm{~m}$ ). Suecia-Dinamarca. 2000.

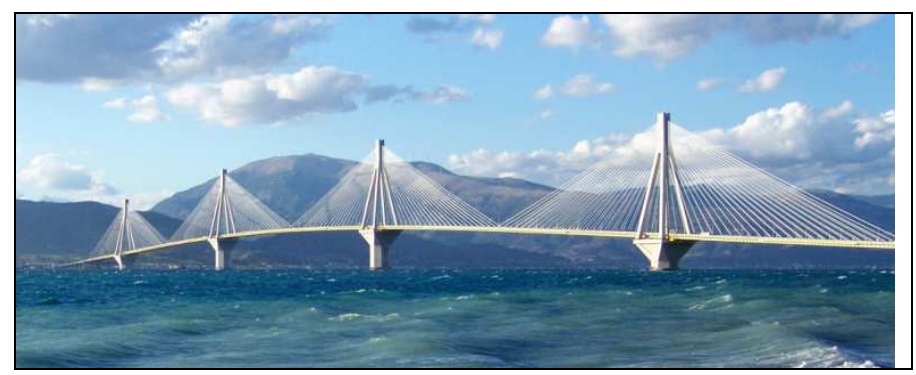

Figura 14. Puente Rion-Antirion sobre el Estrecho de Corinto. $\left(L_{\max }=3 \times 560 \mathrm{~m}\right)$. Grecia. 2004.

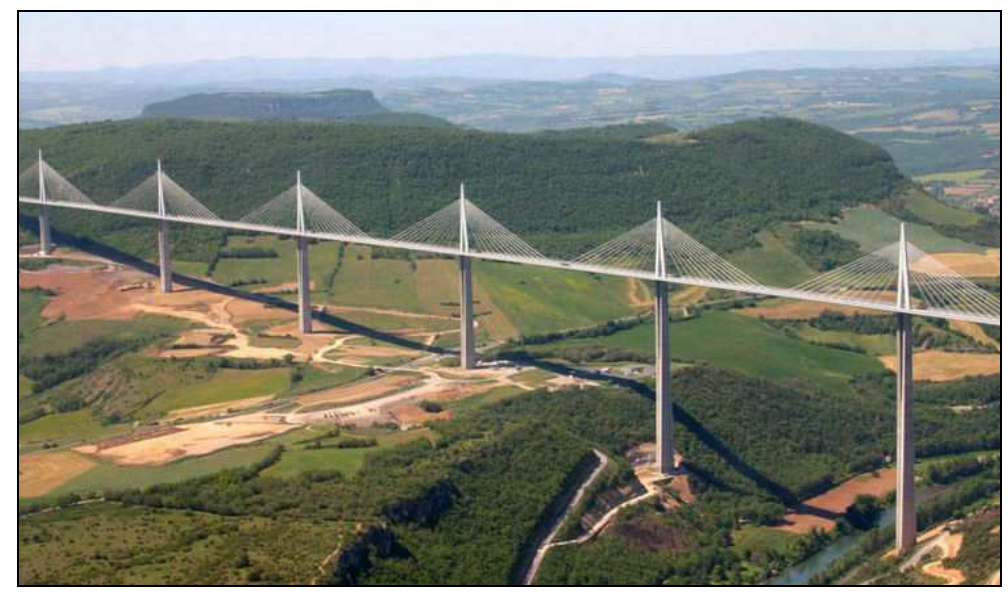

Figura 15. Viaducto de Millau. $\left(L_{\max }=6 \times 342 m\right)$. Francia. 2004.

Finalmente hay que destacar que, aunque hasta hace poco tiempo el rango de luces se extendía hasta los $890 \mathrm{~m}$ del Puente Tatara en Japón (Figura 16), recientemente se han inaugurado el Puente Stonnecutters en Hong Kong (2009 con 1018 m de luz), en China el Puente Sutong sobre el rio Yangtze de $1088 \mathrm{~m}$ de luz (Figura 17) y finalmente el Puente 
de la isla Russky en Vladivostok (2012 con 1105 m de luz, Figura 18), que representa el record mundial en el momento de escribir este documento. Es decir, que las luces máximas que se pueden alcanzar con esta tipología estructural se extiende hoy en día claramente más allá de los $1000 \mathrm{~m}$.

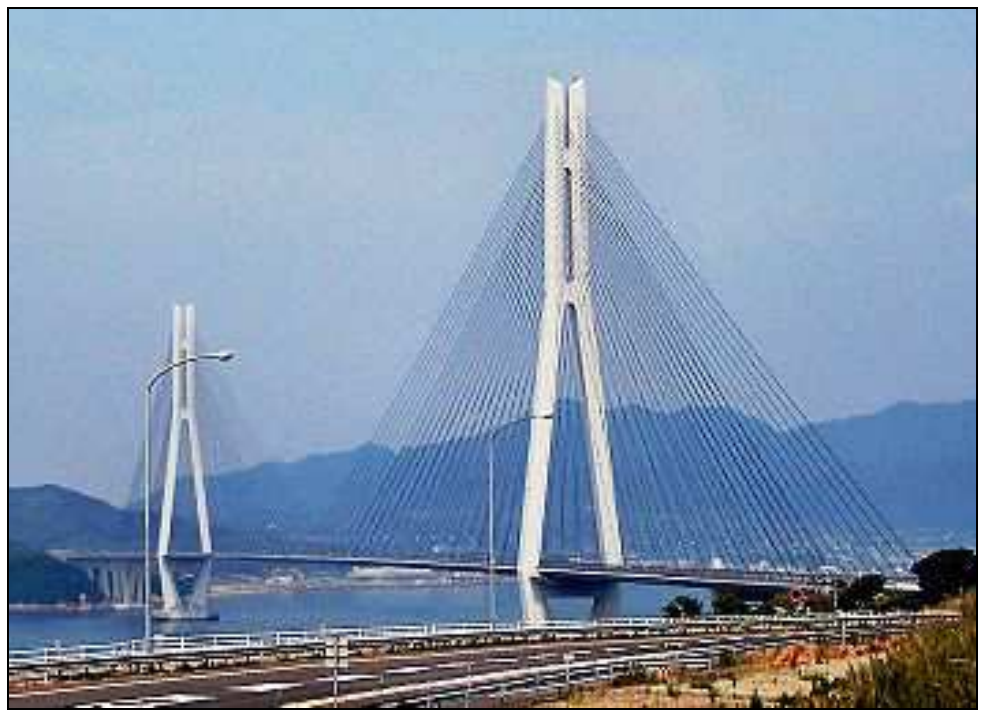

Figura 16. Puente Tatara sobre el mar de Seto $\left(L_{\max }=890 \mathrm{~m}\right)$. Hiroshima. Japón. 1999.

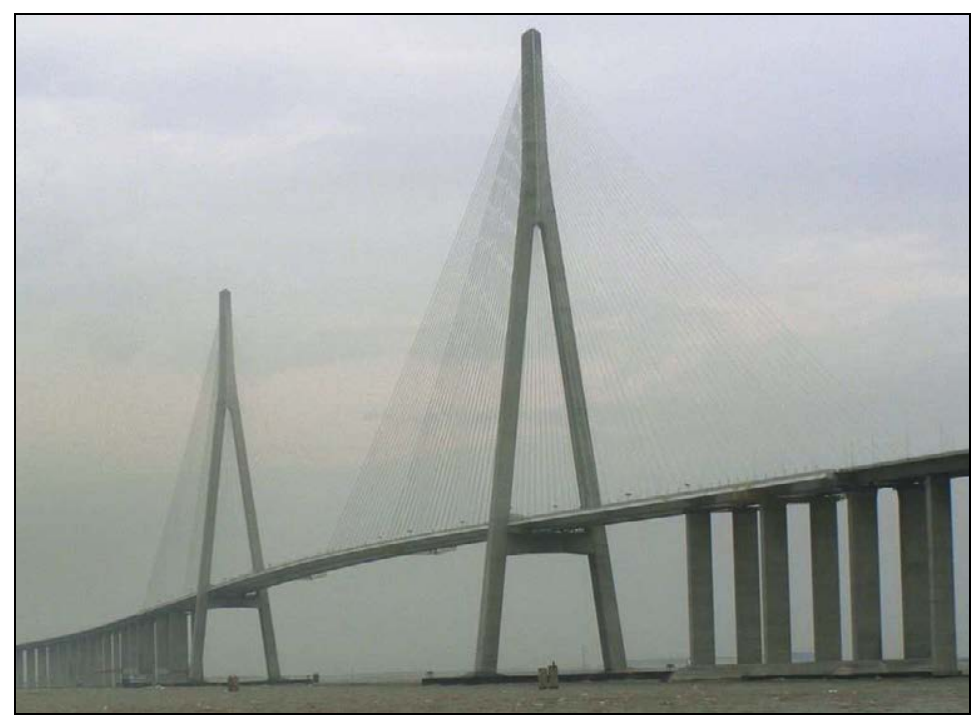

Figura 17. Puente Sutong sobre el Yangtze $\left(L_{\max }=1088 \mathrm{~m}\right)$. China. 2009. 


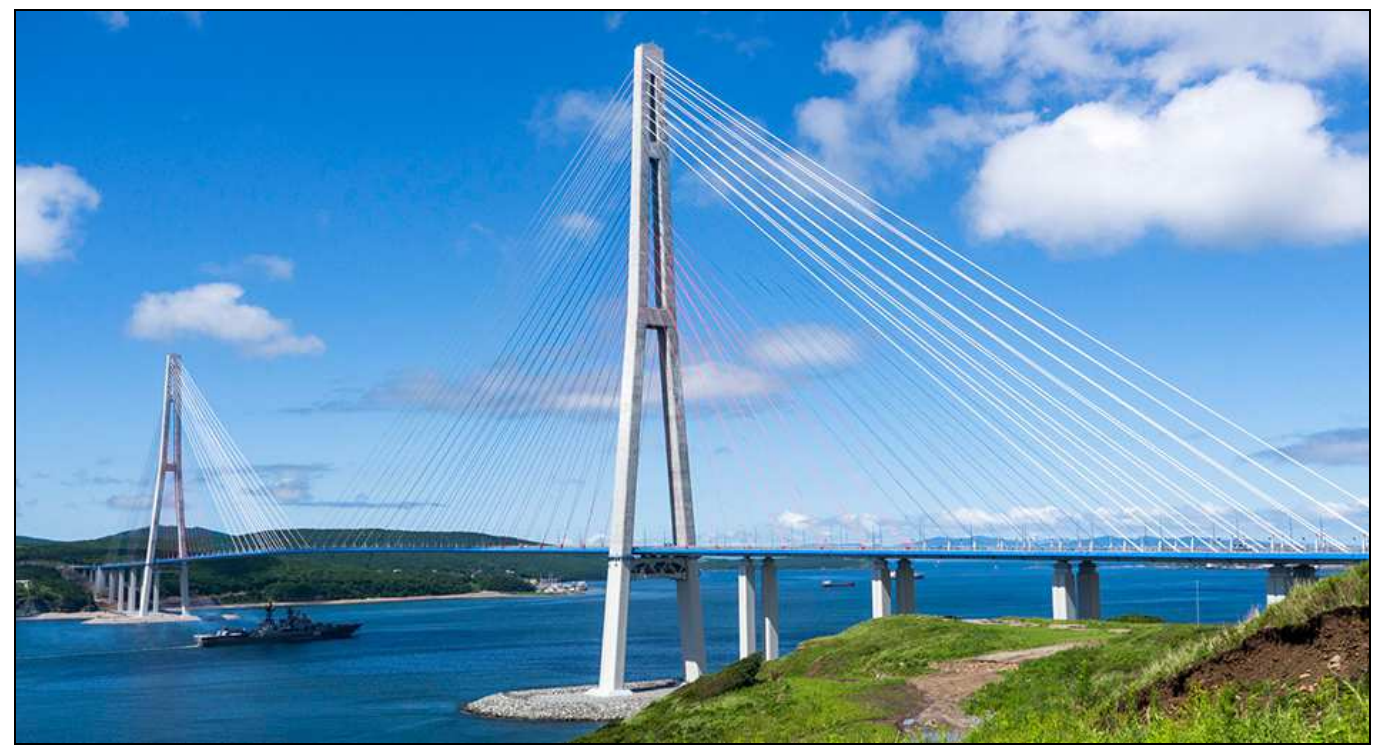

Figura 18. Puente Russky ( $L_{\max }=1105 \mathrm{~m}$ ). Vladivostok. Rusia. 2012.

En el caso de España, las primeras realizaciones importantes con esta tipología de puentes son: el Puente de Sancho el Mayor en Navarra sobre el río Ebro (1978 con 146 m de luz, Figura 19) proyectado por la oficina de C. Fernández Casado y el Puente sobre el estrecho de Rande en Pontevedra (1978 con 400 m de luz, Figura 20) de F. de Miranda y F. del Pozo.

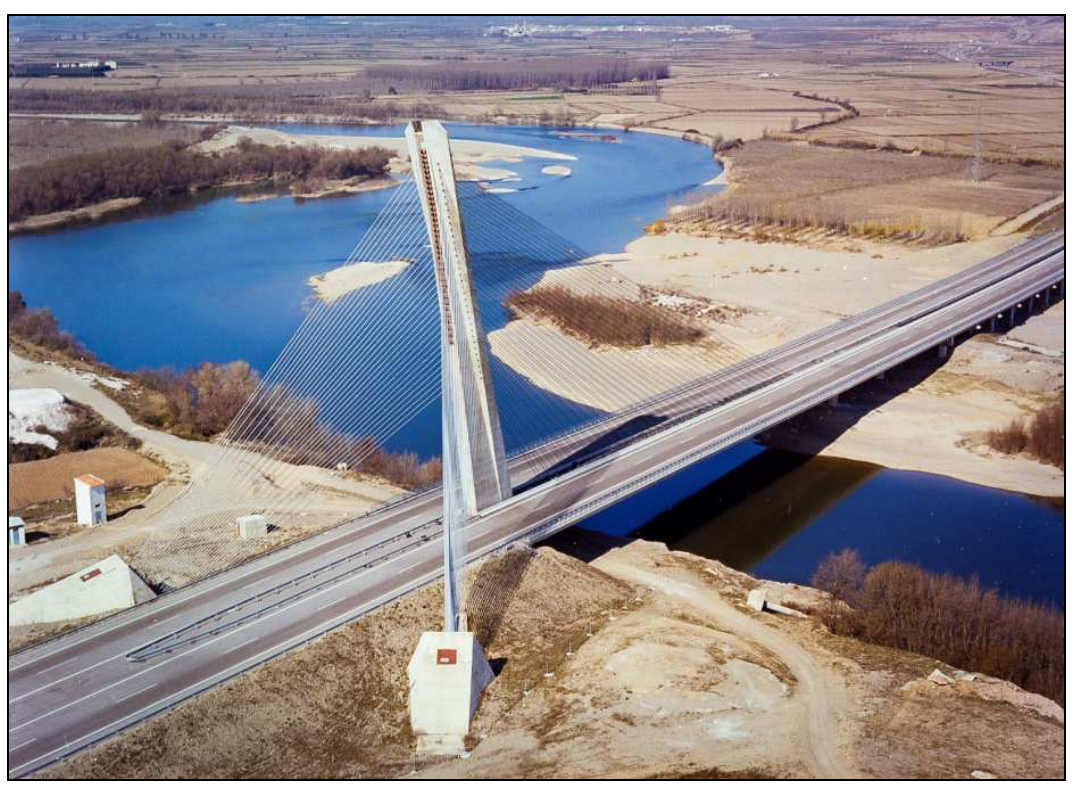

Figura 19. Puente Sancho el Mayor sobre el Ebro $\left(L_{\max }=146 \mathrm{~m}\right)$. Castejón. España. 1978. 


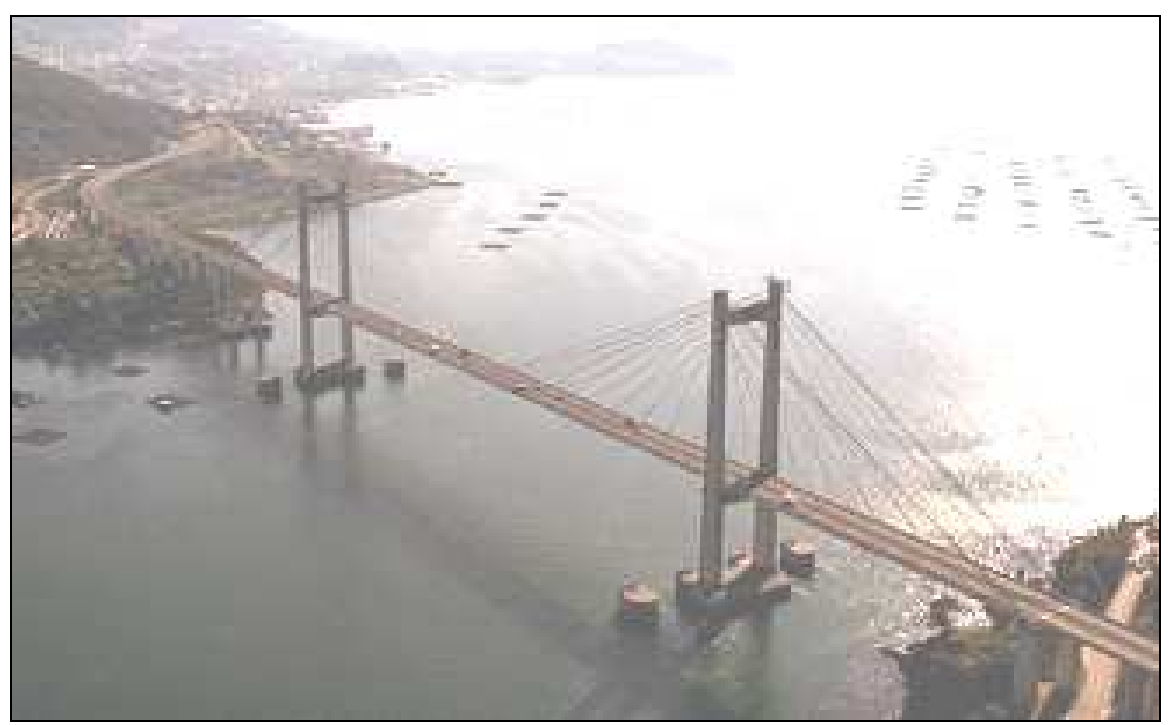

Figura 20. Puente de Rande $\left(L_{\max }=400 \mathrm{~m}\right)$. Vigo. España. 1978.

Otras realizaciones importantes en España las constituyen: el Puente Fernando Reig en Alcoy (1986 con $132 \mathrm{~m}$ de luz) [41][42], el Puente de Sama de Langreo (1990 con $130 \mathrm{~m}$ de luz)[71], el Puente del Centenario en Sevilla (1992 con $265 \mathrm{~m}$ de luz)[80], el Puente de Ayamonte (1992 con $320 \mathrm{~m}$ de luz), el Viaducto de las Arenas (1994, con 4 vanos de $105 \mathrm{~m}$ de luz)[8], el Puente de Colindres (1994 con 2 vanos de $125 \mathrm{~m}$ ), el $4^{\circ}$ Puente sobre el río Guadiana en Badajoz (1994 con 136 m de luz) [85][85], el Puente sobre el río Lérez en Pontevedra (1996 con $125 \mathrm{~m}$ de luz) [36], el Viaducto sobre el río Iregua (2000 con 120 m de luz) [30], el Puente sobre el río Besaya (2006 con 135 m de luz)[38], el Puente de Talavera de la Reina (2011 con 318 m de luz), y el Puente Príncipe de Viana sobre el río Segre en Lleida (2012 con 86+75 m de luz)[72].

Pero habría que destacar como principal realización de esta tipología en España el Puente sobre el embalse de Barrios de Luna en León (1984 con 440 m de luz, Figura 21)[68], diseñado igualmente en la oficina de proyectos de C. Fernández Casado, y que constituyó en su momento el record del mundo de luz en puentes atirantados.

Indicar que esta última estructura recientemente se ha visto superada por el Nuevo puente sobre la Bahía de Cádiz (2015 con 540 m de luz, Figura 22) [65][66], inaugurado en Septiembre de 2015. 


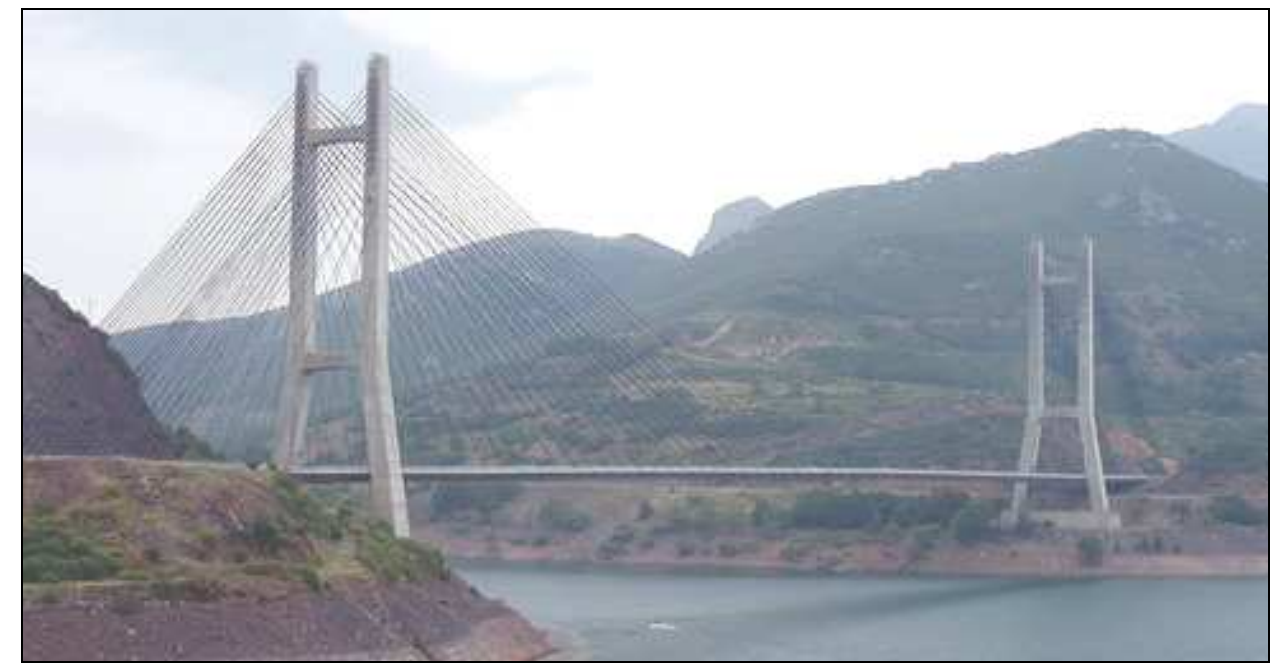

Figura 21. Puente sobre el embalse de Barrios de Luna $\left(L_{\max }=440 \mathrm{~m}\right)$. España. 1984.

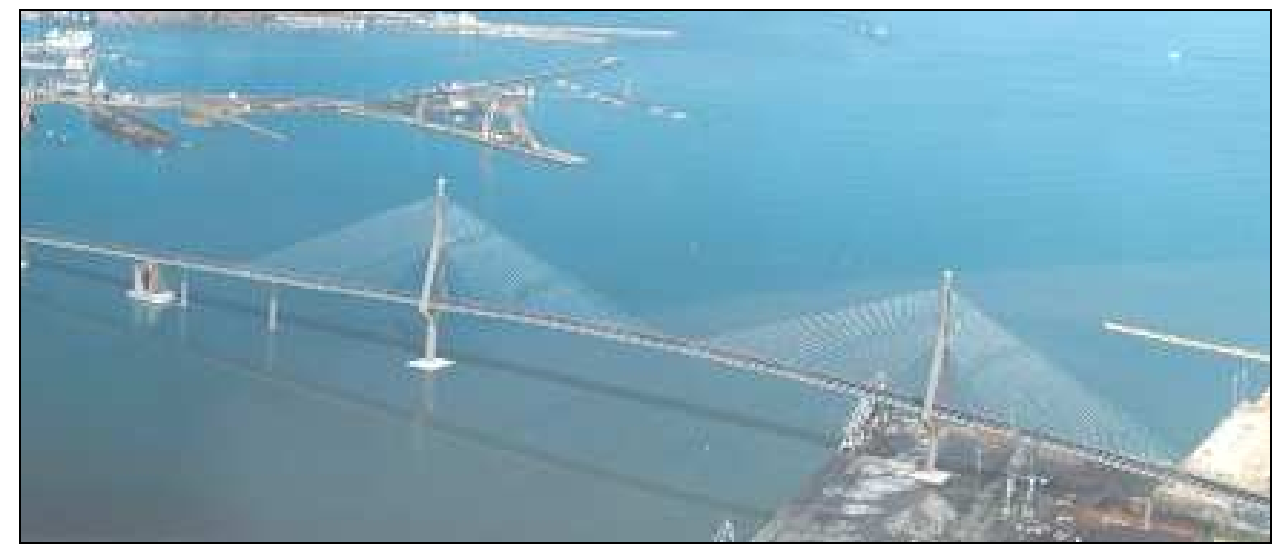

Figura 22. Nuevo puente sobre la Bahía de Cádiz $\left(L_{\max }=540 \mathrm{~m}\right)$. España. 2014. 


\subsection{MORFOLOGIA DE LOS PUENTES ATIRANTADOS.}

La morfología de estas estructuras proviene del planteamiento que dio origen a esta tipología de puentes, los cuales se desarrollaron para salvar luces medias o grandes, generadas al suprimir los apoyos intermedios, gracias a la presencia de tirantes que fijaban el tablero a los pilonos. En esencia, se trataba de sustituir los apoyos eliminados por las coacciones verticales que imponen los tirantes.

Dentro de la propia morfología de estas estructuras se debe así contemplar la influencia de la geometría del tablero, de las pilas, del esquema elegido para el atirantamiento y de las vinculaciones establecidas entre tablero y pilas, para así poder conocer cómo será su comportamiento frente a la actuación de las cargas aplicadas sobre el conjunto. De cada uno de estos aspectos se procede a continuación a realizar una pequeña descripción de las diferentes alternativas existentes para su diseño, así como de las repercusiones, a nivel del comportamiento estructural del puente, que tiene cada una de ellas. Todo este planteamiento se realiza de cara a una mejor comprensión e interpretación de los resultados que se obtengan en esta investigación.

\subsubsection{Tipología de los atirantamientos.}

En primer lugar, para el caso del esquema del atirantamiento se plantean tres tipologías fundamentales: atirantamiento en arpa, en abanico o en semi-arpa. En la primera de ellas todos los tirantes se colocan paralelos. En la segunda los tirantes se anclan con una separación regular en el tablero y se concentran en el extremo superior del fuste de la pila. Por último, el caso del esquema en semi-arpa se sitúa como un caso intermedio entre los dos anteriores.

Estructuralmente la efectividad de los tirantes es mayor en el caso de los tirantes en abanico según Podolny\&Scalzy [84], ya que su oblicuidad respecto del tablero es menor que en los otros casos, sobretodo en el caso de los tirantes más cortos, además de que su configuración geométrica es más estable al conformar cada pareja de tirantes consecutivos un triángulo. Por este motivo, los axiles obtenidos en la solución en abanico serán menores que en la solución en arpa y por lo tanto el consumo de acero en los tirantes será más reducido. 
Tiene en contra esta disposición la dificultad de la ejecución del nudo de anclaje de los tirantes en la pila, por la concentración que se produce en este punto de todos ellos, lo que obligará a un diseño muy cuidadoso de esta zona. La tendencia en la actualidad es el empleo de los esquemas en semi-arpa que consigue un equilibrio entre las ventajas e inconvenientes de las dos tipologías anteriores.
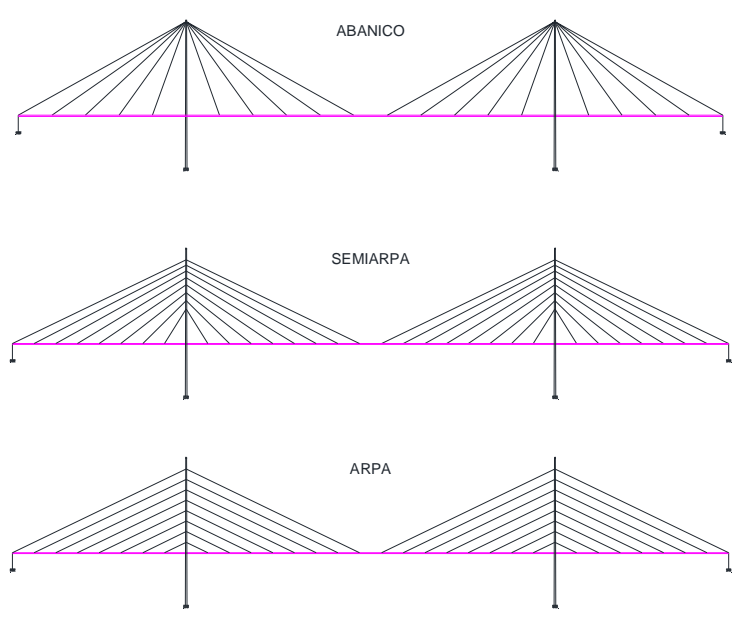

Figura 23. Esquemas posibles de atirantamientos.

Por otra parte, la separación entre anclajes originalmente se estableció en valores elevados, que, aunque reducía el número de éstos a colocar, implicaba tableros con una gran rigidez a flexión para poder resistir los importantes esfuerzos que sobre ellos se generaban. Hoy en día la tendencia es la contraria, al fijarse separaciones entre tirantes del orden 4 a 10 m en el caso de tableros de hormigón, con el objetivo de reducir el volumen del tablero, aun a costa de incrementar el número de tirantes.

Estas separaciones vendrán también condicionadas por el tipo de tablero y el método constructivo elegido, ya que por ejemplo, para el caso de tableros de hormigón construidos por avance en voladizo, las distancias entre anclajes vendrán condicionadas por la capacidad del carro de avance a utilizar, es decir por la longitud de dovela a ejecutar en cada fase en voladizo.

Espacialmente el atirantamiento se puede producir en un solo plano, anclándose al eje del tablero, caso en el que es necesario contar en éste con secciones cerradas, o por lo menos con suficiente inercia a torsión para soportar la disposición de cargas excéntricas, debidas bien a la actuación de sobrecargas no centradas, bien por la aplicación de cargas transversales de viento o sismo. La otra posibilidad es la disposición de dos planos de atirantamiento, anclándose éstos en ambos bordes del 
tablero, por lo que, en este caso, sí podría plantearse usar una sección abierta con inercia a torsión reducida, ya que las cargas excéntricas se resistirán por el par de fuerzas generado por la acción de los tirantes.

Un condicionante de partida para la elección de la distribución espacial de los tirantes puede ser la configuración geometrica del tráfico a soportar por el puente. Por ejemplo, si el tráfico a soportar corresponde a una calzada con sólo 2 carriles de tráfico rodado de doble sentido, se deberán proyectar dos planos de atirantamiento, mientras que en el caso de que dicho tráfico se disponga en calzadas de 2 o más carriles es posible plantear la disposición de un sólo plano central de tirantes.

A su vez esta disposición espacial no solo condiciona la geometría del tablero, si no que el pilono deberá tener una geometría compatible con este esquema. En definitiva que será en gran medida la configuración del atirantamiento elegido la que determine la geometría del tablero y pilono.

\subsubsection{Tipologia de los tableros.}

La tipología de los tableros empleados en los puentes atirantados se puede analizar tanto desde el punto de vista geométrico como desde el punto de vista del tipo del material empleado en su conformación.

Como se acaba de señalar en el epígrafe anterior, desde el punto de vista geométrico, la tipología de los tableros diseñados depende a su vez directamente de la configuración de los tirantes proyectada en cada caso particular.

Por ejemplo, si se adopta una geometría para los tirantes consistente en 2 planos independientes (Figura 24), bien sea anclados a pilonos de 2 fustes o bien a un único pilono central en forma de $\mathrm{A}$, los anclajes sobre el tablero deberán situarse en ambos bordes de la calzada, resultando así la necesidad de plantear en dichos puntos un elemento resistente dispuesto en sentido longitudinal que recoja todos estos anclajes. Por ello, en este caso, normalmente se proyectará una sección transversal con vigas de borde de gran rigidez, sobre las que acometerán un conjunto de vigas transversales que permitirán a su vez el apoyo de la superficie de rodadura. Dicha superficie será materializada generalmente mediante una losa de hormigón, o bien en ciertos casos mediante una losa ortótropa metálica.

Adicionalmente, en este caso también, al disponerse de 2 planos de tirantes con capacidad para absorber mediante un par de fuerzas las torsiones generadas sobre el 
tablero, no es necesario que la sección transversal del tablero tenga a su vez rigidez a torsión, por lo que las secciones abiertas, consistentes en vigas de borde (bijácenas), tendrán un buen funcionamiento.
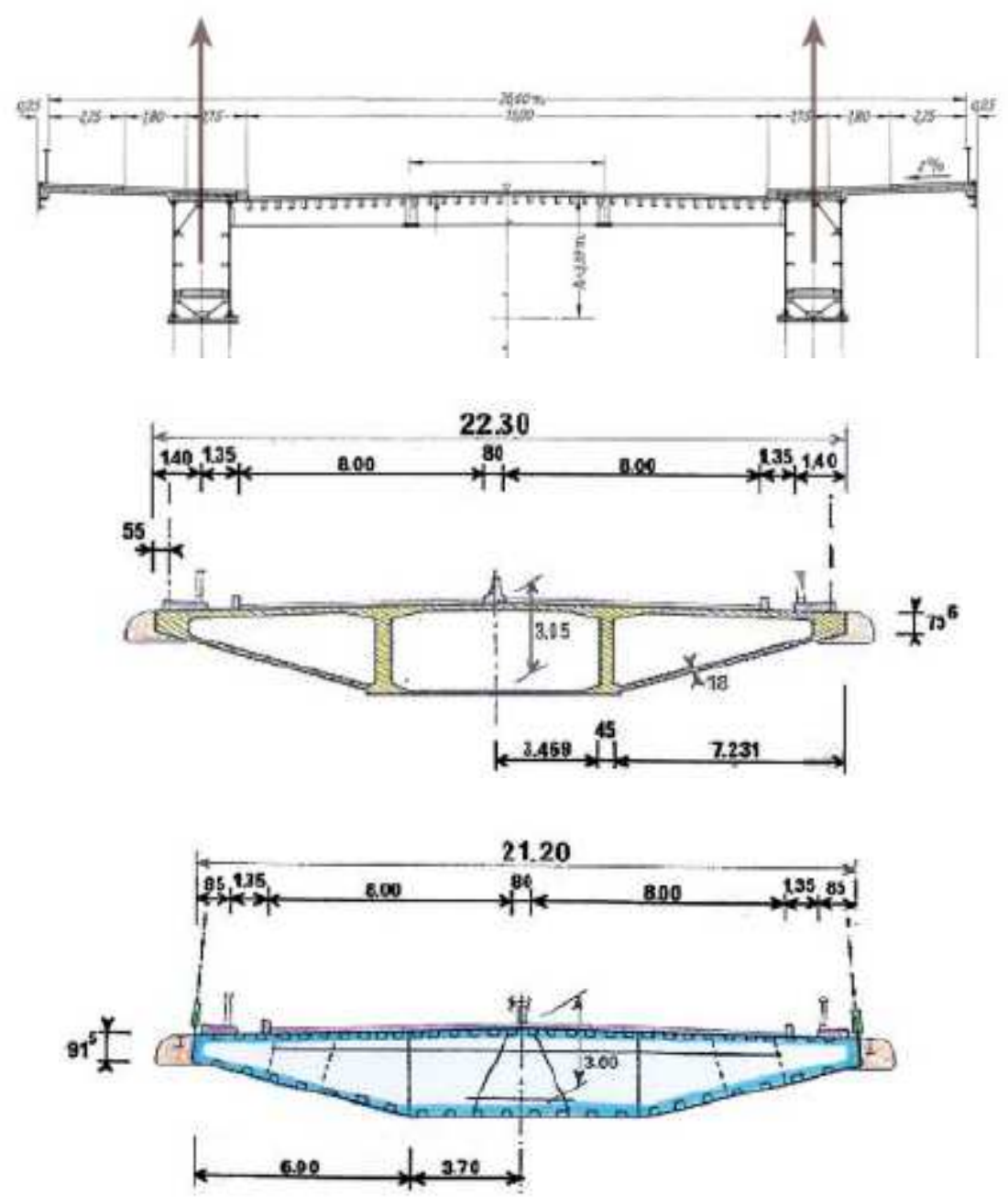

Figura 24. Tablero con doble plano de atirantamiento sustentado en los bordes. Sección transversal bijácena del Knie bridge y sección en cajón de hormigón y metálica del tablero del Puente de Normandía.

En cambio, si la configuración de cables elegida es un solo plano de tirantes (Figura 25), fijados a un pilono central o a una pila en $A$, estos mismos cables deberán anclarse a su vez en el eje del tablero, necesitándose en este caso de una sección cerrada tipo cajón que proporcione la rigidez a torsión necesaria, dado que con el esquema de tirantes elegido no es posible absorber la posible excentricidad de las cargas verticales y horizontales. 

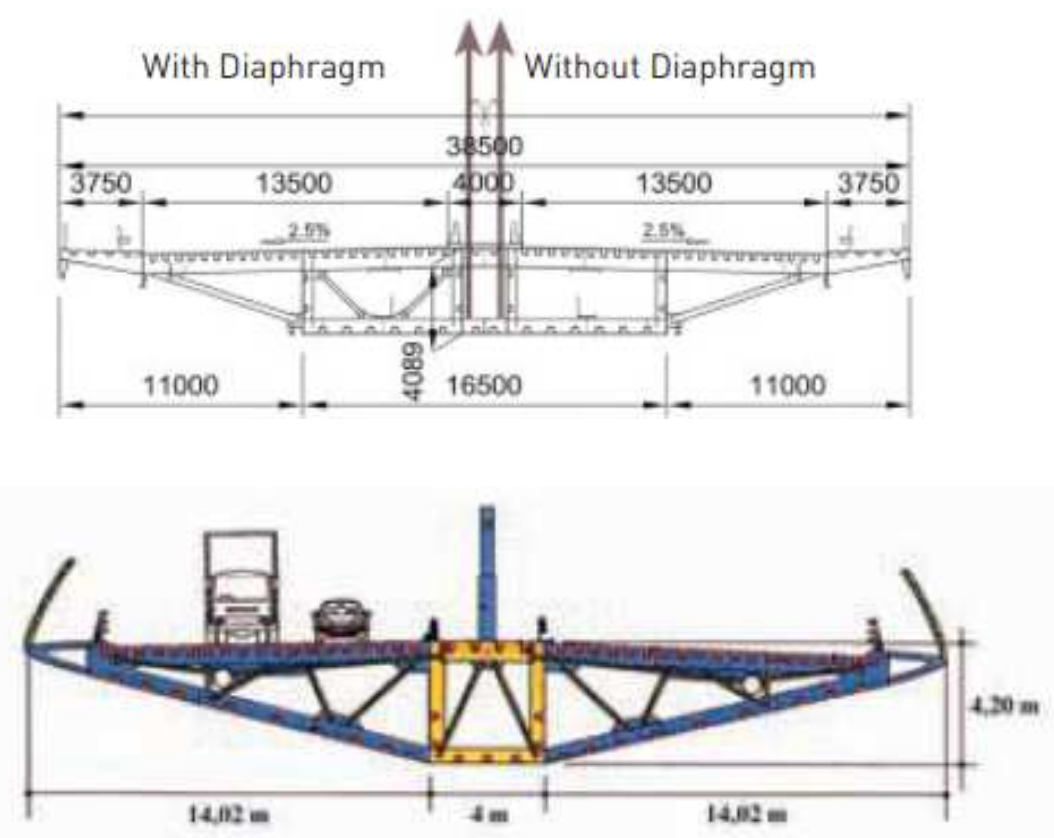

Figura 25. Tableros con plano único de atirantamiento central, Flehe Bridge y Viaducto de Millau.

En esta última alternativa se necesitará adicionalmente dotar a la sección de cierta rigidez frente la flexión transversal, como por ejemplo triangulando la sección cajón o dotándola de un alma justo bajo el anclaje de los cables, de tal forma que el efecto puntual de la introducción de la carga de los tirantes se evite, haciendo colaborar al conjunto de la sección.

Desde el punto de vista de los materiales que pueden conformar el tablero se pueden plantear alternativas de hormigón, metálicas o mixtas, como en cualquier tablero de otro tipo de puentes.

Respecto de los tableros metálicos o mixtos, éstos pueden adoptar diferentes configuraciones geométricas, con secciones cerradas o abiertas como se observa en la Figura 26 y en la Figura 27. Hay que indicar, que, debido a su coste, las soluciones con cajón metálico hoy en día, salvo en el caso de puentes de grandes luces, han sido desbancadas en gran medida por las de tipo mixto, en las que son metálicas tanto las vigas longitudinales como transversales que conforman el tablero, siendo de hormigón la losa de compresión del tablero que conforma la plataforma de rodadura. 


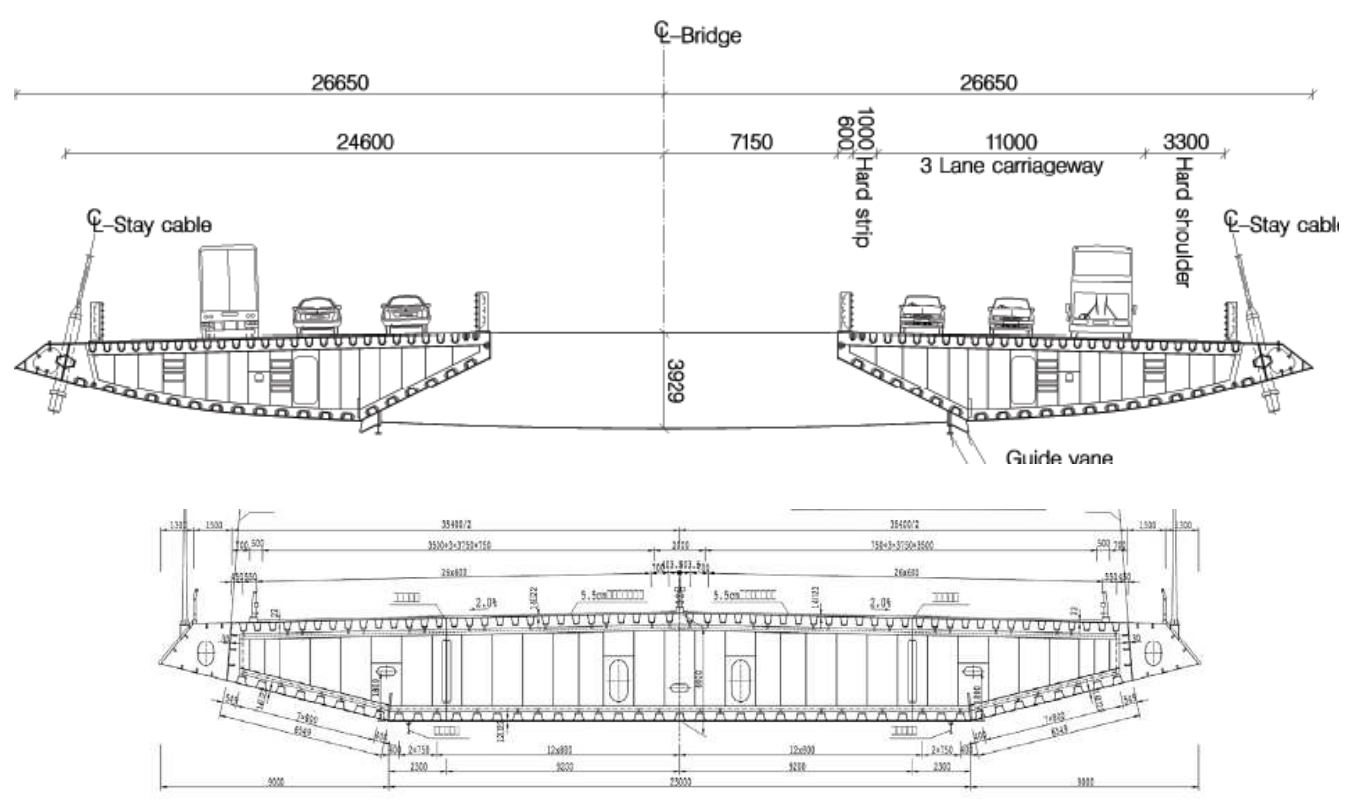

Figura 26. Tipologías de tableros metálicos. Puente Stonecutters y Puente Sutong.

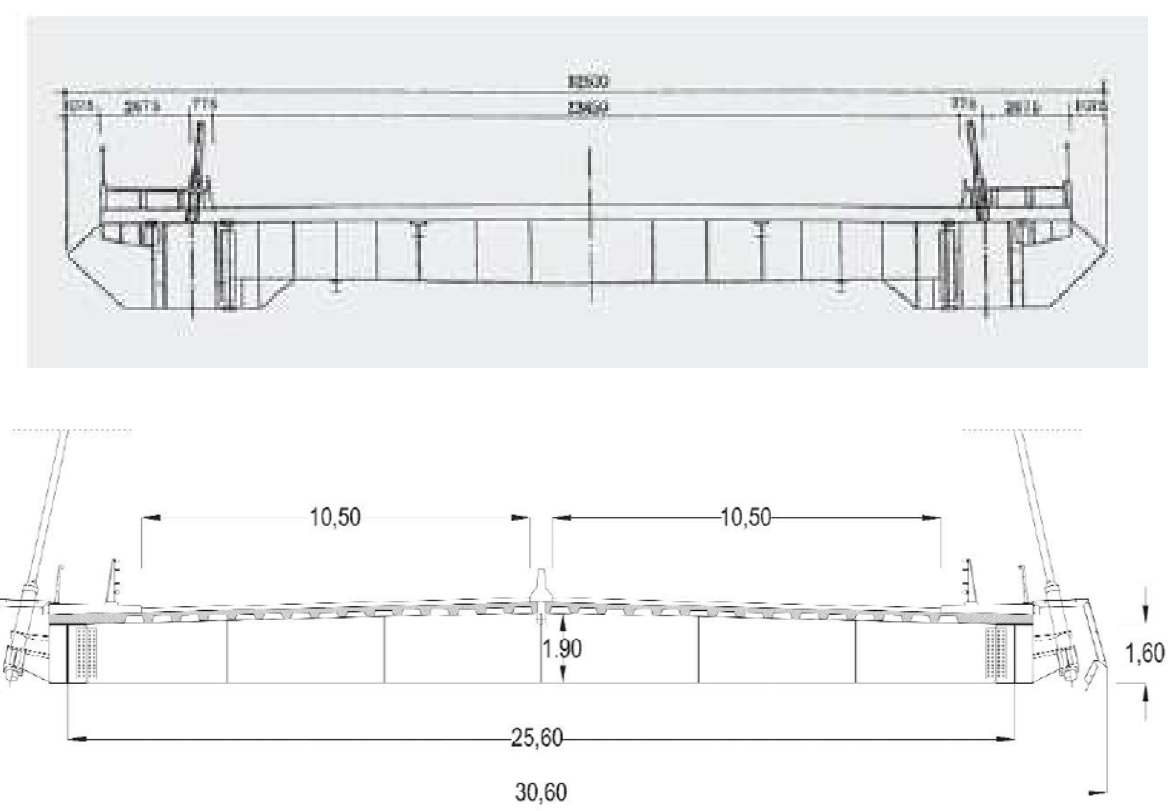

Figura 27. Tipologías de tableros mixtos. Puente YangPu y Puente en Waterford [11].

Las soluciones con tablero de hormigón cuentan con una gran libertad formal para conformar secciones abiertas, cerradas tipo cajón o incluso como losa maciza o aligerada. En los siguientes esquemas se muestran distintas geometrías posibles de tableros ya ejecutados (Figura 28). 

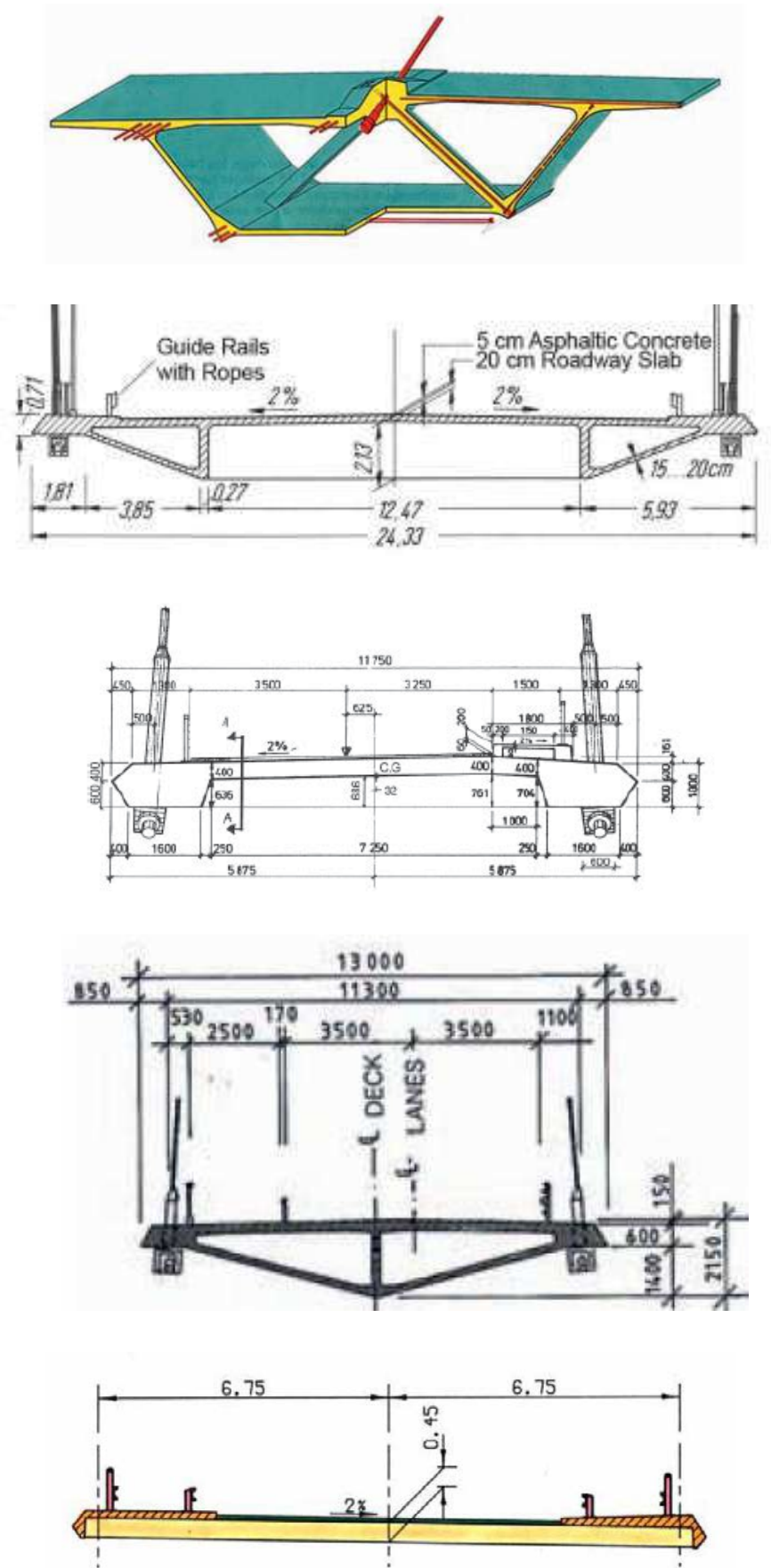

Figura 28. Tipologías de tableros de hormigón. Puente Brotonne, Puente Pasco-Kennewick,

Puente Helgeland, Puente Skarnsundet y Puente Evripos.

Queda así por definir que rango de aplicación tiene cada una. Cada solución aporta una ventaja en función de las características del material que la conforma: en el caso de los tableros metálicos su escaso peso propio los hace adecuados para las situaciones en los que esta carga pueda ser determinante, como en puentes de grandes luces, mientras que las secciones de hormigón cuentan con su economía de 
costes, con una gran capacidad para resistir las importantes compresiones que generan los axiles de los tirantes en las zonas del tablero cercanas a las pilas, así como con un peso que puede servir de contrapeso al vano principal en los vanos de compensación de puentes asimétricos.

M. Schlaich [93], M. Virlogueux [100] y H. Svensson [99] plantean que los tableros de hormigón tienen ventaja en coste respecto de las otras alternativas en el caso de un rango de luces de hasta aproximadamente $400 \mathrm{~m}$. Por encima de esta magnitud el incremento del peso propio y su influencia sobre el nivel de esfuerzos final obtenido hacen que empiece a ser más rentable el empleo de soluciones metálicas o mixtas.

Como se ha indicado, en los tableros de hormigón, dada su gran sección, difícilmente se producirán problemas de inestabilidad en las cercanías de los pilonos, por lo que en dicha zona generalmente son más adecuados. Por contra, la predicción y el control de las deformaciones del tablero durante las fases de construcción son mucho más complicadas, dado el efecto del comportamiento reológico del hormigón y la dificultad para determinar con precisión un módulo de deformación de este material.

Para evitar en parte estos inconvenientes y aprovechar las ventajas de la sección de hormigón R.Walther [103] planteó el uso de tableros con secciones relativamente esbeltas, con la intención de aprovechar la capacidad del hormigón a compresión, pero dotando al tablero de la flexibilidad necesaria, buscando así como consecuencia aumentar la efectividad del atirantamiento.

Un inconveniente fundamental de disponer tableros flexibles, es que al reducir la rigidez del tablero se incrementarán las deformaciones debidas a las cargas permanentes, que a su vez podrían aumentar los efectos de las deformaciones diferidas. Así, si se proyecta una sección de gran esbeltez, se deberá cumplir con el condicionante de que el equilibrio de fuerzas entre los axiles de los tirantes y las cargas aplicadas sobre el tablero, generen sobre éste fuerzas centradas en su sección, es decir que se deberá adoptar una geometría en alzado que permita mantener dicha resultante en la dirección del eje del tablero. De esta manera se minimizan las flexiones generadas en el tablero e, indirectamente a su vez, la redistribución de estos esfuerzos debido a los efectos diferidos. Como consecuencia de todo ello, con esta configuración se ven reducidos los efectos de la fluencia sobre las deformaciones, consiguiéndose así un control más ajustado de las deformaciones durante la construcción. 
Dos ejemplos claros de este tipo de soluciones son: el Puente Diepolsau en Suiza, con proyecto de R. Walther, $97 \mathrm{~m}$ de luz y una losa de canto 0'55 m, y en segundo lugar el Puente Evripos en Grecia (Figura 29), con proyecto de M. Schlaich, con un tablero de sólo $45 \mathrm{~cm}$ de canto para una luz de $215 \mathrm{~m}$.

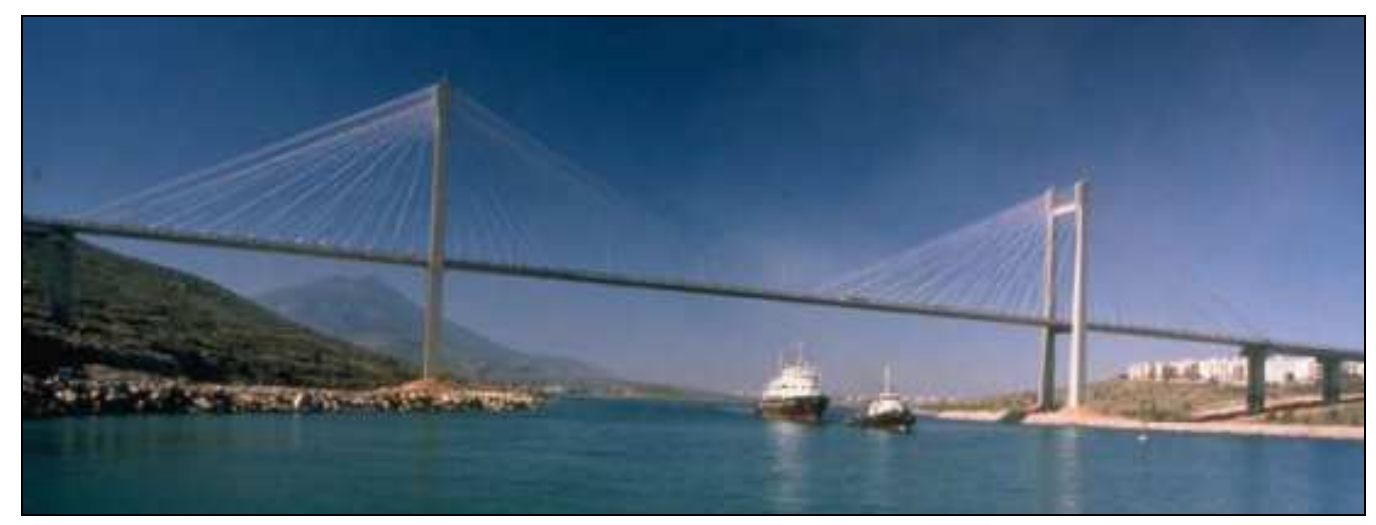

Figura 29. Puente Evripos $\left(L_{\max }=215 m\right)$. Grecia. 1993.

Otros dos ejemplos significativos de cómo aprovechar las características de los diferentes materiales que componen el tablero son:

- En primer lugar el diseño elegido para el tablero del Puente de Normandía. En éste se proyectó una solución con tablero de hormigón en la zona cercana a las pilas y en los vanos de acceso y compensación, mientras que en las zonas de centro de vano se adoptaron secciones metálicas (Figura 24), dado su menor peso propio.

- $\quad$ En segundo lugar el caso del Puente de Barrios de Luna [68] (Figura 21), en que se utilizó una sección de hormigón aligerada en la zona central para reducir el efecto del peso propio, manteniéndose la sección completa en las zonas próximas a las pilas, donde el volumen de compresiones en el tablero era mayor.

A su vez, el tipo de material que conforme el tablero tiene una implicación importante en cuanto al proceso de tesado de los tirantes. M. Schlaich [93] [93] indica que, mientras que en el caso de los tableros de hormigón el proceso de tesado de los tirantes se debe reducir a una sola fase, siempre que sea posible, para así simplificar el proceso constructivo, en el caso de los tableros metálicos o mixtos el proceso de tesado puede ser recomendable efectuarlo en 2 fases. Esta afirmación se justifica dado que el efecto del peso propio es menor en este tipo de tableros. Se puede por ello plantear la aplicación de un primer tesado parcial que compense sólo los efectos debidos al peso propio del tablero, para proceder posteriormente a realizar el tesado 
final cuando la ejecución de la estructura se haya concluido y se encuentren aplicadas todas las cargas permanentes. Es más, incluso plantea que se pueda intentar compensar parte de las sobrecargas que habrá de soportar la estructura en la situación de servicio, aunque la eficacia de esta acción dependerá de la rigidez del tablero y de su luz.

En resumen, el material elegido para conformar la sección del tablero afecta de varias maneras al comportamiento estructural del conjunto:

- En primer lugar, los tableros de hormigón serán más pesados e implicarán una mayor importancia de las cargas permanentes respecto de las sobrecargas, y por ello a su vez, normalmente el proceso constructivo será determinante en su diseño. En el caso de los tableros metálicos y mixtos los pesos propios son menores y las sobrecargas serán más condicionantes al aplicarse en la situación final.

- En segundo lugar, disponer tableros con secciones con una rigidez elevada a flexión, como puede ocurrir en el caso de proyectarse un tablero de hormigón en sección cajón, implicarán que gran parte de los esfuerzos sean absorbidos directamente por este elemento, de tal manera que la efectividad de los tirantes será menor que en el caso de tableros más flexibles.

En general un tablero más rígido, a igualdad del resto de parámetros resistentes, provocará que la eficacia del mecanismo resistente mediante flexión del propio tablero sea cada vez más importante y por lo tanto los momentos flectores alcanzados en este elemento serán de mayor cuantía conforme aumente la inercia de la sección transversal.

Es decir que el dimensionado de la sección transversal, además de cumplir los condicionantes geométricos que se hayan impuesto en cada caso particular, debe encontrar un punto de equilibrio entre disponer de la suficiente flexibilidad para absorber la menor cuantía posible de esfuerzos de flexión longitudinal, favoreciendo la eficacia del atirantamiento, y contar a su vez con la rigidez necesaria para limitar las deformaciones verticales que se pudieran generar.

\subsubsection{Tipología de pilonos y vinculación tablero-pilono.}

Se describen sucintamente en este epígrafe las diferentes morfologías de los pilonos, las cuales dependen básicamente del esquema de atirantado elegido y de la luz del 
tablero. También en este apartado se describen las posibles vinculaciones a disponer entre tablero y pilono a plantear, y la repercusión sobre el comportamiento del conjunto que tendrán cada una.

Como se ha adelantado, la geometría del pilono vendrá determinada por el esquema de atirantamiento elegido. En el caso de atirantamiento en abanico, al concentrarse los tirantes sobre una zona muy reducida, se requerirá la disposición de una pieza especial en cabeza de los fustes de los pilonos con capacidad para albergar los anclajes de todos los tirantes. Un ejemplo típico en este sentido es el caso de los pilonos del Puente Pasco-Kennewick (Figura 32).

Por contra, si el esquema es en arpa o semiarpa los tirantes se repartirán en toda la altura y el efecto local causado por el tesado de los tirantes sobre el pilono será más reducido que en el caso anterior.

Por otra parte también será condicionante en la forma del pilono el número de planos de atirantamiento. Una tipología habitual para el caso del uso de 2 planos de atirantamiento suele ser el uso de 2 fustes verticales, los cuales pueden quedar arriostrados o no mediante un travesaño transversal dispuesto a una cierta altura, conformándose en este último caso un pilono en forma de $\mathrm{H}$. Una alternativa a la solución anterior, planteada para conseguir centrar las acciones de los tirantes sobre el plano del fuste, dado que éstos quedarán por el exterior del borde del tablero, es lograr una separación variable entre los fustes, mayor por debajo de la rasante del tablero y menor por encima de éste. De esta forma se consigue que la zona donde se materializan los anclajes de los cables quede en la vertical del borde del tablero, consiguiéndose así que no se produzca ningún efecto transversal sobre los fustes del pilono. Esta solución se empleó en el diseño de los pilonos del Puente de Barrios de Luna (Figura 21).

Si el puente es de gran luz, se puede plantear la sustitución de los pilonos en $\mathrm{H}$ por pilonos en forma de A, en los que los anclajes se concentran en la zona superior donde se localiza el fuste único, consiguiéndose así reducir el coste de estos elementos. La dificultad de esta solución radica en la concentración de anclajes que se produce en esta zona, así como en los posibles efectos en dirección transversal que genera la acción de los tirantes y en la complejidad que supone la ejecución de los fustes inclinados que requerirán de algún tipo de sostenimiento provisional hasta que su configuración sea estable. 
Finalmente, para el caso del uso de un solo plano de atirantamiento central, las alternativas en relación a la morfología del fuste del pilono posibles son dos: la primera de ellas sería la opción de erigir un fuste único centrado, lo que implicaría separar el tablero en 2 mitades a la altura del paso por este punto, tal y como se ha planteado en el Puente Stonecutters (Figura 26), mientras que la alternativa más utilizada, y planteada por primera vez en el Puente Severin en Colonia, es la disposición de una pila A que permita el paso del tablero bajo las patas de la $\mathrm{A}$.

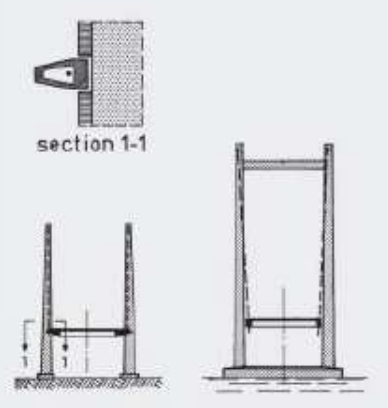

low level bridges (rivers]

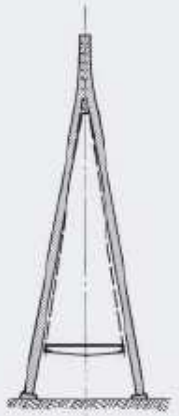

\section{.} high level (sea)

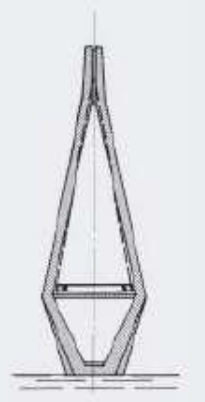

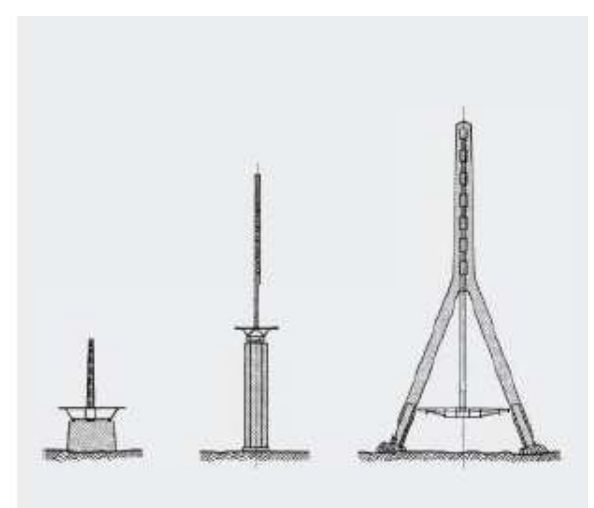

Figura 30. Esquemas de pilonos para atirantamiento en 2 planos (pilonos exentos, en $\mathrm{H}$ en $\mathrm{Y}$ invertida y en rombo) y para atirantamiento en 1 sólo plano (pilonos exentos y pilono en $Y$ invertida). Esquemas tomados de Svensson [99].

Es importante destacar el juego de reparto de esfuerzos entre tablero y pilono que se establece en función de la rigidez relativa del pilono respecto del tablero. En concreto se deduce que un aumento de la rigidez en los pilonos implica una mayor efectividad del atirantamiento, lo que a su vez posibilitará la reducción de la rigidez necesaria en el tablero.

Por último hay que indicar que, salvo en las primeras realizaciones de puentes atirantados y en algún caso de puentes de gran luz en que se han proyectado fustes metálicos, lo habitual es que los fustes de los pilonos se ejecuten de hormigón.

En relación a la vinculación vertical entre tablero y pilono existen varias alternativas a plantear, siendo las más habituales, bien de dejar colgado el tablero de los cables en la zona bajo el pilono, introduciendo una vinculación entre ambos sólo para los desplazamientos horizontales, o bien apoyar el tablero sobre un travesaño que una ambas patas o fustes del pilono. Esta última alternativa tiene la desventaja de que en dicha sección los esfuerzos generados serán muy elevados, al ser este punto de apoyo un "punto duro" en relación a todo al resto del tablero que está apoyado de forma elástica sobre los anclajes de los cables. Por este motivo, y en función del nivel de 
esfuerzos que se deseen alcanzar, se deberá plantear una separación hasta los primeros cables que consiga homogeneizar los esfuerzos de flexión que aparecen en el tablero entre esos primeros tirantes y el apoyo en el pilono.

En el caso de pilonos de fuste único se puede incluso plantear la posibilidad de solidarizar el tablero y el pilono por encima de éste, quedando el conjunto apoyado en las pilas que se disponen por su parte inferior. Más complicada, y por lo tanto de más rara aplicación, es la posibilidad de empotrar el conjunto tablero-pilono en las propias pilas. Hay que indicar que esta última alternativa rigidiza en gran medida el tablero y por lo tanto reduce la efectividad del atirantamiento, pero por otra parte son muy adecuadas para el caso de puentes continuos, tal y como señala Rodado [90].

Por otra parte, en sentido transversal la vinculación habitual entre tablero y pilonos es disponer algún tipo de tope que haga las funciones de coacción a la deformación transversal del tablero. De esta manera, los desplazamientos transversales debidos al viento o sismo quedan coaccionados y se pueden transmitir desde el tablero hasta el pilono.

\subsection{PROCESO CONSTRUCTIVO.}

En primer lugar hay que señalar que algunos autores, tal y como Troitsky\&Lazar [56], Behin\&Murray [13], y Reddy\&Gabhoussy [89], plantearon en su momento que el proceso constructivo en estos puentes es esencial para el dimensionado de cada uno de sus elementos, ya que, normalmente, es en las fases de montaje de la estructura cuando se alcanzan esfuerzos de gran magnitud que condicionan todo el diseño. Es por ello que, para cualquier estudio que se realice sobre estas estructuras, se ha de tener en cuenta la evolución del esquema resistente y de cargas aplicadas a lo largo de todo el proceso constructivo.

Además, otro de los aspectos fundamentales en el diseño de un puente atirantado es garantizar una cierta geometría final al final de su ejecución, la cual a su vez también dependerá del proceso seguido durante todas las fases de construcción.

Por estos motivos, y de cara al desarrollo del estudio realizado en adelante, es interesante hacer un análisis de los procesos más habituales tanto de ejecución del tablero como de instalación de los tirantes, quedando los métodos de ejecución de los pilonos, pilas y estribos fuera del ámbito de esta investigación ya que, en general, no 
difieren de los planteados para otro tipo de puentes y además no influirán en los planteamientos y resultados que se obtengan en este estudio.

\subsubsection{Proceso constructivo del tablero.}

De forma muy resumida se puede indicar que los procesos constructivos que principalmente se aplican en estas estructuras son cuatro distintos: ejecución sobre cimbra, ejecución por voladizos sucesivos, empuje del tablero y construcción apeada con posterior giro o ripado del tablero.

El primero de todos estos procedimientos consistirá en la ejecución, en primer lugar de las pilas y estribos, para a continuación o bien cimbrar sobre el terreno y hormigonar el tablero completo, en el caso de tableros de hormigón in situ, o bien montar las distintas dovelas sobre apoyos provisionales, en el caso de tableros metálicos, mixtos o de hormigón prefabricado. De esta manera en una fase posterior de ejecución, una vez completado y dispuesto el tablero sobre apeos, se procede al tesado de forma consecutiva de los cables, lo que permite a su vez su descimbrado y puesta en carga. Este procedimiento requiere de la posibilidad de la instalación de la cimbra o apeos provisionales, por lo que sólo se podrá emplear si no existe una gran altura entre el tablero y el terreno, si el puente se ejecuta sobre terreno competente y si no hay ningún condicionante que impida la ocupación provisional de esa superficie bajo el tablero.

Hay que señalar que el empleo de este procedimiento, para la ejecución del vano principal, implicará en cierto modo una pérdida de la justificación de la necesidad de una tipología como la atirantada, ya que dicha tipología se justifica técnica y económicamente cuando es necesario cubrir vanos de grandes dimensiones, y esta condición de partida queda en cuestión cuando se ejecutan apoyos provisionales o se disponen cimbras sobre el terreno. Por este motivo, este método constructivo no es el más extendido en su aplicación, salvo para la ejecución de los vanos de compensación, los cuales en muchos casos disponen de pilas definitivas intermedias para fijar los puntos de anclaje de los cables de retenida y aumentar la eficacia del atirantamiento.

El procedimiento de ejecución por voladizos desde el pilono es el de uso más común y el que conjuga la esencia de la necesidad de una tipología como la atirantada, ya que independiza la ejecución del tablero respecto del terreno. En dicho método las 
dovelas irán completando el tablero en voladizo desde el pilono, ejecutándose todas ellas de forma que se equilibren los pesos, a ambos lados del pilono.

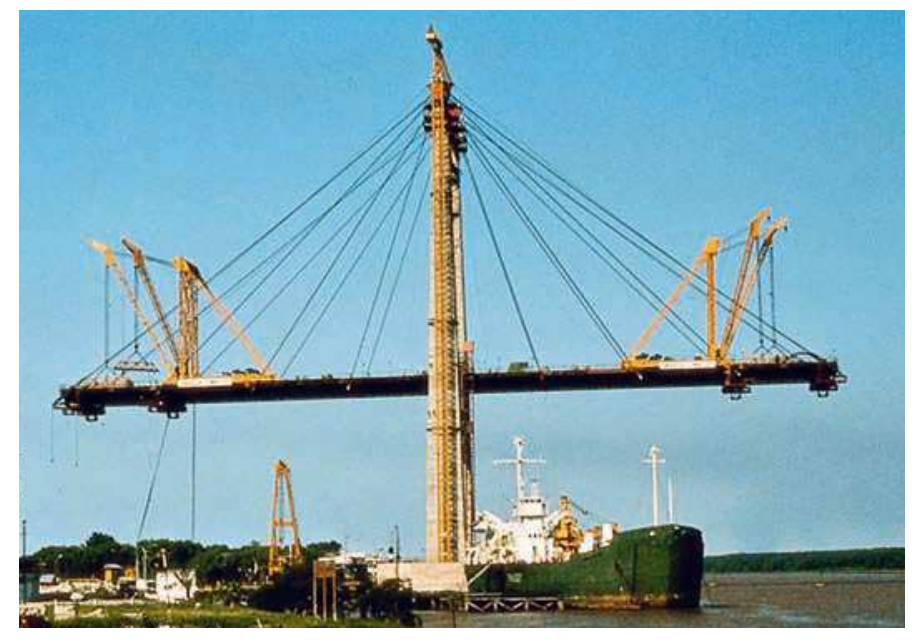

Figura 31. Ejecución por voladizos desde el pilono del Puente Posadas-Encarnación en Argentina.

Con esta solución se puede plantear o bien la ejecución de dovelas de hormigón in situ, hormigonadas mediante el uso de carros de avance, o bien el montaje de elementos prefabricados que serán izados para conformar el tablero, como por ejemplo en el caso de tableros metálicos, figura 31, o elementos de hormigón prefabricados, Figura 32. Se irán de esta forma completando las distintas dovelas desde el pilono hasta, avanzando hacia el centro del vano, cerrar éste contra el voladizo del pilono opuesto o bien al alcanzar la pila o estribo que sirven de apoyo extremo.

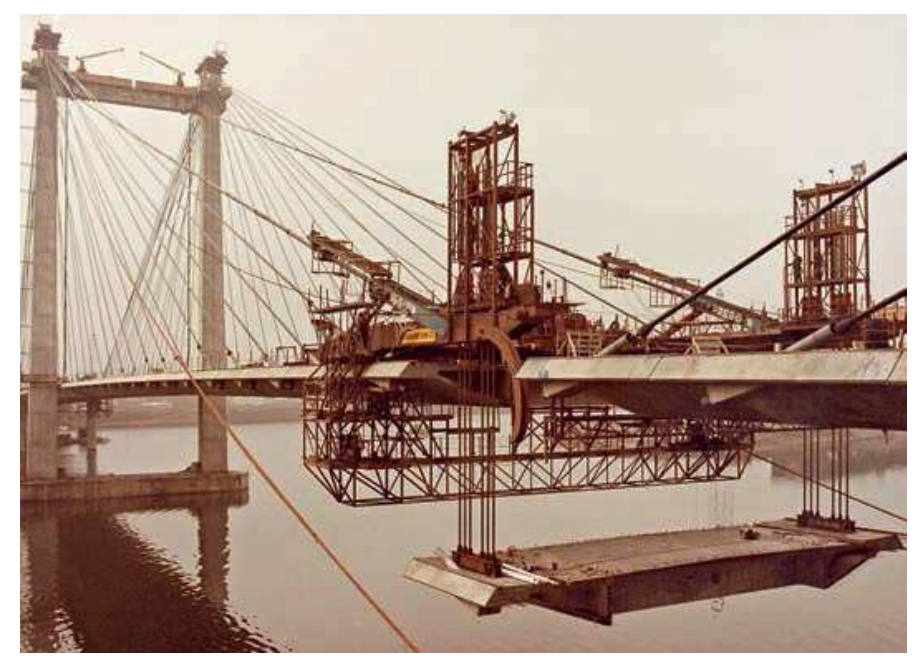

Figura 32. Montaje de dovela de hormigón prefabricada en el Puente Pasco-Kennewick en USA. 
Esta solución es la que se ha elegido para la estructura estudiada en esta investigación, ya que se considera la más común en su práctica, y por lo tanto la que mayor número de aplicaciones puede tener.

A su vez, al utilizar este método constructivo se podría plantear el uso, si fuera necesario, de algún procedimiento que limite los esfuerzos y deformaciones generadas durante la construcción, como podría ser el atirantado provisional del carro, de tal manera que se reduzcan las flexiones y flechas en el voladizo generado por la dovela en ejecución. Esta solución se ha planteado en numerosas ocasiones, incluso, como en el caso del Puente Helgeland [99], utilizando los propios cables definitivos como arriostramiento provisional del carro de avance.

También se pueden plantear dos alternativas para mejorar este proceso [35]:

- En primer lugar un proceso de sobretesado y destesado alternativo de los 2 últimos cables dispuestos en cada fase para compensar los esfuerzos máximos generados en las fases de construcción. Esta solución fue empleada en el Puente de Barrios de Luna [69].

- En segundo lugar, la ejecución de sólo el núcleo de la sección del tablero, la cual se completaría posteriormente en una segunda fase mediante el añadido de costillas o jabalcones que permitan alcanzar el ancho completo de la sección. De esta forma se consigue que no actúe el peso propio de la sección completa sobre el carro de avance, aligerando el volumen de este elemento auxiliar y los efectos que produce la ejecución en voladizo.

Hay que señalar que, en los casos estudiados en este documento, no se ha introducido ninguna de las alternativas anteriores ya que introducirían una complicación adicional en el cálculo que no aportaría nada al objeto del estudio, además de que para las dimensiones de la estructuras analizadas a continuación tampoco estarían justificadas técnicamente.

Los 3 últimos métodos constructivos reseñados son de más rara aplicación, ya que requieren de condiciones y medios de ejecución más complejos, y por lo tanto se han llevado a la práctica en contadas ocasiones. Tres ejemplos singulares, uno de cada uno de estas alternativas, pueden ser:

- La alternativa consistente en el empuje del tablero desde los estribos hasta cerrar el vano principal se restringe al caso de tableros metálicos, y la realización más 
importante hasta el día de hoy con este método es el del Viaducto de Millau (Figura 33). En dicho puente se llevó a cabo el empuje simultáneo de tablero y pilonos, dada la altura de las pilas que restringía la posibilidad de ejecutar apoyos provisionales, resultando, los que fue finalmente necesario disponer en el centro de los vanos, de unas dimensiones muy considerables.

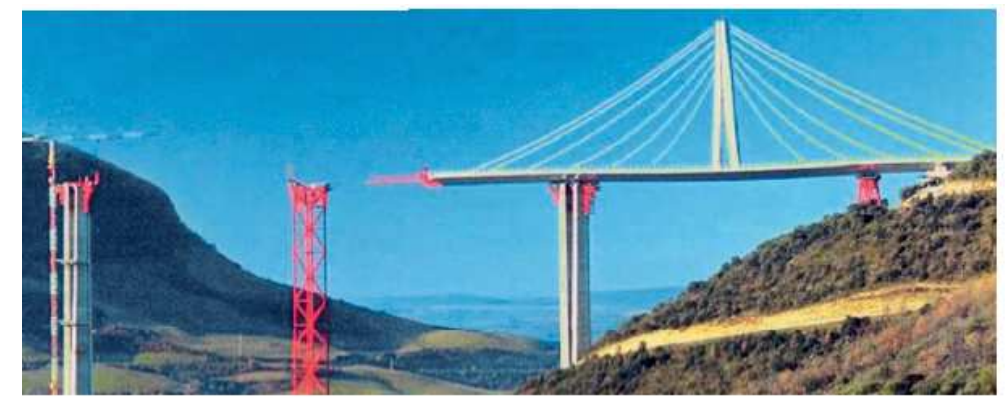

Figura 33. Fase de empuje del tablero y pilono del Viaducto de Millau.

En España un ejemplo de este tipo de proceso constructivo es el llevado a cabo para la ejecución del Viaducto de Arbizelai en Arrasate-Mondragón[39].

- En el caso de los tableros ripados el ejemplo más notable es el Puente de Oberkassel en Dusseldorf (Figura 34). En este caso se ejecutó el nuevo tablero adyacente al puente existente que se venía a sustituir y que por lo tanto era necesario ripar para colocar en la misma posición del original. Esta maniobra se pudo realizar dado que tanto tablero como pilono eran metálicos.

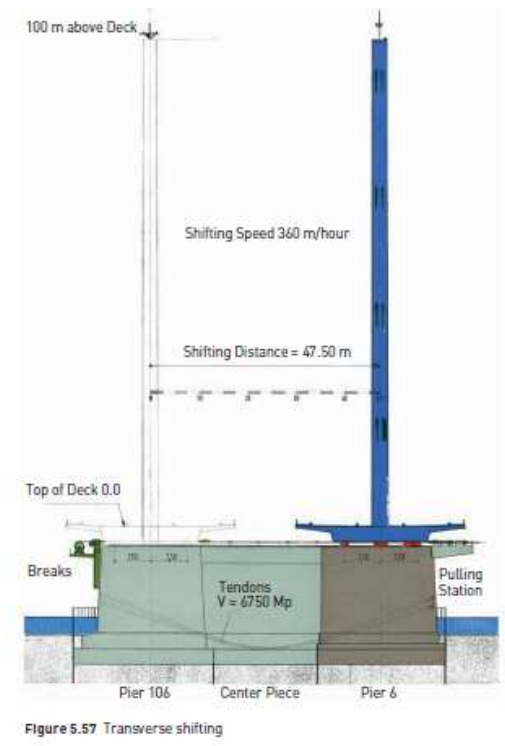

Figura 34. Posición provisional donde se ejecutó el puente de Oberkassel y posición definitiva una vez ripado el tablero [99]. 
- En el caso de los puentes construidos sobre cimbra y posteriormente girados hasta su posición definitiva, el caso más notable es el del Puente Ben-Ahin (Figura 35) sobre el Mosa en Bélgica. Este puente con un sólo pilono, fue ejecutado cimbrado en paralelo al cauce, para posteriormente descimbrarlo mediante el tesado de los cables y rotarlo sobre el eje del pilono hasta que alcanzó la orilla opuesta del río.

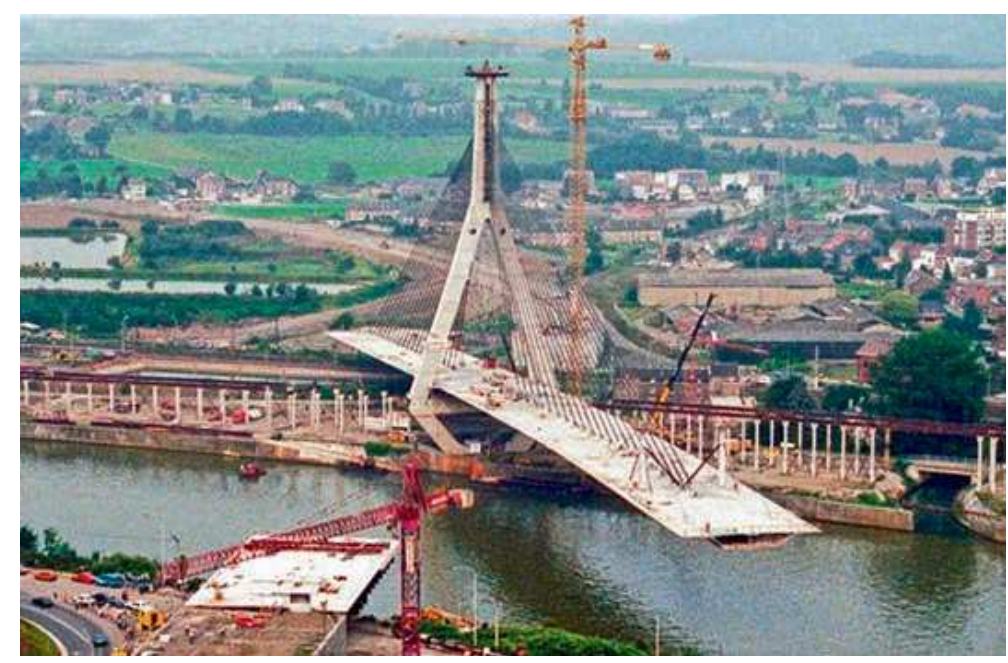

Figura 35. Rotación del tablero y pilono del Puente de Ben-Ahin [76].

Por otro lado, es importante remarcar que, hoy en día, la tendencia en el proyecto de estas estructuras se centra en la realización de un seguimiento muy riguroso de la geometría y de su evolución en las distintas fases de montaje, para conocer en cada caso las configuraciones geométricas que se están produciendo y por ejemplo, en el caso de tableros de hormigón, condicionar la posición de los carros de avance a la situación creada en cada etapa de construcción, de cara al ajuste final geométrico pretendido.

Claramente orientados en este sentido se encuentran las realizaciones de puentes con tablero de cantos muy reducidos que ya se han mencionadol, en los cuales se pretenden conseguir dos objetivos simultáneos: por un lado reducir los pesos propios de las secciones del tablero al mínimo posible, de tal manera que las flexiones generadas sobre este elemento, entre cada pareja de cables, sean lo más limitadas posibles, así como por otra parte conseguir mitigar los efectos de la fluencia al haber reducido las flexiones en el tablero, dada la disminución de la cuantía de las cargas permanentes y al centrar la resultante de las fuerzas aplicadas en su eje.

\footnotetext{
${ }^{1}$ Puente de Diepolsau ( Walther ) y puente Evripos ( Schlaich )
} 
En definitiva, estas propuestas parten de una filosofía basada en buscar soluciones que reduzcan el efecto de la fluencia, es decir, evitar el problema antes de que éste se produzca, ya que los cálculos y predicciones sobre el comportamiento reológico del hormigón siempre tendrán una elevada incertidumbre.

\subsubsection{Métodos de puesta en carga de los tirantes.}

La operación de puesta en carga de un tirante debe buscar, conocido el valor teórico de la fuerza aplicada en el cable en la fase de su colocación, que sobre la estructura se alcancen los niveles de esfuerzos y las deformaciones planteadas teóricamente. Así este proceso de puesta en carga tendrá dos vertientes diferenciadas, por un lado el análisis teórico de la puesta en tensión de los cables en el modelo de cálculo, y por otra parte la materialización de esa puesta en carga del cable en obra.

Los posibles métodos de instalación de los tirantes según Marchetti\&Lecinq [73] [74] son tres diferentes:

- En primer lugar el método en fuerzas o tensiones, basado en la introducción de un cierto axil de tesado en cada cable a instalar, controlando, mediante un manómetro, la presión aplicada en el gato de tesado.

A nivel teórico este método conllevará la simulación de la colocación del tirante mediante la introducción de un par de fuerzas en los puntos de anclaje y en la dirección del cable, con valor correspondiente al del axil de montaje establecido para cada tendón.

Hay que señalar que, con este método de instalación de cables, el proceso de ejecución deberá tener un control exacto de las cargas aplicadas en cada fase de construcción (pesos de elementos auxiliares como carros de avance, utillaje o acopios de material, así como del efecto de la temperatura ambiente), para que los axiles de tesado a introducir tengan en cuenta todas estas acciones. Así, los posibles errores generados en el momento del tesado podrán ser debidos, además de por una mala evaluación de las cargas actuantes sobre la estructura en el momento de la colocación del cable, a la lectura errónea o a una mala calibración del manómetro del gato de tesado.

Es importante tener en cuenta también que, en puentes de grandes luces, la flexibilidad del tablero en las fases de construcción es muy elevada, por lo que pequeñas variaciones en las tensiones de los cables generarán importantes 
movimientos verticales. La máxima precisión en los gatos de tesado suele quedarse en el aproximadamente el 2-3\%, pero para esos valores las deformaciones producidas pueden ser ya importantes. Incluso, ocurrirá que, en una misma estructura, las deformaciones que se midan en las primeras fases de construcción serán mucho más pequeñas que en las fases finales, al variar la flexibilidad de la propia estructura según la evolución del proceso constructivo.

Es por todo ello que para un seguimiento adecuado del proceso de puesta en tensión de los cables en estructuras de grandes luces es necesario llevar un control geométrico de las deformaciones verticales del tablero. Bien es cierto también que este control geométrico podrá ser realizado de forma tanto más aproximada cuanto mayor sea la flexibilidad de la estructura, pero establecer el límite entre qué estructuras son muy flexibles y cuáles no, no es sencillo de determinar.

Otro inconveniente de este método de control de la puesta en carga de los tirantes es que hay que tener muy claro qué parte de las deformaciones producidas se deben al peso propio del tablero, qué parte al peso de los equipos colocados sobre éste, qué parte a la temperatura ambiental, qué parte al soleamiento o al gradiente térmico, etc. Es más, en tableros metálicos el modulo de elasticidad del acero no introducirá ninguna incertidumbre en dichas medidas, pero en el caso de los tableros de hormigón el módulo de deformación siempre será un parámetro de muy difícil evaluación.

- El segundo método es el denominado método en deformaciones, en el que se propone adoptar, como medida de referencia para el proceso de tesado, las deformaciones a aplicar en los cables al colocarlos.

Analíticamente este proceso se simulará en el modelo de cálculo en primer lugar colocando la barra correspondiente al cable a instalar, para a continuación aplicarle un acortamiento, mediante por ejemplo una variación de temperatura, que represente el proceso de tesado.

Este método es el que proponen utilizar tanto Virlogeux [101] como el SETRA [102], denominándolo método de las predeformaciones. En concreto, este método se basa en la definición de las denominadas longitudes en vacío o neutras de los cables, que son aquellas longitudes que posee cada uno de los cables cuando sobre ellos no actúa ningún axil ni su peso propio. A partir de esta 
longitud, que se puede considerar una magnitud intrínseca de la estructura y que por lo tanto no depende del nivel de cargas aplicados sobre ella, se puede proceder a determinar tanto los axiles de tesado de los cables, como los correspondientes a su fase final, simplemente estableciendo el incremento de longitud (predeformación) necesario alcanzar en cada caso para generar la configuración geométrica requerida.

La ventaja de este método radica en el carácter intrínseco a cada estructura de la longitud en vacío, por lo que se independiza de las condiciones ambientales (temperatura) y del nivel de cargas aplicado, con la consiguiente facilidad de los cálculos a realizar para el seguimiento del proceso constructivo. Por otra parte, en este caso los posibles errores de instalación de los tirantes se reducirán a una mala evaluación de su geometría real o de la medición de los alargamientos de cada uno de los tirantes.

El principal inconveniente, como se comprobará a lo largo de este estudio, es que la incertidumbre en la posición de los anclajes de los cables influye de forma muy acusada en el procedimiento. En el caso de puentes con tableros metálicos y de estructuras donde se usen cables prefabricados esta cuestión puede ser de menor importancia, pero en el caso de puentes con tableros de hormigón, la incertidumbre en la posición de los anclajes será del orden de centímetros ,cuestión ésta que invalidará la aplicabilidad de este método, tal y como señalan Marchetti\&Lecinq [73], Goodyear [47] y Gabrow [45].

- El último método propuesto para la instalación de los cables es el denominado método de la tensión de referencia [102] [73], método en el que se comparará las tensiones aplicadas en los tirantes durante su proceso de puesta en carga, con la tensión generada a partir de la aplicación de las predeformaciones referidas a la longitud en vacío de cada cable, incluyendo los efectos de la variación de la temperatura del cable y variación de la posición de los anclajes respecto de la situación de referencia.

Este método se simulará analíticamente introduciendo en el modelo de cálculo unas fuerzas de tesado, pero esta vez en relación con la tensión de referencia antes definida.

Se pretende con este método evitar los inconvenientes antes señalados para el método en deformaciones y adoptar sus ventajas. Este método se utilizará en 
este estudio y se comprobará que aun aplicándolo se sigue teniendo que controlar la posición de los anclajes para no introducir por este motivo errores en la colocación de los cables.

Como resumen de todos estos métodos se puede concluir que, en general, será necesario realizar un control tanto en tensiones como en deformaciones del tablero, alargamiento de los cables y posición de los anclajes, para, en la medida de lo posible, tener todos los parámetros que influyen en el proceso de colocación de los cables monitorizados, de tal manera que toda esta información pueda servir para controlar y ajustar el proceso constructivo.

Un ejemplo interesante de este proceso de monitorización es el que se llevó a cabo durante la construcción del Puente sobre el río Iregua [78].

\subsection{COMPORTAMIENTO ESTRUCTURAL.}

Se describe en este epígrafe de forma breve cual es la respuesta resistente de un puente atirantado frente a las cargas exteriores aplicadas. En concreto este apartado se centra en la descripción del comportamiento frente a cargas verticales, ya que el estudio realizado a continuación únicamente abordará este tipo de acciones, no habiéndose contemplado por ello en esta descripción ni el comportamiento frente a cargas horizontales ni frente a efectos dinámicos.

El comportamiento resistente de este tipo de estructuras se basa en la conjunción de 2 mecanismos resistentes: el primero constituido por la propia eficacia del atirantamiento y el segundo constituido por la capacidad de resistencia a flexión del tablero. El comportamiento global de la estructura será la conjunción de ambos, dependiendo de la configuración geométrica y mecánica de cada caso en concreto.

El esquema de atirantamiento depende a su vez de varios factores como son la propia rigidez de los tirantes, el ángulo de ataque de los cables sobre el tablero, la rigidez de los pilonos y la existencia de cables de contrarresto anclados a contrapesos o puntos fijos.

Así, en primer lugar, hay que hacer notar que serán mucho más eficaces los cables cortos que los cables largos. Además, en este mismo sentido, en el caso de los esquemas de atirantamiento en abanico, dichos cables cortos contarán con ángulos respecto del tablero mucho mayores que en los cables más largos extremos, por lo 
que su efectividad se verá así aumentada en doble medida. En el caso de disponer esquemas de atirantamiento en arpa este último efecto no existirá, ya que el ángulo entre cables y tablero es constante en todo el desarrollo de éste.

Adicionalmente hay que tener en cuenta que la eficacia de un cable depende de la propia rigidez del punto de anclaje en el pilono. Por otra parte, se ha comentado que frente a la actuación de cargas repartidas a todo lo largo del tablero, éste se comportará de forma similar a una viga continua sobre fundación elástica, con apoyos en los puntos de anclaje de los tirantes, los cuales, si las separaciones entre ellos son homogéneas a ambos lados del pilono, producirán un efecto sobre la pila que se reducirá a la generación de un axil de compresión y no generarán desplazamientos horizontales de los anclajes en el pilono. Pero podemos encontrar dos situaciones que nos alejan de esta configuración de equilibrio y que provocarán una disminución de la rigidez de dicho punto de anclaje del tirante:

- En primer lugar la actuación de cargas repartidas sobre puentes no simétricos, es decir con vanos laterales con luces menores de la mitad del vano principal. Este aspecto provocará que las cargas uniformes, aplicadas en toda la longitud del tablero, tengan en el vano principal una mayor cuantía que en los vanos de compensación. Esta situación obligará a fijar los puntos de anclaje de los cables que soportan el tramo del vano principal no equilibrado por el de compensación para garantizar la eficacia del atirantamiento de este tramo. La forma de limitar los desplazamientos de estos puntos pueden ser tres diferentes: aumentar la rigidez de los pilonos, incluir cables de contrarresto en los vanos cortos anclados a un macizo de contrapeso o disponer de apoyos intermedios en el vano de compensación que permitan fijar los extremos de los cables que llegan a ese punto e indirectamente del punto del pilono en el que se anclan.

- $\quad$ Por otra parte, la presencia de cargas aplicadas de forma alterna sobre un vano u otro. Estas, en el caso de ser aplicadas sobre el vano principal llevará de nuevo a las soluciones anteriores, pero al ser aplicadas sobre los vanos de compensación sólo podrán ser equilibradas mediante el atirantamiento si se dota al pilono de rigidez suficiente. Si no se opta por esta solución, la imposibilidad de resistir estas acciones mediante axiles en los cables implicará la necesidad de incrementar la rigidez del tablero en el vano de compensación. Implícitamente, la conclusión a la que se llegaría siguiendo este razonamiento es, que si no se quiere penalizar en exceso el dimensionado de los pilonos, es mejor 
adoptar un esquema longitudinal con vanos descompensados, de tal forma que las flexiones que se produzcan en los vanos de compensación queden amortiguadas por su menor longitud en relación al vano principal. Este es uno de los motivos por los que en general se recomiendan relaciones entre la luz del vano principal y la de los de compensación del orden de 0'2 a 0'4 [69].

De los planteamientos anteriores se deduce que un puente con vanos simétricos implicará la necesidad disponer pilas más rígidas que si el puente no es simétrico, para así reducir, en la medida de lo posible, los movimientos horizontales de los anclajes de los cables que reducen la efectividad del atirantamiento. Una alternativa más adecuada y muy extendida en la actualidad consiste en la ya comentada de disponer pilas intermedias en el vano de compensación.

Un problema relacionado con los planteamientos anteriores es la solución a adoptar en el caso puentes atirantados continuos, donde en los pilonos intermedios es necesario encontrar algún mecanismo de rigidización de la pila que garantice la eficacia del atirantamiento de los vanos intermedios para el caso de cargas alternadas. Un estudio tipológico en este sentido es el, ya señalado, realizado por Rodado [90], donde se analizaron y evaluaron las ventajas de diferentes soluciones para este problema.

Por otra parte se ha señalado la importancia de la rigidez del tablero frente al comportamiento global de la estructura, de manera que, el juego de rigideces establecido junto con el atirantamiento, conformará el reparto de esfuerzos en ambos elementos. Es más, aumentar la rigidez del tablero puede provocar que se incrementen los esfuerzos de flexión sobre éste, los cuales a su vez necesitarán para resistirse de una mayor dimensión y por lo tanto rigidez. Este proceso puede llegar a penalizar en exceso al tablero y desaprovechará el mecanismo de atirantamiento. En general, como ya se ha comentado anteriormente, hoy en día la tendencia es la ejecución de tableros dotados de la mayor esbeltez posible, siendo dimensionados o bien por la necesidad de resistencia a compresión en la zona del pilono, o bien por las exigencias de los efectos aeroelásticos, en el caso de los puentes de gran luz.

Como se indicó en [67], puede ocurrir incluso que el canto de la sección del tablero venga determinado por el ancho de éste, ya que esta dimensión normalmente será de mayor cuantía que la separación entre cables que condiciona el cálculo longitudinal. Es decir, que en el establecimiento de la rigidez del tablero puede influir tanto el mecanismo resistente longitudinal, como el transversal. Por este motivo la 
presencia de tableros muy anchos pueden llevar a disponer secciones de gran inercia, que implicarán a su vez una gran rigidez longitudinal, lo que provocará que los tirantes sean menos eficaces a la hora de absorber esfuerzos. Comentar eso si que, en el caso de disponer el tablero una sección tipo bijácena, el anterior planteamiento no será cierto, ya que el incremento del ancho del tablero implicará el aumento de la capacidad de las vigas transversales pero no siempre de las vigas longitudinales.

Por otro lado hay que señalar que en los puentes construidos por voladizos sucesivos ejecutados desde el pilono, durante las fases de construcción se plantearán las dos consideraciones particulares siguientes:

- En primer lugar que al ejecutarse las dovelas de forma alterna, existirá una cierta descompensación de los esfuerzos generados por los tirantes en el pilono, lo que impondrá una dimensión mínima para resistir estos esfuerzos en dicho elemento.

- Por otra parte, que en dichas fases de construcción, y más aún en las fases finales de ese proceso, la estructura provisional será extremadamente flexible, por lo que este aspecto deberá tenerse en cuenta durante los cálculos a realizar, de tal manera que se consiga finalmente la geometría que se ha considerado como objetivo final para la rasante del tablero.

Por último, en el caso de plantearse la ejecución del tablero mediante apoyos provisionales sobre el terreno el comportamiento en fases de construcción es completamente diferente al anteriormente expuesto, ya que el tablero se encontrará ejecutado en su totalidad apoyado cuando se procede al tesado sucesivo de los tirantes, los cuales irán sustituyendo de forma progresiva la reacción introducida por los apoyos provisionales. Es decir que en este caso la flexibilidad del tablero en las fases provisionales es mucho más reducida que en el caso anterior. Señalar que LozanoGalant [59] ha analizado en sus investigaciones el comportamiento de puentes atirantados ejecutados mediante este procedimiento planteando diversos métodos de cálculo para su análisis.

\subsection{MÉTODOS DE CÁLCULO.}

Las estructuras que se analizan en este estudio tienen una serie de particularidades que hacen especial su cálculo. Principalmente los puentes atirantados tienen las características estructurales singulares siguientes: 
- En primer lugar su alto grado de hiperestatismo dado su compleja configuración geométrica. Esta característica complica e invalida en gran medida los análisis simplificados que se pudieran efectuar.

- En segundo lugar, pero no menos importante, es su carácter evolutivo con el tiempo al considerarse el proceso constructivo. Es decir que si se analiza el proceso de ejecución de estas estructuras deberá tenerse en cuenta un gran número de configuraciones geométricas y estructurales diferentes, siendo además los resultados obtenidos en las sucesivas fases de ejecución dependientes de los resultados de las anteriores fases consideradas.

- Por último, hay que considerar también, como ya se ha señalado, que en general durante las fases de construcción la rigidez del tablero es muy reducida y por la tanto las estructuras parciales serán muy deformables.

Las tres características anteriores condicionan los estudios que se realicen en relación a estructuras de este tipo y siempre deberán ser tenidas en cuenta, tanto en los planteamientos y análisis previos, como en el cálculo y en la interpretación de los resultados de dichos cálculos. Por este motivo es imprescindible la consideración de todas las fases constructivas para poder conocer la evolución de los esfuerzos y deformaciones en la estructura, ya que los esfuerzos pésimos en ciertos elementos no se producirán en la situación final sino que podrán producirse en alguna fase intermedia de construcción.

El problema fundamental del cálculo de estos puentes consiste en la determinación de las tensiones o fuerzas existentes en los tirantes tanto en las fases finales como en las intermedias de construcción. En concreto, el cálculo de estas estructuras se ha basado siempre en la determinación de los axiles a aplicar a los tirantes en el momento de su colocación (axiles de montaje), de tal forma que en la situación final de servicio queden con el esfuerzo correspondiente al de la situación objetivo que se pretende alcanzar (axiles finales).

De forma esquemática, el proceso de cálculo a seguir consistirá en, primeramente, adoptar y calcular una situación objetivo a alcanzar en la fase de servicio, para a continuación emplear, para la determinación de los axiles de montaje de los cables, alguno de los dos métodos de cálculo siguientes: el denominado método del desmontaje (o método backward) y el denominado método del montaje (o método forward). 
El primero de estos métodos, el método del desmontaje de la estructura (Walther [103], Manterola[69], Gimsing [46]) permite, una vez definida la situación final objetivo en servicio, bajo la actuación de las cargas permanentes e incluso de parte de las cargas variables, proceder a reproducir el proceso constructivo en orden inverso, de tal manera que el axil que tiene cada tirante en el momento de su desmontaje coincidirá con el que es necesario aplicarle en el instante de su colocación para así poder alcanzar la situación objetivo elegida al final del proceso de construcción real.

Este método requiere por lo tanto reproducir todo el proceso constructivo, fase por fase, para conocer todos los axiles iniciales de cada tirante, siendo necesario desarrollar el proceso completo para conocer estos valores. El principal inconveniente de este método es que se desarrolla en sentido contrario al proceso real de construcción, por lo que la consideración de los efectos dependientes del tiempo que se generan en el hormigón es complicada.

Por otro lado el método del montaje (Wang [106] y [105]) reproduce el proceso constructivo en el sentido de construcción real, por lo que los efectos reológicos dependientes del tiempo generados en el hormigón sí se pueden introducir con sencillez en los cálculos. La dificultad de este método es que se requiere un proceso iterativo para alcanzar la situación objetivo elegida en servicio.

Normalmente se predimensionarán los tirantes bajo la actuación del nivel de cargas que se considere adecuado en la situación objetivo, para a continuación aplicar dichas cargas sobre la estructura. Las deformaciones generadas en los puntos de anclaje se utilizarán para calcular el acortamiento que es necesario generar en los tirantes para devolver los anclajes a su posición original. Esta deformación se traducirá en el valor de los axiles de montaje en la primera iteración del cálculo por el procedimiento del montaje. En las sucesivas iteraciones se calcularán los movimientos de los anclajes respecto de su posición definitiva y a partir de ellos se calcularán los incrementos de carga en los tirantes que es necesario añadir al valor inicial adoptado. Este proceso se continuará hasta hacer converger los resultados con la precisión requerida.

Hay que señalar que, en relación a los efectos a largo plazo de los fenómenos reológicos del hormigón es importante considerar una situación objetivo final que permita valorar su cuantía, de tal manera que, adoptando la configuración oportuna de axiles en tirantes y geometría del tablero, se puedan compensar dichos efectos. 
En general, lo más habitual es que el cálculo de cualquier puente atirantado utilice de ambos métodos [103], el del desmontaje para la obtención de los axiles de montaje de los cables y el de montaje para el seguimiento y monitorización de los efectos dependientes del tiempo y el seguimiento de la ejecución real en obra.

Hay que recordar que Lozano-Galant en sus extensos estudios ([60], [61], [62] y [63]) ha desarrollado todos estos procedimientos de forma específica para el caso de puentes ejecutados sobre apoyos provisionales, planteando para ello un método de desmontaje, otro de montaje e incluso el que se ha denominado como método directo, el cual permite calcular cada fase individual del proceso constructivo, utilizando el concepto de longitud neutra de un tirante, sin necesidad de pasar por todas las fases anteriores. Comenta este autor, eso sí, que este método sólo es adecuado a puentes con tableros metálicos o mixtos, ya que en él no se pueden implementar directamente los efectos reológicos del hormigón.

\subsubsection{Situación final objetivo y axiles de montaje de los tirantes.}

Como se ha indicado anteriormente, la situación final objetivo se define como aquella configuración geométrica y en esfuerzos idónea, cuando se analice la estructura terminada o en servicio, tanto desde el punto de vista resistente como funcional, para lo que además deberá incorporar los efectos diferidos del hormigón en el caso de los tableros de hormigón o mixtos.

Los criterios más habituales para definir la situación objetivo, tal y como indican Lozano-Galant [59] y Quintana [88], son criterios basados en alguna condición bien a nivel de esfuerzos en el tablero o a nivel de deformaciones en este mismo elemento. Otros criterios se basarán en alguna consideración de tipo energético, como puede ser la minimización de la energía elástica de deformación. Como se señala en [91], los métodos del primer tipo resultarán más adecuados a estructuras simétricas o casi simétricas, mientras que los métodos energéticos resultarán más adecuados en el caso de tableros asimétricos.

Así, un primer criterio utilizado para obtener una valor inicial aproximado de los axiles finales actuantes sobre los tirantes es el denominado método del tablero articulado (Gimsing [46], Svensson [99]), que consiste básicamente en considerar muy pequeña o nula la rigidez a flexión del tablero, de tal forma que en los puntos de anclaje de los cables se pueda plantear la existencia de una rótula frente al giro. De esta forma se puede calcular la carga correspondiente a absorber por cada tirante al descomponer 
dicha acción en la dirección del tirante y en la dirección del eje del tablero. Este método es válido para el caso de los tirantes intermedios pero produce desviaciones respecto de los valores correctos en el caso de los tirantes extremos cercanos a los estribos y pilas. Gimsing [46] planteó una serie de formulaciones en este sentido que permitían incorporar la rigidez de tablero y pilas ya desde este primer tanteo.

Una mejora respecto del método anterior es el denominado método del péndulo planteado por Virlogeux [101] y recogido en las recomendaciones de tirantes del SETRA [102]. Este método propone partir de la misma consideración anterior, en la que la rigidez del tablero se considera nula en los puntos de anclaje de los tirantes, pero tiene en cuenta la geometría en alzado del tablero y las distintas fases de construcción, de tal manera que la carga correspondiente a aplicar sobre cada tirante, en el momento de su puesta en carga, se descomponga en un axil de tracción según la dirección teórica del tirante y en un axil centrado de compresión en el tablero. Virlogeux [101] indica que de esta forma, al centrar los axiles con el eje del tablero, los efectos diferidos que se pudieran producir, en el caso de los tableros de hormigón o mixtos, se reducen en gran medida. Se consigue así adicionalmente que la configuración geométrica final del tablero se desvíe en la menor medida de la teórica.

Una alternativa a este método es el método de anulación de los desplazamientos de los puntos de anclaje de los cables, propuesto por Wang [106]. Este método se puede desarrollar usando tanto el método del montaje como el del desmontaje, buscándose alcanzar deformaciones verticales nulas en los puntos de anclaje de los cables. Este cálculo se realiza en base a la matriz de rigidez condensada que reproduce la flexibilidad de cada punto de anclaje respecto a su desplazamiento vertical. De esta manera, utilizando dicha matriz, se puede conseguir, mediante un proceso iterativo, una configuración de axiles en los tirantes que permita minimizar las deformaciones verticales de estos puntos.

El anterior método tiene como principales inconvenientes tanto la no consideración de la influencia de la rigidez del pilono, como la no consideración de la influencia de la geometría en alzado del tablero respecto los esfuerzos sobre el mismo generados.

Para evitar los inconvenientes del método anterior, Chen [23] plantea en cambio que para calcular los axiles de los tirantes basándonos en la anulación de los desplazamientos verticales del tablero, se tenga en cuenta tanto la geometría del tablero como la rigidez del pilono. Este último aspecto se considera que es especialmente importante en el caso de los puentes asimétricos, en los que 
adicionalmente a la condición de desplazamientos en el tablero habrá que plantear alguna condición respecto de los flectores generados en el pilono. Con este condicionante adicional se consigue reducir la cuantía a largo plazo de los efectos diferidos del hormigón sobre la geometría final del tablero.

En general, y en especial para el caso de los tableros de hormigón, se puede utilizar el método de anulación de reacciones bajo los anclajes de los tirantes (Manterola [69], Walther [103], Schlaich [93], Svensson[99], El Araby[31]), es decir, adoptar como situación final objetivo aquella que resulte en una configuración de esfuerzos similar a la de la viga continua que representa el tablero apoyado sobre cada punto de anclaje de un tirante. De esta manera la reacción que se produce en cada apoyo deberá ser descompuesta en un axil de tracción en la dirección del tirante y en un axil de compresión en el tablero. Tanto Gimsing [46] como Svensson [99] consideran que este método es especialmente adecuado para tableros de hormigón, pero puede no llevar a la situación óptima en el caso de tableros metálicos o mixtos.

En concreto Gimsing [46] propone que en el caso de tableros metálicos se podría alcanzar cualquier configuración de flectores que permita encajar el rango de tensiones generados en las diferentes secciones del tablero dentro de un intervalo válido. Para conseguir este objetivo plantea realizar un proceso iterativo utilizando un método de desmontaje del tablero.

Por otra parte, con este método, y en el caso de tableros de hormigón, Schlaich [93] indica que se alcanzará una configuración de flectores en el tablero en la situación de servicio que es independiente del módulo de elasticidad y de la rigidez del tablero. De esta forma, si consideramos el efecto de la fluencia, de forma simplificada, como una variación del módulo de elasticidad del tablero, al alcanzarse esta configuración al final del proceso constructivo la actuación de la fluencia no provocará redistribución de esfuerzos de flexión.

También en [93] se indica que este razonamiento es válido si la rigidez del tablero es a su vez constante, lo que no sería estrictamente cierto si las cuantías de armado longitudinal dispuestas para resistir la flexión longitudinal son distintas en ambas caras del tablero, ya que en este caso se producirá una variación de la profundidad de la fibra neutra que invalidaría este planteamiento. Es por este motivo que se insiste en la necesidad de conseguir centrar los axiles de compresión en la sección de hormigón de forma que las cuantías de armado a disponer en ambas caras de la sección no 
difieran en gran medida y por lo tanto se pueda considerar la posición de la fibra neutra constante a lo largo de toda la longitud del tablero.

En resumen Schlaich [93] propone tener en cuenta sólo los efectos de la fluencia correspondientes al acortamiento a largo plazo que se producirá sobre el tablero y despreciar los generados por la flexión del propio tablero, si la configuración adoptada para la situación de servicio es equivalente a la de la viga continua. Así la forma de considerar el efecto del acortamiento del tablero debido a la fluencia será plantear la ejecución de las distintas dovelas con una longitud mayor de la teórica, de tal manera que se pueda compensar el acortamiento generado a largo plazo en ellas debido a la aplicación del axil de compresión introducido por el tesado de los tirantes.

Otro método utilizado para la determinación de los axiles de los tirantes es el denominado método de la fuerza unidad estudiado por Pedrozzi [82], Gabrow [45] y por Bokan y Janjic ([54], [16] y [17]) donde las fuerzas de los tirantes son obtenidas a partir de una serie de parámetros (normalmente flectores en el tablero) que han de ser alcanzados en la configuración final de servicio. De esta forma, conocida esa situación objetivo, se propone la aplicación de unas cargas unitarias en cada cable que permite obtener su correspondiente efecto en las secciones elegidas como de control. Resolviendo a continuación el sistema de ecuaciones resultante de la suma de efectos individuales, se pueden obtener los valores de los axiles de los tirantes que permiten alcanzar la situación adoptada como objetivo. Este proceso se puede aplicar tanto a la fase final de construcción como a cualquiera de las fases intermedias.

Otros métodos utilizados para definir la situación objetivo a alcanzar en la situación de servicio pueden ser los basados en criterios de minimización, adoptándose normalmente como criterio la energía de deformación como parámetro a minimizar. En este sentido tanto primeramente Furukawa [44], como posteriormente Kasuga [55], y finalmente Scotti [94] han planteado estas soluciones, añadiendo los dos últimos el efecto reológico a largo plazo del hormigón en la función a minimizar. Kasuga [55] añade que para tener en cuenta el efecto de la reología del hormigón en las deformaciones del tablero es preciso llevar a cabo un control de las deformaciones distinto del utilizado en el caso de tableros metálicos.

Igualmente también se han planteado por algunos investigadores como Negrao y Simoes [79] [95] [96] definir las situaciones objetivo a partir de la optimización de ciertos parámetros elegidos como los significativos del comportamiento de la estructura, es 
decir usar métodos de optimización para calcular los axiles iniciales en los cables. Se define así una función multicriterio que contiene todos los parámetros adoptados en el análisis y que será la que deba minimizarse para alcanzar el objetivo prefijado. Estos investigadores incluyen en su análisis variables asociadas al coste de las soluciones evaluadas.

En este mismo sentido, recientemente en la Universidad de la Coruña Baldomir [12] propuso minimizar el volumen total de acero en los tirantes, analizando un caso concreto de puente atirantado. Señalan, eso sí, que el procedimiento seguido al venir marcado por las condiciones de contorno preestablecidas permite obtener una solución mínima pero no la solución que alcance el mínimo absoluto, por lo que la solución adoptada dependerá en gran medida de los datos de partida que se utilicen, siendo además muy sensible el cálculo realizado a dichos datos de partida.

Igualmente podemos reseñar la investigación realizada en este ámbito por Zhang [108] que evalúa la aplicación del criterio de optimización que posee el programa comercial MiDAS para la obtención de los axiles de montaje, en un caso real para un puente en China.

Siguiendo en esa misma línea de investigación, se ha desarrollado por parte de Hassan [49] [50] un método de optimización que evita los inconvenientes de los anteriormente reseñados. El planteamiento propuesto es similar, al buscar optimizar una función que permita obtener un mínimo del área de los tirantes, pero utilizando para ello el uso de funciones tipo B-spline para representar de forma continua dichas áreas a lo largo del tablero. A la vez se propone el uso de algoritmos genéticos para mejorar la convergencia y reducir el tiempo de cálculo necesario para obtener dichas soluciones. Así, dado que el procedimiento se ha conseguido depurar respecto de los casos anteriores, se pueden incluir además como condiciones de contorno del problema no sólo la limitación de flechas en el tablero sino también en el pilono.

\subsubsection{Métodos de cálculo probabilistas.}

Todos los métodos anteriores, de una $U$ otra manera, proponen un cálculo determinista de los axiles de los tirantes, pero existen otros métodos basados en la consideración no determinista o aleatoria de ciertos parámetros de la estructura a partir de los cuales proceder a los cálculos correspondientes. 
Estos métodos guardan una cierta relación con la investigación desarrollada en esta tesis, por lo que es interesante dar una rápida visión de los desarrollos realizados en este sentido.

En primer lugar, Cheng [23] [24][25] ha planteado la determinación de los axiles en los tirantes a partir de un planteamiento probabilista, basado en la incertidumbre existente acerca del conocimiento de ciertos parámetros de cálculo. En su estudio este autor ha analizado tanto el efecto de las incertidumbres planteadas sobre la fiabilidad de la estructura como sobre los valores finales de los propios axiles de los tirantes. En concreto en su estudio Cheng utilizó un método mejorado que combinaba el uso de la generación aleatoria de variables mediante el método de Montecarlo y el análisis en fiabilidad según el método FOSM (First order second moment) junto con el uso de las superficies de respuesta, denominando así a su método método MCS-RSF.

Es interesante conocer el orden de magnitud de la variabilidad de los parámetros empleados por este autor, que quedan reflejados en la siguiente tabla:

\begin{tabular}{|c|c|c|c|c|c|}
\hline Random variables & & Substructures & $\mu$ & $\sigma$ & Distribution \\
\hline \multirow[t]{2}{*}{ Elastic modulus } & $E_{g}$ & Girder & $4.32 \mathrm{e} 6 \mathrm{ksf}$ & $0.432 \mathrm{e} 6 \mathrm{ksf}$ & Normal \\
\hline & & Cables & $4.32 \mathrm{e} 6 \mathrm{ksf}$ & $0.432 \mathrm{e} 6 \mathrm{ksf}$ & Normal \\
\hline \multirow{3}{*}{$\begin{array}{l}\text { Cross-sectional } \\
\text { areas }\end{array}$} & $A_{g}$ & Girder & $3.44 \mathrm{ft}^{2}$ & $0.172 \mathrm{ft}^{2}$ & Normal \\
\hline & $A_{c 1}$ & $\begin{array}{l}\text { Cables } \\
\text { (exterior cables) }\end{array}$ & $0.452 \mathrm{ft}^{2}$ & $0.0226 \mathrm{ft}^{2}$ & Normal \\
\hline & $A_{c 2}$ & $\begin{array}{l}\text { Cables } \\
\text { (interior cables) }\end{array}$ & $0.174 \mathrm{ft}^{2}$ & $0.0087 \mathrm{ft}^{2}$ & Normal \\
\hline $\begin{array}{l}\text { Sectional } \\
\text { moment } \\
\text { of inertia }\end{array}$ & $I_{g}$ & Girder & $131.0 \mathrm{ft}^{4}$ & $6.55 \mathrm{ft}^{4}$ & Normal \\
\hline \multirow[t]{3}{*}{ Dead load } & $W_{g}$ & Girder & $6.0 \mathrm{kips} / \mathrm{ft}$ & $0.48 \mathrm{kips} / \mathrm{ft}$ & Normal \\
\hline & $w_{c 1}$ & $\begin{array}{l}\text { Cables } \\
\text { (exterior cables) }\end{array}$ & $0.221 \mathrm{kips} / \mathrm{ft}$ & $0.01768 \mathrm{kips} / \mathrm{ft}$ & Normal \\
\hline & $w_{c 2}$ & $\begin{array}{l}\text { Cables } \\
\text { (interior cables) }\end{array}$ & $0.085 \mathrm{kips} / \mathrm{ft}$ & $0.0068 \mathrm{kips} / \mathrm{ft}$ & Normal \\
\hline
\end{tabular}

Figura 36. Parámetros aleatorios propuestos por Cheng en su investigación [23].

En su estudio, en el cual se compararon los resultados obtenidos con los indicados en su día por Wang [106], se consideraron los efectos de segundo orden, deduciéndose que el efecto sobre las tensiones en los tirantes es muy pequeño, y solo el cálculo considerando grandes deformaciones y efecto P- $\Delta$ en las pilas y tablero tiene alguna importancia sobre los axiles medios de los tirantes. Sí en cambio el efecto de la variación aleatoria de las cargas permanentes del tablero tenía una relación directa sobre los resultados en tirantes, tanto en su valor medio como en su desviación típica. Se concluyó por lo tanto que es este parámetro el que influye de forma mucho más apreciable que la variación del resto de parámetros resistentes de tablero y pilas sobre 
los axiles en los tirantes, y por lo tanto es sobre él sobre el que habrá que centrar la mayor atención para conseguir ajustar los valores de los axiles teóricos con los que realmente se produzcan en obra.

Los anteriormente mencionados métodos de análisis provienen de los desarrollos realizados en relación a formulaciones para el estudio de la fiabilidad de las estructuras, cuya origen en la aplicación a las estructuras de cables se remontan a las investigaciones de Bruneau [19], el cual evaluó la fiabilidad de un puente atirantado mediante la introducción de variables aleatorias utilizando el método FOSM. Dicho análisis contaba con el inconveniente de que los resultados en fiabilidad no indicaban de qué variables aleatorias del cálculo eran más dependientes los resultados obtenidos, dado que aportaba un valor global de la fiabilidad. Posteriormente Imai\&Frangopol [53] aplicaron estos métodos para la evaluación de la fiabilidad de los puentes colgantes. Boucher\&Bourgound [18] mejoraron los anteriores procedimientos mediante el uso de las denominadas superficies de respuesta, las cuales evitaban la definición implícita de las funciones de los estados límite que con los métodos anteriores era necesario emplear, salvo que se abordara el problema utilizando directamente técnicas de simulación de Montecarlo, las cuales cuentan con el inconveniente de su enorme consumo de tiempo de computación.

Un paso más allá en esta línea de investigación lo ha dado Cheng Su [22], en cuyo trabajo ha propuesto una mejora sobre el método utilizado por Cheng [24] para el análisis en fiabilidad. Propuso igualmente un método basado en el uso de las técnicas de Montecarlo para la generación aleatoria de variables conjuntamente con el uso del método de la superficie de respuesta (Método RSMCM). La evolución planteada en este estudio consistió en la depuración del proceso de generación de la superficie de respuesta, basándose en este caso en el uso de funciones de $4^{\circ}$ grado en lugar de funciones lineales con la intención de reducir los errores que se cometían por esta causa. Una de las conclusiones más significativas que se obtiene de este estudio es que, para mantener un mismo nivel de fiabilidad en todas las zonas de la estructura, es necesario admitir distintas tolerancias a los errores de ejecución dependiendo de la fase de ejecución en la que nos encontremos. Hay que comentar que es interesante que esta última conclusión haya sido refrendada en la investigación desarrollada a continuación.

Los valores empleados para los parámetros aleatorios empleados y su distribución se resume en el siguiente cuadro: 


\begin{tabular}{llcc}
\hline Random variable & $\begin{array}{l}\text { Type of } \\
\text { distribution }\end{array}$ & Mean value & $\begin{array}{c}\text { Coefficient } \\
\text { of variation }\end{array}$ \\
\hline$G_{1}, G_{2}: \mathrm{kN} / \mathrm{m}$ & Normal & $2.03 \times 10^{2}$ & 0.01 \\
$T_{1}, T_{2}: \mathrm{kN}$ & Normal & See Table 4 & 0.03 \\
$M_{1}, M_{2}: \mathrm{kNm}$ & Normal & $3.36 \times 10^{4}$ & 0.03 \\
$M_{1}^{0}, \mathrm{M}_{2}^{0}: \mathrm{kNm}$ & Normal & $2.72 \times 10^{3}$ & 0.03 \\
$E_{1}: \mathrm{Pa}$ & Normal & $2.00 \times 10^{13}$ & 0.05 \\
$E_{2}: \mathrm{Pa}$ & Normal & $4.13 \times 10^{10}$ & 0.10 \\
$E_{3}: \mathrm{Pa}$ & Normal & $1.98 \times 10^{11}$ & 0.05 \\
$A_{1}: \mathrm{m}^{2}$ & Lognormal & 1.85 & 0.05 \\
$A_{2}: \mathrm{m}^{2}$ & Lognormal & $2.58 \times 10^{1}$ & 0.10 \\
$A_{3-1}: \mathrm{m}^{2}$ & Lognormal & $4.65 \times 10^{-3}$ & 0.05 \\
$A_{3-2}: \mathrm{m}^{2}$ & Lognormal & $5.33 \times 10^{-3}$ & 0.05 \\
$A_{3-3}: \mathrm{m}^{2}$ & Lognormal & $6.27 \times 10^{-3}$ & 0.05 \\
$A_{3-4}: \mathrm{m}^{2}$ & Lognormal & $7.65 \times 10^{-3}$ & 0.05 \\
$A_{1-5}: \mathrm{m}^{2}$ & Lognormal & $8.57 \times 10^{-3}$ & 0.05 \\
$A_{3-6}: \mathrm{m}^{2}$ & Lognormal & $9.72 \times 10^{-3}$ & 0.05
\end{tabular}

Figura 37. Parámetros aleatorios propuestos por Cheng Su en su investigación [22].

Hay que reseñar también la investigación realizada por Biondini [14] que evaluó la fiabilidad de un puente atirantado en su situación final, mediante la aplicación de muestras de parámetros aleatorios utilizando un procedimiento optimizado con el uso de algoritmos genéticos.

Finalmente es interesante, dada la similitud con la investigación aquí desarrollada, del análisis desarrollado por Sung [98], donde introduciendo unos parámetros aleatorios en los cálculos de un puente atirantado, como las cargas permanentes, la inercia de la sección del tablero, el módulo de elasticidad del material y las sobrecargas aplicadas llegó a evaluar qué influencia tienen estos parámetros en los resultados finales en esfuerzos y deformaciones de la estructura. A partir de estos mismos resultados este autor realizó un estudio en fiabilidad, utilizando el método FORM, que permite valorar qué significación a nivel de probabilidad de fallo tiene la consideración de los anteriores parámetros aleatorios.

\subsubsection{Efectos no lineales en los cálculos.}

Un aspecto importante en todos los cálculos a realizar sobre este tipo de estructuras es el conocimiento de qué efectos no lineales tienen influencia en su comportamiento, cuándo deben considerarse y en qué medida afectan a los resultados finales.

Entre los principales efectos de segundo orden a tener en cuenta tendríamos, tal y como indicaban Astiz [9] y Wang [104]: el efecto catenaria (cable-sag), el efecto de las grandes deformaciones (Large displacements effect) y el efecto P- $\Delta$ en el pilono (beam-column effect). 
El primero de todos ellos es el más importante y se debe al comportamiento no lineal de los cables debido a su nula resistencia a la flexión (efecto "cable sag" ). Esto implica que bajo la acción del peso propio los cables se incurvarán, de tal manera que cuando apliquemos las tensiones teóricas en sus extremos, parte de las deformaciones medidas se deberán al hecho de vencer esa curvatura, y parte a la entrada en carga propiamente dicha del cable. Es decir las deformaciones medidas serán mayores que para el caso de disposición de una barra rígida, o lo que es lo mismo, que se deberán reducir los módulos de elasticidad de los tirantes por esta causa. Este efecto, que depende de las propias tensiones aplicadas, se puede introducir en los cálculos mediante la adopción del denominado módulo de deformación de Ernst [32], que se formula de la siguiente manera:

[Ec2. 1.]

$$
E_{q q}=\frac{E_{S}}{1+\frac{(w L)^{2} \cdot E_{s}}{12 \cdot \sigma^{3}}}
$$

siendo:

$$
\begin{aligned}
& \sigma \text { - Tensión en el cable } \\
& E_{s} \text { - Módulo de elasticidad del acero } \\
& W \text { - Peso del cable por ml } \\
& \text { L- Longitud del cable }
\end{aligned}
$$

Dicha formulación permite calcular un módulo de deformación reducido debido a la presencia a la curvatura del tirante, parámetro que se deberá obtener realizando un cálculo iterativo, de tal forma que se pueda ajustar su valor en cada tirante en función de la tensión existente en ellos en cada fase del cálculo.

Walther [103] y Arenas [7] indican que la influencia de esta no linealidad es prácticamente despreciable en los cables cortos, y normalmente se puede prescindir de este efecto para el caso de cables de menos de $200 \mathrm{~m}$ de longitud para las situaciones definitivas. Incluso en las etapas de construcción, y siempre que las variaciones de tensión en los cables entre las diferentes fases del proceso de montaje no sean muy importantes, los efectos no lineales debidos a la consideración del cálculo del módulo de Ernst en los tirantes se pueden obviar. Es más, este efecto será más importante en el caso de los puentes con tablero metálico ya que el menor peso propio en este caso de los tableros puede provocar que en las fases iniciales justo después de la colocación de los tirantes, éstos queden con tensiones relativamente reducidas que sí que puedan provocar una cierta curvatura de los cables. En estos 
casos, y aun en los cables más cortos, sí sería necesaria la consideración de los efectos no lineales.

El efecto $P$ - $\Delta$ se produce al considerar la influencia de los fenómenos de $2^{\circ}$ orden, tanto en el pilono como en el tablero, cuando se somete a estos elementos a grandes esfuerzos axiles. Esta situación será tanto más importante en aquellas secciones más solicitadas, como son las secciones de encuentro de tablero y pilono, o como en aquellos elementos de mayor esbeltez, es decir en tableros metálicos. En general para considerar este aspecto se requerirá del uso de la matriz de rigidez geométrica de la estructura.

Por otra parte el efecto de las grandes deformaciones se produce al considerar la influencia de la geometría deformada sobre la propia distribución de esfuerzos en la estructura. Es necesario para ello entonces que la estructura sea muy flexible, condición que se cumple en las fases más avanzadas de construcción, cuando se procede a la ejecución mediante voladizos sucesivos, y cuando el puente tiene unas dimensiones considerables.

Diversas investigaciones han sido llevadas a cabo en relación a evaluar la importancia de estos efectos en los cálculos de estas estructuras como las realizadas por Reddy\&Gaboussy [89], Wang [104], Cheng\&Xiao [25], concluyéndose que su consideración será necesaria cuando analicemos estructuras muy flexibles, como tableros metálicos o puentes de grandes luces, donde la variación entre los resultados de un cálculo lineal de primer orden y un cálculo no lineal pueden ser ya de cierta importancia.

El primero de los anteriores investigadores [89] señaló que se podrían encontrar diferencias de hasta un $9 \%$ entre ambos cálculos (lineal y no lineal). Wang estableció que el principal efecto a considerar es el caso de los grandes desplazamientos, pero señaló que un cálculo no lineal sólo estará justificado para el cálculo de las deformaciones producidas durante la aplicación de las sobrecargas de tráfico, pero no para la determinación de los axiles de los tirantes en la situación final.

Por último Cheng\&Xiao, realizando además un análisis no determinista de la estructura, encontraron que los valores medios de los axiles de los tirantes vendrán condicionados por los efectos P- $\Delta$ y de grandes desplazamientos, pero no así la desviación típica de dichos axiles. En general concluyeron que a efectos de la fiabilidad de la estructura la consideración de las no linealidades se podría evitar. 
En general, y tal y como señala Gabrow [45], la consideración de todos estos efectos no está claro si es necesario en un caso general, aunque si es evidente que su influencia será tanto mayor cuanto más flexible sea la estructura. Así podría deducirse que para puentes de luces cortas o medias, con cables de longitudes menores de 100 $m$, y con tableros de hormigón, las diferencias a encontrar entre un cálculo lineal y un cálculo no lineal serán en principio pequeñas.

Este último autor propone la siguiente tabla como resumen de sus planteamientos:

\begin{tabular}{lccc}
\hline Classification & P-Delta & Non-linear cable & Large displacement \\
\hline $\begin{array}{l}\text { Small structure } \\
\text { Final system }\end{array}$ & $\mathrm{X}$ & & \\
\hline $\begin{array}{l}\text { Small structure } \\
\text { Construction Stage }\end{array}$ & $\mathrm{X}$ & $\mathrm{X}$ & \\
\hline $\begin{array}{l}\text { Large structure } \\
\text { Final system }\end{array}$ & $\mathrm{X}$ & $\mathrm{X}$ & $\mathrm{X}$ \\
\hline Large structure & $10-20 \%$ & $10-20 \%$ & $10 \%$ \\
\hline Construction Stage & $\mathrm{X}$ & $\mathrm{X}$ & \\
\hline
\end{tabular}

Figura 38. Propuesta de consideración de los efectos de 2ํㅡㄹ orden realizada por Gabrow [45]. 


\section{CAPITULO 3.}

\section{COMPORTAMIENTO ESTRUCTURAL DE UN TIRANTE.}

\subsection{INTRODUCCION.}

Se plantea es este capítulo un primer análisis teórico del comportamiento estructural de los tirantes, distinguiendo qué factores afectan al valor final de la tensión en el cable, evaluando un caso sencillo de una estructura con un único tirante. A continuación, y dado que el estudio realizado lo ha sido sobre puentes con tablero de hormigón, se hace una breve reseña de la influencia de los efectos diferidos sobre el tirante. Por último se describen las diferencias entre el método de colocación de los cables mediante fuerzas y mediante deformaciones.

Todo este planteamiento se desarrolla con la intención de presentar un bagaje teórico que nos permita, en los siguientes capítulos, realizar el estudio acerca de la introducción de errores aleatorios en los tirantes, evaluando qué factores influyen en este análisis, cómo se pueden interpretar los resultados y qué conclusiones resultan de todos ellos.

Hay que reseñar que todo el consiguiente desarrollo se ha centrado en el caso de unas tipologías de puentes atirantados concretas, ya que abarcar todo el espectro de posibles soluciones que es posible encontrar en este tipo de estructuras representaría un desarrollo mucho mayor de esta investigación, que por otra parte difuminaría y dificultaría la interpretación de los resultados obtenidos.

Por este motivo se ha decidido plantear el estudio sobre las soluciones que se pueden considerar más habituales en este tipo de estructuras en España. En concreto el estudio se centra en puentes atirantados con las siguientes características:

- Puentes de luces cortas y medias $(100<\mathrm{L}<400 \mathrm{~m})$, ya que representan la mayor parte de las realizaciones ejecutadas hoy en día, más aún en España. Dadas 
estas dimensiones se puede además no considerar la introducción de factores de tipo no lineal (cable-sag, efecto P- $\Delta$, efecto de grandes desplazamientos), sin pérdida de validez de los resultados obtenidos en el estudio, sabiendo que por otra parte estos efectos dificultarán su interpretación. Este último razonamiento se plantea de acuerdo a lo señalado por diferentes autores en el capítulo anterior, los cuales indicaban que, en general, no habrá diferencias apreciables entre un cálculo lineal y un cálculo no lineal en puentes con las dimensiones planteadas en este estudio.

- Puentes con tablero de hormigón. Dado el anterior rango de luces a analizar, las tipologías con tableros de hormigón pueden ser soluciones muy adecuadas, y por ello son muy habituales en la práctica constructiva.

- Puentes con configuración simétrica y desarrollo horizontal en alzado. Estas consideraciones se han realizado para poder simplificar el estudio evitando la influencia de ambas características geométricas.

- Puentes ejecutados por avance mediante voladizos sucesivos desde los pilonos.

Se ha adoptado este procedimiento constructivo dado que es el más habitual y el más lógico plantear para la ejecución de estas estructuras.

Estas cuatro premisas restringen el alcance del estudio realizado, pero tal y como se ha señalado, pretenden dar una visión del problema estudiado en el rango más habitual de soluciones planteadas en la práctica, a sabiendas de que los resultados obtenidos no deberían extrapolarse a otro tipo de soluciones sin tener en cuenta las diferencias específicas con las soluciones aquí estudiadas. Igualmente estas características nos harán incidir sobre ciertos aspectos particulares de consideración en el comportamiento y cálculo de estas estructuras en concreto.

\subsection{AXIL ACTIVO Y AXIL PASIVO DE UN TIRANTE.}

El comportamiento de los tirantes dentro del global de la estructura sigue un esquema complejo, ya que debemos considerar que desde el momento del tesado, reciben no sólo la acción de la propia tensión aplicada por los gatos en sus extremos, si no que a partir de ese momento absorberán una parte del peso propio de las nuevas dovelas hormigonadas. Es decir que se podría descomponer su comportamiento en la suma de dos términos: uno como elemento activo, debido a las cargas de tesado, y otro como 
elemento pasivo, al igual que el resto de partes de la estructura, que debe ser capaz de soportar el peso de la propia estructura.

Estas definiciones se pueden observar más claramente a partir del funcionamiento representado en el siguiente esquema, correspondiente a una viga de un solo vano de longitud $2 \cdot L$, apoyada en un tirante en su punto medio, de tal forma que, suponiendo la ejecución de la viga cimbrada, se instalará el cable sin tensión para a continuación proceder al descimbrado y posterior puesta en carga del tirante, imponiendo la condición a la fuerza de tesado de que sea aquella que consiga anular el desplazamiento vertical del punto de anclaje del cable. Es decir, que el esquema final de la estructura corresponderá al caso de una viga continua de 2 vanos de luces la mitad de la de viga original.
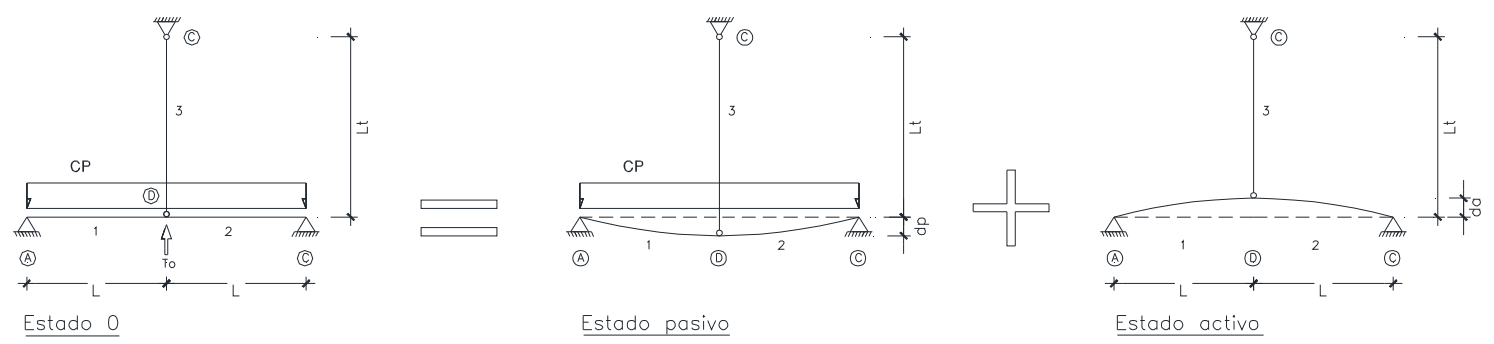

Figura 39. Esquema del comportamiento de un tirante (I).

De esta forma, y según el esquema de la figura anterior, se puede descomponer el esfuerzo del tirante sobre el estado final de la estructura (Estado 0) en la suma de otros 2 estados denominados Estado activo y Estado pasivo, en los que se han separado la actuación de las cargas permanentes por un lado y por otro lado el efecto del tesado del cable, de tal manera que se cumpla la condición de compatibilidad en deformaciones entre tirante y viga, y verifique la anulación del desplazamiento vertical del nudo D (punto de anclaje del tirante). Así resultará que:

$$
\text { [Ec3. 1.] } \mathrm{d}_{0}=\mathrm{d}_{\mathrm{a}}+\mathrm{d}_{\mathrm{p}}
$$

siendo: $\quad$ do $\rightarrow$ Flecha final del nudo $D$.

$$
\begin{aligned}
& d_{a} \rightarrow \text { Flecha en el estado activo del nudo D. } \\
& d_{p} \rightarrow \text { Flecha en el estado pasivo del nudo D. }
\end{aligned}
$$

y al imponer la condición de anulación del desplazamiento en D: $\quad \mathrm{d}_{0}=0$ 
resulta:

$$
\text { [Ec3. 2.] } \mathrm{d}_{\mathrm{p}}=-\mathrm{d}_{\mathrm{a}}
$$

a partir de esa condición de compatibilidad se pueden analizar los alargamientos y los axiles que soporta el tirante siguiendo lo indicado en el siguiente esquema:

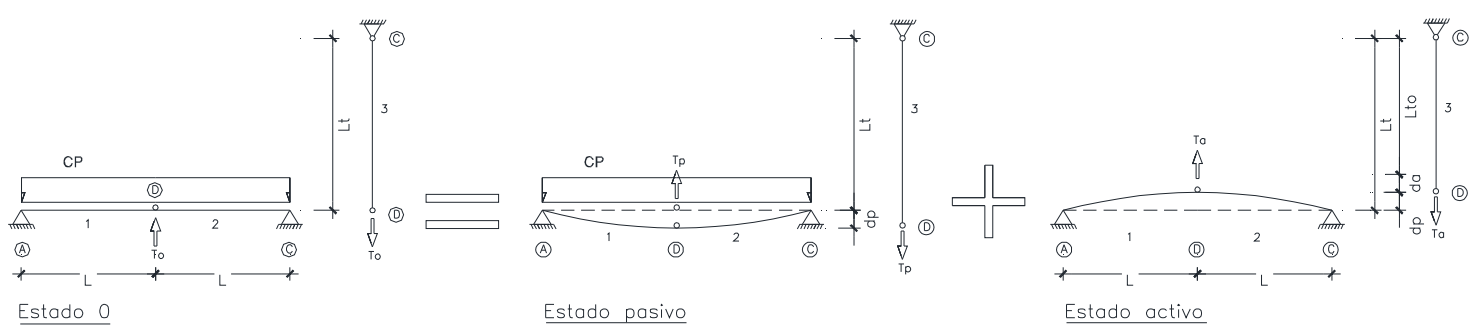

Figura 40. Esquema del comportamiento de un tirante (II).

de donde se deduce que el alargamiento total del tirante (dL) será:

$$
\text { [Ec3. 3.] } d L=d_{p}+d_{a}
$$

y a partir de la longitud final del tirante $\left(L_{+}\right)$, la longitud inicial o longitud neutra $\left(L_{+0}\right)$ de éste entonces resulta:

$$
\text { [Ec3. 4.] } \mathrm{L}_{\mathrm{t} 0}=\mathrm{L}_{\mathrm{t}}-\mathrm{dL}=\mathrm{L}_{\mathrm{t}}-\mathrm{d}_{\mathrm{p}}-\mathrm{d}_{\mathrm{a}}
$$

Implícitamente se observa entonces que el axil final en el tirante se compondrá de la suma de las dos componentes activa y pasiva reseñadas, es decir que:

$$
\text { [Ec3. 5.] } \mathrm{T}_{0}=\mathrm{T}_{\mathrm{a}}+\mathrm{T}_{\mathrm{p}}
$$

siendo: $\quad$ To $\rightarrow$ Tensión final en el cable.

$$
\begin{aligned}
& T_{a} \rightarrow \text { Componente activa de la tensión del tirante. } \\
& T_{p} \rightarrow \text { Componente pasiva de la tensión del tirante. }
\end{aligned}
$$

El anterior esquema, al representar una estructura con un solo cable, no refleja un aspecto importante que más adelante se verá detallado, la influencia de unos tirantes sobre otros, ya que el efecto del tesado de un tirante afectará siempre a los tirantes vecinos. Es decir, este esquema sirve para reflejar bien el diferente origen del esfuerzo 
en un tirante, pero no representa completamente el comportamiento real de un tirante lógicamente.

Hay que señalar que el anterior planteamiento coincide con el indicado, tanto en el Manual de tirantes de ACHE [2], como en las recomendaciones del SETRA [102], en las que se hace hincapié en el diferente origen del axil final en un tirante, distinguiendo la parte debida al reparto de las cargas permanentes de la estructura entre los distintos tirantes, y por otra parte la parte materializada directamente mediante los gatos de tesado de los cables. Esta distinción se señala en ambas normativas que tiene ciertas implicaciones respecto de los formatos de seguridad a aplicar que se detallarán más adelante.

\subsection{FACTORES QUE INFLUYEN EN EL AXIL ACTIVO Y PASIVO.}

A la vista de lo indicado en el epígrafe anterior, una de las primeras necesidades para poder comenzar el análisis que vamos a desarrollar en adelante, será el conocer qué parte, respecto de los esfuerzos finales introducidos sobre ellos, corresponde a la parte activa, y cuál a la pasiva.

En general, este reparto podrá depender de muy diferentes factores, como puede ser, en primer lugar, las rigideces del tablero y de las pilas. Por ejemplo, en el caso de tableros muy rígidos, como puede ocurrir cuando las separaciones entre tirantes son grandes o los anchos del tablero son importantes, y consecuentemente es necesario disponer secciones con cantos importantes, o en el caso de puentes de dimensiones reducidas, donde la preponderancia de la rigidez del tablero respecto del mecanismo resistente de los tirantes es más alta, la propia capacidad del tablero para resistir las cargas aplicadas se traducirá en una menor efectividad de los tirantes. Igual ocurrirá si los pilonos no tienen suficiente rigidez para limitar las deformaciones en los extremos de los cables, lo que también causará una menor efectividad en el aprovechamiento del atirantamiento.

Otros parámetros que pueden influir son: la rigidez del propio tirante, evaluada a partir de la longitud del cable y del área de éste, y el valor de las cargas muertas aplicadas sobre la estructura.

Para valorar todos estos efectos se ha analizado el siguiente esquema estructural, el cual representa de forma simplificada una estructura ejecutada por avance en voladizo, y más en concreto el caso de las 2 últimas dovelas que llegarían al estribo. 
Con este esquema se puede analizar la influencia de todos los anteriores factores, evaluando la importancia relativa de unos respecto de otros a la hora de conformar los dos términos del axil del tirante. Faltaría eso sí, evaluar la influencia de la instalación de un tirante respecto de su vecino, pero este aspecto se comentará en los siguientes capítulos sobre estructuras reales.

El esquema planteado es el siguiente:

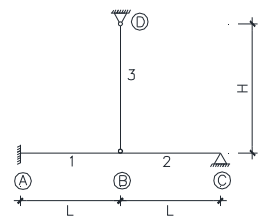

Estado Final

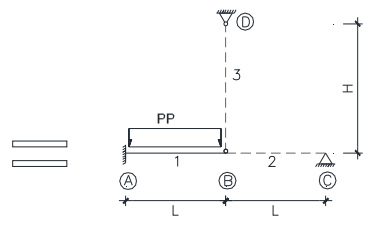

Fase 1 Peso propio
Dovela -1
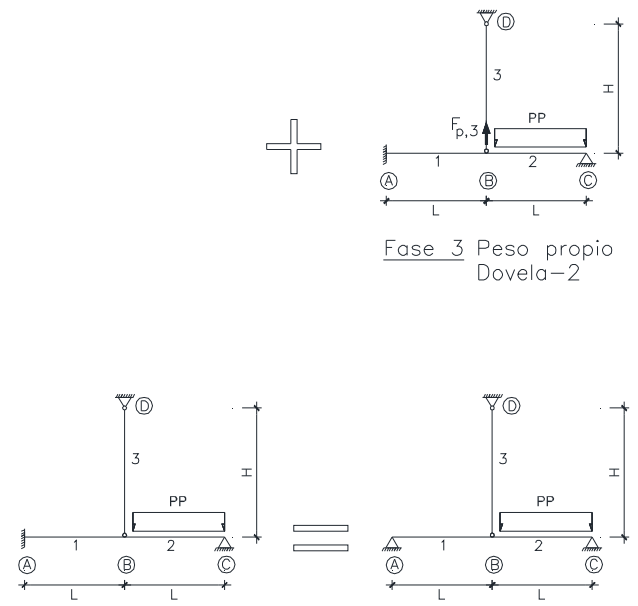

Fase 3

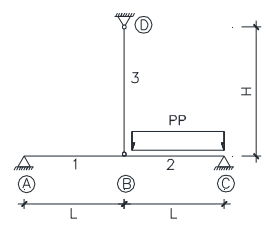

Estado 1

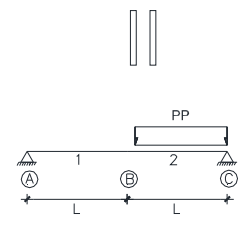

Estado la

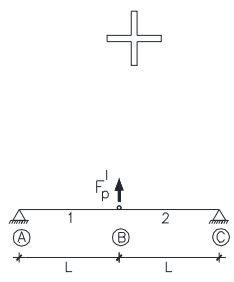

Estado Ib

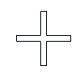

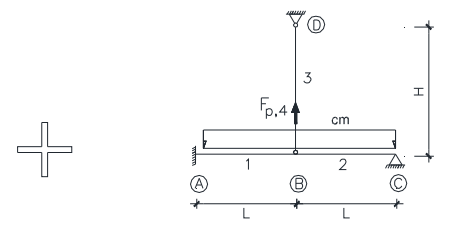

Fase 4 Aplicación

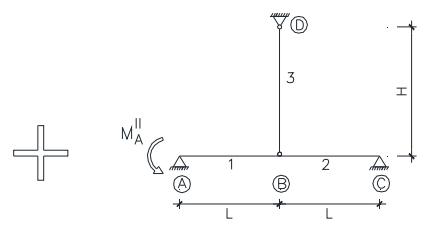

Estado II

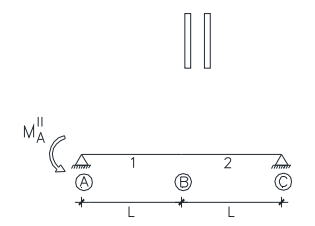

Estado Ila

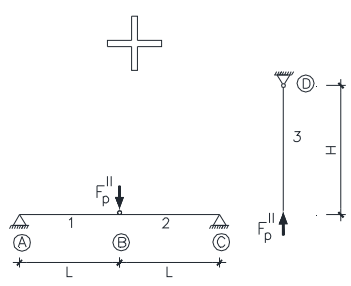

Estado IIb

Figura 41. Esquema estructural del modelo simplificado(I). 


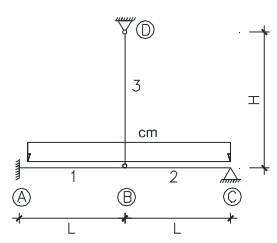

Fase 4

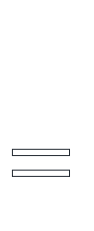

Estado II

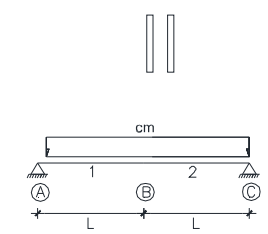

Estado IIla

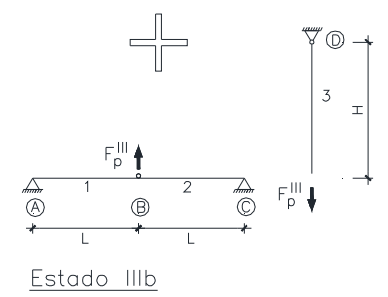

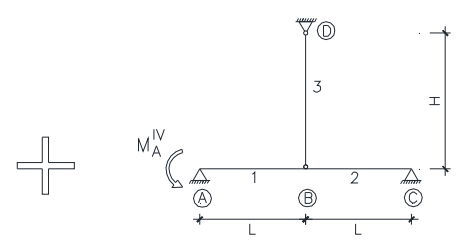

Estado IV

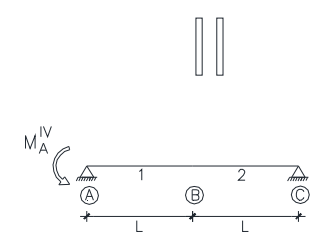

Estado IVa

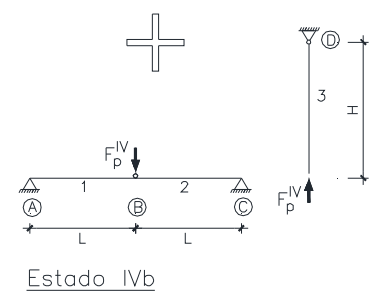

Figura 42. Esquema estructural del modelo simplificado(II).

Utilizando la anterior descomposición en estados podemos plantear las siguientes ecuaciones de compatibilidad en desplazamientos, las cuales permiten obtener las soluciones analíticas de los esfuerzos axiles en el tirante, tanto la parte activa correspondiente al tesado de éste, como la parte pasiva correspondiente a la influencia de los parámetros estudiados. Así entonces:

siendo: $\quad$ pp $\rightarrow$ Peso por $\mathrm{ml}$ de la sección del tablero en $\mathrm{KN} / \mathrm{ml}$.

$\mathbf{c m} \rightarrow$ Carga muerta aplicada sobre el tablero en $\mathrm{KN} / \mathrm{ml}$.

$\mathbf{E}_{c} \rightarrow$ Módulo de deformación del hormigón del tablero en $\mathrm{N} / \mathrm{mm}^{2}$.

$\mathbf{I} \rightarrow$ Inercia del tablero

$E_{s} \rightarrow$ Módulo de elasticidad del acero de los tirantes en $\mathrm{N} / \mathrm{mm}^{2}$.

$\mathbf{A}_{\mathbf{s}} \rightarrow$ Area del tirante en $\mathrm{cm}^{2}$.

$\mathbf{L} \rightarrow$ Separación entre anclajes en $\mathrm{m}$.

$\mathbf{H} \rightarrow$ Altura entre el punto de anclaje en el tablero y en el pilono en $\mathrm{m}$.

$\mathbf{M}_{\mathbf{A}} \rightarrow$ Mto. en nudo A de empotramiento de dovelas ya ejecutadas en $\mathrm{KN} \cdot \mathrm{m}$.

$F_{\mathbf{A}} \rightarrow$ Axil de tesado del tirante en $\mathrm{KN}$.

$F_{\mathrm{p}} \rightarrow$ Parte pasiva total del axil del tirante en $\mathrm{KN}$. 


\section{$\mathbf{F}_{\text {tot }} \rightarrow$ Axil total en el tirante en $\mathrm{KN}$.}

A. Condición de anulación del giro en A para la Fase 3 de aplicación del peso propio de la dovela final. Obtención del momento $M_{A}{ }^{\prime \prime} y$ componente pasiva $F_{p, 3}$.

$$
\text { [Ec3. 6.] } \quad \theta_{A}^{I a}+\theta_{A}^{I b}+\theta_{A}^{I I a}+\theta_{A}^{I I b}=0
$$

siendo los giros de las hipótesis individuales:
[Ec3. 7.] $\theta_{A}^{I a}=\frac{7}{48} \frac{p p \cdot L^{3}}{E_{c} \cdot I}$
[Ec3. 9.] $\quad \theta_{A}^{I I a}=\frac{2}{3} \frac{M_{A}^{I I} \cdot L}{E_{c} \cdot I}$
[Ec3. 8.] $\quad \theta_{A}^{I b}=\frac{1}{4} \frac{F_{P}^{I} \cdot L^{2}}{E_{c} \cdot I}$
[Ec3. 10.] $\theta_{A}^{I I b}=\frac{1}{4} \frac{F_{P}^{I I} \cdot L^{2}}{E_{c} \cdot I}$

con

$$
\begin{aligned}
& \text { [Ec3. 11.] } \quad F_{P}^{I}=\frac{5}{48} \frac{p p \cdot L^{4}}{E_{c} \cdot I} \frac{1}{\left(\frac{H}{E_{s} \cdot A_{s}}+\frac{L^{3}}{6 \cdot E_{c} \cdot I}\right)} \\
& \text { [Ec3. 12.] } \quad F_{P}^{I I}=\frac{M_{A}^{I I} \cdot L^{2}}{4 \cdot E_{c} \cdot I} \frac{1}{\left(\frac{H}{E_{s} \cdot A_{s}}+\frac{L^{3}}{6 \cdot E_{c} \cdot I}\right)}
\end{aligned}
$$

llamando

$$
\text { [Ec3. 13.] } Z=\frac{H}{E_{s} \cdot A_{s}}+\frac{L^{3}}{6 \cdot E_{c} \cdot I}
$$

y además:

$$
\text { [Ec3. 14.] W= } \frac{1}{\frac{2 \cdot L}{3 \cdot E_{c} \cdot I}-\frac{L^{4}}{16 \cdot E_{c}^{2} \cdot I^{2} \cdot Z}}
$$

resulta:

$$
\text { [Ec3. 15.] } \quad M_{A}^{I I}=W \cdot\left(\frac{7}{48} \frac{p p \cdot L^{3}}{E_{c} \cdot I}-\frac{F_{P}^{I} \cdot L^{2}}{4 \cdot E_{c} \cdot I}\right)
$$


es decir que conocido el valor de $\mathrm{Fp}_{\mathrm{p}}$ ', que depende únicamente de las características geométricas y mecánicas del esquema calculado, así como del peso propio, se puede calcular el valor de $M_{A}{ }^{\prime \prime}$, y a su vez se puede obtener entonces:

$$
\text { [Ec3. 16.] } \quad F_{P, 3}=\frac{1}{Z}\left(\frac{5}{48} \frac{p p \cdot L^{4}}{E_{c} \cdot I}-\frac{1}{4} \frac{M_{A}^{I I} \cdot L^{2}}{E_{c} \cdot I}\right)
$$

B. Condición de anulación del giro en A para la Fase 4 de aplicación de las cargas muertas sobre el tablero. Obtención del momento $M_{A}^{\prime V}$ y de la componente pasiva $F_{p, 4}$.

$$
\text { [Ec3. 17.] } \quad \theta_{A}^{I I I a}+\theta_{A}^{I I I b}+\theta_{A}^{I V a}+\theta_{A}^{I V b}=0
$$

siendo:

[Ec3. 18.] $\theta_{A}^{I I I a}=\frac{1}{3} \frac{\mathrm{cm} \cdot \mathrm{L}^{3}}{E_{c} \cdot I}$ [Ec3. 20.] $\theta_{A}^{I V a}=\frac{2}{3} \frac{M_{A}^{I V} \cdot L}{E_{c} \cdot I}$

[Ec3. 19.] $\theta_{A}^{I I I b}=\frac{1}{4} \frac{F_{P}^{I I I} \cdot L^{2}}{E_{c} \cdot I}$ [Ec3. 21.] $\theta_{A}^{I V b}=\frac{1}{4} \frac{F_{P}^{I V} \cdot L^{2}}{E_{c} \cdot I}$

con

[Ec3. 22.] $\quad F_{P}^{I I I}=\frac{5}{24} \frac{\mathrm{cm} \cdot \mathrm{L}^{4}}{E_{c} \cdot I} \frac{1}{Z}$ [Ec3. 23.] $\quad F_{P}^{I V}=\frac{M_{A}^{I V} \cdot L^{2}}{4 \cdot E_{c} \cdot I} \frac{1}{Z}$

resulta:

[Ec3. 24.]

$$
\begin{aligned}
& M_{A}^{I V}=W \cdot\left(\frac{1}{3} \frac{\mathrm{cm} \cdot L^{3}}{E_{c} \cdot I}-\frac{F_{P}^{I I I} \cdot L^{2}}{4 \cdot E_{c} \cdot I}\right) \\
& F_{P, 4}=\frac{1}{Z}\left(\frac{5}{24} \frac{\mathrm{cm} \cdot L^{4}}{E_{c} \cdot I}-\frac{1}{4} \frac{M_{A}^{I V} \cdot L^{2}}{E_{c} \cdot I}\right)
\end{aligned}
$$

C. Condición de anulación de desplazamientos verticales en el nudo B como la suma de movimientos de las fases de construcción, para la obtención del axil de tesado $F_{A}$.

[Ec3. 26.] $\quad \delta_{B}^{1}+\delta_{B}^{2}+\delta_{B}^{3}+\delta_{B}^{4}=0$

siendo los desplazamientos verticales de cada estado los siguientes: 

[Ec3. 27.] $\delta_{B}^{1}=-\frac{p p \cdot L^{4}}{8 \cdot E_{c} \cdot I}$
[Ec3. 29.] $\delta_{B}^{3}=-\frac{F_{P, 3} \cdot H}{E_{s} \cdot A_{s}}$
[Ec3. 28.] $\delta_{B}^{2}=\frac{F_{a} \cdot L^{3}}{3 \cdot E_{c} \cdot I}$
[Ec3. 30.] $\delta_{B}^{4}=-\frac{F_{P, 4} \cdot H}{E_{s} \cdot A_{s}}$

resultando entonces que el axil necesario introducir en el tirante para anular el desplazamiento del nudo B al final del proceso constructivo resulta:

[Ec3.31.] $\quad F_{a}=\frac{3 \cdot E_{c} \cdot I}{L^{3}}\left[\frac{p p \cdot L^{4}}{8 \cdot E_{c} \cdot I}+\frac{\left(F_{P, 3}+F_{P, 4}\right) \cdot H}{E_{s} \cdot A_{s}}\right]$

y la parte pasiva del axil final del tirante:

[Ec3. 32.] $\quad F_{P}=F_{P, 3}+F_{P, 4}$

A partir de esta formulación se procede a realizar un estudio paramétrico, que se presenta en los siguientes epígrafes, con la intención antes señalada. En este estudio se resuelven las ecuaciones anteriores para los distintos casos analizados manteniendo constantes los valores de los módulos de elasticidad del acero y de deformación del hormigón:

- $\mathrm{E}_{\mathrm{c}}=30.000 \mathrm{~N} / \mathrm{mm}^{2}$

- $E_{s}=200.000 \mathrm{~N} / \mathrm{mm}^{2}$

\subsubsection{Variación del $\% F_{p} / F_{\text {tot }}$ en función de la separación entre anclajes.}

El primer parámetro analizado ha sido la separación entre anclajes $L$, que también podría definirse como la longitud de las dovelas. Para ello se ha calculado el porcentaje del axil pasivo del tirante respecto del axil total para una serie de valores de este parámetro, en concreto se han adoptado valores desde $4^{\prime} 0$ a $11^{\prime} 0 \mathrm{~m}$, con la intención de cubrir un rango que pueda presentarse en la realidad.

Adoptando para el resto de parámetros:

Sección tablero 10'00x1'00 m: Que corresponde a un tablero tipo losa aligerada para un ancho de calzada de $10^{\prime} 0 \mathrm{~m}$. 
$\checkmark H=28^{\prime} 9 \mathrm{~m}$, altura que correspondería al caso de un cable extremo de un tablero con pilono central de $50 \mathrm{~m}$ de luz y ángulo $30^{\circ}$.

$\checkmark$ Las áreas de los cables se han ajustado de tal forma que en cada caso calculado las tensiones en el acero queden en el mismo intervalo de valores en el rango de $\sigma_{s} \approx 500-600 \mathrm{~N} / \mathrm{mm}^{2}$.

$\checkmark \mathrm{cm}=28^{\prime} 5 \mathrm{KN} / \mathrm{ml}$ que puede suponer un valor razonable para el ancho de tablero considerado de $10^{\prime} 0 \mathrm{~m}$.

Se adjunta gráfico con los valores obtenidos:

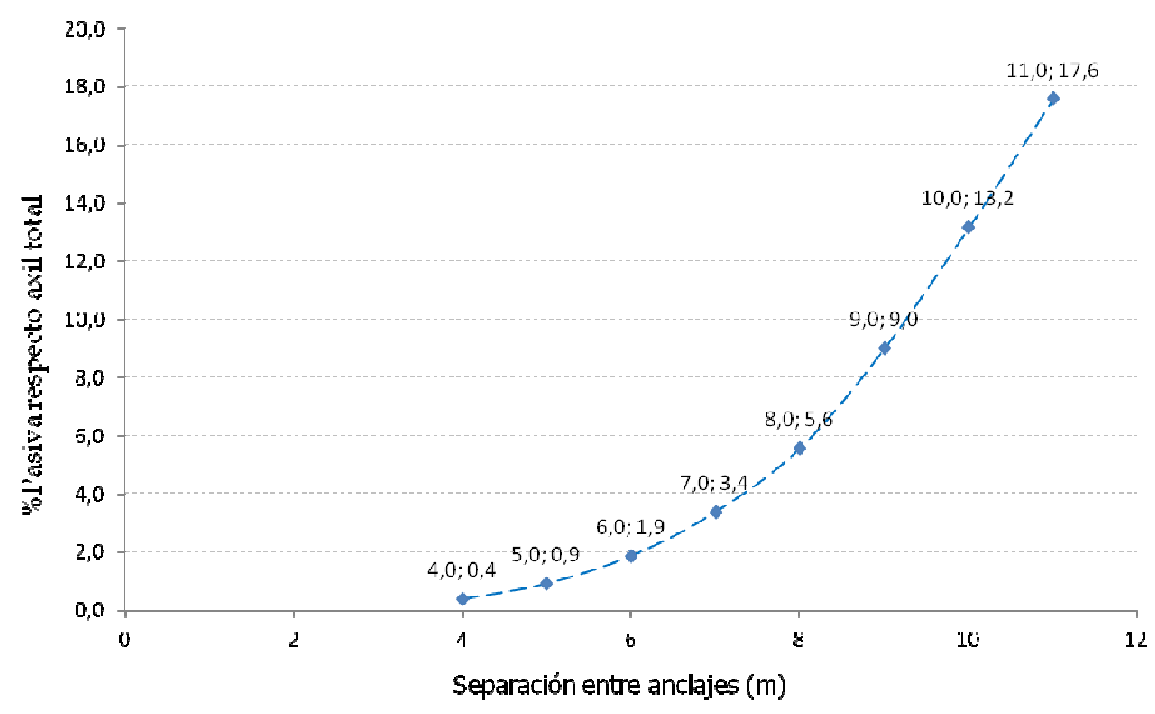

Figura 43. Relación del axil pasivo con la separación entre anclajes.

Del gráfico anterior se deduce que el axil pasivo para el caso analizado presenta siempre porcentajes relativamente bajos $(<20 \%)$ y existe una relación no proporcional entre la separación establecida y los valores obtenidos, creciendo de forma más importante cuanto mayor es la separación entre anclajes. Este resultado se justifica por el hecho de que a mayor separación entre anclajes, para la misma inercia del tablero, significa una mayor flexibilidad de éste, y por lo tanto una mayor eficacia relativa del sistema de atirantamiento. Adicionalmente implicará un aumento de la parte pasiva del tirante respecto de la parte activa.

\subsubsection{Variación del $\% F_{p} / F_{\text {tot }}$ en función de la longitud del tirante.}

Se ha procedido a evaluar este parámetro pensando en la influencia que puede tener la rigidez del tirante sobre los resultados, es decir se ha analizado este aspecto para 
conocer la influencia de la diferente longitud de los tirantes dentro del conjunto de los cables dispuestos, siendo por lo tanto en ese aspecto más flexibles los cables extremos que los cables iniciales.

Indirectamente, en este caso se puede proceder también a evaluar la influencia de la luz del tablero sobre los resultados obtenidos en el axil pasivo, dada la relación existente entre esta magnitud y la longitud de los cables a disponer.

En este caso se han adoptado para el resto de parámetros los siguientes valores constantes en todos los cálculos:

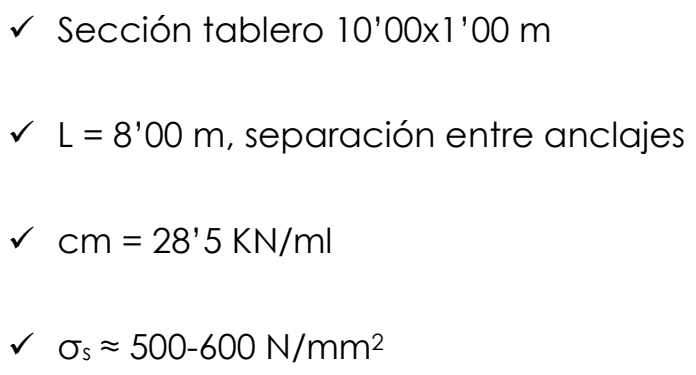

Se incluye gráfico donde se resumen los cálculos realizados, y donde se remarca el resultado que ya fue obtenido en el anterior epígrafe, de tal forma que conocido ese valor se puede tener una idea del rango de variación que provoca este parámetro.

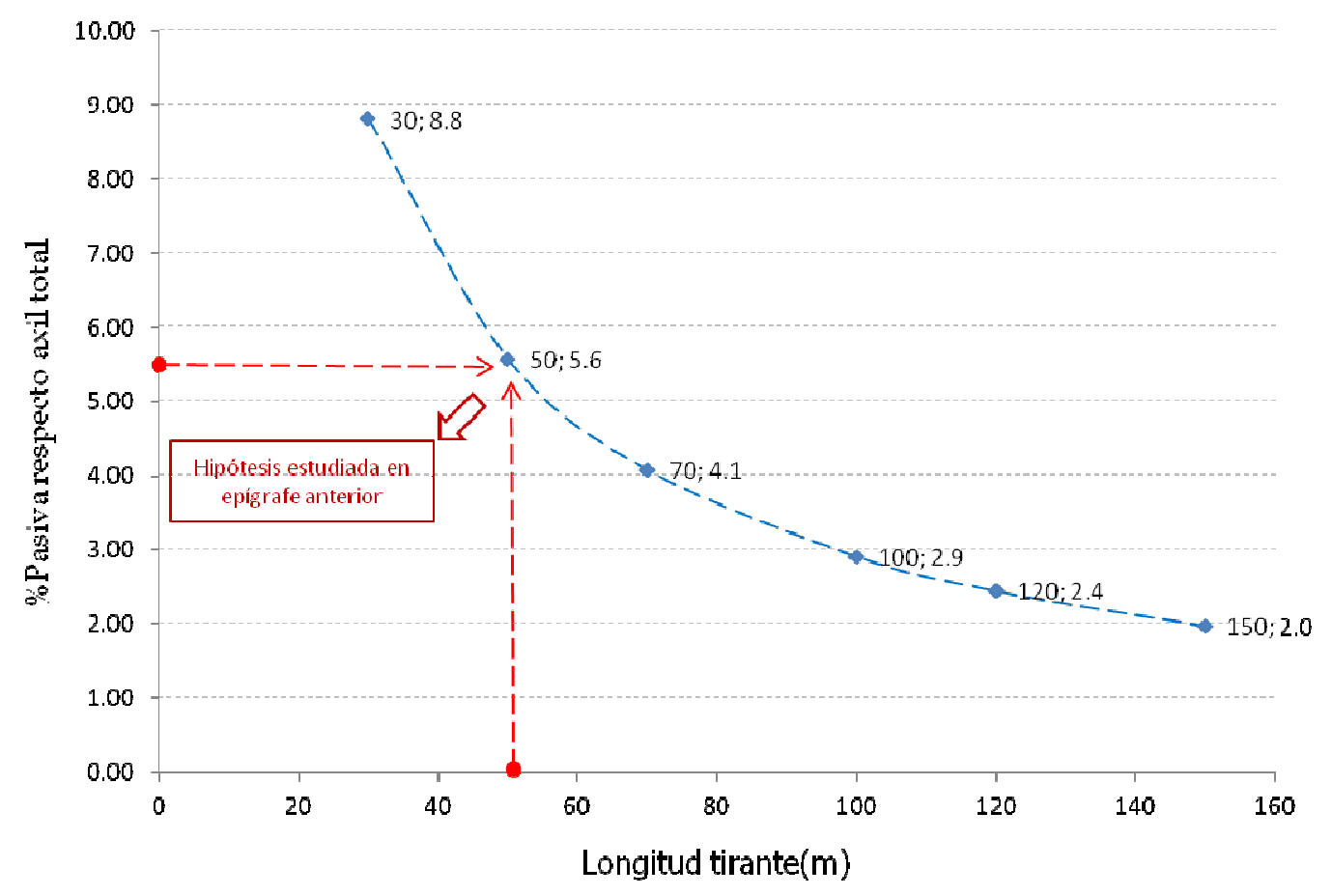

Figura 44. Relación del axil pasivo con la longitud del tirante. 
En este caso se observa que aumentando la longitud del tirante, es decir disminuyendo la rigidez del tirante, se produce una reducción de la parte pasiva del tirante, reducción que no es proporcional siendo más acusada para el caso de los tirantes más cortos. Este resultado significa que los cables cortos tendrán un mayor porcentaje de axil pasivo que los cables largos. Hay que decir de cualquier manera que los porcentajes respecto del axil total son reducidos ya que varían del 2 al $9 \%$.

\subsubsection{Variación del $\% F_{p} / F_{\text {tot }}$ en función del ancho del tablero.}

Se pretende en este caso evaluar la variación del axil pasivo que genera la modificación del ancho del tablero. Este parámetro influye en la formulación planteada de dos maneras diferentes:

$\checkmark \quad$ En primer lugar, y supuesto el tablero como una losa de hormigón, el canto se ha ajustado a la dimensión transversal de éste. En concreto se ha establecido una relación 1/10 para el cociente entre el canto y ancho del tablero. Como ya se ha indicado este aspecto puede que no sea realista en el caso de tableros bijácena y será sólo cierto para el caso de tableros tipo losa.

$\checkmark$ En segundo lugar, y como consecuencia del anterior condicionante, incrementando el peso propio del tablero

$\checkmark \quad$ Por último, aumentando la inercia longitudinal del tablero.

El rango de valores calculados para el ancho del tablero varía desde 6'00 hasta 16'0 $m$, valores que se ajustan a las dimensiones normalmente ejecutadas en este tipo de estructuras. En el caso del resto de parámetros implicados en los cálculos resulta:

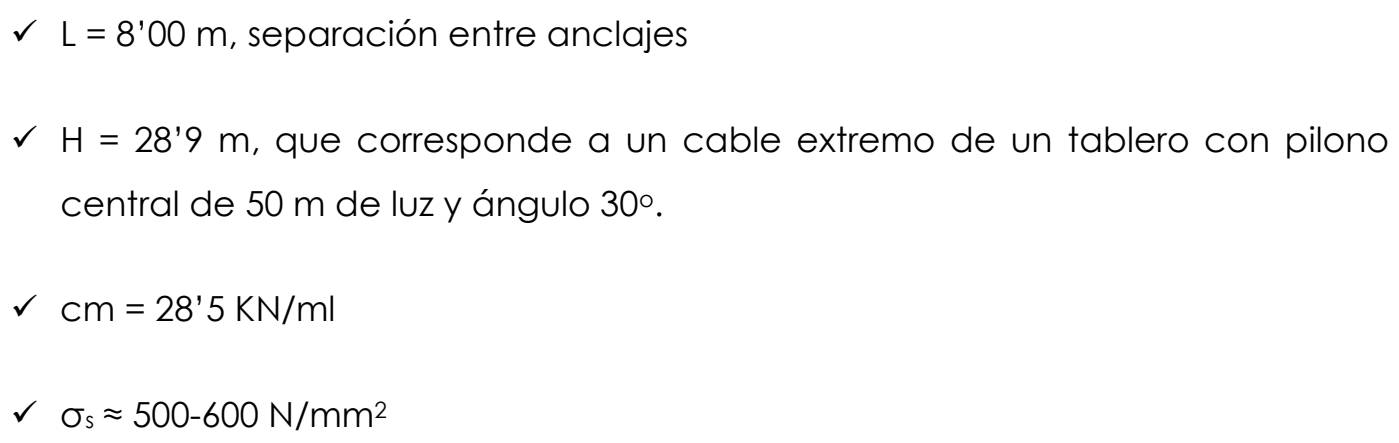

Así los resultados gráficamente resultan: 


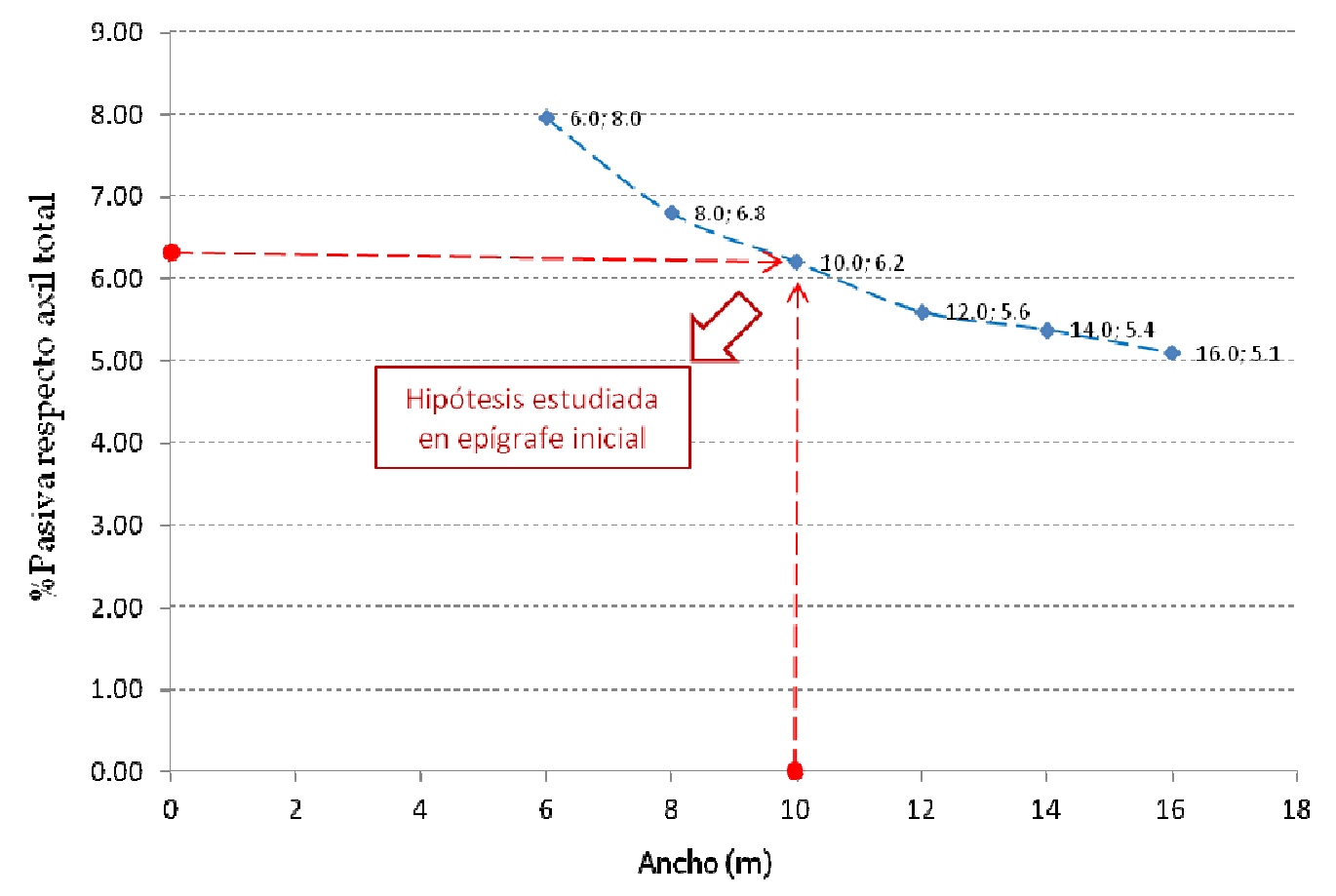

Figura 45. Relación del axil pasivo con el ancho del tablero. Caso con canto ajustado al ancho.

De los resultados anteriores se desprende que el rango de variación del axil pasivo es de entre un $8^{\prime} 0$ a 5'0\% del axil total, siendo menor este porcentaje cuanto mayor es el ancho del tablero, es decir cuánto más rígido es el tablero.

Este mismo análisis se puede realizar manteniendo constante el canto del tablero, pero no así el peso propio y la inercia de éste, ya que este parámetro se hace variar de forma proporcional al ancho del tablero. El resto de parámetros utilizados son:

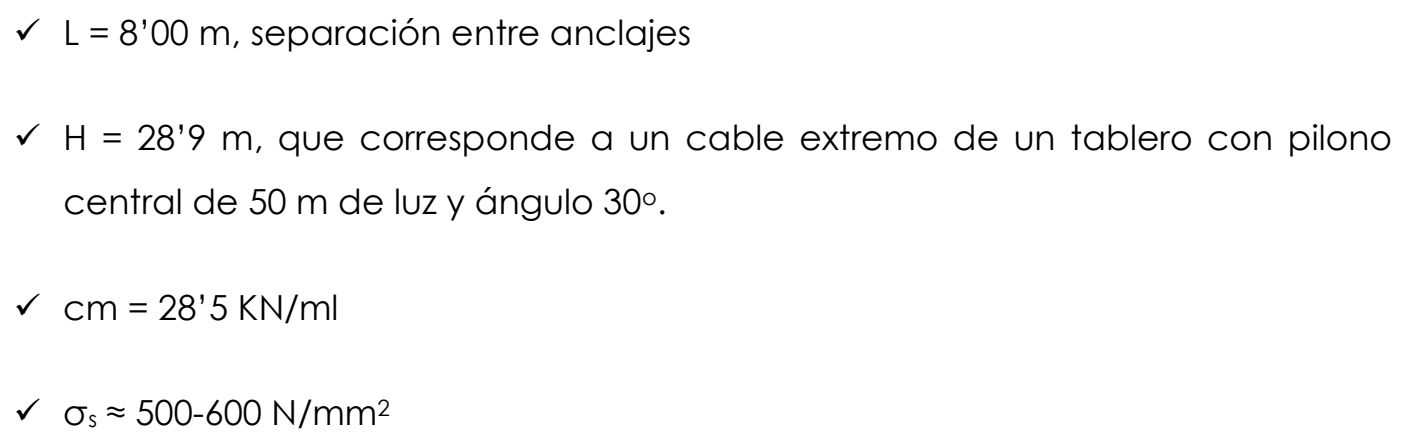

De esta forma los resultados que se reflejan en la Figura 46 , y que muestran que apenas hay variación respecto del caso anterior en cuanto a porcentaje de axil pasivo generado en los tirantes. 


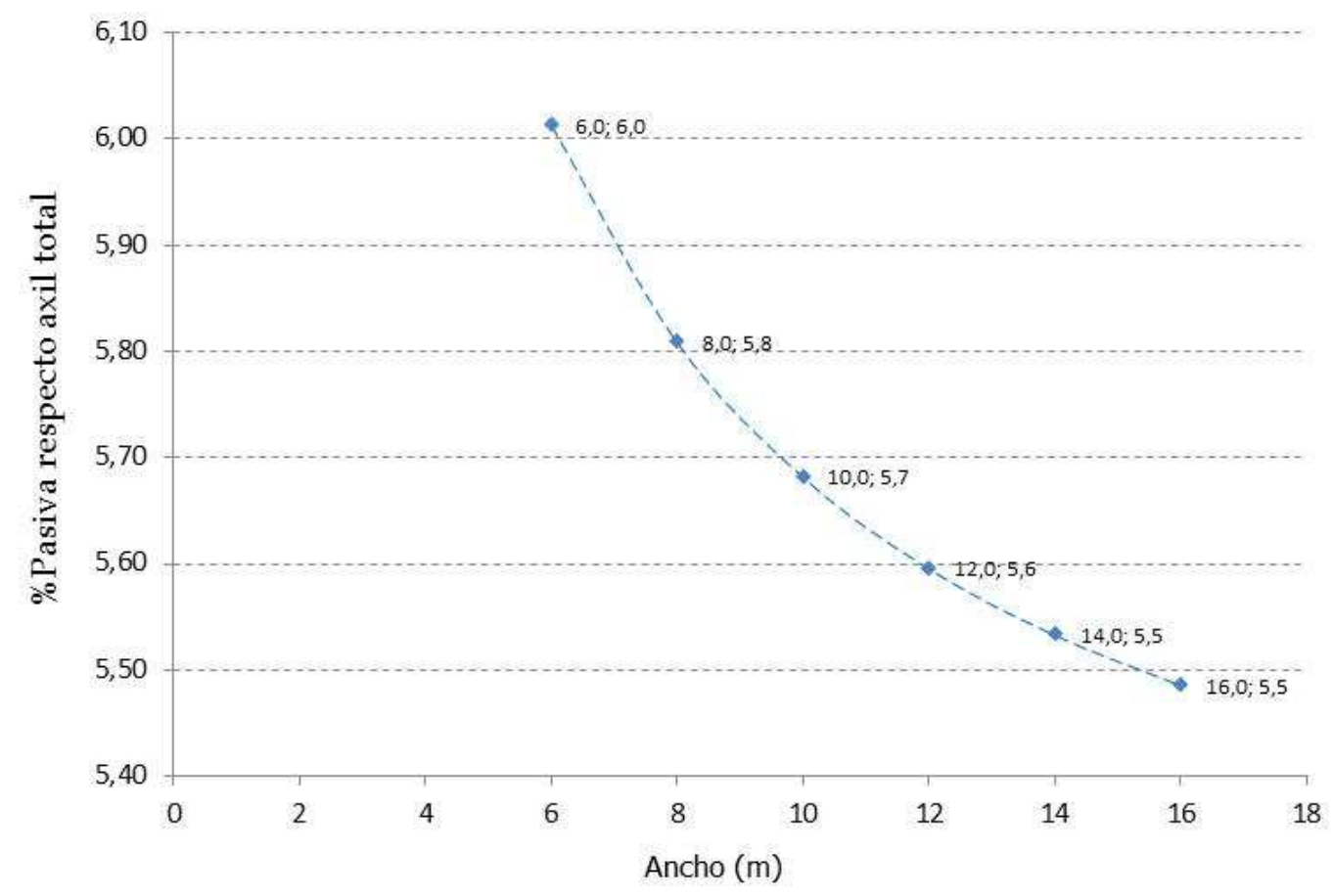

Figura 46. Relación del axil pasivo con el ancho del tablero. Caso con canto constante del tablero.

\subsubsection{Variación del $\% F_{p} / F_{\text {tot }}$ en función de la carga muerta aplicada.}

En este caso se pretende analizar cómo influye el valor de carga muerta aplicada. De esta forma, este parámetro se hace variar en un rango de valores de $10^{\prime} 0$ a 80'0 KN/ml.

En el caso del resto de parámetros:

$\checkmark L=6$ '00 m, Separación entre anclajes

Sección tablero 10'00×1'00 m

$\checkmark H=28^{\prime} 9 \mathrm{~m}$, que corresponde a un cable extremo de un tablero con pilono central de $50 \mathrm{~m}$ de luz y ángulo $30^{\circ}$.

$\checkmark \sigma_{s} \approx 500-600 \mathrm{~N} / \mathrm{mm}^{2}$ 


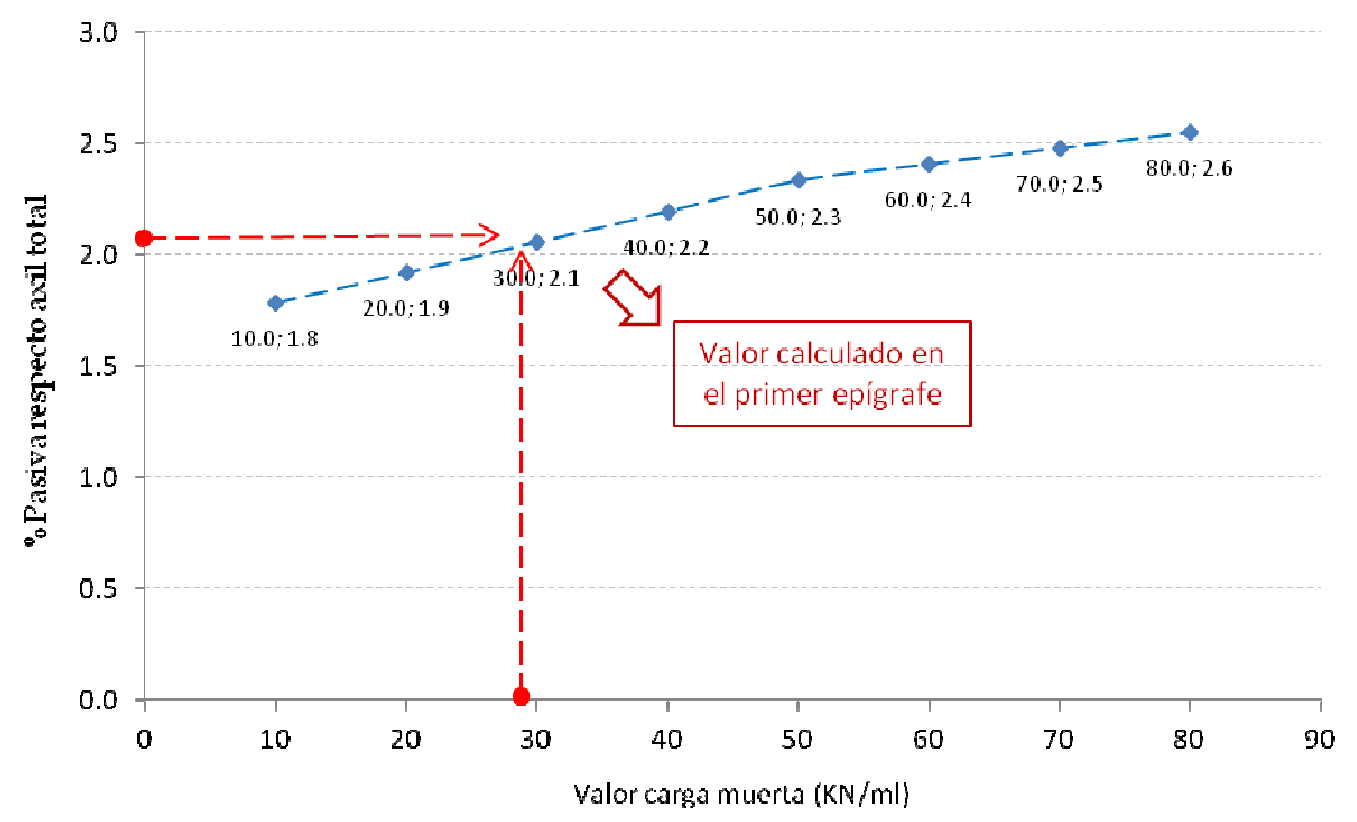

Figura 47. Relación del axil pasivo con el valor de la carga muerta aplicada en el tablero.

De estos resultados se concluye que este parámetro aporta un rango de variación muy estrecho de entre $1^{\prime} 5$ al 2'5\%, siendo el valor del axil pasivo creciente con el aumento de la carga muerta.

Tal vez este aspecto sea más adecuado analizarlo en función de la relación entre la carga muerta y el peso propio del tablero, habiéndose entonces analizado en ese caso relaciones de entre un $4 \%$ y un $30 \%$, siendo el valor adoptado como referencia en el resto de análisis realizados del $11 \%$.

\subsubsection{Variación del $\% F_{p} / F_{\text {tot }}$ en función de la tensión generada en el tirante.}

Se busca en este caso analizar en función del grado de aprovechamiento de los tirantes que repercusión tiene sobre el axil pasivo. El aprovechamiento de los tirantes se mide en función de la tensión generada en los cables, cuyo rango se establece entre 200 y $800 \mathrm{~N} / \mathrm{mm}^{2}$. Se adoptan para el resto de parámetros los siguientes valores:

$\checkmark L=6 ’ 00 \mathrm{~m}$, Separación entre anclajes.

$\checkmark$ Sección tablero 10’00x1'00 m.

$\checkmark H=28 ' 9 \mathrm{~m}$, que corresponde a un cable extremo de un tablero con pilono central de $50 \mathrm{~m}$ de luz y ángulo $30^{\circ}$. 


$$
\checkmark \mathrm{cm}=28 ' 5 \mathrm{KN} / \mathrm{ml}
$$

Se adjunta gráfico con los resultados obtenidos:

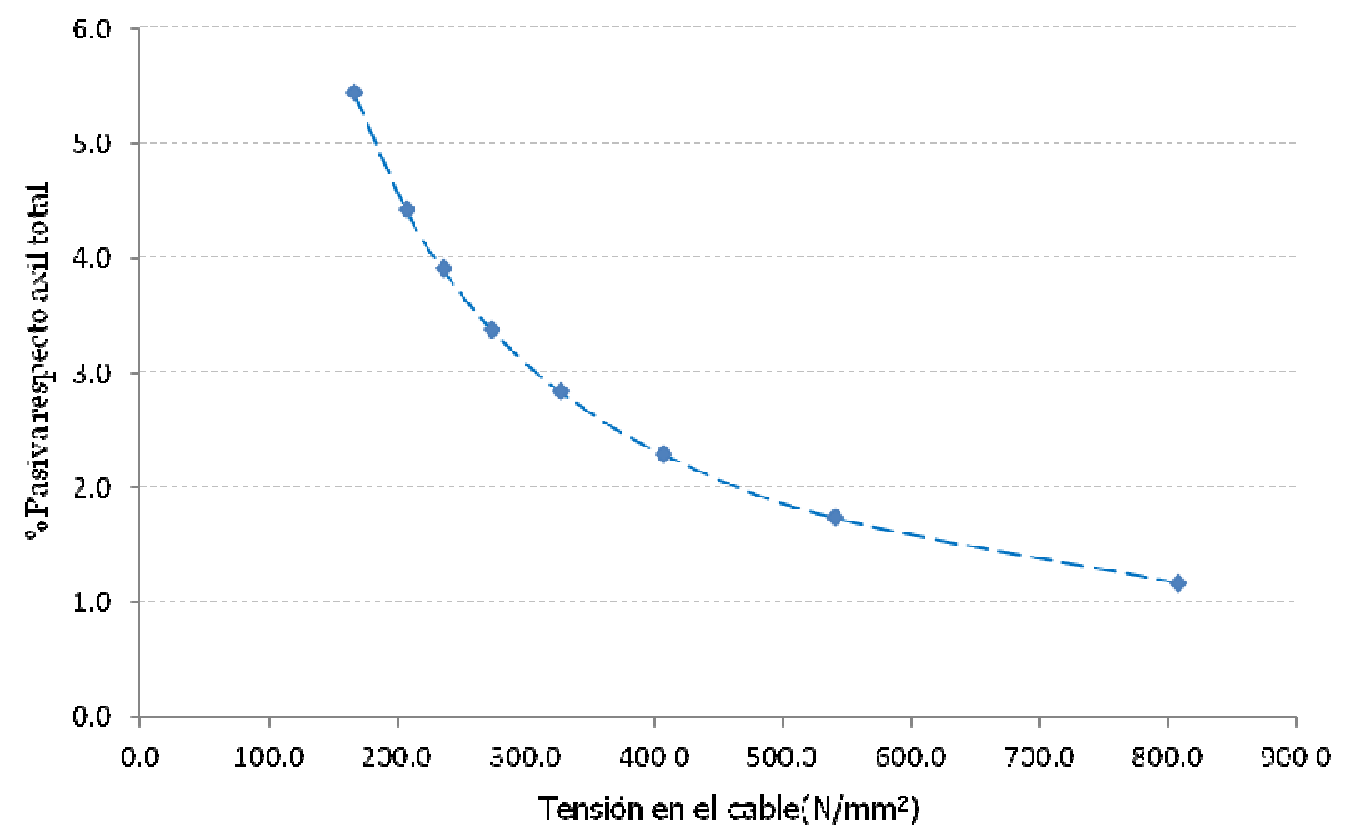

Figura 48. Relación del axil pasivo con el valor de la tensión del tirante.

En este caso se puede observar que la disminución de la tensión de trabajo de los tirantes supone un incremento del axil pasivo de los tirantes, siendo esta dependencia no lineal, tanto más acusada cuanto menor es la tensión de trabajo. El rango de variación obtenido es de entre un $1^{\prime} 0$ a un $5^{\prime} 5 \%$. Un tirante con una tensión baja de trabajo significa un tirante sobredimensionado, o bien un cable más rígido de lo necesario. De esta forma se demuestra que aumentando la rigidez del tirante se aumenta la capacidad de éste de absorber esfuerzos después de haber sido tesado, es decir que tiene más capacidad para captar axil pasivo.

\subsubsection{Variación del $\% F_{p} / F_{\text {tot }}$ en función de la rigidez del tablero.}

En este último apartado del estudio paramétrico se analiza la influencia de la variación de la rigidez del tablero. En este caso la variación de la inercia de la sección no implica un incremento del peso propio del tablero. El rango de variación de la rigidez del tablero se establece entre 0' 40 y $5^{\prime} 0 \mathrm{~m}^{4}$.

Se adoptan para el resto de parámetros los siguientes valores: 
$\checkmark L=8^{\prime} 00 \mathrm{~m}$, Separación entre anclajes.

$\checkmark$ Area de la sección del tablero 10'00 m².

$\checkmark H=28^{\prime} 9 \mathrm{~m}$, que corresponde a un cable extremo de un tablero con pilono central de $50 \mathrm{~m}$ de luz y ángulo $30^{\circ}$.

$\checkmark \mathrm{cm}=28^{\prime} 5 \mathrm{KN} / \mathrm{ml}$

Se adjunta gráfico con los resultados obtenidos:

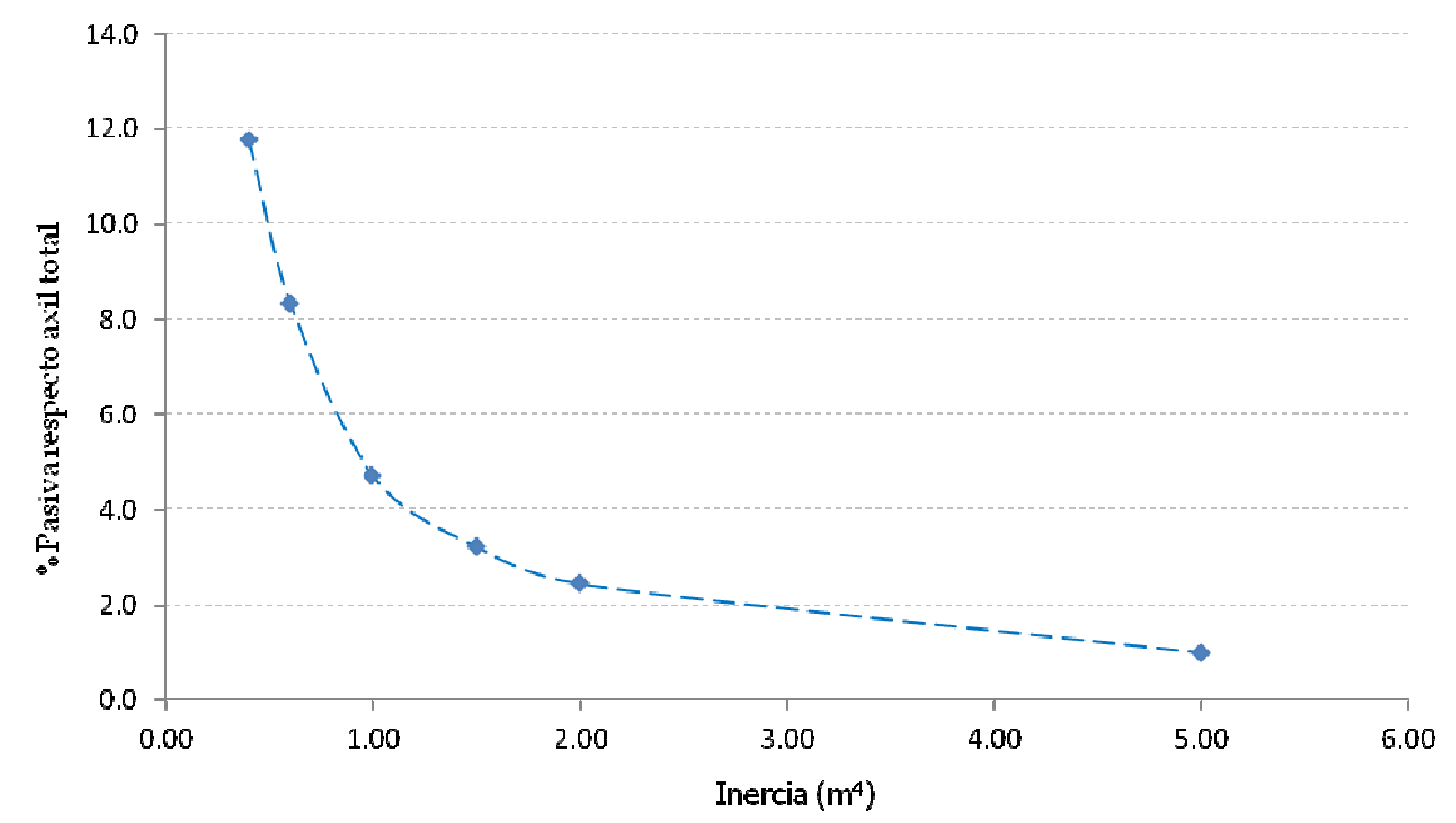

Figura 49. Relación del axil pasivo con la rigidez del tablero.

De estos resultados se concluye que el aumento de la rigidez del tablero, que supone un descenso de la eficacia relativa del sistema de atirantamiento respecto del mecanismo de resistencia a flexión del tablero, implica una disminución del axil pasivo del tirante. Esta disminución de nuevo vuelve a ser no proporcional, siendo más acusada para el caso de las inercias reducidas. El rango de los axiles pasivos obtenidos abarca desde un máximo del $12 \%$ para la inercia menor hasta un $2 \%$ para la inercia mayor calculada. 


\subsubsection{Análisis de los resultados anteriores.}

De todos los anteriores resultados se puede concluir que, sabiendo que el efecto de los cables adyacentes no se ha tenido en cuenta y por lo tanto el valor de la parte pasiva del axil del tirante está infraponderado:

- Que para todos los casos analizados el axil pasivo en ningún caso supone más de un $20 \%$ del axil total, quedando en la mayoría de los casos por debajo del 10\%. es decir supone un porcentaje reducido del axil total de los tirantes.

- Que el incremento de la separación entre anclajes genera el efecto más acusado de aumento del porcentaje de la parte pasiva respecto del axil total en el tirante de todos los casos analizados.

- Que el aumento de la longitud del cable y el aumento de la rigidez del tablero, es decir la disminución de la eficacia del mecanismo resistente del atirantamiento del tablero, conlleva una disminución del porcentaje de la parte pasiva respecto del axil total del tirante. Indirectamente se deduciría que a mayor luz del puente menor será la parte pasiva del axil absorbido por los tirantes a igualdad del resto de parámetros analizados.

- Que el aumento de las cargas muertas implica un aumento, pero poco importante, del porcentaje de la parte pasiva del axil del tirante.

- Que el aumento de la tensión en el tirante implica un decremento de la parte pasiva del axil del tirante, dado que dicho aumento de la tensión deriva de una menor área del tirante colocado y por lo tanto una menor rigidez y eficacia del sistema de atirantamiento.

- La importancia relativa de los anteriores factores es mayor en el caso de la separación entre anclajes y del valor de las tensiones en los tirantes.

- En definitiva se puede indicar que, conforme disminuye la rigidez del tablero frente al sistema de atirantamiento, mayor es el porcentaje de axil pasivo en el cable, es decir que conforme aumenta la eficacia del atirantamiento aumenta a su vez la capacidad de absorber esfuerzos por parte del tirante después de ejecutado el tesado (esfuerzo pasivo). 


\subsection{EFECTOS REOLOGICOS DEL HORMIGON.}

Como ya se ha indicado anteriormente, este estudio se ha centrado en el caso concreto de puentes atirantados de luces cortas o medias, siendo en general las soluciones con tablero de hormigón muy adecuadas para estas tipologías. Por este motivo es fundamental conocer cómo afecta al comportamiento de los tirantes y de la estructura la consideración de los fenómenos reológicos que toda estructura de hormigón comporta.

Según Arenas [7], en el caso de puentes atirantados con tablero de hormigón, habrá que tener en cuenta los efectos que provocará la fluencia sobre el proceso de puesta en carga del tirante. Para ello, hay que analizar el efecto de los tirantes sobre el tablero, partiendo de la hipótesis de que los axiles a aplicar sobre ellos deberán coincidir con aquellos que provoquen sobre el tablero unas reacciones verticales equivalentes a las producidas en él por la acción de las cargas permanentes, considerando a éste como una viga continua apoyada en cado uno de los puntos de anclaje de los cables. A partir de esta idea, se podría estudiar el efecto de la aplicación de una tensión mayor o menor de la teórica anteriormente definida.

Si el esfuerzo aplicado es mayor que el teórico, en principio, se podría pensar que el efecto que resultaría, debido a la acción de la fluencia, sería un incremento de las deformaciones a tiempo infinito. Pero hay que tener en cuenta que el efecto del cable sobre el tablero es una deformación impuesta, por lo que se trata de un caso de fluencia bajo deformación constante y no bajo carga constante.

En realidad el efecto a largo plazo será una relajación de las tensiones en los cables, que tenderán a aproximarse a aquella correspondiente al estado ideal de la viga continua. De forma similar ocurrirá si la tensión aplicada es inferior a la teórica, produciéndose en este caso un incremento de las tensiones en los cables que tendería de nuevo al valor teórico correspondiente a la configuración del tablero como viga continua.

La conclusión de este comportamiento es el hecho de que, para puentes con tableros de hormigón, los incrementos de esfuerzos sobre I configuración de viga continua, tienden a anularse a largo plazo, por lo que no deben introducirse pensando en generar situaciones que puedan compensar las flexiones que se produzcan en servicio debido a la actuación de las sobrecargas. Sí en cambio, hay que tener en cuenta que 
las flechas a tiempo infinito no variarán respecto de las que se hayan producido inicialmente.

Strassky [97] señaló en cambio que una cierta redistribución de esfuerzos podrá llegar a producirse debido al acortamiento por fluencia del tablero, el cual provocará a su vez una reducción en la longitud de los tirantes instalados y por lo tanto una reducción de su esfuerzo axil generándose entonces una cierta redistribución de esfuerzos en la estructura. En cualquier caso este efecto será pequeño y casi con seguridad despreciable, aunque siempre podrá plantearse su corrección mediante un ajuste final de los axiles de los tirantes o retesado.

Schlaich [93] resumió las anteriores consideraciones postulando que la mejor forma de reducir, y en la medida de lo posible evitar, los efectos de la fluencia en el comportamiento de la estructura será plantear alcanzar, en la situación final del proceso constructivo, una configuración similar a la de la viga continua, dado que, en dicha configuración, los esfuerzos obtenidos no dependen del módulo de deformación del hormigón, y la fluencia puede asimilarse a una modificación de este parámetro con el tiempo.

Para poder cumplir con el objetivo indicado, se requiere además que los esfuerzos axiles aplicados sobre el tablero queden centrados con su eje, de tal forma que no provoquen incrementos de las flexiones y por lo tanto variación entre las armaduras dispuestas en la cara superior e inferior, evitándose así la variación a lo largo del puente de la profundidad de la fibra neutra y por lo tanto de la inercia.

Con los anteriores condicionantes el efecto de la fluencia se deberá tener en cuenta sólo en lo relativo al acortamiento que genera a largo plazo en las dovelas del tablero y en el alzado del pilono que señalaba Strassky, pero este efecto a su vez se evitará ejecutando estos elementos con una longitud incrementada en la dimensión que está previsto que les afecte la fluencia.

Es necesario comentar también que El Araby [31] ha desarrollado una investigación relacionada con la evaluación de los efectos reológicos del hormigón, pero en este caso más orientada al caso de tableros mixtos y puentes extradosados.

Finalmente es interesante reseñar los resultados obtenidos por Park [81] en relación a la significación sobre la desviación en de las tensiones en los tirantes si se consideran los 
efectos de la fluencia y retracción del hormigón, estimando que esas diferencias podrían llegar a quedar en el entorno de un máximo del 10\%.

\subsection{DETERMINACION DE LOS AXILES DE LOS CABLES.}

Se procede en este epígrafe a detallar y analizar el proceso de cálculo y colocación de los cables en los puentes atirantados desde el punto de vista estructural, es decir se va analizar cómo se simula la introducción de un esfuerzo de tesado de los cables en el modelo de cálculo, y qué valor tiene ese esfuerzo.

En concreto en este epígrafe se va a detallar primeramente como se ha procedido a calcular los axiles teóricos en la situación final, posteriormente se detalla cómo se obtienen los axiles de tesado de los cables y que métodos se han utilizado para ello. Por último, se procede a describir la diferencia entre el proceso de instalación de los cables en fuerzas y en deformaciones.

\subsubsection{Cálculo de los axiles finales de los cables.}

Para el cálculo de los axiles teóricos en la situación final se ha utilizado el método de anulación de las reacciones bajo los apoyos del tablero, es decir, que dichos axiles se han obtenido suponiendo que el comportamiento del tablero en la situación final equivale al caso de una viga continua sobre los apoyos en los anclajes del tablero.

En este caso esta hipótesis se considera suficiente por varios motivos:

$\checkmark \quad$ En primer lugar porque las estructuras a calcular lo son con tablero de hormigón, por lo que, como se ha comentado en el capítulo anterior no tendría sentido buscar configuraciones de esfuerzos al final del proceso constructivo distintas de la de la viga continua, ya que a tiempo infinito la redistribución de esfuerzos debida a la fluencia tenderá a anular las diferencias introducidas inicialmente.

$\checkmark \quad$ En segundo lugar, porque como las rasantes de las estructuras estudiadas serán siempre horizontales, no es necesario emplear métodos más sofisticados como el de anulación de los desplazamientos verticales.

El cálculo de esta situación de referencia o situación final objetivo, consistirá en:

- La determinación de las reacciones en los apoyos de la viga continua y su proyección en la dirección del eje de cada tirante para obtener el valor del axil 
final en este elemento. Además, la proyección de ese axil en la dirección del eje del tablero permite a su vez conocer la ley de axiles generada finalmente sobre éste.

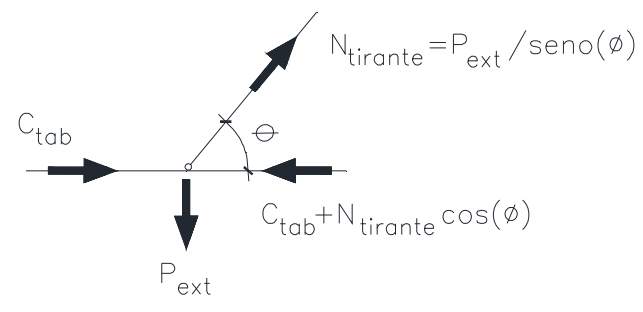

Figura 50. Equilibrio de fuerzas entre axiles en tablero y tirantes y reacción de la viga continua sometida a peso propio y carga muerta.

- El cálculo de los esfuerzos flectores, cortantes y axiles en el pilono generados a su vez por los axiles de los tirantes.

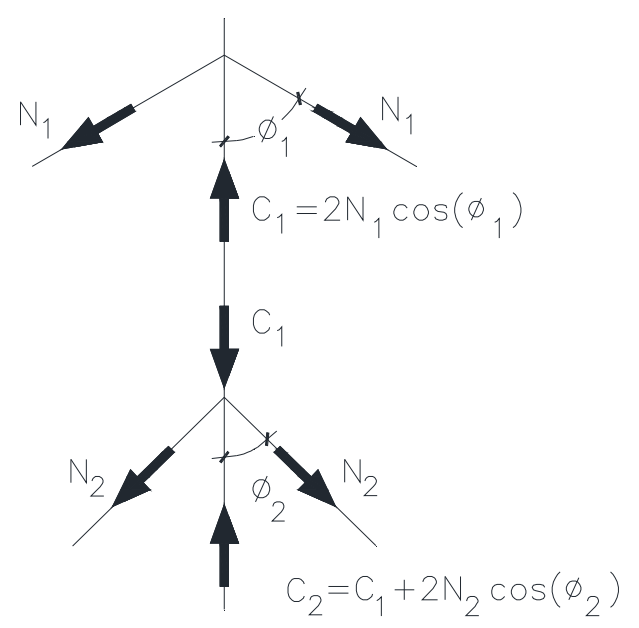

Figura 51. Equilibrio de fuerzas entre axiles en tirantes y pilono.

El esquema anterior será distinto en el caso de que el tablero no sea simétrico, resultando una cierta descompensación entre los axiles que llegan al pilono desde ambos lados, siendo necesario para compensar este efecto, bien aumentar la rigidez del pilono para limitar los desplazamientos horizontales de éste, bien disponer cables de retenida en el vano corto o apoyos intermedios en este elemento para llevar las cargas descompensadas sobre el pilono hasta puntos fijos.

- Es necesario también en este caso determinar la deformación final de la estructura en cada uno de sus elementos, ya que esta situación de referencia será la de partida del proceso de desmontaje que se describe a continuación, 
para el cual es necesario conocer no solo la situación objetivo en esfuerzos si no también en deformaciones.

\subsubsection{Cálculo de los axiles o deformaciones de montaje de los tirantes.}

Sabiendo que el proceso constructivo a analizar, en todos los casos en estudio, corresponde a la ejecución del tablero por dovelas mediante el avance en voladizo desde el pilono, una vez obtenidos los axiles finales correspondientes a la situación objetivo, es necesario obtener los axiles o deformaciones a aplicar a los tirantes en el instante de su colocación para alcanzar, una vez terminado el proceso constructivo, la situación objetivo definida anteriormente.

En concreto el método de cálculo aplicado en este estudio es el denominado proceso de desmontaje (método backward), que como se ha comentado anteriormente está basado en la obtención de los axiles de montaje de los cables, calculados mediante un proceso de cálculo inverso al que se seguiría durante la ejecución real de la estructura. De tal manera que el axil que posee el tirante justo antes de anular su presencia en el modelo de cálculo será el axil a aplicar en el instante de su colocación.

De esta forma la secuencia de dicho proceso de desmontaje será:

1) Partir del Estado final en esfuerzos y deformaciones calculado anteriormente en la situación objetivo de la viga continua.

2) A continuación se comienzan a retirar sucesivamente todas las cargas actuantes sobre la estructura, en orden inverso al que se colocan en su montaje, comenzando con la retirada de la carga muerta.

3) En este desmontaje, para la eliminación de cada tirante, se parte de la estructura existente en ese momento, anulando la barra del tirante. Sobre esta estructura se aplica en los nudos extremos de dicha barra, dos cargas iguales y contrarias al axil del tirante.

4) Para el desmontaje de las barras del tablero, se aplica una carga igual y contraria a su peso propio, sin llegar a anular la barra. De esta forma, al final del desmontaje, la estructura resultante tiene todas las barras activas salvo los tirantes que se encuentran todos anulados. 
La elección en nuestro caso del método backward para realizar este cálculo se ha tomado sabiendo que en los cálculos desarrollados a continuación no se han tenido en cuenta los efectos reológicos del hormigón, y por lo tanto este método tiene una ventaja significativa sobre el método forward, que, como se ha indicado en el capítulo anterior, requeriría de un proceso iterativo de ajuste de los axiles de montaje, hasta alcanzar aquellos valores en todos los tirantes que lleven a una configuración en la situación final similar o suficientemente aproximada a la definida como objetivo.

\subsubsection{Métodos de colocación de los tirantes.}

Es en este punto donde el estudio se ha realizado utilizando dos métodos de colocación de los tirantes diferentes, el primero de ellos el basado en fuerzas y el segundo el basado en deformaciones.

En el primer caso la variable utilizada como referencia para el montaje de un tirante es el valor de la fuerza inicial a aplicar sobre el cable, es decir que, en este caso, la referencia para el operario que instalaría el cable sería el manómetro que mediría la presión introducida en el gato de tesado, de tal forma que, controlando dicha medida, finalmente el cable quede con un axil de tracción igual al teóricamente planteado.

En definitiva, este método se basa en la aplicación de una fuerza como medida para conseguir que la instalación de los tirantes sea correcta, por este motivo a este método le denominaremos método del desmontaje aplicando fuerzas a los cables o bien simplemente método en fuerzas.

Como ya se ha comentado en el capítulo anterior, el principal inconveniente de este método consiste en la propia característica del parámetro a controlar, el cual no es un valor intrínseco a la estructura. Es decir que la fuerza a aplicar en un tirante, para alcanzar la situación final objetivo, depende de las cargas realmente aplicadas en la estructura en ese momento, tanto de los pesos propios de los elementos estructurales, como de las cargas muertas de los equipos colocados sobre el tablero, así como del efecto de la temperatura ambiental y de los diferentes efectos del soleamiento sobre los elementos de la estructura.

Desde este punto de vista entonces cualquier error en la evaluación de los pesos propios, cargas muertas, de la temperatura ambiental o del efecto de la diferencia de temperaturas entre elementos estructurales (tirantes, tablero y pilono) puede llevar a 
que, aún aplicando exactamente el axil teórico de tesado, no se alcance la situación teórica objetivo.

Haciendo el planteamiento inverso, se podría pensar que se introduzcan unos errores en la instalación de los cables que implicarán unos efectos similares a los indicados en el párrafo anterior. Por este motivo en este trabajo se ha desarrollado una metodología para generar unos axiles de montaje de los cables erróneos, proceso que se describe en el siguiente capítulo.

Una forma de evitar la influencia en parte de estas incertidumbres puede ser la adelantada en 2.3.2, basada en el uso de la denominada en [102] como tensión de referencia definida como:

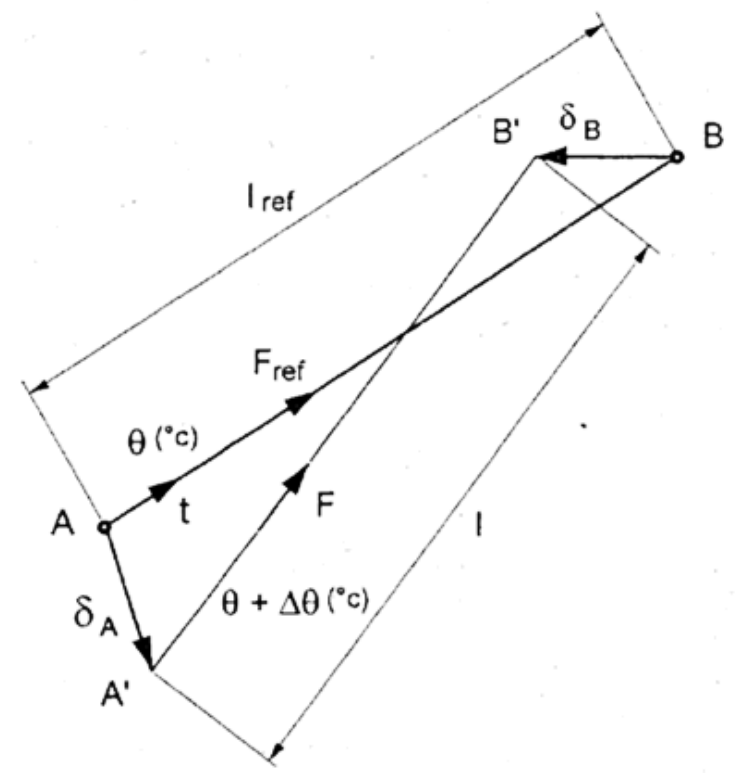

Figura 52. Esquema tomado de [102] para la definición de tensión de referencia..

[Ec3. 33.]

$$
F_{r e f}=F-\frac{E \cdot A}{L_{r e f}}\left[\left(u_{B}-u_{A}\right) \cdot \cos \alpha+\left(v_{B}-v_{A}\right) \cdot \operatorname{sen} \alpha\right]-E \cdot A \cdot \Delta T \cdot \alpha
$$

siendo: $\quad F \rightarrow$ Tensión en fase a calcular

Lref $\rightarrow$ Longitud teórica del cable en la situación de referencia.

$U_{A}, U_{B}, V_{A}$ y $V_{B} \rightarrow$ Desplazamientos de los nudos extremos (anclajes) del tirante.

$\cos \alpha$ y sen $\alpha \rightarrow$ Angulos que forman las distintas orientaciones de los ejes de los cables. 
$\alpha \rightarrow$ Coeficiente de dilatación térmico del acero de los cables.

Con este método se define un parámetro intrínseco a la estructura que permite calcular una fase determinada sin tener que pasar por el proceso de cálculo de las anteriores o posteriores. Por este mismo motivo no es un método adecuado para el uso en tableros de hormigón dado que no se podrá considerar la actuación de la reología del hormigón en los cálculos.

Sí es un método muy útil para la realización del control del tesado en obra y del cálculo de los posibles ajustes que hubiera que realizar respecto de la situación teórica.

Por otro lado, se ha implementado también el método en deformaciones. Este proceso se basa en las recomendaciones para estructuras atirantadas del SETRA [102], que plantean ejecutar las estructuras atirantadas mediante un método que utilice un parámetro intrínseco a cada estructura.

Así, en aquel documento, redactado bajo la dirección de M.Virlogeux, se adopta un parámetro como referencia que se señala que es independiente tanto de las cargas aplicadas como de los efectos de la temperatura. Este parámetro es la denominada longitud en vacío o neutra de un cable, que será aquella longitud que tendrá el cable a instalar cuando se mida sin el efecto de su peso y en posición horizontal. Implícitamente, al utilizar este parámetro estamos enfocando la aplicación de este método al caso de cables prefabricados, es decir de aquellos tipos de cables que se suministran a obra con una longitud fija ya determinada, como pueden ser los tipos de cables cerrados (locked coil cables) o los cables compuestos por alambres de alta resistencia a la fatiga (wire-cables), pero no así los cables más convencionales como pueden ser los compuestos de un conjunto de cordones trenzados (strand-cables).

La longitud neutra (Lo) se calculará a partir del axil de cada tirante en la situación final, es decir se obtienen igualmente a partir de la configuración del tablero final supuesta como una viga continua. Conocido el valor de dicho axil, se puede calcular el incremento de longitud que habría que dar al cable de longitud Lo para alcanzar el axil teórico en la situación definitiva. Esta medida es la que se denomina predeformación del cable, que, en otras palabras, no es más que el alargamiento que debe tener el cable de longitud Lo para que en la situación final del proceso constructivo posea un axil equivalente a la proyección, en la dirección del tirante, de la reacción vertical de la viga continua que representa el tablero en situación final. 
Analíticamente, y a partir de las definiciones anteriores, se puede plantear la siguiente formulación.

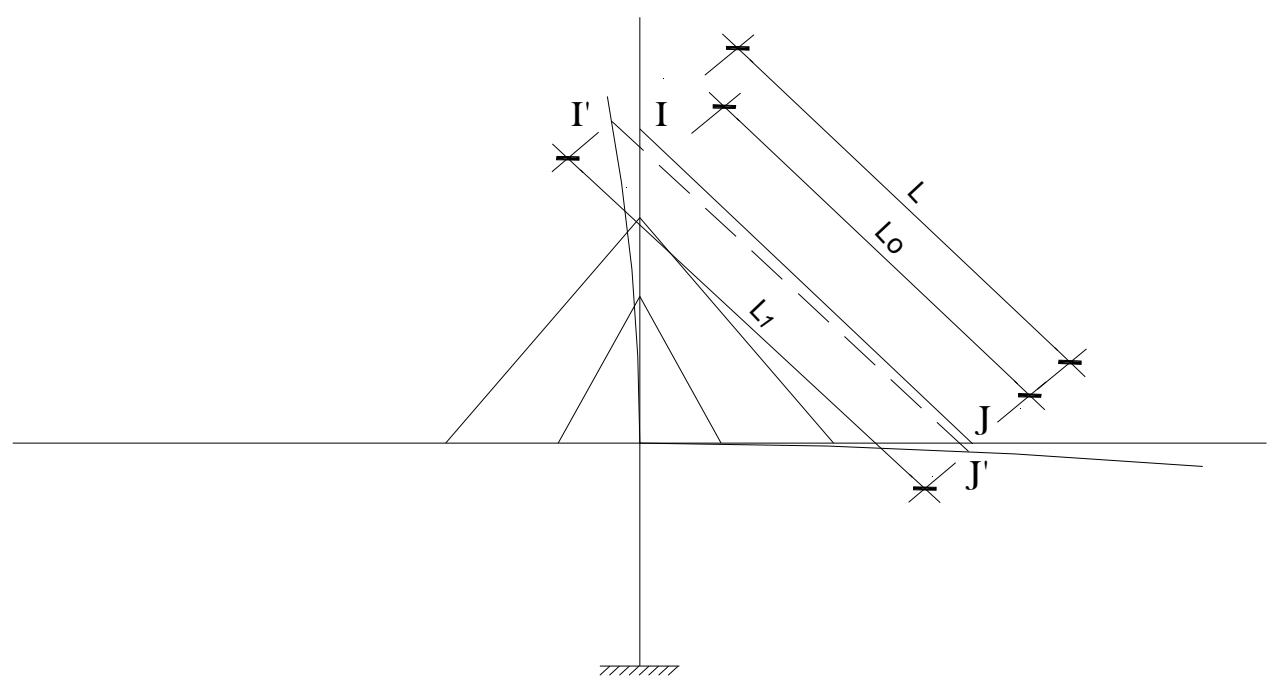

Figura 53. Esquema repreentativo de la longitud neutra $\left(L_{0}\right)$, de la distancia real entre anclajes de un cable $\left(L_{1}\right)$ y de la distancia teórica entre anclajes $(L)$.

Siendo:

$\mathbf{L} \rightarrow$ Distancia entre los nudos extremos del tirante, I-J, en la estructura sin deformar

$L_{0} \rightarrow$ Longitud neutra del tirante.

$\boldsymbol{\delta}_{\text {pred }} \rightarrow$ Predeformación del tirante. (Obtenida a partir del esfuerzo del tirante correspondiente a la proyección de la reacción sobre apoyos de la viga continua)

$\mathbf{L}_{\mathbf{1}} \rightarrow$ Distancia entre los nudos I-J en la estructura deformada previa al tesado del tirante

$\Delta \mathrm{L}_{1} \rightarrow$ Variación de la distancia entre los nudos I-J en la fase previa a su tesado.

$\Delta \mathbf{L}_{2} \rightarrow$ Variación de la distancia entre los nudos I y J debido a dos fuerzas unidad actuando en la dirección del tirante, sobre la estructura existente antes de la colocación del propio tirante.

resulta entonces:

[Ec3. 34.] $\quad \mathrm{L}_{0}=\mathrm{L}-\delta_{\text {pred }}$

[Ec3. 35.] $\quad \mathrm{L}_{1}=\mathrm{L}+\Delta \mathrm{L}_{1}$ 


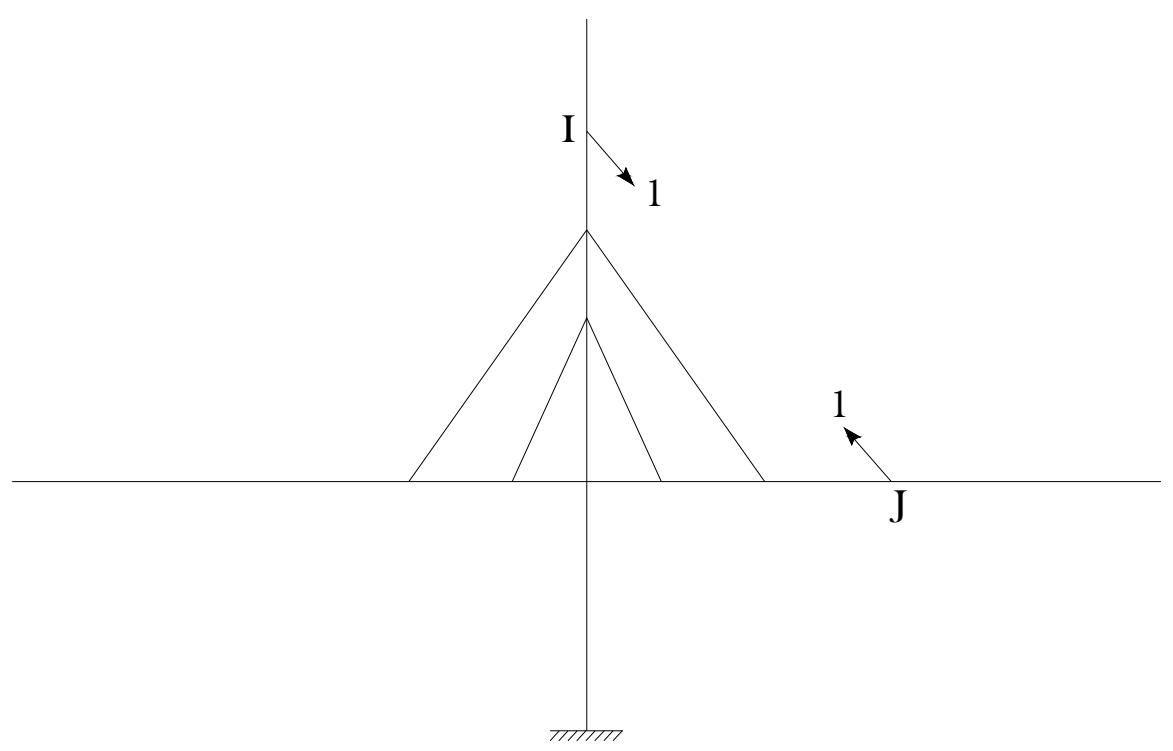

Figura 54. Esquema representativo de la aplicación de cargas unitarias en la dirección del tirante para el cálculo de la distancia $\Delta L_{2}$.

Si se tesa el tirante hasta que los extremos del tramo de longitud inicial Lo, alcancen los anclajes I y J, el valor de la fuerza de tesado, N, se obtiene igualando por un lado la longitud alcanzada por el tramo "Lo" después de su tesado:

$$
\text { [Ec3. 36. }] \quad L_{0}+\frac{N \cdot L_{0}}{E A}
$$

y por otro lado, evaluando la nueva distancia entre los nudos I-J después de aplicar la fuerza N sobre la estructura previa resulta:

$$
\text { [Ec3.37.] } L_{1}+N \cdot \Delta L_{2}
$$

igualando ambos términos obtenemos:

$$
\text { [Ec3. 38.] } L_{0}+\frac{N \cdot L_{0}}{E A}=L_{1}+N \cdot \Delta L_{2}
$$

y operando se obtiene:

$$
\text { [Ec3. 39.] } N\left(\frac{L_{0}}{E A}-\Delta L_{2}\right)=L_{1}-L_{0}=L+\Delta L_{1}-\left(L-\delta_{\text {pred }}\right)
$$


[Ec3. 40.]

$$
N=\frac{\delta_{\text {pred }}+\Delta L_{1}}{\frac{L_{0}}{E A}-\Delta L_{2}}=\frac{E A\left(\delta_{\text {pred }}+\Delta L_{1}\right)}{L_{0}-E A \cdot \Delta L_{2}}=\frac{E A\left(\delta_{\text {pred }}+\Delta L_{1}\right)}{L-\delta_{\text {pred }}-E A \cdot \Delta L_{2}} \approx \frac{E A\left(\delta_{\text {pred }}+\Delta L_{1}\right)}{L-E A \cdot \Delta L_{2}}
$$

Se llega así a obtener una expresión analítica del valor del axil de tesado equivalente a la introducción de una deformación en los cables. Como se puede observar en esta formulación, el axil de montaje equivalente depende de:

- La medida de la longitud neutra Lo, que se refleja en el término $\delta$ pred.

- La posición de los anclajes de los cables, que se refleja en el segundo término del numerador, es decir $\Delta \mathrm{L}_{1}$.

- La propia rigidez de la estructura, que queda reflejada en el término $\Delta \mathrm{L}_{2}$ del denominador.

En este caso el operario encargado de la colocación del cable debería haber marcado la longitud neutra y la deformación a introducir en el momento de la instalación del cable, calibrando en este caso el proceso de montaje mediante la medición de estas longitudes. Adicionalmente tal y como se plantea en la anterior formulación será necesario que se controle la posición de los anclajes de los tirantes, las cuales afectan de igual forma a la longitud del cable a colocar, a través del término $\Delta \mathrm{L}_{1}$.

Como ya se ha comentado, asociado a este parámetro, se puede definir el otro método de instalación de los cables denominado método de las deformaciones.

En nuestro caso el planteamiento realizado ha sido diferente ya que se ha planteado emplear la longitud neutra de los tirantes como parámetro de referencia de los cables, el cual combinado con un primer proceso de desmontaje de la estructura permite conocer las deformaciones de montaje a aplicar a los distintos cables sin realizar sucesivas iteraciones en el proceso de montaje hasta obtener dichos valores. Podemos decir entonces que en nuestro caso hemos empleado un método mixto, entre los 3 existentes según Lozano-Galant [59], métodos backward, forward y directo, ya que el proceso de desmontaje (Backward) nos permite obtener la deformada con la que es necesario realizar el montaje (Forward) de la estructura, utilizando a su vez el concepto de longitud neutra (Directo).

En definitiva el proceso de cálculo realizado es la siguiente: 
- Se calcula la situación final objetivo con los mismos condicionantes que en el método del desmontaje, obteniéndose así las predeformaciones que los cables deben alcanzar en la situación final objetivo. A partir de estas predeformaciones se obtienen las longitudes neutras de todos los tirantes.

- Calculada la situación objetivo anterior, se procede a realizar un proceso de desmontaje similar al empleado con el método en fuerzas, el cual nos permite calcular las deformaciones de montaje de los cables y nos llevaría a la conformación de un estado inicial, denominado Estado 1, donde se calculan las deformadas iniciales de todos los elementos.

- $\quad$ Partiendo así de este estado inicial (Estado 1), en el que los movimientos de los nudos son los que aparecen en la fase final del desmontaje y los esfuerzos en barras y reacciones en apoyos son todos nulos, se obtiene entonces la estructura de la que se parte para realizar todo el proceso de montaje. Esta estructura inicial tiene todas las barras de los tirantes anuladas, así como las dos que simulan los apoyos de neopreno en los estribos 1 y 2. Por otro lado, están dispuestos los apoyos provisionales para la construcción de las dovelas 1 del lado izquierdo y derecho.

- Sobre esta estructura se van aplicando sucesivamente las cargas correspondientes a los pesos propios de cada dovela y montando los tirantes.

- Para el montaje del tirante, se realiza el cálculo de su axil de montaje equivalente a la predeformación a aplicar en el instante de su colocación, teniendo en cuenta la posición de los nudos que une dicho cable en ese instante, y su predeformación o longitud neutra.

- Se finaliza el montaje aplicando la carga muerta sobre el conjunto del tablero para alcanzar la situación final objetivo.

La aplicación de dos métodos diferentes para simular la colocación del tirante lo ha sido para conocer qué influencia tiene la aplicación de uno u otro método sobre la ejecución de la estructura, pensando que en ambos casos se producirán errores durante la colocación de los cables. 


\section{CAPITULO 4.}

\section{INFLUENCIA DEL METODO DE INSTALACION DE LOS}

\section{CABLES.}

\subsection{INTRODUCCION.}

Se comienza en este capítulo a desarrollar la investigación que se ha ido planteando anteriormente, ahora ya sobre modelos reales. Se describe en primer lugar el modelo teórico de partida utilizado como origen de todos los siguientes estudios, describiéndose su geometría y su modelización, así como los resultados obtenidos teóricos que servirán de referencia en los posteriores desarrollos.

A continuación se procede a estudiar y valorar los posibles errores que se pueden producir durante la colocación de un cable, detallando el origen de estos procesos erróneos y el valor máximo que la distintas normativas permiten para los casos que analizamos.

Adicionalmente, y con la intención de simular esos procesos erróneos de montaje, se ha desarrollado un procedimiento de introducción de los errores de ejecución sobre los tirantes de forma aleatoria.

Previamente a la introducción de dichos errores se realiza un análisis comparativo de los métodos de instalación de cables mediante el método de las fuerzas y el de las deformaciones, evaluando si los errores máximos que indica la normativa para ambos casos resultan de igual repercusión.

Finalmente se procede a aplicar los procesos de montaje erróneos sobre el modelo indicado, bien mediante el método en fuerzas, bien mediante el método en deformaciones, analizando que diferencias se encuentran en los resultados de cada uno de ellos. 


\subsection{ESTRUCTURA TEORICA DE PARTIDA.}

El estudio realizado lo ha sido sobre un modelo de cálculo que tiene su origen en el proyecto de un puente para una carretera de 2 carriles, el cual formaba parte de un proyecto constructivo asociado a las obras del Embalse de Giribaile que desarrolló el Ministerio de Fomento en los años 90.

La estructura originaria consistía en un puente atirantado de 2 vanos con pilono central, con luces de $91^{\prime} 00+85^{\prime} 00 \mathrm{~m}$ (Figura 55). El tablero se constituía por una losa de hormigón de 13'80 m de ancho, planteándose dicha losa aligerada con canto mínimo 0'90 m en los bordes y 1'05 m en el centro de la sección, disponiéndose en ella 11 aligeramientos de $\varnothing 0^{\prime} 65 \mathrm{~m}$ (Figura 56).

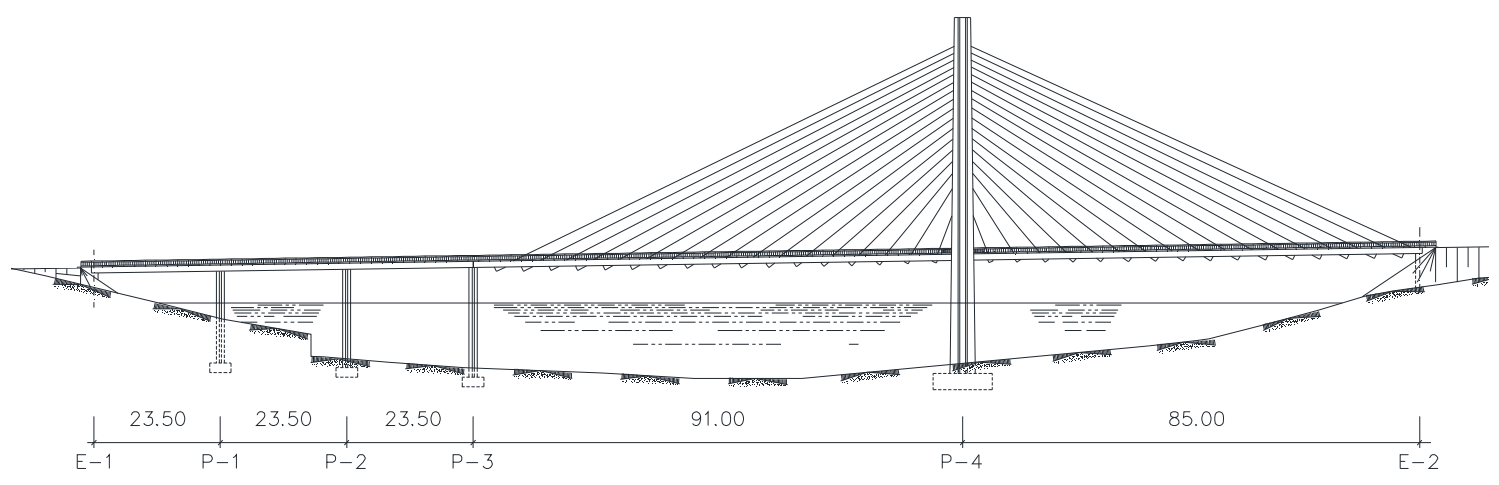

Figura 55. Alzado del puente modelizado.

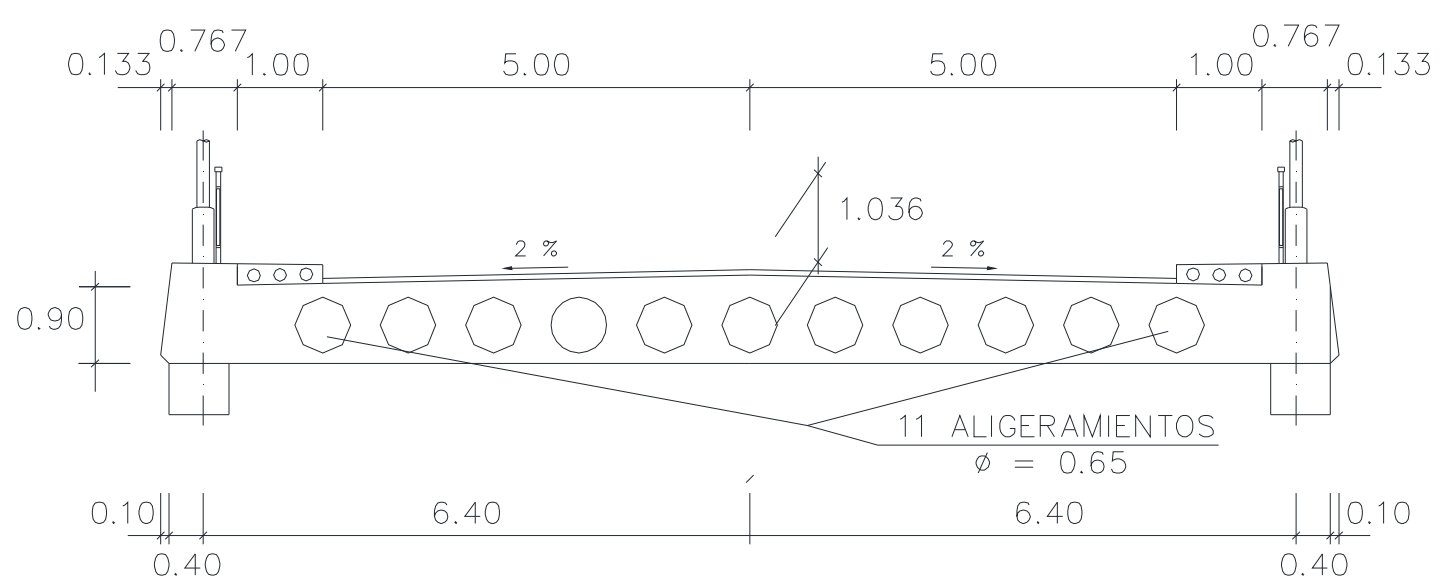

Figura 56. Sección del tablero del puente modelizado. 
Con la configuración antes esquematizada, el tablero resulta ligeramente asimétrico respecto de la posición del pilono, ya que en el vano izquierdo se dispone una dovela más que en el vano derecho.

Respecto del esquema de atirantamiento adoptado, el tablero se apoyaba sobre dos familias de cables dispuestos en ambos bordes del tablero, consistentes en 34 cables cada una, que quedan anclados en el tablero cada 5'00 m.

Los cables se anclaban en su extremo superior en el pilono central mediante una disposición en semiarpa, arrancando el más corto (cable 2) desde la cota +12'90 m respecto del eje del tablero, situándose los sucesivos por encima a separaciones decrecientes entre ellos, que varían de 2'917 m para el inferior, hasta 1'25 m para los cables superiores. Siguen así los cables un esquema que incluye un cable de retenida en el vano derecho (18D) para equilibrar el peso de la dovela extrema del vano izquierdo. La separación de los anclajes en el tablero se estableció en 5'00 m.

El pilono a su vez se conformaba por una pila en $\mathrm{H}$ de 2 fustes, de 68' $85 \mathrm{~m}$ de altura total (44'02 m por encima de la rasante del tablero). La sección de ambos fustes era variable, siendo de sección hueca por debajo del puntal intermedio y maciza por encima del puntal.

Los apoyos extremos del tablero se realizaban, en el caso del estribo izquierdo, en una pila intermedia de altura $21^{\prime} 00 \mathrm{~m}$ y canto 2'00 m, que servía a su vez como apoyo del vano final del viaducto de acceso, y en un estribo cerrado para el caso del estribo derecho, con altura 10'00 m y canto 4'00 m.

Las áreas de los tirantes se han establecido de forma que las tensiones generadas en cada uno de ellos quedan cercanas a los $450 \mathrm{~N} / \mathrm{mm}^{2}$ en la situación objetivo, al final del proceso constructivo y al haber dispuesto las cargas muertas sobre el tablero. Este valor se considera razonable si se plantea utilizar cables de características mecánicas convencionales.

Se adjunta tabla con los valores obtenidos en cada cable, incluyendo su longitud y el ángulo con el eje del tablero: 


\begin{tabular}{|c|c|c|c|c|}
\hline Barra & Tirante & Longitud (m) & Angulo & Area $\left(\mathrm{cm}^{2}\right)$ \\
\hline 98 & $2-D$ & 13.8 & -68.8 & 35.7 \\
\hline 99 & 2-I & 13.8 & 248.8 & 35.7 \\
\hline 100 & $3-D$ & 18.7 & -57.7 & 39.4 \\
\hline 101 & 3-1 & 18.7 & 237.7 & 39.4 \\
\hline 102 & 4-D & 23.3 & -49.9 & 43.6 \\
\hline 103 & 4-I & 23.3 & 229.9 & 43.6 \\
\hline 104 & 5-D & 28.0 & 44.5 & 47.5 \\
\hline 105 & 5-I & 28.0 & 224.5 & 47.5 \\
\hline 106 & 6-D & 33.0 & -40.7 & 51.1 \\
\hline 107 & 6-1 & 33.0 & 220.7 & 51.1 \\
\hline 108 & 7-D & 38.0 & -37.9 & 54.3 \\
\hline 109 & 7-I & 38.0 & 217.9 & 54.3 \\
\hline 110 & B-D & 43.1 & -35.8 & 57.0 \\
\hline 111 & $8-1$ & 43.1 & 215.8 & 57.0 \\
\hline 112 & 9-D & 48.3 & -34.0 & 59.6 \\
\hline 113 & 9-1 & 48.3 & 214.0 & 59.6 \\
\hline 114 & 10-D & 53.4 & -32.6 & 61.9 \\
\hline 115 & $10-1$ & 53.4 & 212.6 & 61.9 \\
\hline 116 & $11-D$ & 58.5 & -31.3 & 64.1 \\
\hline 117 & $11-\mid$ & 58.5 & 211.3 & 64.1 \\
\hline 118 & $12-D$ & 63.6 & -30.2 & 66.3 \\
\hline 119 & 12-ل & 63.6 & 210.2 & 66.3 \\
\hline 120 & 13-D & 68.7 & -29.1 & 68.6 \\
\hline 121 & 13-1 & 68.7 & 209.1 & 68.5 \\
\hline 122 & 14-D & 73.7 & -28.1 & 70.5 \\
\hline 123 & 14-I & 73.7 & 200.1 & 70.8 \\
\hline 124 & $15-D$ & 78.8 & -27.3 & 73.3 \\
\hline 125 & الـ15 & 78.8 & 207.3 & 72.3 \\
\hline 126 & $16-D$ & 83.8 & -26.5 & 72.2 \\
\hline 127 & $16-1$ & 83.8 & 206.5 & 76.2 \\
\hline 128 & $17-D$ & 88.8 & -25.8 & 86.1 \\
\hline 129 & $17-1$ & 68.6 & 205.8 & 70.6 \\
\hline 130 & $10-\mathbb{D}$ & 93.9 & -25.1 & 86.1 \\
\hline 131 & 18- إ & 93.9 & 205.1 & 98.1 \\
\hline
\end{tabular}

Tabla 1. Denominación y áreas de los tirantes de la estructura Modelo 90.

La estructura reseñada podría considerarse como la mitad del desarrollo de una estructura simétrica de 3 vanos, sobre 2 pilonos, en la que se generarían unas luces 90+180+90 m. Se pretende hacer ver así que la estructura analizada correspondería entonces al caso de un puente de luces cortas o medias, y los resultados y conclusiones presentados en adelante habrá que restringirlos a este tipo de puentes.

Para facilitar la identificación de cada una de las distintas estructuras calculadas en esta investigación, en adelante, cuando se haga referencia a esta estructura se la denominará Modelo 90.

Para representar el comportamiento de la estructura se ha generado un modelo plano de elementos tipo barra que representa el conjunto de la estructura (Figura 57). La 
modelización de la estructura se ha realizado utilizando un programa de cálculo matricial de estructuras de barras ${ }^{2}$ que permite además la simulación de las diferentes fases del proceso constructivo de forma secuencial, mediante el uso de archivos de tipo lógico que organizan y programan en un solo cálculo la realización del proceso completo. En el anexo A.l se incluyen dichos archivos utilizados.

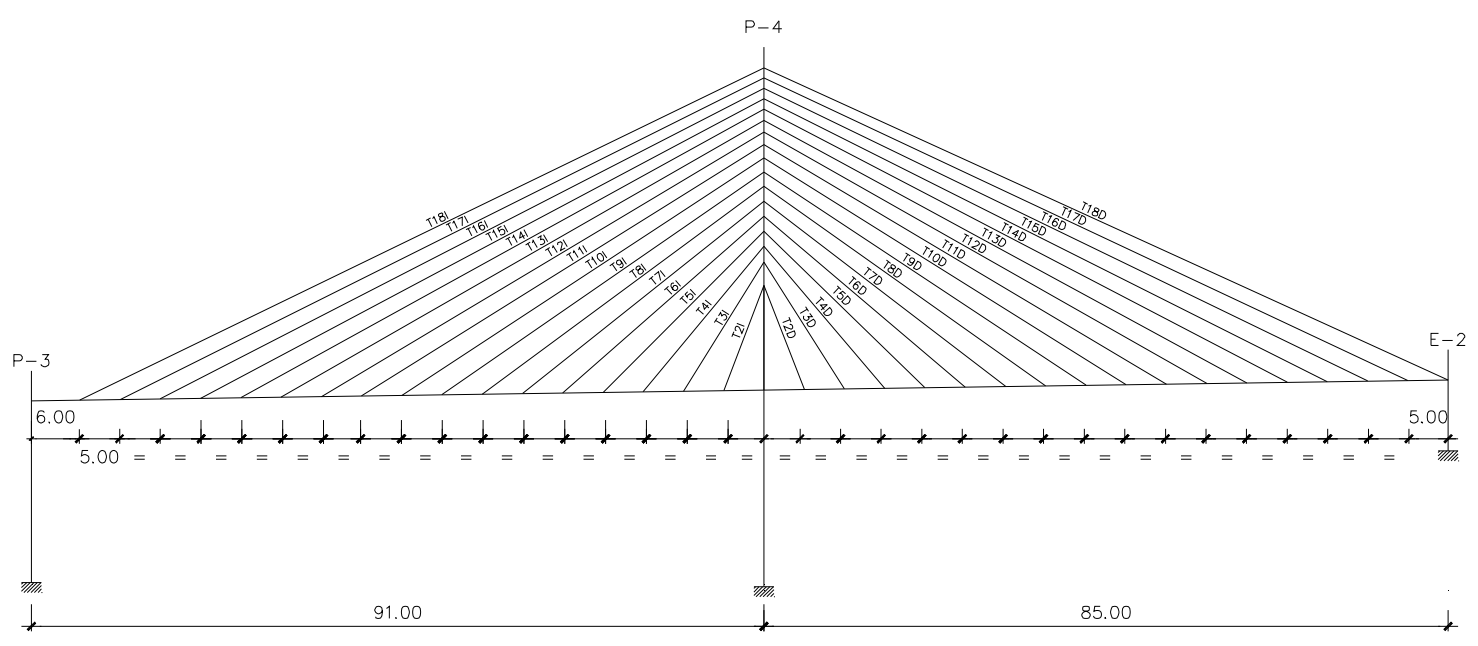

Figura 57. Esquema del modelo de cálculo de la estructura analizada.

Hay que señalar que se ha utilizado un modelo plano en 2D para los cálculos, a pesar de que el puente analizado está formado por 2 planos de atirantamiento, ya que el estudio realizado sólo se ha centrado en comportamiento de la estructura en su plano frente a la actuación de cargas contenidas en éste, no habiéndose tenido en cuenta, dado el objetivo de esta investigación, el comportamiento frente a acciones transversales.

Por otra parte hay que Indicar que se ha considerado, para cada una de las dovelas del tablero, su discretización mediante dos barras de 2'00 y 3'00 m de longitud respectivamente cada una de ellas, de tal manera que los anclajes de los cables al tablero se realizan en su nudo intermedio, quedando éstos por lo tanto a 2'00 m del frente de la dovela.

Las vinculaciones establecidas en el modelo de la estructura han consistido en empotramientos en la cimentación de ambos estribos, así como en el arranque del

\footnotetext{
${ }^{2}$ El programa de cálculo es el denominado ESTRE que ha sido desarrollado por la Oficina técnica de Acciona Infraestructuras, y que es utilizado desde hace tiempo para el cálculo de puentes y estructuras modelizables mediante barras.
} 
pilono. La vinculación entre pilono y tablero se establece coartando tanto los desplazamientos horizontales como verticales, pero sí permitiendo el giro del tablero en ese punto, de tal manera que se consigue así simular el apoyo de este elemento sobre una riostra transversal dispuesta entre los 2 fustes del pilono.

En el anexo A.l se ha incluido un listado de todas las anteriores características descritas con las que se ha conformado el modelo de cálculo de esta estructura.

Por último añadir que, para simular los apoyos extremos del tablero sobre los estribos, se han creado 2 barras sin capacidad de absorción de momentos (inercia nula) que representen los apoyos de neopreno.

El proceso constructivo previsto para la ejecución de esta estructura consiste en la ejecución de la siguiente secuencia de operaciones:

- $\quad$ Ejecución de cimentaciones pilas y estribos.

- Ejecución de las dovelas 11 y ID sobre cimbra y tesado de los cables 21 y 2D. Que consta de las siguientes subfases:

$\checkmark \quad$ 1.- Ejecución sobre la riostra de pilas y sobre apoyos provisionales de las dovelas 1Izq. y 1-Der.

$\checkmark \quad$ Instalación del tirante 21 y eliminación del apoyo provisional extremo de la dovela dovela 1-lzq.

$\checkmark \quad$ Instalación del tirante 2D y eliminación del apoyo provisional extremo de la dovela dovela 1-Dcha.

$\checkmark \quad$ Colocación sobre las dovelas-1 de los carros de avance incluyendo el peso propio de las dovelas 2

$\checkmark \quad$ Desplazamiento del carro de avance izquierdo, aplicándose entonces las cargas de peso propio de la dovela 2-Izq. y montaje del tirante 31.

$\checkmark \quad$ Desplazamiento del carro de avance derecho, aplicándose entonces las cargas de peso propio de la dovela 2-Dcha. y montaje del tirante 3D.

- Avance en voladizo para la ejecución de las restantes dovelas siguiendo un esquema secuencial como el siguiente para la fase " $n$ ":

> Hormigonado de las dovelas " $n$ ", derecha e izquierda.

> $\quad$ Tesado del cable "n" izquierda.

> Tesado del cable " $n$ " derecha. 
- El proceso constructivo finaliza con la ejecución de la dovela 18 Izquierda de apoyo sobre la pila, dado que la estructura no es completamente simétrica.

\subsection{CALCULO DE LA ESTRUCTURA TEORICA.}

Como se ha indicado en el epígrafe 3.5.1, el primer paso en el cálculo de la estructura teórica que nos servirá de referencia es el cálculo de los axiles finales de los tirantes en la situación objetivo adoptada, correspondiente a aquella configuración del tablero con comportamiento como viga continua, apoyada elásticamente sobre los puntos de anclaje de los tirantes.

Para ello se ha partido de dicha situación objetivo, en esfuerzos y deformaciones, para determinar los axiles de los tirantes en la situación final. En todos los tirantes la condición establecida de descomposición de la reacción en cada apoyo de la viga continua permite obtener dicho axil, salvo en el caso del último tirante a instalar, el cable 18D de retenida, donde adicionalmente se establece la condición de anulación de los momentos en el pilono, a la altura del encuentro con el tablero.

De este estado por lo tanto se obtienen:

$\checkmark \quad$ Esfuerzos en tablero en situación final, axiles como descomposición de la acción de los tirantes, y flectores y cortantes como resultado de la actuación de las cargas permanentes sobre la viga continua.

$\checkmark \quad$ Deformaciones en tablero bajo la actuación de las cargas permanentes.

$\checkmark \quad$ Axiles finales en tirantes.

Con estos 3 conjuntos de datos se conforma el denominado "Estado 0 inicial", estado utilizado para el comienzo del proceso de desmontaje de la estructura, que permitirá obtener los axiles iniciales de los tirantes. Partiendo por lo tanto de este estado, se procede a realizar de forma secuencial dicho proceso habiéndose utilizado para ello unos archivos "Iógicos" programados en VBasic que permiten automatizar todo el proceso. El listado del archivo lógico empleado se incluye en el anexo A.2 a este documento.

La secuencia de operaciones del desmontaje resulta:

1.Eliminación de la carga muerta. 
2.Retirada de los apoyos en estribos del vano izquierdo y derecho.

3.Eliminación de la dovela 181.

4.Eliminación del tirante 18D.

5. Retirada del tirante 181.

6.Eliminación de las dovelas 17D y 171.

7.Retirada del tirante 17D.

8. Retirada del tirante 171.

9.Eliminación de las dovelas 16D y 161.

$[\ldots]$

y sucesivamente hasta eliminar todas las barras del tablero del modelo de cálculo.

Utilizando entonces esta secuencia de operaciones, se obtienen los axiles de montaje de los tirantes a partir del valor que posee cada tirante justo antes de su anulación en en el modelo de cálculo de la estructura.

A continuación se ha procedido a determinar las situaciones teóricas de referencia para los cálculos a realizar posteriormente, es decir se ha procedido a obtener los esfuerzos en los diferentes elementos estructurales como resultado del proceso teórico de construcción, el cual se ha simulado mediante un proceso de montaje, igualmente automatizado utilizando un archivo lógico. Dicho archivo, como ya se ha comentado, se adjunta en el anexo A.2 de esta tesis.

Los resultados obtenidos lo son, tanto en las situaciones intermedias de dicho proceso, como en la situación final del proceso constructivo al aplicar la carga muerta sobre el tablero, así como en las situaciones de servicio, bajo la actuación de la sobrecarga de tráfico rodado.

Algunos de estos resultados se representan en las siguientes figuras, incluyéndose sólo aquellos que se han considerado más representativos del comportamiento de la estructura. En concreto, en la primera de ellas (Figura 58) se representan los axiles de montaje, los máximos y mínimos de construcción, los axiles finales al terminar el proceso constructivo y los máximos y mínimos de servicio. En la segunda de ellas (Figura 59) se representan las envolventes de flectores en el tablero, tanto los máximos como los mínimos de construcción, los máximos y mínimos de servicio, al aplicar las sobrecargas de tráfico en el tablero, y la ley de flectores al final del proceso constructivo. En la 
última de ellas se representan los flectores en el pilono en las mismas situaciones anteriores (Figura 60).

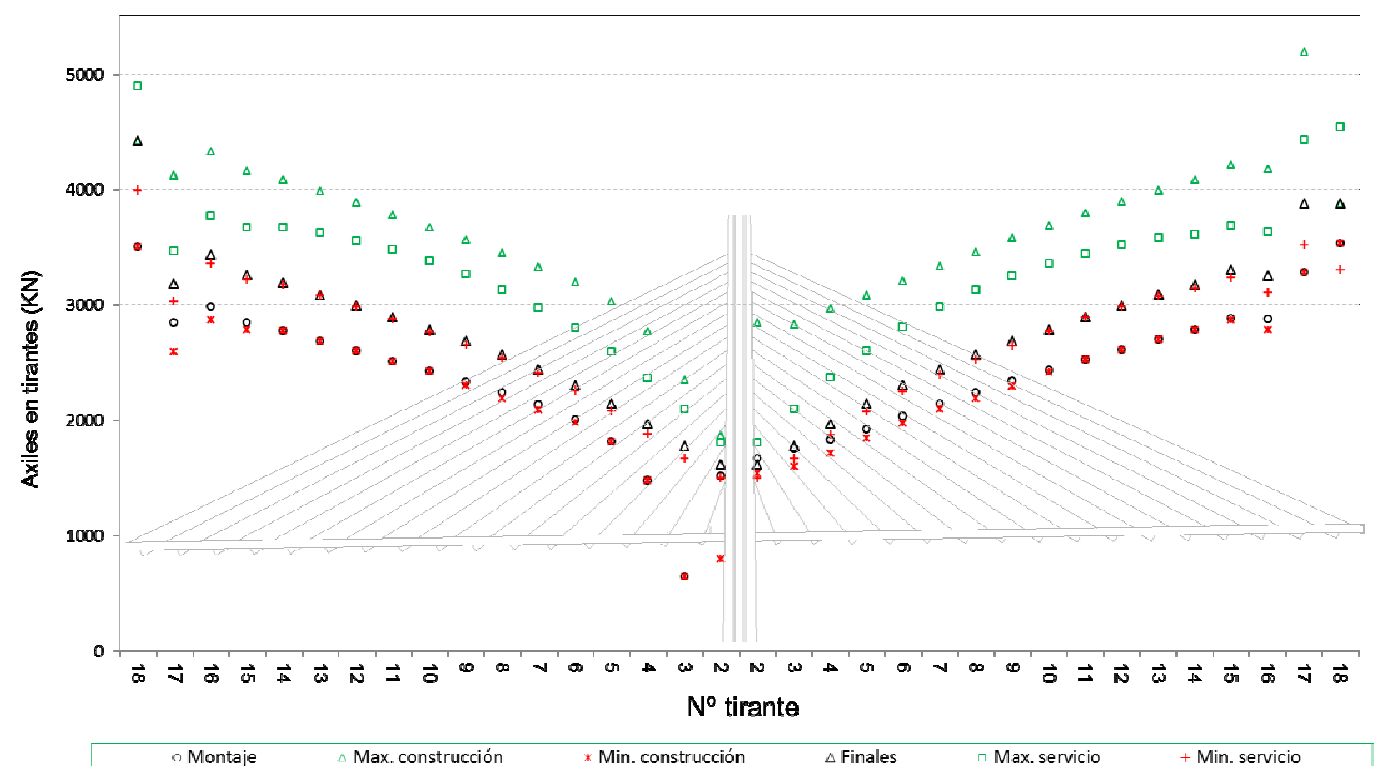

Figura 58. Axiles en tirantes en fase de inicial de instalación, máximos y mínimos de las fases de construcción, en la fase final del proceso constructivo y máximos y mínimos en las situaciones de servicio.

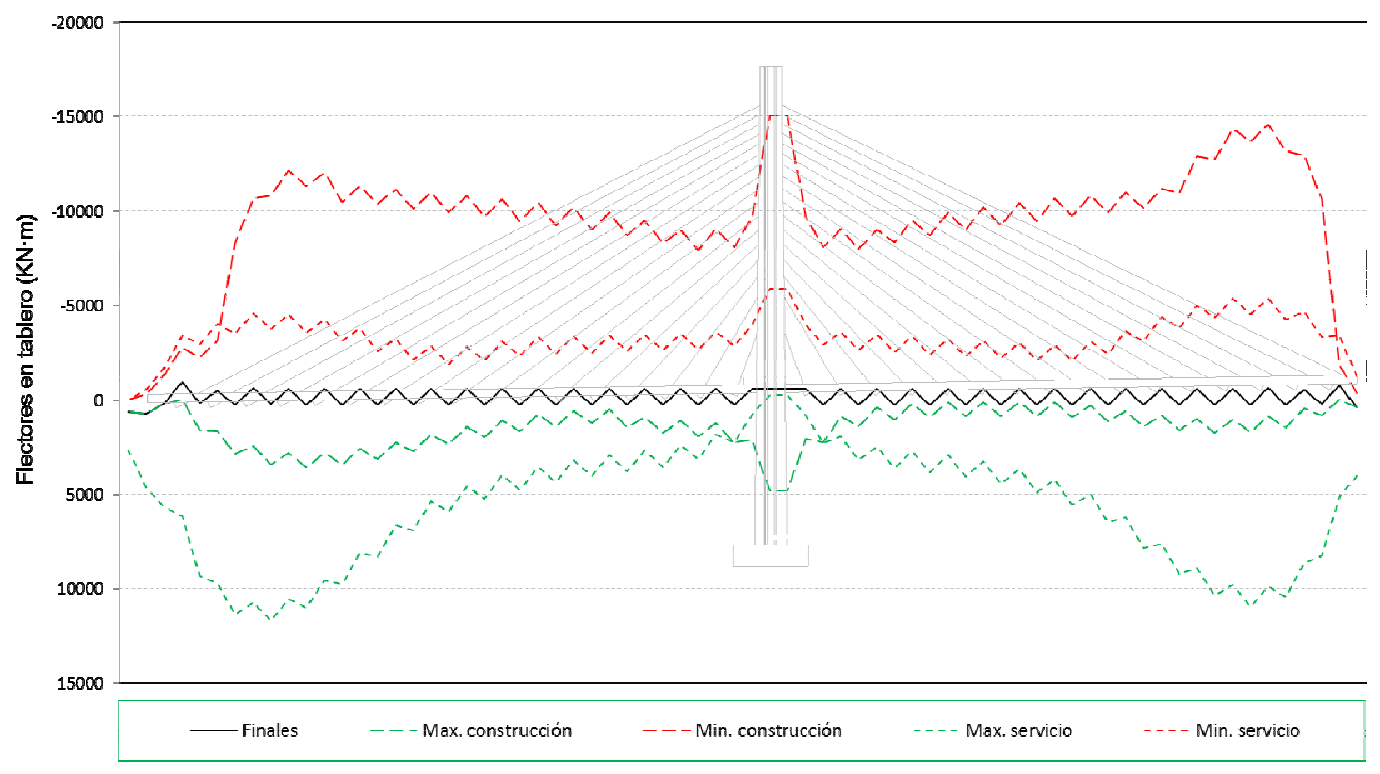

Figura 59. Envolvente de flectores en el tablero en las fases de construcción, final y en servicio. 


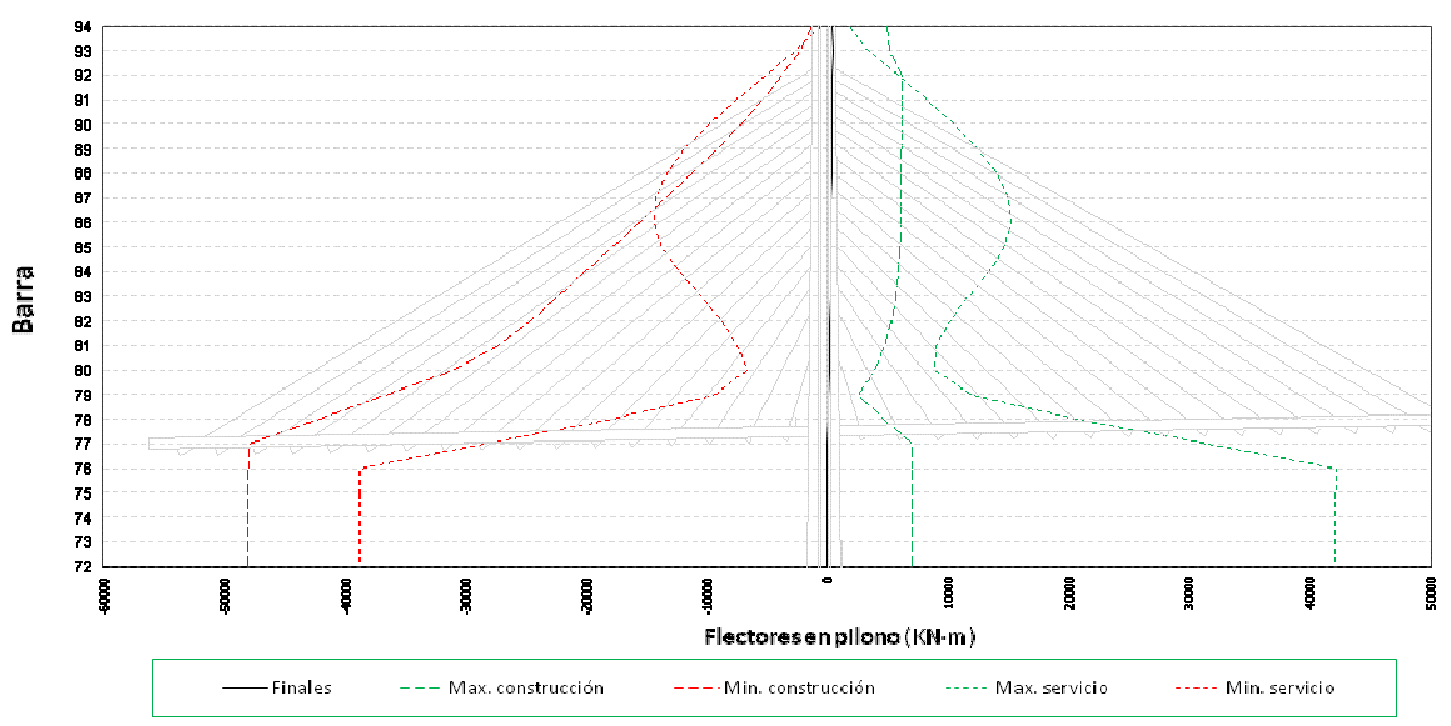

Figura 60. Envolvente de flectores en el pilono en las fases de construcción, final y en servicio.

De los resultados aquí representados se pueden extraer varias conclusiones en relación a los axiles en tirantes y a los flectores en el tablero y pilono. En el primer caso se puede deducir que:

- Los axiles de montaje de los tirantes son siempre menores que los axiles en la situación final.

- Los máximos axiles en las fases de construcción y en servicio son superiores a los axiles en la situación final. En concreto las mayores diferencias entre los axiles en la situación final y en las situaciones de servicio se producen en el tramo de tablero más flexible, es decir hacia los 3/4 de la luz principal.

- Los mínimos axiles en las fases de construcción y en servicio, salvo algún caso puntual, en general son superiores a los axiles en el instante del montaje. Es decir que el mínimo axil que soportan cada tirante desde su instalación es el axil de montaje.

- Siendo la separación entre cables uniforme y a su vez las cargas permanentes que le corresponde soportar a cada cable del mismo orden en todos ellos, en general se observa que los axiles en los tirantes crecen conforme nos alejamos del pilono. Esto es debido a la disminución del ángulo de los cables respecto del tablero, y por lo tanto al reducirse su eficacia para soportar la misma reacción vertical, conforme más alejados del pilono se encuentre su anclaje. 
Por otra parte, en el caso de los resultados en flectores en el tablero, se observa que mientras que la situación final de construcción representa unos valores iguales a los de la situación objetivo teórica planteada como viga continua, y por lo tanto sus valores absolutos resultan relativamente acotados, la envolvente total de flectores se conforma por los mínimos de la situación de construcción y por los máximos de la situación de servicio. Esta cuestión se fundamenta en las dos siguientes causas:

- En primer lugar porque el mínimo flector negativo, en cada dovela, a lo largo de todo el proceso constructivo se produce en la sección de anclaje del cable instalado sobre la última dovela terminada, cuando se ejecuta el hormigonado de las dovelas adyacentes y por lo tanto actúa todo su peso sobre dicha sección de anclaje. Es importante señalar que en este caso, ese valor del esfuerzo depende en gran medida del peso de la dovela y no tanto del axil en los tirantes, por lo que en los resultados sucesivos se observará que la influencia de los errores aleatorios que se introduzcan en los cálculos será pequeña en este aspecto concreto.

- En segundo lugar, la envolvente de flectores positivos queda determinada por la actuación de las sobrecargas sobre la estructura terminada, momento en el cual la flexibilidad de la estructura frente a este tipo de carga descentrada, respecto del pilono, genera los valores máximos que componen la envolvente de flectores positivos. Este efecto es más acusado en la zona cercana al centro de vano y en el tramo en el que los ángulos de los cables son ya reducidos, es decir se produce en el entorno de los 3/4 de la luz principal.

En relación a las envolventes de flectores en el pilono se puede indicar que:

- Las flectores en el pilono en la situación final son prácticamente nulos, ya que con esa condición se han calculado los axiles finales de los tirantes.

- La envolvente de flectores en fase de construcción condiciona la envolvente total de flectores negativos, dada la secuencia de construcción elegida, al ejecutarse primero las dovelas del vano izquierdo, quedando por encima de las de servicio con la actuación de las sobrecargas.

- Las envolventes de flectores positivos en cambio quedan condicionadas por las situaciones de servicio. 
Por último se ha analizado qué ocurre en esta estructura con el reparto del axil del tirante entre la parte pasiva y la parte activa, es decir, se va a comparar el incremento de axil que se produce desde el momento de la instalación de cada cable laxil activo), respecto del axil final que resulta en cada uno de ellos. Se obtiene así la siguiente gráfica (Figura 61):

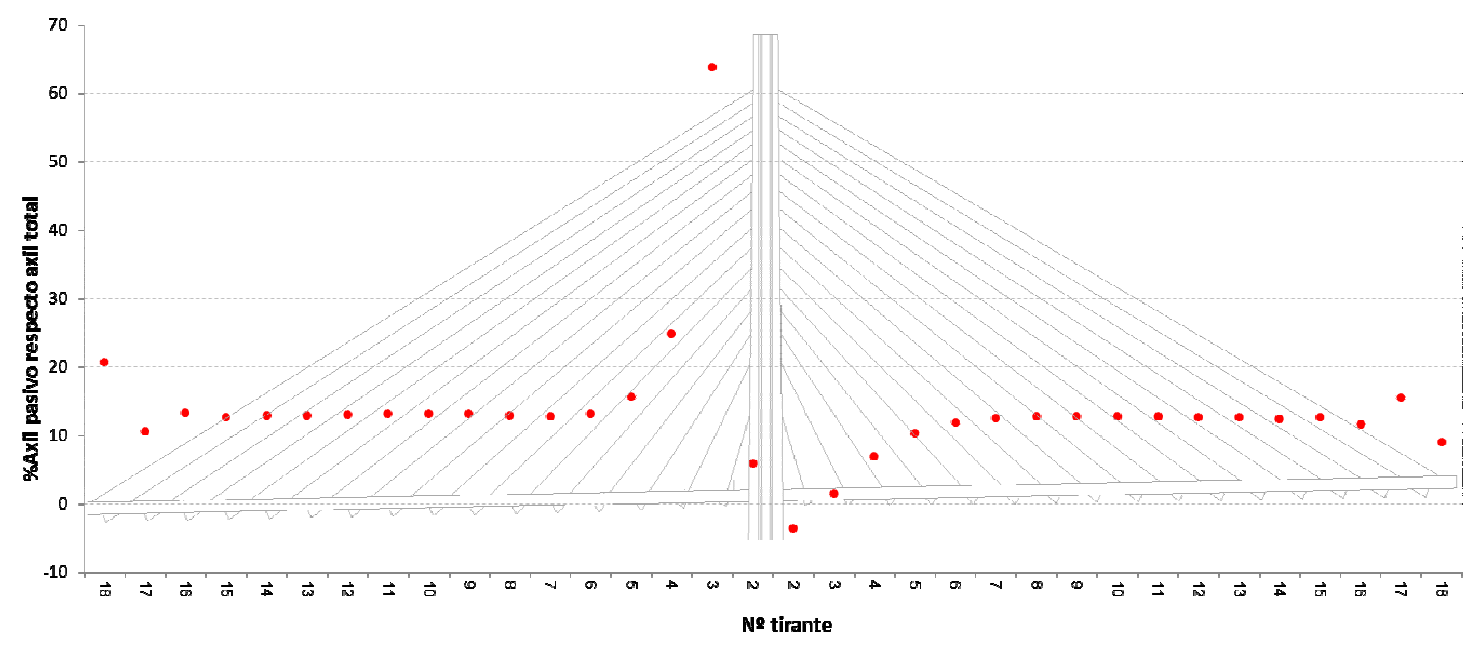

Figura 61. Porcentaje del axil pasivo respecto del axil total en los diferentes tirantes.

Del gráfico anterior se puede deducir:

- Que el porcentaje del axil pasivo es bastante constante en la mayor parte de los tirantes representando del orden de un $12 \%$ del axil total, lo que representa un valor reducido.

- Que en los tirantes iniciales, debido al proceso constructivo planteado, se alcanzan valores distintos del resto. En concreto en los tirantes 41 y 31 tienen porcentajes mayores, mientras que los tirantes $21,2 D$ y $3 D$ tienen porcentajes menores, quedando incluso el tirante $2 D$ con un axil final menor que el de montaje. Esta cuestión llevaría a concluir que el tratamiento de estos cables iniciales en relación a este aspecto debería hacerse de forma diferenciada respecto del resto.

- Que el efecto de la colocación de tirantes adyacentes a los ya colocados representa un incremento del porcentaje de axil pasivo del orden del $11 \%$, ya que como se deduciría de lo indicado en el epígrafe 3.3, para el caso de separaciones de tirantes del orden de 5'00 m, los gráficos allí incluidos señalaban un porcentaje de en torno al 1\%, siendo por lo tanto la diferencia 
hasta el 12\% alcanzado el incremento generado por la colocación de más de un cable.

- En los tirantes extremos 181 y 18D también se produce una singularidad en los resultados que tiene la siguiente justificación: en primer lugar que el axil del tirante 181 queda muy condicionado por el peso de la dovela extrema asimétrica y por ello su axil pasivo es mayor que en los cables adyacentes. Por otro lado, el cable 18D sólo sirve de contrarresto de la dovela asimétrica anteriormente indicada y no recibe la acción de ninguna dovela por parte del vano derecho, por lo que su componente pasiva es menor que los cables vecinos.

Finalmente recordar que esta estructura corresponde al caso de un puente simétrico con tablero de hormigón y un solo pilono, pudiéndose extrapolarse fácilmente estos resultados al caso de un puente de idénticas características pero con doble pilono y luz principal, es decir al caso de un puente con distribución de luces 90+180+90m.

\subsection{EVALUACIÓN DE LOS ERRORES DE EJECUCIÓN.}

El objetivo de este estudio ha sido analizar qué efecto tienen sobre la estructura final la introducción de estos errores, por lo que es necesario definir y detallar qué tipo de errores se considerará que se pueden producir, de qué tipo pueden ser, así como cuantificar un error máximo por encima del cual los sistemas de control de la obra sean capaces de detectarlo y corregirlo.

Los métodos de instalación de los cables en los puentes atirantados básicamente son dos: el primero de ellos se basará en la aplicación de una tensión determinada sobre el cable, correspondiente al axil de montaje calculado, que es el método denominado en fuerzas. En este método se contralará la presión marcada en el manómetro de los gatos de tesado, y es durante este proceso en el que podrán producirse errores. Hay que señalar que este método es muy sensible a las cargas aplicadas sobre la estructura en el momento de la instalación del cable, por lo que los pesos de los equipos auxiliares colocados sobre el tablero deben ser evaluados con mucha precisión. Igualmente el efecto de la temperatura deberá ser tenido en cuenta a partir de su medición y ajustando los cálculos al instante del tesado.

El segundo método de instalación de los cables es el método en deformaciones, en el que, haciendo uso de la definición de longitud en vacío del cable, se calculan las 
deformaciones a aplicar a los cables en el instante de su puesta en obra, siendo esta longitud, la medida que servirá para verificar su puesta en tensión. Este método cuenta con la ventaja de que al ser la longitud en vacío un parámetro intrínseco de la estructura, y establecer la colocación del cable mediante una medida que es función de ese parámetro, los efectos de las cargas aplicadas en el instante de colocación del cable y los efectos de la temperatura se pueden obviar.

Uno de los objetivos de este estudio va a ser analizar qué diferencias se pueden llegar a producir si aplicamos uno u otro método sobre una estructura determinada.

\subsubsection{Tipos de errores.}

Por otra parte, durante el proceso de colocación y tesado de los tirantes se podría plantear la existencia de diferentes tipos de errores. En principio, para el caso de los tirantes en estudio, los errores debidos a las pérdidas se considerarán despreciables. No será así en el caso de los errores en la fuerza de aplicación en los tirantes o bien en las deformaciones a generar sobre ellos, así como los errores debidos al replanteo de los anclajes. Es decir se va a considerar la posibilidad de la existencia de una instalación errónea de los cables, ya sea por una mala medición de la presión aplicada al gato de tesado, ya sea al medir los alargamientos de los cables o al colocar los cajetines de anclaje de los tirantes.

En primer lugar hay que distinguir entonces dos conceptos, por un lado el error de exactitud y por otro el error de precisión. Se define "precisión" como la desviación máxima que pueden presentar las diferentes medidas efectuadas con un mismo aparato de medida, mientras que se puede definir la "exactitud", que tendría ese mismo aparato de medida, como el error que pudiera tener la lectura efectuada respecto del valor real aplicado al cable.

Se puede concluir entonces, que el error debido a la exactitud de la medida, al ser un error sistemático y de valor constante en todas las mediciones realizadas, podría corregirse directamente realizando un control adecuado de la estructura, mientras que los errores de precisión no será posible eliminarlos, dado su carácter aleatorio, por lo que la estructura se podrá ejecutar incluyendo estos errores.

Por otra parte, en base a lo establecido en la "Teoría matemática de errores" [4] [27], éstos mismos se indica que podrán ser de dos tipos: Errores sistemáticos y errores aleatorios. Los primeros estarían relacionados con la exactitud del sistema de medida, 
mientras que los segundos serían los relacionados con la precisión de dichas medidas. Es entonces sobre estos últimos sobre los que se centrará el estudio realizado a continuación.

También en base a dicha "Teoría de errores" se demuestra que, si la causa del fallo en la precisión está originada por la adición de varios errores elementales, pudiendo considerarse cada uno de ellos como una variable aleatoria independiente, el error total de precisión resultante, según el "Teorema Central del Límite", tendrá una distribución aproximadamente normal. Es decir, que si se pretende simular la existencia de estos fallos se deberá plantear un algoritmo que permita generar muestras aleatorias de valores erróneos que tengan una distribución normal, con media el valor teórico de la medida realizada y desviación típica a determinar.

Como se ha indicado anteriormente, los procesos de cálculo erróneos se van a basar en la aplicación de, o bien esfuerzos de tesado erróneos, aplicando el método de las fuerzas, o bien errores en la medición de deformaciones o en la posición de anclajes utilizando el método en deformaciones.

\subsubsection{Algoritmo para la generación de muestras de valores aleatorios.}

Se necesita entonces, para proceder a simular un montaje erróneo de los cables de un puente atirantado, definir una herramienta que permita la generación de variables aleatorias según una distribución normal. Para ello se ha utilizado un algoritmo, basado en el "Teorema central del límite" [15][87], que se obtiene a partir de la siguiente formulación:

Dada una serie de variables aleatorias independientes $\left\{r_{1}, r_{2}, \ldots, r_{i}, \ldots, r_{N}\right\}$ todas ellas con la misma distribución con media $\theta$ y desviación típica $\sigma^{2}$, se verifica que la esperanza y varianza de la suma de todas ellas cumple:

$$
\begin{array}{ll}
\text { [EC4. 1.] } & E\left[\sum_{i=1}^{N} r_{i}\right]=N \cdot \theta \\
\text { [EC4. 2.] } & V\left[\sum_{i=1}^{N} r_{i}\right]=N \cdot \sigma^{2}
\end{array}
$$

Aplicando entonces el "Teorema central del límite": 


$$
\text { [EC4. 3.] } \operatorname{Lim}_{N \rightarrow \infty} P\left(a<\frac{\sum_{i=1}^{N} r_{i}-N \cdot \theta}{\sqrt{N} \cdot \sigma}<b\right)=\frac{1}{\sqrt{2 \cdot \pi}} \cdot \int_{a}^{b} e^{-\frac{1}{2} \cdot z^{2}} \cdot d z
$$

definiéndose la variable aleatoria z normal estándar como:

$$
\text { [EC4. 4.] } \quad \frac{\sum_{i=1}^{N} r_{i}-N \cdot \theta}{\sqrt{N} \cdot \sigma}=z
$$

y sabiendo además que la mayor parte de los lenguajes de programación definen una función "random" ( RND en VBasic o similar) que permite la generación de números aleatorios según variables con distribución uniforme, y conociendo que para dichas distribuciones, en un cierto intervalo [a,b], los valores de la media y desviación típica resultan:

$$
\text { [Ec4. 5.] } \quad \mu=\frac{a+b}{2} \quad \text { [Ec4. 6.] } \quad \sigma^{2}=\frac{(b-a)^{2}}{12}
$$

que se puede particularizar para el caso en el que las variables aleatorias $r_{i}$ se definan en el intervalo $[0,1]$, sus medias y desviaciones típicas resultan:

$$
\mu=1 / 2 \quad \sigma=1 / \sqrt{ } 12
$$

Si este conjunto de $\mathrm{N}$ variables aleatorias se quiere asimilar a una variable aleatoria $\mathrm{x}_{\mathrm{i}}$ con media $\mu_{x}$ y varianza $\sigma_{x}^{2}$ resulta entonces, sustituyendo en la ecuación [Ec4. 4.] los valores anteriores que:

$$
\text { [Ec4. 7.] } z=\frac{\sum_{i=1}^{N} r_{i}-\frac{N}{2}}{\sqrt{\frac{N}{12}}}=\frac{x-\mu_{x}}{\sigma_{x}}
$$

y operando en esta expresión:

[Ec4. 8.]

$$
x=\sigma_{x} \cdot \sqrt{\frac{12}{N}} \cdot\left[\sum_{i=1}^{N} r_{i}-\frac{N}{2}\right]+\mu_{x}
$$


Es decir, se ha obtenido una expresión que permite calcular valores de una variable aleatoria con distribución normal, con media $\mu_{x}$ y desviación típica $\sigma_{x}$ a partir de la generación de $\mathrm{N}$ valores aleatorios con distribución uniforme en el intervalo $[0,1]$.

Es más, si se el número de valores a generar con la función RND es 12 y como media $\mu_{x}$ se adopta 1, resulta entonces la expresión simplificada:

$$
\text { [Ec4. 9.] } \quad x=\sigma_{x} \cdot\left[\sum_{i=1}^{12} r_{i}-6\right]+1
$$

Esta expresión indica que dados 12 números aleatorios (generados mediante la función RND de VBasic), restando a su suma 6, multiplicando este valor por la desviación típica, y sumando a este resultado 1, se verifica que los valores que se obtienen siguen una distribución normal, pudiendo plantearse además estos valores como un coeficiente de error, en tanto por un uno, de una cierta cantidad a la que se quiera ponderar.

\subsubsection{Errores máximos admisibles.}

Utilizando el algoritmo anterior, se pueden generar valores aleatorios de las magnitudes que se están analizando según una distribución normal estándar, lo que supone, que teóricamente se puede presentar todos aquellos valores que se encuentren representados en la curva de Gauss. Esto implicaría la posible generación de errores con valores muy altos, que en la realidad, durante el proceso de instalación de los cables, se detectarían con facilidad y se podrían corregir inmediatamente. Este estudio se ha querido centrar en analizar el comportamiento de la estructuras atirantadas bajo la introducción de errores aleatorios que no sean detectables, y por lo tanto corregibles, de tal manera que se necesita poder truncar las anteriores series de valores aleatorias en aquellos límites de error que se consideren inadmisibles a la hora de la puesta en obra.

Por ello, se ha realizado un análisis de las diferentes tolerancias existentes en la normativa actual que serían de aplicación en este caso referentes a los errores de montaje de los cables, suponiéndose así que en obra deberán usarse elementos de control suficientes como para no permitir la aplicación de valores superiores a los máximos en ellas contemplados.

Realizando entonces una rápida revisión de la normativa vigente hoy en día, se encuentra que se establecen una serie de limitaciones a los errores máximos admisibles 
a poderse producir durante los procesos de ejecución de las estructuras atirantadas, dividiéndose en errores sobre los procesos de montaje utilizando la medida de la fuerza provocada por el gato de tesado, errores en la medida de las deformaciones generadas en el cable, una vez que se introduce la fuerza de tesado de forma progresiva, e incluso existen también limitaciones a los errores en la geometría de un determinado elemento de hormigón, que representarán la tolerancia en el replanteo de los anclajes.

La primera referencia analizada son las "Manual de Tirantes" de ACHE [2], que señalan que en la instalación de cables se podrán producir desviaciones máximas de hasta un $\pm 5 \%$ respecto del valor teórico del axil de tesado. Otra referencia, aunque no es de aplicación para el caso de los cables de los puentes atirantados, son las recogidas en la EHE08 [1], que indica en su artículo 35.1, en relación a los sistemas de pretensado convencionales, que se deberán utilizar durante las fases de tesado de los cables, elementos de medida de dichas tensiones aplicadas que garanticen una precisión mínima del $2 \%$. Sí sería una referencia más adecuada para nuestro caso el "Manual de tirantes" del SETRA [102], donde se señala que la desviación máxima en la tensión a aplicar a los cables puede ser del $\pm 2 \%$ respecto del valor teórico. Igualmente en las recomendaciones del PTI [86] se indica un valor del error máximo admisible en el valor de la fuerza de tesado de los cables de un $2^{\prime} 5 \%$.

Respecto de errores en la medida de los alargamientos de los cables, la principal referencia analizada es también el "Manual de tirantes" del SETRA, en el cual se establece que el error máximo en la medida de la longitud de un tirante será el 0'01\% de la longitud en vacío del cable. Señalar que parecería razonable, tal y como posteriormente se va a comprobar en esta investigación, definir un valor límite para el caso de los cables cortos como complemento de la anterior condición, ya que además en ellos se puede producir alguna singularidad como las que más adelante se detallan en esta investigación.

Por último, en relación a los errores admisibles en la posición de los anclajes, dado que se analiza el caso de estructuras con tablero y pilono de hormigón, se han estudiado las tolerancias establecidas en la normativa vigente de hormigón estructural (EHE08, $\mathrm{EC} 2, \mathrm{ACl}-318, \ldots$ ) en lo referente a las tolerancias en los encofrados y en las dimensiones finales de las piezas de hormigón. En general, los valores obtenidos hablan todos ellos de errores admisibles del orden de 1 ó 2 centímetros, valores que se juzgan adecuados para conseguir un buen control de ejecución de una pieza de hormigón. 
Del análisis de los anteriores valores se deduce que es mucho más exigente la condición en longitud de cables que la condición en la posición de anclajes, ya que por ejemplo, si se analizara el caso de un tirante intermedio de un puente de luces medias, el cual podría tener un longitud aproximada de $50 \mathrm{~m}$, en ese caso el error en longitud admisible resultaría:

$$
\Delta \mathrm{L}=0^{\prime} 0001 \cdot 50 \cdot 10^{3}=5 \mathrm{~mm}
$$

Es decir, que como más adelante se va a comprobar, en el caso de los puentes con tablero de hormigón, la instalación de los cables a partir de las deformaciones necesarias a aplicar sobre ellos, necesitaría de un control de ejecución de los encofrados y geometría final de los cajetines de anclaje de los cables, o bien del posicionado de los bastidores de anclaje metálicos si los anclajes se llevan todos ellos contra un elemento de este tipo, que está muy por encima del control habitualmente exigido para las estructuras de hormigón.

Es más, esta condición en la posición de los anclajes será tanto más limitativa cuanto más pequeña sea la longitud del cable, es decir en el caso de puentes de pequeña luz y más aun en el caso de sus tirantes más cortos.

\subsubsection{Verificación de la normalidad de las muestras.}

Hasta este punto de desarrollo del procedimiento de cálculo, se ha procedido a definir un algoritmo que permite obtener, a partir de un conjunto de valores aleatorios de una variable con distribución normal, muestras truncadas al eliminar los valores extremos obtenidos por encima de los límites marcados como admisibles por la normativa. Pero ahora deberá comprobarse que las muestras, que originalmente se consideraban con distribución normal, después de ser truncadas siguen teniendo una distribución aproximadamente normal.

Para verificar la validez de la hipótesis de normalidad de la serie de todos los valores generados, se ha utilizado el denominado test de normalidad de Anderson-Darling [5].

En dicho test se definen las siguientes hipótesis de contraste:

- Ho - La serie de valores analizada tiene una probabilidad menor del 1-p\% de no seguir una distribución normal. 
- $\mathrm{H}_{1}$ - La serie de valores analizada tiene una probabilidad mayor del 1-p\% de no seguir una distribución normal.

Siendo el valor del p\% el nivel de confianza del resultado del test.

A continuación, y una vez ordenada la serie de datos obtenida, se calcula el parámetro "A" mediante la siguiente expresión:

[Ec4. 10.] $\quad A^{2}=\sum_{i=1}^{n}(1-2 i) \cdot \frac{1}{n} \cdot\left[\ln F\left(x_{i}\right)+\ln \left(1-F\left(x_{n-i-1}\right)\right)\right]-n$

Donde los diferentes $F\left(x_{i}\right)$ se obtienen de la integración de la función de distribución normal $N(0,1)$ :

$$
\text { [EC4. 11.] } \quad F(x)=\frac{1}{\sqrt{2 \cdot \pi}} \cdot \int_{-x}^{x} e^{-t^{2} / 2} \cdot d t
$$

Señalar que a su vez, para la obtención de dichos valores se ha programado un algoritmo de integración numérica mediante diferencias finitas (aplicando la fórmula del trapecio), que permite obtener los valores de dicha función de distribución mediante incrementos suficientemente pequeños.

A su vez se procederá a calcular el parámetro CV, que es función del nivel de confianza p\% con el que se quiera obtener el resultado del test. Habiéndose en este caso adoptado un nivel de confianza del 95\%, resulta entonces la fórmula para obtener el valor de CV la siguiente:

$$
\text { [Ec4. 12.] } \quad C V=0^{\prime} 752 /\left(1+\frac{0^{\prime} 75}{n}+\frac{2^{\prime} 25}{n^{2}}\right)
$$

siendo " $n$ " el $n$ de elementos de la muestra analizada

Conocido el valor de los parámetros A y CV para la muestra analizada, la hipótesis $\mathrm{H}_{0}$ se verifica entonces si dicho parámetro $\mathrm{A}^{2}$ alcanza un valor menor que el de CV.

Así, siguiendo el criterio anterior, sólo se dan por válidas aquellas series de datos generadas que cumplan la condición de normalidad anterior. En el caso de que la serie de datos analizada no verifique esta condición, la serie de datos se descartará y se generará una nueva, hasta obtener una serie que sí cumpla este criterio. 
El algoritmo programado para la aplicación de este procedimiento se ha incluido en el anexo A.3, incluido al final de este documento.

\subsubsection{Desviación tipica de las muestras de valores aleatorios.}

Llegados a este punto es necesario establecer el valor de la desviación típica de las muestras para tener completamente definida la distribución de estas variables.

El criterio para definir dicha desviación típica ha sido obtener el mayor valor de este parámetro que permite llegar a la convergencia, en un número de iteraciones razonable, del proceso de verificación de la normalidad de las muestras con el "Test de Anderson-Darling". Se consigue así obtener el valor de la máxima dispersión de los resultados respecto del valor medio, con la intención de poder con ellos simular un proceso lo más "erróneo" posible, pero dentro de los límites admisibles por la normativa, y así poder proceder a aplicarlos sobre una estructura real para conocer qué efecto produce (Figura 62).

En concreto, en este estudio se analizaron los valores de las desviaciones típicas función del número de iteraciones del proceso de Anderson-Darling para el caso de la aplicación del método en fuerzas, resultando el siguiente cuadro:

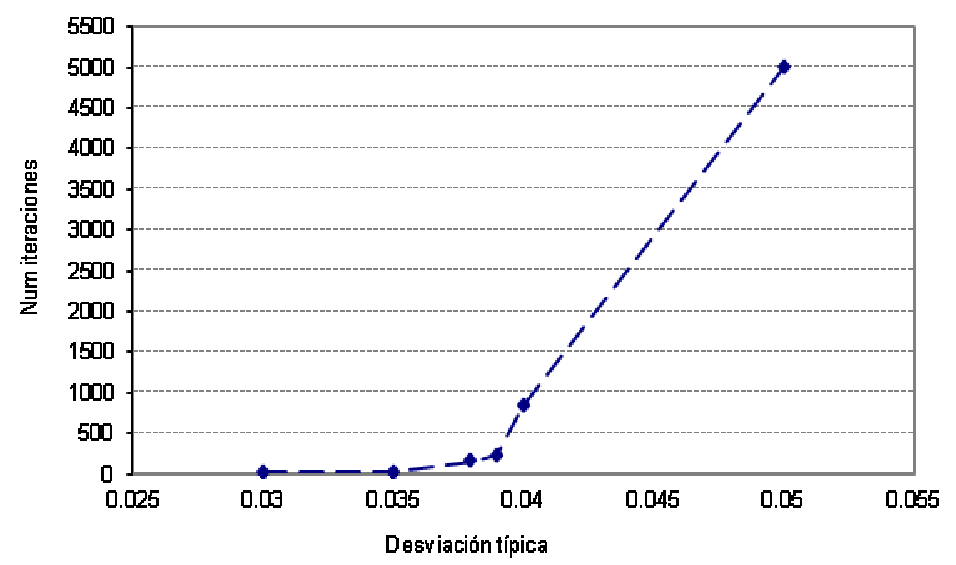

Figura 62. Relación entre el número de iteraciones necesarias para la convergencia del Test de Anderson-Darling y la desviación típica de las muestras.

A partir de estos valores se ha decidido adoptar una desviación típica para las muestras del $4 \%$ para el caso de instalación de los cables mediante fuerzas. Por otro lado, haciendo un análisis similar, para el caso de la puesta en obra de los cables mediante deformaciones, se ha adoptado un valor de la desviación típica del 0'008\%, 
cuyo orden de magnitud está en relación con el de las predeformaciones máximas admisibles según el SETRA [102] en longitudes que se fijaban en el orden del 0'01\%.

\subsubsection{Definición del tamaño de las muestras aleatorias.}

Se puede decir que, el estudio del comportamiento de estas estructuras bajo la actuación de errores aleatorios, se va a realizar utilizando un método de aproximación a la solución similar a los planteamientos que se realizan con el Método de Montecarlo para resolver numerosos problemas matemáticos, en los que la solución analítica no es posible conocerla a priori. Se utiliza entonces la generación de variables aleatorias para poder obtener soluciones particulares, el promedio de las cuales, cuando se han generado un número suficiente de resultados, se puede ajustar a la solución exacta del problema con bastante aproximación. Es por esta cuestión que es muy importante la definición del tamaño de las muestras a usar, motivo por el cual en este epígrafe se detalla el procedimiento utilizado para establecer dicho parámetro.

Hay que tener en cuenta que, a mayor número de elementos en la muestra, los resultados obtenidos se acercarán en mayor proporción al resultado exacto, mientras que por otra parte, ese incremento del número de elementos de la muestra implica un proceso de cálculo más laborioso y más complicado de hacer converger, al aplicar el test de Anderson-Darling.

Así este parámetro se ha de establecer fijando un número lo suficientemente grande de elementos por muestra como para que los resultados que se obtengan puedan ser relativamente aproximados, y lo suficientemente pequeño para minimizar el tiempo que implica el proceso de cálculo. Es decir, las muestras tendrán el menor número posible de elementos, de tal forma, que los resultados obtenidos se puedan ya calificar como cercanos a los que se obtendrían con un número muy elevado de ítems.

Con el objetivo de determinar este valor, se ha procedido a realizar un análisis generando muestras compuestas por un número distinto de elementos (en concreto se han generado muestras compuestas por un número de elementos de 20, 50, 100 y 200), de tal manera que a partir de estos resultados se pueda deducir en qué punto la aproximación a la solución correcta se considera adecuada.

A continuación se adjuntan los resultados obtenidos, utilizando el método de colocación de los cables mediante fuerzas, al aplicar sobre la estructura las diferentes muestras con el número de elementos que se ha indicado anteriormente. Hay que 
indicar además que, para cada número de elementos a calcular, se han generado 3 muestras, para poder acotar de mejor manera los resultados.

Así, se presentan a continuación resultados de los valores característicos superiores e inferiores de los axiles finales de los cables ${ }^{3}$, así como de los axiles característicos superiores de los máximos de montaje y los característicos inferiores de los mínimos de montaje, ambos para el caso del cable 10D (Figura 63).

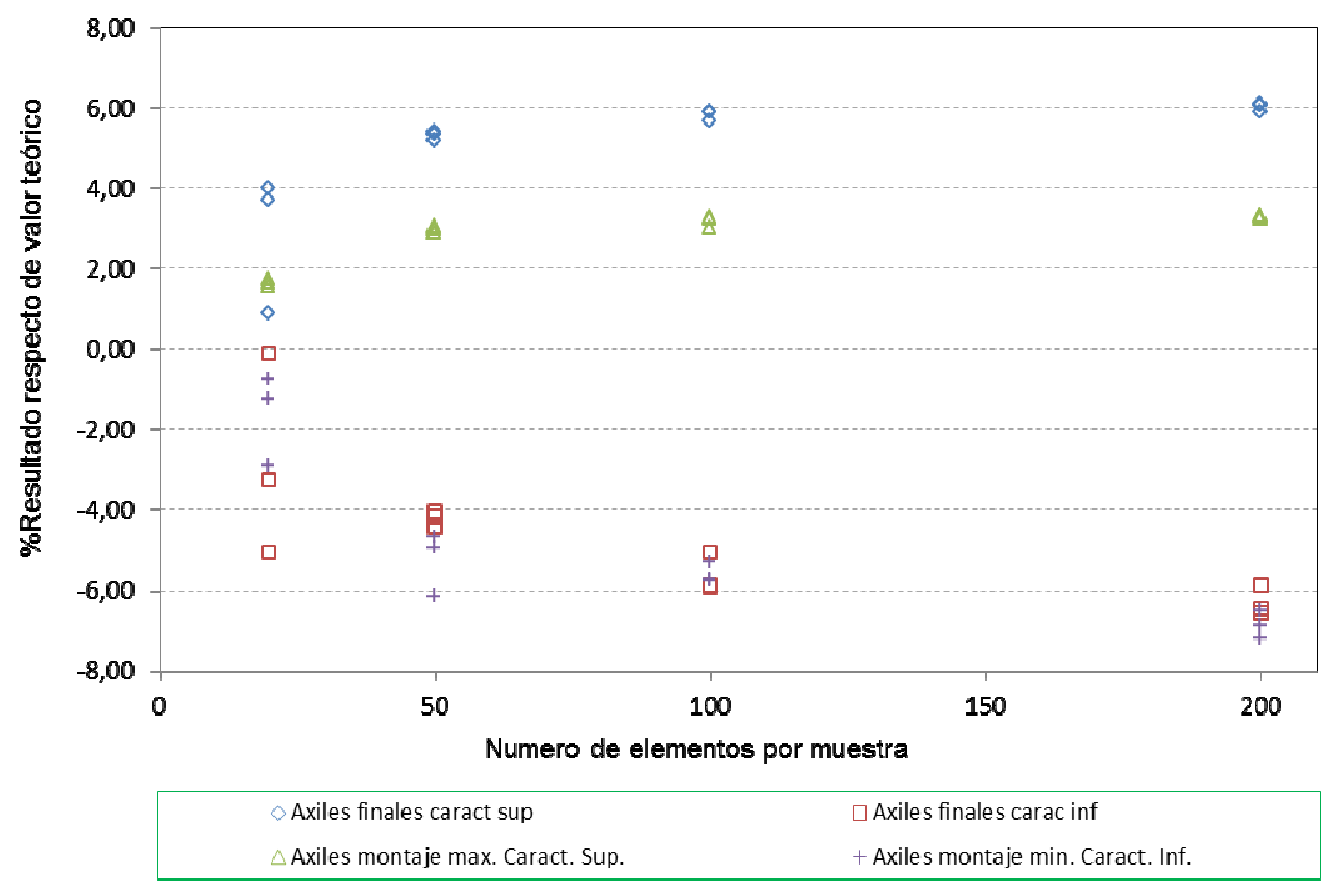

Figura 63. Desviación máxima de los resultados (en tanto por ciento) respecto de los valores teóricos conforme se aumenta el número de elementos de la muestra. Cable 10D.

Por otra parte se representan también, para todos los cables de la estructura, los valores de los axiles finales (Figura 64):

\footnotetext{
${ }^{3}$ En el apartado 4.6, correspondiente al análisis de los resultados obtenidos, se detallarán en concreto la definición de estos resultados.
} 
(a) Muestras con 25 elementos cada una.

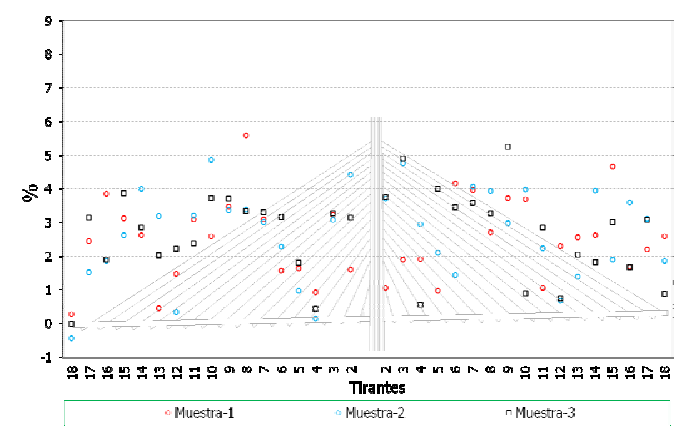

(c) Muestras con 100 elementos cada una.

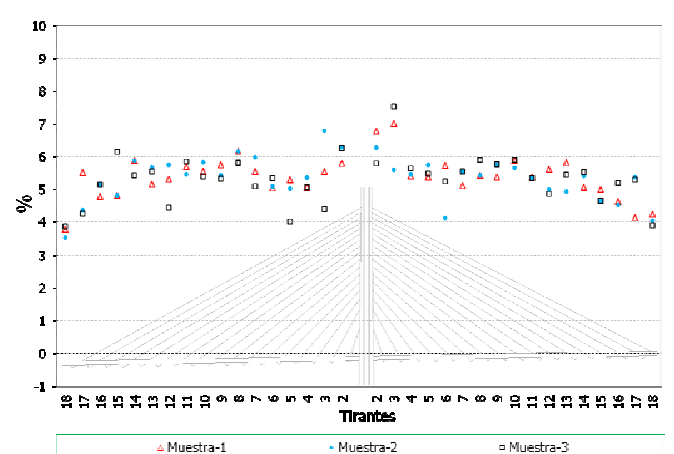

(b) Muestras con 50 elementos cada una.

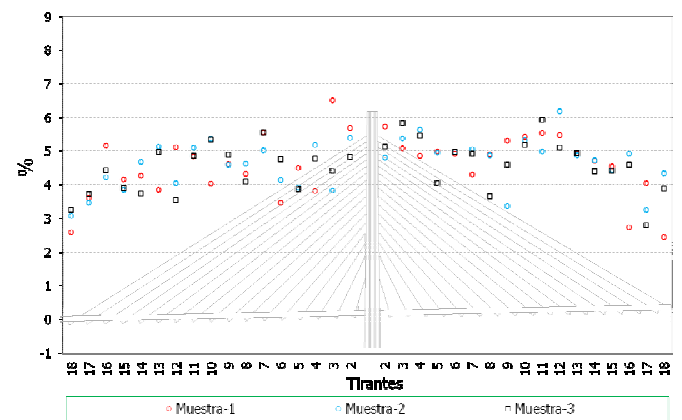

(d) Muestras con 200 elementos cada una.

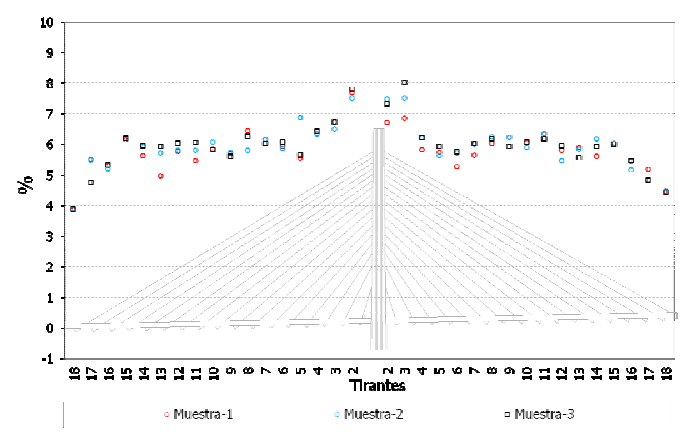

Figura 64. Desviación de los resultados obtenidos (en tanto por ciento) respecto de los valores teóricos conforme se aumenta el número de elementos de la muestra. Axiles finales en todos los tirantes.

A la vista de todos estos resultados parece claro que un número de 100 elementos por muestra aporta resultados lo suficientemente aproximados, no mereciendo la pena aumentar el número de elementos hasta valores del orden de 200 items o superiores, ya que el incremento de la precisión en los resultados es pequeño en proporción al incremento del volumen de cálculos a realizar. 


\subsubsection{Validación del método de generación de aleatorios.}

En los epígrafes anteriores, se ha obtenido un algoritmo que permite generar valores aleatorios según una distribución normal con media el valor teórico analizado, y a su vez se ha propuesto un método que permite garantizar que las muestras anteriores se conforman por valores que quedan dentro de los límites establecidos por la normativa.

Así el esquema de cálculo planteado sigue el siguiente diagrama de flujo:

Subrutina Cálculo Coeficientes de ponderación aleatorios de los tirantes (Por el método del límite central)

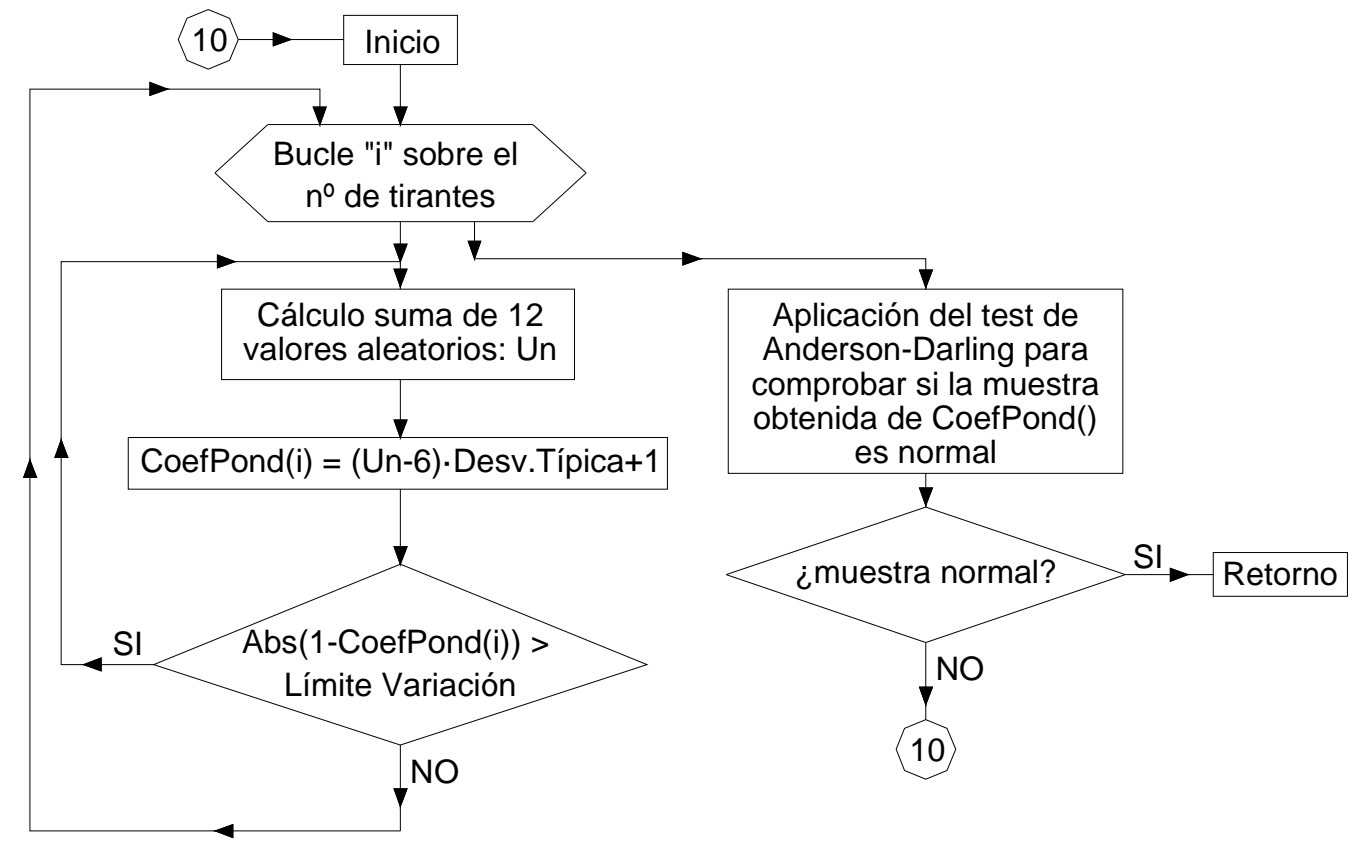

Para verificar el resultado del desarrollo teórico anterior, se presentan a continuación (Figura 65) los resultados de la aplicación de todo este procedimiento sobre el 1er elemento de la $1^{\text {a }}$ muestra. En concreto, se adjunta primeramente un gráfico donde se representan los valores de los axiles de montaje de todos los cables, donde se observa que, efectivamente, los valores aleatorios obtenidos en este caso quedan dentro del rango de error $( \pm 5 \%)$ que admite [2]. Como conclusión del resultado de este gráfico se observa que estos valores serían admisibles, y por lo tanto sería posible aplicarlos sobre la estructura real en estudio. 


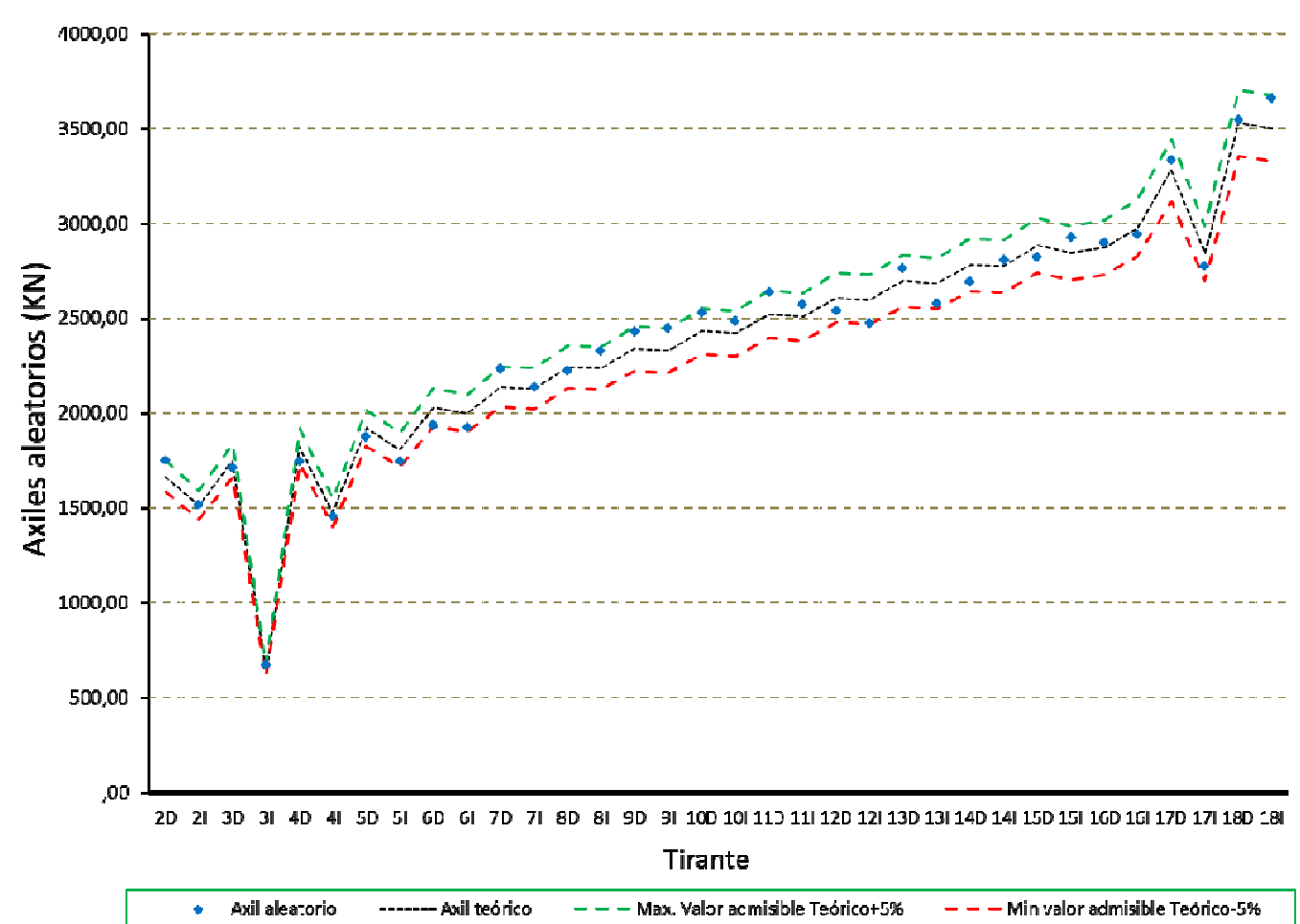

Figura 65. Gráfico de los axiles de montaje en todos los cables para el $1^{\text {er }}$ elemento de la $1^{a}$ muestra.

A continuación se presenta una tabla donde se incluyen todos los valores correspondientes al gráfico anterior, en el que se añade la cuantía de la variación respecto de los valores teóricos, en valor absoluto y en porcentaje, y donde se calculan también la media de las diferencias en valor absoluto y la desviación típica de los porcentajes de dichas diferencias. 


\begin{tabular}{|c|c|c|c|c|c|}
\hline \multicolumn{6}{|c|}{ Axiles de montaje del primer elemento de la 1 a muestra } \\
\hline \multicolumn{3}{|r|}{$(\mathrm{KN})$} & $(\mathrm{KN})$ & (KN) & \multirow[b]{2}{*}{ \%Variaciór } \\
\hline Barra & Tirante & Axil aleatorio & Axil teórico & Variación & \\
\hline 98 & $2 \mathrm{D}$ & $1.747,4$ & $1.668,3$ & $-79,2$ & $-4,7$ \\
\hline 99 & 21 & $1.511,9$ & $1.515,6$ & 3,7 & 0,2 \\
\hline 100 & $3 \mathrm{D}$ & $1.708,7$ & $1.748,8$ & 40,1 & 2,3 \\
\hline 101 & 31 & 665,4 & 643,0 & $-22,4$ & $-3,5$ \\
\hline 102 & $4 \mathrm{D}$ & $1.740,9$ & $1.825,3$ & 84,4 & 4,6 \\
\hline 103 & 41 & $1.450,1$ & $1.473,8$ & 23,7 & 1,6 \\
\hline 104 & $5 \mathrm{D}$ & $1.871,1$ & $1.921,0$ & 49,9 & 2,6 \\
\hline 105 & 51 & $1.738,0$ & $1.807,3$ & 69,4 & 3,8 \\
\hline 106 & $6 \mathrm{D}$ & $1.931,2$ & $2.029,1$ & 97,9 & 4,8 \\
\hline 107 & 61 & $1.921,2$ & $2.000,0$ & 78,8 & 3,9 \\
\hline 108 & 7D & $2.226,5$ & $2.138,1$ & $-88,4$ & $-4,1$ \\
\hline 109 & 71 & $2.130,1$ & $2.131,0$ & 0,9 & 0,0 \\
\hline 110 & $8 D$ & $2.218,2$ & $2.241,3$ & 23,1 & 1,0 \\
\hline 111 & 81 & $2.322,0$ & $2.235,7$ & $-86,2$ & $-3,9$ \\
\hline 112 & 9D & $2.426,9$ & 2.339,1 & $-87,8$ & $-3,8$ \\
\hline 113 & 91 & $2.440,3$ & $2.330,4$ & $-109,9$ & $-4,7$ \\
\hline 114 & $10 \mathrm{D}$ & $2.521,8$ & $2.431,1$ & $-90,6$ & $-3,7$ \\
\hline 115 & 101 & $2.480,0$ & $2.419,8$ & $-60,3$ & $-2,5$ \\
\hline 116 & 11D & $2.633,2$ & $2.521,2$ & $-111,9$ & $-4,4$ \\
\hline 117 & 111 & $2.564,1$ & $2.508,6$ & $-55,5$ & $-2,2$ \\
\hline 118 & $12 \mathrm{D}$ & $2.534,3$ & $2.609,3$ & 75,1 & 2,9 \\
\hline 119 & 121 & $2.469,5$ & $2.597,5$ & 128,0 & 4,9 \\
\hline 120 & 13D & $2.754,8$ & $2.698,5$ & $-56,2$ & $-2,1$ \\
\hline 121 & 131 & $2.573,9$ & $2.685,1$ & 111,2 & 4,1 \\
\hline 122 & $14 \mathrm{D}$ & $2.688,4$ & $2.778,9$ & 90,5 & 3,3 \\
\hline 123 & 141 & $2.798,6$ & $2.775,9$ & $-22,7$ & $-0,8$ \\
\hline 124 & 15D & $2.817,9$ & $2.884,0$ & 66,2 & 2,3 \\
\hline 125 & 151 & $2.918,7$ & $2.845,0$ & $-73,7$ & $-2,6$ \\
\hline 126 & $16 \mathrm{D}$ & $2.891,1$ & $2.875,5$ & $-15,6$ & $-0,5$ \\
\hline 127 & 161 & $2.934,1$ & $2.979,0$ & 45,0 & 1,5 \\
\hline 128 & $17 \mathrm{D}$ & $3.328,8$ & $3.279,6$ & $-49,2$ & $-1,5$ \\
\hline 129 & 171 & $2.765,5$ & $2.841,5$ & 76,0 & 2,7 \\
\hline 130 & 18D & $3.541,8$ & 3.529,9 & $-11,9$ & $-0,3$ \\
\hline 131 & 181 & $3.656,6$ & $3.502,1$ & $-154,5$ & $-4,4$ \\
\hline & & lia V.Abs \%de & ción en las & uestras $=$ & 2,8 \\
\hline & & Media \%de & iación en las & nuestras = & $-0,09$ \\
\hline
\end{tabular}

Tabla 2. Valores de los axiles de montaje de todos los cables correspondientes al primer ítem de la primera muestra calculada.

Con la intención de confirmar la anterior conclusión, se pueden también representar los valores de los diferentes axiles de montaje a aplicar sobre un cable concreto (caso del cable 5I), en este caso para cada uno de los 100 elementos calculados en la $1^{a}$ muestra. Resulta así el siguiente gráfico (Figura 66): 


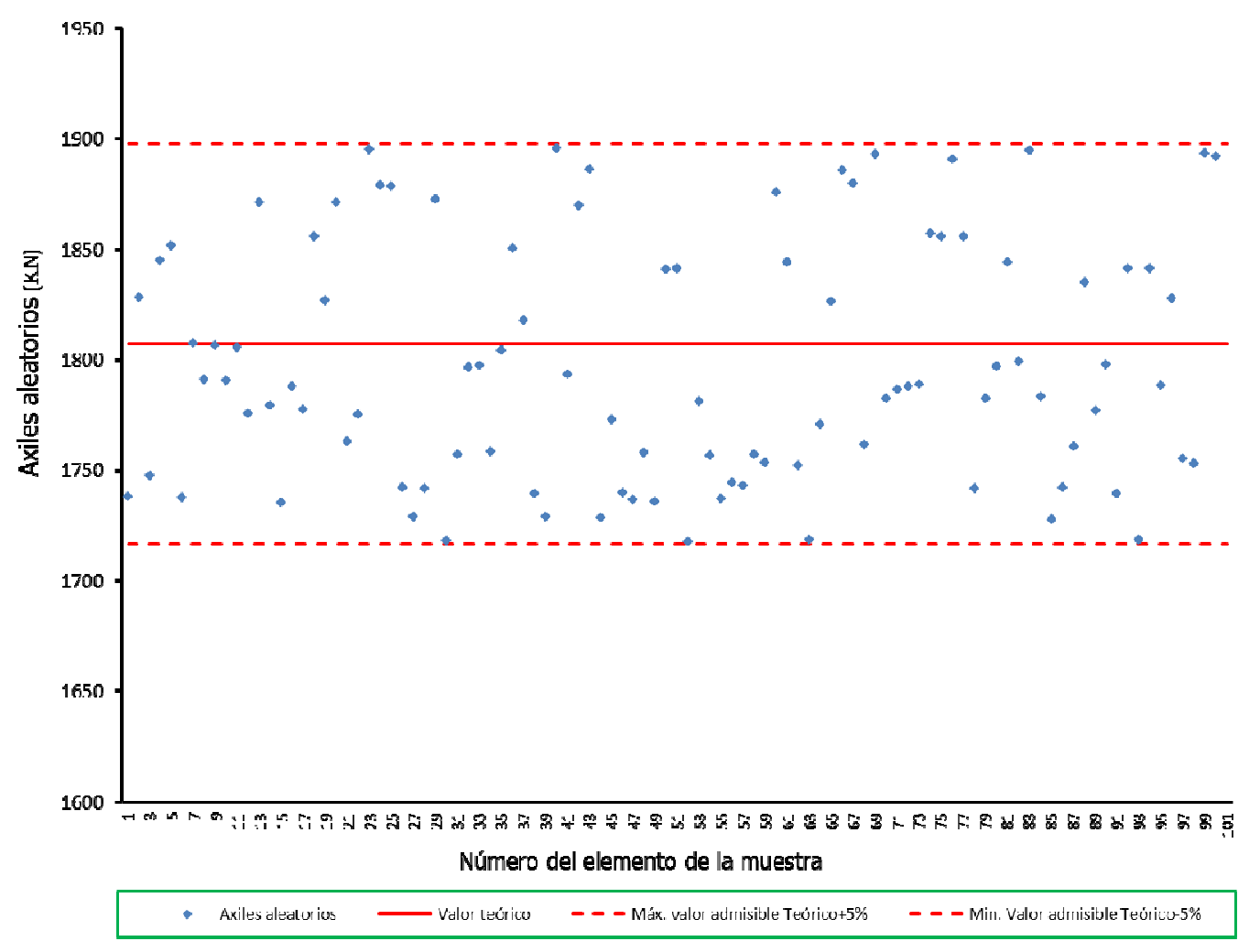

Figura 66. Gráfico de los axiles de montaje en el cable 51 , para el $1^{\text {er }}$ elemento de la 19 a muestra.

De los datos anteriores se extrae la conclusión que las series de valores obtenidas con el proceso planteado, que siguen distribuciones aproximadamente normales, podrían producirse en la realidad sobre la estructura sin que se considerase necesaria su eliminación, ya que están siempre contenidas dentro de los límites admisibles establecidos por la normativa.

\subsection{COMPARATIVA DE LOS MÉTODOS DE COLOCACIÓN DE LOS CABLES.}

Dado que en adelante se van a comparar los 2 métodos de colocación de cables antes señalados en 2.3.2, es necesario conocer si las limitaciones previas marcadas fijadas sobre ambos tienen una repercusión similar, de tal forma que los resultados obtenidos con ellos sean homogéneos y por lo tanto comparables.

Para evaluar si la cuantía de los errores máximos admisibles a introducir en cada método de cálculo tiene una repercusión similar en los procesos que se desarrollarán a continuación, se ha procedido a realizar el siguiente análisis comparativo entre ambos métodos.

En concreto el análisis realizado ha consistido en, a partir de las predeformaciones teóricas necesarias para alcanzar en la situación final el estado de viga continua, calcular la longitud 
en vacío o neutra de cada cable Lo, y obtener a continuación la cuantía del error máximo que permite la normativa sobre esta medida. Esta medida del error en longitud se puede transformar en un error en fuerzas al conocer el área de cada tirante y así obtener el porcentaje que, este error máximo en la medida de los alargamientos del cable, significa en porcentaje de los axiles teóricos de montaje de los cables.

Es decir:

[Ec4. 13.] \%Error max. respecto de $\mathrm{F}_{0}=\mathrm{F}_{0, \text { Error }} / F_{0} \cdot 100$

siendo:

$\mathrm{F}_{0, \text { Error }} \rightarrow$ Error equivalente en fuerzas al máximo admisible en deformaciones.

resulta:

$$
\text { [Ec4. 14.] } \quad \mathrm{F}_{0, \mathrm{Error}}=\mathrm{E} \cdot \mathrm{A} \cdot \Delta_{\mathrm{L}, \mathrm{error}} / \mathrm{L}
$$

con:

Fo $\rightarrow$ Axil teórico de montaje de un cable.

$E \rightarrow$ Modulo de elasticidad del tirante: $\quad E_{s}=200.000 \mathrm{~N} / \mathrm{mm}^{2}$

$A \rightarrow$ Área de los tirantes.

$L \rightarrow$ Longitud teórica del tirante o distancia entre los puntos de anclaje del cable.

$\Delta_{\llcorner} \rightarrow$ Predeformación del tirante para alcanzar una configuración similar a la de la viga continua en la situación final, y obtenida como se ha señalado en los epígrafes anteriores.

$\Delta$ L.error $\rightarrow$ Error en la medida de las alargamientos de los cables en el momento de su instalación. Se adopta el criterio de las recomendaciones del SETRA francés que implican un porcentaje del error del 0'01\% de Lo.

En el siguiente cuadro se detallan los resultados obtenidos relativos a la implicación de los errores en deformaciones respecto de los errores máximos en fuerzas: 


\begin{tabular}{|c|c|c|c|c|c|c|c|c|c|c|c|c|c|}
\hline \multirow{4}{*}{$\begin{array}{c}\text { Es }= \\
\text { Tirante }\end{array}$} & \multirow{4}{*}{$\begin{array}{l}1,95 E+08 \\
\text { Barra }\end{array}$} & \multirow{4}{*}{$\begin{array}{c}\mathrm{KN} / \mathrm{m}^{2} \\
\text { Area } \\
\mathrm{cm}^{2}\end{array}$} & \multirow{4}{*}{$\begin{array}{l}\text { Axil fase } \\
\text { final }\end{array}$} & \multirow{2}{*}{\multicolumn{3}{|c|}{ Axil fases montaje }} & \multicolumn{3}{|c|}{ nax sobre long según norma francesa = } & \multirow{4}{*}{$\begin{array}{c}0,01 \\
\text { Pred.montaje } \\
\Delta \mathrm{l}_{\mathrm{m}, \mathrm{i}} \\
\mathrm{mm}\end{array}$} & \multirow{4}{*}{$\begin{array}{l}\% \cdot L_{0} \\
\text { Error mx pred. } \\
\quad m m\end{array}$} & \multirow{4}{*}{$\begin{array}{l}\text { Error max } \\
\text { equival ente } \\
\text { en F's } \\
\text { KN }\end{array}$} & \multirow{4}{*}{$\begin{array}{l}\text { \%Errormax } \\
\text { respecto } \mathrm{F}_{\mathrm{g}}\end{array}$} \\
\hline & & & & & & & Long, final & Predefor. & Long.vació & & & & \\
\hline & & & & Inicial & $\operatorname{Max}$ & Min & $\mathbf{L}_{\mathbf{i}}$ & $\Delta \mathrm{L}_{-}$ & $L_{0, j}$ & & & & \\
\hline & & & & \multicolumn{2}{|c|}{$\mathrm{KN}$} & & $\mathrm{m}$ & $\mathrm{mm}$ & $\mathrm{m}$ & & & & \\
\hline $18-D$ & 130 & 86,1 & 3875,4 & 3529,9 & 3875,4 & 3529,9 & 98,899 & 216,7 & 98,682 & $-216,7$ & 9,4 & 167,9 & 4,76 \\
\hline 17-D & 128 & 86,1 & 3875,9 & 3279,6 & 5188,9 & 3279,6 & 88,847 & 205,0 & 88,642 & $-205,0$ & 8,9 & 168,0 & 5,12 \\
\hline $16-D$ & 126 & 72,2 & 3248,1 & 2875,5 & 4171,4 & 2782,3 & 83,808 & 193,4 & 83,614 & $-193,4$ & 8,4 & 140,8 & 4,89 \\
\hline $15-D$ & 124 & 73,4 & 3300,7 & 2884,0 & 4209,3 & 2863,4 & 78,760 & 181,8 & 78,579 & $-181,8$ & 7,9 & 143,0 & 4,96 \\
\hline 14-D & 122 & 70,5 & 3171,5 & 2778,9 & 4082,5 & 2778,9 & 73,715 & 170,1 & 73,545 & $-170,1$ & 7,4 & 137,4 & 4,95 \\
\hline 13-D & 120 & 68,5 & 3084,9 & 2698,5 & 3992,8 & 2698,5 & 68,670 & 158,6 & 68,511 & $-158,5$ & 6,9 & 133,5 & 4,95 \\
\hline $12-D$ & 118 & 66,3 & 2984,7 & 2609,3 & 3890,5 & 2609,3 & 63,607 & 146,8 & 63,460 & $-146,8$ & 6,3 & 129,3 & 4,96 \\
\hline 11-D & 116 & 64,1 & 2886,5 & 2521,2 & 3788,9 & 2521,2 & 58,516 & 135,0 & 58,381 & $-135,0$ & 5,8 & 125,1 & 4,96 \\
\hline $10-D$ & 114 & 61,9 & 2785,1 & 2431,1 & 3683,4 & 2414,4 & 53,400 & 123,2 & 53,277 & $-123,2$ & 5,3 & 120,7 & 4,96 \\
\hline $9-D$ & 112 & 59,6 & 2680,2 & 2339,1 & 3573,8 & 2289,4 & 48,260 & 111,4 & 48,148 & $-111,4$ & 4,8 & 116,1 & 4,97 \\
\hline $8-D$ & 110 & 57,0 & 2566,3 & 2241,3 & 3455,8 & 2185,2 & 43,128 & 99,5 & 43,029 & $-99,5$ & 4,3 & 111,2 & 4,96 \\
\hline 7-D & 108 & 54,3 & 2441,3 & 2138,1 & 3332,2 & 2090,6 & 38,016 & 87,7 & 37,928 & $-87,7$ & 3,8 & 105,8 & 4,95 \\
\hline 6-D & 106 & 51,1 & 2299,7 & 2029,1 & 3205,0 & 1972,9 & 32,973 & 76,1 & 32,897 & $-76,1$ & 3,3 & 99,6 & 4,91 \\
\hline 5-D & 104 & 47,6 & 2139,6 & 1921,0 & 3083,2 & 1843,3 & 28,038 & 64,7 & 27,973 & $-64,7$ & 2,8 & 92,7 & 4,83 \\
\hline 4-D & 102 & 43,6 & 1960,9 & 1825,3 & 2968,2 & 1711,6 & 23,277 & 53,7 & 23,224 & $-53,7$ & 2,3 & 85,0 & 4,66 \\
\hline 3-D & 100 & 39,4 & 1774,6 & 1748,8 & 2830,3 & 1597,8 & 18,699 & 43,1 & 18,656 & $-43,1$ & 1,9 & 76,9 & 4,40 \\
\hline $2-D$ & 98 & 35,7 & 1608,2 & 1668,3 & 2839,9 & 1534,0 & 13,835 & 31,9 & 13,803 & $-31,9$ & 1,4 & 69,7 & 4,18 \\
\hline $2-1$ & 99 & 35,7 & 1608,2 & 1515,6 & 1862,0 & 793,7 & 13,835 & 31,9 & 13,803 & $-31,9$ & 1,4 & 69,7 & 4,60 \\
\hline $3-1$ & 101 & 39,4 & 1774,6 & 643,0 & 2346,2 & 643,0 & 18,699 & 43,1 & 18,656 & $-43,1$ & 1,9 & 76,9 & 11,96 \\
\hline $4-1$ & 103 & 43,6 & 1960,9 & 1473,8 & 2769,2 & 1473,8 & 23,277 & 53,7 & 23,224 & $-53,7$ & 2,3 & 85,0 & 5,77 \\
\hline 5-I & 105 & 47,6 & 2139,6 & 1807,3 & 3024,4 & 1807,3 & 28,038 & 64,7 & 27,973 & $-64,7$ & 2,8 & 92,7 & 5,13 \\
\hline $6-1$ & 107 & 51,1 & 2299,7 & 2000,0 & 3192,2 & 1979,1 & 32,973 & 76,1 & 32,897 & $-76,1$ & 3,3 & 99,6 & 4,98 \\
\hline 7-1 & 109 & 54,3 & 2441,3 & 2131,0 & 3327,8 & 2084,7 & 38,016 & 87,7 & 37,928 & $-87,7$ & 3,8 & 105,8 & 4,96 \\
\hline $8-1$ & 111 & 57,0 & 2566,3 & 2235,7 & 3449,2 & 2184,4 & 43,128 & 99,5 & 43,029 & $-99,5$ & 4,3 & 111,2 & 4,97 \\
\hline $9-1$ & 113 & 59,6 & 2680,2 & 2330,4 & 3563,8 & 2296,5 & 48,260 & 111,4 & 48,148 & $-111,4$ & 4,8 & 116,1 & 4,98 \\
\hline 104 & 115 & 61,9 & 2785,2 & 2419,8 & 3671,5 & 2419,8 & 53,400 & 123,2 & 53,277 & $-123,2$ & 5,3 & 120,7 & 4,99 \\
\hline 114 & 117 & 64,1 & 2886,3 & 2508,6 & 3776,4 & 2508,6 & 58,516 & 135,0 & 58,381 & $-135,0$ & 5,8 & 125,1 & 4,99 \\
\hline 124 & 119 & 66,4 & 2985,6 & 2597,5 & 3879,4 & 2597,5 & 63,607 & 146,8 & 63,460 & $-146,8$ & 6,3 & 129,4 & 4,98 \\
\hline $13 \mathrm{H}$ & 121 & 68,5 & 3081,7 & 2685,1 & 3979,1 & 2685,1 & 68,670 & 158,5 & 68,511 & $-158,5$ & 6,9 & 133,5 & 4,97 \\
\hline 144 & 123 & 70,8 & 3183,9 & 2775,9 & 4083,4 & 2775,9 & 73,715 & 170,1 & 73,545 & $-170,1$ & 7,4 & 138,0 & 4,97 \\
\hline $15-$ & 125 & 72,3 & 3253,1 & 2845,0 & 4157,3 & 2783,8 & 78,760 & 181,8 & 78,579 & $-181,8$ & 7,9 & 141,0 & 4,95 \\
\hline $16-1$ & 127 & 76,2 & 3430,8 & 2979,0 & 4325,7 & 2867,9 & 83,808 & 193,4 & 83,614 & $-193,4$ & 8,4 & 148,7 & 4,99 \\
\hline 174 & 129 & 70,6 & 3176,4 & 2841,5 & 4119,1 & 2586,5 & 88,847 & 205,0 & 88,642 & $-205,0$ & 8,9 & 137,7 & 4,84 \\
\hline $18-1$ & 131 & 98,1 & 4414,0 & 3502,1 & 4414,0 & 3501,4 & 98,899 & 216,7 & 98,682 & $-216,7$ & 9,4 & 191,3 & 5,46 \\
\hline
\end{tabular}

Tabla 3. Axiles teóricos finales y de montaje de los tirantes y obtención de los errores equivalentes en fuerzas, correspondientes a un proceso de instalación de los cables mediante el método de las deformaciones con el error máximo admitido por la Normativa del SETRA[102]. 
Los resultados obtenidos relativos a la implicación de los errores incluidos en la tabla anterior se representan gráficamente a continuación (Figura 67):

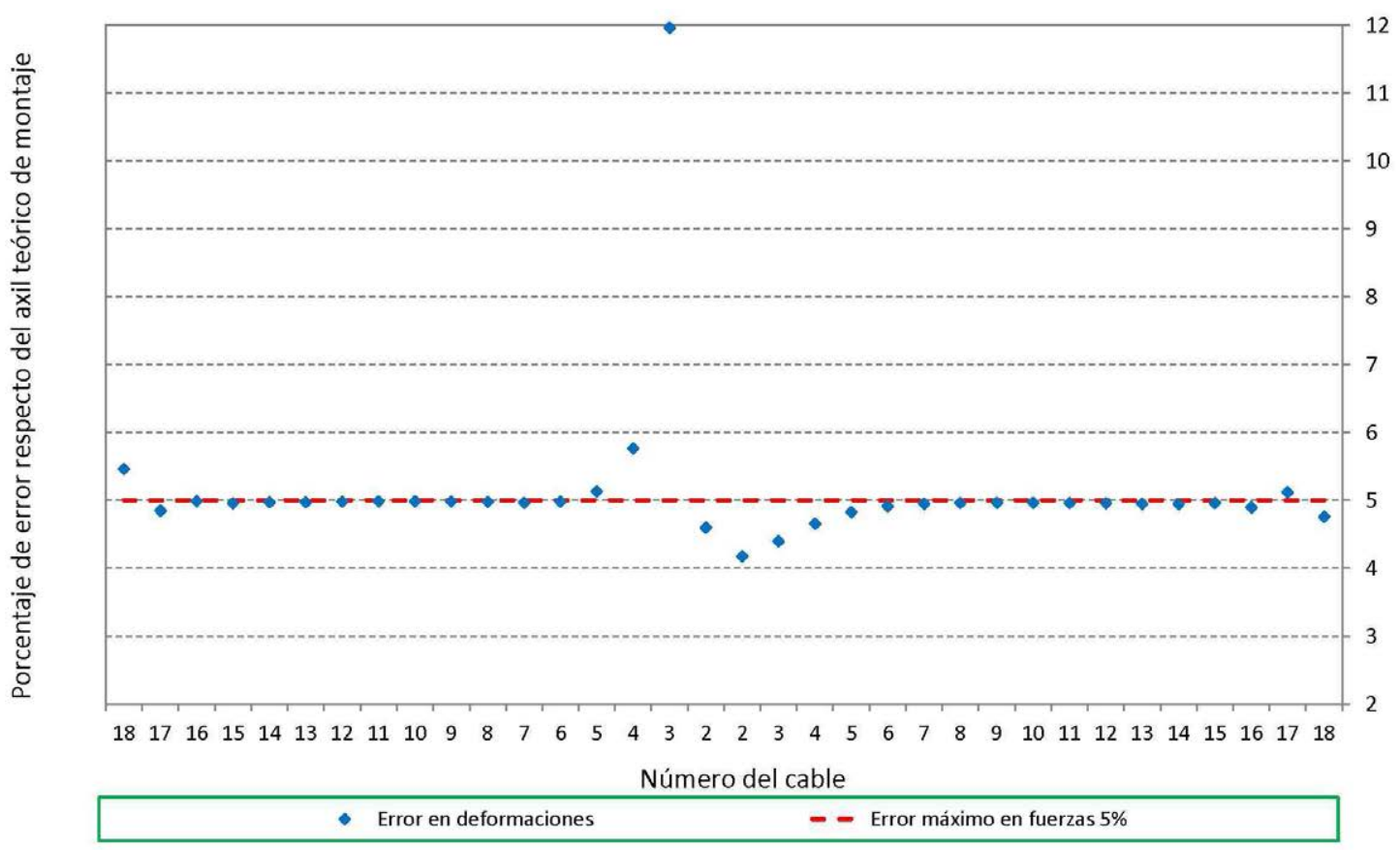

Figura 67. Porcentaje de error, respecto del axil teórico de montaje $\left(F_{0}\right)$, del axil de montaje equivalente al aplicar el error máximo admisible en la medida de los alargamientos $\left(F_{0, e r r}\right)$.

De los resultados anteriores se puede concluir que:

$\checkmark \quad$ La implicación de los errores en deformaciones es similar a la planteada en el caso de la introducción de los errores en fuerzas. Resultaran errores máximos del orden del 5\%.

$\checkmark \quad$ En general los valores obtenidos en cada cable son similares salvo en el entorno del cable 3-I. Esta diferencias se debe a que el análisis realizado lo es en porcentaje del axil teórico de montaje de los cables, teniendo este cable, debido al proceso constructivo proyectado, un axil muy pequeño, lo que a su vez conlleva que los errores planteados en deformaciones signifiquen un porcentaje muy alto respecto de las fuerzas iniciales de tesado teóricas.

A la vista de estas conclusiones quedaría confirmado que los porcentajes de error máximo indicados por las normativas respecto de los axiles teóricos de montaje de los cables y respecto de las deformaciones teóricas a introducir en el momento de su colocación tienen una cuantía similar, es decir que los efectos esperables debidos a estas limitaciones tienen que ser muy parecidos, salvo en algún caso particular, como el ya señalado, que habría que estudiar en concreto. 


\subsection{RESULTADOS OBTENIDOS.}

Se procede en este epígrafe a desarrollar los resultados correspondientes a la aplicación de los errores aleatorios de montaje, bien por el método de las fuerzas o bien por el método de las deformaciones. En el primer caso, como ya se comentó, se introducirán errores en la fuerza de tesado, mientras que en el segundo caso se introducirán tanto errores en la medida de las deformaciones de los cables como en la posición de los anclajes. Así los análisis realizados han sido:

- Error en la medida de las fuerzas de tesado.

- Error en la medida de las deformaciones de tesado.

- Error en la posición de los anclajes.

En resumen hay que señalar que los resultados obtenidos lo han sido a partir de la generación de 3 muestras de 100 series de valores aleatorios de los axiles o predeformaciones de montaje de los tirantes cada una. Aclarar que se ha decidido generar 3 muestras en cada caso estudiado, dado que de cada una de ellas se obtendrá un valor representativo de los 100 resultados obtenidos para poder facilitar su interpretación, y así existirá una forma de comparar dichos valores representativos entre sí. De esta manera, finalmente ha resultado que se han realizado 900 cálculos individuales de la estructura para cada situación estudiada.

\subsubsection{Definición de valor característico y de los esfuerzos e hipótesis a calcular.}

Por el motivo anteriormente señalado, se ha definido un valor representativo de cada muestra de parámetros obtenidos como el valor característico, el cual corresponde a aquel valor que o bien es superado por el $95 \%$ de los elementos de la muestra, definiéndose este valor como característico inferior, o bien supera al $95 \%$ de los elementos de la muestra, definiéndose este a su vez como característico superior.

A continuación pasan a presentarse los resultados que se han considerado más representativos, como son los axiles en los tirantes, y los flectores en el tablero y pilono. Para cada uno de ellos se han analizado las siguientes situaciones:

a) Situación final. Correspondiente al final del proceso de construcción y aplicación de la carga muerta sobre el tablero, la cual se deberá comparar con la situación teórica objetivo definida en nuestro caso (viga continua).

b) Situación de construcción. Correspondiente a la envolvente de cada una de las diferentes fases de construcción del tablero. 
c) Situación de servicio. Correspondiente la envolvente de la aplicación de las sobrecargas de tráfico sobre la estructura terminada.

\subsubsection{Errores en fuerzas de tesado.}

Se adjuntan a continuación resultados de la aplicación, mediante un proceso de cálculo de montaje de la estructura, de los errores aleatorios sobre las fuerzas teóricas de montaje de los cables, que quedaron representadas en la Figura 58.

Así el cálculo de los procesos constructivos "erróneos" parte de las series de variables aleatorias acotadas generadas con el algoritmo anteriormente indicado en 4.4, y de su aplicación sobre los valores teóricos de los axiles de montaje, de tal manera que:

$$
\text { [Ec4. 15.] } \quad \mathrm{F}_{\text {err, } \mathrm{i}}^{\mathrm{N}}=\mathrm{F}_{\text {teor, }} \cdot \mathrm{VA}^{\mathrm{N}}{ }_{\mathrm{i}}
$$

siendo: $\quad \mathrm{FN}_{\mathrm{N} \text { er, }} \rightarrow$ valor del axil erróneo $\mathrm{N}$-ésimo del cable i.

Fteor, $\rightarrow$ valor del axil teórico del cable i.

$\mathrm{VAN}_{\mathrm{i}} \rightarrow$ coeficiente aleatorio de ponderación $\mathrm{N}$-ésimo del cable i.

El algoritmo utilizado para la generación de estos errores aleatorios se incluye en el anexo A.3.

Se han generado para ello 3 muestras de 100 elementos correspondientes a axiles de montaje erróneos de cada cable, que se aplicarán sobre la estructura al simular su proceso constructivo.

Los resultados representados lo son de tirantes (Figura 68 a Figura 73), tablero (Figura 74 a Figura 76) y pilono (Figura 77 a Figura 78). 
4.6.2.1 Axiles en tirantes.

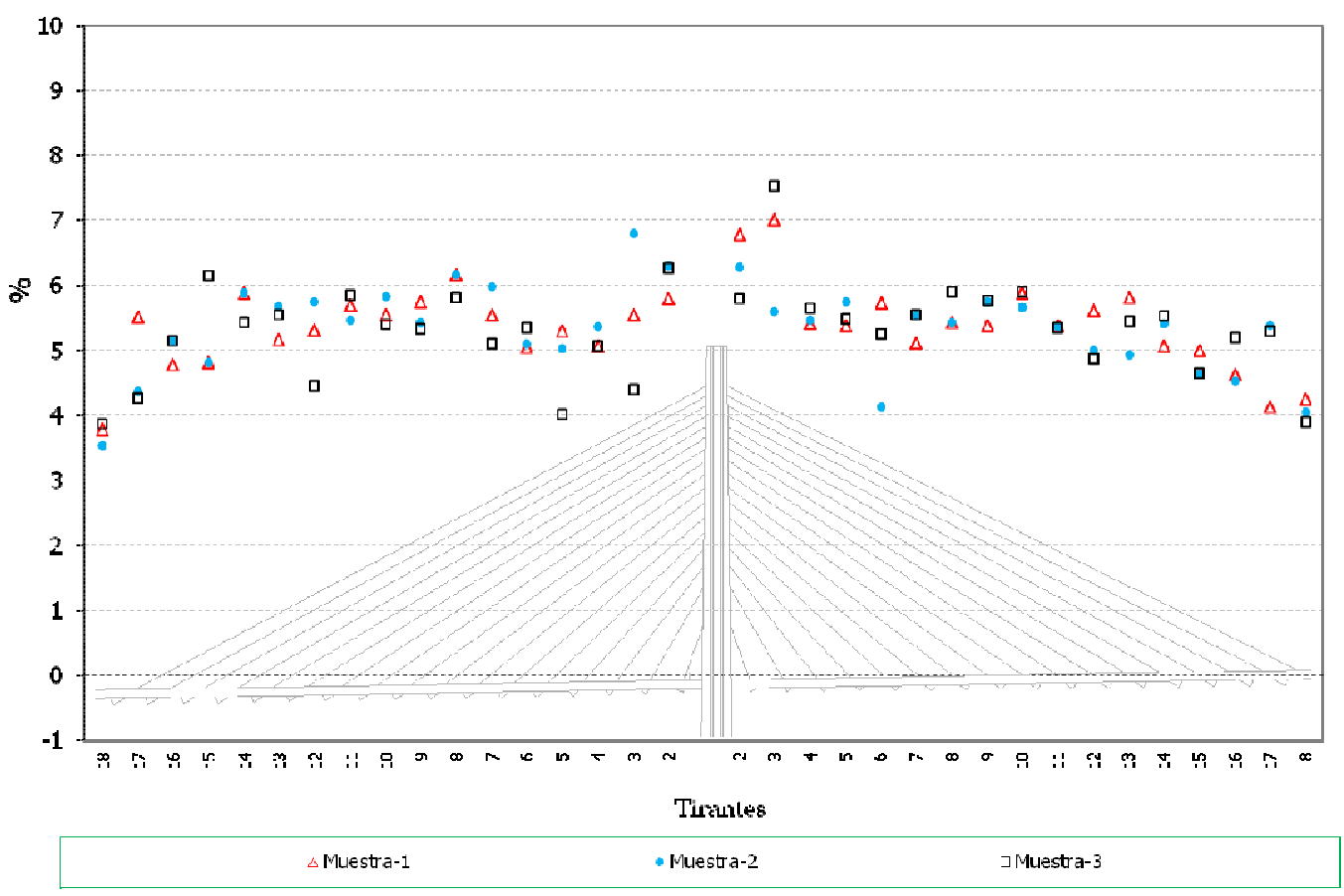

Figura 68. Axiles finales en los tirantes. Diferencia en $\%$ de los valores caracteristicos superiores respecto de los teóricos.

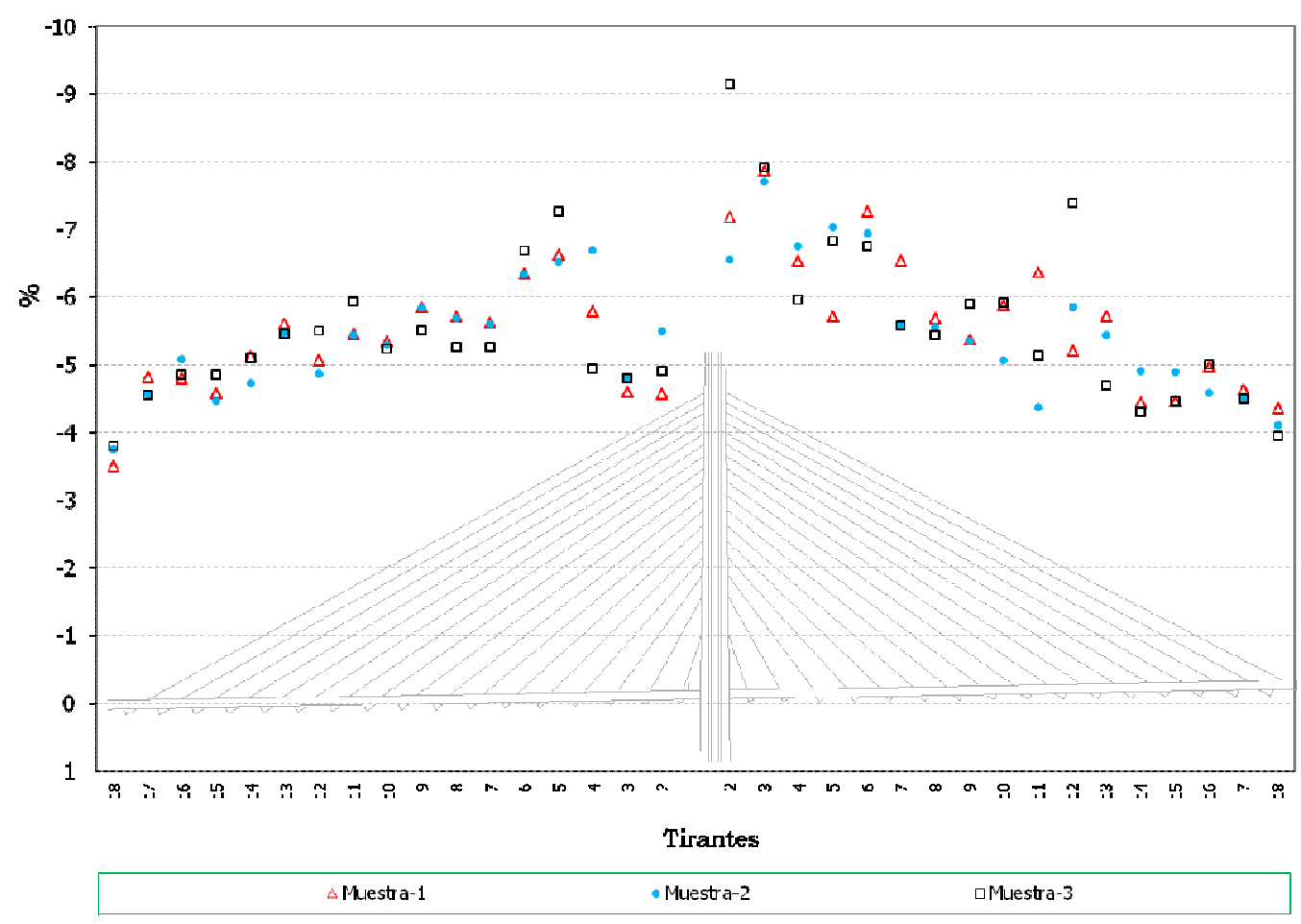

Figura 69. Axiles finales en los tirantes. Diferencia en \% de los valores característicos inferiores respecto de los teóricos. 


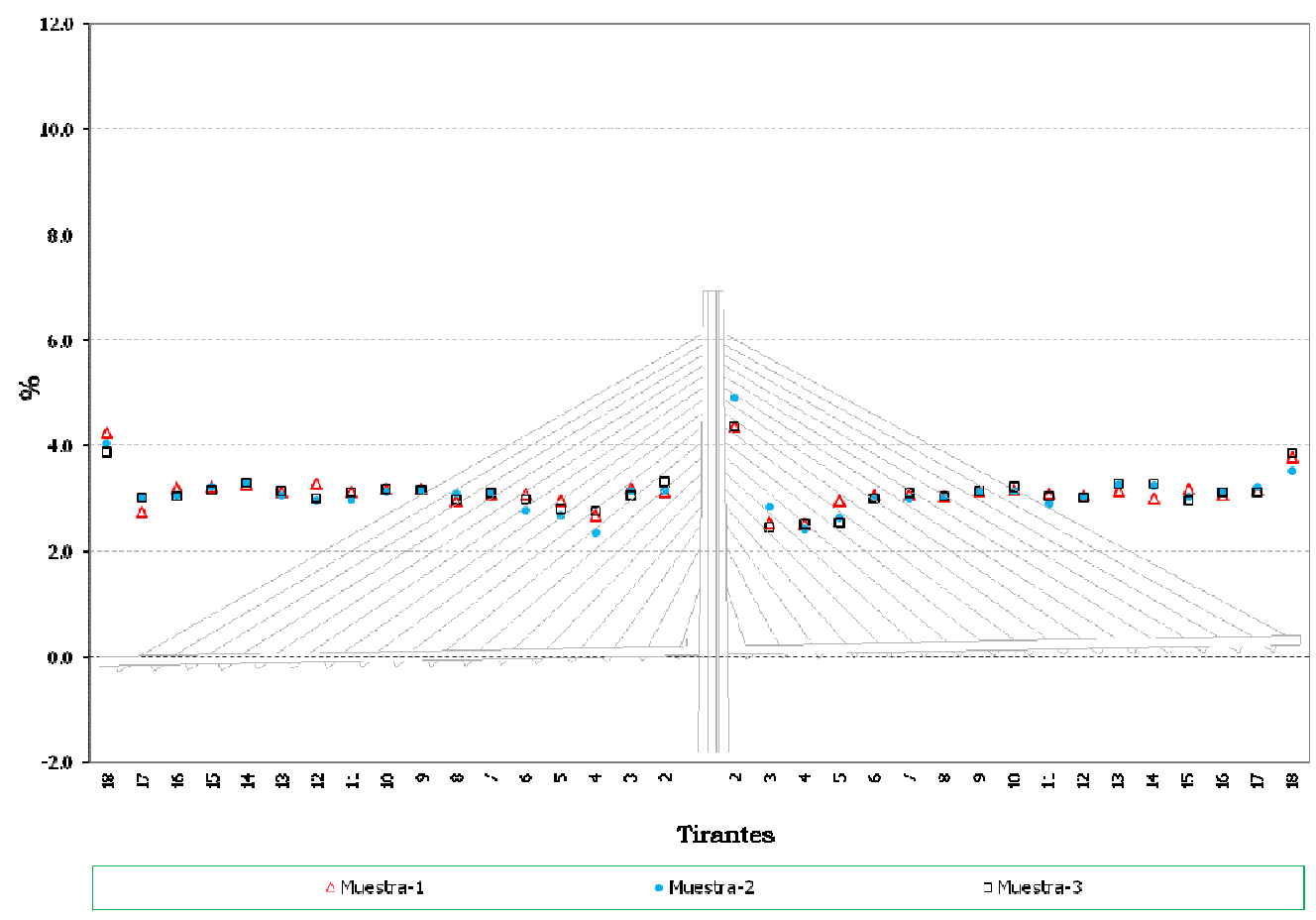

Figura 70. Axiles en los tirantes máximos de construcción. Diferencia en \% de los valores característicos superiores respecto de los teóricos.

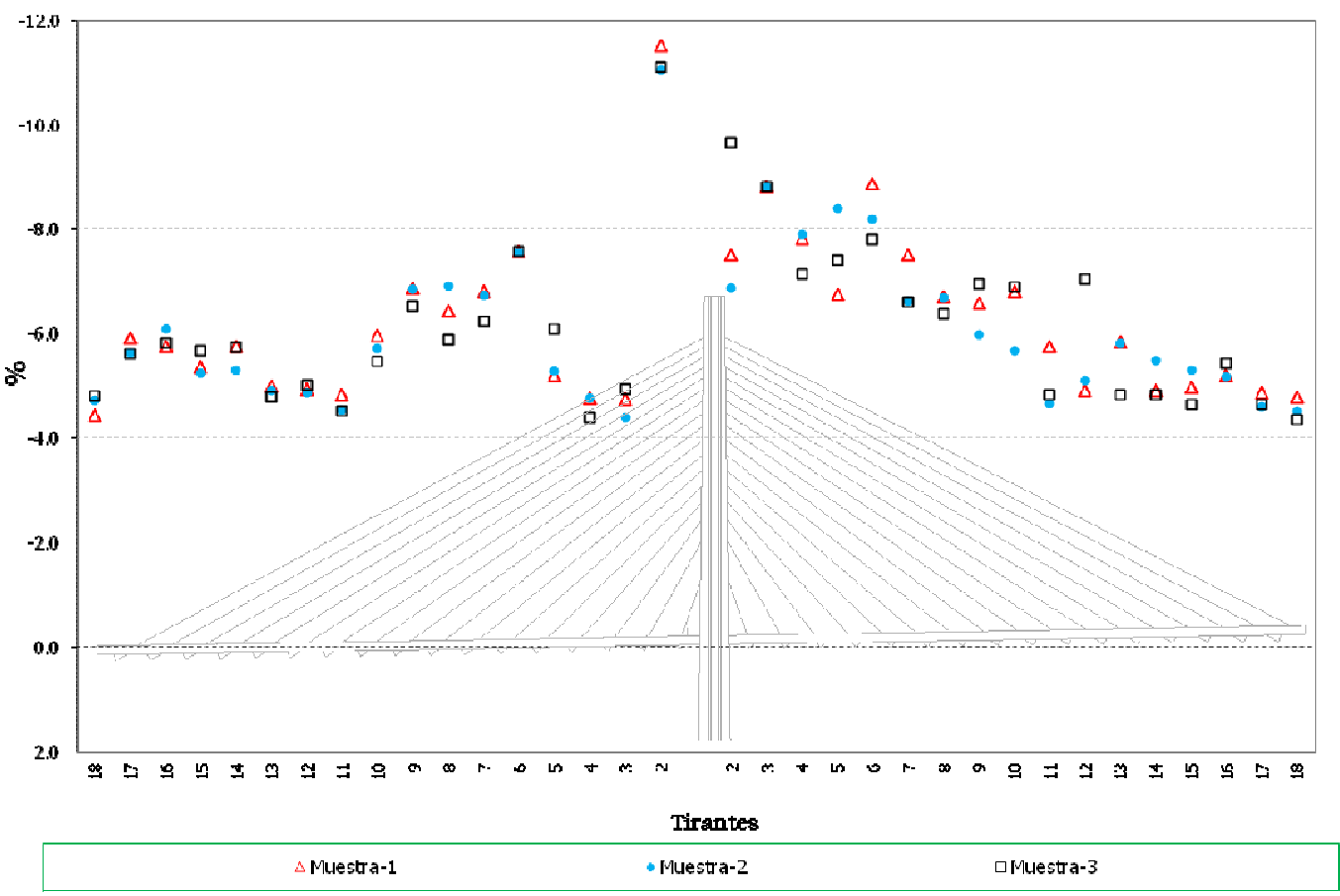

Figura 71. Axiles en los tirantes mínimos de construcción. Diferencia en \% de los valores característicos inferiores respecto de los teóricos. 


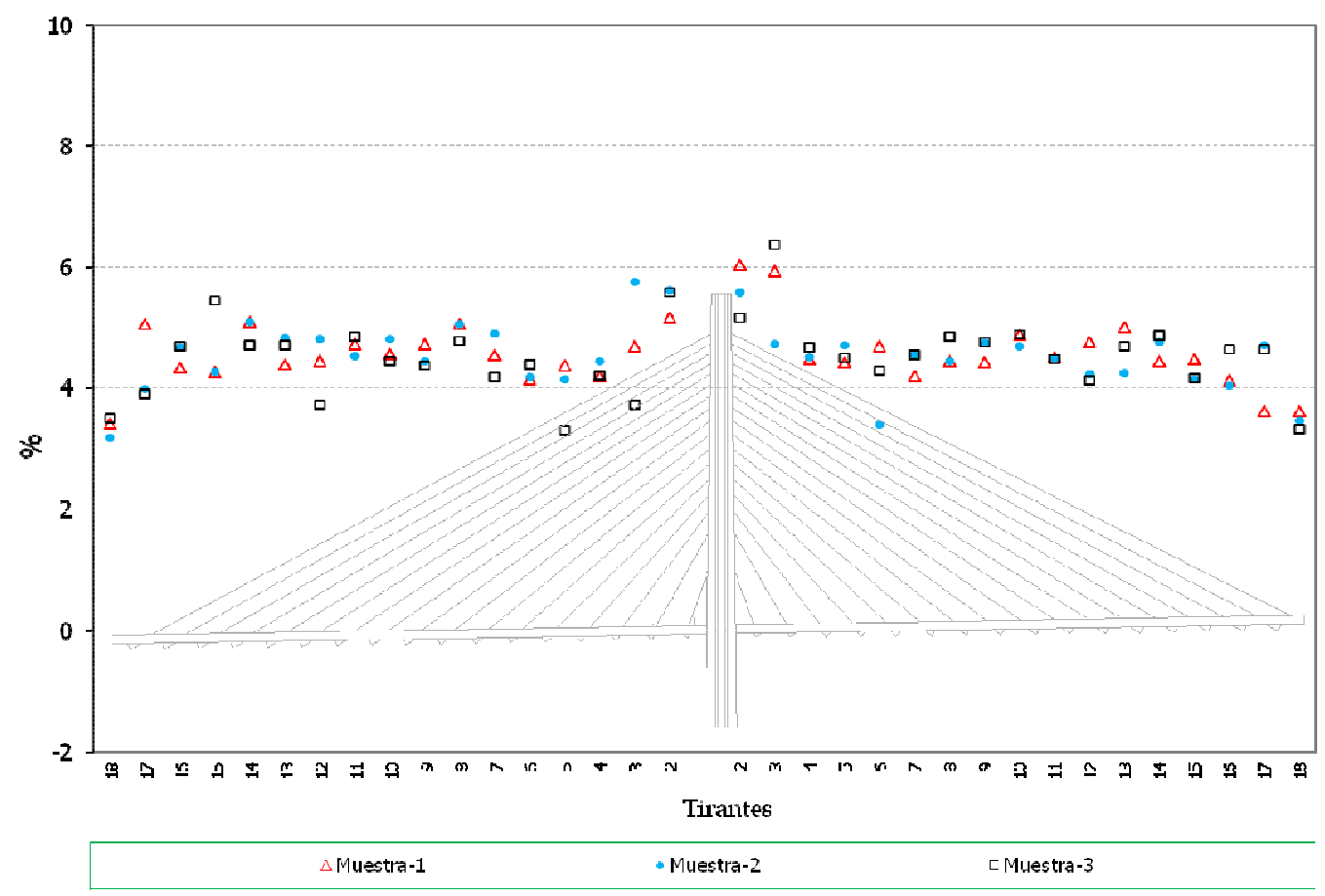

Figura 72. Axiles en los tirantes máximos de servicio. Diferencia en \% de los valores característicos superiores respecto de los teóricos.

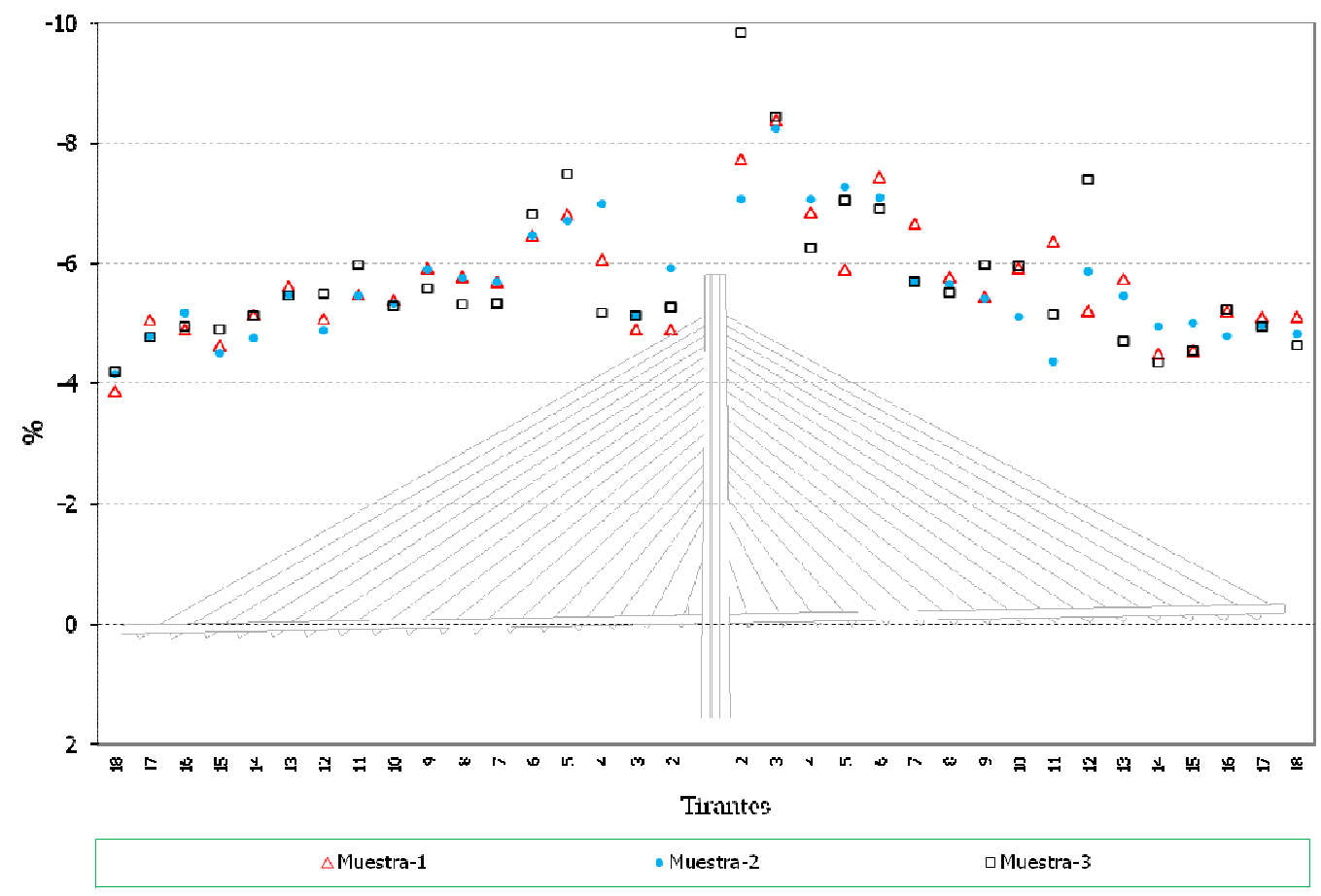

Figura 73. Axiles en los tirantes mínimos de servicio. Diferencia en \% de los valores característicos inferiores respecto de los teóricos. 


\subsubsection{Flectores en tablero.}

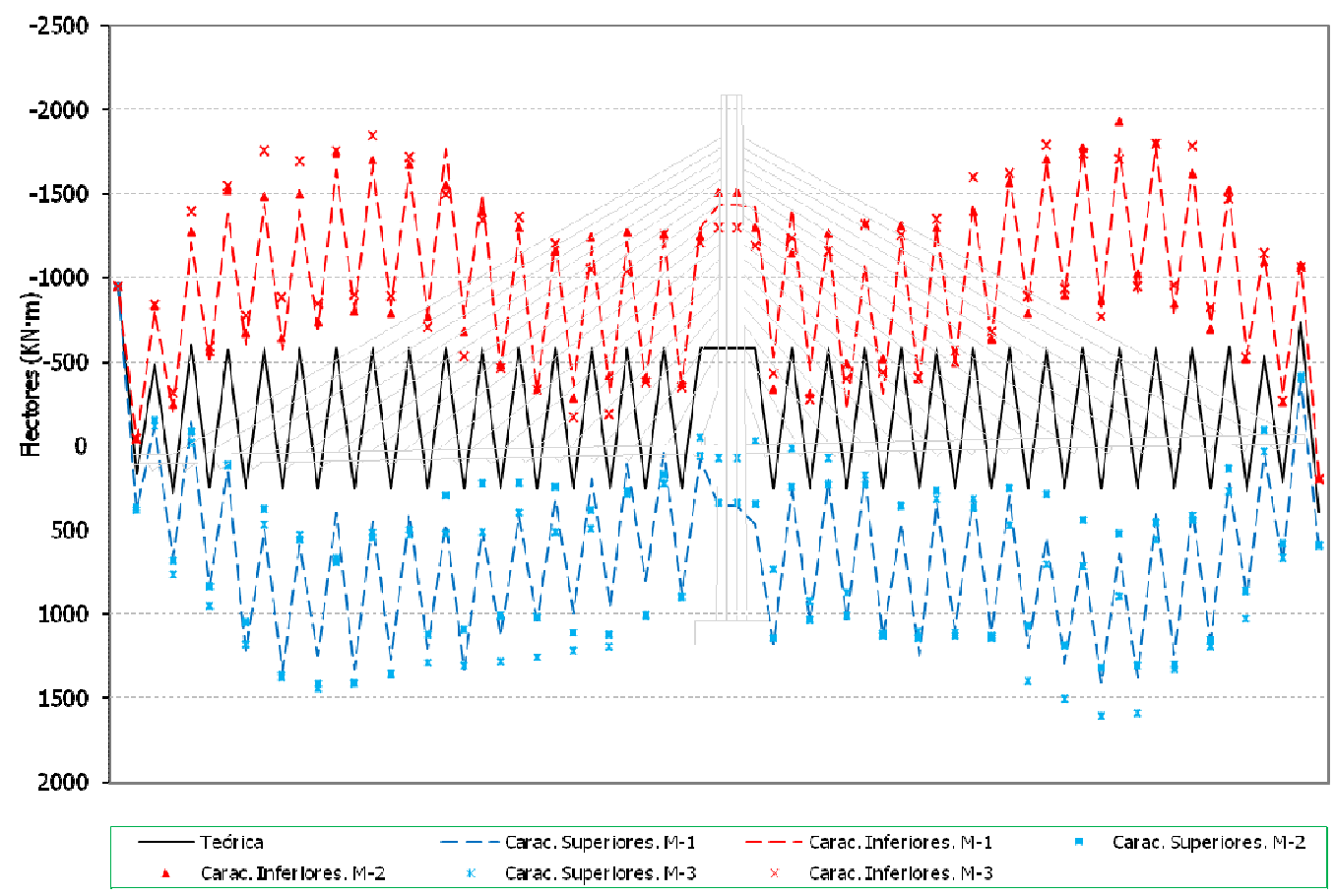

Figura 74. Flectores en tablero en situación final. Valores característicos superiores e inferiores.

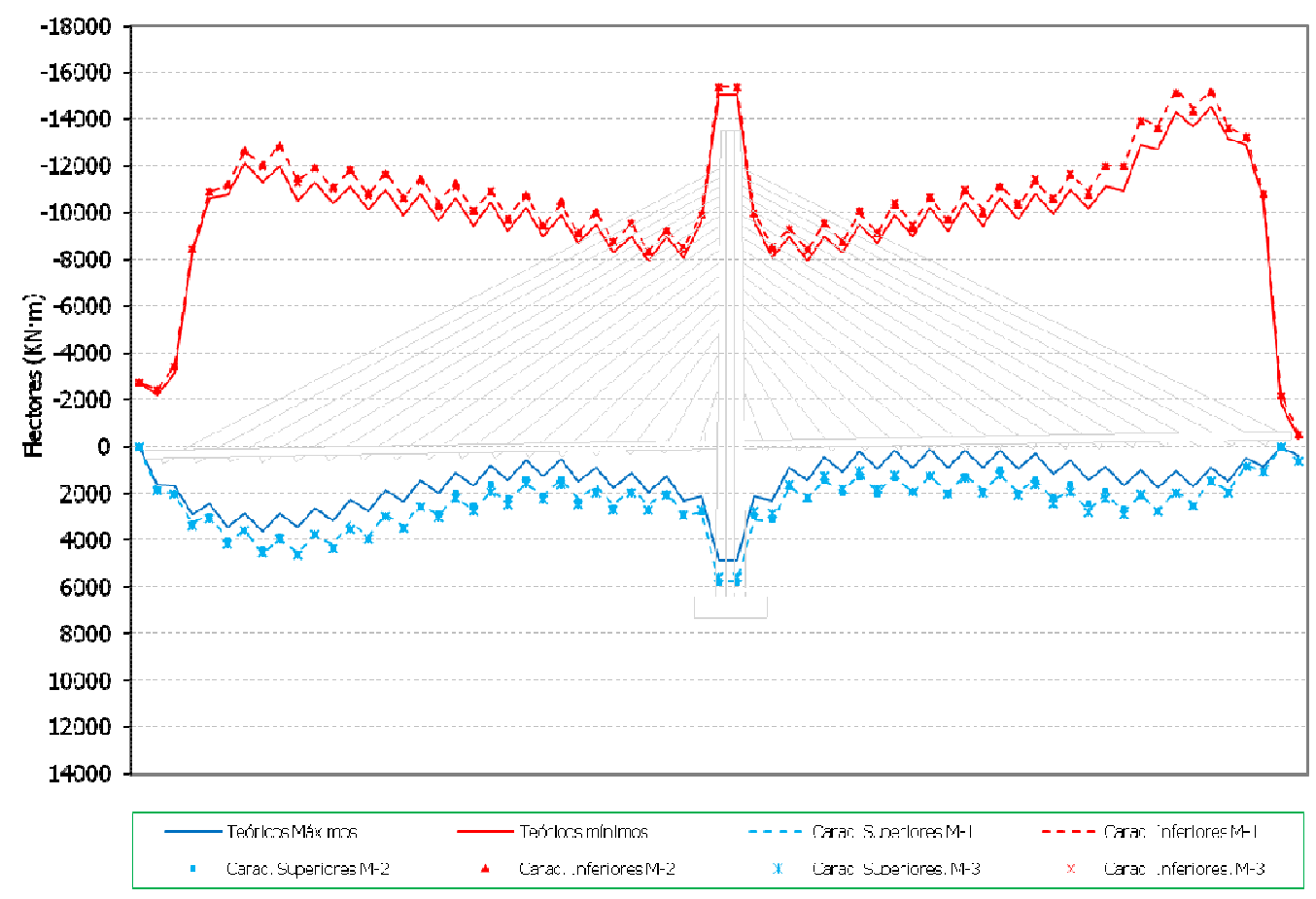

Figura 75. Flectores en tablero en fases de construcción. Valores característicos superiores e inferiores. 


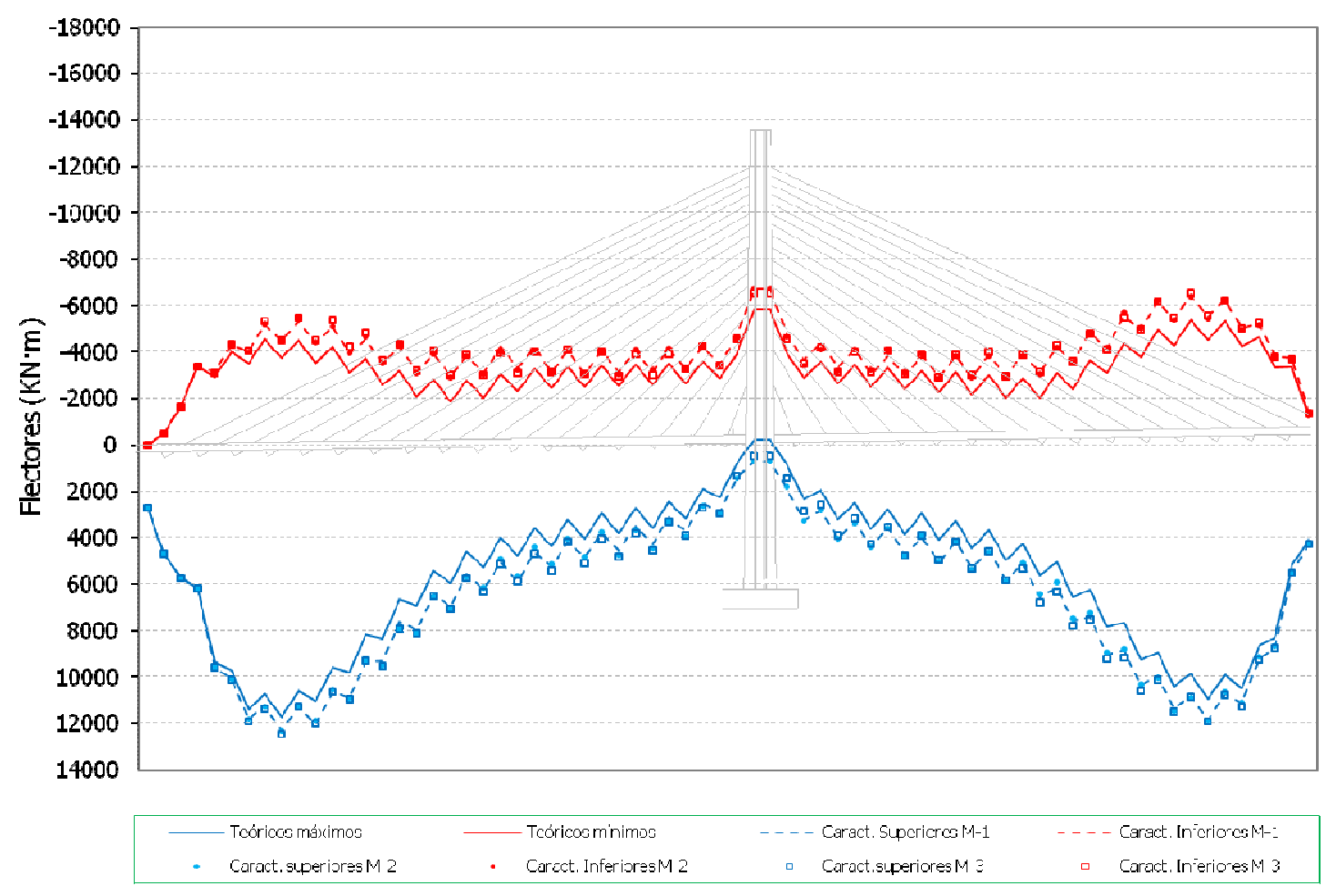

Figura 76. Flectores en tablero en fases de servicio. Valores caracteristicos superiores e inferiores.

\subsubsection{Flectores en el pilono.}

Se incluyen en este apartado sólo los resultados considerados más significativos en este elemento, que en concreto son la envolvente de flectores mínimos en fase de construcción y la envolvente de máximos en servicio. 


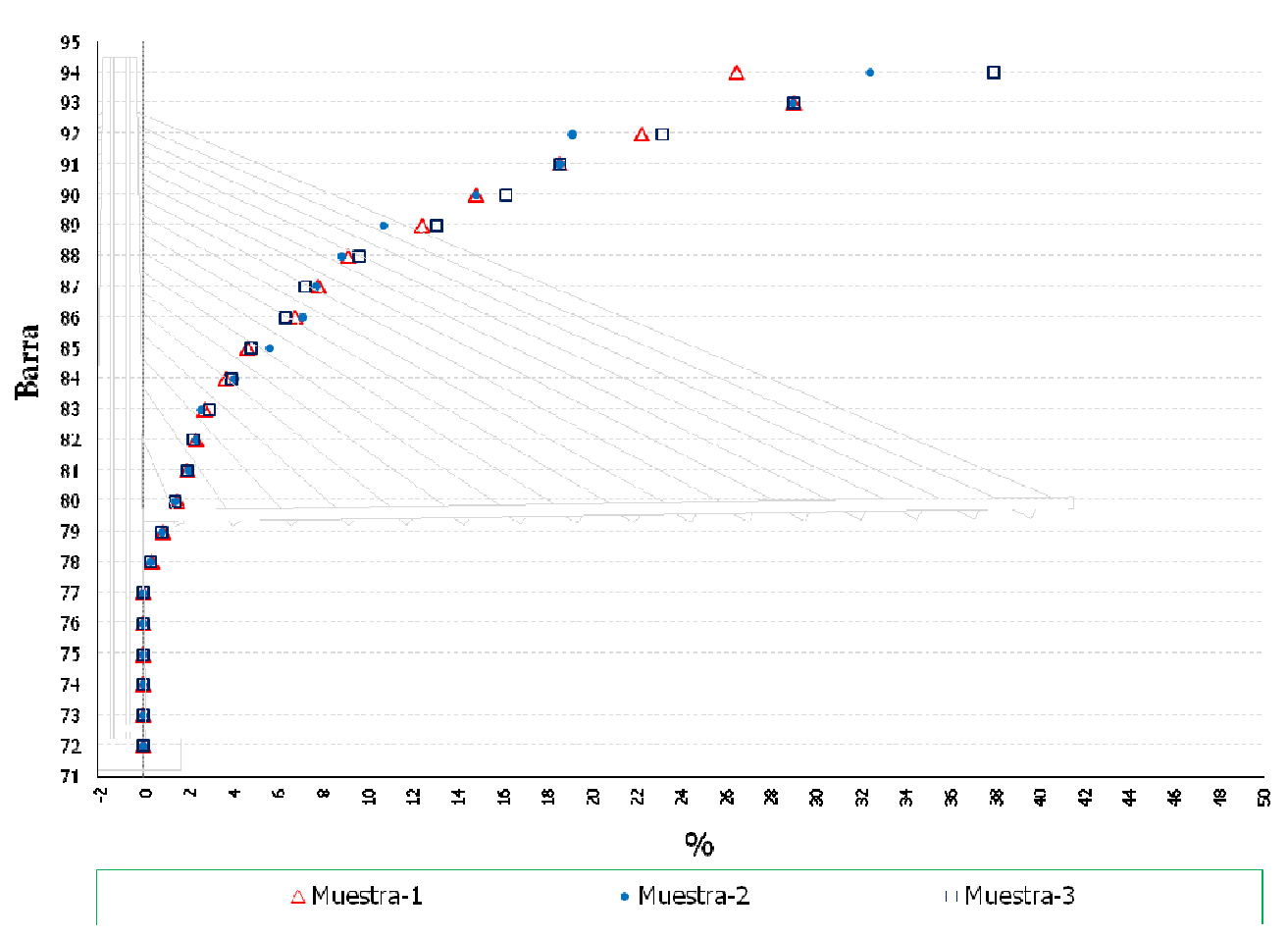

Figura 77. Flectores en pilono mínimos en fases de construcción. Diferencias en \% de los valores característicos inferiores respecto de los teóricos.

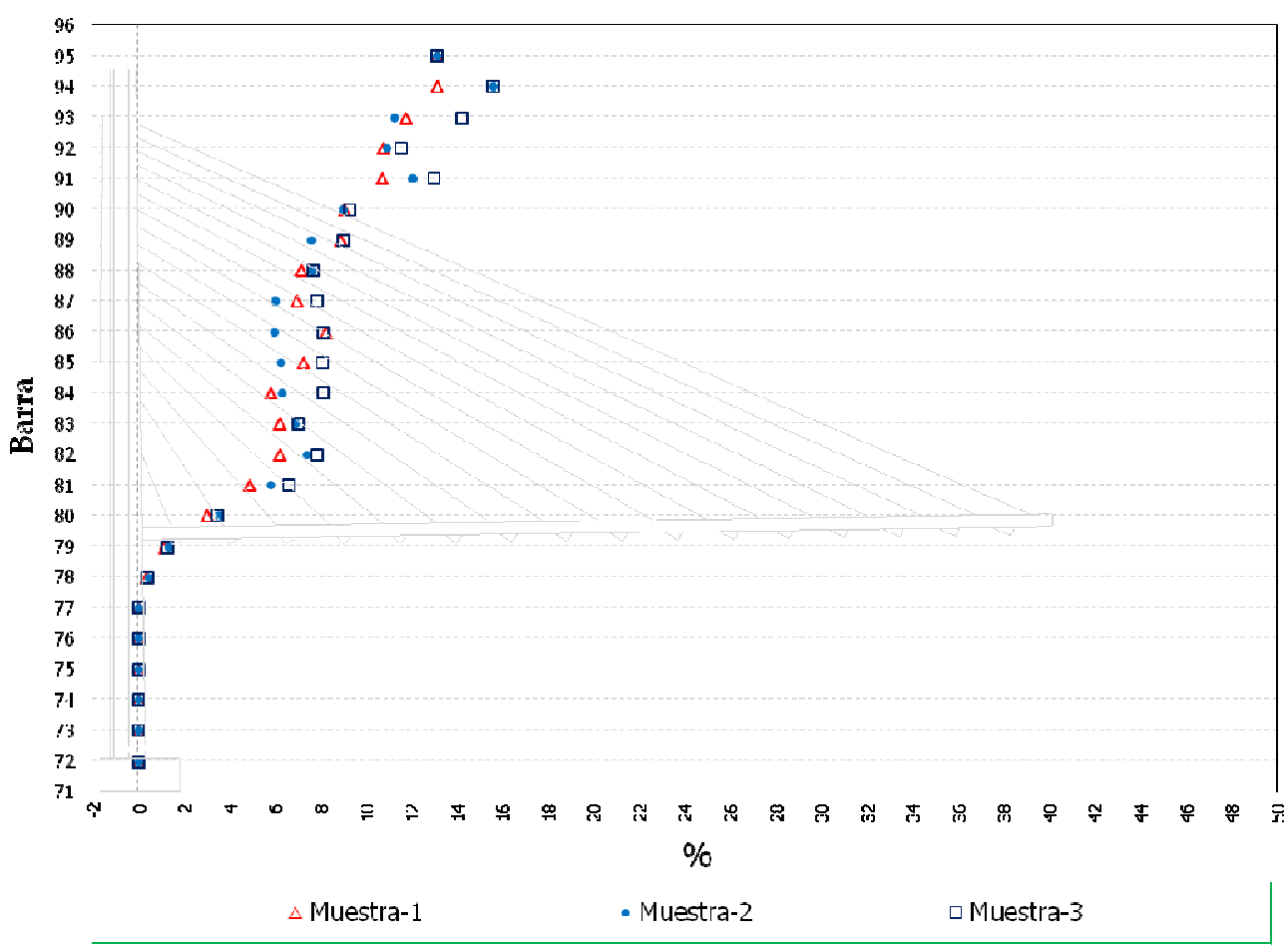

Figura 78. Flectores en pilono máximos en fases de servicio. Diferencias en \% de los valores característicos superiores respecto de los teóricos. 


\subsubsection{Errores en alargamientos de los cables.}

Se adjuntan a continuación resultados de la aplicación de los errores aleatorios sobre las deformaciones teóricas de montaje de los cables, también mediante un proceso de cálculo del montaje de la estructura. Así el cálculo de los procesos constructivos "erróneos" tiene como base de partida en este caso la siguiente formulación:

$$
\text { [EC4. 16.] } \quad \delta^{\mathrm{N}}{ }_{\text {err, }}=\delta_{\text {teor }, i}+\left(\mathrm{VA}^{\mathrm{N}}{ }_{\mathrm{i}}-1\right) \cdot \mathrm{L}_{\mathrm{i}}
$$

siendo: $\quad \delta^{N}$ err,i $\rightarrow$ valor de la deformación errónea $\mathrm{N}$-ésima del cable i.

$\delta_{\text {teori }} \rightarrow$ valor de la deformación de montaje teórica del cable i.

$\mathrm{VAN}_{\mathrm{i}} \rightarrow$ coeficiente aleatorio de ponderación $\mathrm{N}$-ésimo del cable i.

$\mathrm{Li}_{\mathrm{i}} \rightarrow$ longitud neutra o en vacío del cable $\mathrm{i}$.

El algoritmo utilizado para la generación de estos errores aleatorios se incluye en el anexo A.3.

Se han generado en este caso también 3 muestras de 100 elementos correspondientes a deformaciones de montaje erróneos de cada cable, que se aplicarán sobre la estructura al simular su proceso constructivo.

Los resultados representados lo son de tirantes (Figura 79 a Figura 84), tablero (Figura 85 a Figura 87) y pilono (Figura 88 a Figura 89). 
4.6.3.1 Axiles en tirantes.

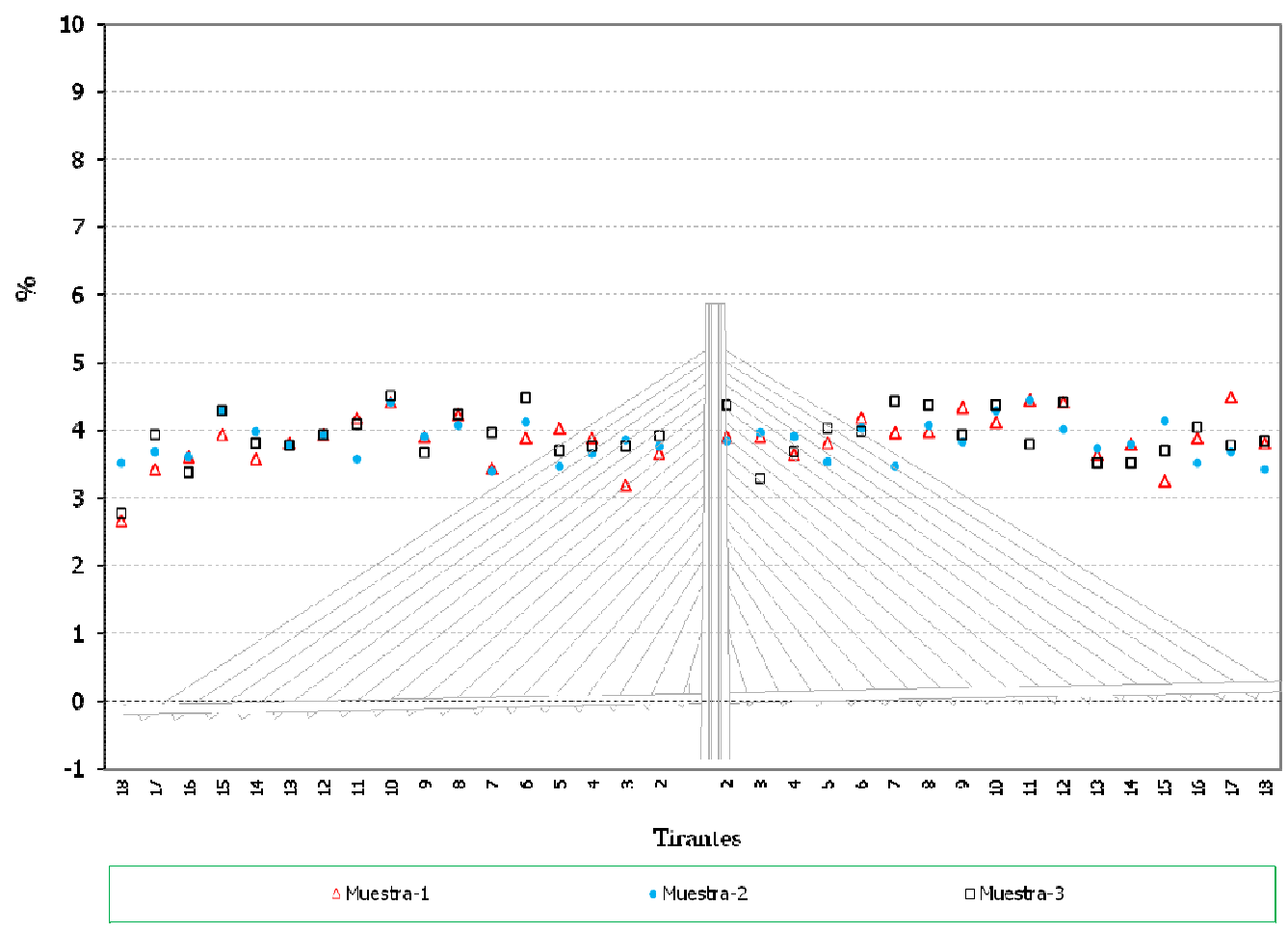

Figura 79. Axiles finales en los tirantes. Diferencia en \% de los valores característicos superiores respecto de los teóricos.

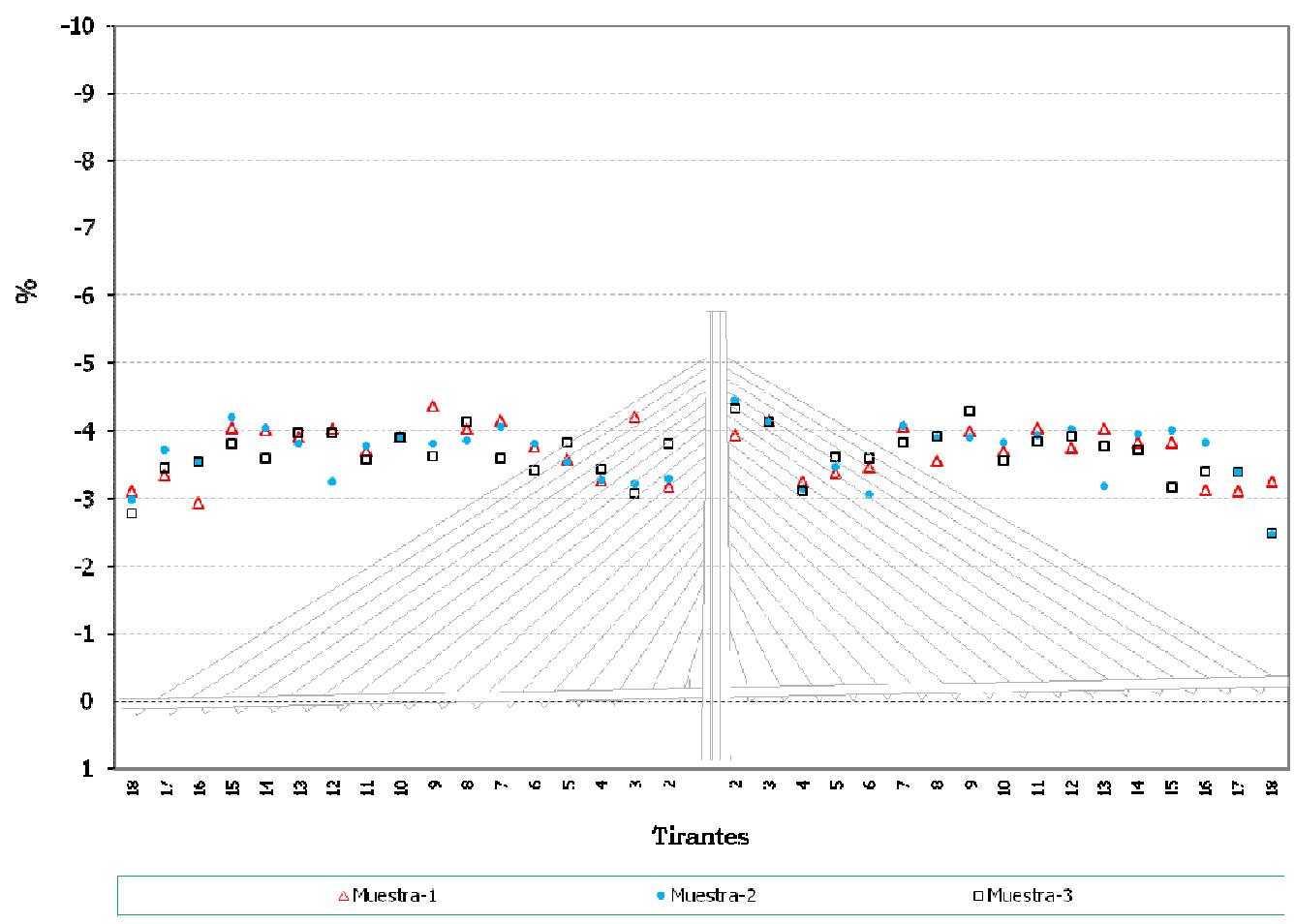

Figura 80. Axiles en los tirantes máximos de construcción. Diferencia en \% de los valores característicos superiores respecto de los teóricos. 


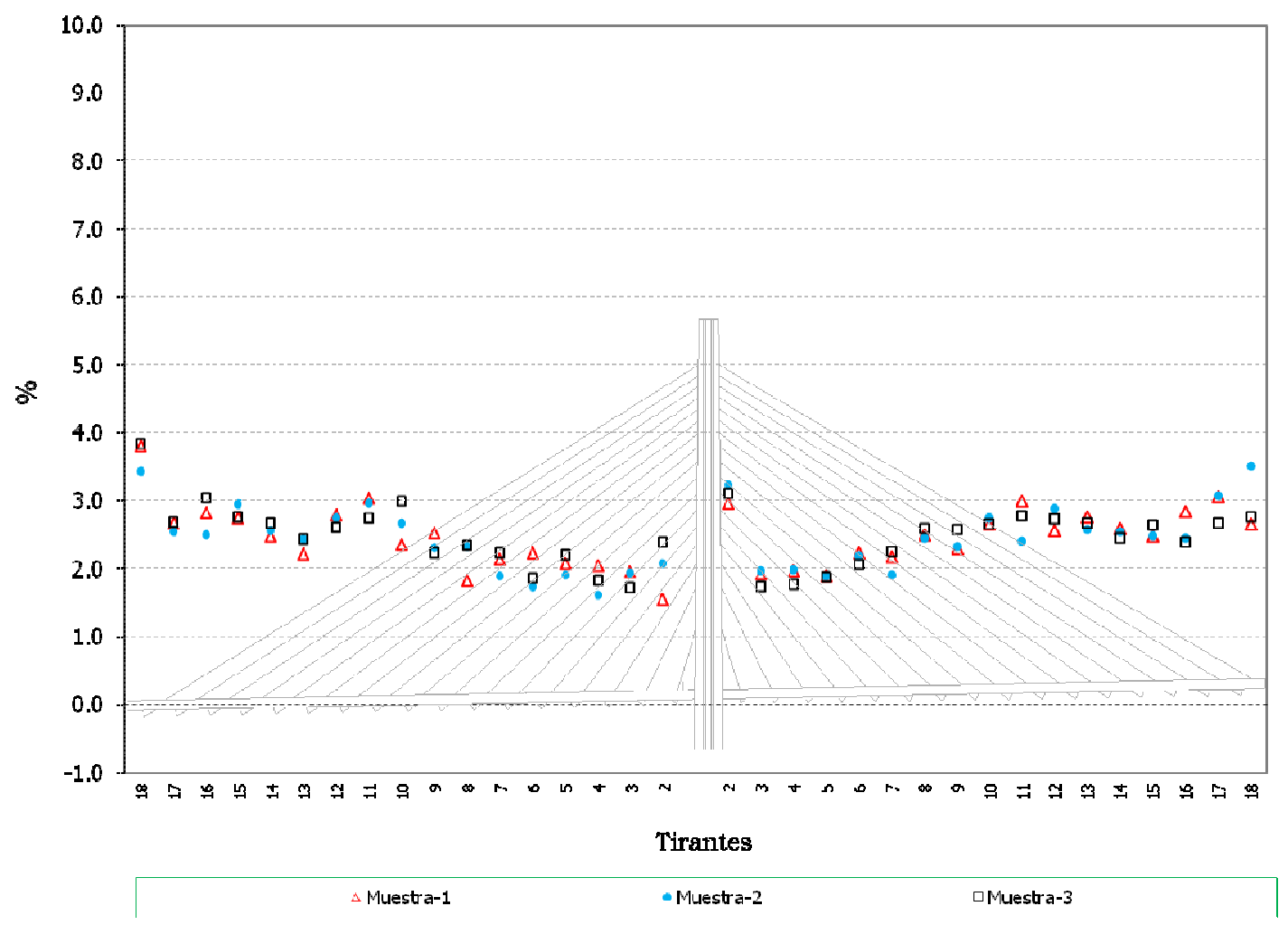

Figura 81. Axiles en los tirantes máximos de construcción. Diferencia en \% de los valores característicos superiores respecto de los teóricos.

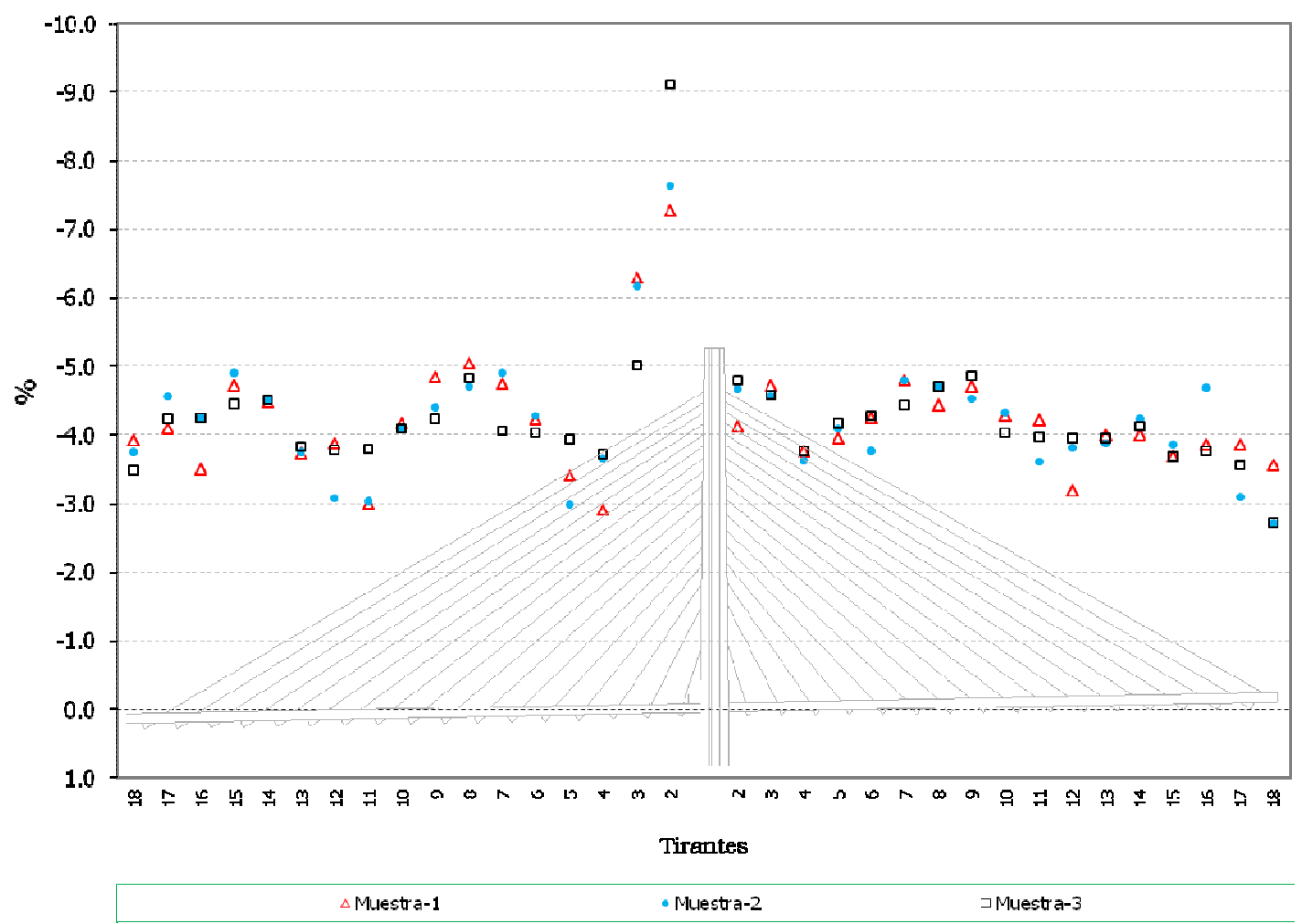

Figura 82. Axiles en los tirantes mínimos de construcción. Diferencia en \% de los valores característicos inferiores respecto de los teóricos. 


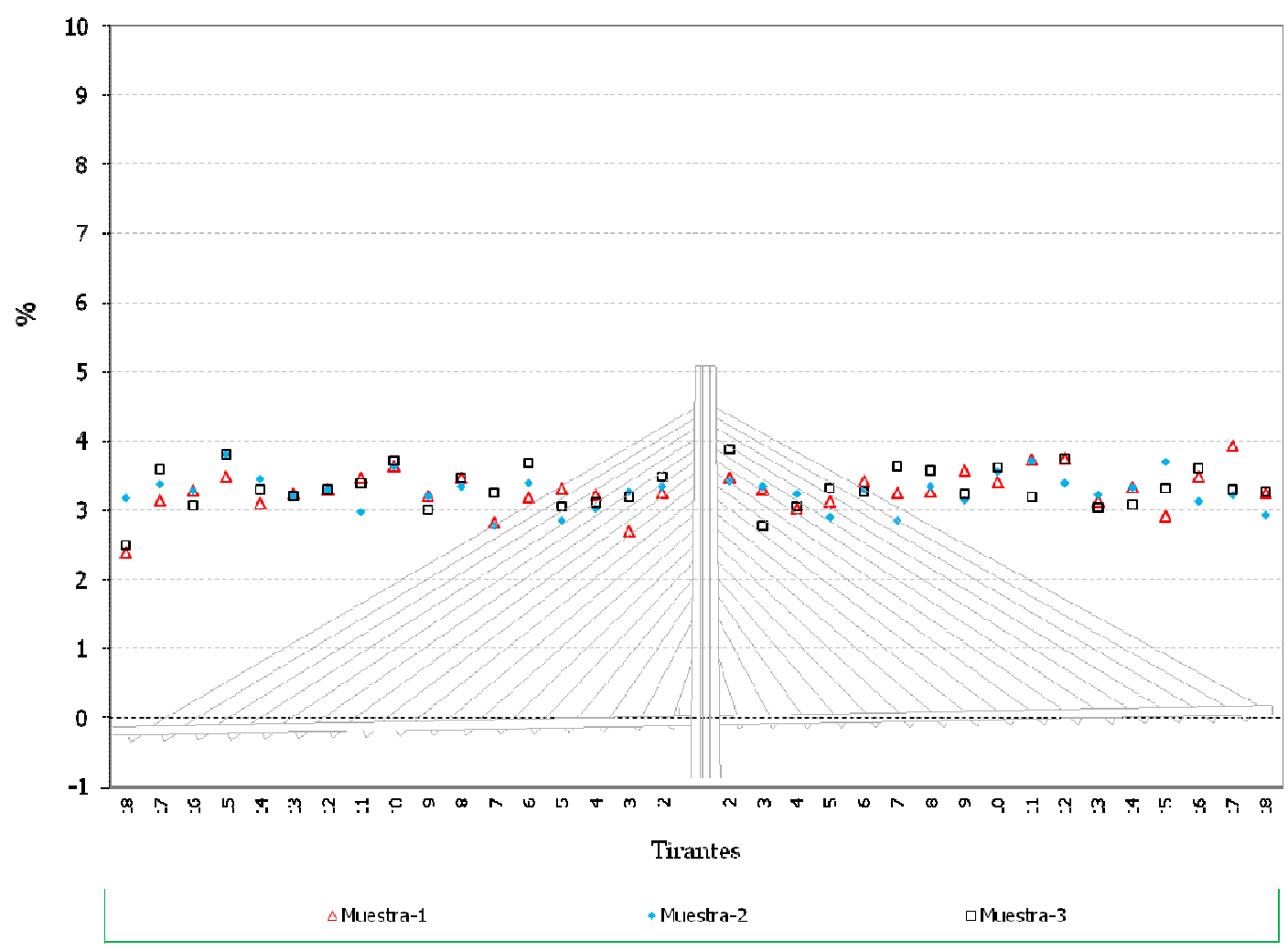

Figura 83. Axiles en los tirantes máximos de servicio. Diferencia en \% de los valores característicos superiores respecto de los teóricos.

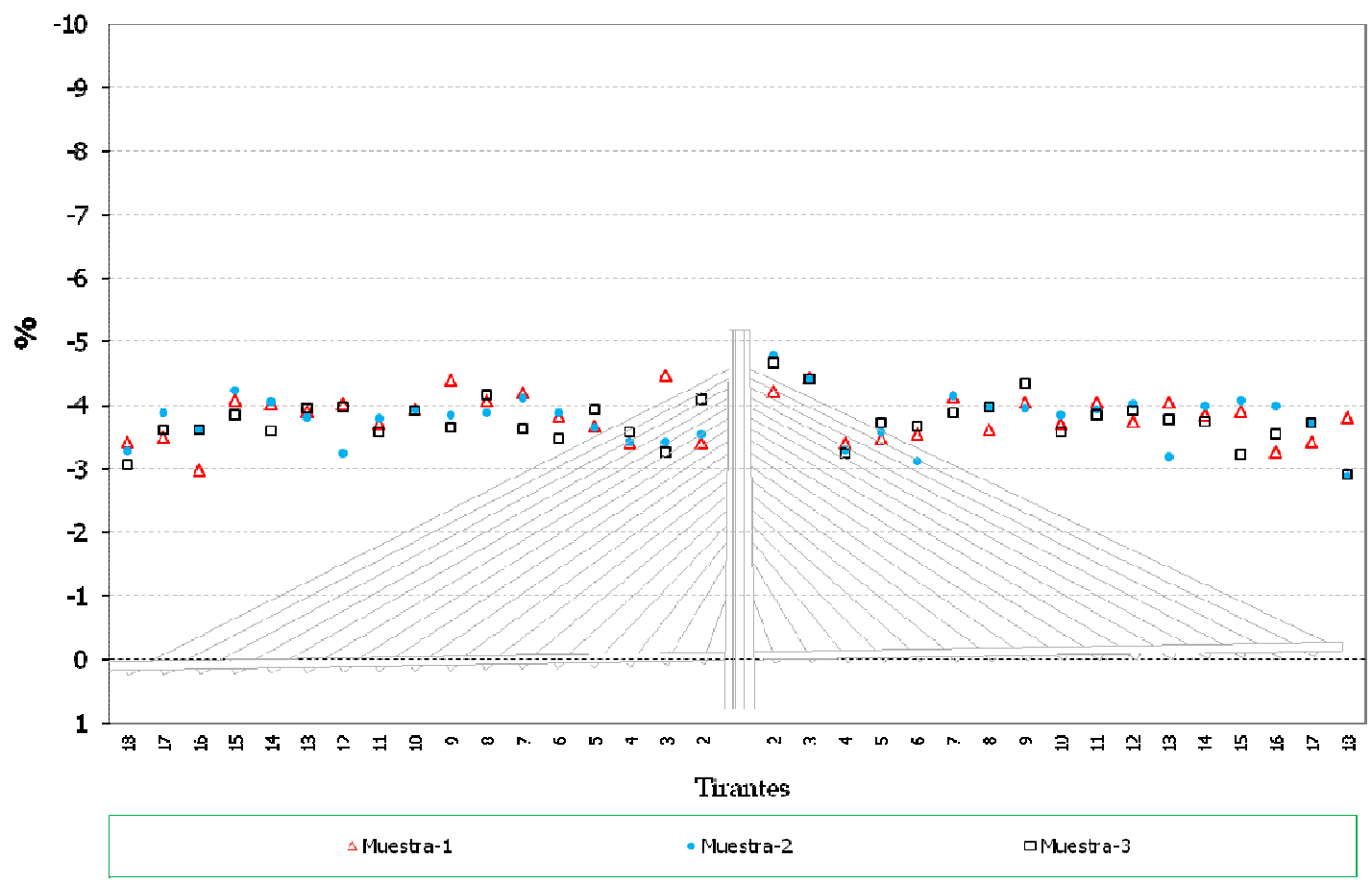

Figura 84. Axiles en los tirantes mínimos de servicio. Diferencia en \% de los valores característicos inferiores respecto de los teóricos. 


\subsubsection{Flectores en tablero.}

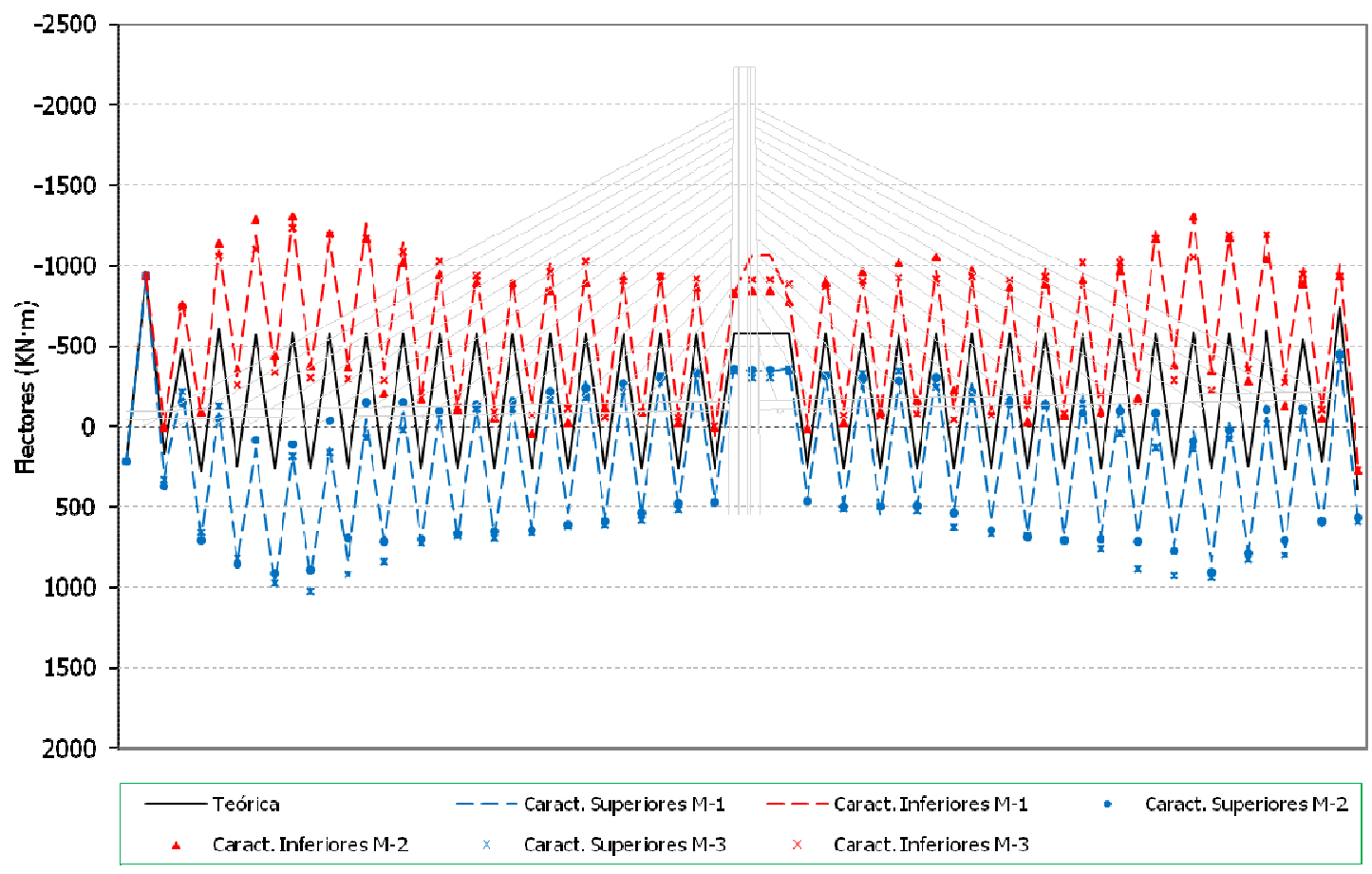

Figura 85. Flectores en tablero en situación final. Valores característicos superiores e inferiores.

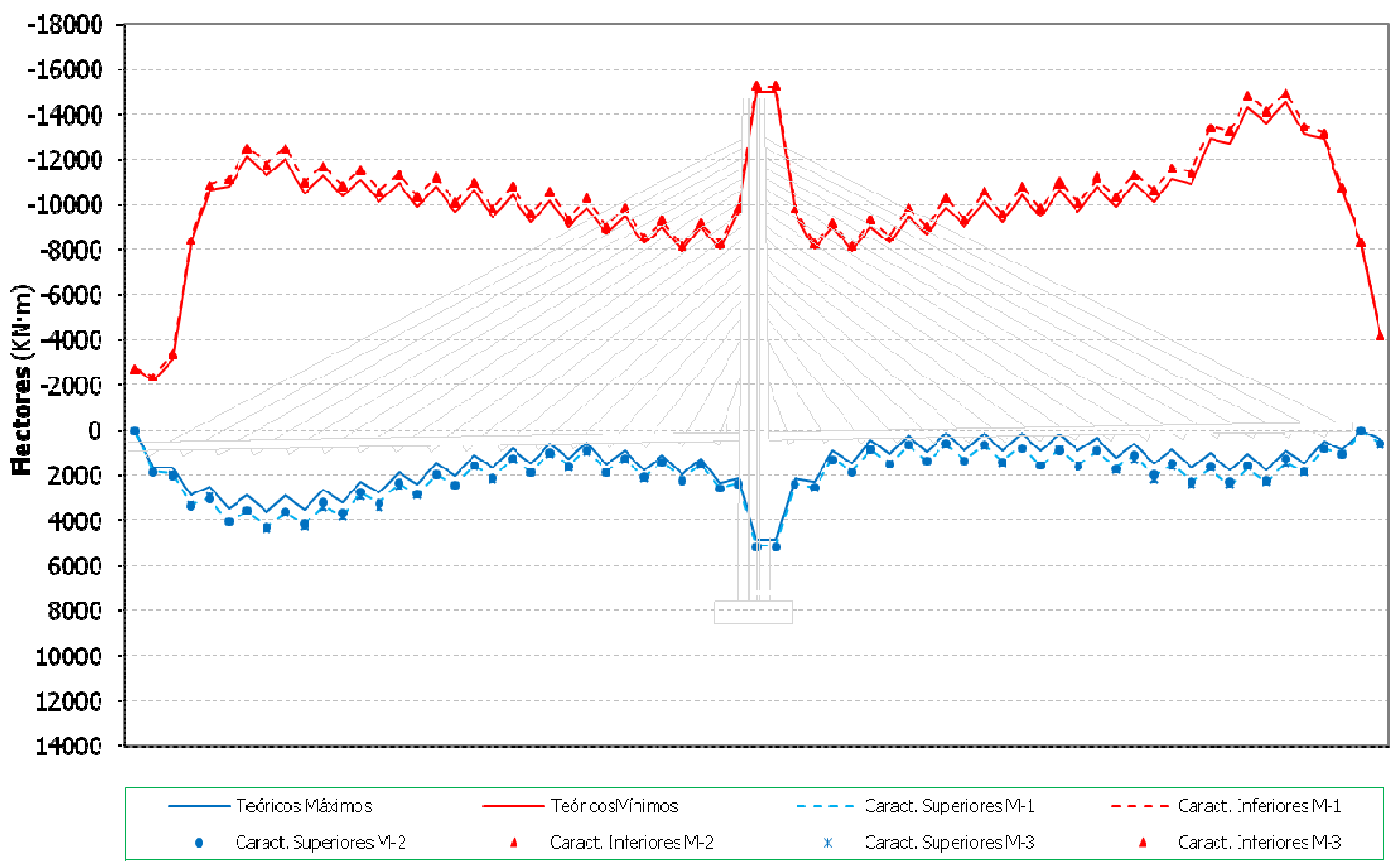

Figura 86. Flectores en tablero en fases de construcción. Valores característicos superiores e inferiores. 


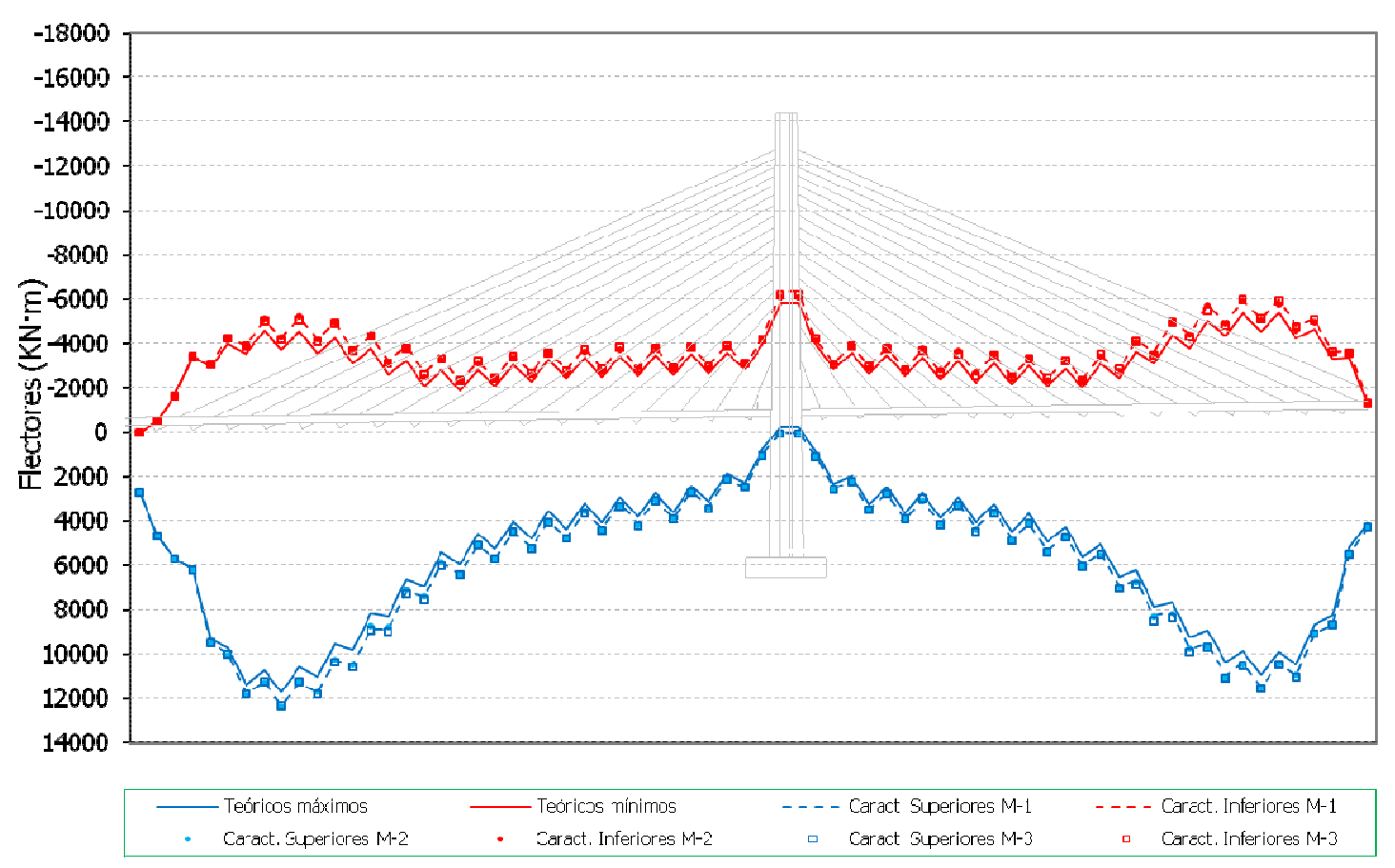

Figura 87. Flectores en tablero en fases de servicio. Valores característicos superiores e inferiores.

\subsubsection{Flectores en pilono.}

Se incluyen en este caso sólo los valores más representativos correspondientes a las envolventes de mínimos de construcción y máximos de servicio. 


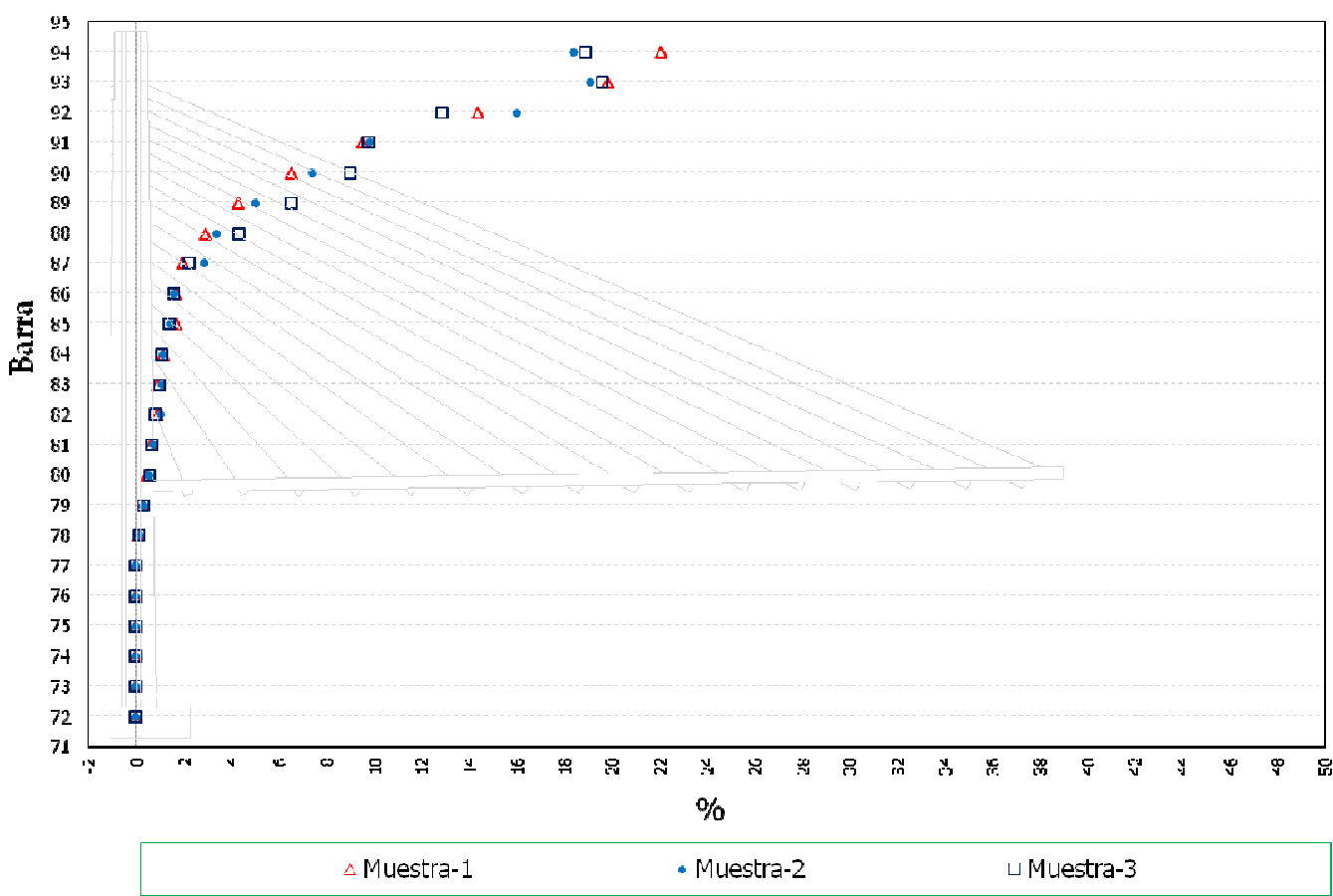

Figura 88. Flectores en pilono mínimos en fases de construcción. Diferencias en \% de los valores característicos inferiores respecto de los teóricos.

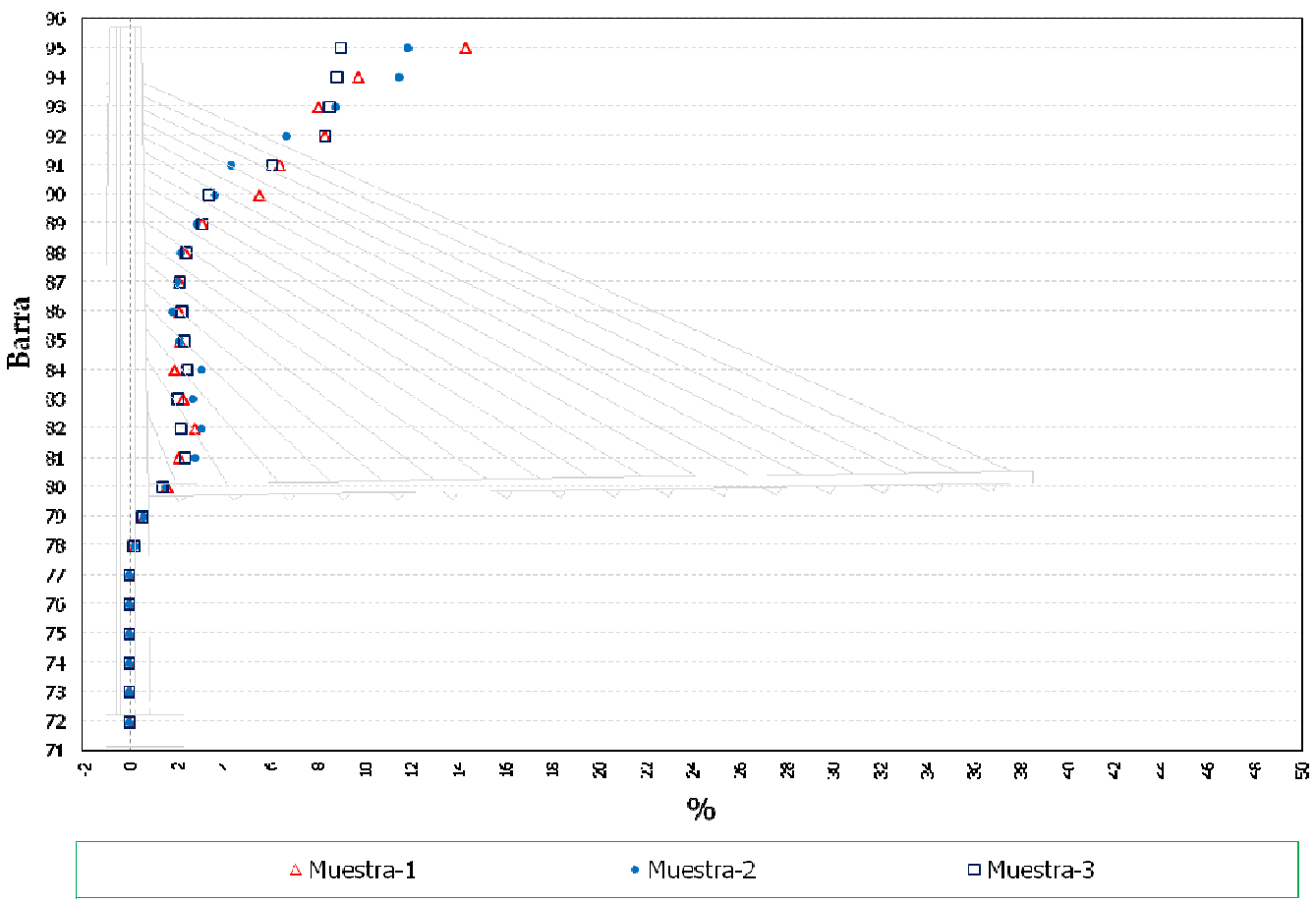

Figura 89. Flectores en pilono máximos en fases de servicio. Diferencias en \% de los valores característicos superiores respecto de los teóricos. 


\subsubsection{Errores en la posición de los anclajes}

El error en la posición en los anclajes se ha considerado que puede producirse con un rango de $\pm 1 \mathrm{~cm}$, habiéndose traducido dicho error en la posición del inicio y final del cable como un error en la longitud a medir de éste. De esta forma, el proceso seguido para la introducción de estos errores sobre el proceso constructivo es igual al planteado en el caso anterior de error en la medida de la longitud de los cables.

Se presentan a continuación los resultados obtenidos de los axiles de los tirantes y de los flectores del tablero y pilono.

\subsubsection{Axiles en tirantes.}

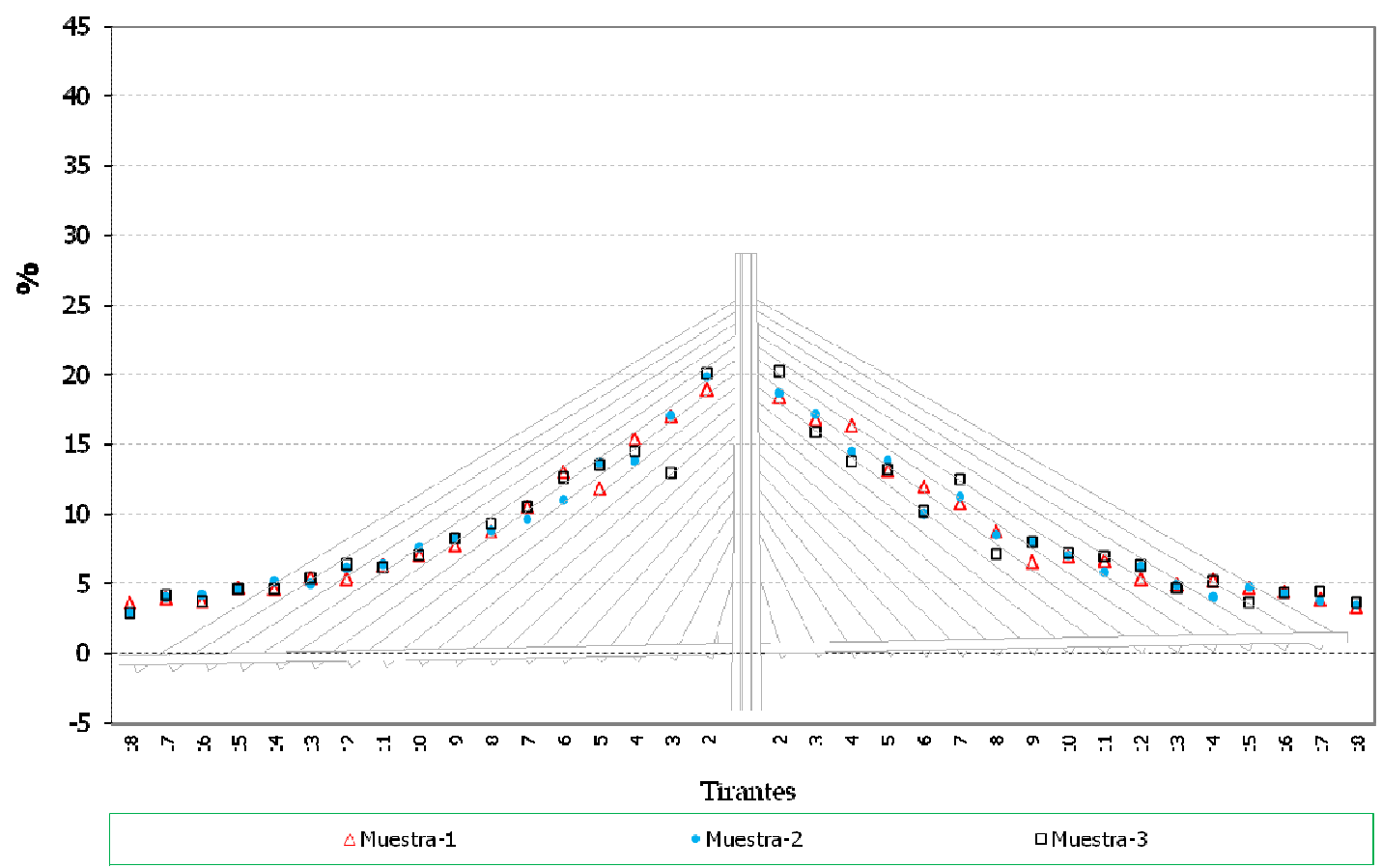

Figura 90. Axiles finales en los tirantes. Diferencia en \% de los valores característicos superiores respecto de los teóricos. 


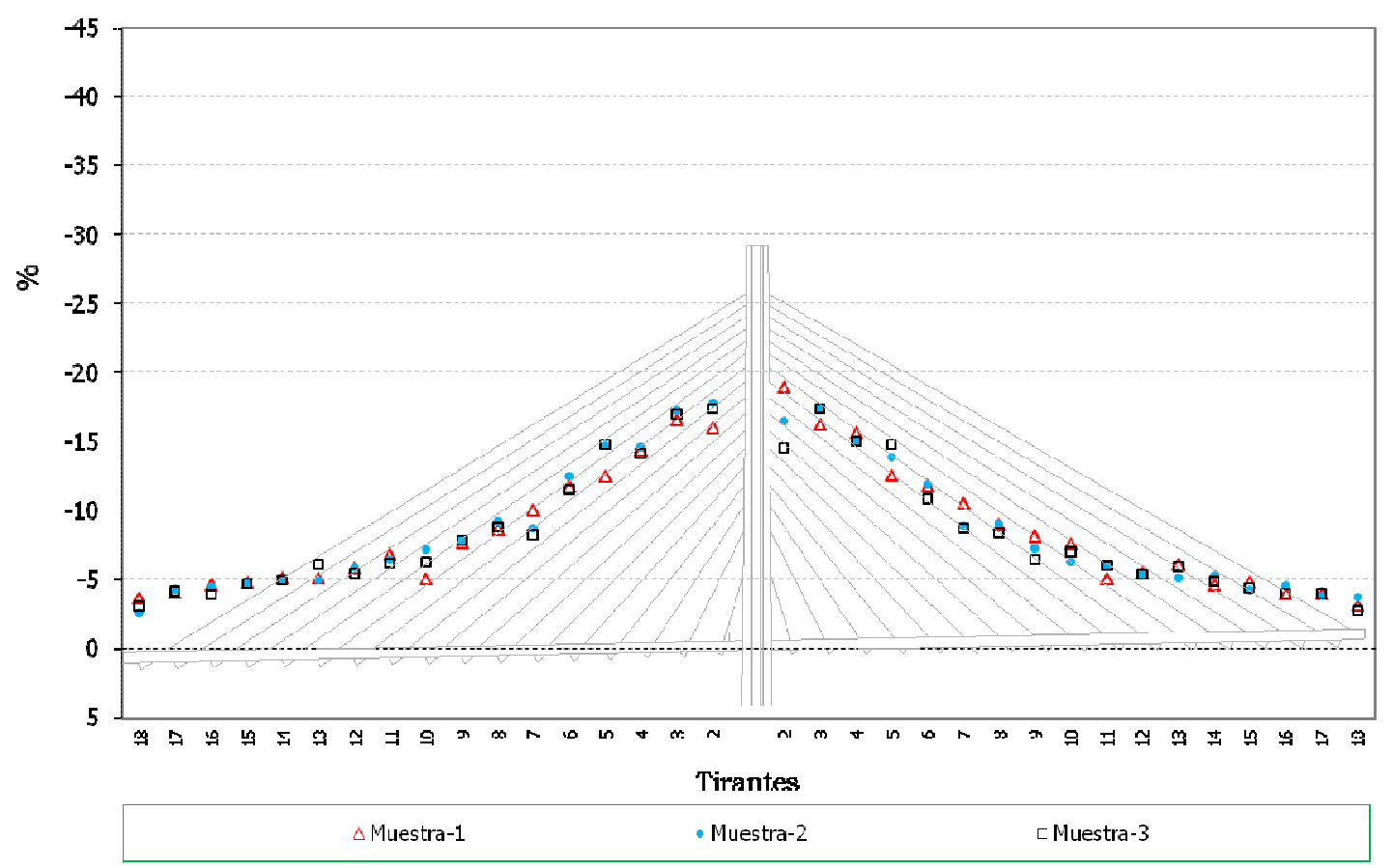

Figura 91. Axiles finales en los tirantes. Diferencia en \% de los valores característicos inferiores respecto de los teóricos.

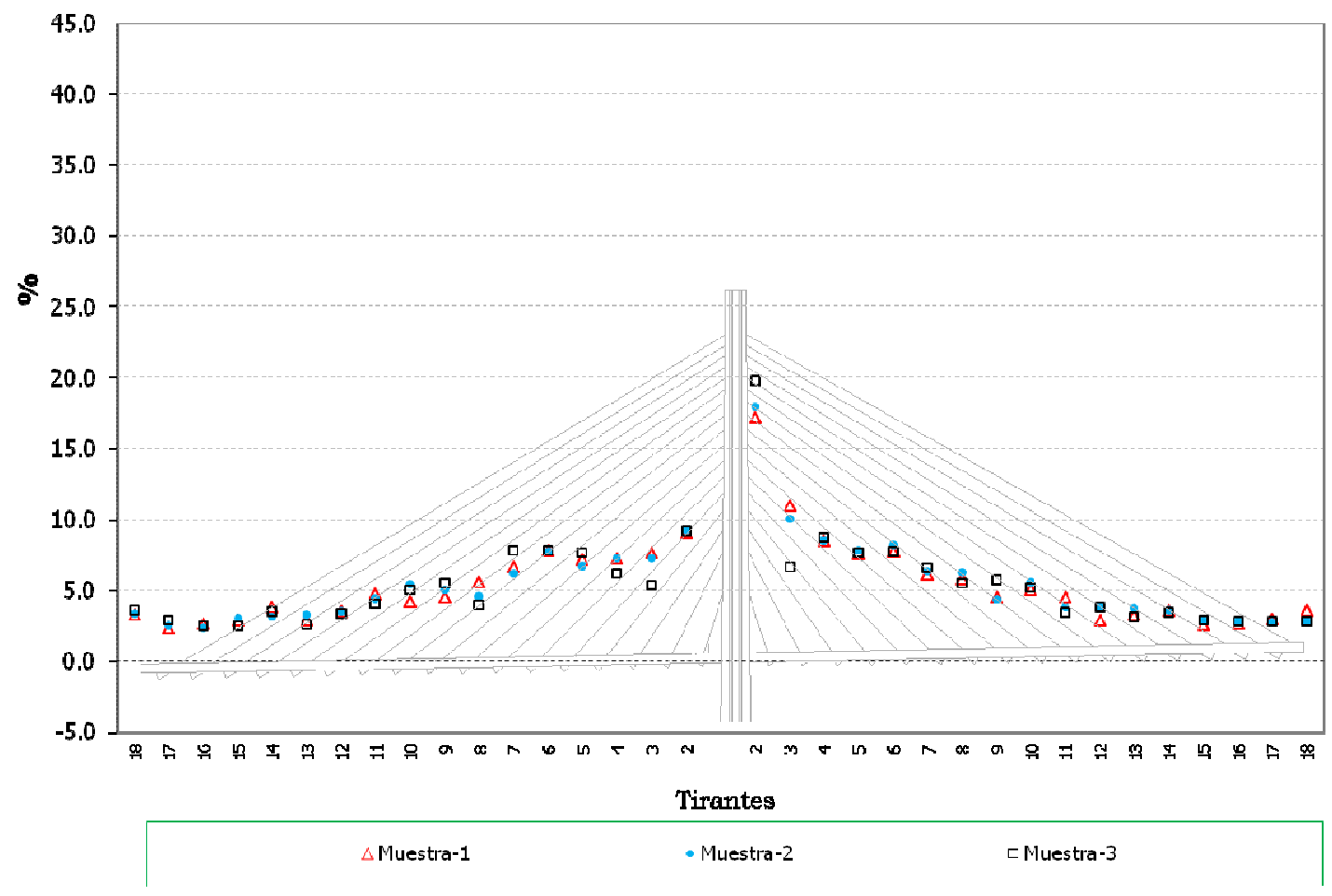

Figura 92. Axiles en los tirantes máximos de construcción. Diferencia en \% de los valores característicos superiores respecto de los teóricos. 


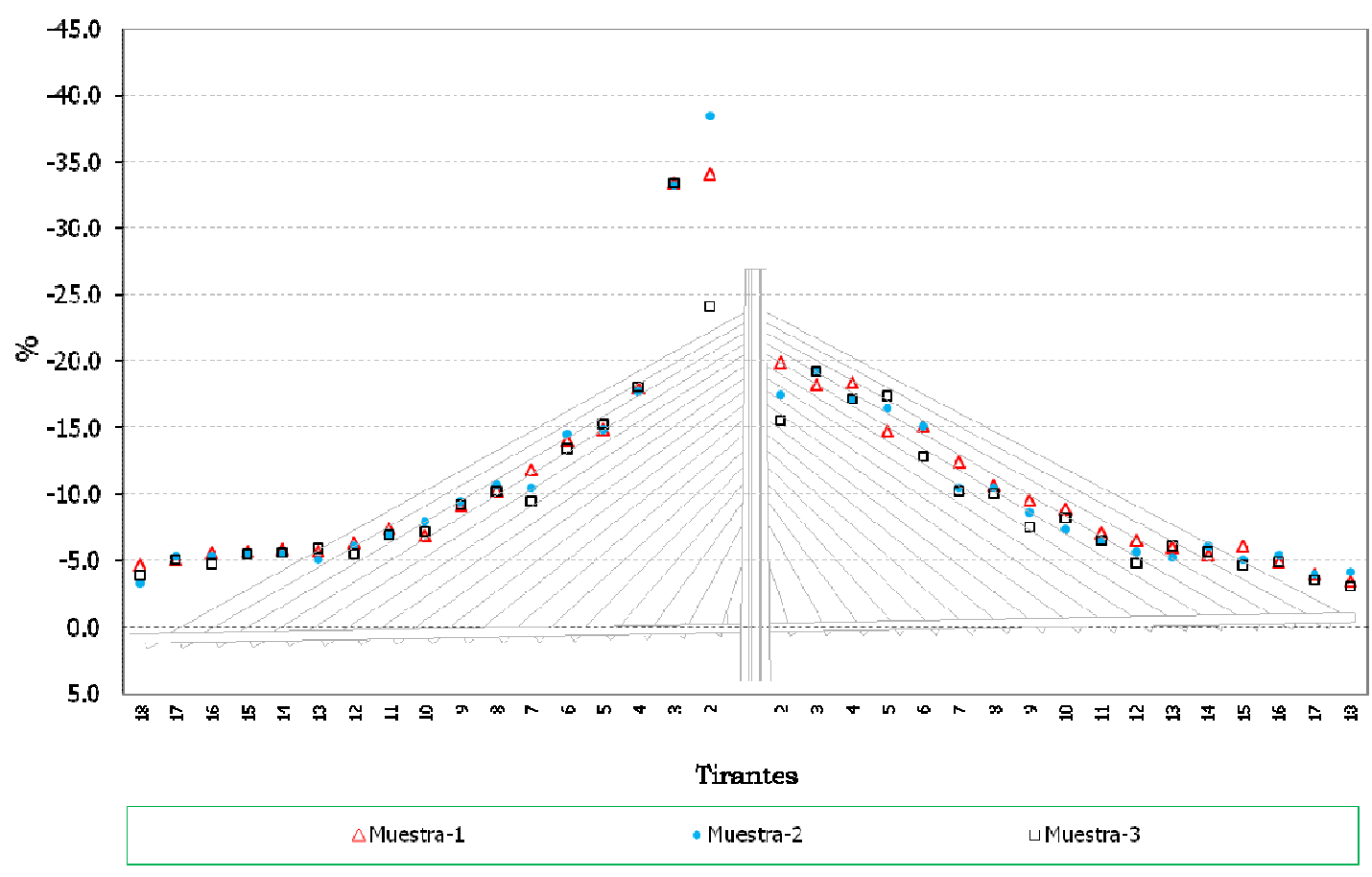

Figura 93. Axiles en los tirantes mínimos de construcción. Diferencia en \% de los valores característicos inferiores respecto de los teóricos.

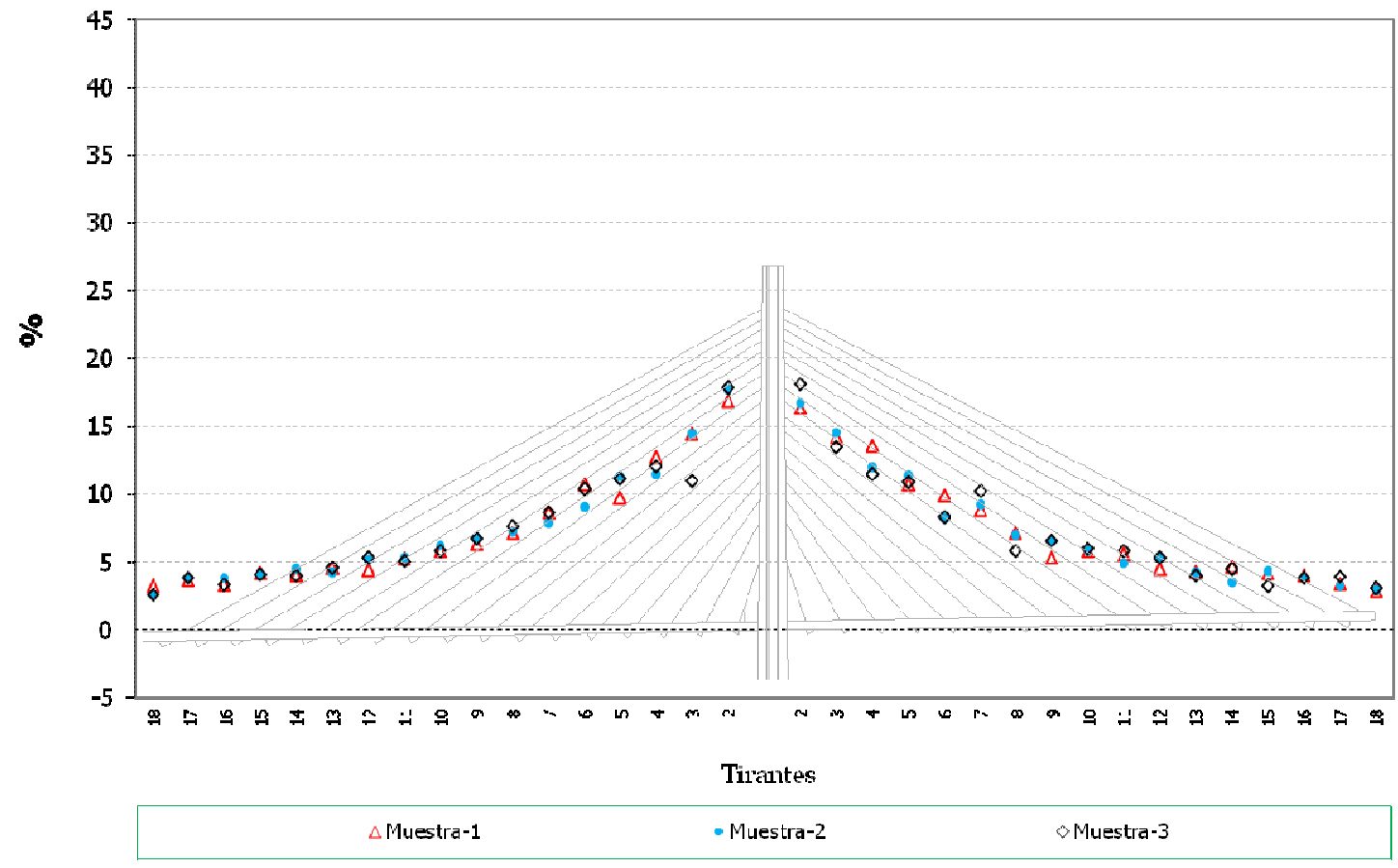

Figura 94. Axiles en los tirantes máximos de servicio. Diferencia en \% de los valores característicos superiores respecto de los teóricos. 


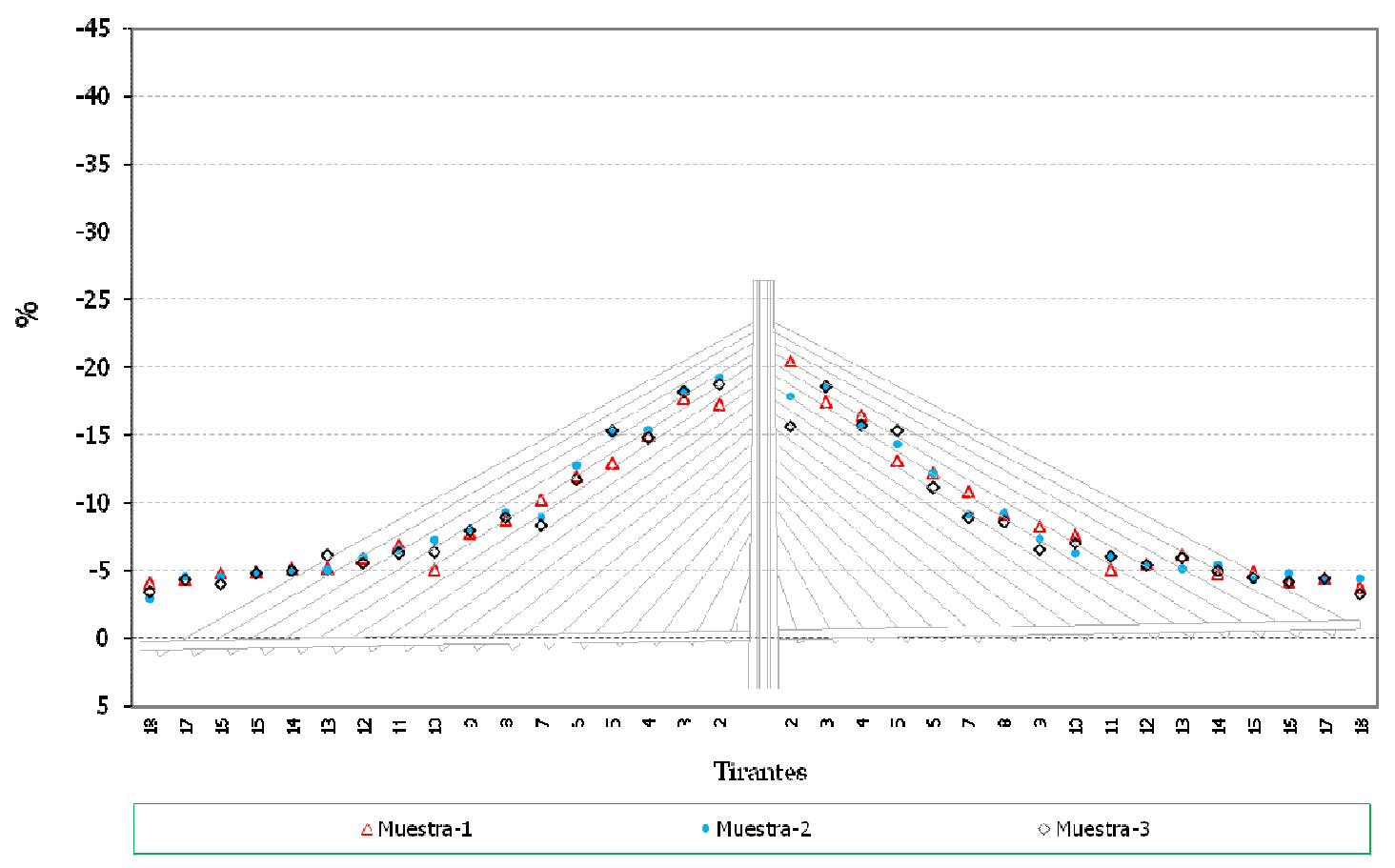

Figura 95. Axiles en los tirantes mínimos de servicio. Diferencia en \% de los valores característicos inferiores respecto de los teóricos.

\subsubsection{Flectores en tablero.}

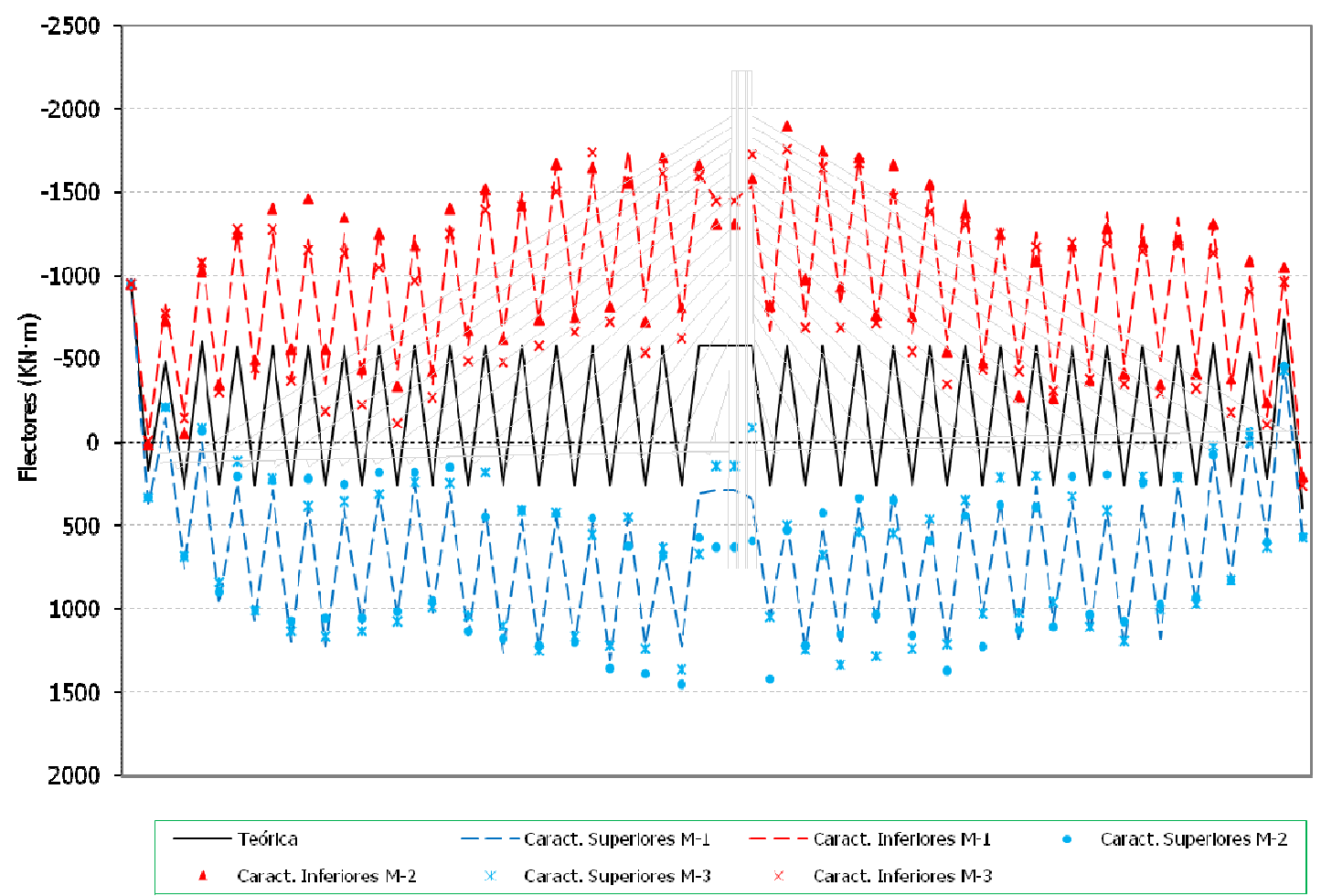

Figura 96. Flectores en tablero en situación final. Valores característicos superiores e inferiores. 


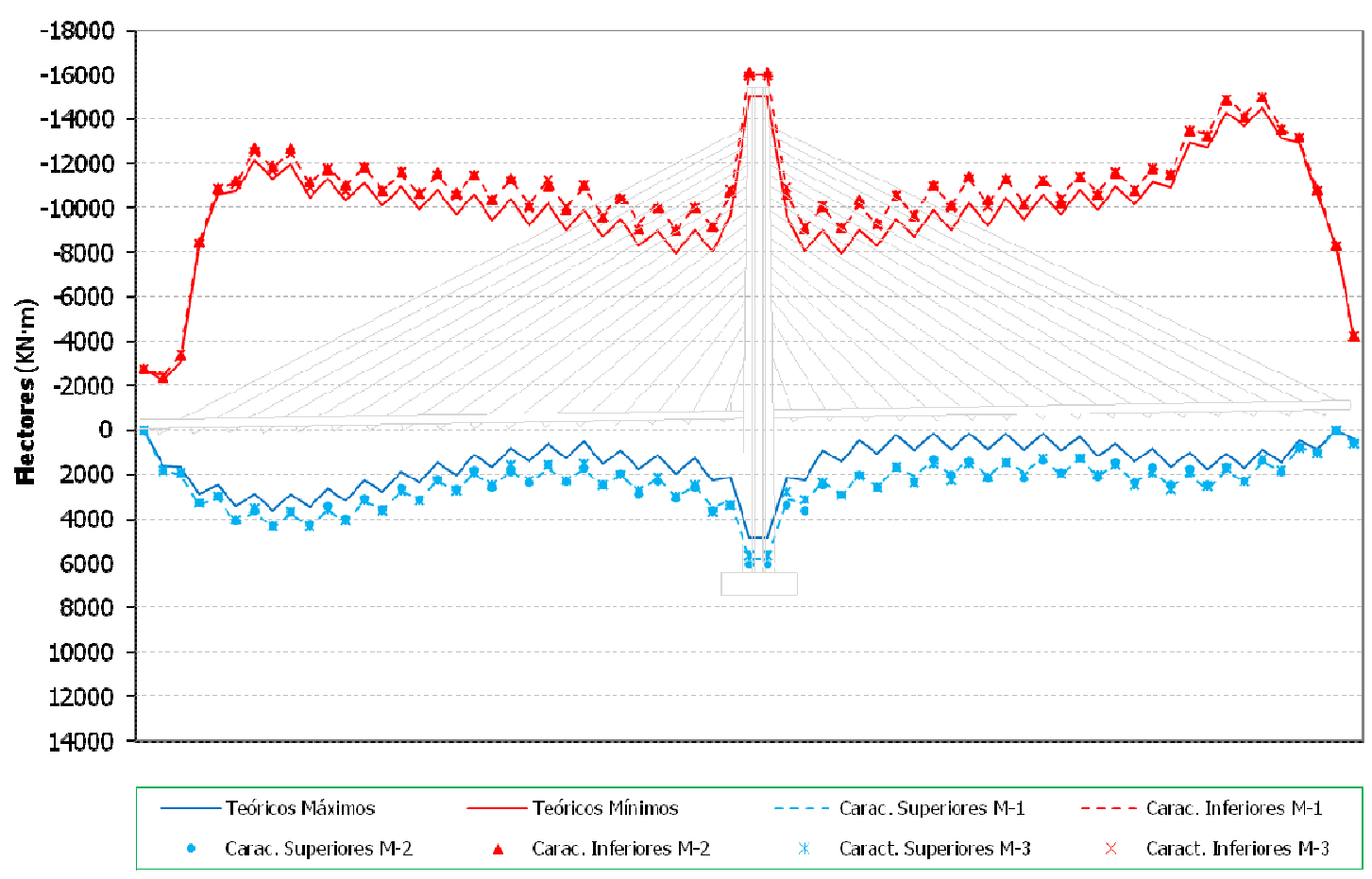

Figura 97. Flectores en tablero en fases de construcción. Valores característicos superiores e inferiores.

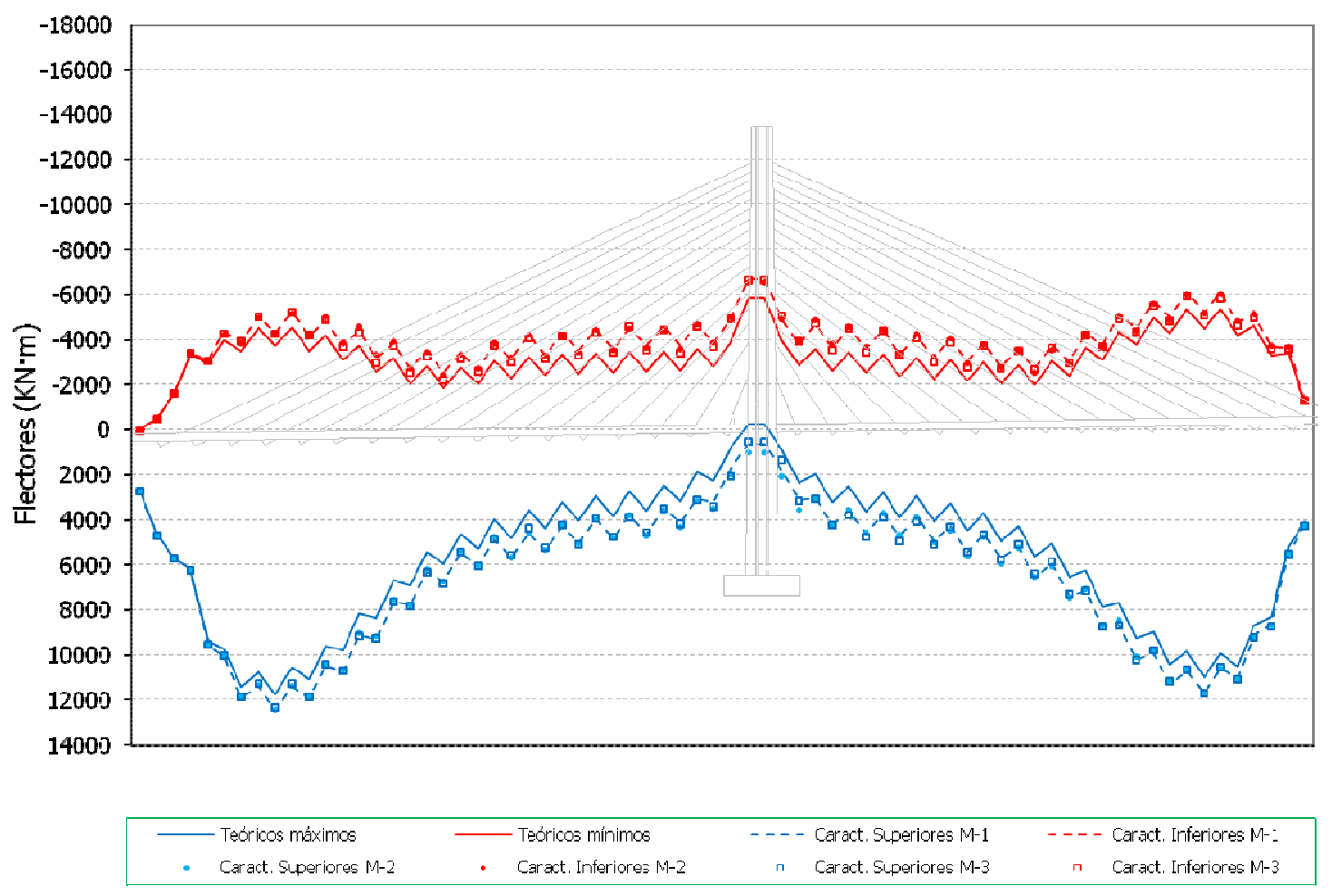

Figura 98. Flectores en tablero en fases de servicio. Valores característicos superiores e inferiores. 


\subsubsection{Flectores en el pilono.}

Se adjuntan resultados en este caso de las envolventes de mínimos de construcción y máximos de servicio, que son aquellas que se consideran más representativas:

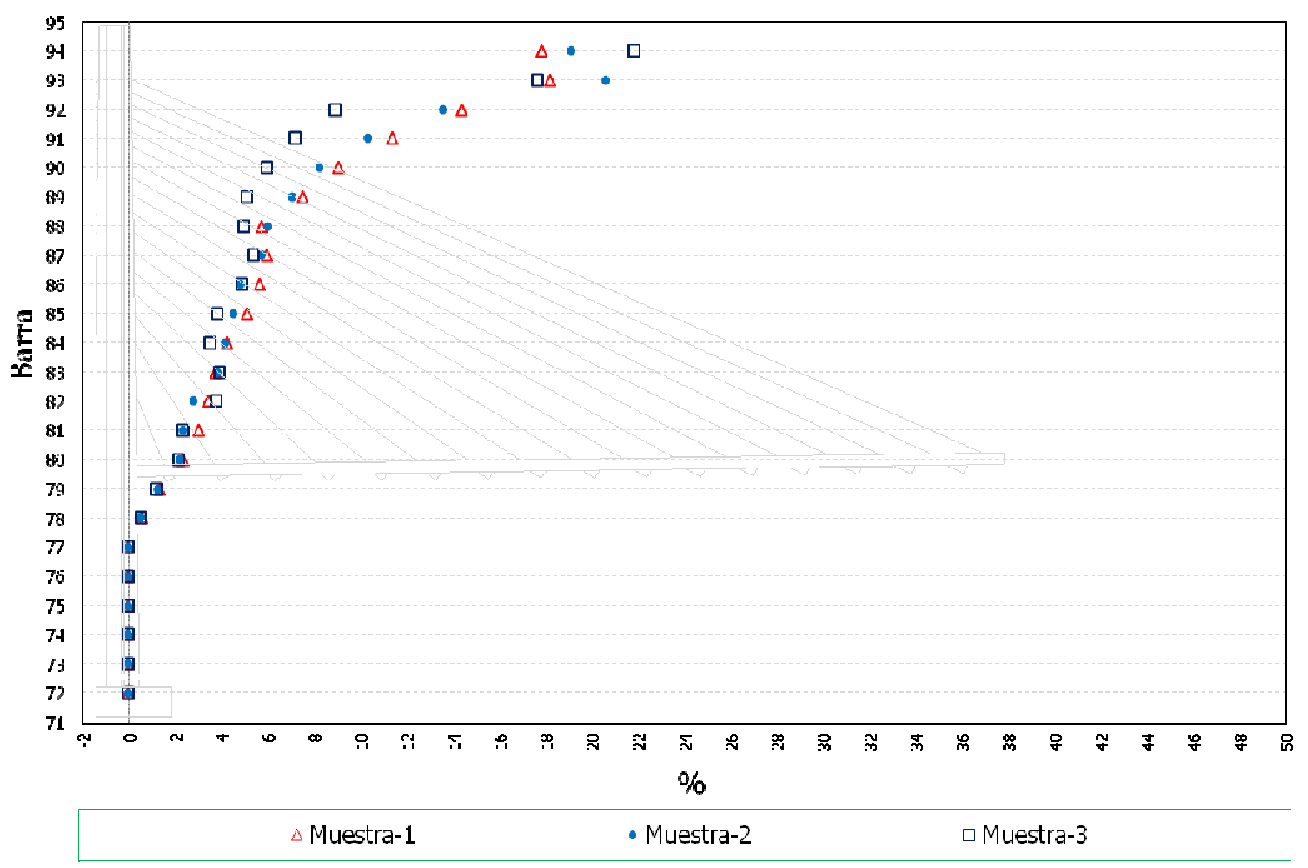

Figura 99. Flectores en pilono mínimos en fases de construcción. Diferencias en \% de los valores característicos inferiores respecto de los teóricos.

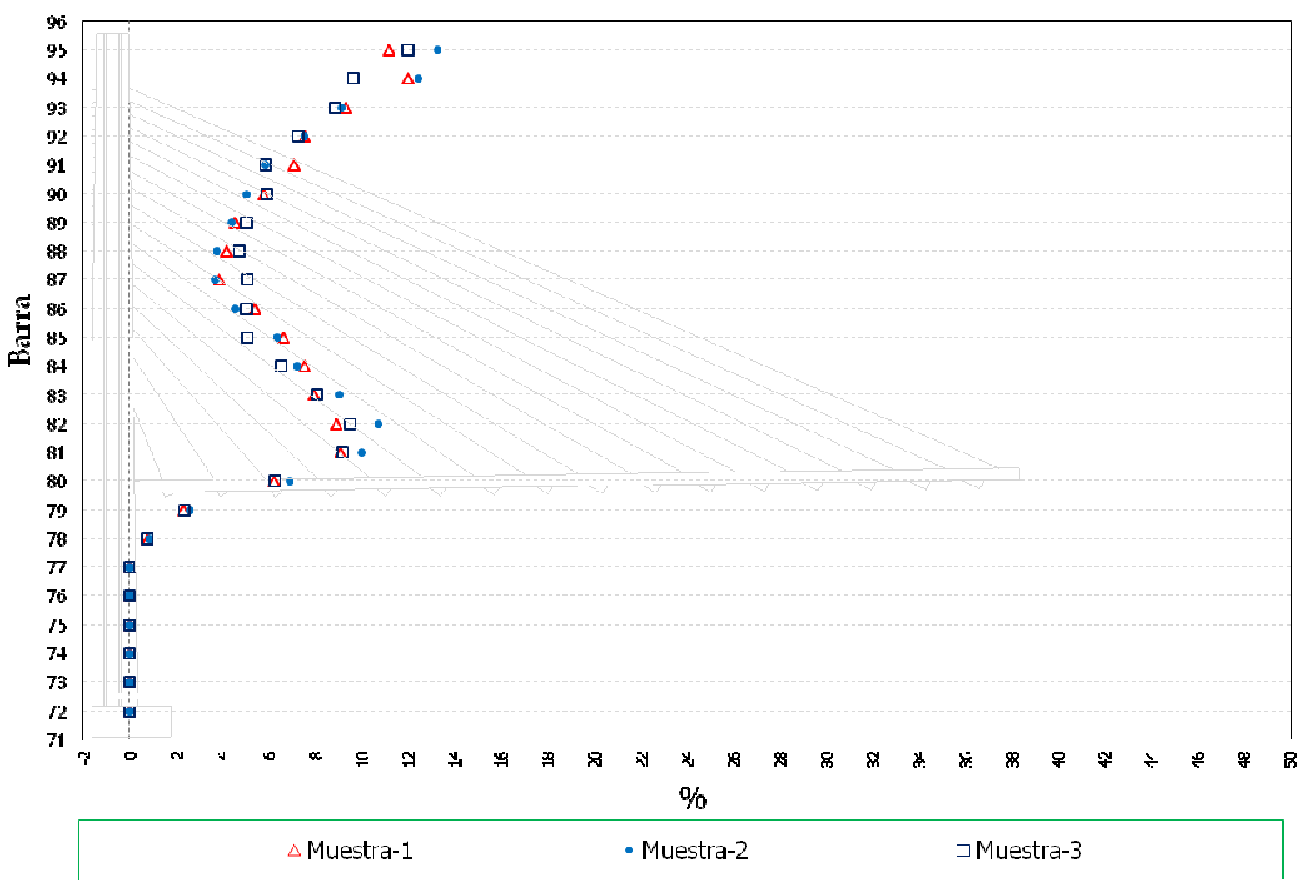

Figura 100. Flectores en pilono máximos en fases de servicio. Diferencias en \% de los valores característicos superiores respecto de los teóricos. 


\subsection{ANÁLISIS DE RESULTADOS.}

\subsubsection{Resultados con errores en fuerzas de tesado.}

En el caso de los axiles en los tirantes se puede indicar que:

- Los errores en los axiles en la fase final alcanzan valores de hasta un 8\% del valor teórico, siendo mayores los errores en el caso de los valores característicos inferiores.

- En el caso de las envolventes de axiles en las fases de construcción, se observa claramente que el axil máximo queda muy condicionado por el peso de las dovelas y por lo tanto, el efecto aleatorio introducido en los cables no tiene apenas significación en este caso. No ocurre así en el caso de los axiles mínimos de construcción, donde los errores llegan a alcanzar porcentajes de hasta un $10 \%$.

- Por otra parte en el caso de los axiles en servicio, ocurre algo similar al caso de los axiles finales, siendo mayor el efecto de la aleatoriedad sobre los axiles mínimos en lugar de los máximos, dado que en estos últimos influye, en cierta medida, el valor de la sobrecarga aplicada, sobre la que no existe incertidumbre. Los errores en el caso de los axiles mínimos alcanzan hasta un $10 \%$.

- Por otra parte, los errores en todos los casos parecen mayores en los primeros cables instalados, donde el efecto de la menor longitud del cable supone una mayor repercusión de las imperfecciones en la ejecución simuladas.

- $\quad$ Finalmente indicar que las diferencias entre los valores obtenidos con las muestras diferentes calculadas no son significativas, mostrando unas tendencias más o menos de igual cuantía, lo que estará avalado por su carácter aleatorio y por lo tanto validaría estos resultados obtenidos.

Por otra parte en el caso de los resultados en flectores del tablero:

- Se observa que existe una gran influencia en la situación final del proceso constructivo, que queda relativamente alejado de la situación objetivo de la viga continua.

- En cambio, de cara a las envolventes que verdaderamente dimensionarían el tablero, mínimos de construcción y máximos de servicio, las diferencias obtenidas con la introducción de errores de montaje resultan del orden del 5-7\% para los flectores mínimos de construcción y del orden de hasta un 20-30\% en el caso de los máximos de construcción de servicio. 
- Señalar eso sí, que la cuantía de las desviaciones es más o menos uniforme en todo el tablero, pero esta cuantía representa valores muy altos porcentualmente en la zona de tablero donde los valores teóricos son más bajos.

Por último, en el caso de los flectores en el pilono se puede indicar que:

- En primer lugar, los flectores en las barras del pilono situados por debajo del tablero no se ven afectados por los errores de montaje de los cables, permaneciendo iguales en las 3 muestras generadas a los valores teóricos. Dicho resultado era previsible dada la conexión entre tablero y pilono.

- En las barras situadas por encima del tablero las diferencias aumentan de forma sustancial con la altura del pilono para los flectores mínimos en fases de construcción, alcanzando porcentajes cercanos al $40 \%$ en las barras superiores. Hay que indicar de nuevo que estos resultados lo son en porcentaje, resultando por ello unos valores muy elevados en el caso del extremo superior del pilono donde los valores teóricos de los flectores son muy pequeños.

- En cuanto a los flectores máximos en servicio, se observa un crecimiento del error de hasta un $10 \%$ en las primeras barras sobre el tablero respecto del valor teórico, manteniéndose constante esta proporción en la zona central, para volver a elevarse alcanzado errores de hasta un $20 \%$ en las 4 últimas barras.

\subsubsection{Resultados con errores en deformaciones.}

En el caso de los axiles en los tirantes:

- Los errores en los axiles en la fase final alcanzan valores de hasta un 4\% del valor teórico, siendo similares los niveles de error para el caso de los máximos y mínimos de la situación final.

- Al igual que en el caso anterior, se observa que los axiles mínimos de construcción tienen porcentajes de error, de hasta un $8 \%$, superiores a los de los máximos.

- En el caso de los axiles en servicio los valores de los errores finalmente generados en los axiles en los tirantes quedan en el orden de entre un 3 y un $4 \%$, siendo algo mayores en el caso de los mínimos.

Por otra parte en el caso de los resultados en flectores del tablero:

- También se observa que existe una gran influencia en la situación final del proceso constructivo, que queda relativamente alejado de la situación objetivo de la viga 
continua. El efecto es más acusado en la zona situada a 3/4 de la luz, donde el esquema de atirantamiento es menos eficaz y la flexibilidad del tablero por lo tanto elevada.

- Los efectos en las envolventes de construcción y servicio son más reducidos que en el caso anterior, encontrándose las mayores desviaciones en aquella zona del tablero donde la rigidez del sistema de atirantamiento es más reducida (en los 3/4 de luz).

Por último, en el caso de los flectores en el pilono se puede indicar que:

- La forma de la distribución de los errores son semejantes a las obtenidas en el caso de errores en fuerzas.

- En primer lugar la no existencia de errores en los flectores de las barras situadas bajo el tablero.

- Crecimiento muy acusado, a partir de aproximadamente la mitad de altura del pilono, para llegar a alcanzar un $22 \%$ de diferencia en la barra superior. Que, eso si, es un porcentaje inferior al obtenido al aplicar el método en fuerzas.

- Respecto de flectores máximos en servicio, aunque la forma de la distribución de errores con la altura es semejante a la obtenida por el método de las fuerzas, el valor en la zona intermedia se sitúa en el $4 \%$ y los máximos superiores alcanzan el $14 \%$.

\subsubsection{Resultados con errores en posición de anclajes.}

En el caso de los axiles en los tirantes:

- Los errores en los axiles finales son mucho más acusados en el caso de los tirantes cortos, donde se llegan a alcanzar desviaciones de hasta un $20 \%$.

- En el caso de los axiles de construcción, la envolvente de máximos queda acotada en su desviación, pero en el caso de la envolvente de mínimos, los errores en los tirantes más cortos quedan con errores de hasta un $30 \%$.

- Los axiles en servicio muestran un comportamiento similar al caso de los axiles finales del proceso de construcción.

- En todos los casos se observa una cuantía de errores muy superior en los cables instalados, dado que la cuantía de error máximo admisible adoptada es constante en todos ellos, representando así un efecto mucho mayor en los cables cortos iniciales que 
en los cables finales. Es decir que este resultado llevaría a la idea de que debería ser necesario un control de ejecución más riguroso en los cables iniciales que en los finales.

En el caso de los flectores en el tablero:

- Los flectores en la situación final resultan con una desviación importante respecto de la situación teórica de la viga continua. Este efecto es más acusado en los tirantes iniciales donde el error de posición en los anclajes es más acusado.

- En las envolventes de flectores en la situación de construcción y servicio el efecto de la aleatoriedad queda amortiguado y al igual que ocurre con los tirantes se acentúa en la zona de tablero donde anclan los cables más cortos.

Por último en el caso de los flectores en el pilono se puede indicar que:

- Comparando con los casos anteriores de introducción de errores en fuerzas y deformaciones, se aprecian diferencias en la forma de las distribuciones de errores en las barras situadas por encima del tablero.

- $\quad$ Para los flectores mínimos en las fases de construcción se produce un crecimiento inicial de hasta el $8 \%$ en la zona intermedia, para alcanzar de forma exponencial el 22\% en la barra superior, justo bajo los tirantes más largos 18D y 181.

- En cuanto a los flectores máximos en servicio, se alcanza en la $4^{a}$ barra por encima del tablero un porcentaje máximo de en torno al 12\%, para disminuir en las 6 barras siguientes hasta el 5\% de error, y volver a aumentar hasta llegar al 16\% respecto del valor teórico.

\subsubsection{Comparación de resultados.}

A la vista de los resultados anteriores se puede concluir que:

- $\quad$ El método que introduce errores en fuerzas de tesado produce en general mayores desviaciones en los esfuerzos en tirantes, tablero y pilono que el caso de los errores en deformaciones. Estas diferencias resultan:

$\checkmark$ Relativamente acotadas en tirantes.

$\checkmark$ Importantes en la situación final del tablero, lo que tendrá implicaciones en la configuración geométrica finalmente alcanzada, pero no en el dimensionamiento de este elemento. 
$\checkmark$ Acotadas en la mayor parte del tablero para las envolventes que lo dimensionarían (mínimos de construcción y máximos de servicio).

$\checkmark$ Significativas en aquellas zonas de tablero en las que las envolventes aportan valores teóricos reducidos.

Igualmente en el pilono se puede hacer unos razonamientos similares a lo señalado para el tablero.

- La introducción de errores en el replanteo de los anclajes provoca, para el caso de error de hasta $1 \mathrm{~cm}^{4}$, esfuerzos en los tirantes con mucha mayor desviación que las generadas en los métodos anteriores, no así en el caso de los flectores en el tablero. Es decir, que como ya indicaban diversos autores [73][45], estos errores invalidan la aplicación del método en deformaciones salvo que se realice un control muy preciso del replanteo de dichos puntos durante todo el proceso constructivo.

- $\quad$ Es importante también hacer notar que el número de iteraciones que ha sido necesario realizar para obtener muestras normales es muy sensible a los valores adoptados para la desviación típica de las muestras. Por otra parte sabiendo que a menor desviación típica la muestra quedará menos dispersa respecto del valor medio, esta cuestión puede ser la causa de las diferencias encontradas entre el método de introducción de errores en fuerzas y en deformaciones. Ya que, aunque se ha intentado ajustar el valor de la desviación típica adoptada en cada caso para que se alcanzaran el mismo número de iteraciones en la obtención de muestras, sin embargo, dada la sensibilidad a este parámetro del proceso, finalmente las muestras en deformaciones han necesitado un número de iteraciones de en torno a 600, mientras que en el caso de las fuerzas, estos valores se han quedado en unas 500. Se podría así pensar que al ser más difícil encajar las muestras en deformaciones estas se habrán desviado porcentualmente menos respecto del valor teórico.

\footnotetext{
${ }^{4}$ Este valor requerirá de un control geométrico de la ejecución muy exigente, dado que las tolerancias habituales para la ejecución de los elementos de hormigón raramente alcanzan esta precisión, tanto por las propias características del material como por su forma de ejecución.
} 


\subsection{ANÁLISIS EN DEFORMACIONES.}

Como complemento al anterior análisis en esfuerzos, se presenta en este epígrafe un estudio de la implicación en deformaciones verticales que tiene la introducción de errores aleatorios sobre el montaje de los cables.

De esta forma, se han estudiado en las 2 fases del proceso constructivo que se consideran más representativas de cara al análisis a realizar, representándose la deformada completa del tablero correspondiente a la máxima y mínima deformación característica. Se adopta dicho valor característico según las definiciones empleadas en el análisis en esfuerzos, con la intención de tener valores de las deformaciones verticales del tablero correspondientes a la introducción de errores de tesado de cuantía la indicada en los epígrafes anteriores y en relación con los valores de resultados presentados en esfuerzos.

Para cada una de las fases estudiadas se han evaluado los valores obtenidos en el nudo extremo de la dovela en la que se tesa el tirante correspondiente a esa fase, adoptándose dicho nudo como punto de referencia de la deformada del tablero completo y tomándose el valor característico en ese punto como base para la obtención de las deformadas que se analizan a continuación. Es decir que en cada fase estudiada se adopta un nudo de referencia, nudo en el que se evalúan los 100 valores de las deformaciones obtenidas en una muestra, eligiéndose el valor característico de todos ellos, y representándose la deformada del tablero correspondiente a ese elemento de la muestra en todos los nudos del tablero.

En concreto se han elegido 4 fases del proceso correspondientes a la instalación de 2 cables simétricos cortos (cables 7 Izquierda y 7 Derecha) y a la colocación de los 2 cables simétricos más largos (cables 18 Izquierda y 18 Derecha). Se pretende, de este modo, poder valorar qué cuantía de deformaciones verticales supone los errores aleatorios en las fases iniciales, cuando la deformabilidad del tablero no es aún elevada, y en las fases finales, cuando el tablero será muy flexible y por lo tanto sensible a la aplicación de estas fuerzas erróneas.

Los valores representados lo han sido analizando una sola de las muestras de valores aleatorios obtenidos anteriormente, ya que no se considera necesario, dada la intención con la que presentan estos resultados, incluir el análisis de las otras muestras 2 generadas.

De esta forma, en concreto las fases analizadas y los nudos correspondientes utilizados como referencia de los resultados son:

- Fase 24 - Tesado del cable 7 Izquierdo. Nudo de referencia 39.

- Fase 26 - Tesado del cable 7 Derecho. Nudo de referencia 38. 
- Fase 68 - Tesado del cable 18 Izquierdo. Nudo de referencia 95.

- Fase 70 - Tesado del cable 18 Derecho. Nudo de referencia 91.

Se pasa a continuación a presentar estos resultados, mostrándose en la parte superior de cada gráfico la deformada completa del tablero, correspondiente a la máxima y mínima deformación vertical característica en el nudo representativo de la fase analizada. En la parte inferior de este mismo gráfico se representan las diferencias con la deformada teórica de la fase del proceso constructivo analizada.

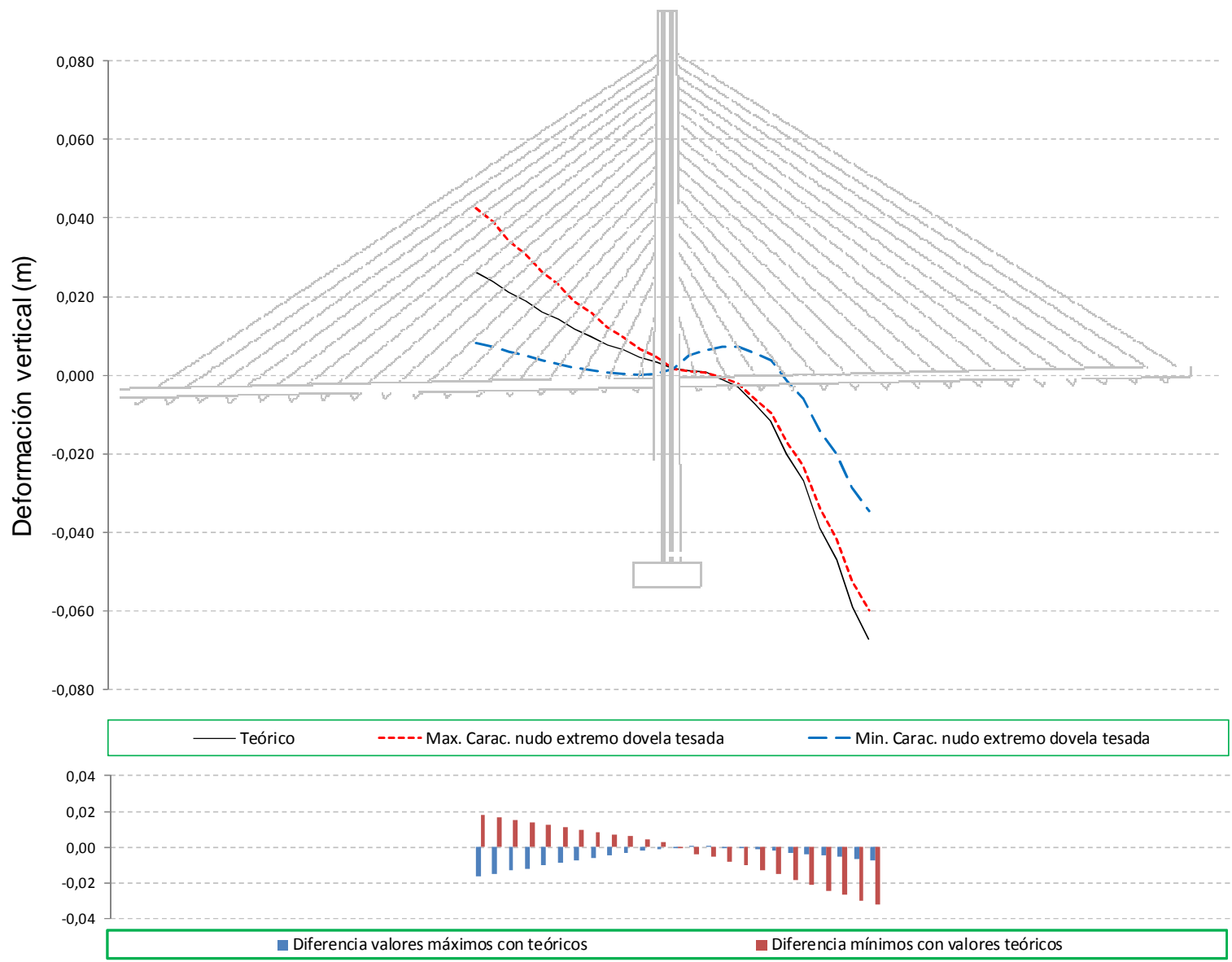

Figura 101. Deformaciones verticales del tablero para el caso de la fase 24 del proceso constructivo. Resultados correspondientes a la deformada obtenida a partir del valor característico del nudo 39 extremo de dovela tesada con el tirante 7 Izquierdo.

Hay que comentar que los resultados obtenidos llevan incorporado el efecto de la aleatoriedad de todos los axiles de montaje de los tirantes. Este aspecto puede causar que, por ejemplo en el caso del gráfico anterior, la deformada teórica en el extremo opuesto a la dovela tesada donde se localiza el nudo de referencia quede por debajo de las deformadas correspondientes al máximo y mínimo característico. Esta situación hay resaltar que sólo se ha producido en el análisis de las fases iniciales, en las que el efecto de la aleatoriedad es 
importante en relación al valor de las deformaciones verticales debidas al giro como sólido rígido del tablero alrededor del pilono, no siendo así en el caso de las fases avanzadas del proceso constructivo.

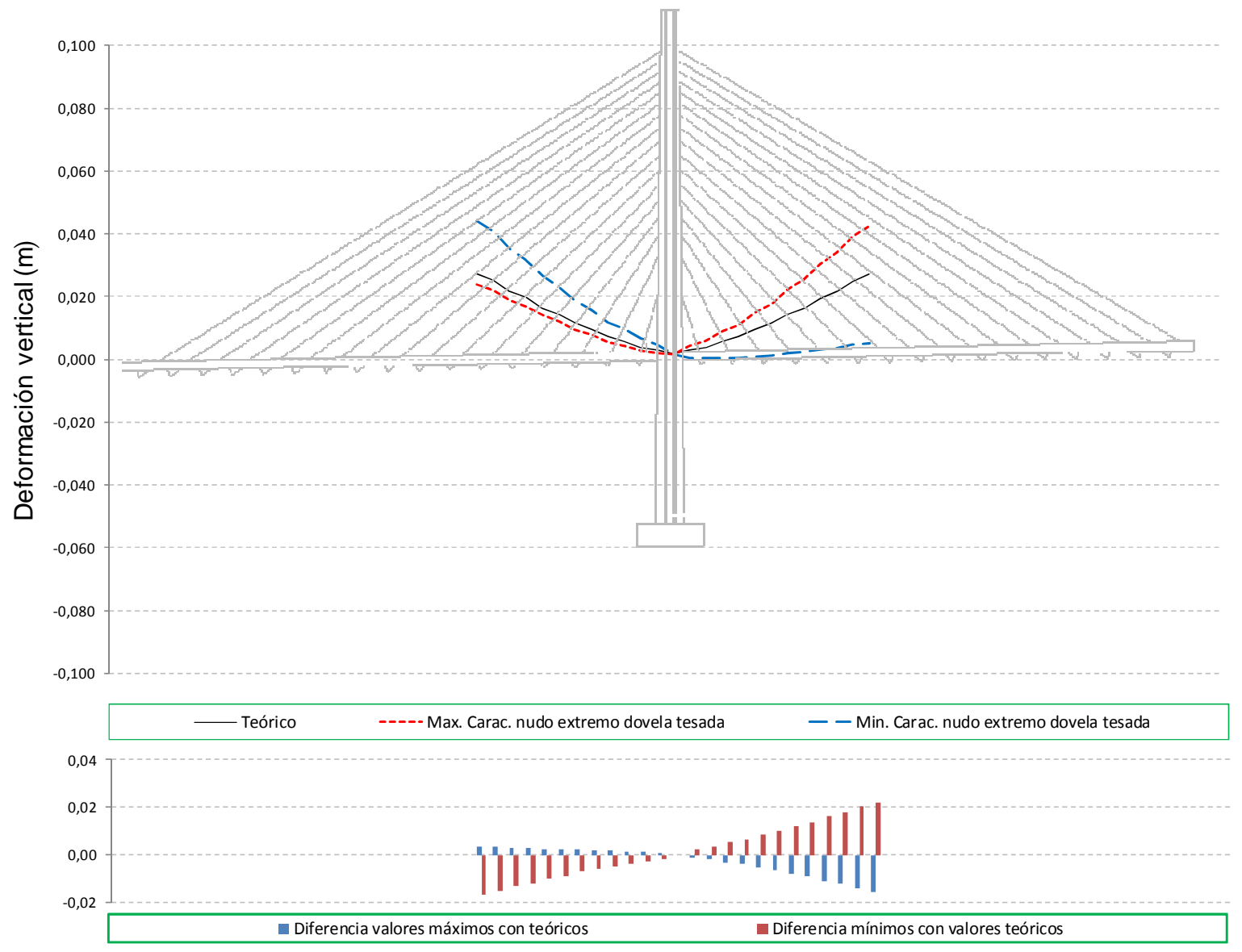

Figura 102. Deformaciones verticales del tablero para el caso de la fase 26 del proceso constructivo. Resultados correspondientes a la deformada obtenida a partir del valor característico del nudo 38 extremo de dovela tesada con el tirante 7 Derecho. 


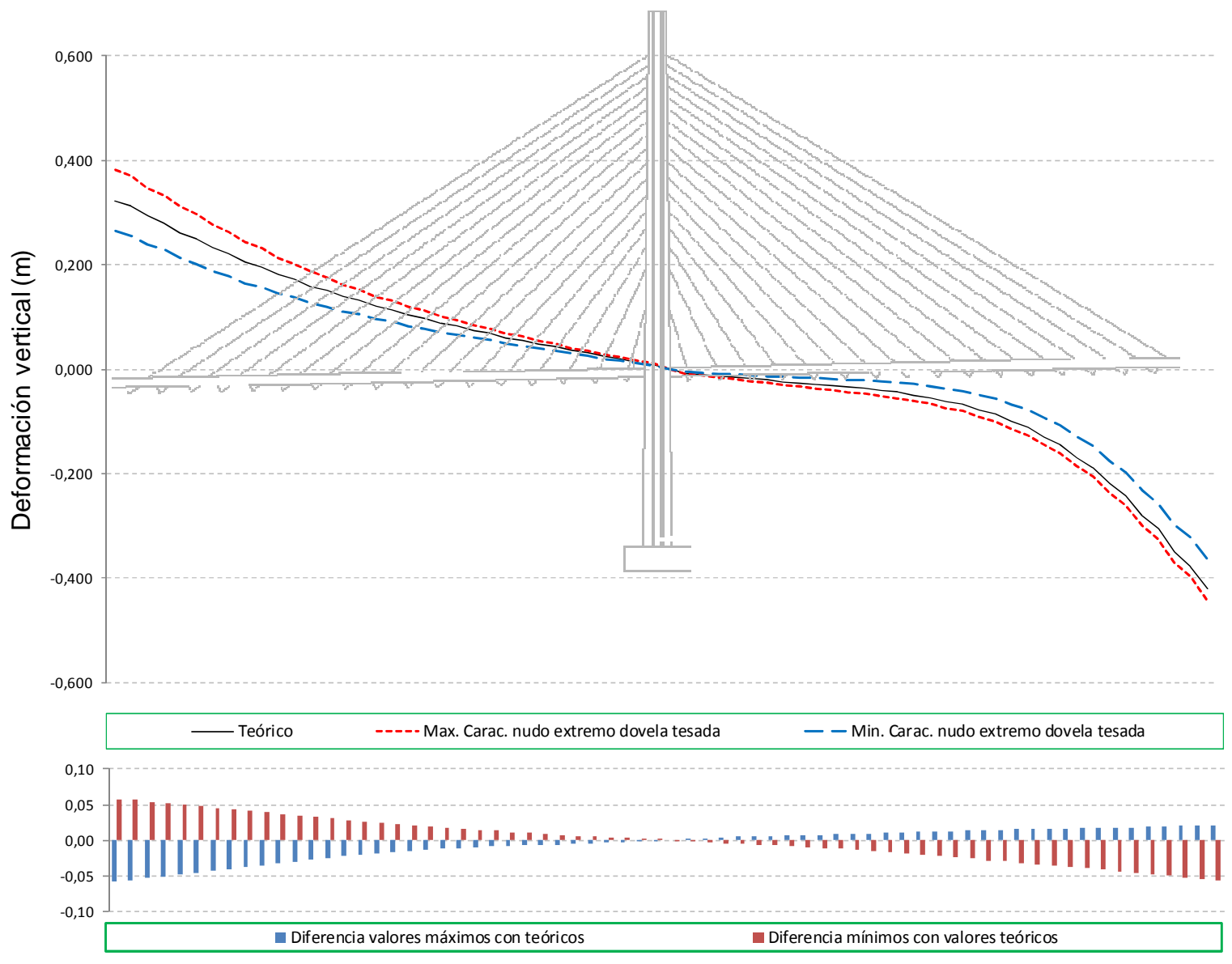

Figura 103. Deformaciones verticales del tablero para el caso de la fase 68 del proceso constructivo. Resultados correspondientes a la deformada obtenida a partir del valor característico del nudo 95 extremo de dovela tesada con el tirante 18 Izquierdo. 


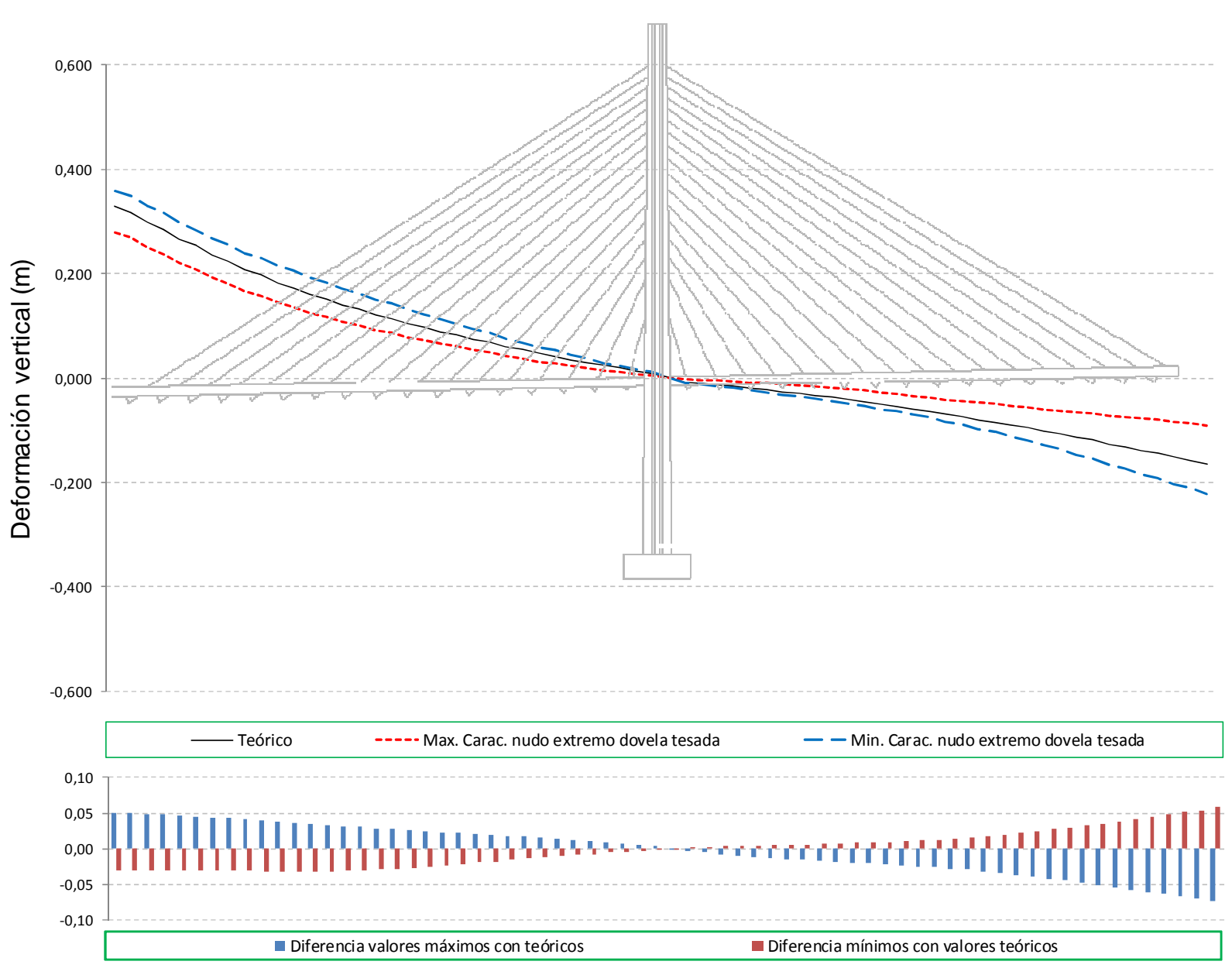

Figura 104. Deformaciones verticales del tablero para el caso de la fase 70 del proceso constructivo. Resultados correspondientes a la deformada obtenida a partir del valor característico del nudo 91 extremo de dovela tesada con el tirante 18 Derecho.

De todos los anteriores resultados se puede concluir que:

- La deformada obtenida a partir del valor característico máximo, generado en el nudo representativo de la fase del proceso constructivo analizada, lleva a una deformación por debajo del valor teórico en el extremo opuesto del tablero. Esta cuestión se justifica por el hecho de las deformadas no sólo tienen una componente parabólica correspondiente al caso de ménsula empotrada, si no que estas deformaciones incorporan un cierto giro alrededor del punto de encuentro del tablero y pilono.

- A la inversa resulta analizando la deformación mínima característica del nudo representativo. 
- En las primeras fases de cálculo las deformaciones, en el extremo del tablero ejecutado, son del orden de centímetros, siendo las diferencias respecto del valor teórico menores de 2'0 centímetros.

- En las fases finales del proceso constructivo las deformaciones son mucho más altas, dada la gran flexibilidad de la estructura, con valores de hasta $40 \mathrm{~cm}$, siendo las diferencias con los valores teóricos del orden de $5^{\prime} 0 \mathrm{~cm}$.

Todos estos resultados muestran que en las primeras fases del proceso constructivo será muy difícil detectar los errores en el montaje de los cables, mediante el seguimiento topográfico del alzado del tablero construido, ya que diferencias como las indicadas se pueden producir muy fácilmente por otros motivos además de por un proceso de montaje erróneo.

En las fases finales en cambio, si se podrá más fácilmente detectar estos errores controlando la nivelación del tablero, con lo que también sería más sencillo pensar que podría intentar corregirse este error.

La forma de corregir estas deformaciones erróneas, una vez detectada y conocida su causa, será proceder a compensar dichas desviaciones mediante el posicionado del carro de avance de las dovelas que queden por ejecutar. De esta forma, y como se indica en [102], no se introducirán esfuerzos parásitos sobre la estructura pero si quedarán las deformaciones producidas hasta el momento de la detección del proceso erróneo marcadas en la configuración geométrica final del tablero. Es decir que éstas se podrán compensar en parte pero en ningún caso eliminarlas por completo.

Indica además [102], que en ningún caso se deberá intentar corregir estas deformaciones mediante el ajuste de las fuerzas aplicadas en los cables. Igualmente comenta que si las dovelas no fueran hormigonadas "in situ" y fueran prefabricadas, éstas deberían ejecutarse con un control geométrico muy preciso, ya que en este caso no se podrán corregir las deformaciones que se pudieran producir sin introducir sobre el tablero esfuerzos parásitos. 


\section{CAPITULO 5.}

\section{INFLUENCIA DEL NUMERO DE CABLES DE LA ESTRUCTURA.}

\subsection{INTRODUCCION}

En este nuevo capítulo, una vez analizada en el capítulo anterior la estructura original aplicando unos procesos de montaje erróneos sobre los tirantes, siguiendo los métodos en fuerzas y deformaciones de instalación de los cables, pasamos a realizar un nuevo estudio en ese sentido analizando la influencia del número de cables en los resultados.

Se pretende así, con este nuevo análisis, completar las conclusiones obtenidas anteriormente, valorando qué implicación tiene la reducción del número de tirantes respecto de la solución original, a igualdad del resto de parámetros del cálculo, dado que en este caso reducimos el número de elementos a los que aplicar el esfuerzo aleatorio, pero en cambio por otro lado sobre ellos se concentrarán esfuerzos de tesado de mayor cuantía.

El desarrollo seguido en este capítulo ha sido similar al del capítulo anterior, describiéndose en primer lugar la estructura que se va a calcular, obteniéndose los resultados teóricos que en ella resultarían de un proceso de montaje exacto, para a continuación proceder a aplicar los procesos de montaje erróneos procedimentados en el capítulo anterior, con errores aleatorios en fuerzas de tesado o en deformaciones de montaje de los tirantes, evaluándose así los resultados obtenidos en cada caso y comparándolos con los del capítulo anterior.

Finalmente se obtienen conclusiones de los resultados aquí presentados, analizando no sólo la influencia del método de instalación de los cables sobre esta estructura, sino que además se va a proceder a compararlos con los ya conocidos del capítulo anterior, de tal forma que se pueda tener una visión de en qué medida el número de tirantes modifica las conclusiones indicadas en aquel. 


\subsection{ESTRUCTURA A ANALIZAR.}

Como ya se ha indicado, la geometría de la estructura a estudiar en este capítulo es idéntica a la del capítulo anterior, con la única diferencia en el número de tirantes, el cual se ve reducido a la mitad al eliminarse uno de cada dos tirantes de la estructura original. Resulta así una separación entre anclajes de tirantes en el tablero ampliada hasta 10'0 m. Por otra parte, señalar que, respecto de la estructura anteriormente calculada, no se han modificado ni la geometría del pilono ni la sección del tablero. Este último parámetro se decide no modificarlo en base a que al ser el ancho del tablero original de 13'80 m, manteniéndose idéntico al de la estructura del capítulo anterior, será esta dimensión la que siga condicionando el canto del tablero, al seguir siendo esta medida mayor que la distancia entre los anclajes de los cables.

Esta condición se ha adoptado en concordancia con el tipo de sección planteada para el tablero, tipo losa aligerada, pudiendo no ser realista para otros tipos de secciones. En cualquier caso, y dado el objetivo que se busca con esta investigación, no se considera necesario modificar la sección transversal en aras a simplificar la interpretación de los resultados, así como a facilitar la comparación con lo mostrado en el capítulo anterior.

Para facilitar la interpretación de los resultados obtenidos y poder referenciarlos con facilidad a una u otra estructura, en adelante a esta estructura se la denominará Modelo 90M, y así vendrá reflejado en adelante en el texto.

Indicar eso sí, que se han adaptado las áreas de los tirantes para conseguir que sigan soportando en las distintas fases de cálculo un nivel de tensiones similar al planteado en el caso de la estructura del capítulo anterior. De esta forma las propiedades de los distintos cables para este caso resultan: 


\begin{tabular}{|c|c|c|c|c|}
\hline Barra & Tirante & Longitud & Angulo & Area $\left(\mathrm{cm}^{2}\right)$ \\
\hline 88 & 27 & 13,8 & $-68,8$ & 89.2 \\
\hline 99 & 2 & 13,8 & 248,8 & 59,2 \\
\hline 102 & 45 & 23.3 & -49.9 & 66.5 \\
\hline 103 & 4 & 23,3 & 229,9 & 86,5 \\
\hline 106 & 62 & 33.0 & -40.7 & 97.8 \\
\hline 107 & 8 & 33,0 & 220,7 & 97,8 \\
\hline 110 & 85 & 43,1 & $-35,8$ & 10,3 \\
\hline 111 & 8 & 43,1 & 215,8 & $1 \cdot 0,2$ \\
\hline 114 & $10 \mathrm{D}$ & 63,4 & -326 & $1 \cdot 0,0$ \\
\hline 116 & 101 & 63,4 & 2126 & $1 \times 9,2$ \\
\hline 118 & $12 \mathrm{D}$ & 63,6 & $-30,2$ & 129,1 \\
\hline 119 & 12 & 63,6 & 210,2 & 128,2 \\
\hline 122 & $1 \angle D$ & 73,7 & $-28,1$ & 131,5 \\
\hline 123 & 14 & 73,7 & 208,1 & 135,3 \\
\hline 126 & "16D' & 83,8 & $-25,5$ & 162,6 \\
\hline 127 & 18 & 83,8 & 2035 & 147,7 \\
\hline 130 & $18 \mathrm{D}$ & 83,9 & $-25,1$ & 123,2 \\
\hline 13.1 & 18 & 93,9 & 2051 & 134,7 \\
\hline
\end{tabular}

Tabla 4. Denominación y áreas de los tirantes de la estructura Modelo 90M.

El anclaje de estos tirantes se localiza de nuevo a 2'00 m del frente de dovela, que ahora es de 10 '0 m de longitud, quedando modelizado el tablero por el mismo número de barras que en el caso anterior.

Por otra parte igualmente se han mantenido sin modificar tanto el esquema general del proceso constructivo, adaptando las fases al nuevo número de dovelas y cables de este caso pero siendo la secuencia general seguida la misma, como las vinculaciones entre tablero con pilono y estribos.

El modelo de cálculo de la estructura es de nuevo un modelo de barras plano, que se resuelve utilizando el mismo programa de cálculo matricial indicado en el capítulo anterior. En el anexo A.1, incluido al final del documento, se incluyen las características geométricas y mecánicas de los elementos que componen este modelo de cálculo, el cual se representa en el siguiente esquema: 


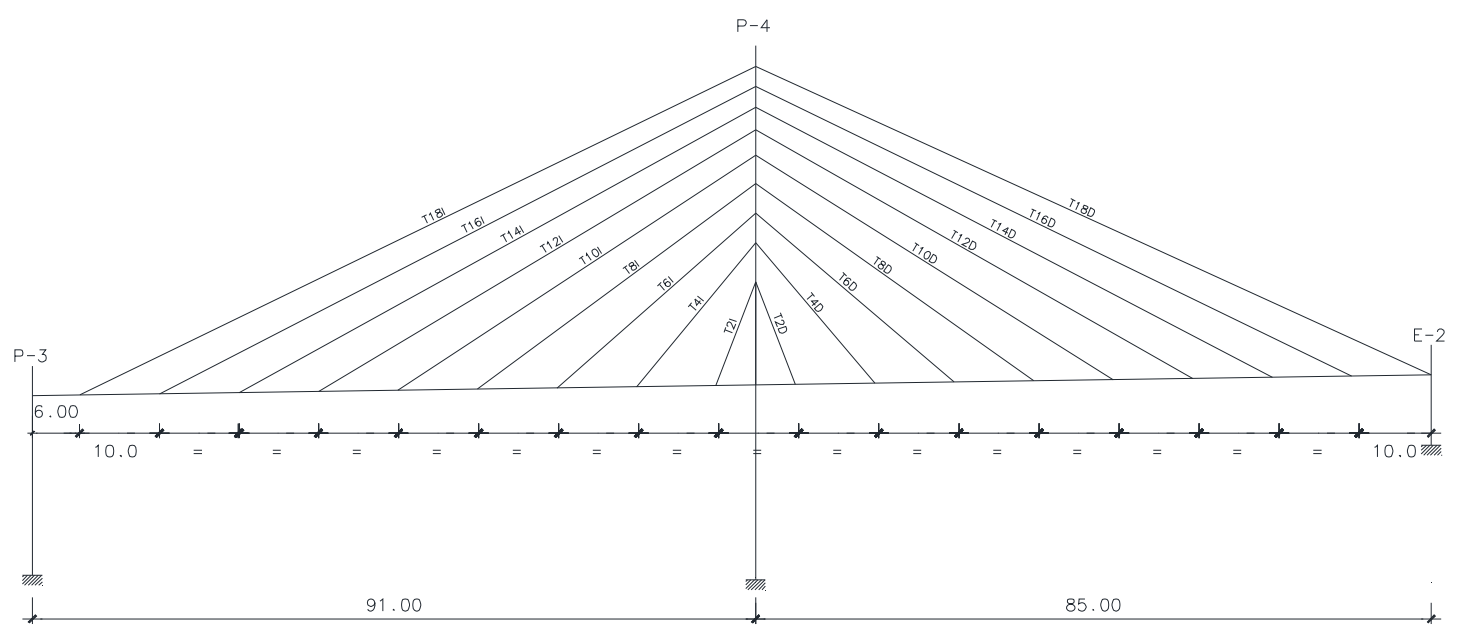

Figura 105. Esquema del modelo de cálculo 90M.

De la misma forma que en el caso anterior, sobre esta estructura se ha procedido a determinar las situaciones teóricas de referencia. Es decir, que se han obtenido los esfuerzos en los diferentes elementos estructurales como resultado del proceso teórico de construcción, el cual se ha simulado, igualmente, mediante un proceso de cálculo del montaje de la estructura automatizado mediante un archivo lógico, similar al utilizado en el capítulo precedente. Los resultados obtenidos utilizando este procedimiento lo son tanto en las situaciones intermedias de dicho proceso, como en la situación final al aplicar la carga muerta sobre el tablero, así como en las situaciones de servicio, bajo la actuación de la sobrecarga de tráfico rodado.

Los resultados que se representan en las siguientes figuras son aquellos que se han considerado más representativos del comportamiento teórico de la estructura. En concreto, en la primera de ellas (Figura 106) se representan los axiles de montaje, los máximos y mínimos de construcción, los axiles finales al terminar el proceso constructivo y los máximos y mínimos de servicio. En la segunda de ellas (Figura 107) se representan las envolventes de flectores en el tablero, tanto los máximos como los mínimos de construcción, los máximos y mínimos de servicio, al aplicar las sobrecargas de tráfico en el tablero, y la ley de flectores al final del proceso constructivo. En la última de ellas se representan los flectores en el pilono en las mismas situaciones anteriores (Figura 108) 


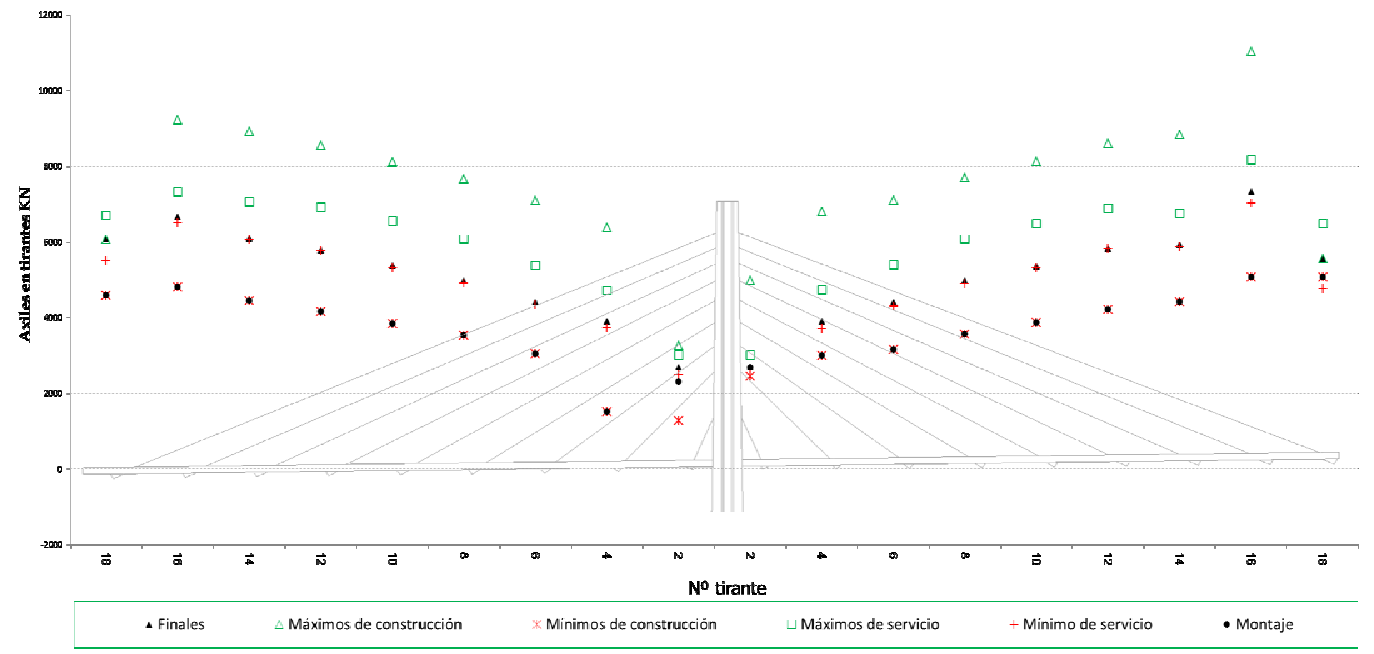

Figura 106. Axiles en tirantes en fase de inicial de instalación, máximos y mínimos de las fases de construcción, en la fase final del proceso constructivo y máximos y mínimos en las situaciones de servicio.

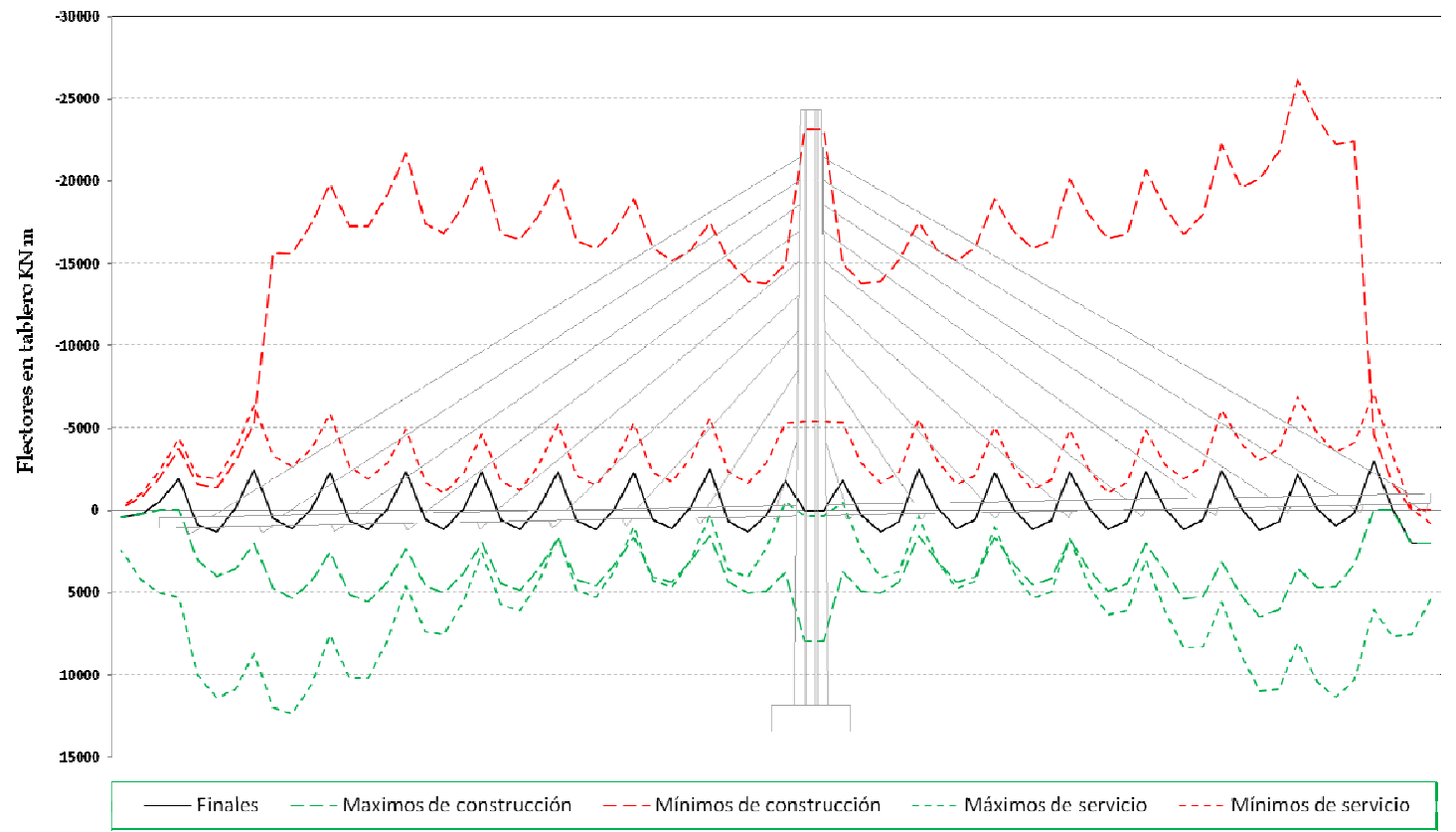

Figura 107. Envolvente de flectores en el tablero en las fases de construcción, final y en servicio. 


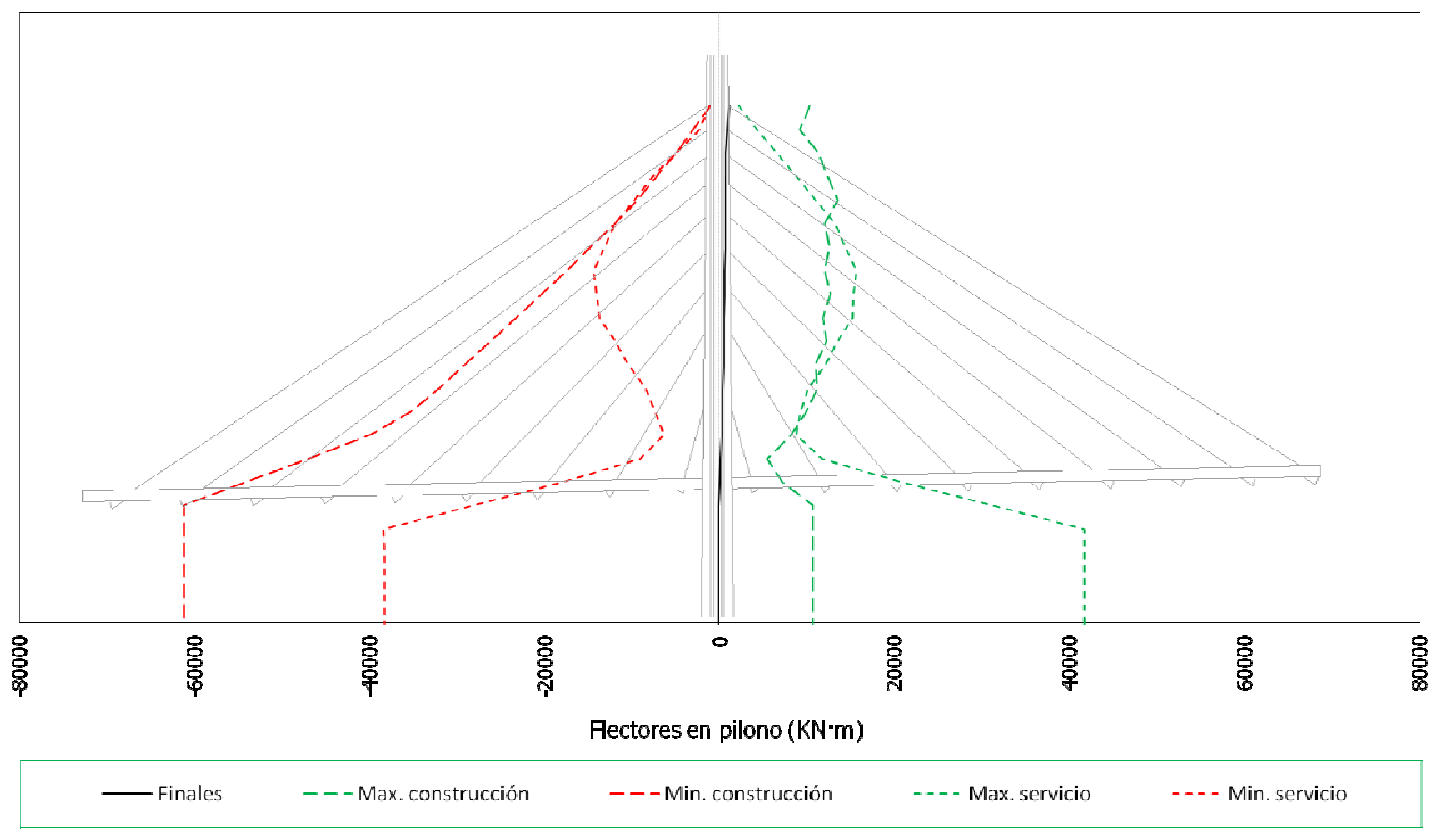

Figura 108. Envolvente de flectores en el pilono en las fases de construcción, final y en servicio.

De los resultados aquí representados se pueden extraer varias conclusiones en relación a los axiles en tirantes y a los flectores en el tablero y pilono. En el primer caso se puede deducir que:

- Que en general, el valor máximo del axil alcanzado en los tirantes se produce durante las fases de construcción, siendo el mínimo el producido en el instante de su colocación, valor este último que por lo tanto siempre es inferior al alcanzado en la situación final del proceso constructivo.

- Los valores obtenidos para los axiles de los tirantes representan en torno al doble de lo obtenido en el caso anterior del Modelo 90, lo que tiene cierto sentido al haberse duplicado la separación entre cables y haberse mantenido las cargas permanentes aplicadas sobre el tablero.

- Que al igual que en el caso anterior se presentan valores singulares en los axiles de los primeros cables 5 , dado el proceso constructivo adoptado para las primeras fases del tablero, así como en los cables extremos, donde igualmente el proceso de construcción y la dovela adicional del vano derecho condicionan los valores teóricos obtenidos.

Por otra parte, en el caso de los resultados en flectores en el tablero, se observa que:

\footnotetext{
${ }^{5}$ Aunque en este caso en menor medida que en el Modelo 90 dado el mayor peso de las dovelas iniciales.
} 
$\checkmark \quad$ La ley de flectores en la situación final de construcción representa unos valores iguales a los de la situación objetivo teórica planteada como viga continua, y por lo tanto sus valores absolutos resultan relativamente acotados. La envolvente total de flectores, al igual que en el Modelo 90, se conforma por los mínimos de la situación de construcción y por los máximos de la situación de servicio.

$\checkmark \quad$ Por otra parte, mientras que la envolvente de flectores positivos adopta valores muy parecidos a los del Modelo 90, con incrementos puntuales dada la mayor separación entre anclajes, en el caso de los flectores negativos los valores son muy superiores a los del Modelo 90, ya que en este caso el peso de las dovelas es muy superior y por lo tanto el valor de este esfuerzo resulta igualmente mucho más alto al estar principalmente dominado por esta acción.

Por último en relación a las envolventes de flectores en el pilono se puede indicar que:

- Al igual que ocurría en el Modelo 90, los flectores en el pilono en la situación final son prácticamente nulos.

- De nuevo la envolvente de flectores en fase de construcción condiciona los flectores negativos, dada la secuencia de construcción elegida de ejecución en primer lugar de las dovelas del vano izquierdo, quedando con valores por encima de las envolventes de servicio al aplicar las sobrecargas de tráfico. Los valores obtenidos son algo mayores que en el Modelo 90, lo que resulta lógico dado el valor más alto de los axiles de los tirantes.

- Las envolventes de flectores positivos en cambio sí queda condicionada por las situaciones de servicio.

- Los valores obtenidos para este caso son similares a los del Modelo 90, ya que al ser la cuantía total de las diferentes cargas aplicadas sobre el tablero (pesos propios, cargas muertas y sobrecargas de tráfico) de igual cuantía, el efecto producido por la transmisión de esas cargas sobre el pilono es similar, aunque está concentrado en menos puntos, dado que ahora el número de tirantes se ha reducido.

Se podría adicionalmente analizar qué ocurre con la parte pasiva y activa del axil de los tirantes. Es decir, que se puede comparar qué incremento de axil se produce desde el momento de la instalación de cada cable, respecto del axil final que resulta en cada uno, resultando así la siguiente gráfica (Figura 109): 


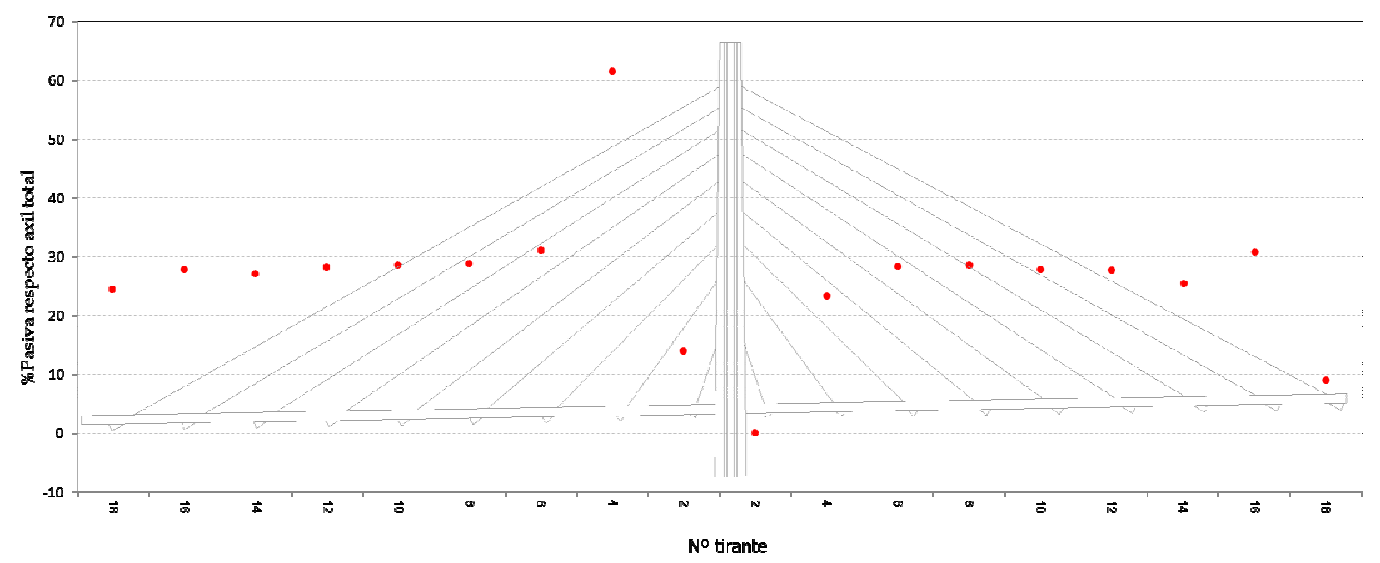

Figura 109. Porcentaje del axil pasivo respecto del axil total en los diferentes tirantes.

Igualmente del gráfico anterior se puede deducir:

- Que la evolución de los porcentajes del axil pasivo respecto del total sigue un esquema similar al del caso del Modelo 90, incluyendo los mismos valores singulares en los cables extremos e iniciales.

- Es significativo que la diferencia entre el axil aplicado al instalar el cable representa en este caso un porcentaje mucho mayor. En el caso del Modelo 90 este porcentaje era de $\approx 13 \%$ y en el caso de Modelo $90 \mathrm{M}$ este porcentaje aumenta hasta el $26 \%$. Es decir que en la componente final del axil del tirante, en este caso, la influencia del peso propio de las dovelas es mucho mayor dada la mayor longitud entre los anclajes de los cables. Se confirma así lo adelantado en el epígrafe 3.3, resultando además que la diferencia en el efecto de la presencia de un solo cable al caso analizado es de un incremento del axil pasivo del $13 \%$.

- Que este caso corrobora lo señalado por del Pozo [29] en relación a la vinculación existente entre el valor de la rigidez del tablero y la componente pasiva del axil del tirante. Es decir, que dado que el tablero dispone de la misma sección, y por lo tanto inercia, que el Modelo 90, habiéndose aumentado, eso sí, la separación entre anclajes, resulta entonces un elemento más flexible que en el caso del capítulo anterior, por lo que los tirantes en proporción deberán ser capaces de absorber una mayor cuantía de las cargas permanentes. En definitiva, que la pérdida de eficacia del mecanismo resistente por flexión del tablero provocará una mayor solicitación pasiva de los cables. 


\subsection{RESULTADOS OBTENIDOS.}

Se procede en este epígrafe a desarrollar los resultados correspondientes a la aplicación de los errores aleatorios de montaje, bien por el método de las fuerzas o bien por el método de las deformaciones. A diferencia del capítulo anterior en este caso no se ha considerado la introducción de errores en la posición de los anclajes dadas las conclusiones allí extraídas. Así los análisis realizados han sido:

- Error en la medida de las fuerzas de tesado.

- Error en la medida de las deformaciones de tesado.

Igualmente se ha utilizado la definición del valor característico ya reseñada, y se han definido las mismas situaciones de cálculo que en el capítulo anterior (situación final, situaciones de construcción y situaciones de servicio).

\subsubsection{Errores en fuerzas de tesado.}

En primer lugar se representan los resultados correspondientes a la introducción de fuerzas de tesado erróneas, habiéndose generado para ello de nuevo 3 muestras de 100 elementos, las cuales se aplicarán sobre la estructura al simular su proceso constructivo. Resulta así que finalmente se habrán realizado 600 cálculos individuales de la estructura.

Los resultados representados lo son de tirantes (Figura 110 a Figura 115), tablero (Figura 116 a Figura 118) y pilono (Figura 119 a Figura 120). 


\subsubsection{Axiles en tirantes.}

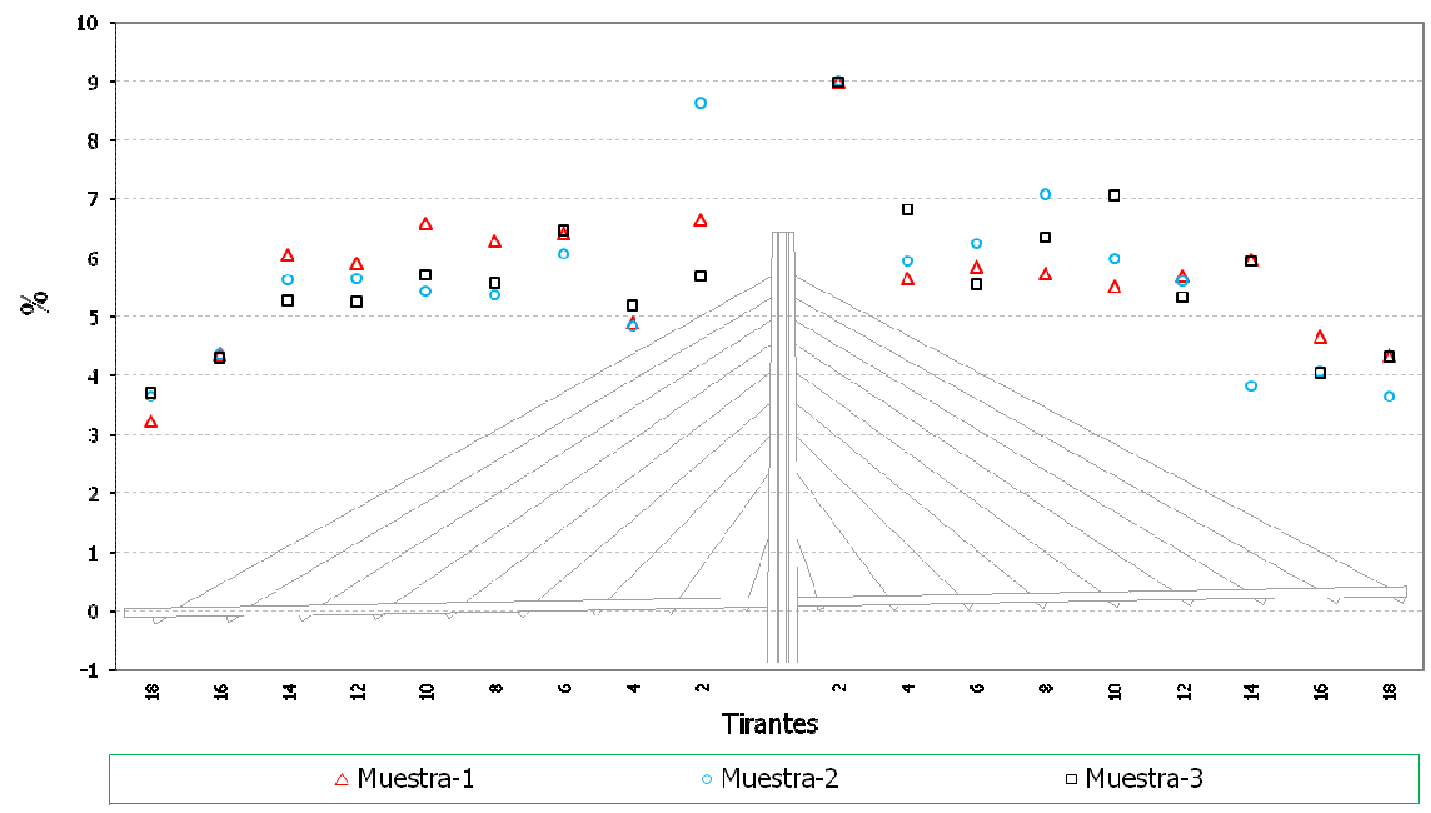

Figura 110. Axiles finales en los tirantes. Diferencia en \% de los valores característicos superiores respecto de los teóricos.

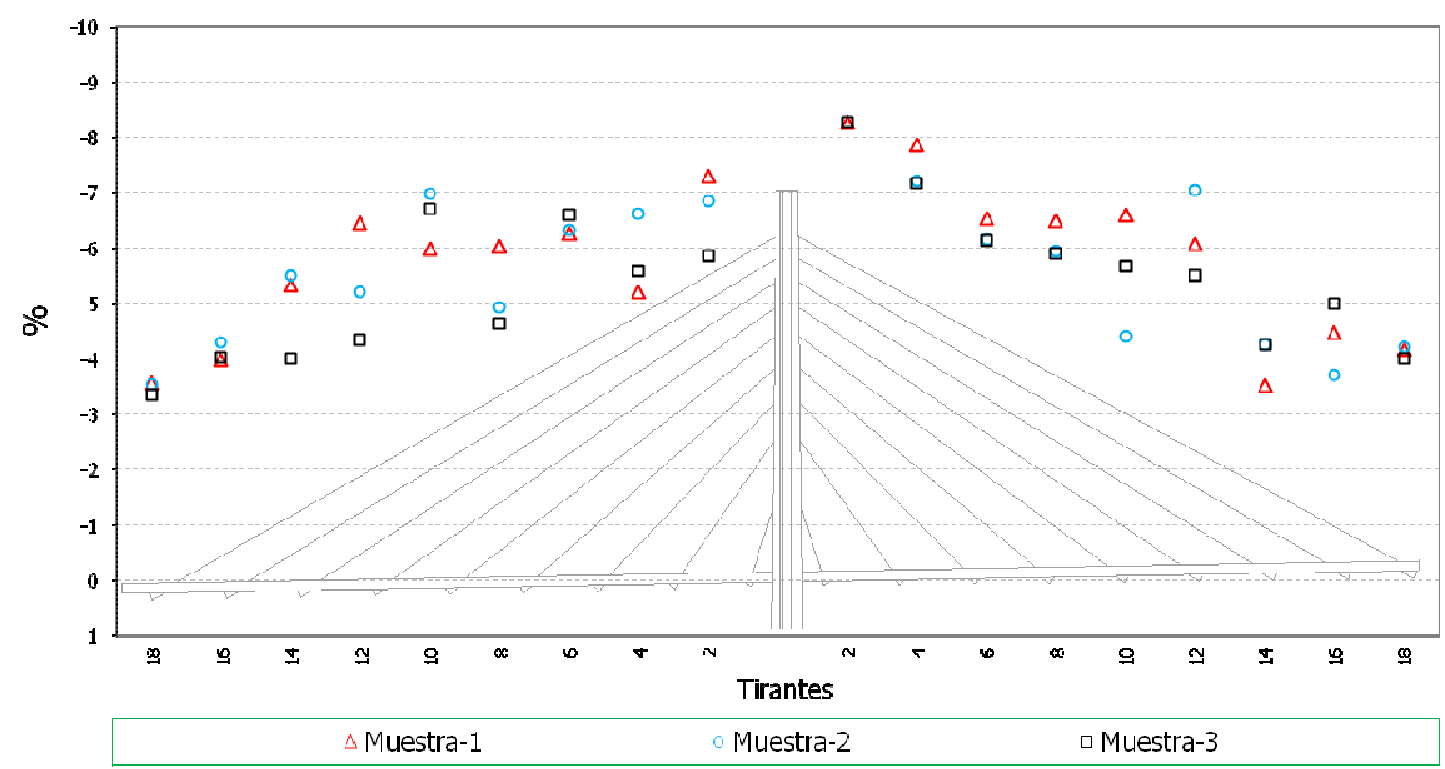

Figura 111. Axiles finales en los tirantes. Diferencia en \% de los valores característicos inferiores respecto de los teóricos. 


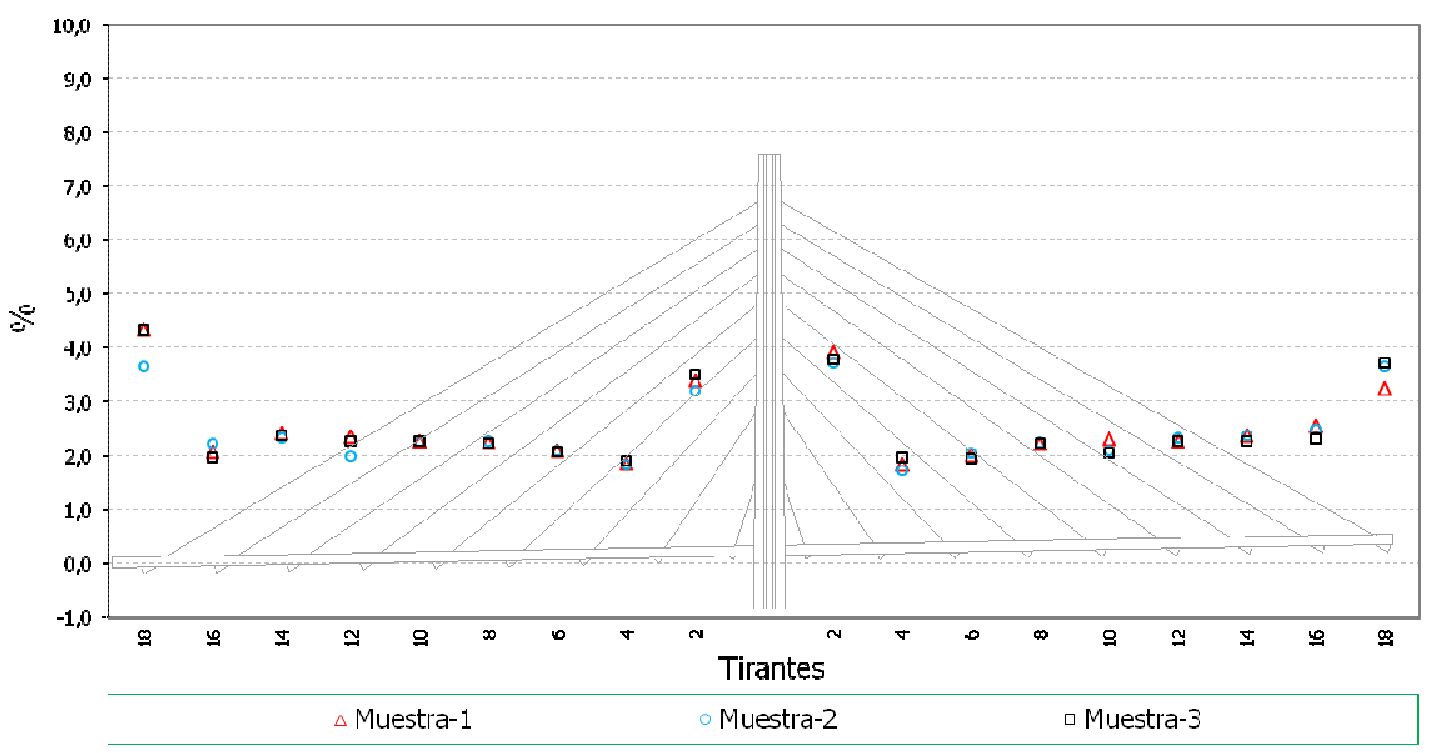

Figura 112. Axiles en los tirantes máximos de construcción. Diferencia en \% de los valores característicos superiores respecto de los teóricos.

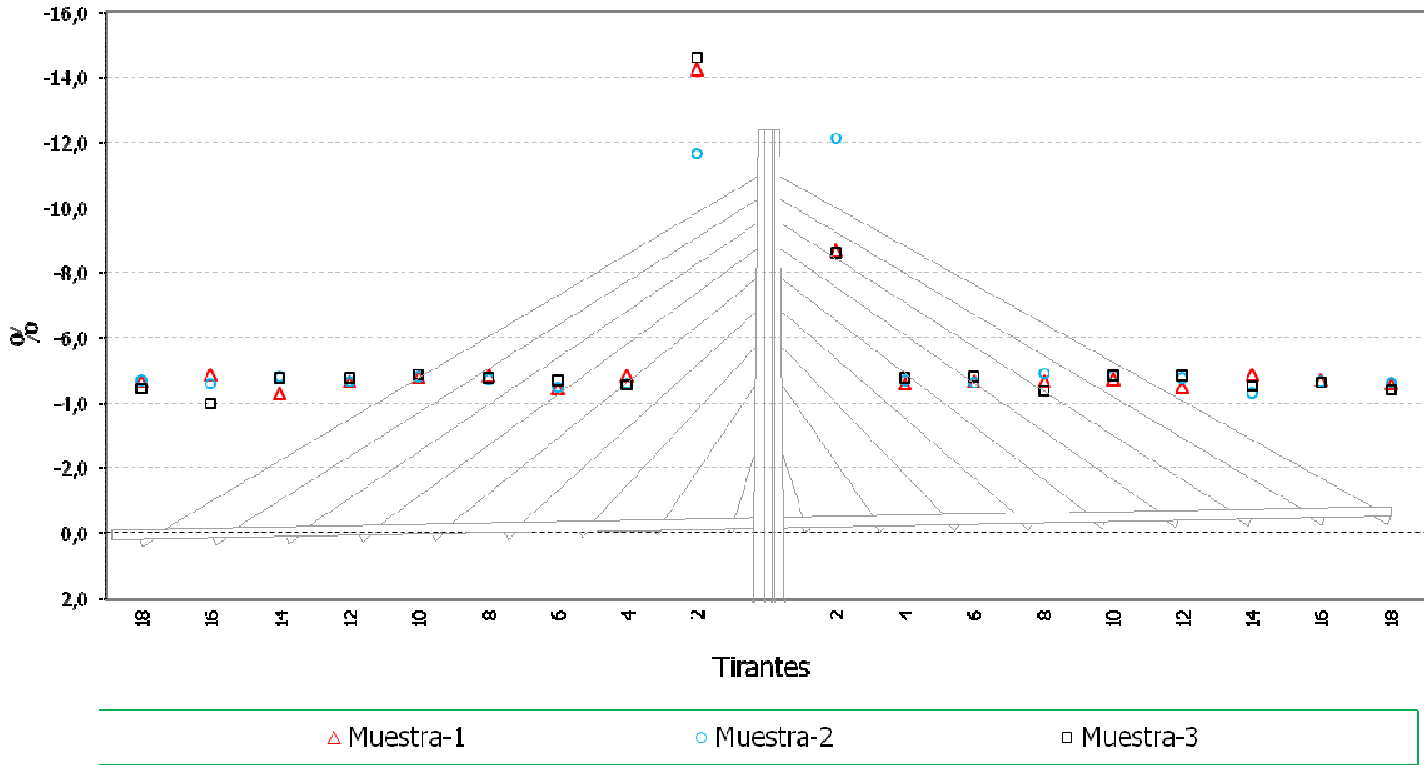

Figura 113. Axiles en los tirantes mínimos de construcción. Diferencia en \% de los valores característicos inferiores respecto de los teóricos. 


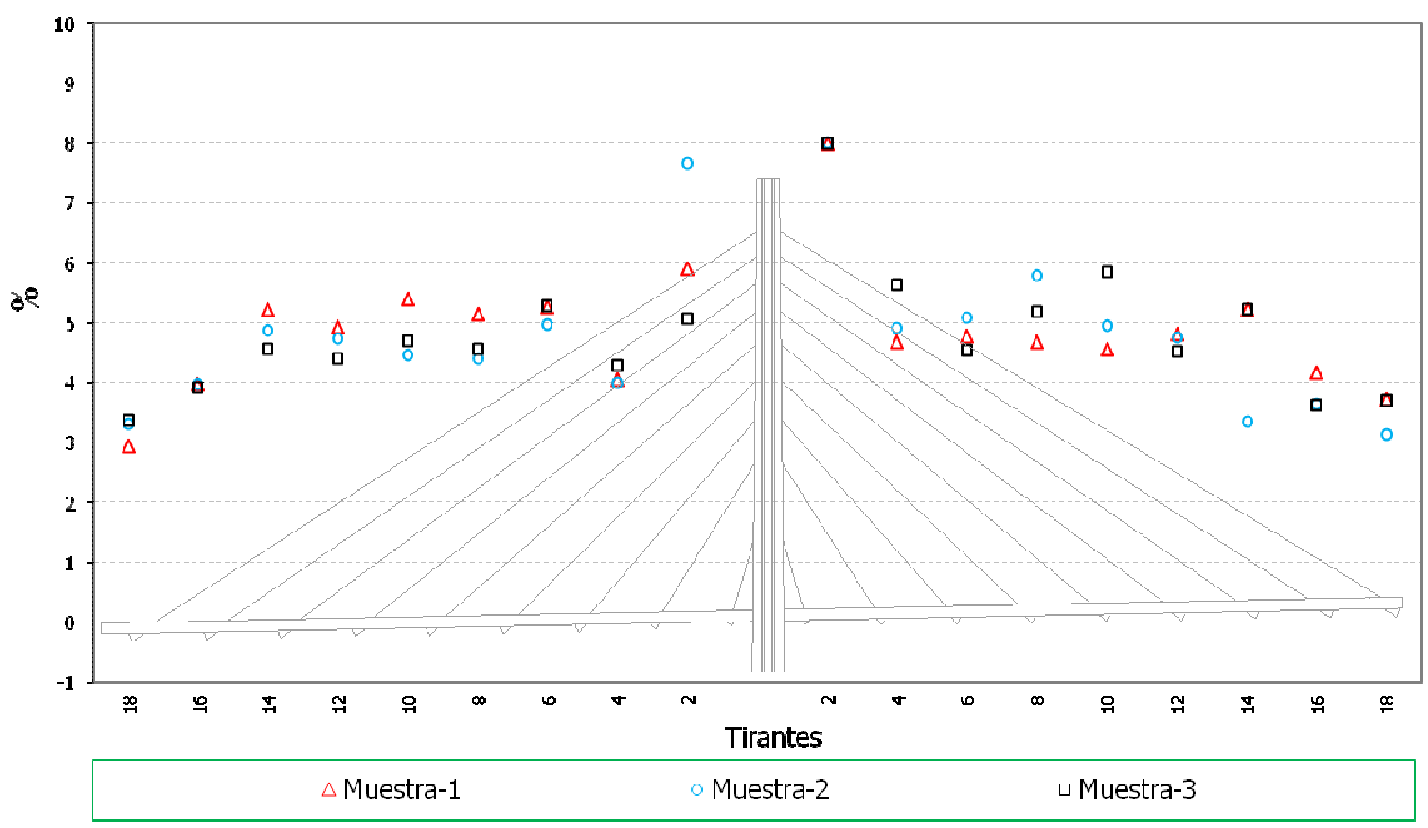

Figura 114. Axiles en los tirantes máximos de servicio. Diferencia en \% de los valores característicos superiores respecto de los teóricos.

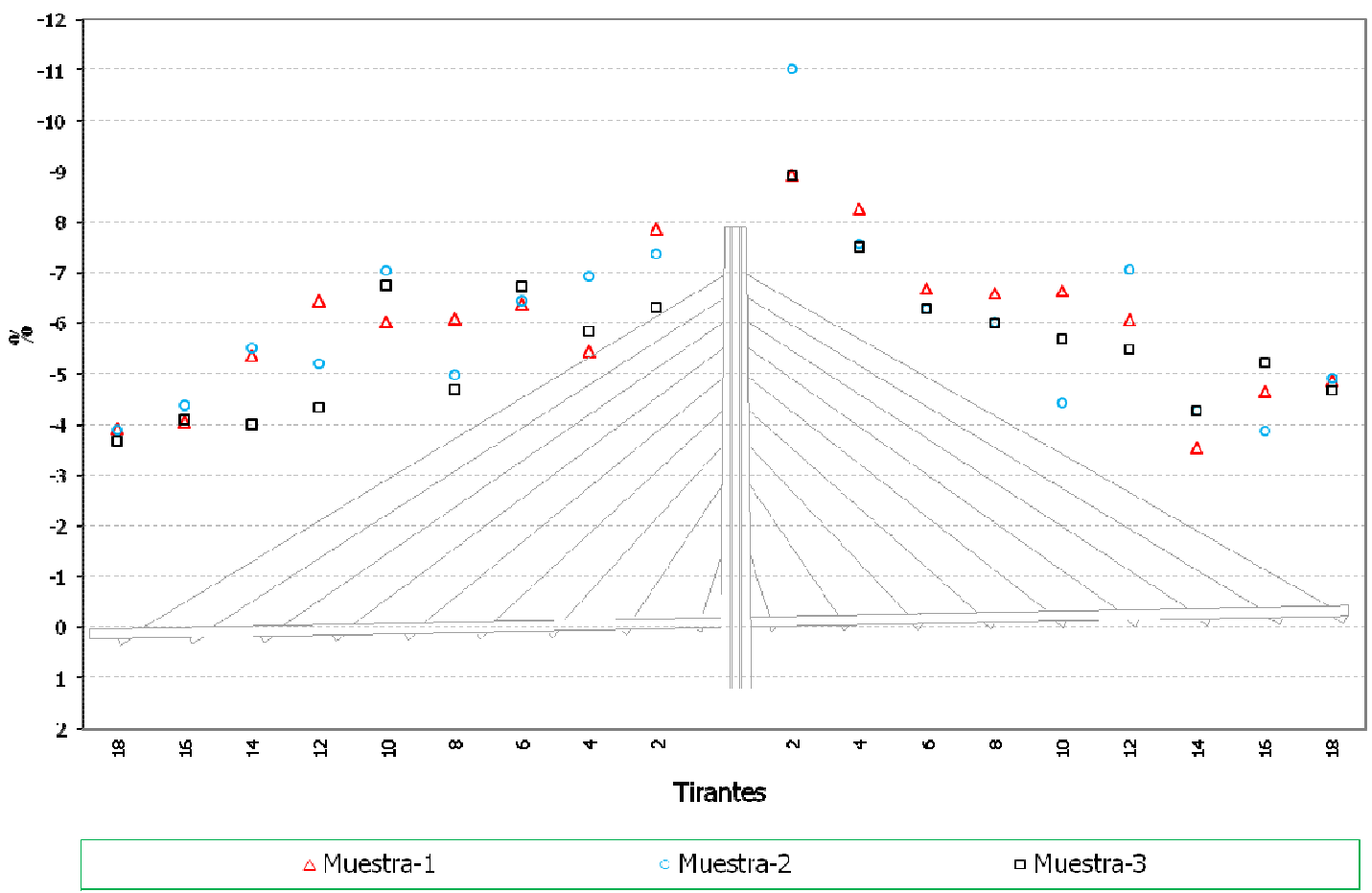

Figura 115. Axiles en los tirantes mínimos de servicio. Diferencia en \% de los valores característicos inferiores respecto de los teóricos. 


\subsubsection{Flectores en tablero.}

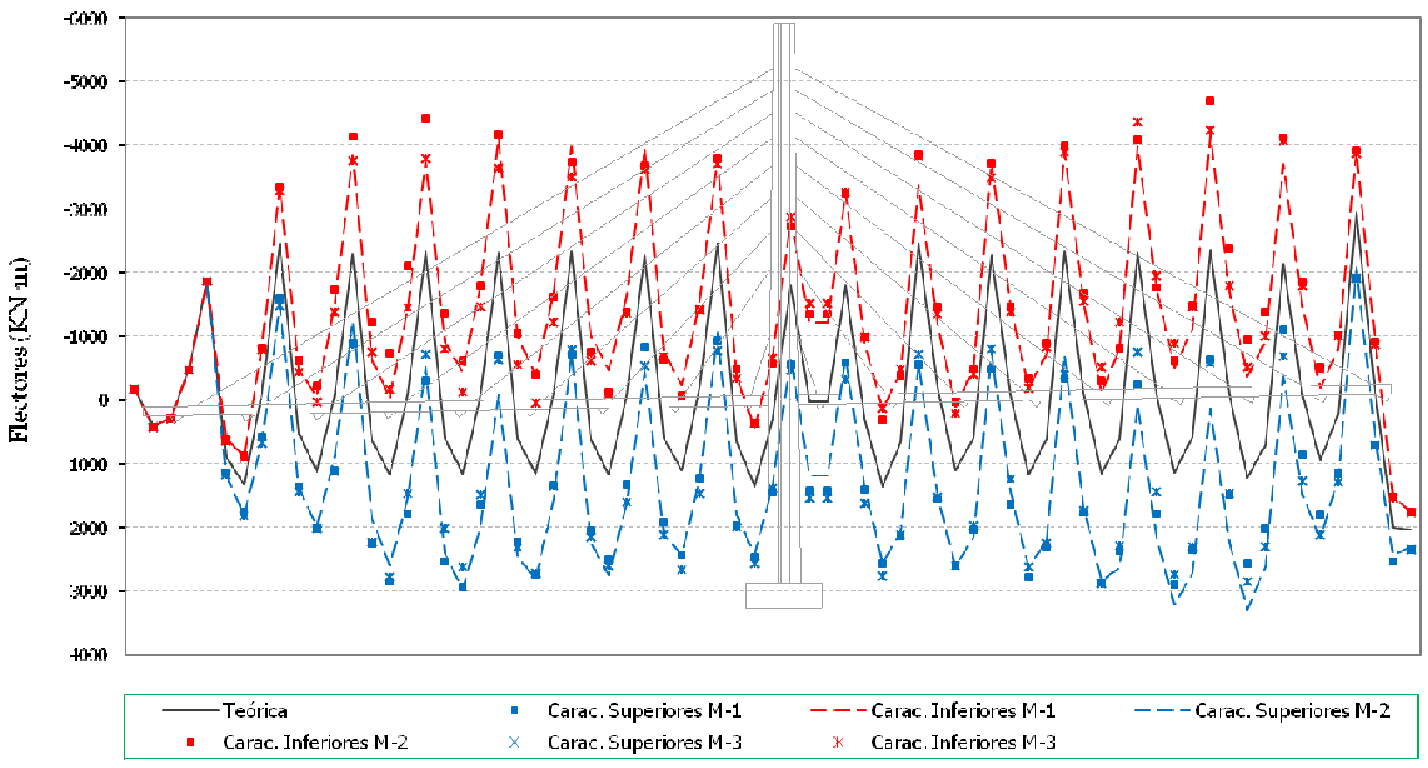

Figura 116. Flectores en tablero en situación final. Valores característicos superiores e inferiores.

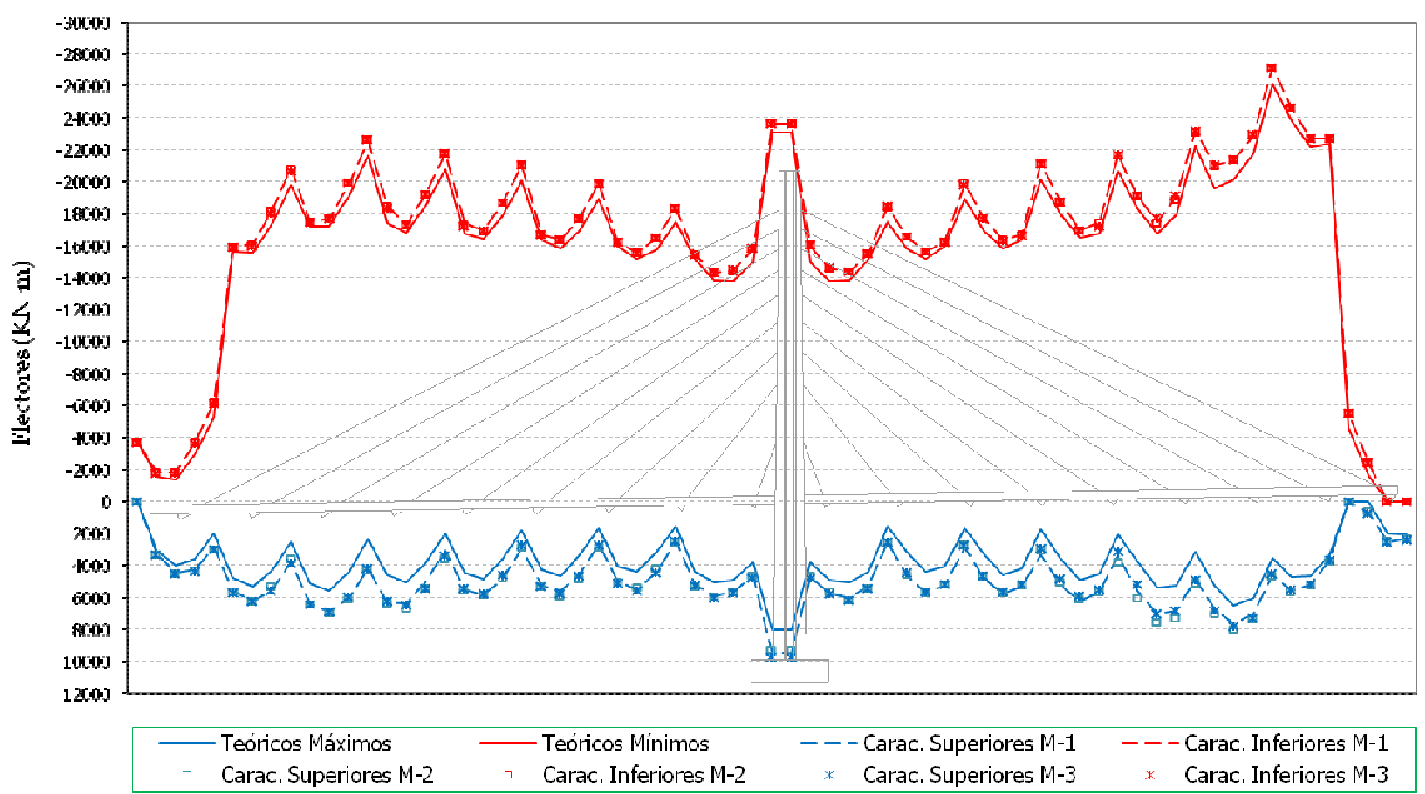

Figura 117. Flectores en tablero en fases de construcción. Valores característicos superiores e inferiores. 


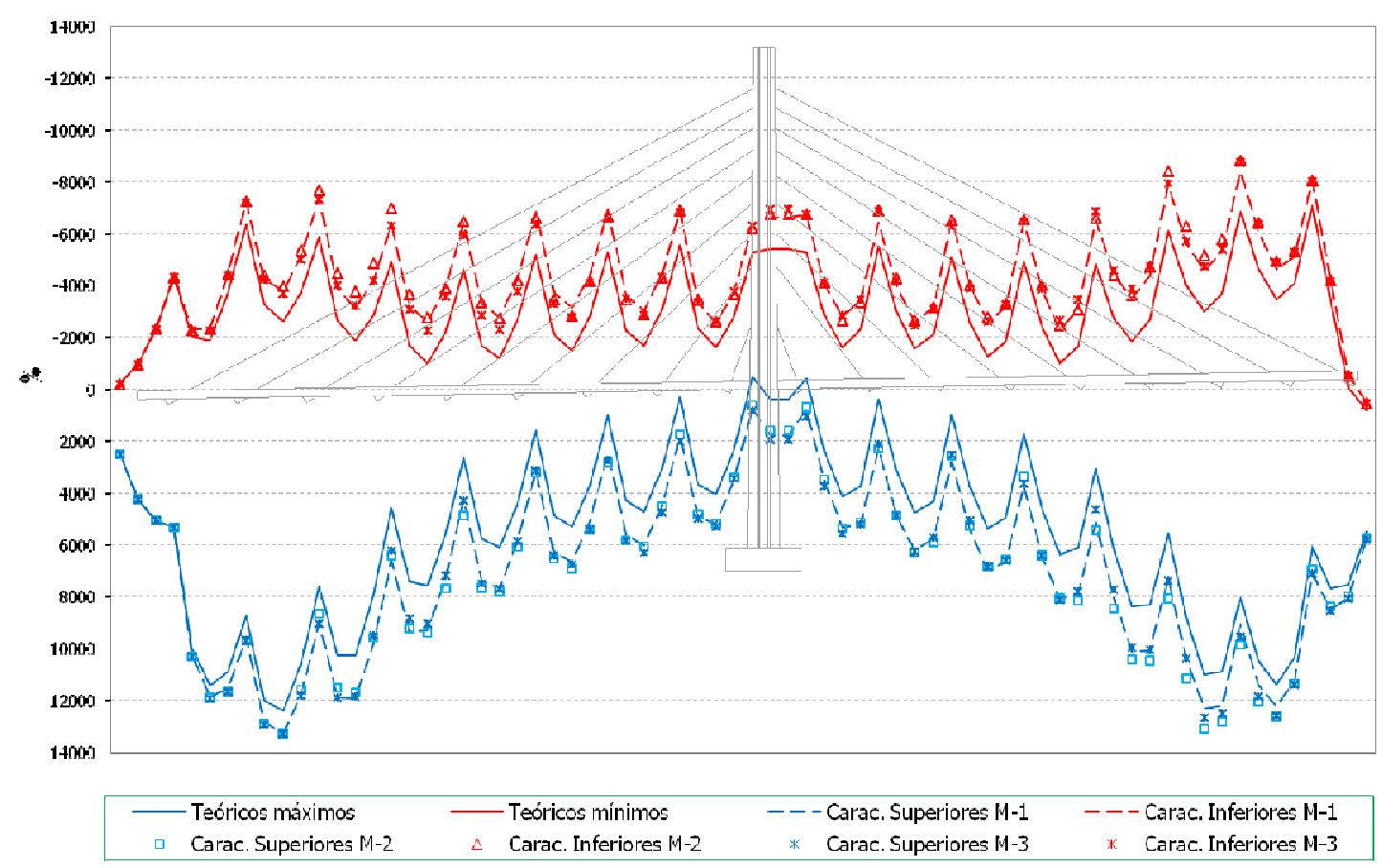

Figura 118. Flectores en tablero en fases de servicio. Valores característicos superiores e inferiores.

\subsubsection{Flectores en el pilono.}

Se incluyen en este apartado los resultados considerados más significativos en este elemento, que en concreto son la envolvente de flectores mínimos en fase de construcción y la envolvente de máximos en servicio. 


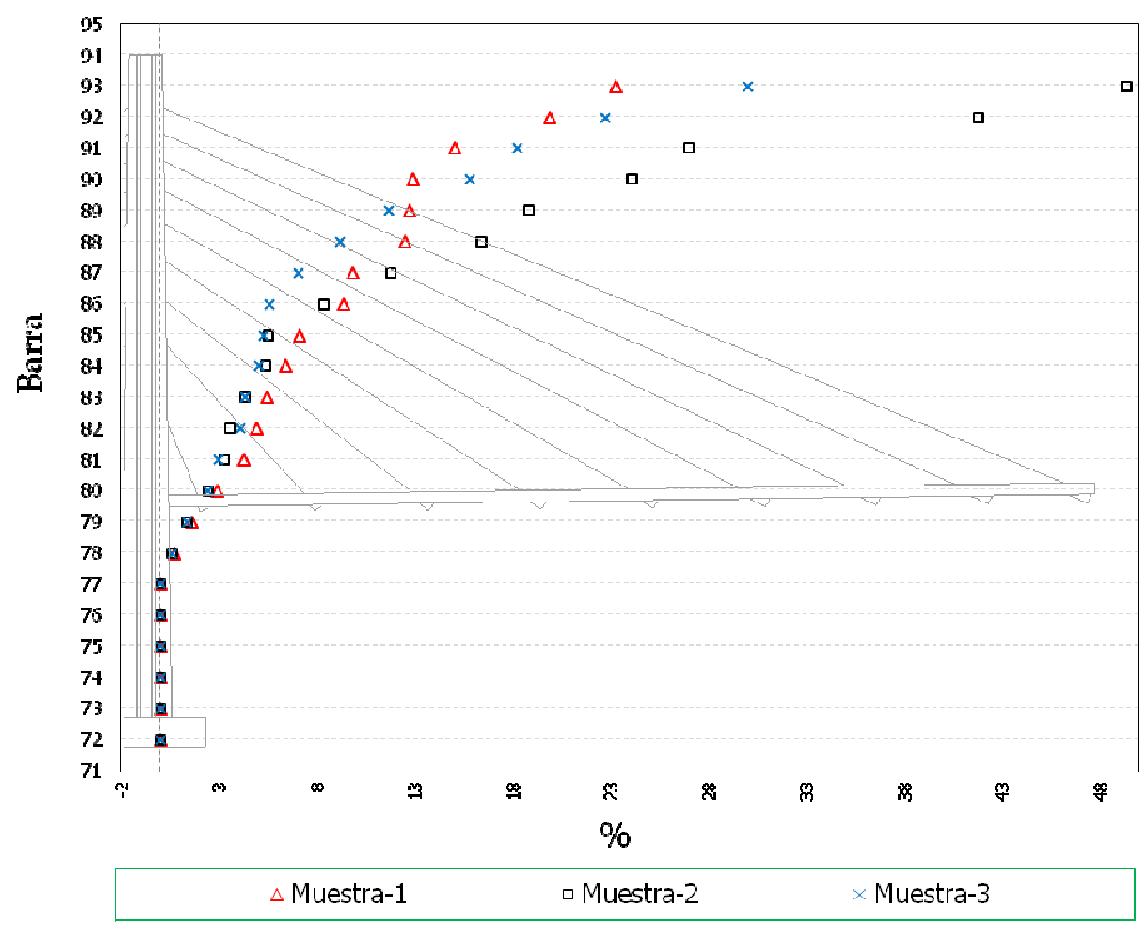

Figura 119. Flectores en pilono mínimos en fases de construcción. Diferencias en \% de los valores característicos inferiores respecto de los teóricos.

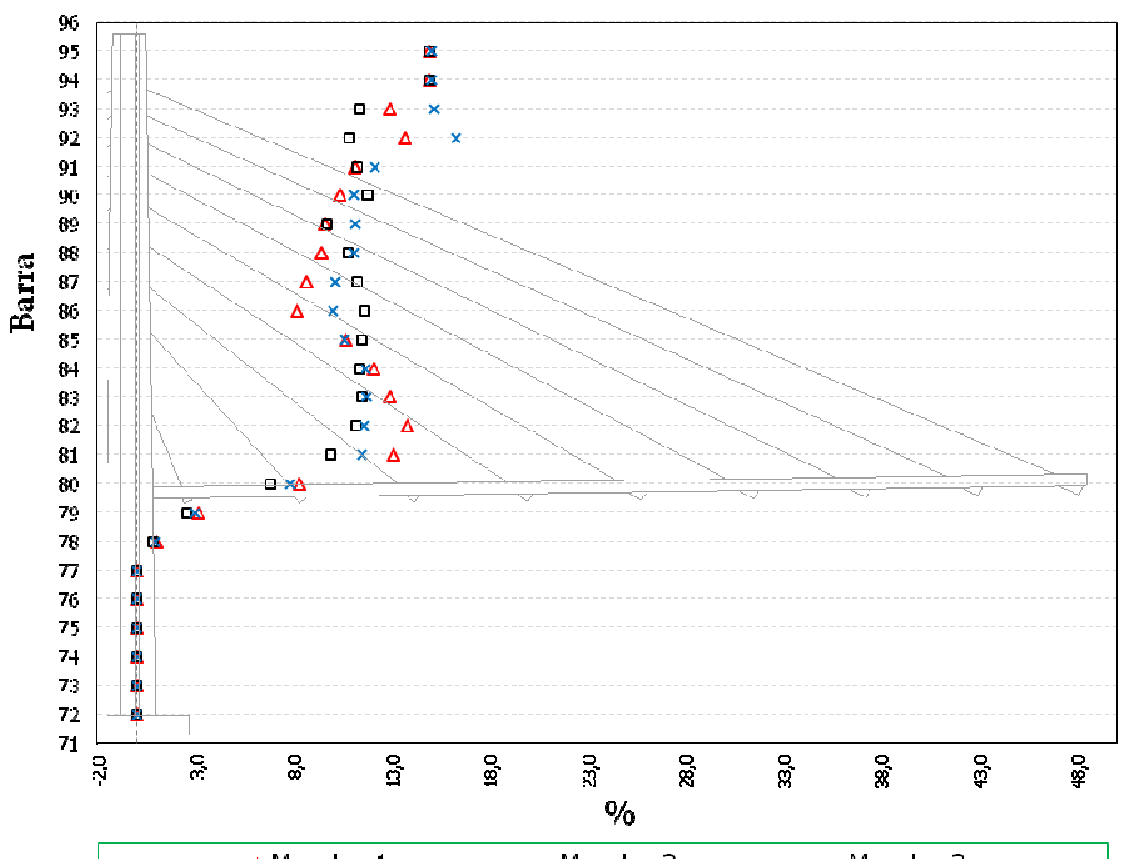

Figura 120. Flectores en pilono máximos en fases de servicio. Diferencias en \% de los valores característicos superiores respecto de los teóricos. 


\subsubsection{Errores en alargamientos de los cables.}

Se adjuntan a continuación resultados de la aplicación de los errores aleatorios sobre las deformaciones teóricas de montaje de los cables.

Los resultados representados lo son de tirantes (Figura 121 a Figura 126), tablero (Figura 127 a Figura 129) y pilono (Figura 130 a Figura 131) .

\subsubsection{Axiles en tirantes.}

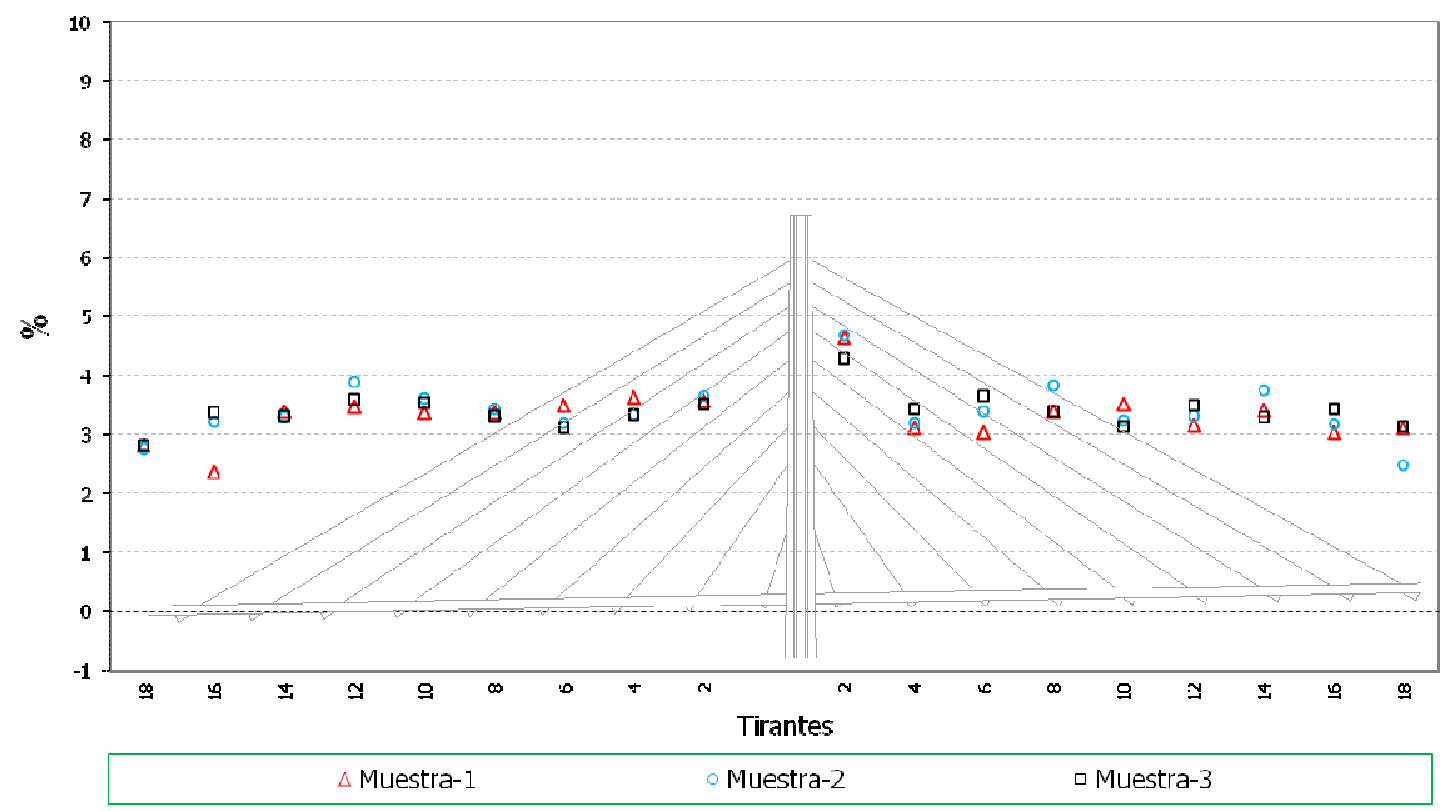

Figura 121. Axiles finales en los tirantes. Diferencia en \% de los valores característicos superiores respecto de los teóricos. 


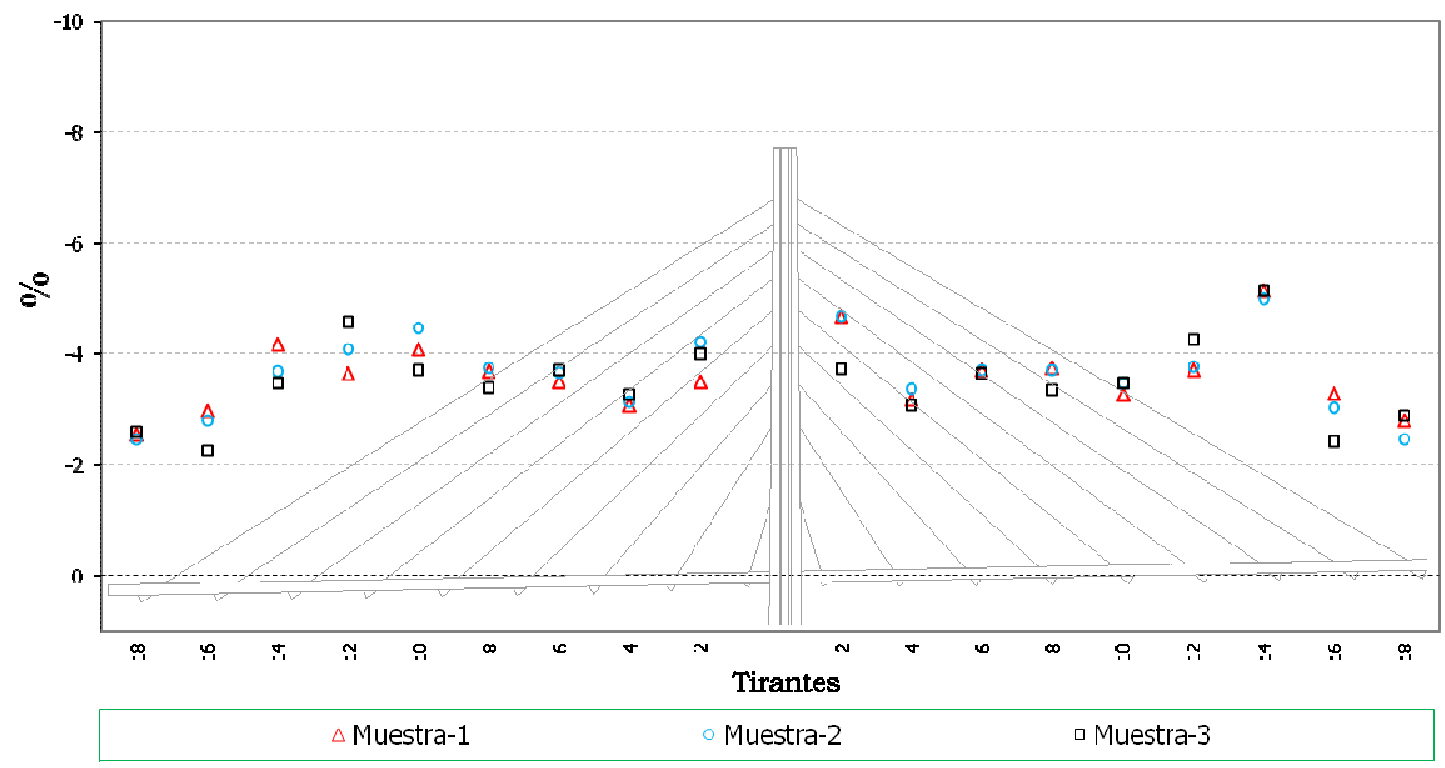

Figura 122. Axiles finales en los tirantes. Diferencia en \% de los valores característicos inferiores respecto de los teóricos

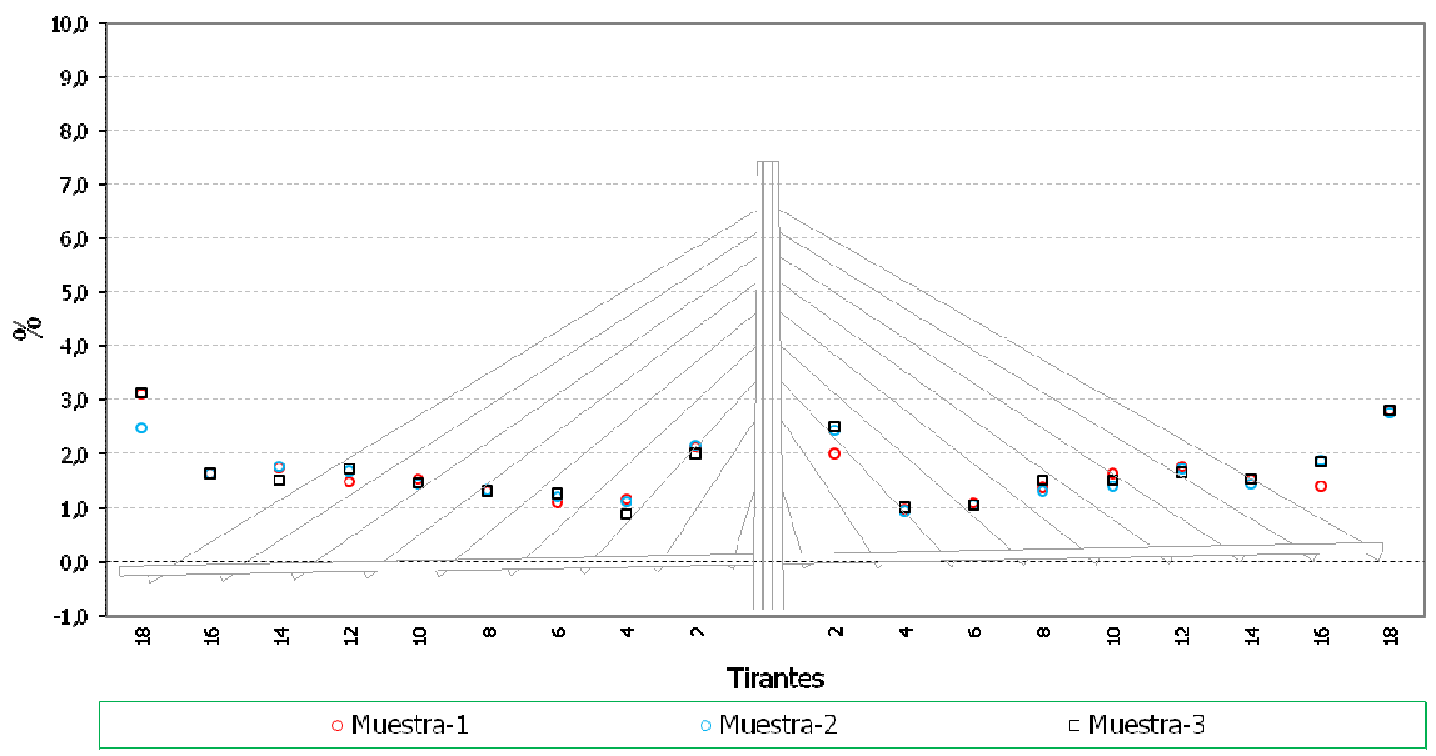

Figura 123. Axiles en los tirantes máximos de construcción. Diferencia en \% de los valores característicos superiores respecto de los teóricos. 


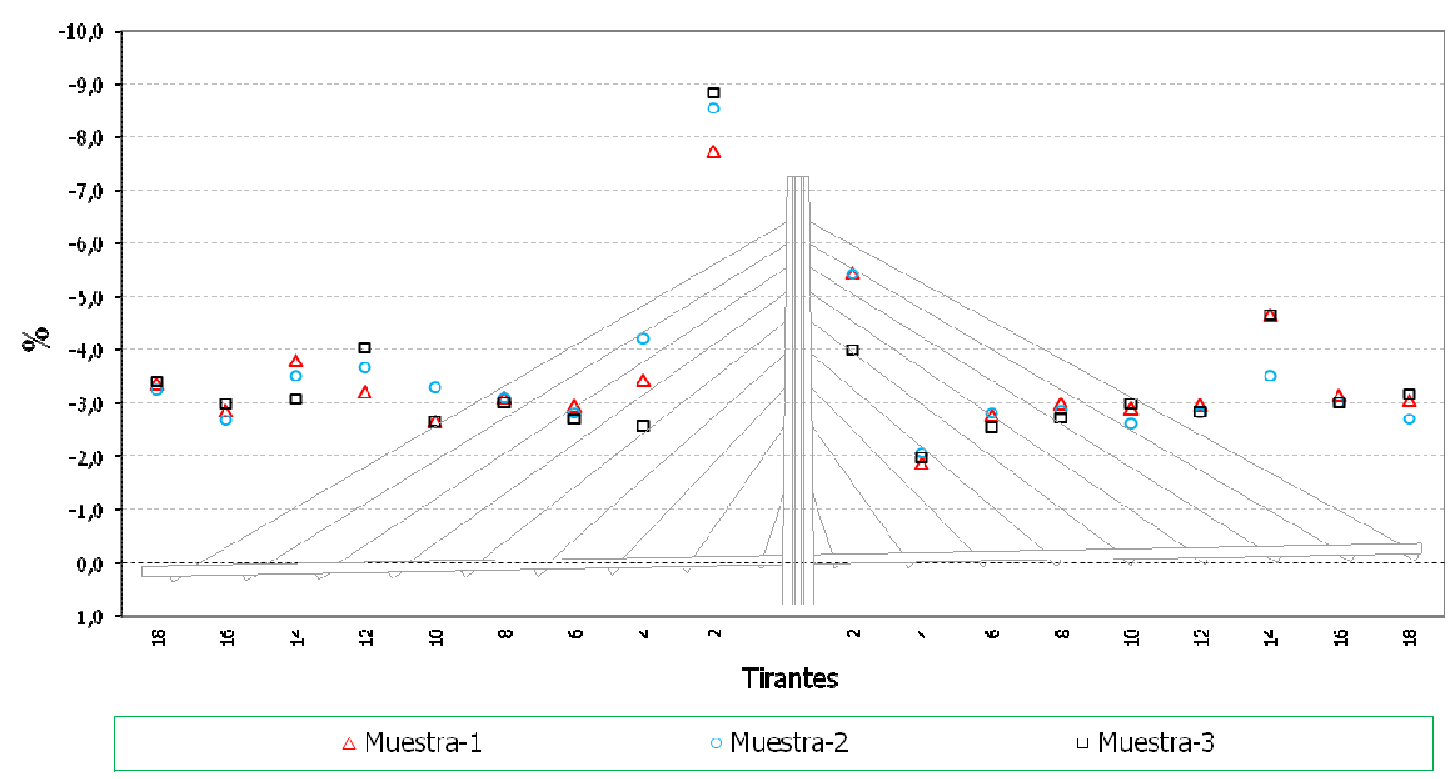

Figura 124. Axiles en los tirantes mínimos de construcción. Diferencia en \% de los valores característicos inferiores respecto de los teóricos.

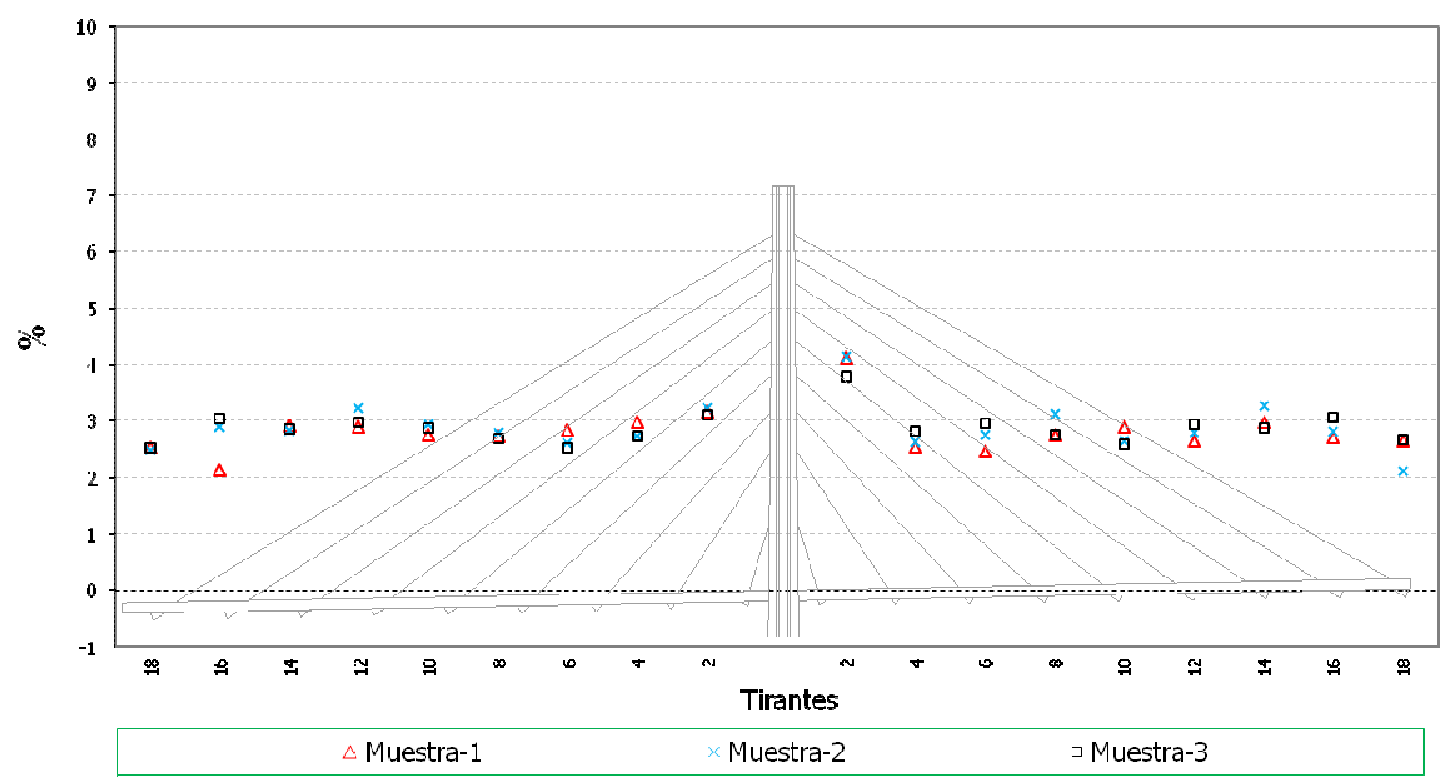

Figura 125. Axiles en los tirantes máximos de servicio. Diferencia en \% de los valores característicos superiores respecto de los teóricos. 


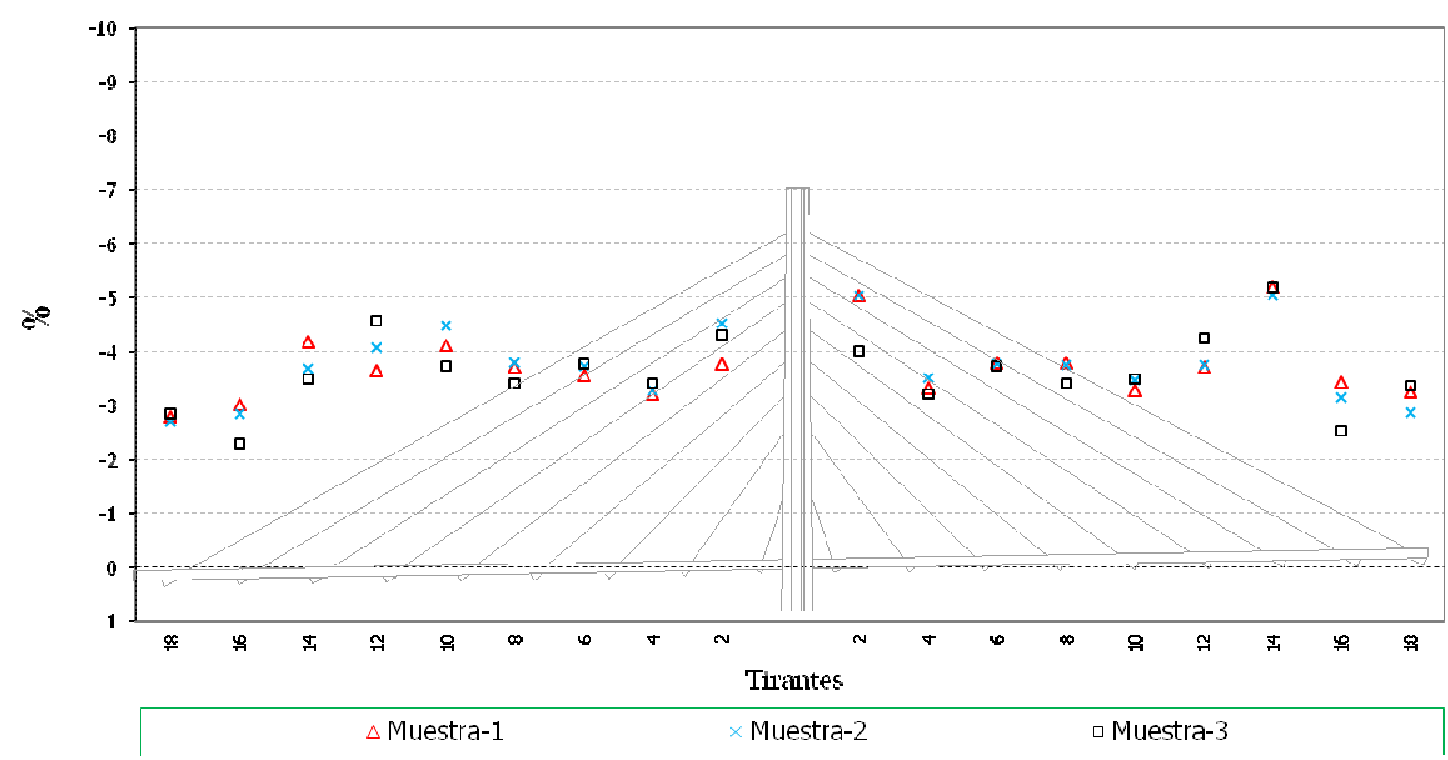

Figura 126. Axiles en los tirantes mínimos de servicio. Diferencia en \% de los valores característicos inferiores respecto de los teóricos.

\subsubsection{Flectores en tablero.}

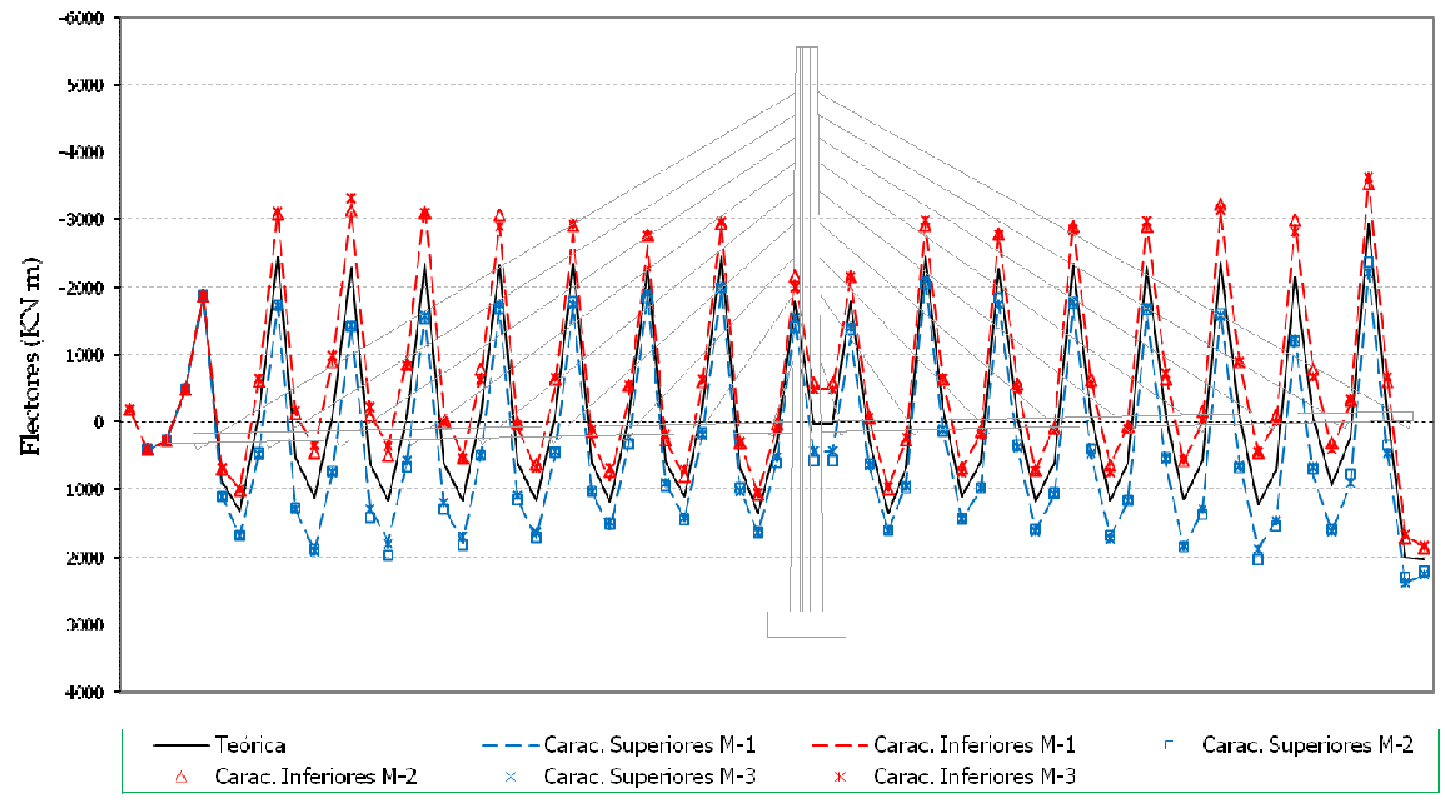

Figura 127. Flectores en tablero en situación final. Valores característicos superiores e inferiores. 


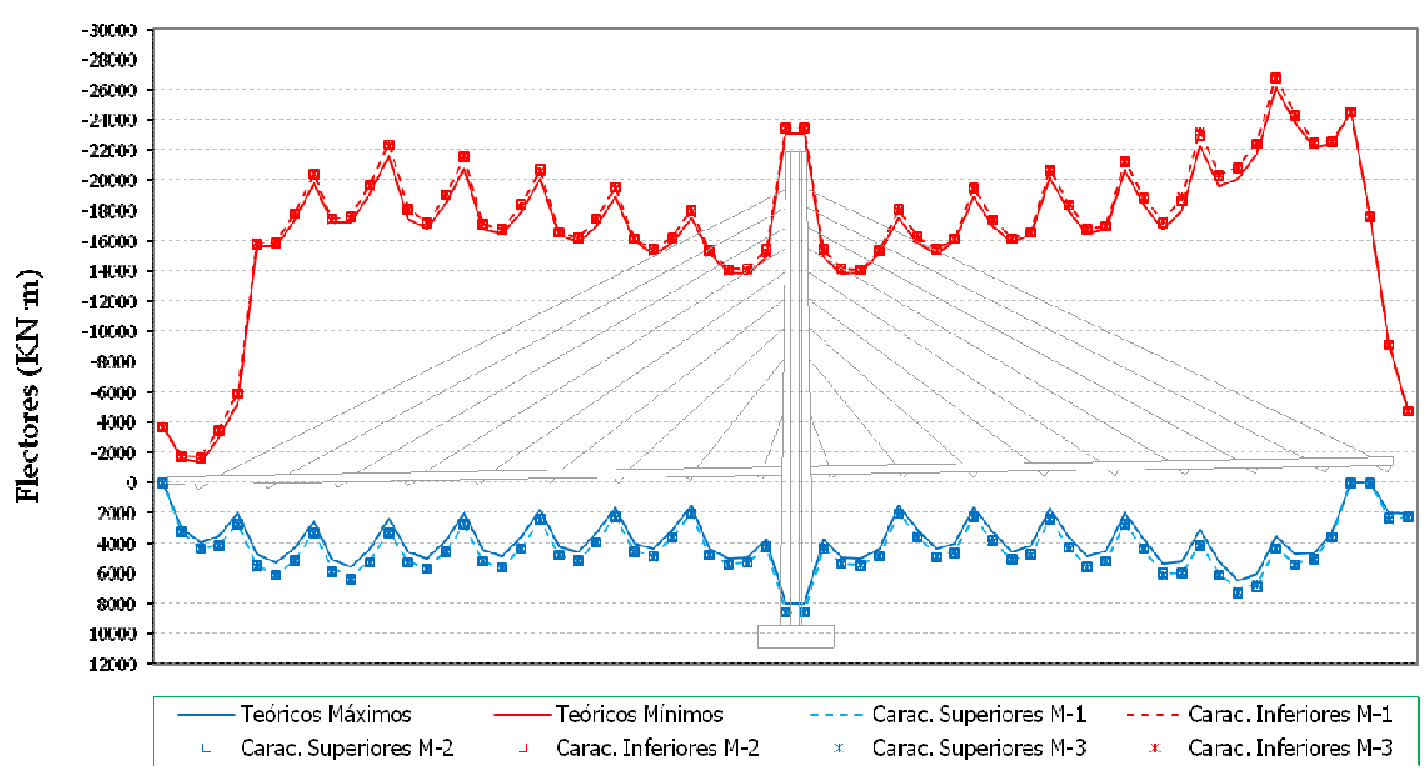

Figura 128. Flectores en tablero en fases de construcción. Valores característicos superiores e inferiores.

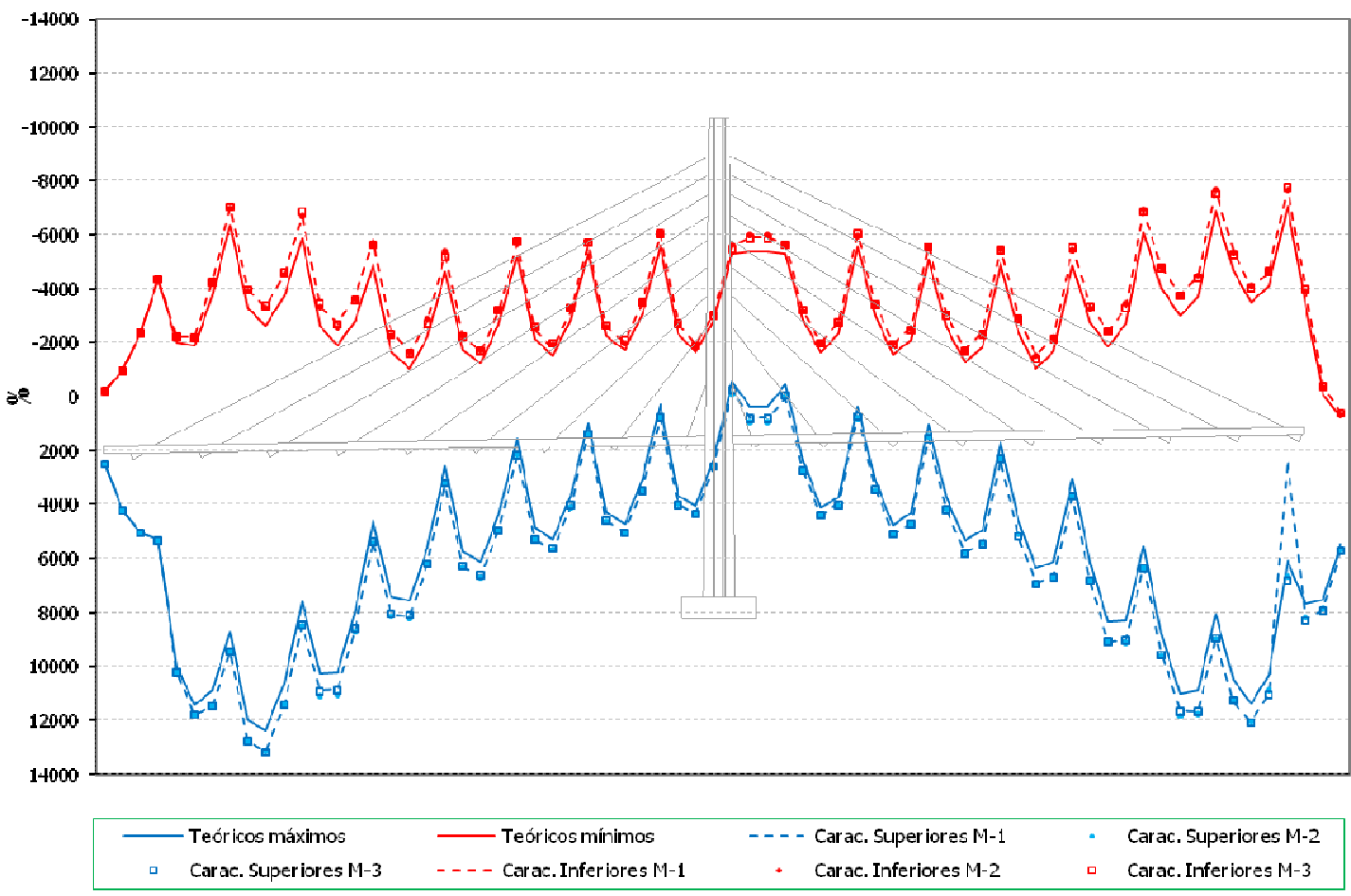

Figura 129. Flectores en tablero en fases de servicio. Valores característicos superiores e inferiores. 


\subsubsection{Flectores en pilono.}

Se incluyen en este caso sólo los valores más representativos correspondientes a las envolventes de mínimos de construcción y máximos de servicio.

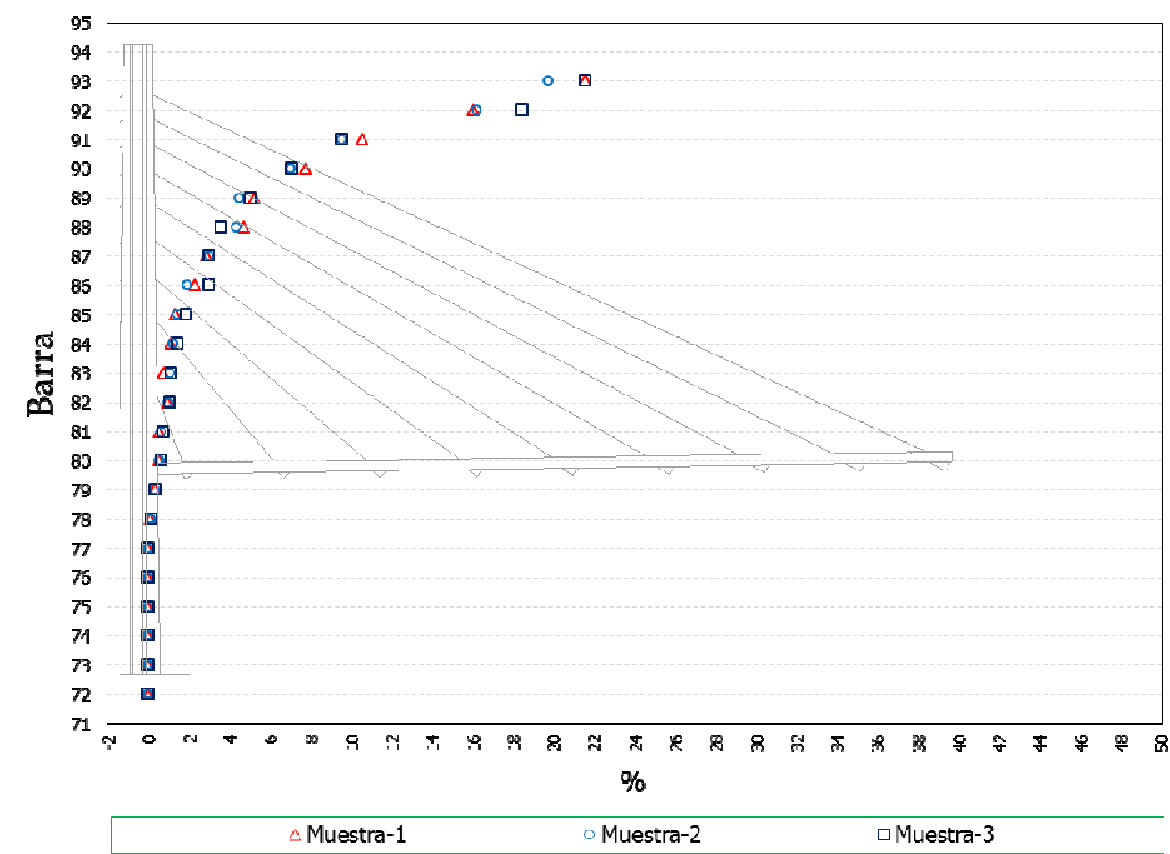

Figura 130. Flectores en pilono mínimos en fases de construcción. Diferencias en \% de los valores característicos inferiores respecto de los teóricos.

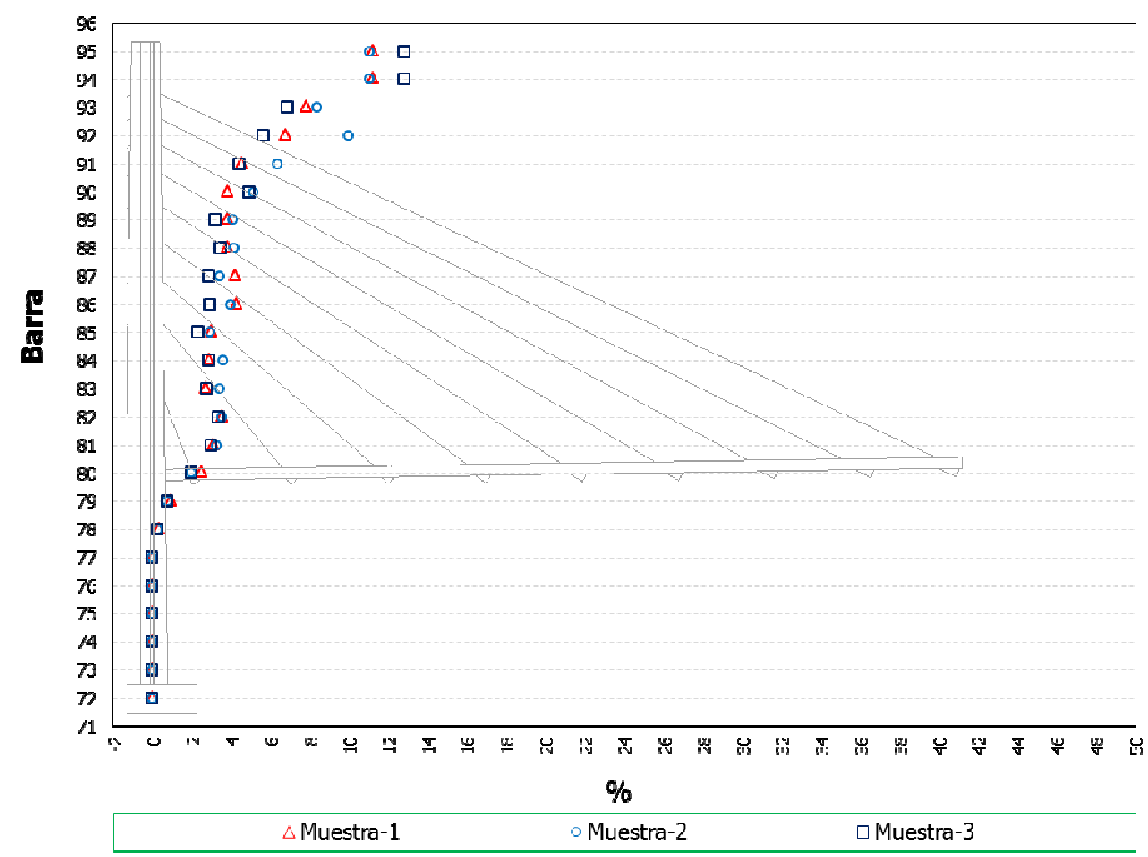

Figura 131. Flectores en pilono máximos en fases de servicio. Diferencias en \% de los valores característicos superiores respecto de los teóricos. 


\subsection{ANALISIS DE RESULTADOS.}

\subsubsection{Resultados con errores en fuerzas.}

En el caso de los axiles en los tirantes se puede indicar que:

- Los axiles finales alcanzan valores erróneos de hasta un 9\% respecto de los valores teóricos, observándose que en los primeros cables instalados este efecto es mayor.

- $\quad$ Igual que ocurre en el Modelo 90, los máximos de construcción quedan condicionados por el peso de las dovelas, valor que no incluye ningún efecto aleatorio, quedando así reflejado en los resultados obtenidos.

- Los valores mínimos de construcción en este caso presentan unas desviaciones muy acusadas en los primeros cables, con porcentajes respecto de los resultados teóricos de hasta un 14\%, frente a desviaciones de hasta el 10\% que se presentaron en el Modelo 90 , resultando sin embargo más acotados en el resto de cables.

- Respecto de los axiles en servicio, en este caso se aprecia una mayor dispersión de resultados que en los resultados del capítulo anterior, con valores de las desviaciones de los axiles mínimos con un máximo de hasta un $11 \%$ frente al 10\% del Modelo 90. Los axiles máximos de servicio se desvían hasta en un $8 \%$ frente a un $6 \%$ obtenido en el modelo del capítulo anterior. De nuevo, al igual que ocurrió en el Modelo 90, el efecto en este caso es más acusado en los primeros cables instalados.

Por otra parte en el caso de los resultados en flectores del tablero:

- De la misma forma que en el Modelo 90, los desviación en los flectores en situación final es muy acusada.

- Por otra parte las envolventes de construcción y servicio obtenidas son muy similares a las del modelo anterior, con desviaciones respecto del valor teórico de entre un 5-7\% para las envolventes de mínimos de servicio y de entre un 20-40\% para las envolventes de máximos.

Por último en el caso de los flectores en el pilono se puede indicar que:

- Se obtienen, de forma muy similar al Modelo 90, con valores de la envolvente de mínimos de construcción con en torno al 10\% de desvío respecto del valor teórico en la parte inferior del fuste, elevándose esos porcentajes hasta un $20 \%$ en la parte superior 
del fuste, donde, como ya se indicó, el resultado es mayor en porcentaje al ser más reducidos los flectores teóricos alcanzados en esa zona.

- Para los máximos de servicio los porcentajes son similares a los obtenidos en el Modelo 90, con valores del orden del $10 \%$ en toda la altura del fuste.

\subsubsection{Resultados con errores en deformaciones.}

En este caso, respecto de los valores obtenidos en los axiles de los tirantes se puede indicar:

- Que, como ocurrió en el Modelo 90, los axiles finales presentan menores dispersiones que en el caso de la introducción de los errores en fuerzas.

- Para los axiles máximos de construcción, los resultados obtenidos son similares a los del modelo anterior, encontrándose de nuevo un efecto muy marcado del peso de las dovelas sobre los valores obtenidos, suavizándose así el efecto introducido de la aleatoriedad de los axiles de los tirantes.

- Los axiles mínimos de construcción presentan valores cercanos al 3\% de desviación respecto de los resultados teóricos, de nuevo de forma similar a lo obtenido en el caso del Modelo 90.

Por otra parte en el caso de los resultados en flectores del tablero:

- Los resultados obtenidos, tanto en la situación final como para las envolventes de construcción y servicio, muestran un comportamiento y desviaciones respecto de los valores teóricos similares al caso del Modelo 90.

- En general el efecto de la aleatoriedad sobre los flectores del tablero es menos acusado aplicando el método en deformaciones que aplicando el método en fuerzas, con porcentajes de error siempre limitados a valores del orden del 3-4\% en el caso de las envolventes de servicio.

Por último en el caso de los flectores en el pilono se puede indicar también que:

- De nuevo los valores obtenidos son similares a los del Modelo 90, presentándose en general porcentajes menores aplicando errores en las deformaciones de montaje de los cables, que aplicando los errores sobre los axiles de tesado de los cables. 


\subsubsection{Comparación de resultados.}

En definitiva se puede concluir, resumiendo lo indicado en los epígrafes anteriores en relación a los resultados obtenidos por ambos métodos de montaje y en relación a los resultados obtenidos en el Modelo 90 que:

- El método de aplicación de errores en las deformaciones de montaje está produciendo menores desviaciones respecto de los valores teóricos que el método de introducción de errores en las fuerzas de tesado.

En este sentido hay que hacer notar la cuestión ya señalada en el apartado 4.7, en relación a la comparación de la dispersión generada por ambos métodos de cálculo.

- Que, en general, los valores obtenidos en el Modelo 90M son similares a los obtenidos en el Modelo 90, por lo que parece que no hay acumulación de los efectos de la aleatoriedad en función del número de cables de la estructura, dado que parece que es la propia aleatoriedad la que suaviza y compensa los errores introducidos en los diferentes cables, independientemente del número de éstos que disponga la estructura.

Es más, incluso en este caso se han obtenido valores en algunos casos de mayores desviaciones porcentuales, sobre todo en el caso de los axiles de los tirantes, lo que parecería significar que a menor número de cables, mayor es la influencia de los errores introducidos, dada la mayor responsabilidad de cada cable individualmente. Bien es cierto también que este efecto se puede deber en parte, en el caso del uso de la introducción de errores en deformaciones, a lo señalado en 4.4 .3 por el SETRA[102] en relación a los errores máximos admisibles.

- $\quad$ Sí sigue siendo apreciable el efecto sobre los axiles de los cables que presentan alguna singularidad debido al proceso constructivo, siendo en este caso el efecto más acusado que en el Modelo 90, dado que los tirantes de esta estructura tienen individualmente en proporción una mayor influencia sobre el comportamiento resistente de la estructura, al ser menor su número. 


\section{CAPITULO 6.}

\section{INFLUENCIA DE LA LUZ DE LA ESTRUCTURA.}

\subsection{INTRODUCCION.}

Se procede en este capítulo a analizar otro parámetro que puede tener influencia en los resultados obtenidos al introducir los errores aleatorios ya comentados en capítulos anteriores. Este nuevo parámetro a analizar es la luz de la estructura, parámetro que tendrá implicaciones tanto en la flexibilidad global de la estructura como en los niveles de esfuerzos alcanzados en los diferentes elementos. Se ha procedido así a calcular una estructura derivada de la originalmente analizada en el capítulo 4.2, manteniendo ciertas características geométricas y mecánicas de aquella, y aumentando la luz de la estructura hasta el doble de la original. Esta modificación, a su vez, implicará cambiar algún otro parámetro geométrico, el cual se describirá en detalle a continuación.

El desarrollo del capítulo es similar al del capítulo anterior, describiéndose en primer lugar la estructura analizada y los resultados teóricos de referencia obtenidos, para a continuación presentar los resultados consecuencia de la introducción de errores aleatorios en el montaje de los cables, tanto por el método de las fuerzas como por el método de las deformaciones. Finalmente se redactan unas conclusiones relativas al caso analizado y a su comparación con los anteriormente ya analizados.

\subsection{ESTRUCTURA A ANALIZAR.}

Como se ha adelantado, la estructura analizada en este capítulo ha sido la correspondiente al caso de una estructura con luz doble de la originalmente estudiada para el Modelo 90, y descrita en el epígrafe 4.2. En definitiva, en esta nueva estructura se ha mantenido en general el esquema resistente de la estructura original en lo referente a número de tirantes y esquema del atirantamiento, ancho del tablero, vinculaciones entre tablero y pilono, etc..

De esta forma, la nueva estructura corresponde al caso de un puente con pilono central de luces $177+170 \mathrm{~m}$, es decir que este caso podría ser equivalente a analizar un puente de 3 
vanos, con 2 pilonos, con distribución de luces 170'0 +354'0 + 170'0 m. Esta distribución de luces quedaría dentro del rango de luces medias o grandes para esta tipología de estructuras con tableros de hormigón.

Con la intención de clarificar la interpretación y comparación de los distintos resultados obtenidos, se denominará en adelante a esta estructura Modelo 180.

A pesar de haber mantenido el número de tirantes del Modelo 90 (18 cables en cada vano), ahora la separación entre anclajes es de $10^{\prime} 0 \mathrm{~m}$, dado que la luz total del tablero se ha doblado. Resultan así dovelas de 10'0 m de longitud, en las que a 2'00 m del frente de dovela se dispone el anclaje del cable correspondiente.

Por otra parte, como el ancho del tablero se sigue planteando para soportar una calzada de doble sentido de circulación con 13'80 m de ancho, el canto del tablero no se ha modificado, basándonos en la hipótesis planteada en el epígrafe 5.2 de que el canto del tablero puede venir condicionado por la dimensión transversal de éste. Por este motivo tanto su inercia como peso propio siguen siendo idénticos a los de los modelos ya analizados.

Este último planteamiento, a sabiendas de que puede no ajustarse por completo a un caso real, se ha decidido adoptarlo para así, al modificar el menor número de parámetros posibles entre los diferentes modelos de cálculo analizados, facilitar la interpretación y comparación de resultados entre todos ellos.

Sí se ha introducido una modificación en este caso en referencia a la altura de la torre, o más concretamente a la relación entre la altura de la torre y la luz del vano principal. Así, la altura del pilono en este caso se establece en una altura de 60'0 m, por encima de rasante del tablero, de tal manera que la relación de esta dimensión respecto de la luz principal es de valor 0'35. Esta relación se ha reducido respecto del caso del Modelo 90, donde esta relación se estableció en un valor de 0’44. Esta cuestión conllevará que los tirantes más largos quedarán más tendidos y serán por lo tanto algo menos eficaces que en los modelos precedentes. Esta característica diferenciadora de este modelo ha sido tenida en cuenta en la interpretación de los resultados que se presentan en lo sucesivo.

En relación también al pilono planteado en este caso hay que señalar que su inercia se ha aumentado respecto de los casos anteriores, ajustándose en proporción a la nueva longitud generada en el pilono. Este aspecto también traerá alguna consecuencia específica en la interpretación de los resultados obtenidos.

Las áreas de los tirantes, para homogeneizarlos con los de los casos ya analizados, se han ajustado de tal forma que las tensiones que soportarán todos ellos queden en valores similares 
a los obtenidos en el resto de estructuras, es decir tensiones de trabajo del orden de $\approx 450$ $\mathrm{N} / \mathrm{mm}^{2}$. Con esta condición han resultado las áreas en cada cable que se sintetizan en el cuadro siguiente:

\begin{tabular}{|c|c|c|c|c|}
\hline Barra & Tirante & Longitud (m) & Angulo & Area $\left(\mathrm{cm}^{2}\right)$ \\
\hline 98 & 2-D & 13.9 & -60.8 & 59.4 \\
\hline 99 & $2-1$ & 13.9 & 246.9 & 59.4 \\
\hline 100 & 3D & 23.3 & -50.0 & 86.4 \\
\hline 101 & अ & 23.3 & 230.0 & 86.4 \\
\hline 102 & 4-D & 33.0 & -40.8 & 97.6 \\
\hline 103 & $4-1$ & 33.0 & 220.8 & 97.6 \\
\hline 104 & 5-D & 43.2 & -35.8 & 110.0 \\
\hline 105 & $5-1$ & 43.2 & 215.8 & 110.0 \\
\hline 106 & 6-D & 53.4 & -32.6 & 119.1 \\
\hline 107 & $6-1$ & 53.4 & 212.6 & 119.1 \\
\hline 108 & 7-D & 63.6 & .30 .2 & 927.8 \\
\hline 109 & $7-1$ & 63.6 & 290.2 & 927.8 \\
\hline 190 & QDD & 73.7 & .26 .2 & 936.0 \\
\hline 111 & $8-1$ & 73.7 & 200.2 & 136.0 \\
\hline 112 & 9-D & 83.8 & -26.5 & 143.8 \\
\hline 113 & $9-1$ & 89.8 & 206.5 & 143.8 \\
\hline 114 & 10-D & 93.9 & -25.2 & 151.0 \\
\hline 115 & 10-I & 93.9 & 205.2 & 151.0 \\
\hline 116 & 11-D & 104.1 & .24 .1 & 157.5 \\
\hline 117 & 111-I & 104.1 & 204.1 & 157.5 \\
\hline 118 & $12-D$ & 114.2 & -23.2 & 163.2 \\
\hline 119 & 12.I & 114.2 & 203.2 & 169.3 \\
\hline 120 & 13-D & 124.4 & -22.4 & 166.5 \\
\hline 121 & $\begin{array}{lll}13-1 \\
\end{array}$ & 124.4 & 202.4 & 968.4 \\
\hline 122 & 14-D & 134.6 & .21 .8 & 972.7 \\
\hline 123 & 14-I & 134.6 & 201.8 & 979.4 \\
\hline 124 & 15-D & 144.8 & .21 .2 & 979.0 \\
\hline 125 & 15-I & 144.8 & 201.2 & 176.3 \\
\hline 126 & 16-D & 165.1 & -20.8 & 175.0 \\
\hline 127 & 16-I & 165.1 & 200.8 & 196.7 \\
\hline 128 & $17-D$ & 165.3 & -20.3 & 200.7 \\
\hline 129 & $17-1$ & 165.3 & 200.3 & 168.2 \\
\hline 130 & 196-D & 175.6 & -20.0 & 211.3 \\
\hline 131 & 18. & 175.6 & 200.0 & 242.3 \\
\hline
\end{tabular}

Tabla 5. Denominación y áreas de los tirantes de la estructura Modelo 180.

De nuevo se recuerda que en el anexo A.l también se incluyen los listados con las características completas de este modelo de cálculo.

El proceso constructivo propuesto sigue siendo el mismo que el indicado para el caso de las estructuras anteriores, ejecutándose en voladizo primeramente la dovela del vano izquierdo y posteriormente la del vano derecho, hasta completar los dos vanos.

Hay que señalar que en esta estructura se sigue manteniendo un esquema ligeramente asimétrico, ya que el vano izquierdo posee una dovela más que el derecho, quedando equilibrado este elemento mediante la disposición de un cable de retenida en el vano derecho (tirante 18D).

El modelo utilizado para el cálculo de esta estructura se conforma por el mismo número de barras y nudos que el Modelo 90 , habiéndose generado igualmente mediante el uso del mismo programa de cálculo matricial de estructuras planas que el utilizado en aquel. El esquema del modelo de cálculo planteado para esta estructura es el siguiente: 


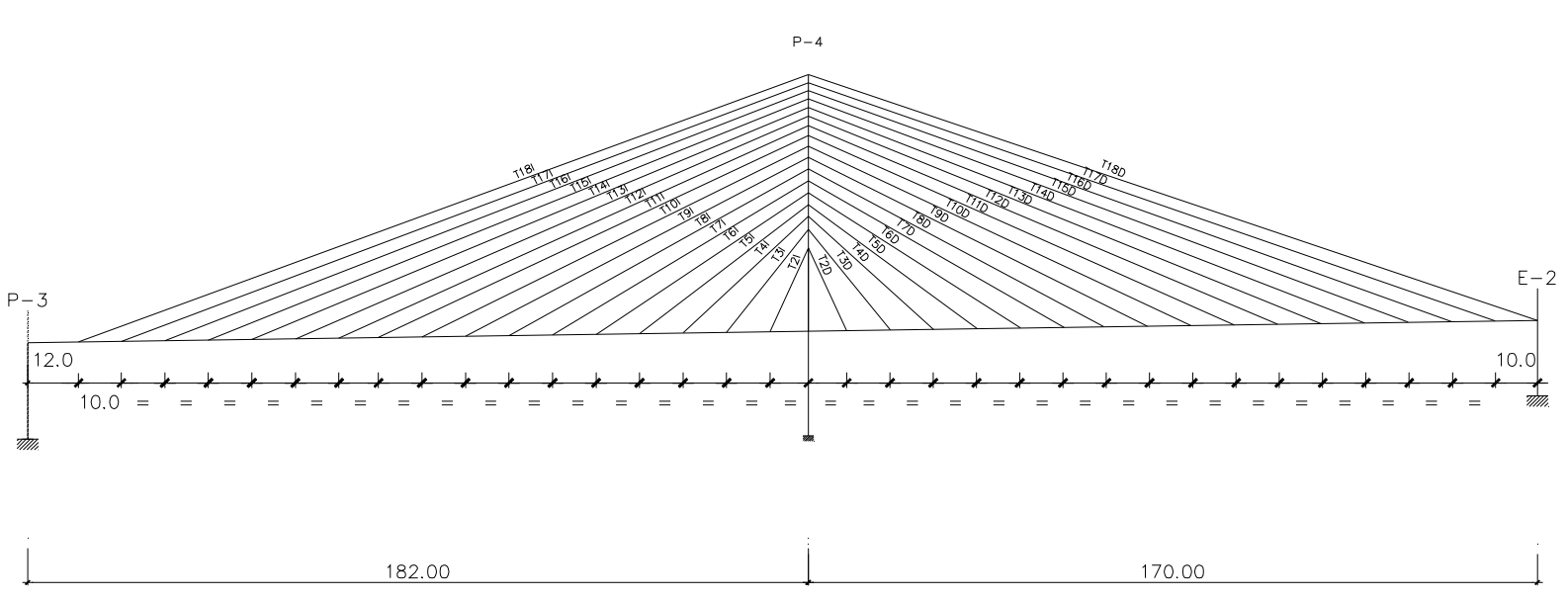

Figura 132. Esquema del modelo de cálculo 180.

Como se ha indicado, con este tipo de estructura se pretende valorar qué efecto tiene la luz total del puente sobre los resultados obtenidos al aplicar los procesos de montaje de los cables erróneos. Para ello se va a proceder a compararlos con los cálculos realizados en los capítulos anteriores.

De nuevo se procede en primer lugar a realizar el cálculo de la estructura teórica, cuyos resultados servirán de referencia a los obtenidos posteriormente, y cuyos resultados más significativos durante el proceso de montaje, en la situación final y en la situación de servicio se presentan a continuación (Figura 133, Figura 134 y Figura 135):

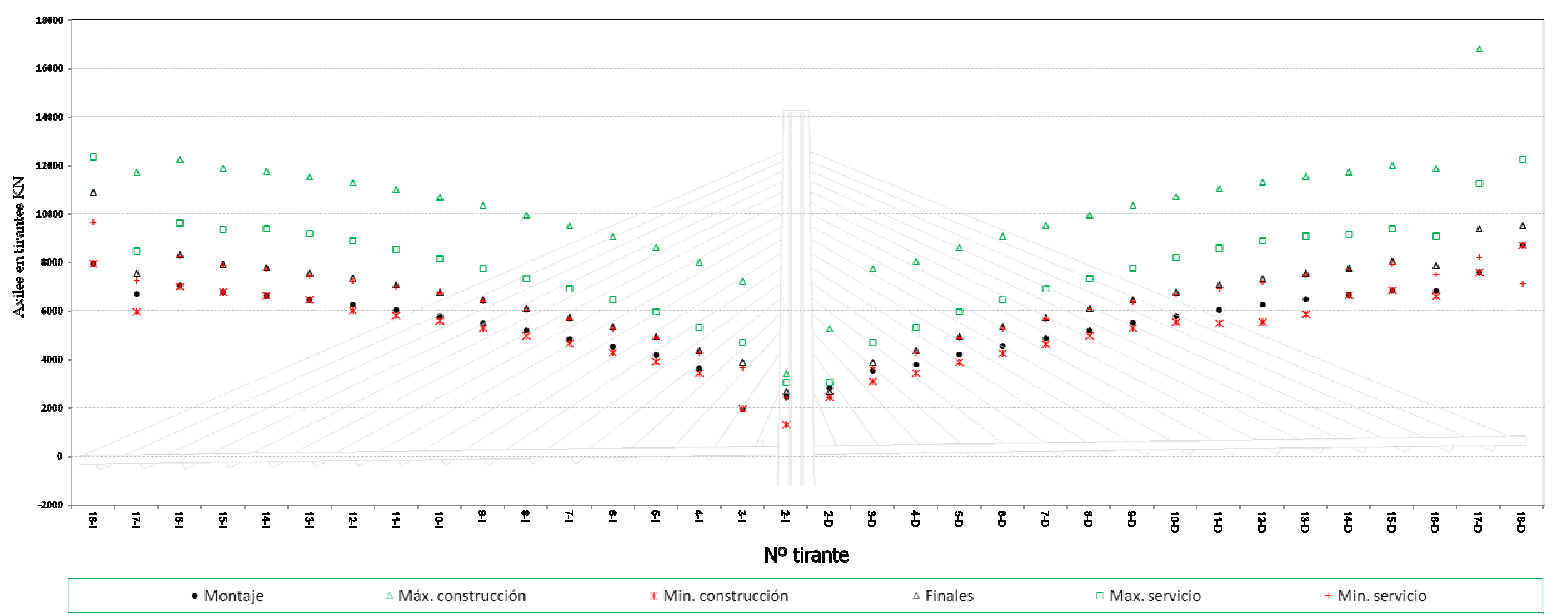

Figura 133. Axiles en tirantes en fase de inicial de instalación, máximos y mínimos de las fases de construcción, en la fase final del proceso constructivo y máximos y mínimos en las situaciones de servicio. 


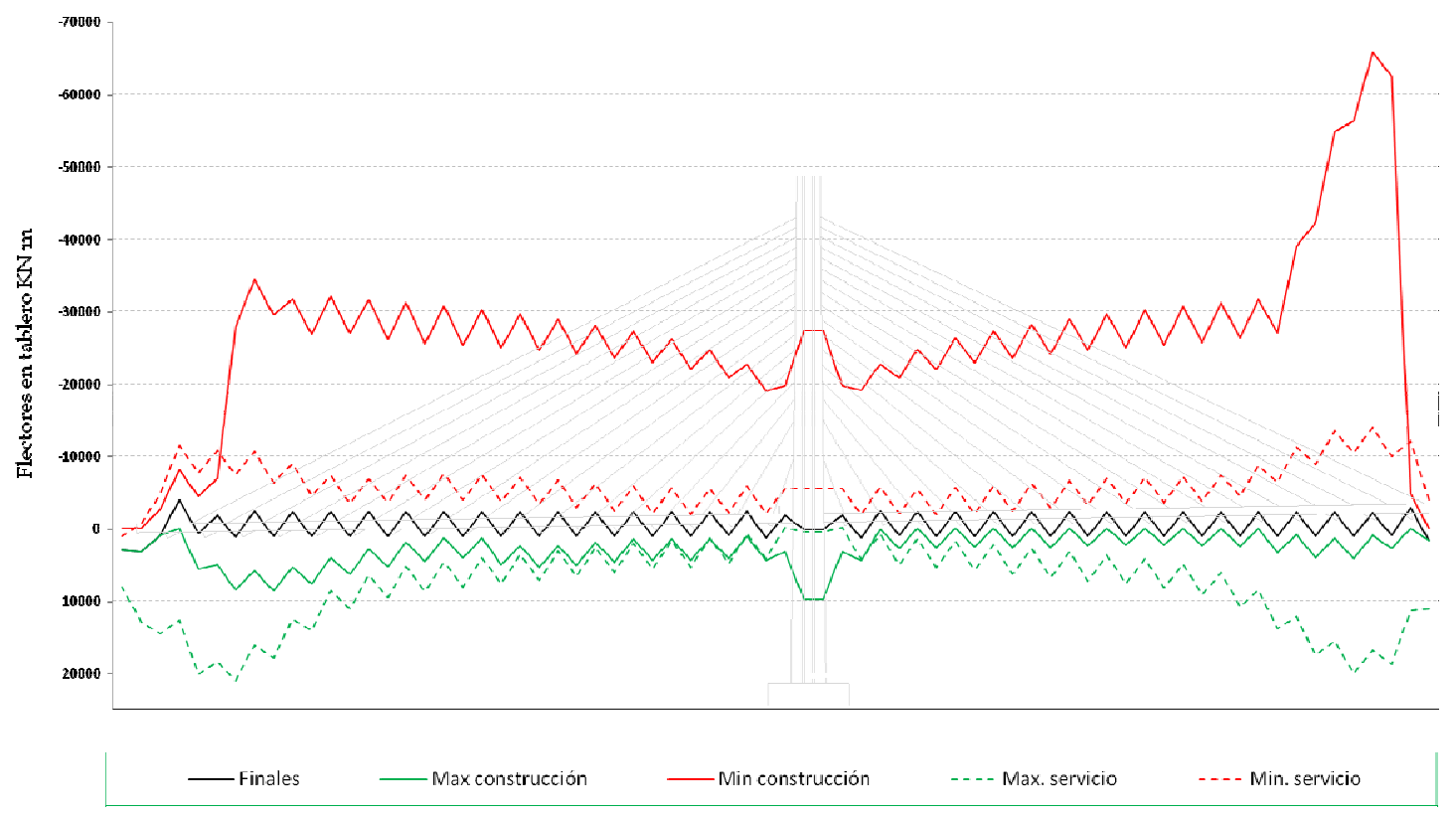

Figura 134. Envolvente de flectores en el tablero en las fases de construcción, final y en servicio.

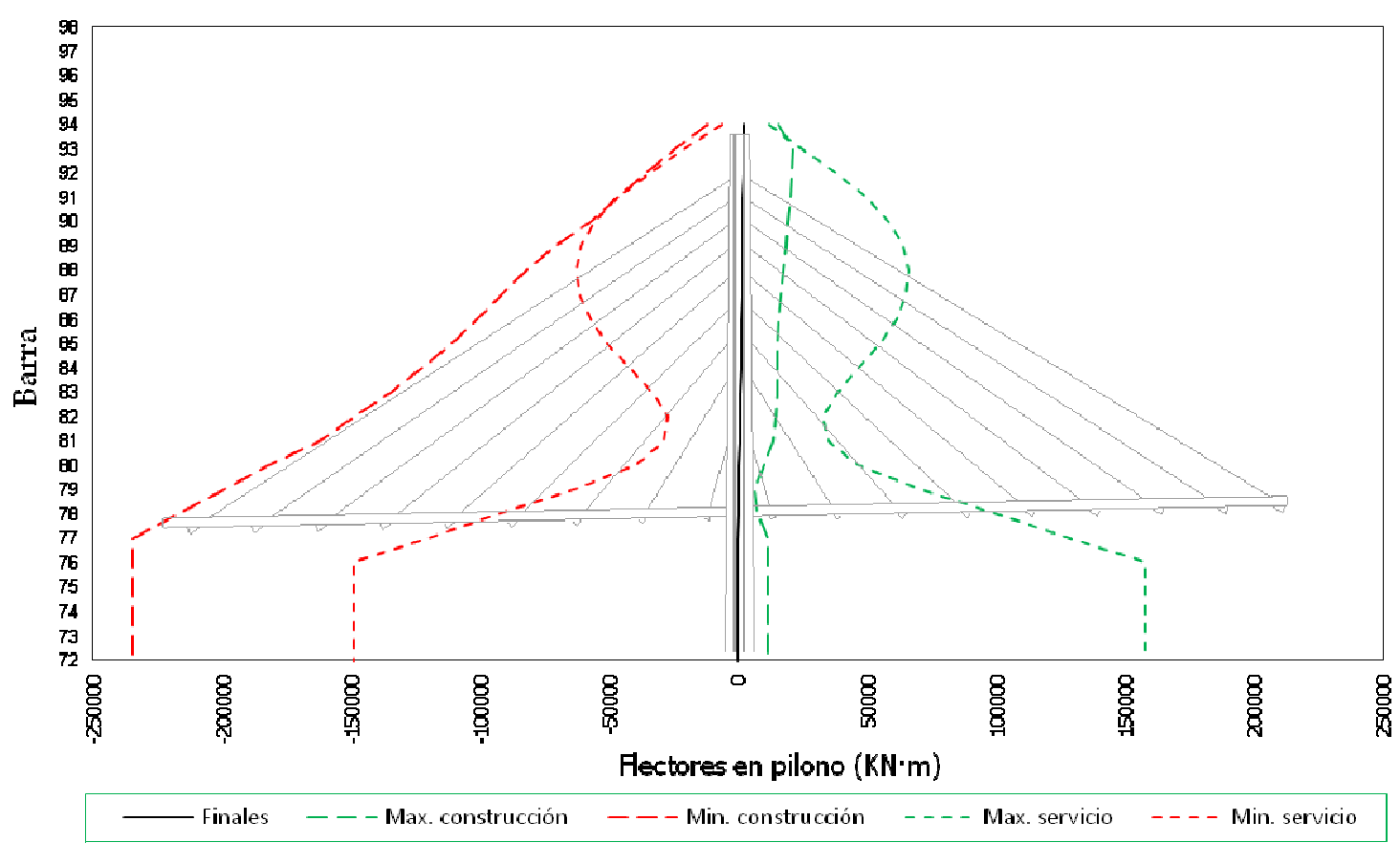

Figura 135. Envolvente de flectores en el pilono en las fases de construcción, final y en servicio.

De todos los resultados anteriormente presentados se pueden extraer las siguientes conclusiones. En primer lugar en el caso de los axiles de los tirantes (Figura 133):

- Que como ocurre en los casos de los Modelos 90 y 90M, los axiles finales son superiores a los de montaje en la mayoría de los casos. 
- Que igualmente a lo ocurrido con los modelos anteriores, el valor máximo del axil de cada tirante se produce, en general, durante las fases de construcción, siendo el mínimo generado en cada cable muy similar al axil de montaje.

- La evolución de los axiles en los tirantes a lo largo de la longitud del tablero queda determinada por la variación de los ángulos que forman los cables con el tablero, de tal manera que a pesar de tener que absorber una carga similar en prácticamente todos, los cables extremos tienen una configuración geométrica más desfavorable, lo que condiciona la generación sobre ellos de unos axiles de mayor cuantía.

- Los valores de los axiles obtenidos son superiores a los del caso del Modelo 90M. Esta cuestión se justifica en el hecho de que, al ser la relación entre la altura de la torre y la luz del tablero de menor cuantía que en el caso anterior, los ángulos que forman los cables con el eje del tablero son más pequeños y por lo tanto su efectividad es menor. Es decir, que a pesar de que cada tirante debe absorber una carga equivalente a la correspondiente a los tirantes del Modelo 90M, en este caso, dada su menor efectividad, esa misma carga provoca un axil mayor en los cables.

Por otra parte, en relación a los flectores en el tablero (Figura 134) se puede indicar que:

- La envolvente de flectores en el tablero se conforma de nuevo por los flectores mínimos generados durante las fases de construcción y los flectores máximos aparecidos en servicio al aplicar las sobrecargas de tráfico.

- La ley final de flectores coincide con la obtenida en el caso de una viga continua de luces 10'0 m, es decir los valores obtenidos serán los mismos en esta situación que los determinados para el Modelo 90M.

- Los valores de los flectores, tanto máximos como mínimos, resultan muy superiores a los obtenidos en los casos anteriores, lo que denota, que además de haberse incrementado de forma considerable la luz de la estructura, el esquema de atirantamiento es menos eficaz, y por ello el tablero debe ser capaz de absorber esfuerzos de mayor cuantía.

Finalmente en el caso del pilono (Figura 135) se puede indicar:

- Las envolventes de flectores del pilono resultan idénticas a las de los modelos anteriores, siendo los valores determinantes en el caso de los flectores mínimos los valores obtenidos en la fases de construcción, mientras que los máximos quedan determinados por las situaciones de servicio. 
- Los flectores en fase final siguen, al igual que en los casos anteriores, dado que el proceso constructivo se sigue planificando para que así ocurra, son de cuantía muy reducida.

- Por otra parte los valores obtenidos en este caso son, tanto en construcción como en servicio, muy superiores a los obtenidos en las estructuras calculadas en los capítulos anteriores.

Adicionalmente se han estudiado las componentes que conforman el axil de los tirantes de esta estructura, tal y como se hizo en los capítulos anteriores. Se adjunta gráfico representando estos valores (Figura 136).

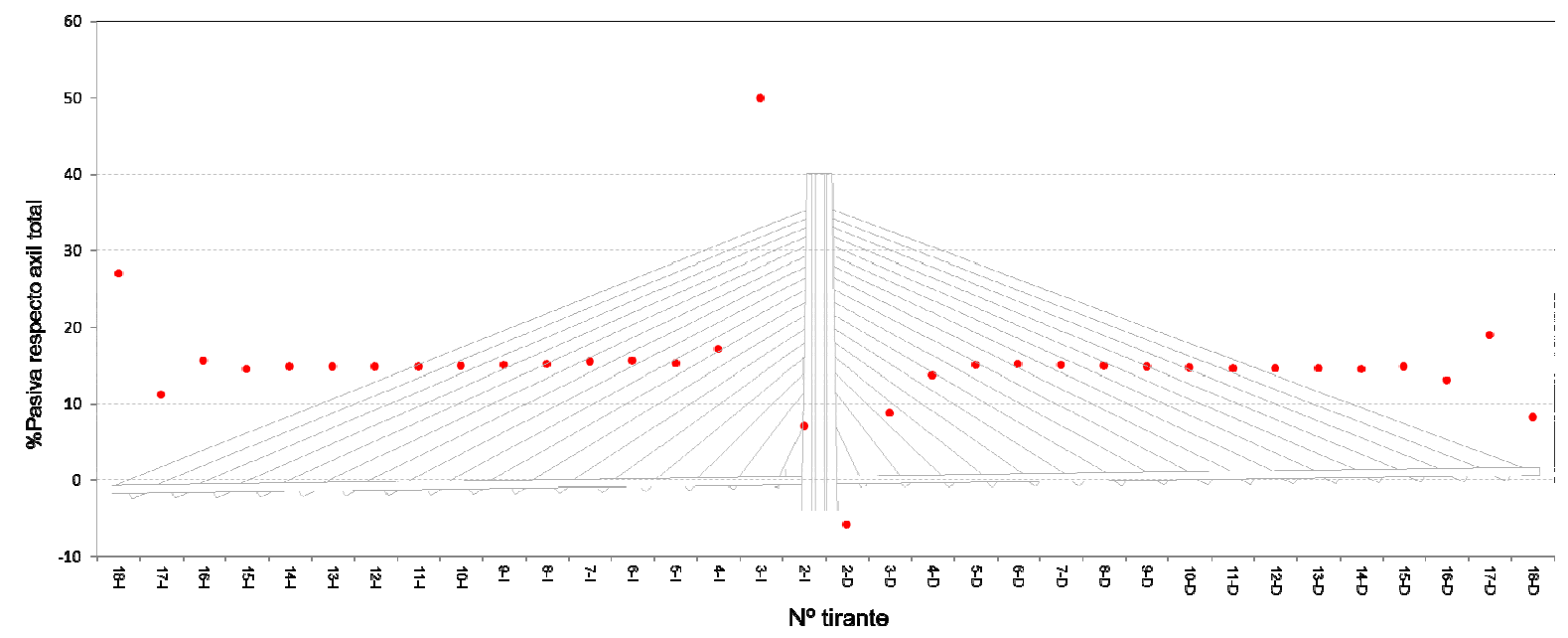

Figura 136. Porcentaje del axil pasivo respecto del axil total en los diferentes tirantes.

De este gráfico se puede concluir que:

- La diferencia entre los axiles de montaje (parte activa del axil) y los finales alcanzan valores cercanos al 15\%, algo mayores que en el Modelo 90, pero menores que en el caso del Modelo 90M.

- Existen peculiaridades en valores obtenidos en el caso tanto de los primeros como de los últimos cables colocados. En este caso, a diferencia de lo ocurrido en los modelos ya calculados, el tirante 18D de retenida presenta una diferencia menos acusada con los porcentajes de axil pasivo que presentan los cables adyacentes.

- La justificación de la obtención en este caso de axiles pasivos en los cables de menor cuantía se justifica en base a la menor eficacia del atirantamiento, dada su configuración geométrica más desfavorable, lo que implica que tengan en 
consecuencia menos capacidad para absorber las cargas exteriores aplicadas, como son los pesos propios y cargas muertas.

Se confirmaron así los resultados obtenidos en el epígrafe 3.3, que indicaban que a mayor luz de la estructura, y por lo tanto longitud de los tirantes, menor será la capacidad de absorber axil pasivo. De ahí las diferencias encontradas con el Modelo $90 \mathrm{M}$.

\subsection{COMPARATIVA DEL METODO DE INSTALACION DE LOS CABLES.}

Se incluye en este apartado, como complemento del análisis desarrollado en el epígrafe 4.5, un estudio en el que se comparan las cuantías de errores generadas, respecto de las fuerzas de tesado teóricas, al aplicar los errores máximos admisibles según el SETRA [102] en la medición de las deformaciones de montaje de los tirantes. Se ha aplicado este estudio al caso de las estructuras Modelo $90 \mathrm{M}$ y Modelo 180 , con la intención de comparar con los obtenidos en el epígrafe reseñado para el Modelo 90, pudiendo de esta forma conocerse si la modificación de la estructura a calcular tiene alguna implicación en este sentido.

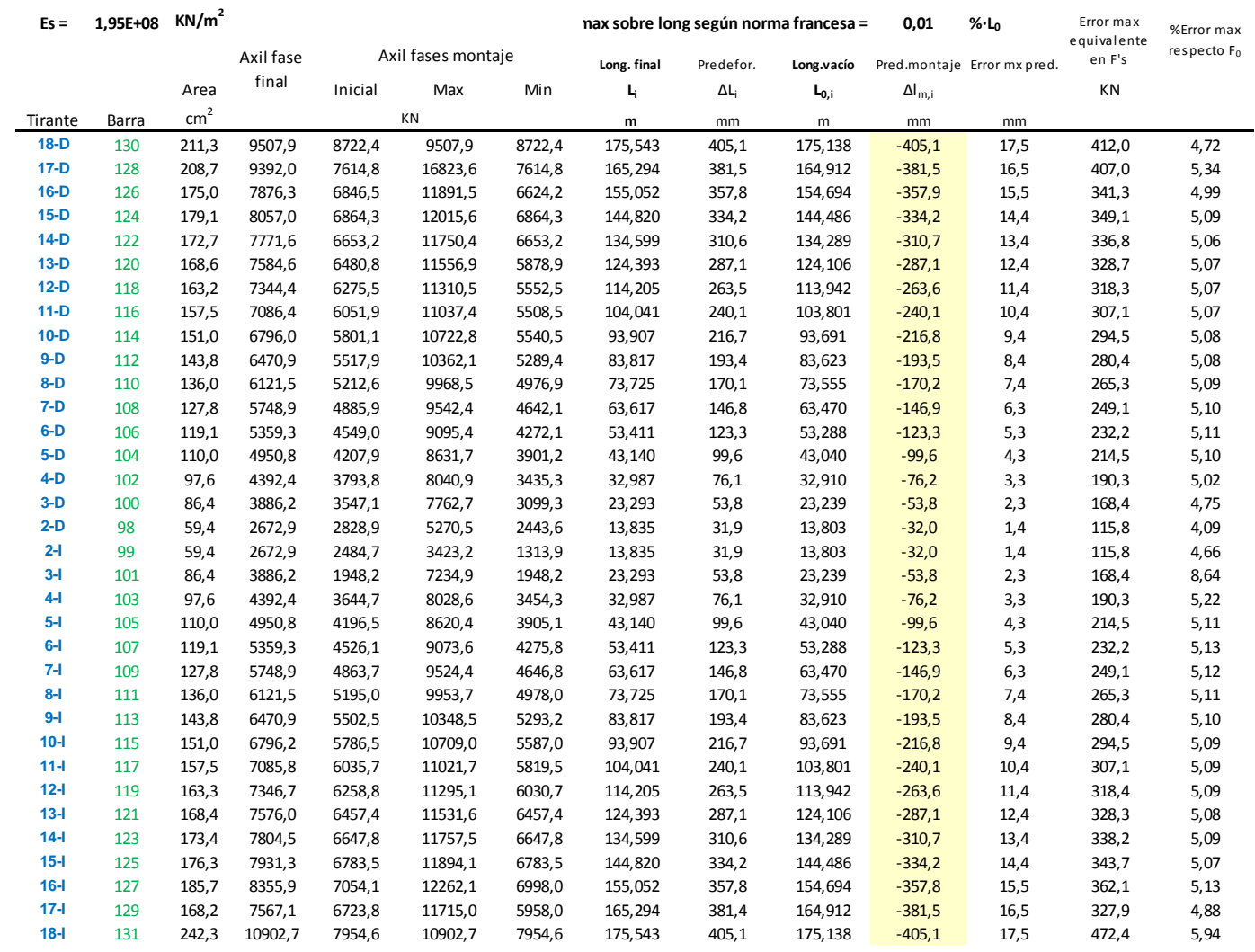

Tabla 6. Axiles teóricos finales y de montaje de los tirantes y obtención de los errores equivalentes en fuerzas, correspondientes a un proceso de instalación de los cables mediante el método de las deformaciones con el error máximo admitido por el SETRA [102] para el caso del Modelo 180. 


\begin{tabular}{|c|c|c|c|c|c|c|c|c|c|c|c|c|c|}
\hline \multirow{4}{*}{$\begin{array}{c}\text { Es }= \\
\text { Tirante }\end{array}$} & \multirow{4}{*}{$\begin{array}{c}1,95 E+08 \\
\text { Barra }\end{array}$} & \multirow{4}{*}{$\begin{array}{l}\mathrm{KN} / \mathrm{m}^{2} \\
\text { Area } \\
\mathrm{cm}^{2}\end{array}$} & \multirow{4}{*}{$\begin{array}{l}\text { Axil fase } \\
\text { final }\end{array}$} & \multirow{2}{*}{\multicolumn{3}{|c|}{ Axil fases montaje }} & \multirow{3}{*}{$\begin{array}{c}\text { nax sobre lo } \\
\text { Long. final } \\
L_{i}\end{array}$} & \multirow{3}{*}{$\begin{array}{l}\text { según no } \\
\text { Predefor. } \\
\quad \Delta \mathrm{L}_{\mathrm{i}}\end{array}$} & \multirow{3}{*}{$\begin{array}{l}\text { Long.vacío } \\
\mathrm{L}_{0, \mathrm{i}}\end{array}$} & \multirow{3}{*}{$\begin{array}{c}\mathbf{0 , 0 1} \\
\text { Pred.montaje } \\
\Delta \mathrm{l}_{\mathrm{m}, \mathrm{i}}\end{array}$} & \multirow{3}{*}{$\begin{array}{l}\% \cdot L_{0} \\
\text { Error mx pred. }\end{array}$} & \multirow{4}{*}{$\begin{array}{l}\text { Error max } \\
\text { equivalente } \\
\text { en F's } \\
\text { KN }\end{array}$} & \multirow{4}{*}{$\begin{array}{l}\text { \%Error max } \\
\text { respecto } \mathrm{F}_{0}\end{array}$} \\
\hline & & & & & & & & & & & & & \\
\hline & & & & Inicial & $\operatorname{Max}$ & $\operatorname{Min}$ & & & & & & & \\
\hline & & & & \multicolumn{2}{|c|}{ KN } & & $\mathrm{m}$ & $\mathrm{mm}$ & $\mathrm{m}$ & $\mathrm{mm}$ & $\mathrm{mm}$ & & \\
\hline 18-D & 130 & 123,4 & 5554,0 & 5054,4 & 5554,0 & 5054,4 & 93,899 & 216,7 & 93,682 & $-216,7$ & 9,4 & 240,7 & 4,76 \\
\hline 16-D & -2 & 162,6 & 7316,7 & 5066,3 & 11023,2 & 5066,3 & 83,808 & 193,4 & 83,614 & $-193,4$ & 8,4 & 317,1 & 6,26 \\
\hline 14-D & -2 & 131,5 & 5919,1 & 4412,4 & 8826,3 & 4412,4 & 73,715 & 170,1 & 73,545 & $-170,1$ & 7,4 & 256,5 & 5,81 \\
\hline 12-D & -2 & 129,2 & 5811,6 & 4202,7 & 8603,4 & 4202,7 & 63,607 & 146,8 & 63,460 & $-146,8$ & 6,3 & 251,8 & 5,99 \\
\hline 10-D & -2 & 119,0 & 5355,2 & 3866,4 & 8130,4 & 3866,4 & 53,400 & 123,2 & 53,277 & $-123,2$ & 5,3 & 232,1 & 6,00 \\
\hline 8-D & -2 & 110,3 & 4963,1 & 3549,1 & 7685,6 & 3549,1 & 43,128 & 99,5 & 43,029 & $-99,5$ & 4,3 & 215,1 & 6,06 \\
\hline 6-D & -2 & 97,8 & 4399,8 & 3157,8 & 7101,6 & 3157,8 & 32,973 & 76,1 & 32,897 & $-76,1$ & 3,3 & 190,7 & 6,04 \\
\hline 4-D & -2 & 86,5 & 3892,7 & 2986,4 & 6794,9 & 2986,4 & 23,277 & 53,7 & 23,224 & $-53,7$ & 2,3 & 168,7 & 5,65 \\
\hline 2-D & -2 & 59,4 & 2674,1 & 2673,7 & 4968,6 & 2438,9 & 13,835 & 31,9 & 13,803 & $-31,9$ & 1,4 & 115,9 & 4,33 \\
\hline $2-1$ & 99 & 59,4 & 2674,2 & 2300,2 & 3249,5 & 1276,1 & 13,835 & 31,9 & 13,803 & $-31,9$ & 1,4 & 115,9 & 5,04 \\
\hline $4-1$ & 2 & 86,5 & 3892,5 & 1500,8 & 6375,8 & 1500,8 & 23,277 & 53,7 & 23,224 & $-53,7$ & 2,3 & 168,7 & 11,24 \\
\hline 6-I & 2 & 97,8 & 4400,4 & 3036,9 & 7089,1 & 3036,9 & 32,973 & 76,1 & 32,897 & $-76,1$ & 3,3 & 190,7 & 6,28 \\
\hline 8-I & 2 & 110,2 & 4960,5 & 3531,4 & 7665,6 & 3531,4 & 43,128 & 99,5 & 43,029 & $-99,5$ & 4,3 & 214,9 & 6,09 \\
\hline I-10 & 2 & 119,2 & 5365,9 & 3834,5 & 8102,1 & 3834,5 & 53,400 & 123,2 & 53,277 & $-123,2$ & 5,3 & 232,5 & 6,06 \\
\hline I-12 & 2 & 128,2 & 5768,8 & 4147,7 & 8541,1 & 4147,7 & 63,607 & 146,8 & 63,460 & $-146,8$ & 6,3 & 250,0 & 6,03 \\
\hline I-14 & 2 & 135,3 & 6089,5 & 4439,8 & 8913,8 & 4439,8 & 73,715 & 170,1 & 73,545 & $-170,1$ & 7,4 & 263,9 & 5,94 \\
\hline I-16 & 2 & 147,7 & 6644,3 & 4802,1 & 9224,7 & 4802,1 & 83,808 & 193,4 & 83,614 & $-193,4$ & 8,4 & 287,9 & 6,00 \\
\hline 18-I & 2 & 134,7 & 6059,3 & 4583,3 & 6059,3 & 4583,3 & 93,899 & 216,7 & 93,682 & $-216,7$ & 9,4 & 262,6 & 5,73 \\
\hline
\end{tabular}

Tabla 7. Axiles teóricos finales y de montaje de los tirantes y obtención de los errores equivalentes en fuerzas, correspondientes a un proceso de instalación de los cables mediante el método de las deformaciones con el error máximo admitido por el SETRA [102] para el caso del Modelo 90M. 


\begin{tabular}{|c|c|c|c|c|c|c|}
\hline \multicolumn{7}{|c|}{$\begin{array}{c}\text { Errores máximos equivalentes en F's introducidos con las predeformaciones } \\
\text { aleatorias respecto de los axiles teóricos de montaje }\end{array}$} \\
\hline Cable & 90 & 180 & $90 \mathrm{M}$ & Max & Media & Min \\
\hline 18-D & 4,76 & 4,72 & 4,76 & 4,76 & 4,75 & 4,72 \\
\hline 17-D & 5,12 & 5,34 & & 5,34 & 5,23 & 5,12 \\
\hline $16-\mathrm{D}$ & 4,89 & 4,99 & 6,26 & 6,26 & 5,38 & 4,89 \\
\hline 15-D & 4,96 & 5,09 & & 5,09 & 5,02 & 4,96 \\
\hline 14-D & 4,95 & 5,06 & 5,81 & 5,81 & 5,27 & 4,95 \\
\hline 13-D & 4,95 & 5,07 & & 5,07 & 5,01 & 4,95 \\
\hline 12-D & 4,96 & 5,07 & 5,99 & 5,99 & 5,34 & 4,96 \\
\hline 11-D & 4,96 & 5,07 & & 5,07 & 5,02 & 4,96 \\
\hline 10-D & 4,96 & 5,08 & 6,00 & 6,00 & 5,35 & 4,96 \\
\hline 9-D & 4,97 & 5,08 & & 5,08 & 5,02 & 4,97 \\
\hline 8-D & 4,96 & 5,09 & 6,06 & 6,06 & 5,37 & 4,96 \\
\hline 7-D & 4,95 & 5,10 & & 5,10 & 5,02 & 4,95 \\
\hline 6-D & 4,91 & 5,11 & 6,04 & 6,04 & 5,35 & 4,91 \\
\hline 5-D & 4,83 & 5,10 & & 5,10 & 4,96 & 4,83 \\
\hline 4-D & 4,66 & 5,02 & 5,65 & 5,65 & 5,11 & 4,66 \\
\hline 3-D & 4,40 & 4,75 & & 4,75 & 4,57 & 4,40 \\
\hline 2-D & 4,18 & 4,09 & 4,33 & 4,33 & 4,20 & 4,09 \\
\hline $2-1$ & 4,60 & 4,66 & 5,04 & 5,04 & 4,77 & 4,60 \\
\hline $3-1$ & 11,96 & 8,64 & & 11,96 & 10,30 & 8,64 \\
\hline $4-1$ & 5,77 & 5,22 & 11,24 & 11,24 & 7,41 & 5,22 \\
\hline $5-1$ & 5,13 & 5,11 & & 5,13 & 5,12 & 5,11 \\
\hline $6-1$ & 4,98 & 5,13 & 6,28 & 6,28 & 5,46 & 4,98 \\
\hline $7-1$ & 4,96 & 5,12 & & 5,12 & 5,04 & 4,96 \\
\hline $8-I$ & 4,97 & 5,11 & 6,09 & 6,09 & 5,39 & 4,97 \\
\hline 9-I & 4,98 & 5,10 & & 5,10 & 5,04 & 4,98 \\
\hline $10-$ I & 4,99 & 5,09 & 6,06 & 6,06 & 5,38 & 4,99 \\
\hline $11-I$ & 4,99 & 5,09 & & 5,09 & 5,04 & 4,99 \\
\hline $12-I$ & 4,98 & 5,09 & 6,03 & 6,03 & 5,36 & 4,98 \\
\hline 13-I & 4,97 & 5,08 & & 5,08 & 5,03 & 4,97 \\
\hline 14-I & 4,97 & 5,09 & 5,94 & 5,94 & 5,33 & 4,97 \\
\hline 15-I & 4,95 & 5,07 & & 5,07 & 5,01 & 4,95 \\
\hline 16-I & 4,99 & 5,13 & 6,00 & 6,00 & 5,37 & 4,99 \\
\hline |I I & 4,84 & 4,88 & & 4,88 & 4,86 & 4,84 \\
\hline 18-I & 5,46 & 5,94 & 5,73 & 5,94 & 5,71 & 5,46 \\
\hline \multicolumn{4}{|c|}{ Estadística por estructura } & & & \\
\hline Estructura & 90 & 180 & $90 \mathrm{M}$ & & & \\
\hline $\operatorname{Max}$ & 11,96 & 8,64 & 11,24 & & & \\
\hline Media & 5,14 & 5,16 & 6,07 & & & \\
\hline Desv tip.: $\sigma$ & 1,23 & 0,67 & 1,39 & & & \\
\hline Min & 4,18 & 4,09 & 4,33 & & & \\
\hline
\end{tabular}

Tabla 8. Tabla resumen de los valores anteriores obtenidos para los Modelos 90M y 180, en comparación con los resultantes del Modelo 90. 


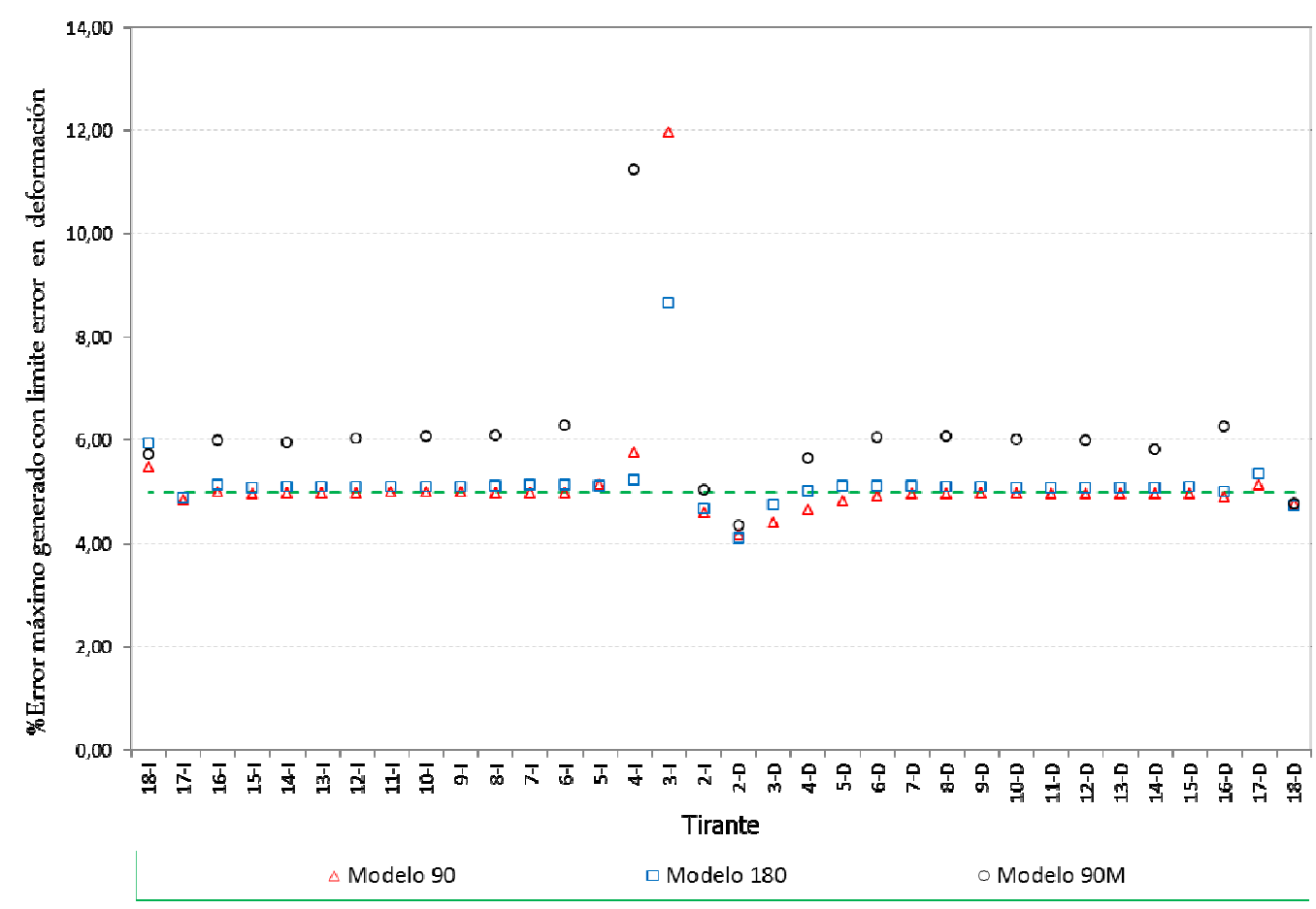

Figura 137. Errores máximos generados en fuerzas respecto del valor teórico en los distintos cables, al aplicar sobre ellos durante su colocación el error máximo admisible en deformaciones según SETRA [102].

De los resultados anteriores se puede concluir que:

- En los modelos de cálculo de la estructura original (Modelo 90) y de la de 180+180 m de Iuz (Modelo 180) la implicación de los errores en deformaciones es similar a la planteada en el caso de la introducción de los errores en fuerzas. Resultando errores máximos del orden del $5 \%$.

- En el caso de la estructura original con la mitad de cables (Modelo 90M), los errores introducidos en deformaciones son algo mayores que los planteados en fuerzas, en concreto los errores en deformaciones suponen un valor medio del $6 \%$ frente al $5 \%$ de 10 producido al aplicar el error máximo en fuerzas. Esta cuestión justificaría en parte las diferencias encontradas entre los resultados obtenidos en 4.6 .3 en el Modelo 90 y en el epígrafe 5.3.2 para el Modelo 90M.

- En general los valores obtenidos en cada cable son similares salvo en el entorno de los cables 31 en las estructuras (Modelo 90) y (Modelo 180), y del 4 l en la estructura (Modelo 90M). Esas diferencias se deben a que el análisis realizado lo es en porcentaje del axil teórico de montaje de los cables, habiendo quedado esos cables por el proceso constructivo con unos axiles muy pequeños, lo que a su vez conlleva a que, los errores 
máximos planteables en deformaciones, signifiquen un porcentaje muy alto respecto de las fuerzas iniciales de tesado teóricas.

\subsection{RESULTADOS OBTENIDOS.}

Se procede en este epígrafe a desarrollar los resultados correspondientes a la aplicación de los errores aleatorios de montaje, bien por el método de las fuerzas o bien por el método de las deformaciones. A diferencia de lo planteado en el capítulo 4, en este caso no se ha considerado la introducción de errores en la posición de los anclajes dadas las conclusiones allí extraídas. Los análisis realizados son similares a los incluidos en capítulos anteriores para las otras estructuras:

- Error en la medida de las fuerzas de tesado.

- Error en la medida de las deformaciones de tesado.

De nuevo, para cada uno de estos cálculos, se han generado 3 muestras de 100 elementos cada una, por lo que en total se han realizado 600 cálculos individuales de la estructura.

Se ha mantenido la definición del valor característico ya reseñada, y se han utilizado las mismas situaciones de cálculo que en el capítulo anterior (situación final, situaciones de construcción y situaciones de servicio).

\subsubsection{Errores en fuerzas de tesado.}

En primer lugar se representan los resultados correspondientes a la introducción de fuerzas de tesado erróneas. Los resultados representados lo son de tirantes (Figura 138 a Figura 143), tablero (Figura 144 a Figura 146) y pilono (Figura 147 a Figura 148). 
6.4.1.1 Axiles en tirantes.

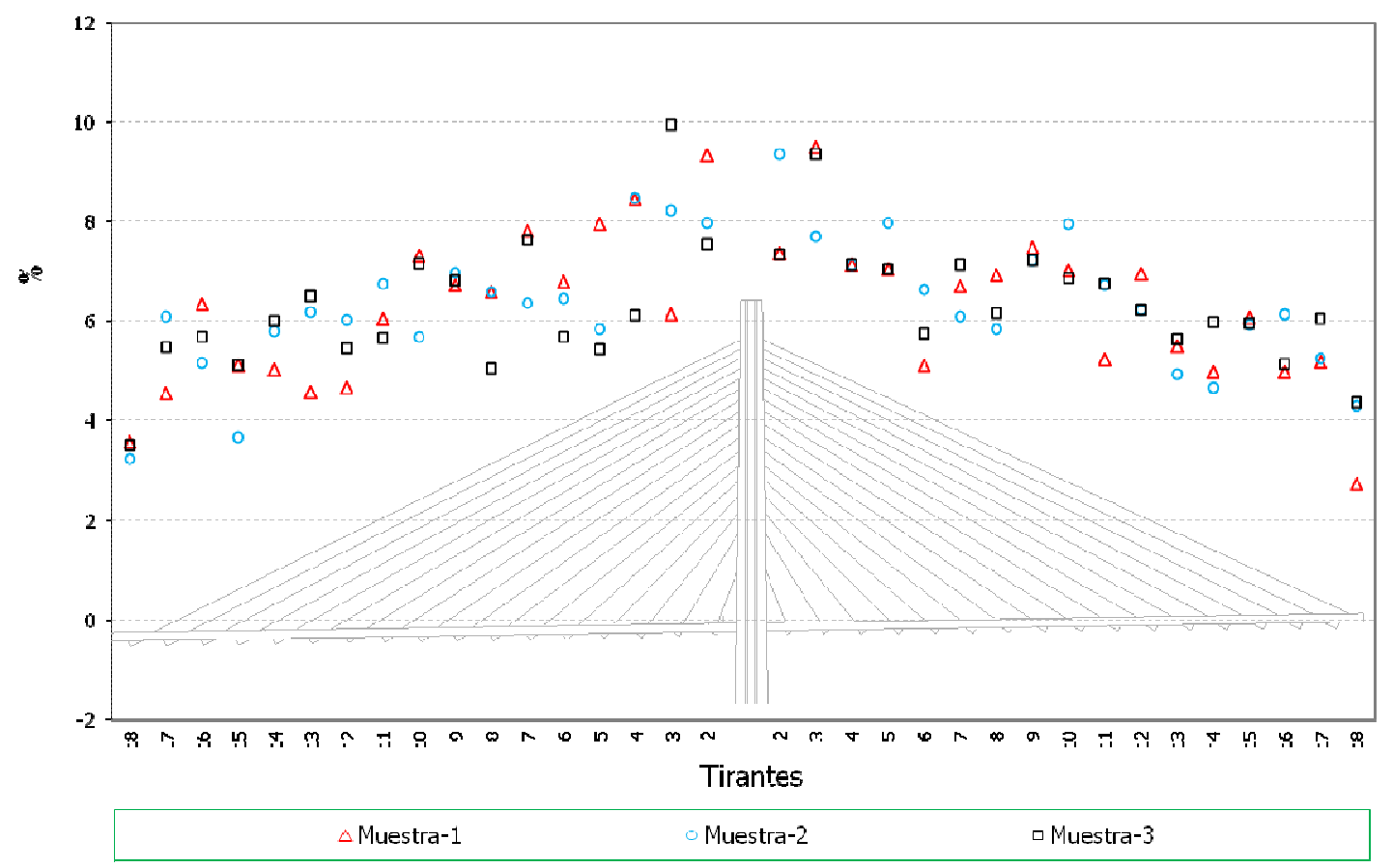

Figura 138. Axiles finales en los tirantes. Diferencia en $\%$ de los valores caracteristicos superiores respecto de los teóricos.

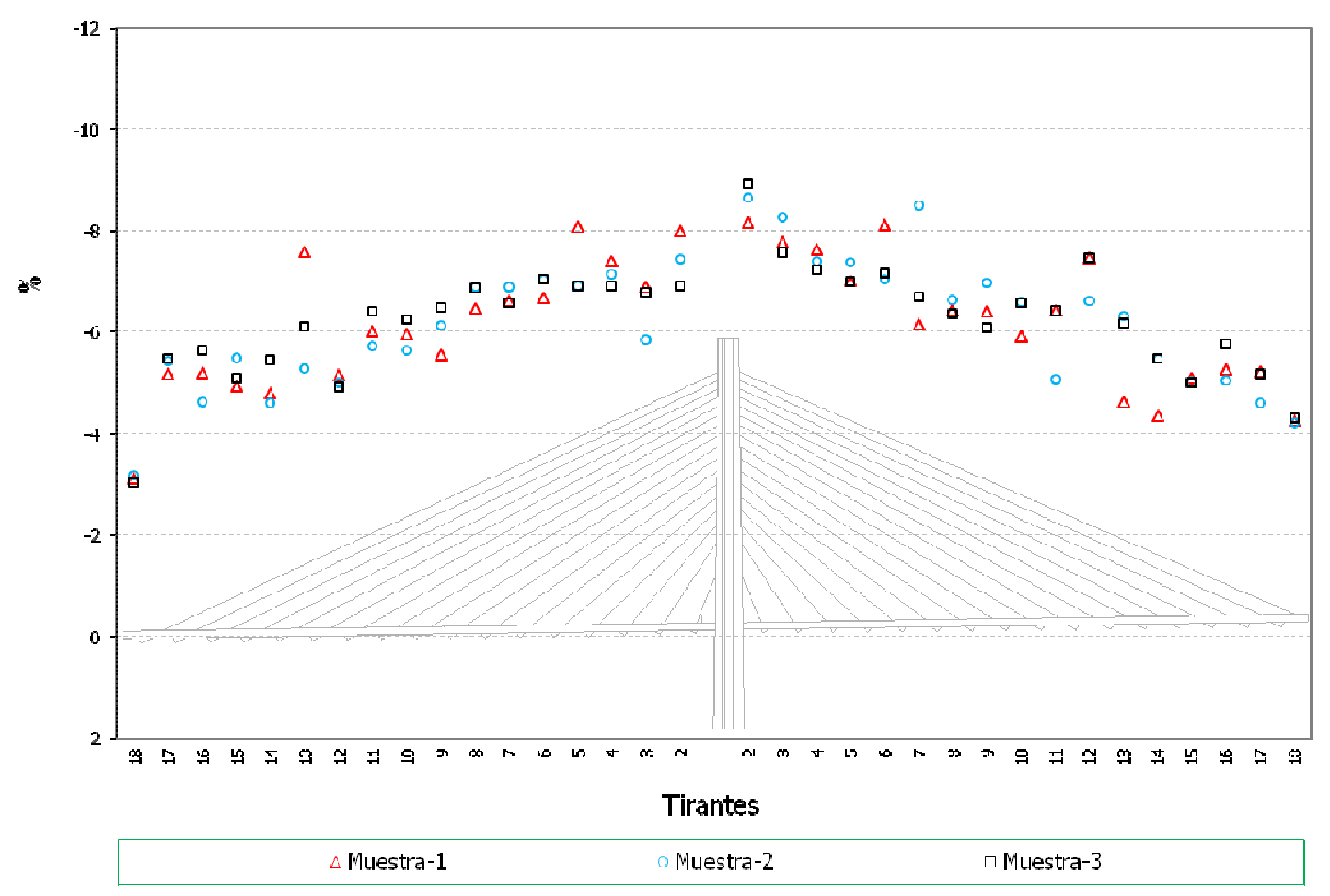

Figura 139. Axiles finales en los tirantes. Diferencia en \% de los valores característicos inferiores respecto de los teóricos. 


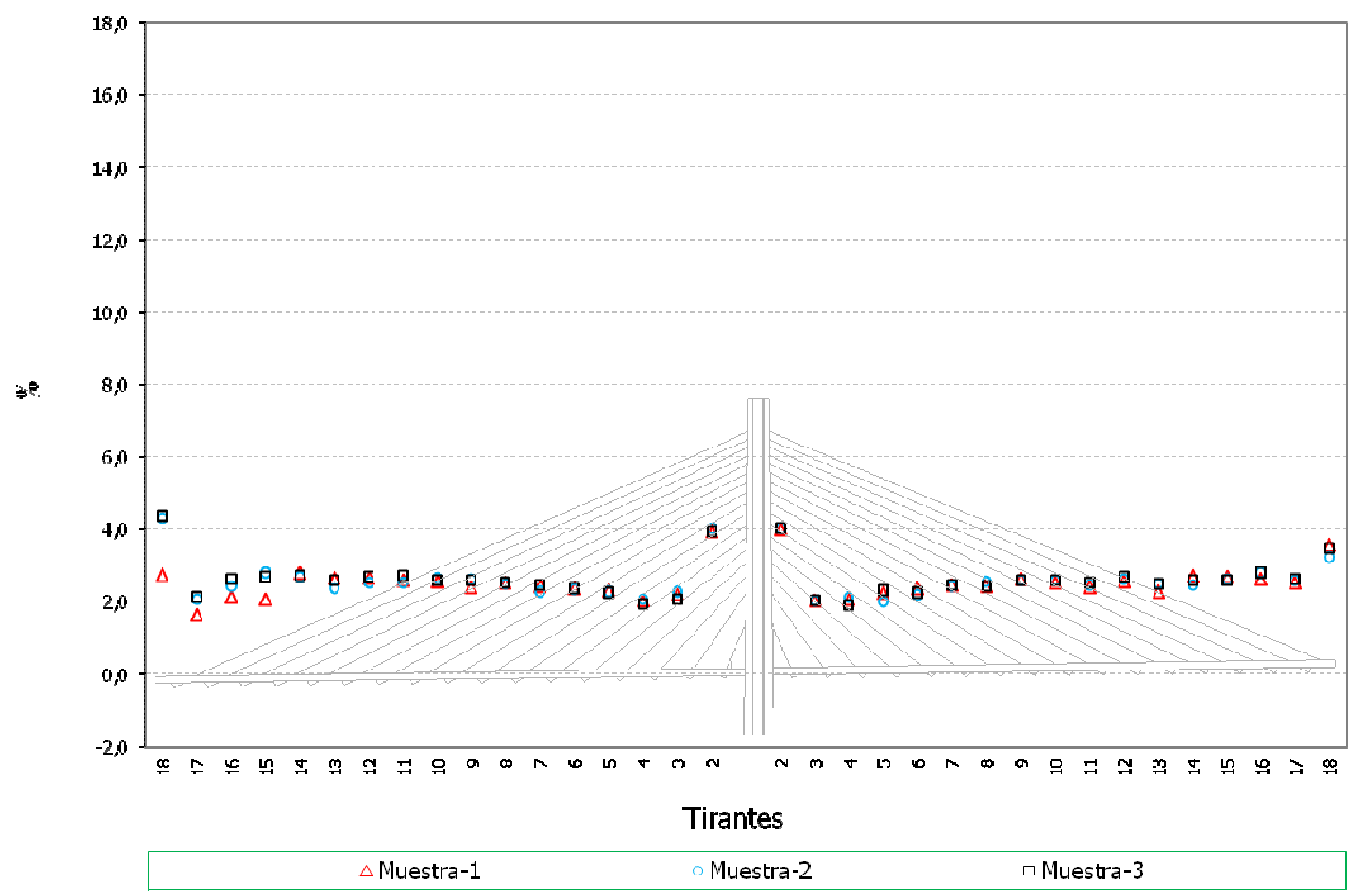

Figura 140. Axiles en los tirantes máximos de construcción. Diferencia en \% de los valores característicos superiores respecto de los teóricos.

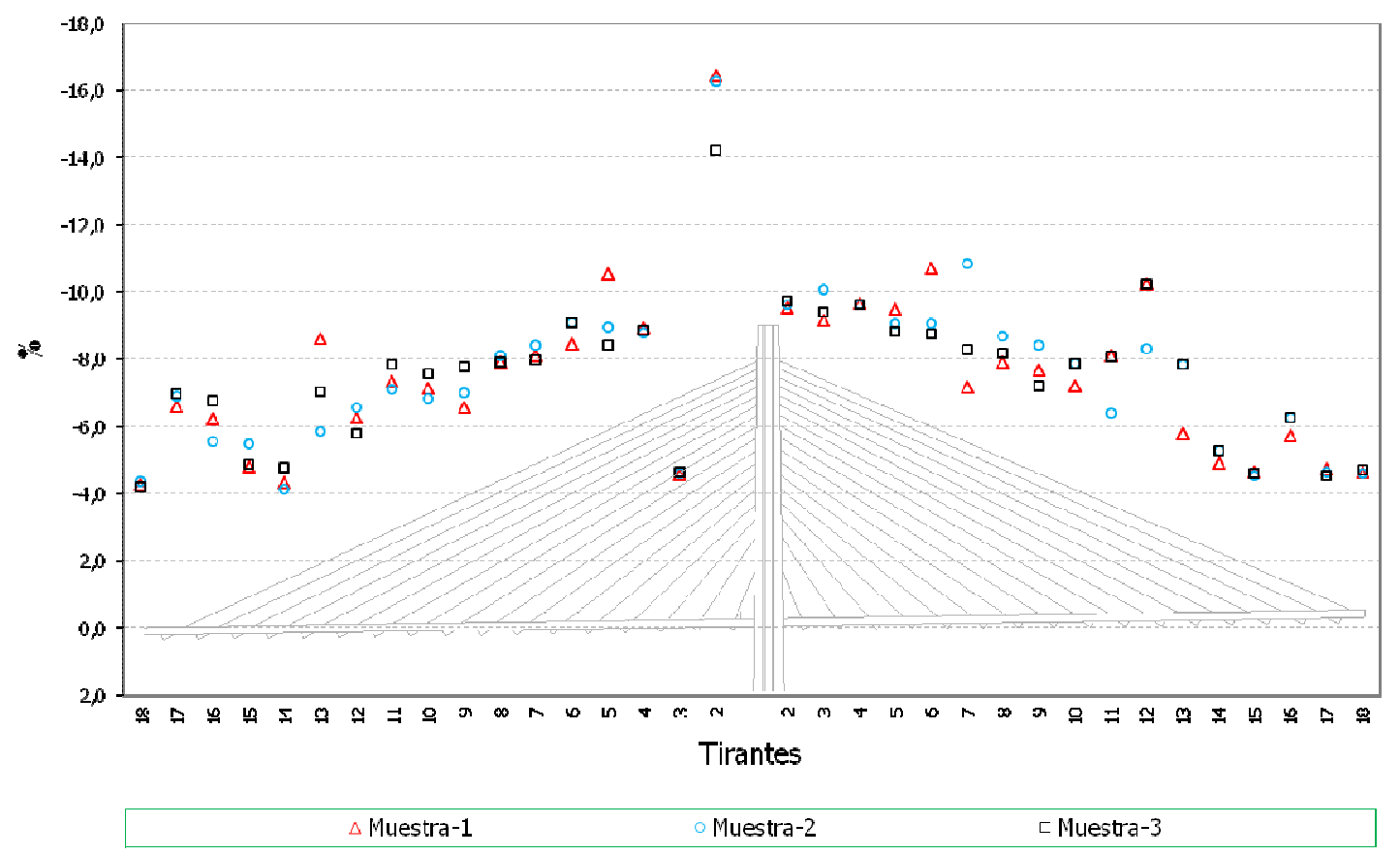

Figura 141. Axiles en los tirantes mínimos de construcción. Diferencia en \% de los valores característicos inferiores respecto de los teóricos. 


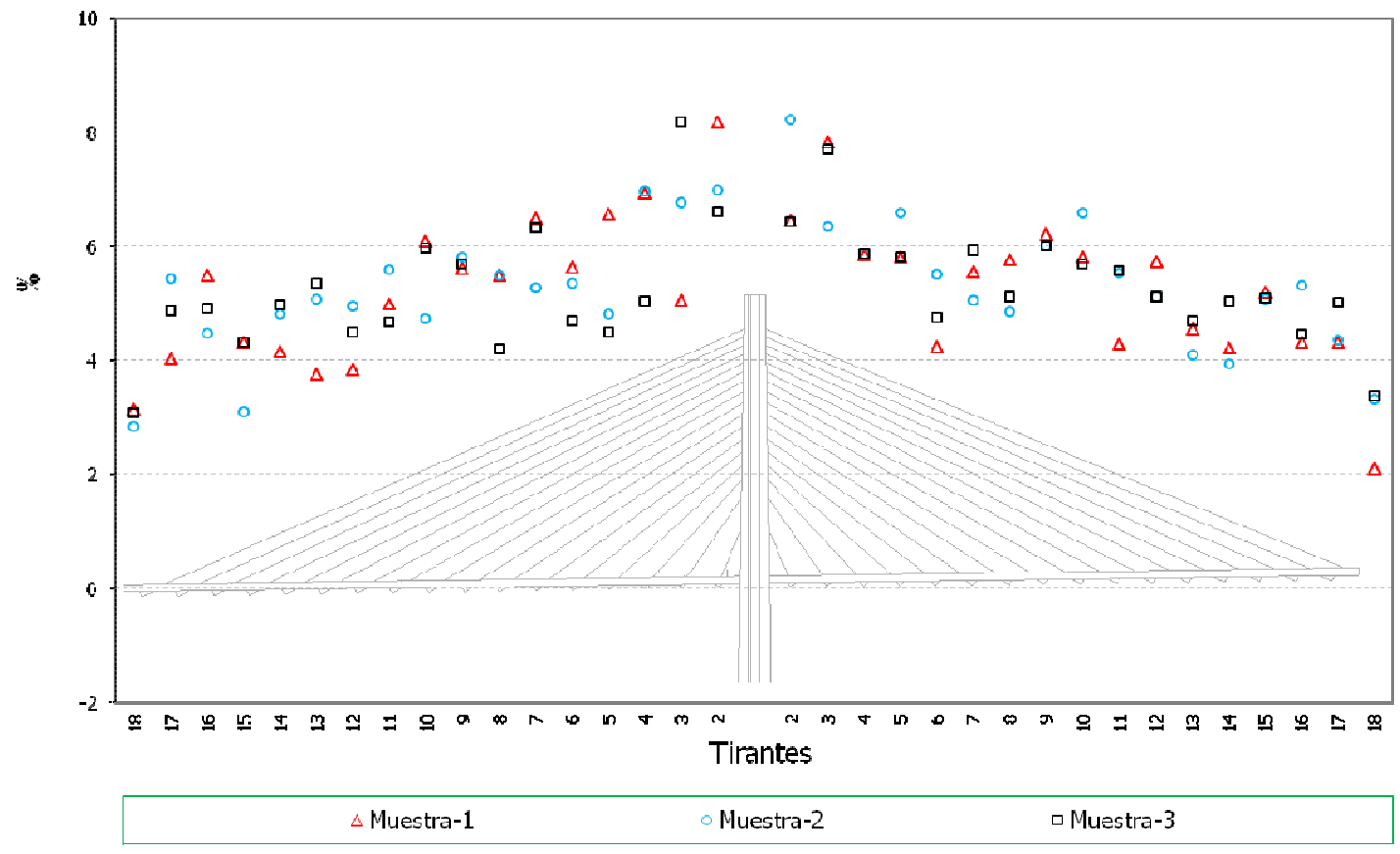

Figura 142. Axiles en los tirantes máximos de servicio. Diferencia en \% de los valores característicos superiores respecto de los teóricos.

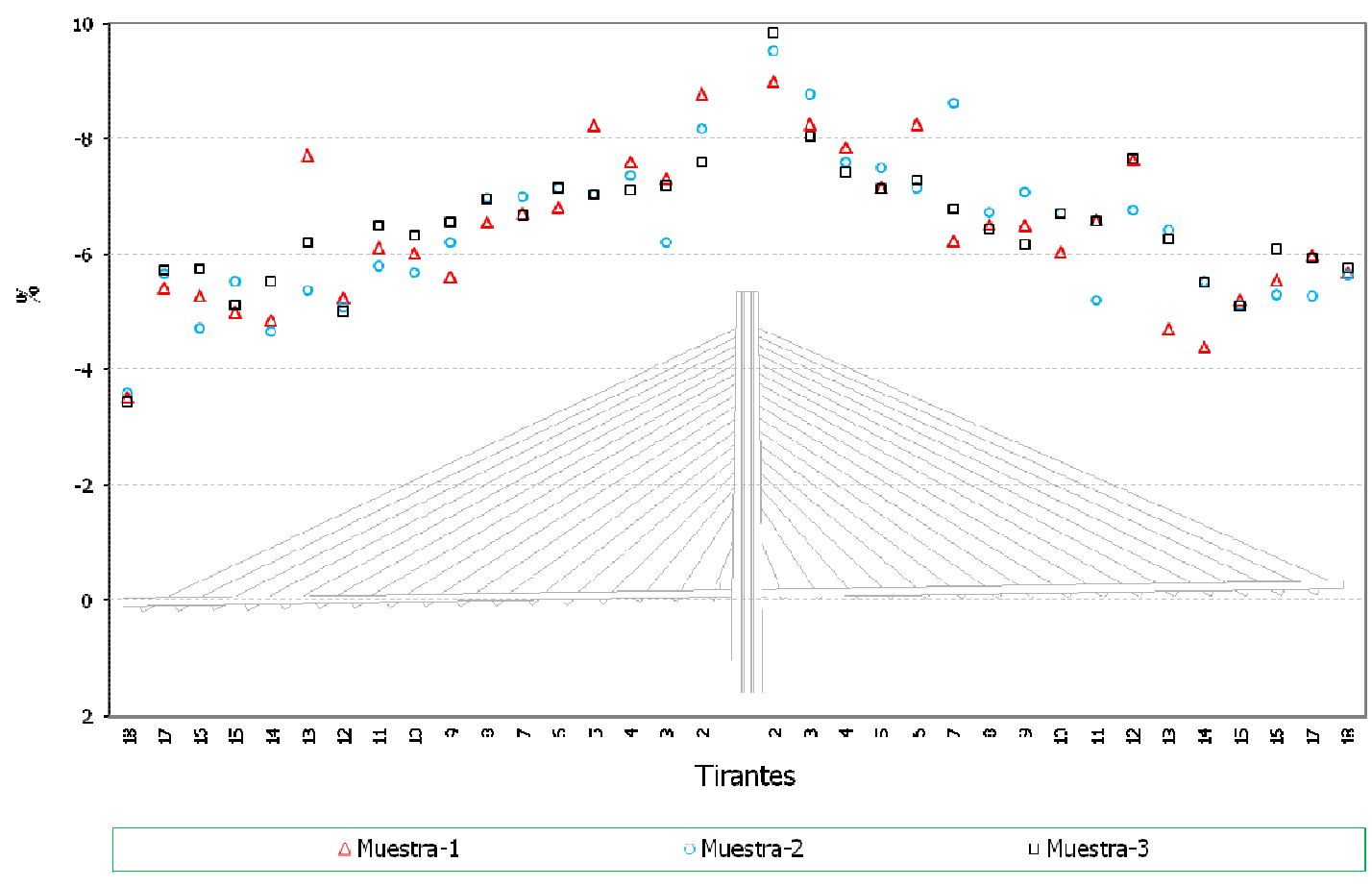

Figura 143. Axiles en los tirantes mínimos de servicio. Diferencia en \% de los valores característicos inferiores respecto de los teóricos. 


\subsubsection{Flectores en tablero.}

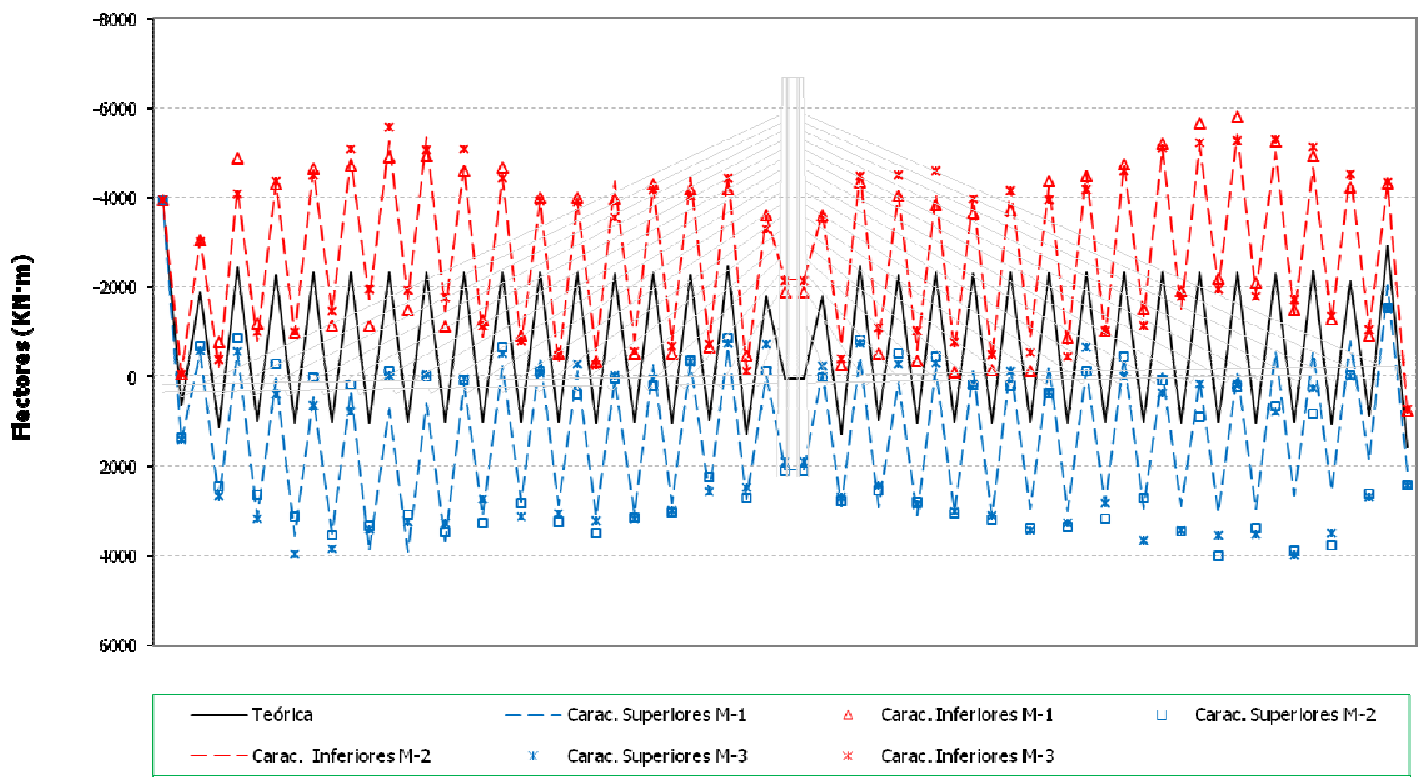

Figura 144. Flectores en tablero en situación final. Valores característicos superiores e inferiores.

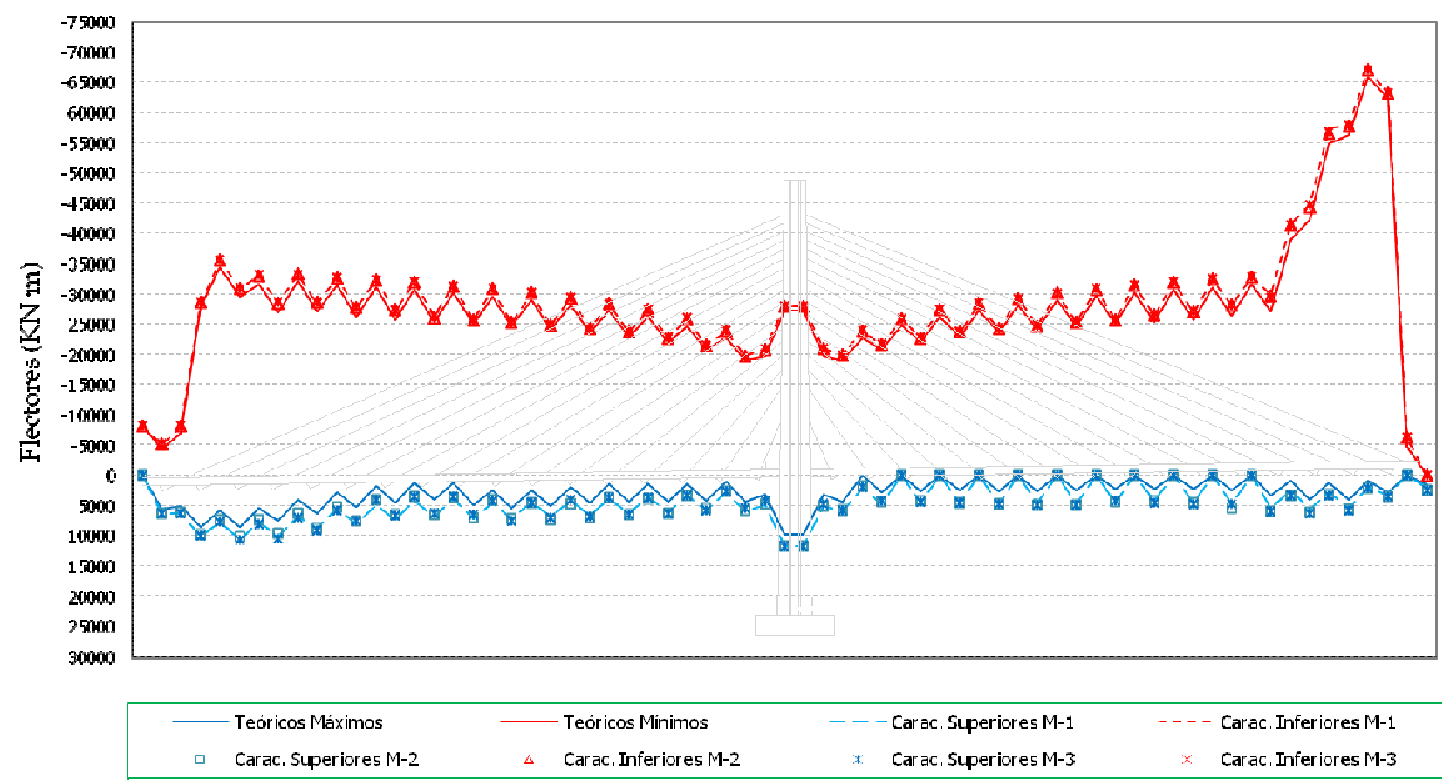

Figura 145. Flectores en tablero en fases de construcción. Valores característicos superiores e inferiores. 


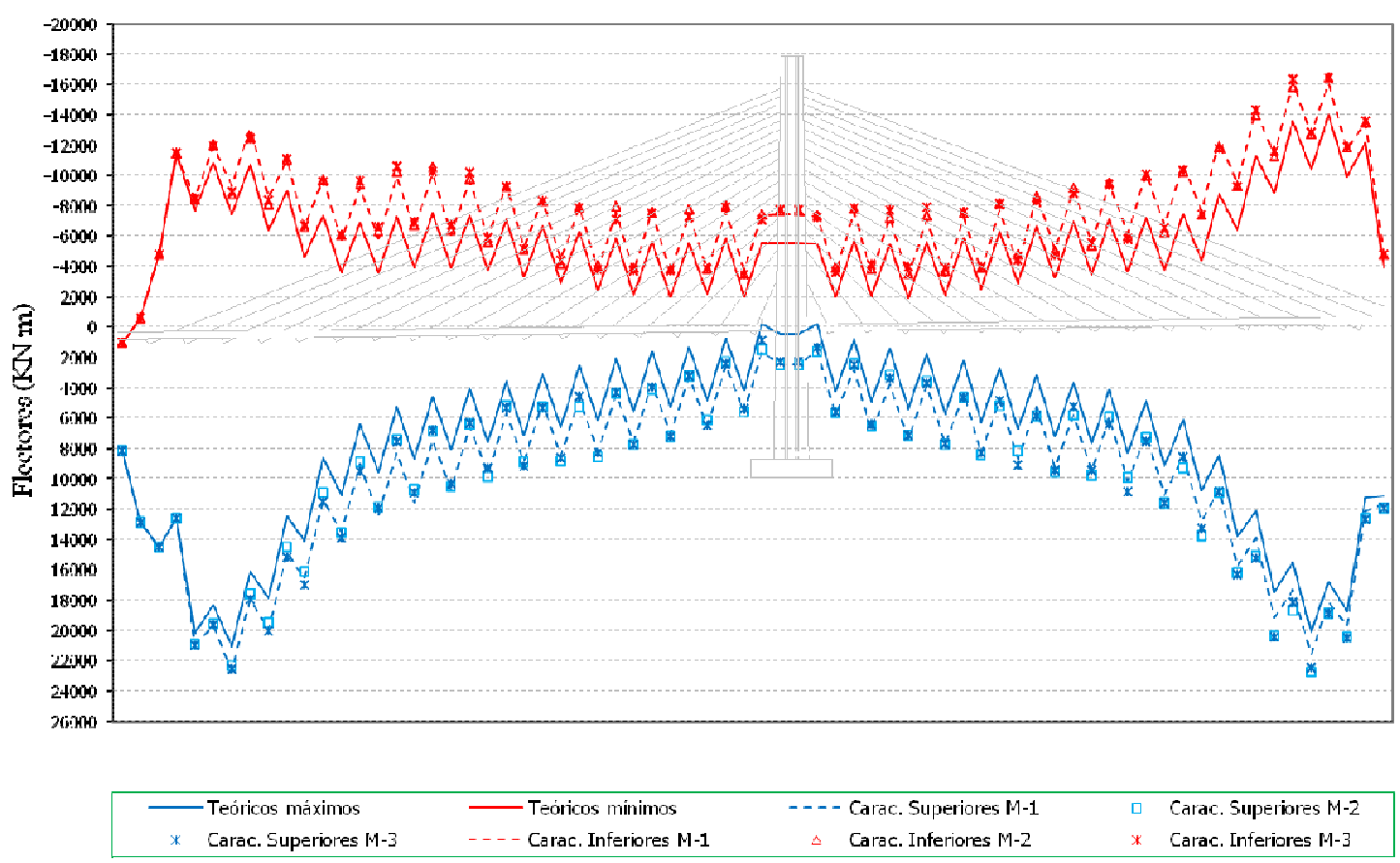

Figura 146. Flectores en tablero en fases de servicio. Valores característicos superiores e inferiores.

\subsubsection{Flectores en el pilono.}

Se incluyen en este apartado sólo los resultados considerados más significativos en este elemento, que en concreto son la envolvente de flectores mínimos en fase de construcción y la envolvente de máximos en servicio. 


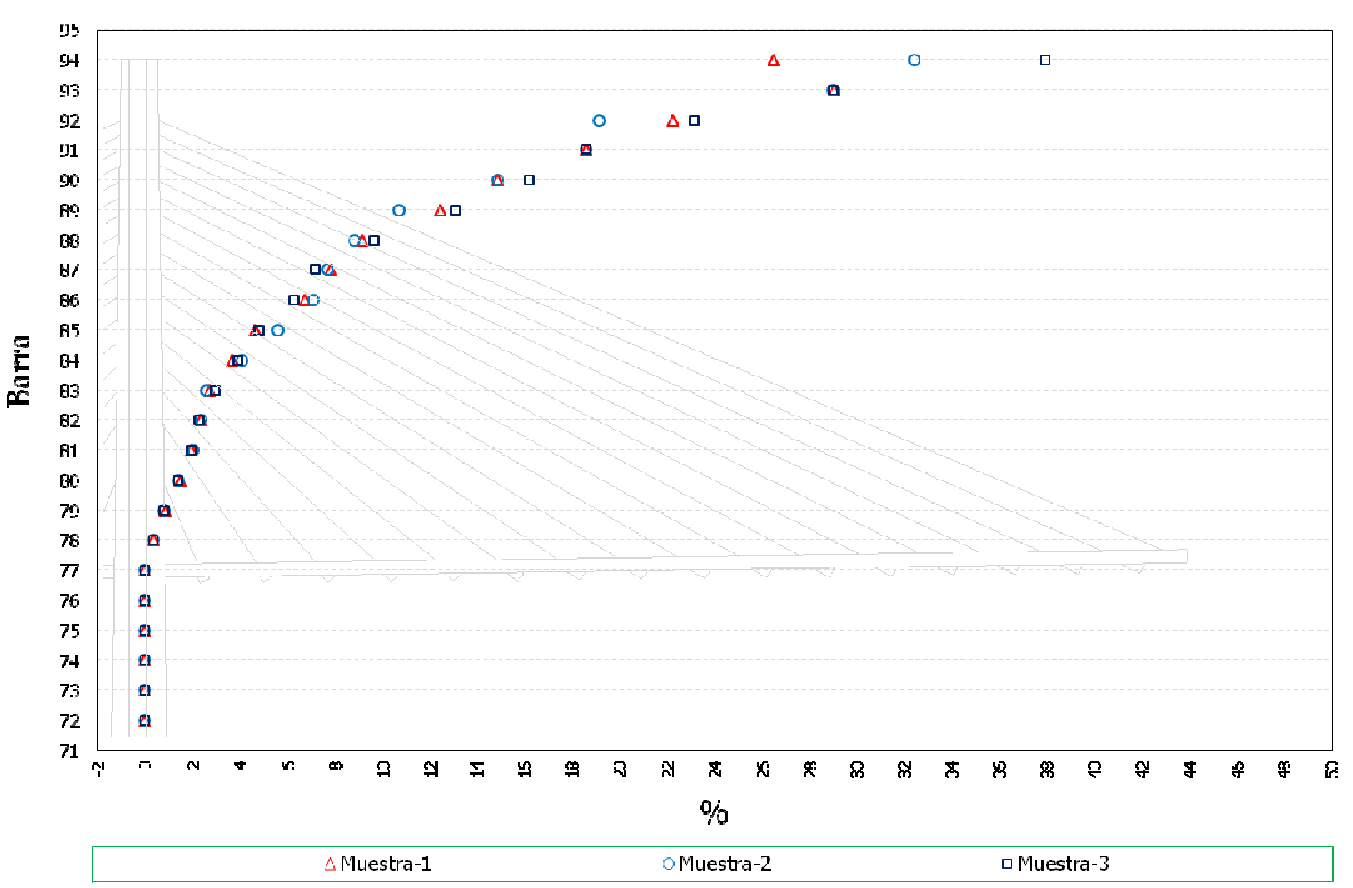

Figura 147. Flectores en pilono mínimos en fases de construcción. Diferencias en \% de los valores característicos inferiores respecto de los teóricos.

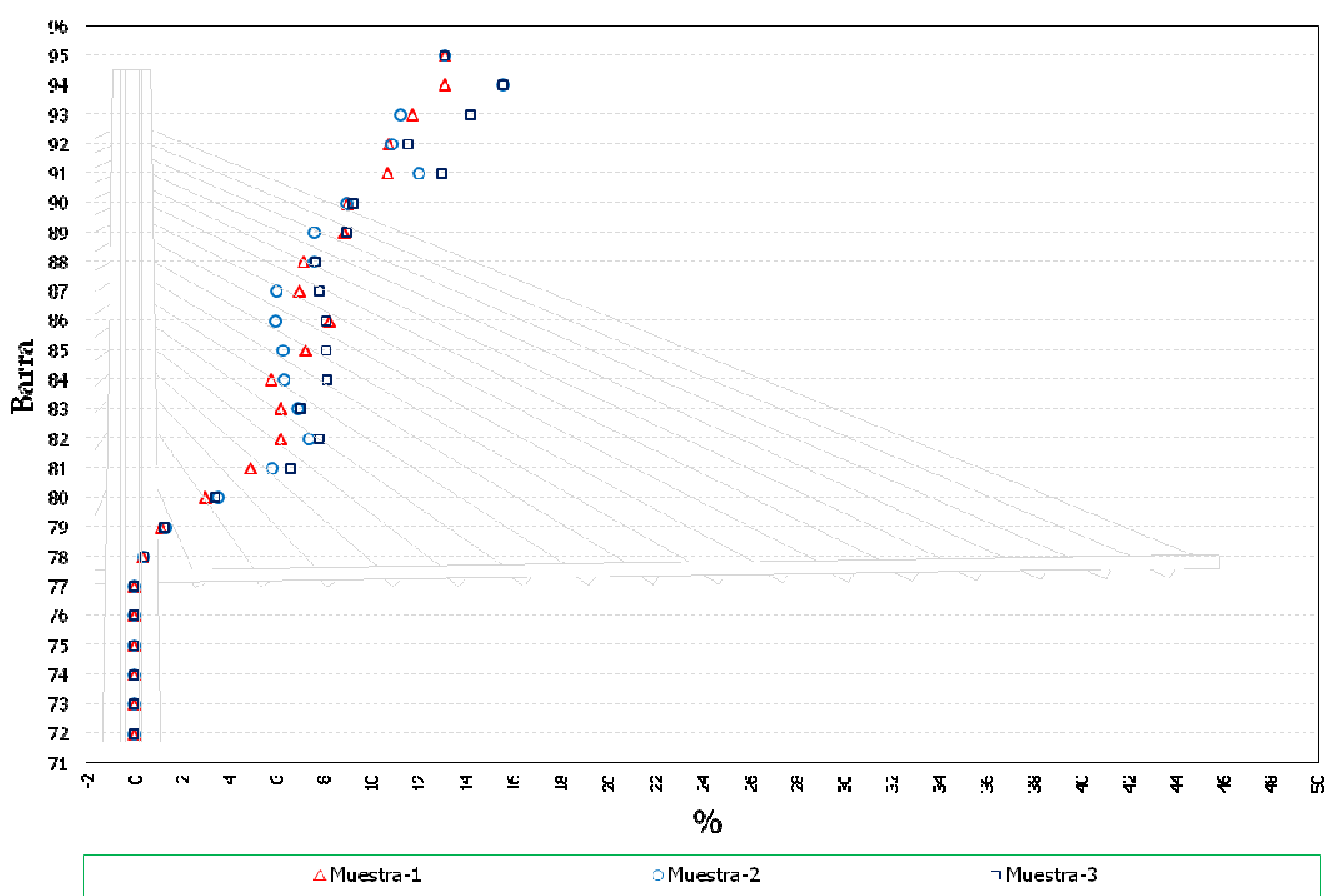

Figura 148. Flectores en pilono máximos en fases de servicio. Diferencias en \% de los valores característicos superiores respecto de los teóricos. 


\subsubsection{Errores en alargamientos de los cables.}

Se adjuntan a continuación resultados de la aplicación de los errores aleatorios sobre las deformaciones teóricas de montaje de los cables.

Los resultados representados lo son de tirantes (Figura 149 a Figura 154), tablero (Figura 155 a Figura 157) y pilono (Figura 158 a Figura 159).

\subsubsection{Axiles en tirantes.}

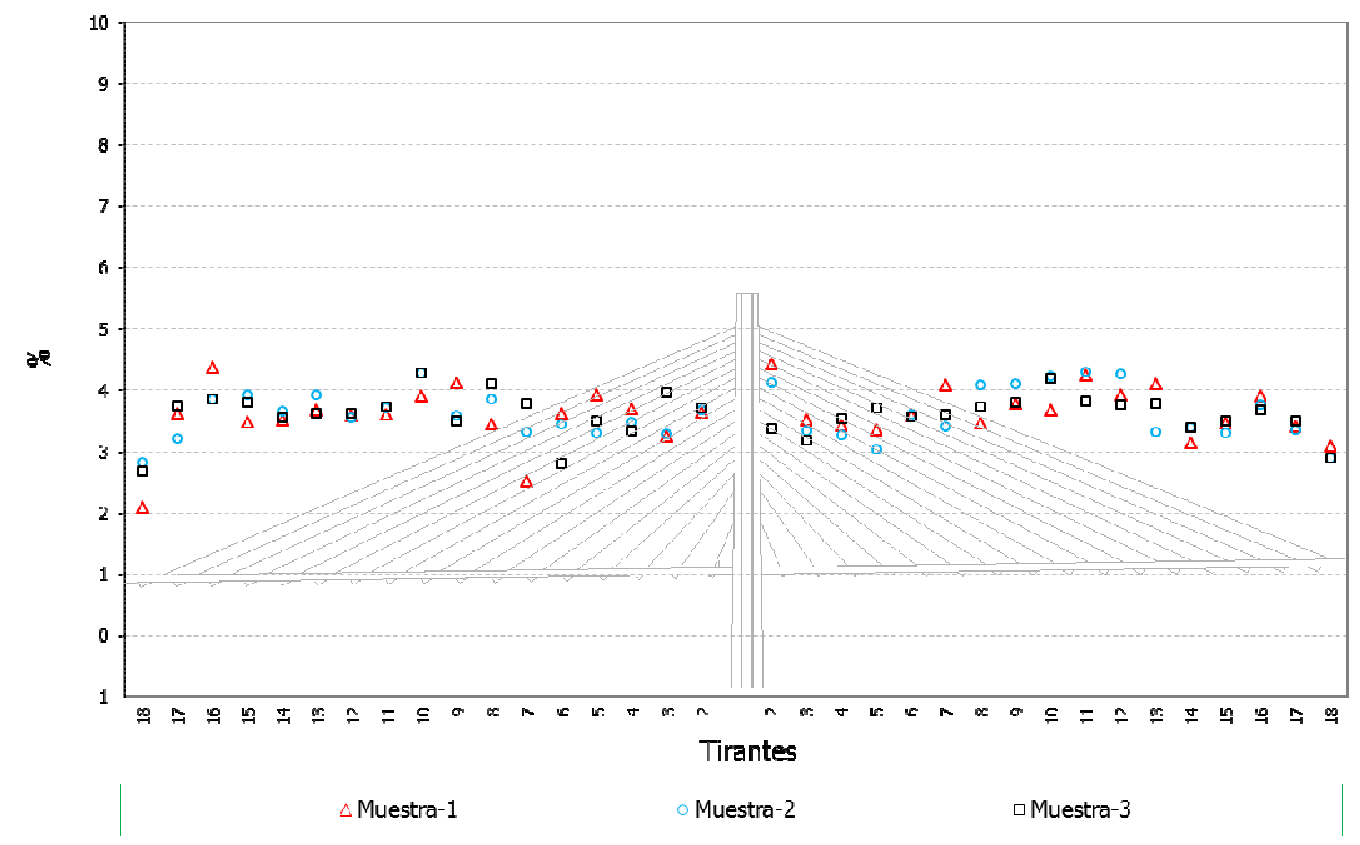

Figura 149. Axiles finales en los tirantes. Diferencia en \% de los valores característicos superiores respecto de los teóricos. 


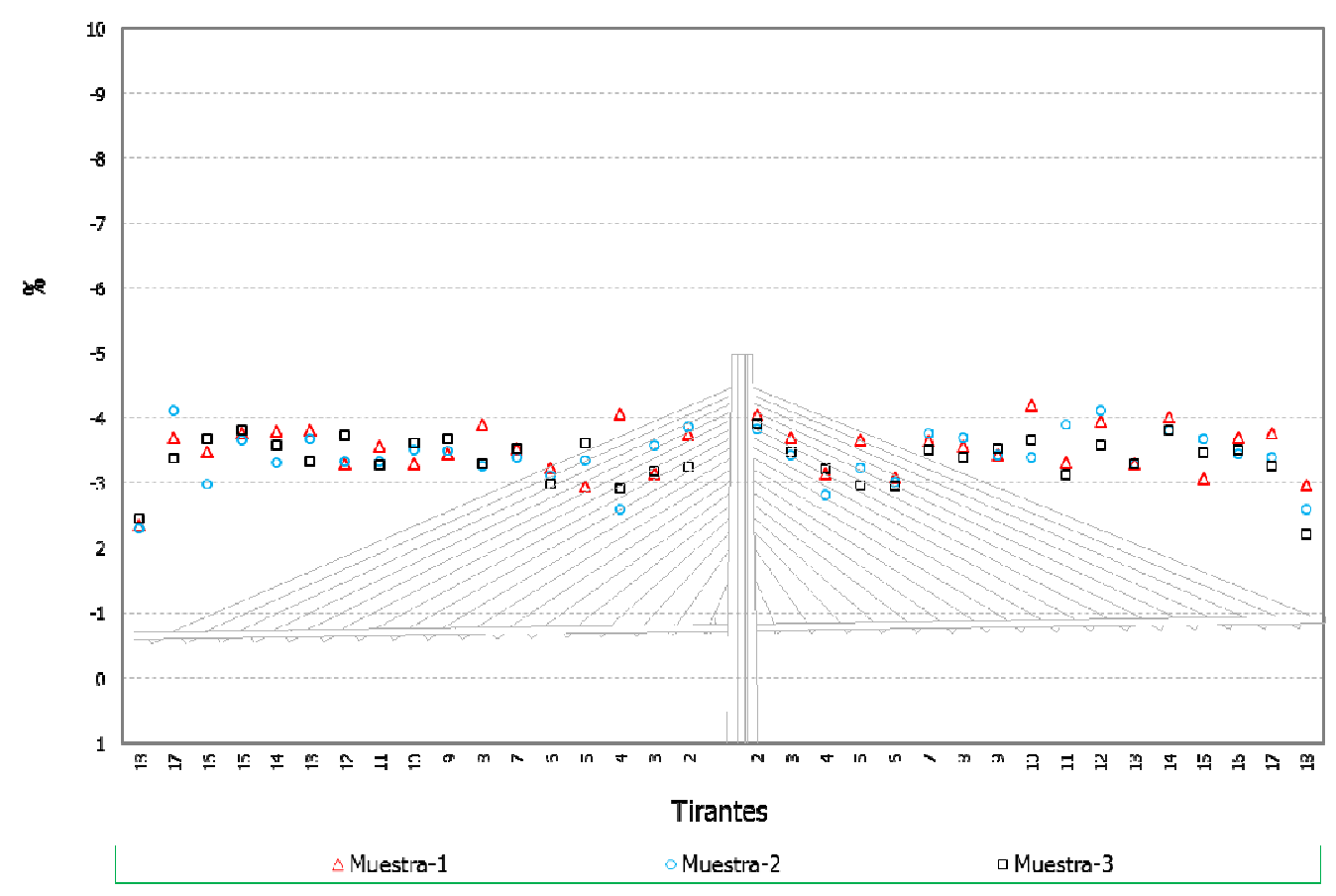

Figura 150. Axiles finales en los tirantes. Diferencia en $\%$ de los valores característicos inferiores respecto de los teóricos.

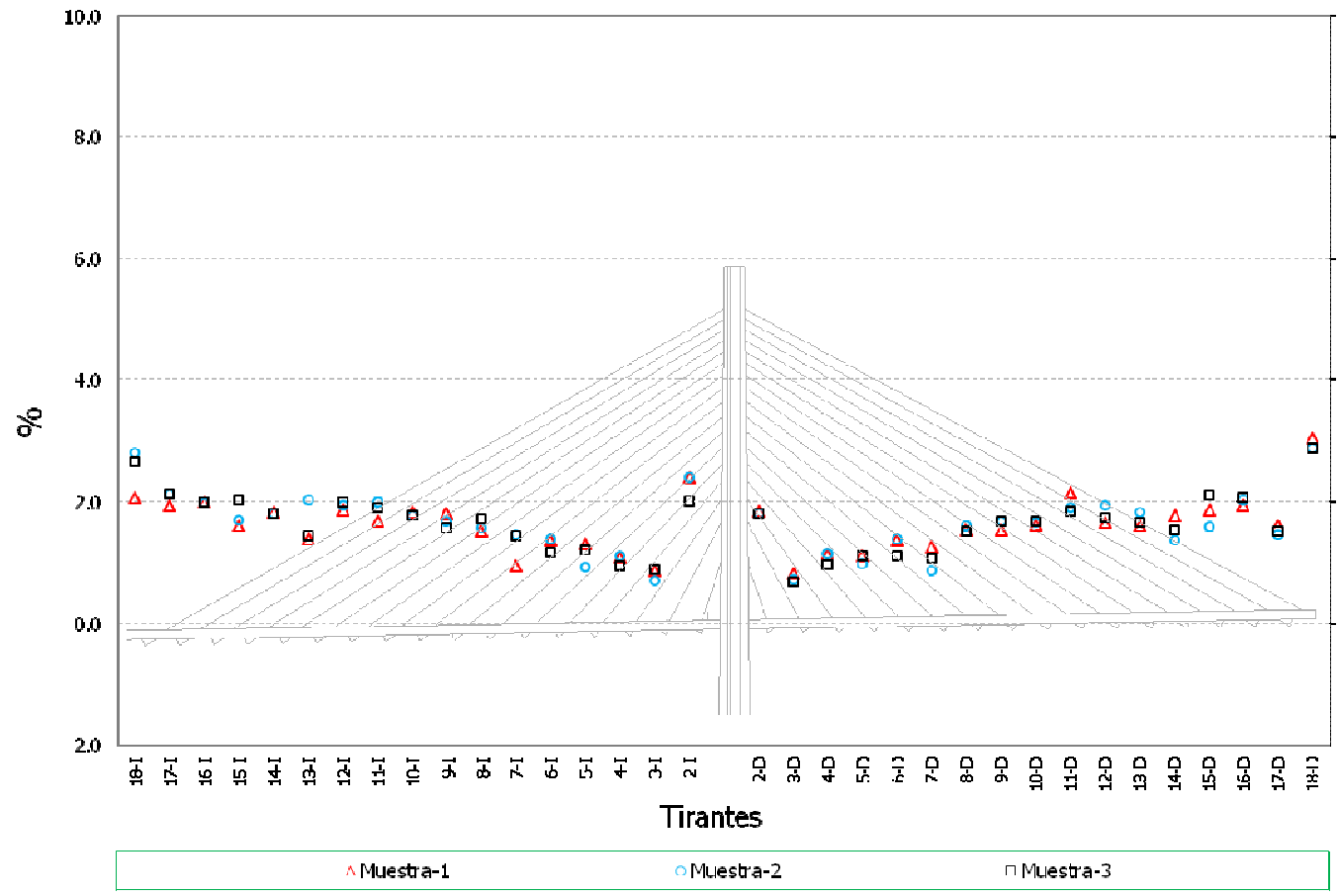

Figura 151. Axiles en los tirantes máximos de construcción. Diferencia en \% de los valores característicos superiores respecto de los teóricos. 


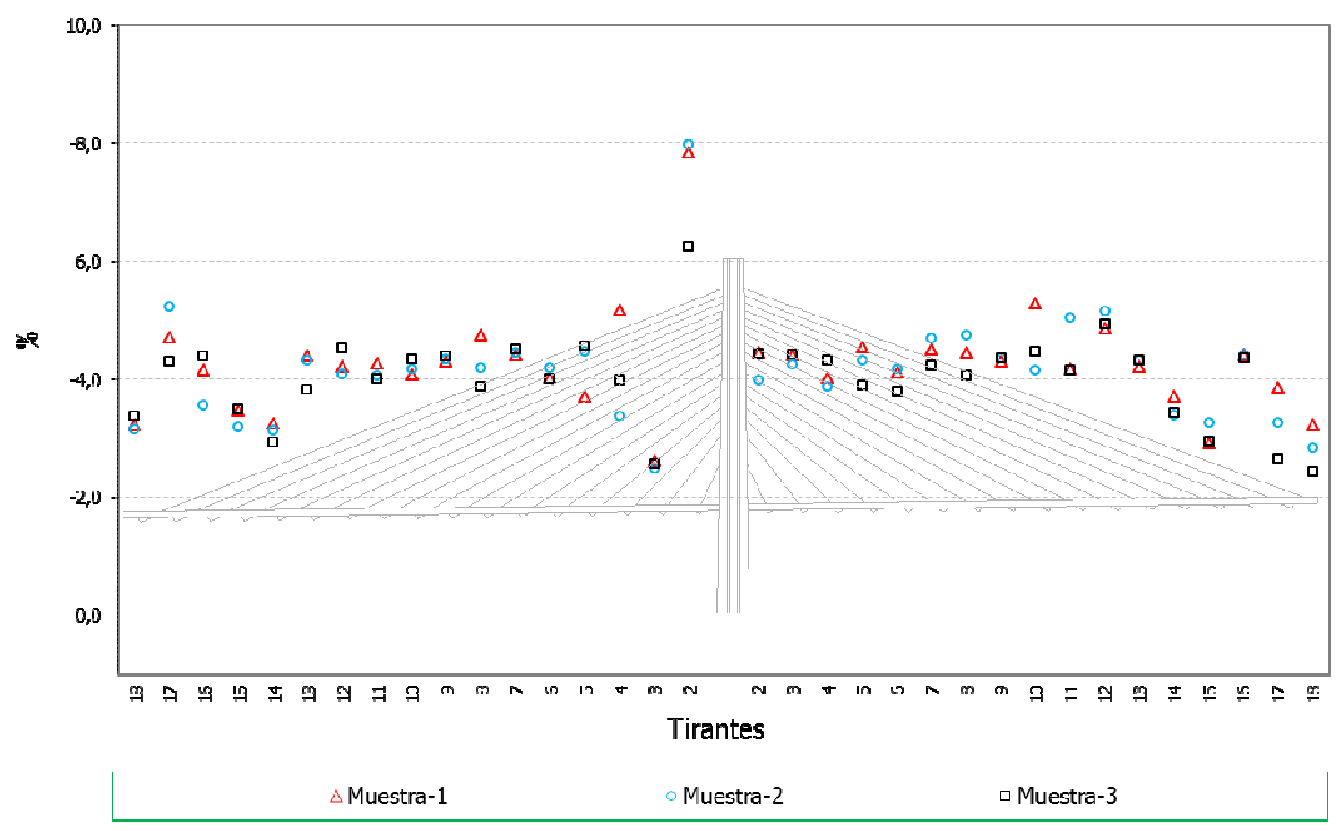

Figura 152. Axiles en los tirantes mínimos de construcción. Diferencia en \% de los valores característicos inferiores respecto de los teóricos.

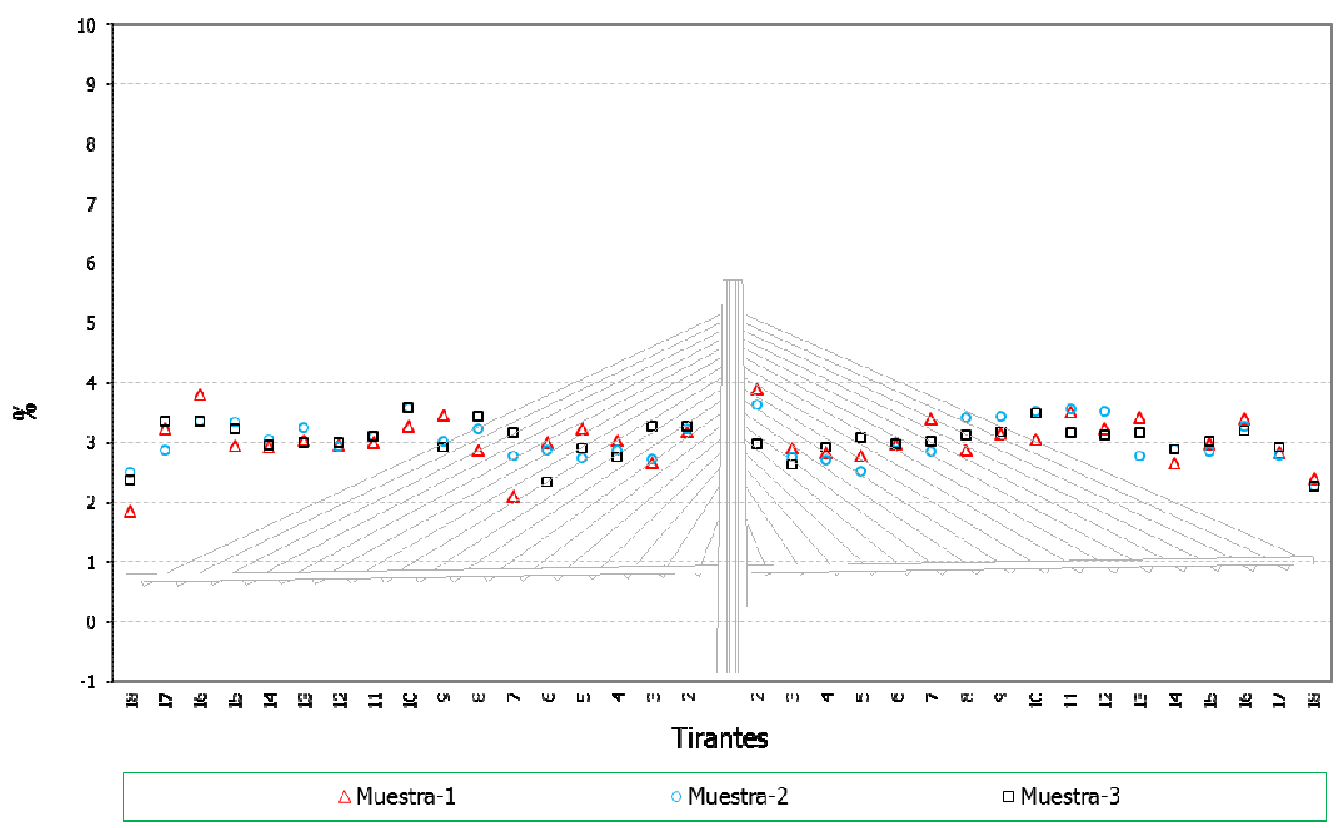

Figura 153. Axiles en los tirantes máximos de servicio. Diferencia en \% de los valores característicos superiores respecto de los teóricos. 


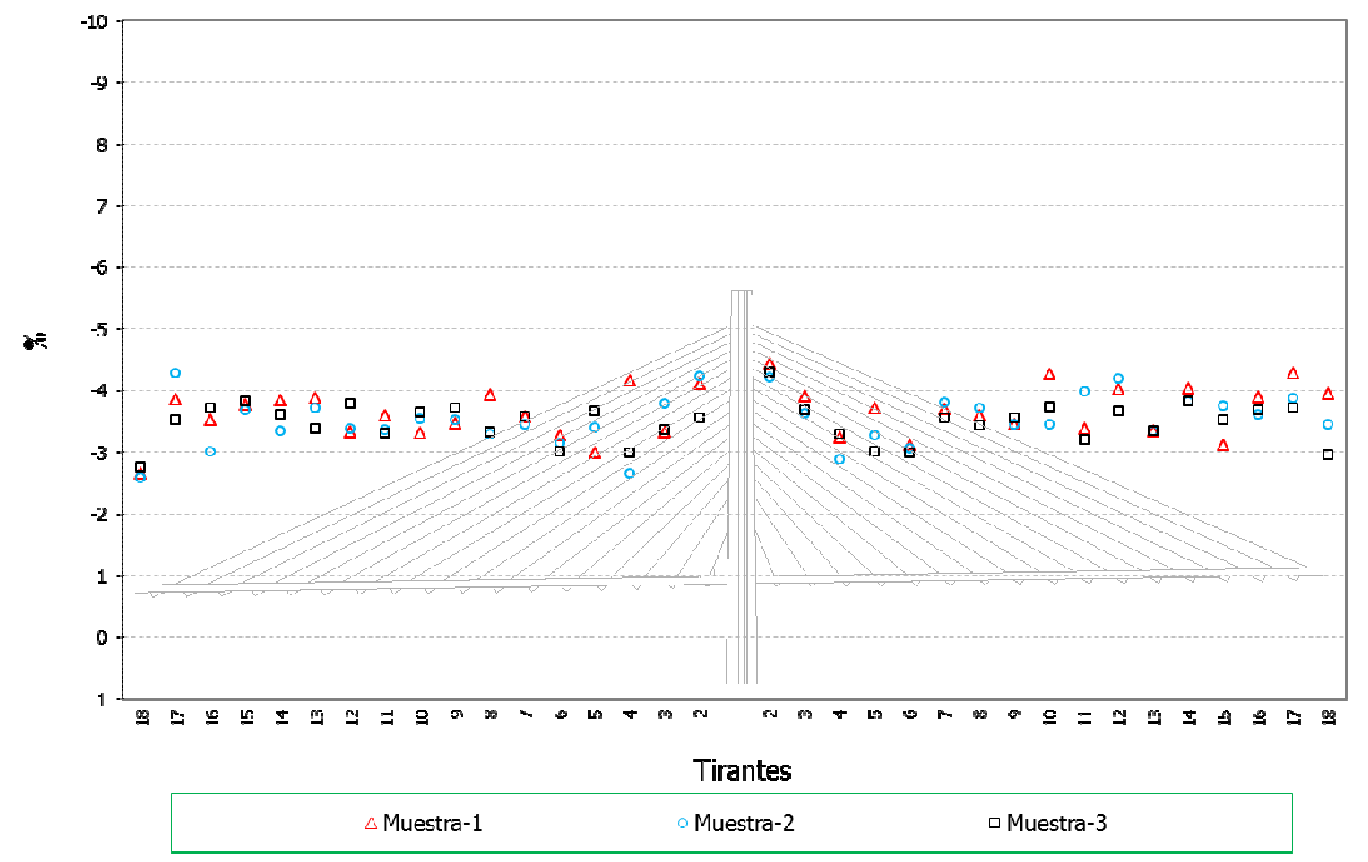

Figura 154. Axiles en los tirantes mínimos de servicio. Diferencia en \% de los valores característicos inferiores respecto de los teóricos.

\subsubsection{Flectores en tablero.}

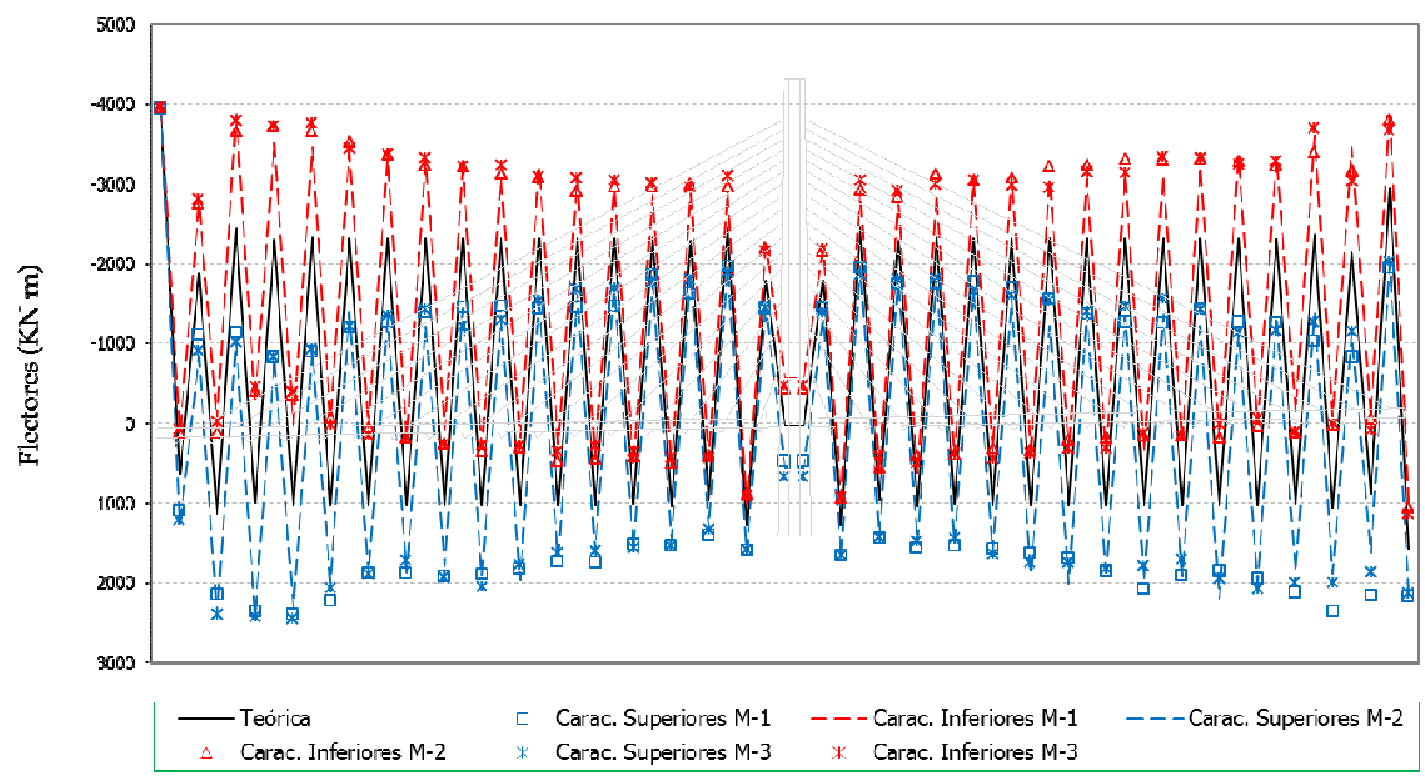

Figura 155. Flectores en tablero en situación final. Valores característicos superiores e inferiores. 


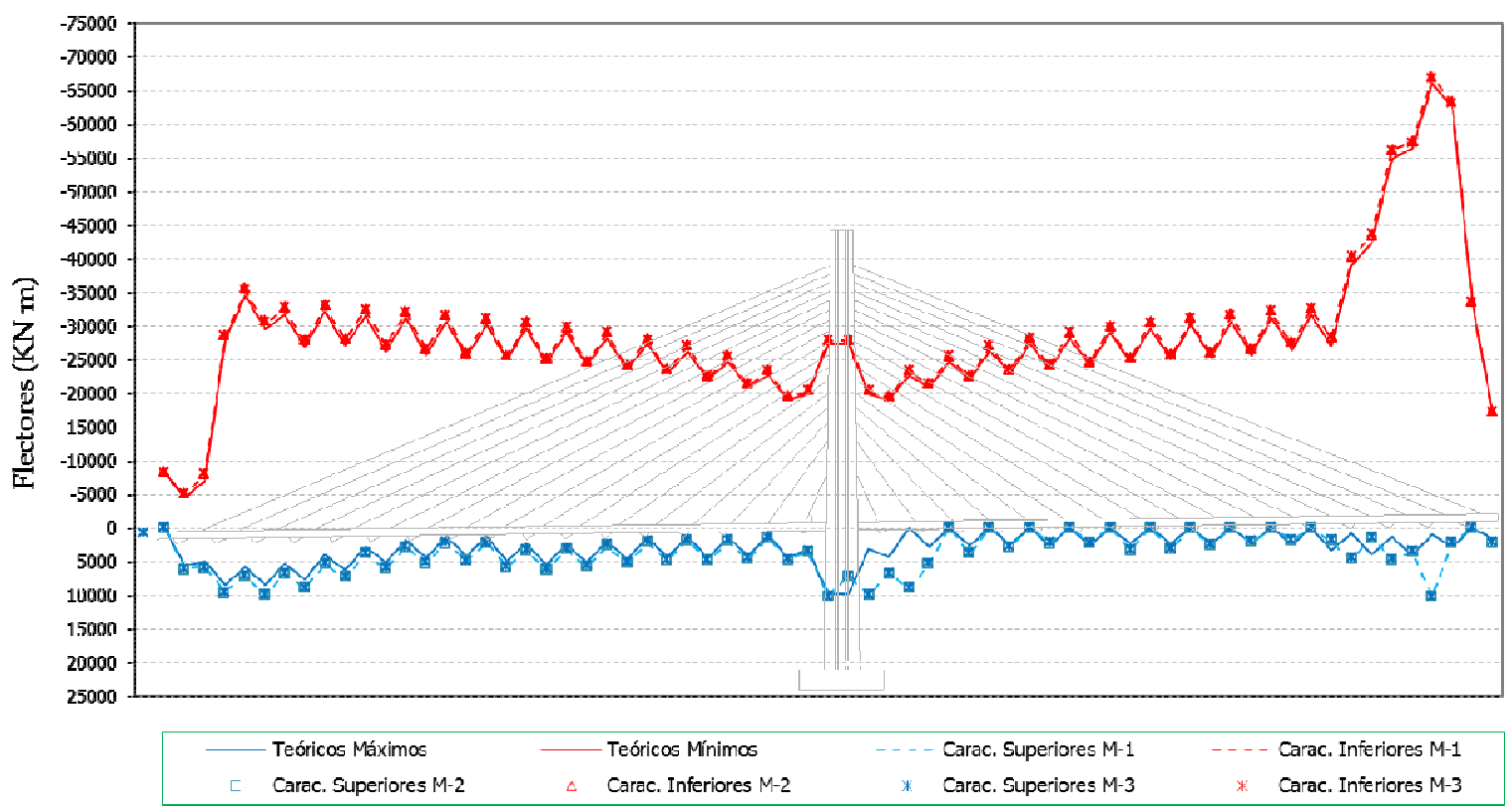

Figura 156. Flectores en tablero en fases de construcción. Valores característicos superiores e inferiores.

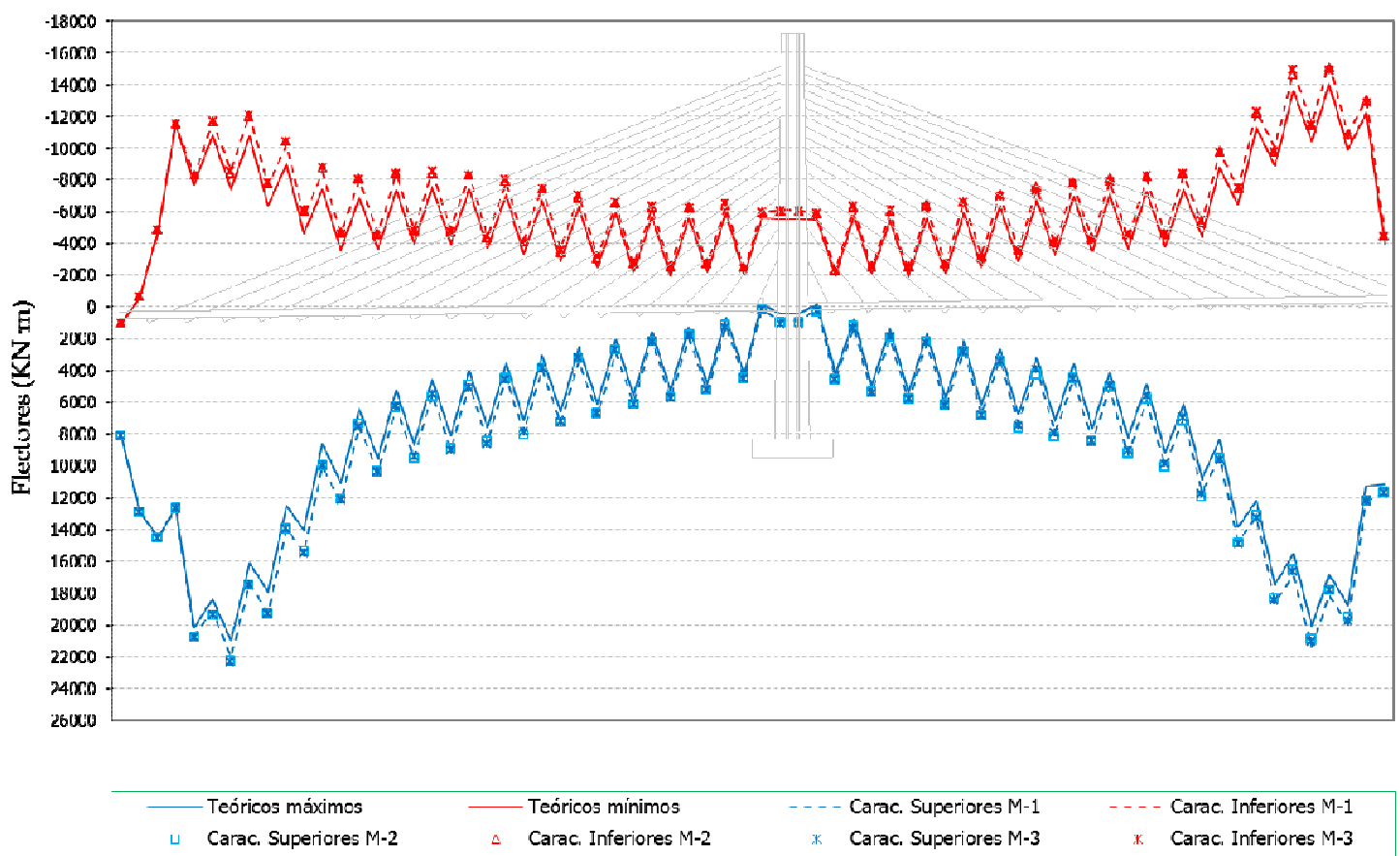

Figura 157. Flectores en tablero en fases de servicio. Valores característicos superiores e inferiores. 


\subsubsection{Flectores en pilono.}

Se incluyen en este caso sólo los valores más representativos correspondientes a las envolventes de mínimos de construcción y máximos de servicio.

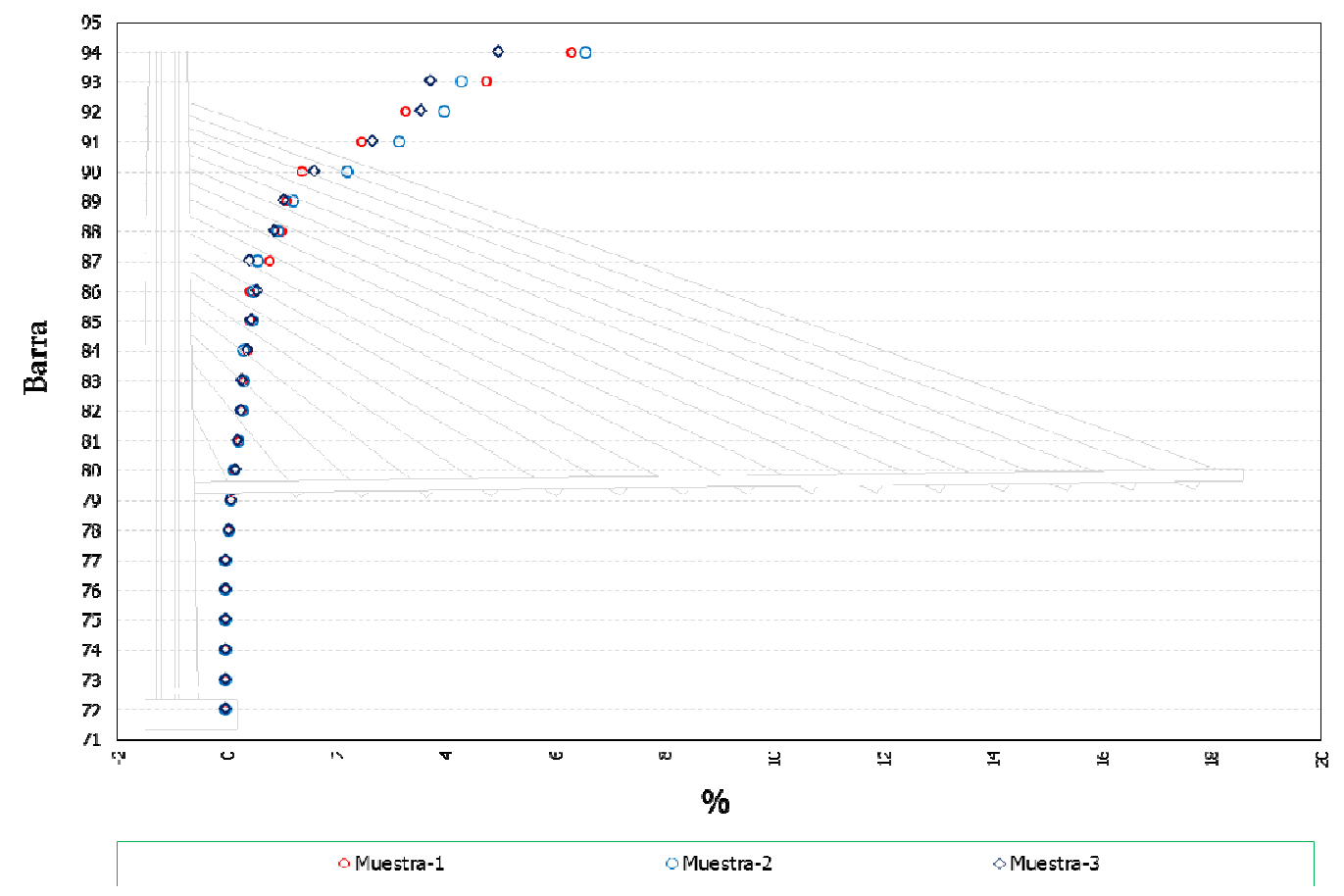

Figura 158. Flectores en pilono mínimos en fases de construcción. Diferencias en \% de los valores característicos inferiores respecto de los teóricos. 


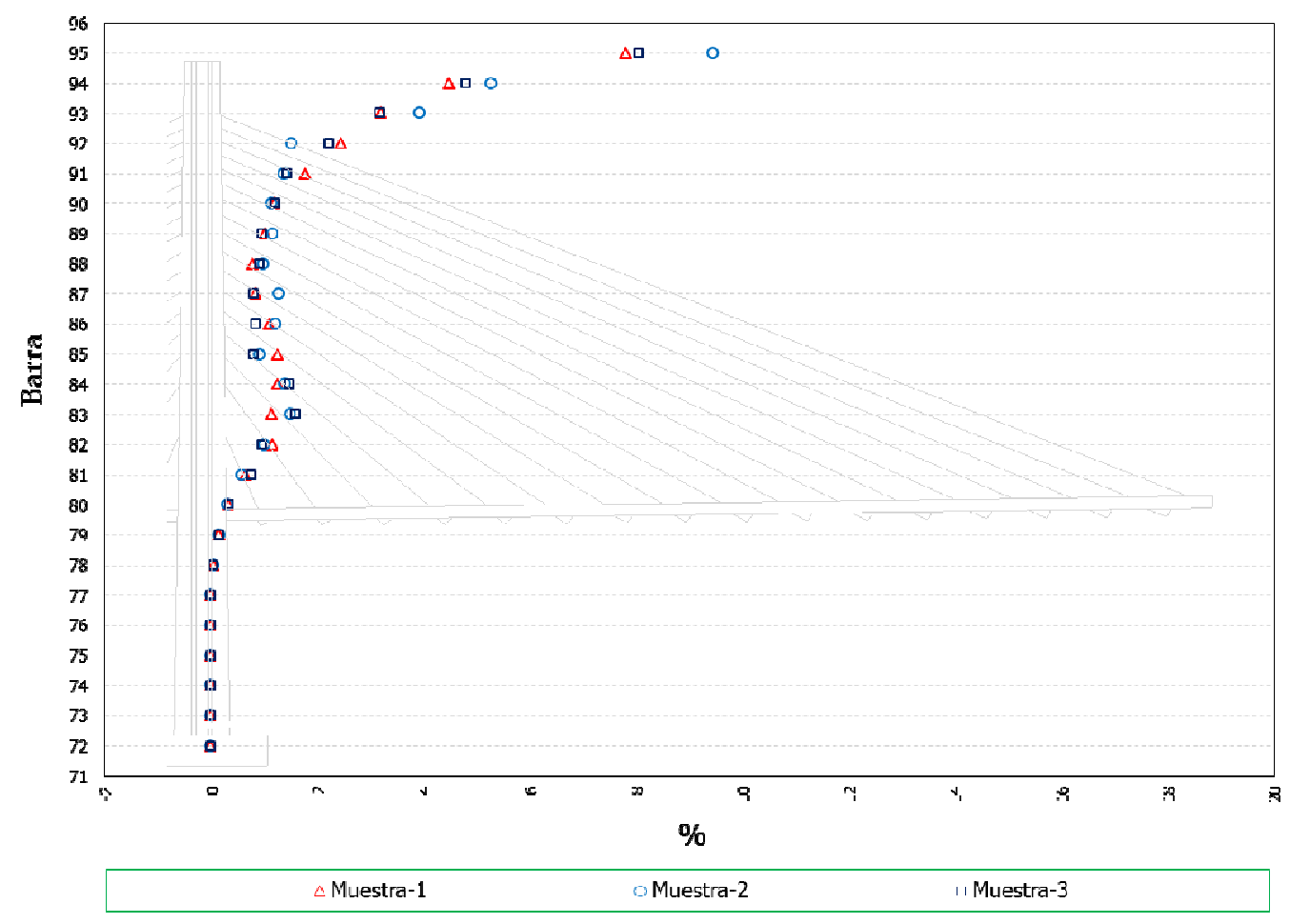

Figura 159. Flectores en pilono máximos en fases de servicio. Diferencias en \% de los valores característicos superiores respecto de los teóricos.

\subsection{ANALISIS DE RESULTADOS.}

Se procede en este epígrafe a detallar las conclusiones obtenidas de los resultados presentados con anterioridad, en los 2 casos de introducción de errores en la colocación de los cables, en fuerzas y deformaciones.

\subsubsection{Resultados con errores en fuerzas.}

A partir de los resultados obtenidos aplicando los errores sobre los axiles de montaje de los tirantes, que se deducen las conclusiones que se detallan a continuación.

En primer lugar sobre los axiles de los tirantes se puede indicar que:

- Los axiles finales alcanzan errores de hasta el 10\% respecto de los valores teóricos, produciéndose al igual que en el caso de los Modelos 90 y $90 \mathrm{M}$ un distribución de errores similar, con mayores cuantías en los primeros cables instalados. En general en este caso la cuantía de los errores generados es menor que en los casos anteriores.

- En los axiles de construcción, también como en los casos anteriores, se muestra un comportamiento diferenciado de los valores máximos y mínimos. Los máximos quedan 
de nuevo condicionados por el valor, no aleatorio, del peso de las dovelas. Los mínimos en cambio alcanzan valores mayores que en el resto de estructuras analizadas, con valores de las desviaciones de hasta un $16 \%$.

- Los axiles de servicio muestran una acumulación de errores en los primeros tirantes, con valores mayores de las desviaciones respecto de los valores teóricos en el caso de los valores mínimos que de los máximos. Si son los valores de los errores obtenidos del mismo orden que en el caso de los Modelos 90 y $90 \mathrm{M}$.

Por otra parte en el caso de los resultados en flectores del tablero:

- De nuevo se muestra unas diferencias muy significativas en el caso de los resultados obtenidos respecto de los flectores teóricos en la situación final.

- En las situaciones de construcción las diferencias con los valores teóricos, como ocurre en el caso de los Modelos 90 y $90 \mathrm{M}$, son reducidas.

- En las situaciones de servicio las diferencias son algo mayores que en construcción respecto de los valores teóricos, al igual que en los casos estudiados en los capítulos anteriores, aunque la magnitud de estas desviaciones siguen siendo reducidas, porcentualmente, salvo en aquellas zonas del tablero donde los valores teóricos son más pequeños.

Por último en el caso de los flectores en el pilono se puede indicar que:

- Igualmente los resultados obtenidos son similares a los obtenidos en los modelos anteriores, tanto en el caso de los mínimos de construcción como en el caso de los máximos de servicio, envolventes que caracterizan el comportamiento del pilono.

- En el caso de los mínimos de construcción, los errores más acusados se producen en la parte superior del pilono, donde los valores teóricos son más pequeños, y por lo tanto los efectos aleatorios tienen una mayor repercusión en porcentaje.

\subsubsection{Resultados con errores en deformaciones.}

A continuación se reseñan las conclusiones deducidas de los resultados obtenidos aplicando los errores sobre las deformaciones de montaje de los cables.

En primer lugar en el caso de los axiles en los tirantes:

- Los errores obtenidos en todos los axiles finales presentan menores dispersiones y diferencias respecto de los resultados teóricos que en la alternativa de introducción de 
los errores de montaje en fuerzas. En este caso además no se observa acumulación de errores en los primeros cables colocados. Por otra parte las cuantías obtenidas son similares a las de los Modelos 90 y $90 \mathrm{M}$.

- En cuanto a los axiles de construcción los errores máximos obtenidos son algo menores que en fuerzas, al contrario de lo que ocurre con los mínimos de construcción. Sír resultan en general desviaciones del mismo orden de magnitud que lo obtenido para los Modelos 90 y $90 \mathrm{M}$.

- Los axiles mínimos de servicio presentan una cuantía de errores del orden del 4\%. Dicho valor es menor que el obtenido con los Modelos 90 y $90 \mathrm{M}$.

Por otra parte en el caso de los resultados en flectores del tablero:

- En la situación final se vuelve a presentar una situación con los valores obtenidos muy lejos de los valores teóricos, aunque de forma algo menos acusada que cuando los errores se han introducido en fuerzas. En este caso, las mayores desviaciones se producen en los extremos del tablero, cuando en el caso de errores en fuerzas las mayores dispersiones de los resultados se producen en la zona situada respecto del pilono a $3 / 4$ de la luz.

- Por otra parte, tanto las envolventes de flectores de construcción como de servicio presentan desviaciones menores que en el caso de la aplicación de fuerzas, y con valores similares al caso de los Modelos 90 y $90 \mathrm{M}$.

Por último en el caso de los flectores en el pilono se puede indicar que:

- Tanto en construcción como en servicio, los efectos introducidos por la aleatoriedad en la medición de las deformaciones en el momento del tesado del cable producen errores de mucha menor cuantía que si el error se produce aplicando fuerzas de tesado a los cables.

- En este caso los errores generados son menores también que los obtenidas al realizar este análisis en las otras estructuras ya analizadas, Modelos 90 y $90 \mathrm{M}$. 


\subsection{ANÁLISIS EN DEFORMACIONES.}

Con la misma intención que el estudio del epígrafe 4.8, se procede en este apartado a evaluar la desviación en las deformaciones verticales del tablero respecto de los valores teóricos al introducir los errores aleatorios. Se analiza este aspecto también en esta estructura, dado que al tener una luz muy superior a las de los casos anteriores, su deformabilidad debe ser en consecuencia muy elevada, y dicha característica deberá reflejarse en los resultados que se obtengan.

Se han estudiado de nuevo las fases que se consideran más representativas del proceso constructivo, analizándose la deformada del tablero completo correspondiente a la máxima y mínima deformación característica. Al igual que en el análisis realizado al Modelo 90, para cada una de las fases estudiadas se ha analizado el nudo extremo de la dovela en la que se acaba de instalar el cable correspondiente, tomándose esos valores como referencia para la obtención de las deformadas que se detallan a continuación.

En concreto se han elegido 4 fases del proceso correspondientes a la instalación de 2 cables simétricos cortos (cables 7 Izquierda y 7 Derecha) y a la colocación de los 2 cables simétricos más largos (cables 18 Izquierda y 18 Derecha). Se pretende así poder valorar que cuantía de deformaciones verticales suponen los errores aleatorios en las fases iniciales, cuando la deformabilidad del tablero no es aun elevada, y en las fases finales, cuando el tablero será muy flexible y por lo tanto sensible a la aplicación de estas fuerzas erróneas.

De esta forma, las fases analizadas y los nudos correspondientes utilizados como referencia de los resultados son:

- Fase 24 - Tesado del cable 7 Izquierdo. Nudo de referencia 39.

- Fase 26 - Tesado del cable 7 Derecho. Nudo de referencia 38.

- Fase 68 - Tesado del cable 18 Izquierdo. Nudo de referencia 95.

- Fase 70 - Tesado del cable 18 Derecho. Nudo de referencia 91.

Los valores representados lo han sido analizando una sola de las muestras generadas anteriormente.

Se pasa a continuación a presentar estos resultados (Figura 160 a Figura 163): 


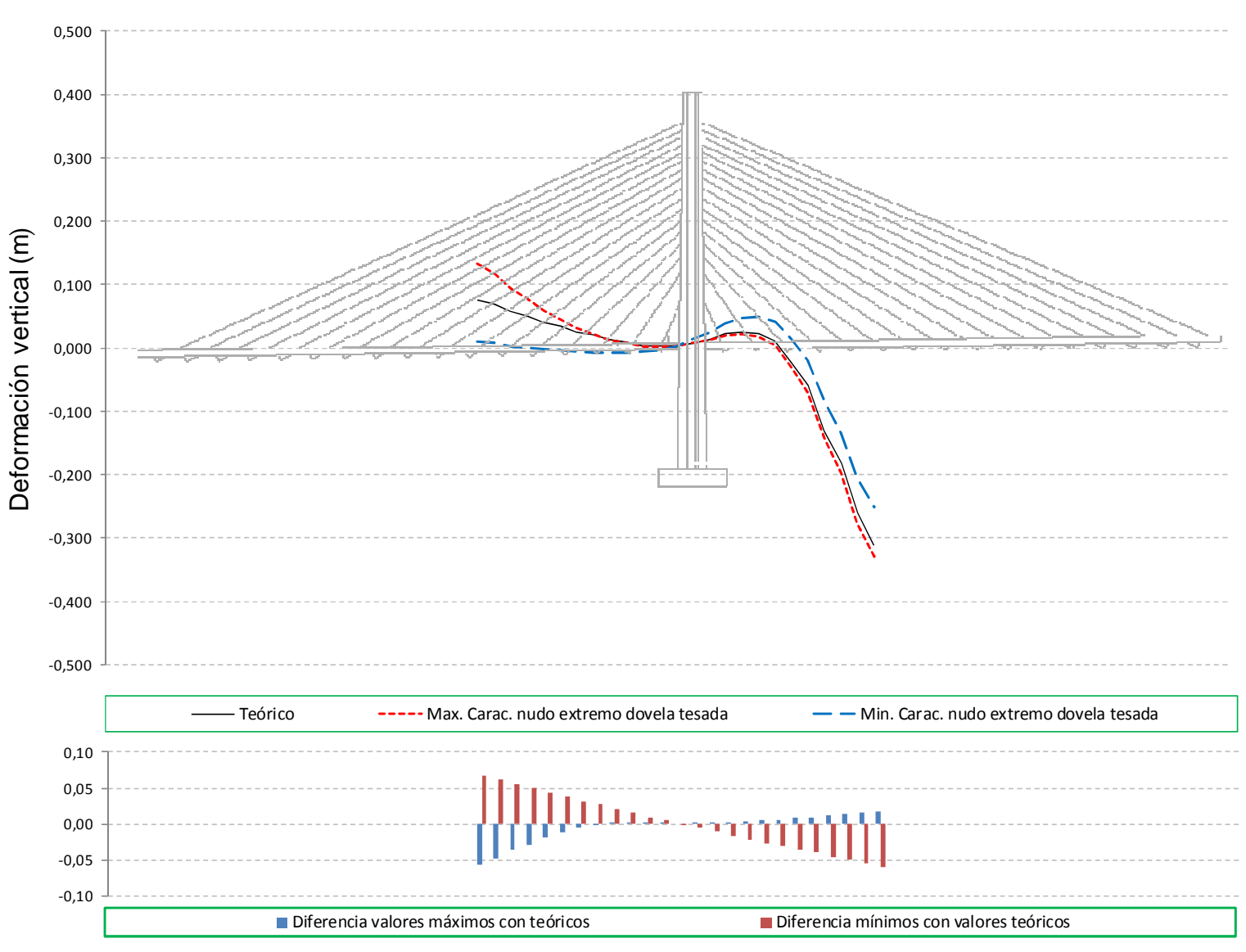

Figura 160. Deformaciones verticales del tablero para el caso de la fase 24 del proceso constructivo. Resultados correspondientes a la deformada obtenida a partir del valor característico del nudo 39 extremo de dovela tesada con el tirante 7 Izquierdo. 


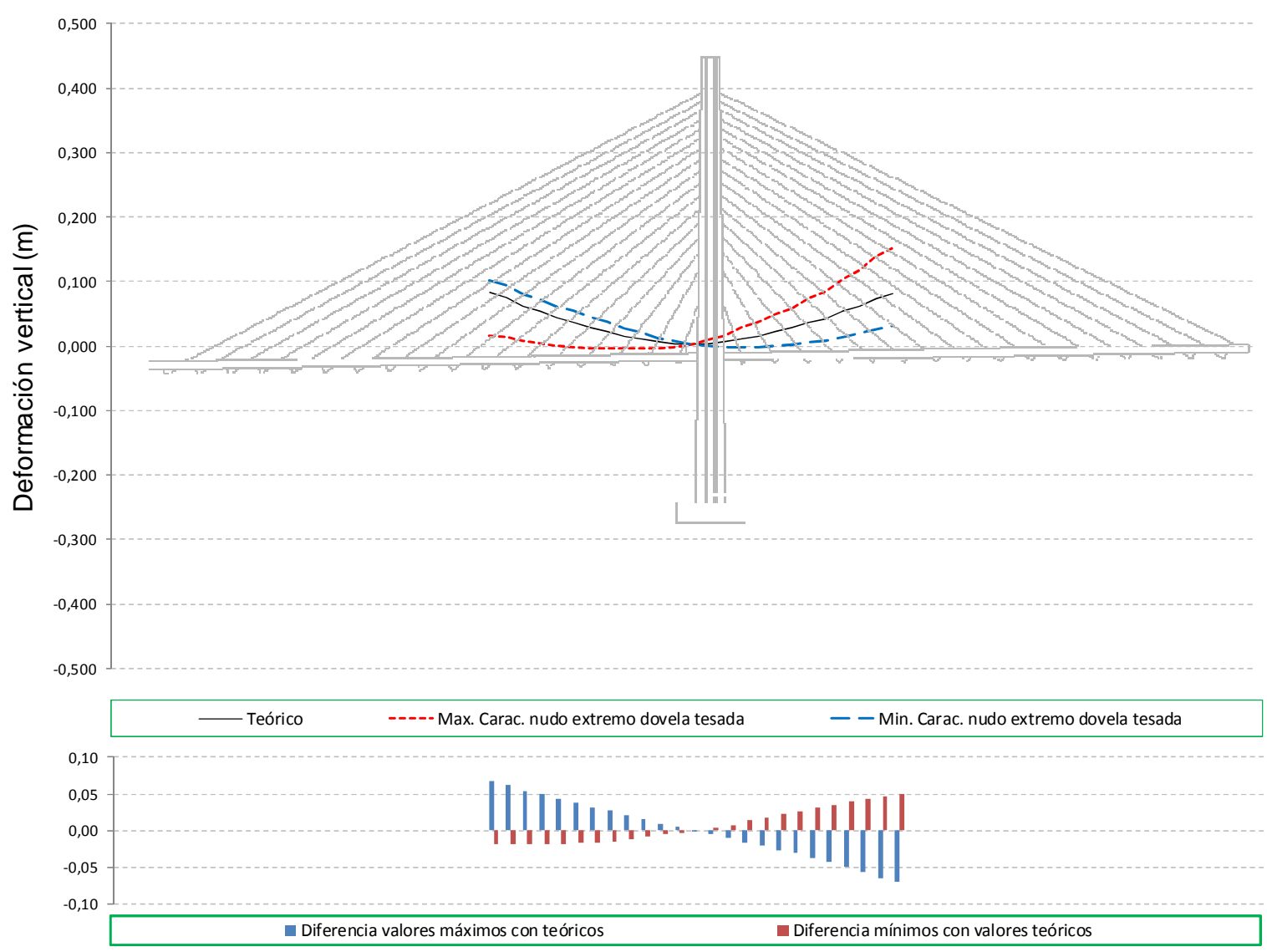

Figura 161. Deformaciones verticales del tablero para el caso de la fase 26 del proceso constructivo. Resultados correspondientes a la deformada obtenida a partir del valor característico del nudo 38 extremo de dovela tesada con el tirante 7 Derecho. 


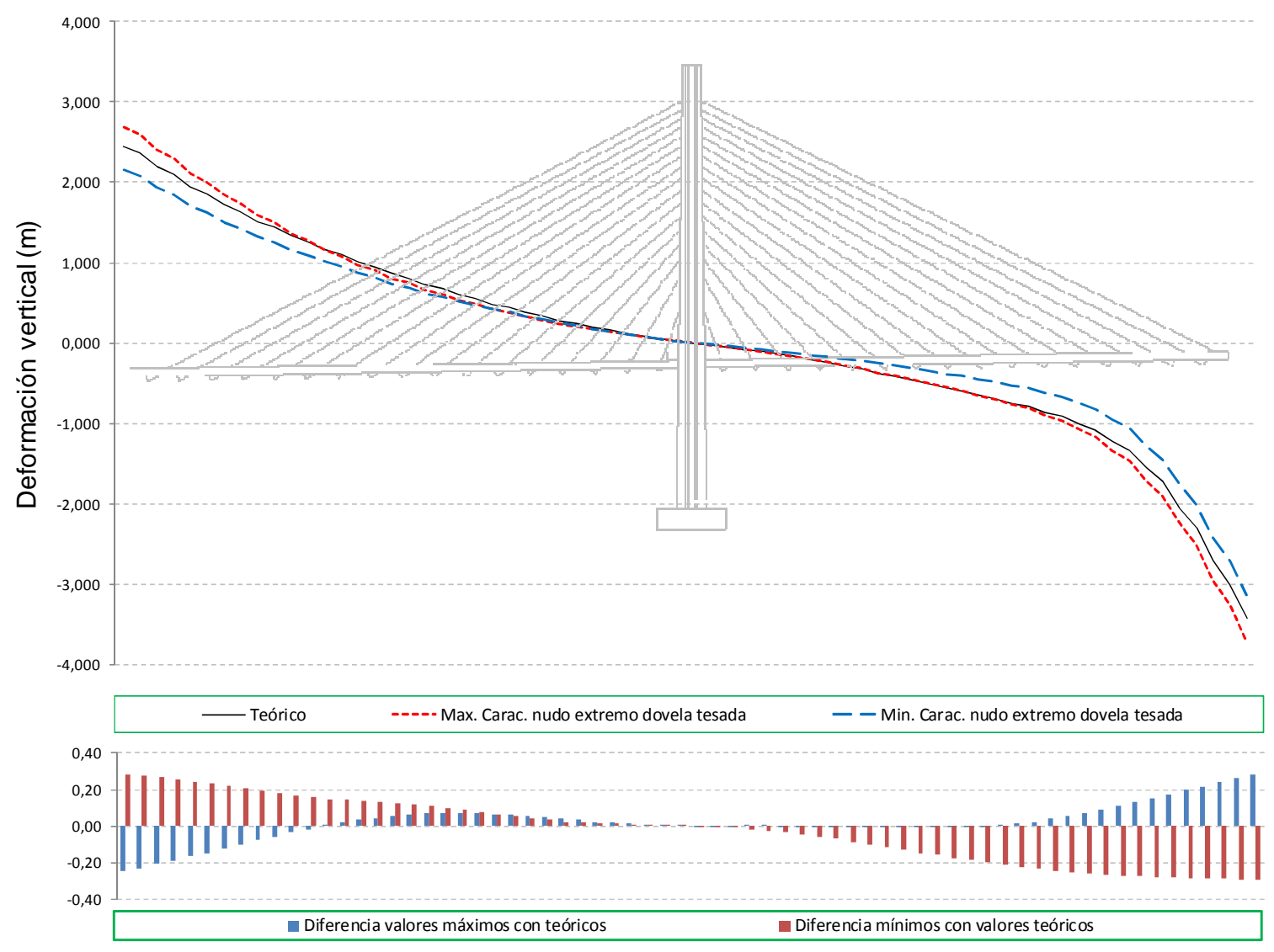

Figura 162. Deformaciones verticales del tablero para el caso de la fase 68 del proceso constructivo. Resultados correspondientes a la deformada obtenida a partir del valor característico del nudo 95 extremo de dovela tesada con el tirante 18 Izquierdo. 


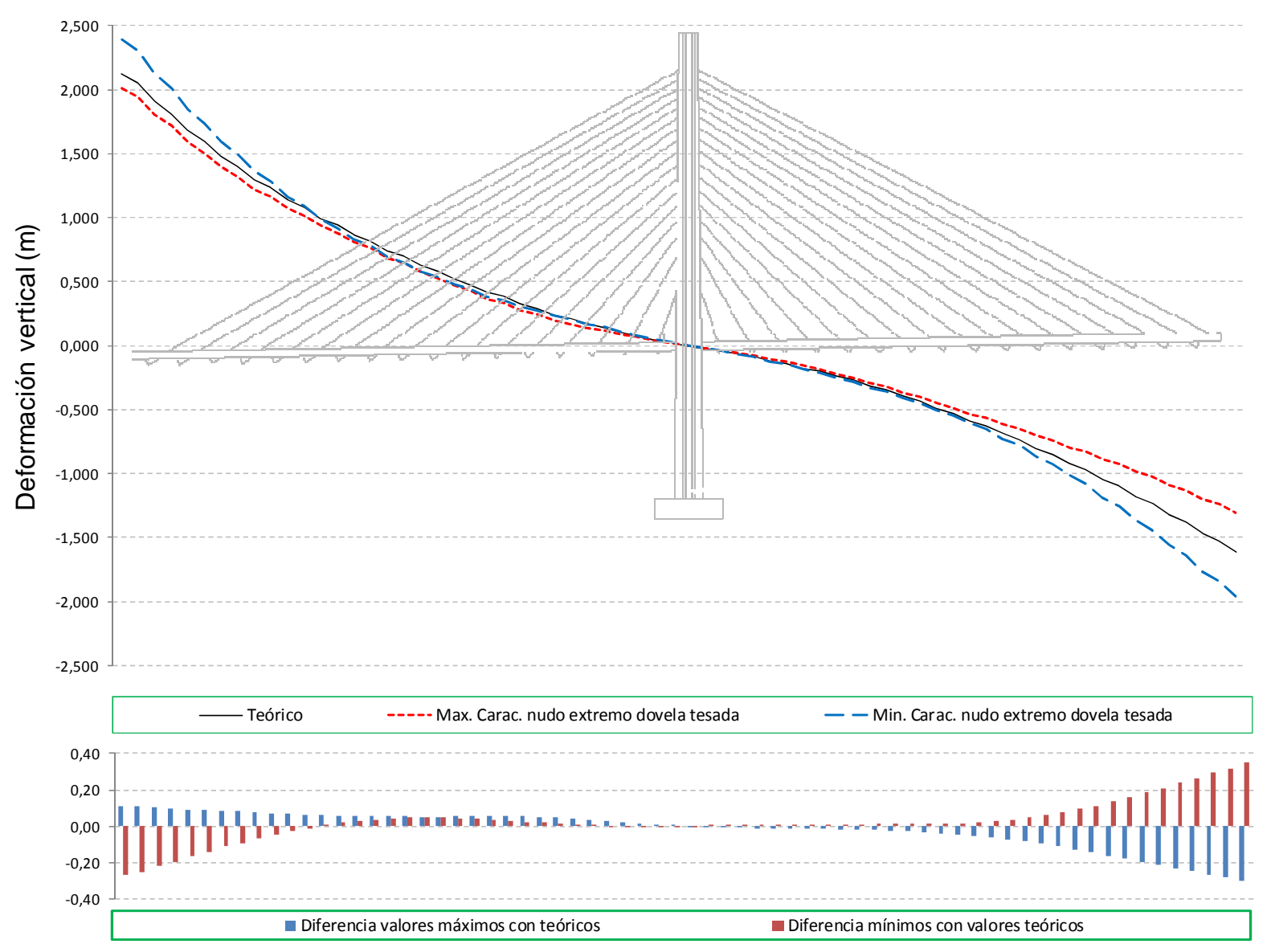

Figura 163. Deformaciones verticales del tablero para el caso de la fase 70 del proceso constructivo. Resultados correspondientes a la deformada obtenida a partir del valor característico del nudo 91 extremo de dovela tesada con el tirante 18 Derecho.

De todos los resultados anteriores se puede concluir lo siguiente:

- Que igual que en el Modelo 90, la deformada del tablero condicionada por el valor máximo característico del nudo representativo de cada fase, presenta en su extremo opuesto un valor por debajo del valor teórico. Este efecto es como ya se ha comentado, debido al giro producido sobre el tablero como sólido rígido alrededor del punto de encuentro del tablero y pilono. De forma contraria ocurre con la deformada asociada al valor característico mínimo del nudo representativo. 
- Las deformadas en las primeras fases resultan con valores del orden de $30-40 \mathrm{~cm}$, mientras que las diferencias con los valores teóricos en cuantías del orden de $5^{\prime} 0 \mathrm{~cm}$.

- En las fases finales las deformaciones verticales son muy elevadas, siendo las diferencias con los valores teóricos de una magnitud de hasta $20 \mathrm{~cm}$. El primer aspecto señalado muestra que la deformabilidad del tablero aquí calculado es superior a la que debería tener una estructura real.

Como conclusión de todos estos resultados se puede señalar que el seguimiento topográfico del tablero debería ser intenso para poder detectar y corregir los efectos de los errores de montaje de los tirantes en las primeras fases del proceso constructivo, dada la pequeña cuantía de dichas deformaciones verticales y la dificultad que se encontraría para determinar el origen de éstas, ya que habría que descartar que se debieran a un origen térmico, reológico o debido a la presencia de alguna carga no controlada sobre el tablero.

Sí en cambio, en las fases más avanzadas del proceso de construcción se podría contar con el seguimiento topográfico como una buena herramienta para la detección de los errores de montaje. Es más, el propio control geométrico en estas últimas fases llevará a limitar los posibles errores en la instalación de los cables de forma más exigente a como establecen la cuantía máxima de errores admisibles establecidos por la normativa.

Igualmente a como se indicó en el epígrafe 4.8, hay que indicar que la forma de compensar estas deformaciones sería el ajuste del carro de encofrado en las fases que quedasen por ejecutar, a pesar de lo cual el tablero quedará con unas ciertas incurvaciones locales debidas al proceso de colocación erróneo del cable y posterior ajuste con el encofrado que no será posible eliminar.

Hay que señalar que las deformaciones que se han obtenido en el cálculo de esta estructura son más elevadas de las que deberían resultar en una estructura real de estas dimensiones. Esta cuestión es debida a varios motivos, como puede ser una rigidez insuficiente en la pila y una altura de pilono reducida respecto de la luz del tablero considerada, en proporción a lo habitualmente adoptado en la práctica.

En cualquier caso se ha decidido mantener estos datos de partida dado el objetivo de este estudio, y con el fin de obtener unos resultados "no reales" pero más fácilmente interpretables y por lo tanto más útiles de cara a la deducción de conclusiones y comparación con los otros modelos de cálculo. 


\section{CAPITULO 7.}

\section{INFLUENCIA DE LA RIGIDEZ DEL TABLERO.}

\subsection{INTRODUCCION}

Habiendo analizado hasta este punto del estudio la influencia del número de tirantes y la luz del tablero en los resultados obtenidos al generar procesos erróneos de montaje de los cables, se procede en este capítulo a evaluar cómo influye en los resultados obtenidos la variación de la rigidez del tablero.

Para ello se ha partido de la estructura original de luces 90+90 m (Modelo 90) procediendo a modificar en ella únicamente la inercia del tablero, manteniendo constantes el resto de parámetros geométricos y mecánicos de la estructura.

El esquema seguido en el desarrollo de este capítulo es similar al planteado en los anteriores, analizándose en primer lugar el comportamiento teórico de la estructura, obteniéndose así los resultados que servirán de referencia en el resto del capítulo. A continuación se ha procedido a aplicar la introducción de los errores aleatorios en el montaje de los tirantes, en este caso, eso sí, sólo aplicando el método en fuerzas, para finalmente analizar todos los resultados obtenidos.

Como novedad en este capítulo se ha incluido un estudio comparativo de los resultados teóricos en función de la modificación aplicada a la inercia del tablero, para poder tener una visión completa de en qué medida este parámetro afecta al comportamiento de la estructura.

Además, en este capítulo se ha añadido una recopilación de los resultados en tirantes de los diferentes modelos estudiados, todos ellos obtenidos aplicando el método en fuerzas, para así poder tener una visión global de los resultados obtenidos a lo largo de este estudio.

Adicionalmente, se han incluido también resultados de las deformaciones verticales generadas durante los procesos de montaje erróneos, de forma que se pueda complementar las conclusiones obtenidas en relación a este aspecto en los capítulos anteriores. 


\subsection{ESTRUCTURA A ANALIZAR.}

La estructura que se propone analizar se basa, como se ha adelantado ya, en la estudiada en el CAPITULO 4 y descrita más en concreto en el epígrafe 4.2. Aquella estructura consistía en un puente atirantado de luces $91^{\prime} 0+85^{\prime} 0 \mathrm{~m}$, con ancho de tablero de $13^{\prime} 80 \mathrm{~m}$ y 18 tirantes dispuestos sobre cada vano.

En este caso, la estructura que se analiza consistirá en un esquema geométrico idéntico, pero incrementando la inercia del tablero respecto de la original. Aunque no es un planteamiento excesivamente realista, se ha decidido mantener el peso propio del tablero aun a pesar de modificar la inercia de la sección. Es decir se está pensando en que en la sección se redistribuye el material pero se mantiene constante su área total.

En concreto se han analizado los 2 casos correspondientes a disponer por un lado una inercia doble de la del Modelo $\mathbf{9 0}$ en el tablero y por otra parte una inercia quíntuple de la de ese mismo modelo original. Se propone así, antes de introducir los procesos erróneos de construcción sobre los modelos de cálculo, analizar la sensibilidad de los resultados teóricos respecto de la modificación de la inercia del tablero.

El proceso constructivo se compondrá de las mismas fases provisionales y se realizará siguiendo la misma secuencia que lo planteado para el Modelo 90.

De esta forma, el modelo de cálculo generado para el cálculo de estas estructuras, salvo la consideración de la inercia del tablero, es idéntico al presentado en la Figura 57. Estos dos nuevos modelos de cálculo se pasarán a denominar en adelante Modelo $\mathbf{9 0 - 2 1}$ para el caso de la inercia doble y Modelo $\mathbf{9 0 - 5 1}$ para el caso de inercia quíntuple.

A partir de todas estas premisas se procede a presentar los valores teóricos obtenidos en estas estructuras, tanto en las fases de construcción, como en la fase final de este proceso y en la fase de servicio al aplicar las sobrecargas de tráfico.

De las características anteriormente señaladas para las estructuras a calcular, se deduce que, si se adopta de nuevo como situación final objetivo la equivalente a la viga continua para el comportamiento del tablero, y dado que, como ya se ha indicado, en esta configuración se concluye que la inercia no tiene influencia en los esfuerzos obtenidos, se puede deducir por lo tanto que la ley de flectores al final del proceso constructivo en estas estructuras serán iguales que la presentada para la estructura Modelo $\mathbf{9 0}$ en la Figura 59. A su vez, dada la anterior consideración, resultará también que los axiles finales en los cables deberán ser los mismos que los obtenidos en aquel caso, representados en la Figura 58. 
Por el contrario, si resultan diferentes los axiles de montaje de cada uno de los cables en comparación con los obtenidos del cálculo del Modelo 90 , quedando así reflejado en la siguiente figura.

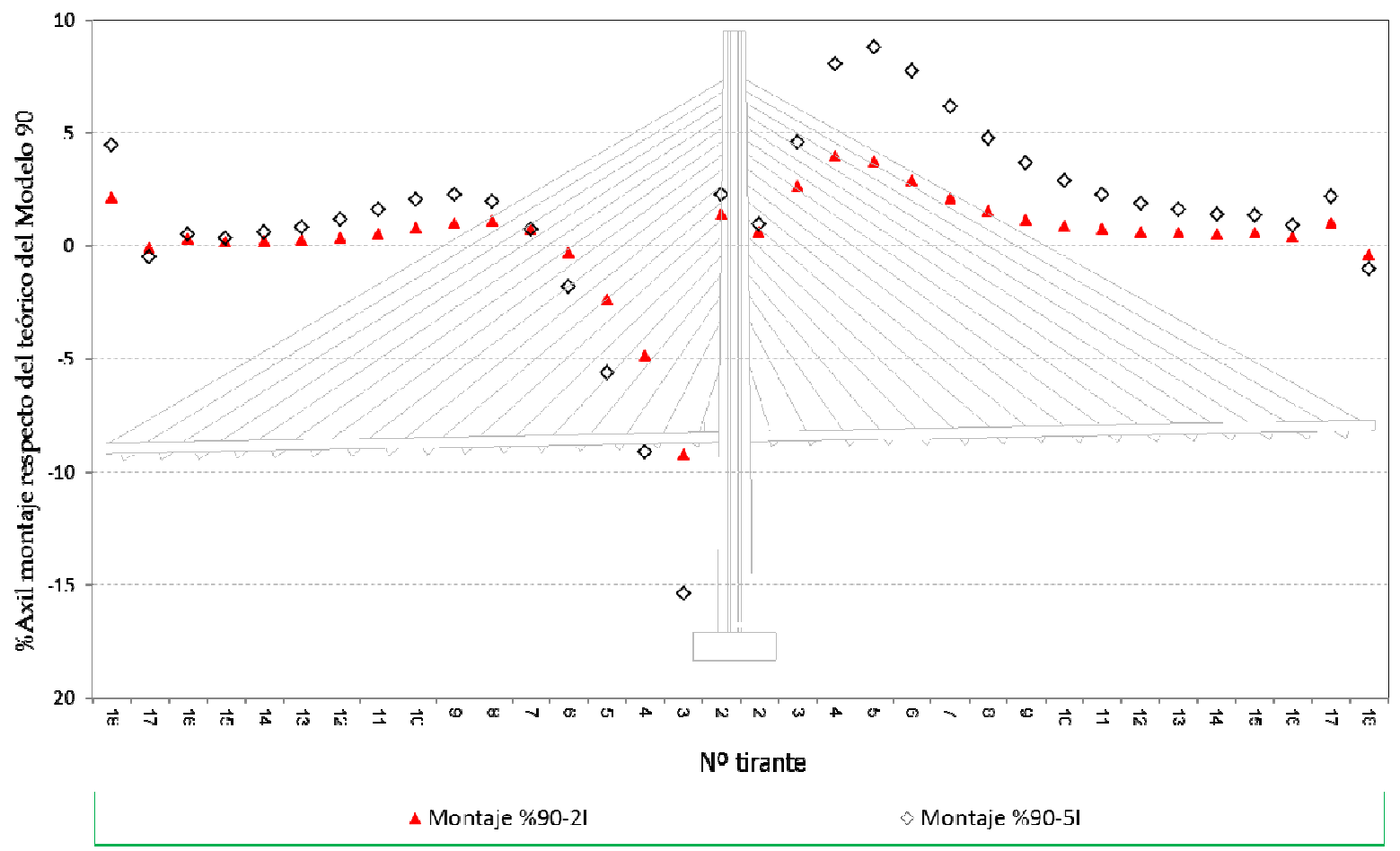

Figura 164. Comparativa de los axiles de montaje entre los modelos 90, 90-2l y 90-5I.

De este gráfico se puede concluir:

- Que la mayoría de los valores muestran una proporcionalidad entre la inercia del tablero y el axil de montaje de los cables.

- $\quad$ Que en general las variaciones de los axiles de montaje son pequeñas, quedando en un rango de entre un $+10 \%$ y un $-15 \%$ respecto de los resultados del Modelo 90 , siendo más apreciables las variaciones en los primeros cables instalados. Este último aspecto se justifica en el hecho de que, al estar el tablero apoyado sobre el pilono y por lo tanto al generarse en esa zona un punto duro con un gran rigidez, adicionalmente, y en la medida en que se aumente aun más la rigidez del tablero, se reducirá en igual medida la eficacia del atirantamiento y por lo tanto se reducirá el axil que absorberá cada tirante dispuesto en esta zona.

- Las anteriores consideraciones no se cumplen en el caso concreto de los primeros cables instalados en el vano izquierdo, dado el particular proceso constructivo propuesto para las primeras fases de ejecución del tablero. 
Se presentan a continuación resultados en tirantes en las fases de construcción y servicio donde se aprecian diferencias en los valores teóricos conforme se aumenta la rigidez del tirante.

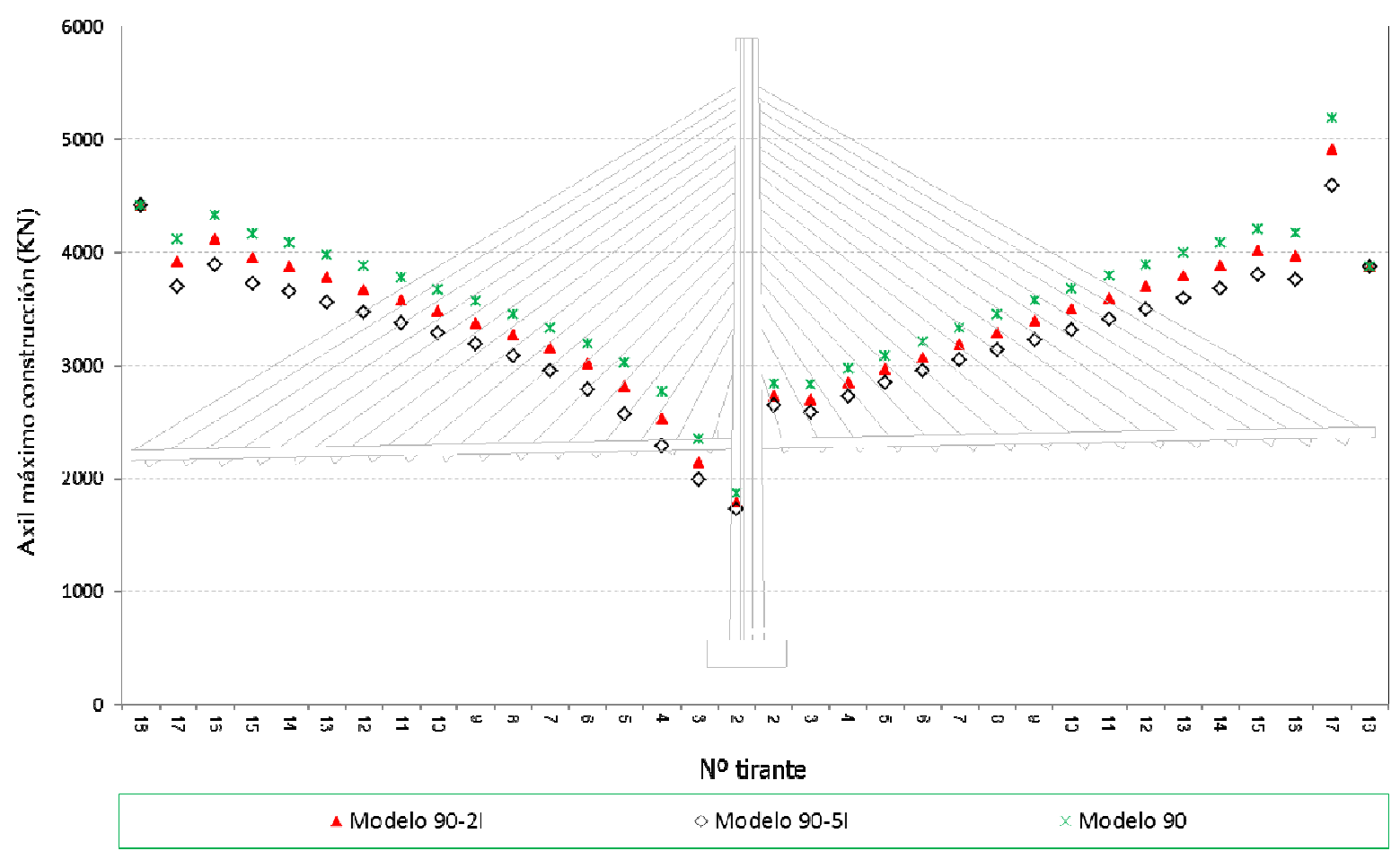

Figura 165. Comparativa de los axiles máximos de construcción en los modelos 90, 90-2l y 90-51.

En el gráfico anterior se observa que, conforme se aumenta la rigidez del tablero, los axiles máximos de construcción de los tirantes disminuyen, siguiendo una distribución en porcentajes similar a la de la figura anterior (Figura 165). De nuevo hay que señalar que la principal causa de estos resultados es que al aumentar la rigidez del tablero, se disminuye la eficacia del atirantamiento y por lo tanto estos elementos tendrán menos capacidad para absorber cargas exteriores.

En el caso de los mínimos de construcción (Figura 166) que se adjuntan a continuación las diferencias con el Modelo 90 son mucho más pequeñas, pero siguen una distribución en porcentajes como la obtenida en los dos casos anteriores de axiles de montaje y máximos de construcción. 


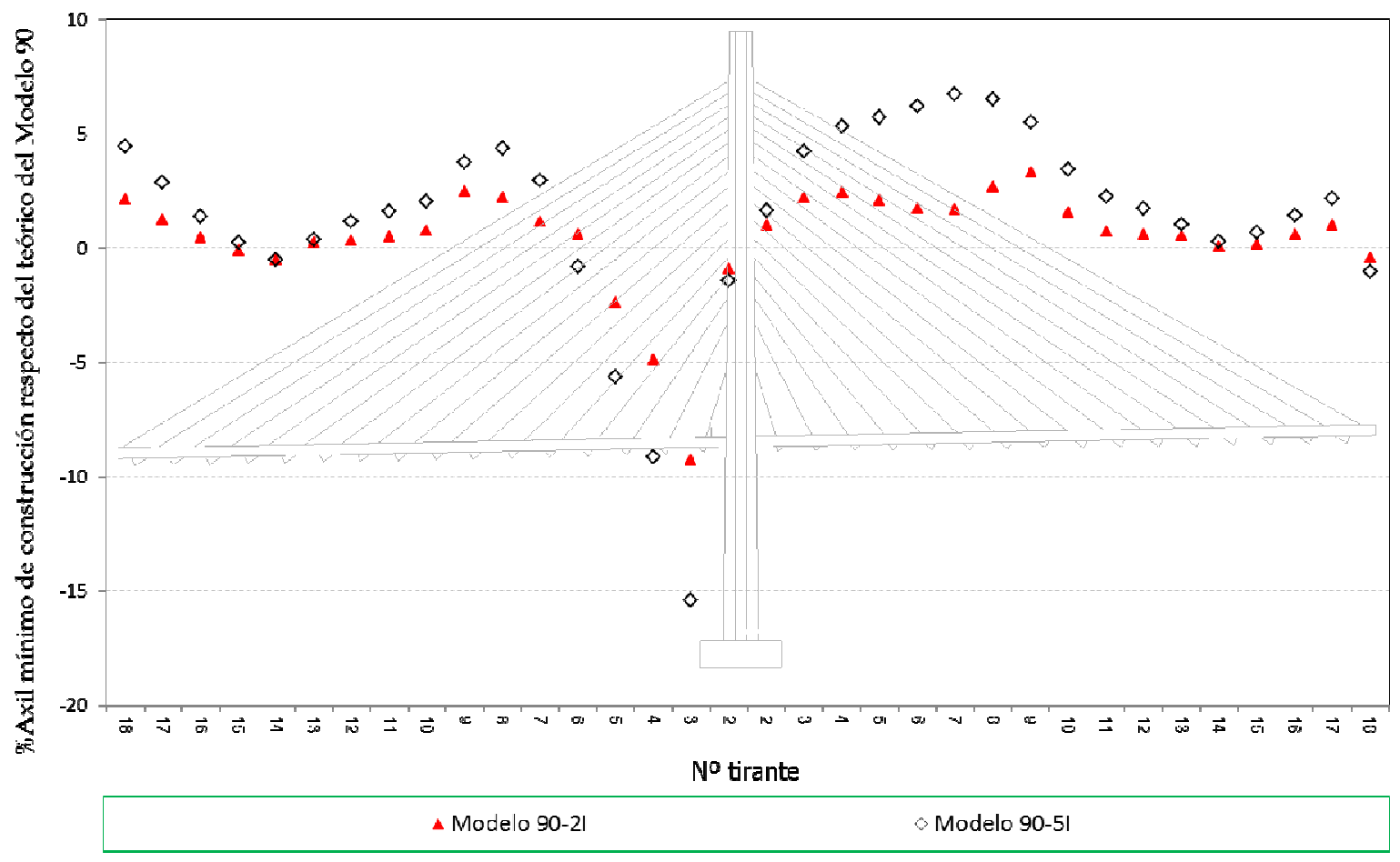

Figura 166. Comparativa de los axiles mínimos de construcción entre los modelos 90, 90-2l y 90-5l.

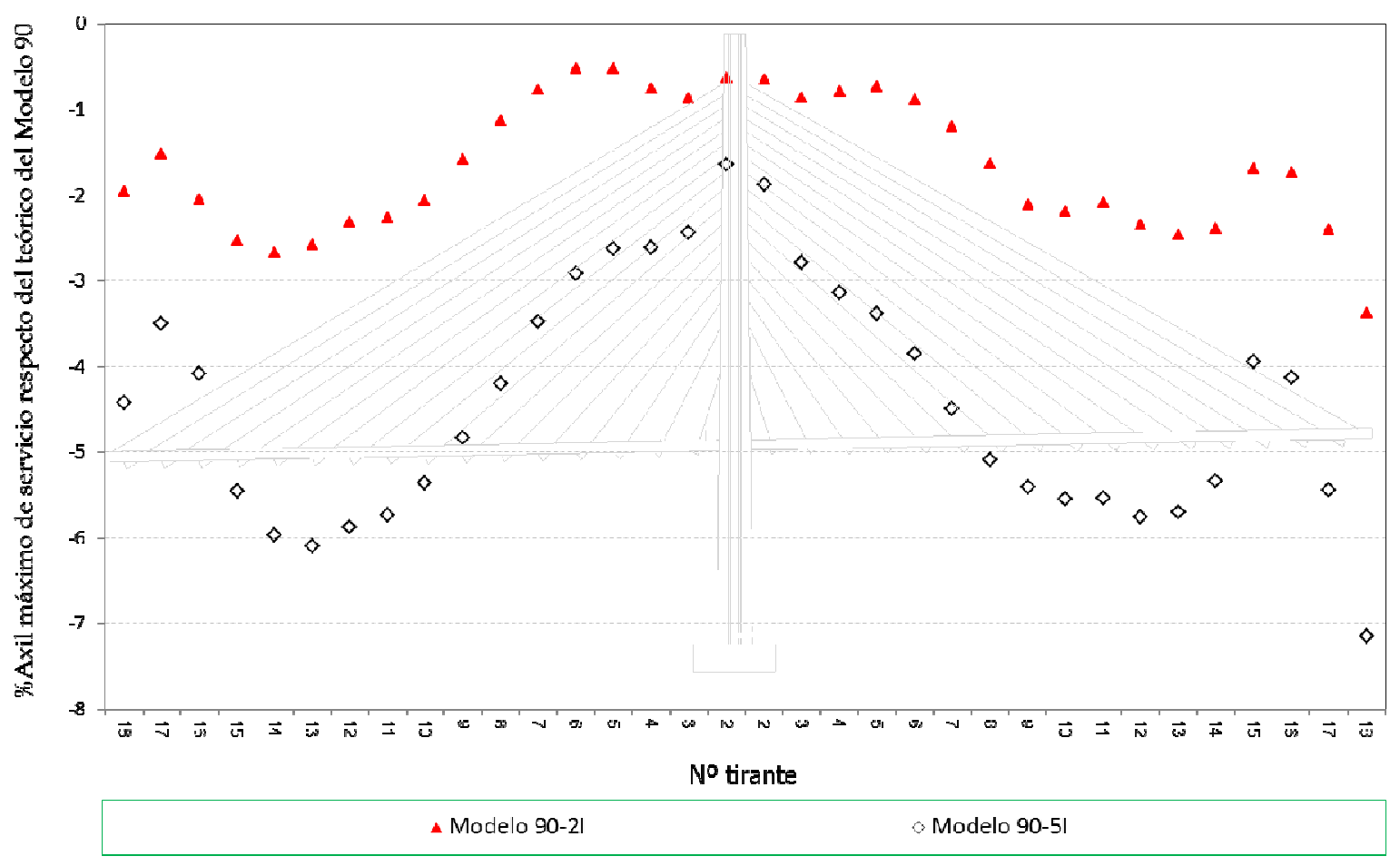

Figura 167. Comparativa de los axiles máximos de servicio entre los modelos 90, 90-2l y 90-5I. 
Por último, en el caso de las situaciones de servicio, los axiles máximos (Figura 167) disminuyen a medida que se produce el aumento de la rigidez del tablero de forma apreciable, por el mismo motivo que se ha señalado para justificar el comportamiento de los axiles máximos de construcción, mientras que los axiles mínimos no sufren apenas modificaciones entre los distintos casos analizados.

A continuación se incluye otro gráfico (Figura 168) donde se representa la variación del axil de los tirantes en función de la inercia del tablero. Para ello se han elegido 3 tirantes que pueden representar de forma bastante adecuada la distribución de éstos a lo largo del tablero, en concreto son los tirantes 5D, 5I, 8D, 8I, 12D y 12l. Así, se representa para todos ellos la evolución del máximo axil de construcción en función de la inercia del tablero en la siguiente figura:

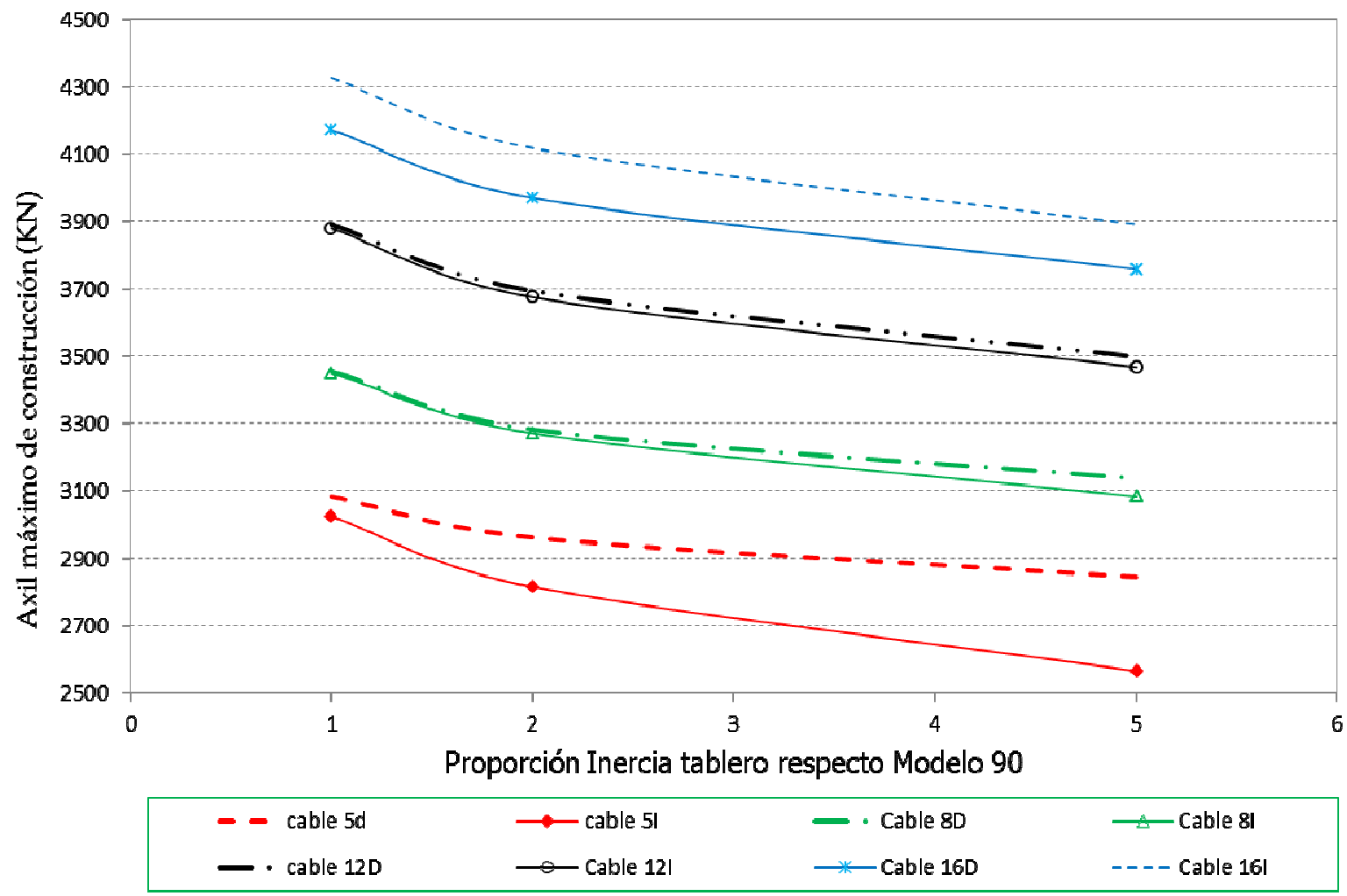

Figura 168. Máximo axil de construcción en los tirantes $5 D, 5 I, 8 D, 81,12 D$ y 121 , en función de la inercia del tablero..

De la figura anterior se deduce que:

- En primer lugar que el aumento de la inercia del tablero implica una disminución del axil del tirante, pero de forma no proporcional a la variación de la inercia.

- En segundo lugar que en los tirantes más cercanos al pilono (5D y 5I), que es la zona del tablero donde más preponderancia tiene su rigidez a flexión frente a la del propio 
atirantamiento, es donde más diferencia encontramos en los tirantes entre las distintas estructuras con tableros de inercias distintas.

- Que también para los tirantes del caso anterior (5D y 5l), el efecto de la variación de la inercia, debido al proceso constructivo adoptado, es mayor en los cables del vano izquierdo que en los del vano derecho.

Finalmente, y para terminar el análisis de los valores teóricos de los axiles de los tirantes en función de la rigidez del tablero, se muestran los resultados en relación a la variación del axil pasivo en los tirantes. Se puede así generar el siguiente gráfico (Figura 169) comparando la evolución de dicha componente del axil de los tirantes en función de la inercia del tablero.

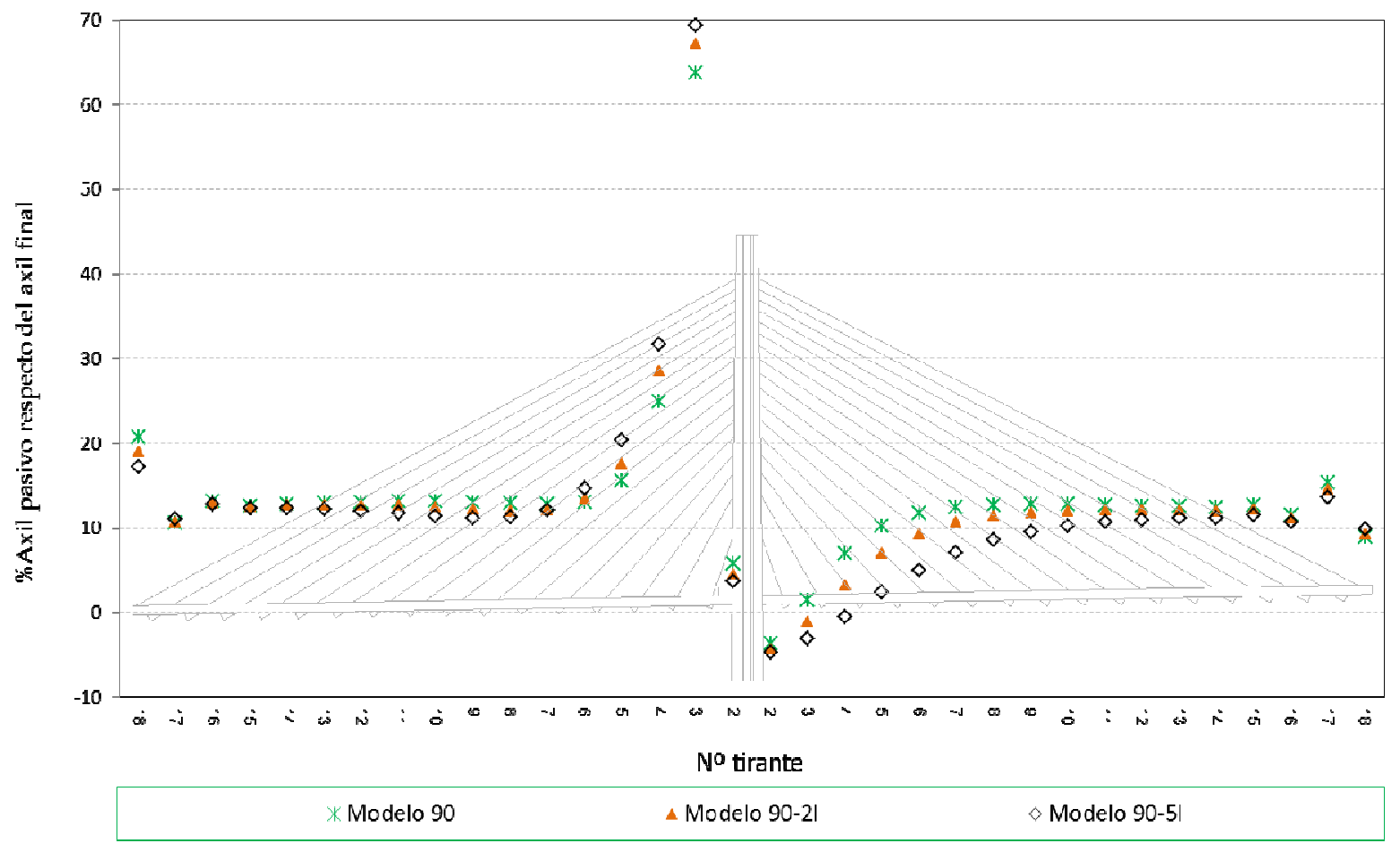

Figura 169. Comparativa de la parte pasiva del axil final de los tirantes en los modelos 90, 90-2l y 90-5l.

En general se observa que las diferencias son pequeñas, siendo la cuantía del axil pasivo del orden del 10\%, salvo en los primeros tirantes del vano derecho, donde se aprecia una disminución de la parte pasiva del axil de los tirantes conforme aumenta la rigidez del tablero. Este efecto aparece debido al proceso constructivo elegido, en el que cuando se instalan los cables del vano izquierdo aún no se han colocado los gemelos del vano derecho, pero si al revés.

Los siguientes resultados que se estudian son los resultados teóricos en flectores en el tablero, comparándose los del Modelo 90-21 y Modelo 90-51, con los del Modelo $\mathbf{9 0}$. Se procede así a 
comparar únicamente las envolventes en construcción y servicio, dado que la situación final teórica como viga continua ya se ha indicado que es idéntica en los tres casos comentados.

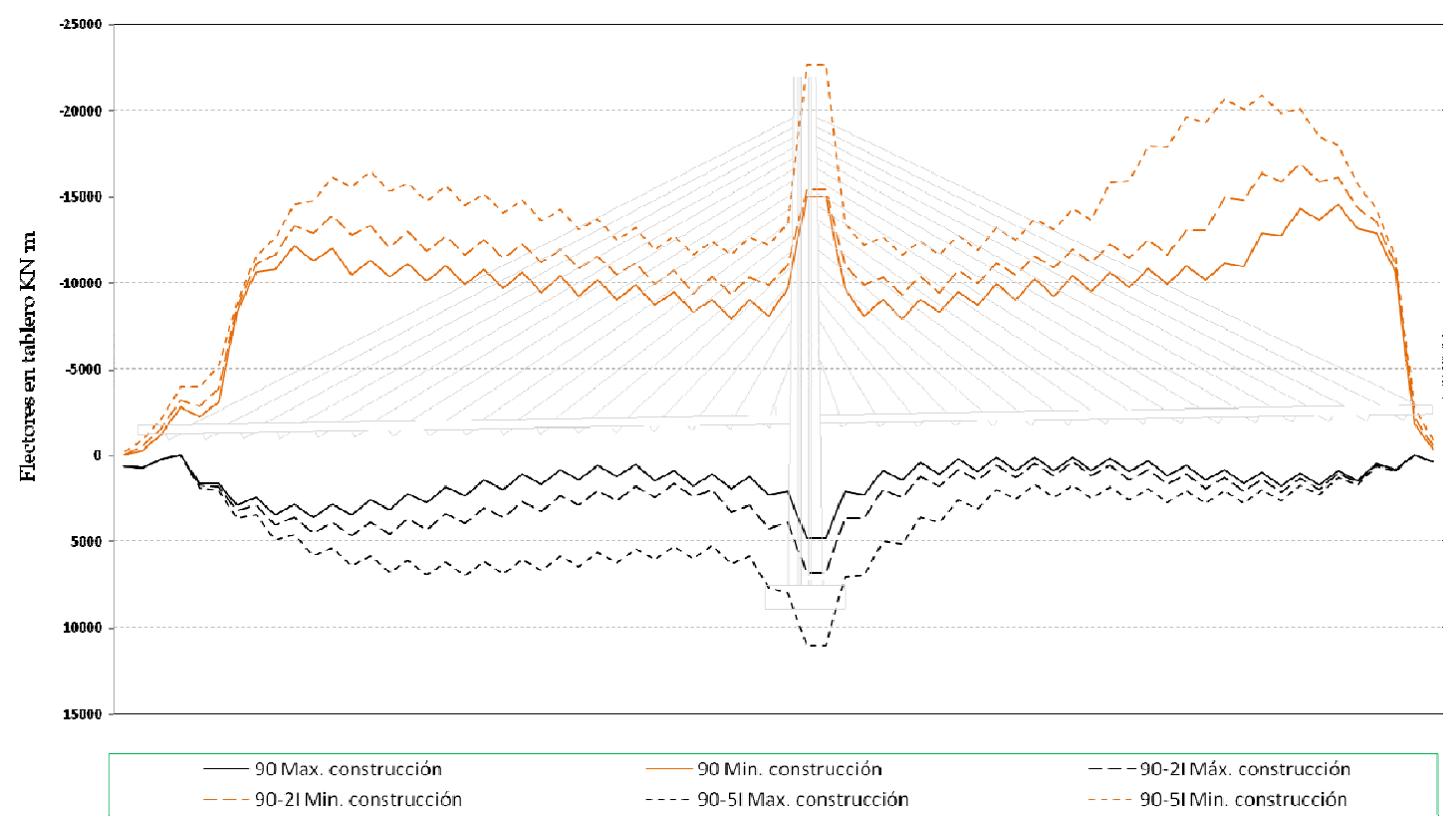

Figura 170. Comparativa de las envolventes de flectores en el tablero para las fases de construcción de los modelos 90, 90-2l y 90-5I. .

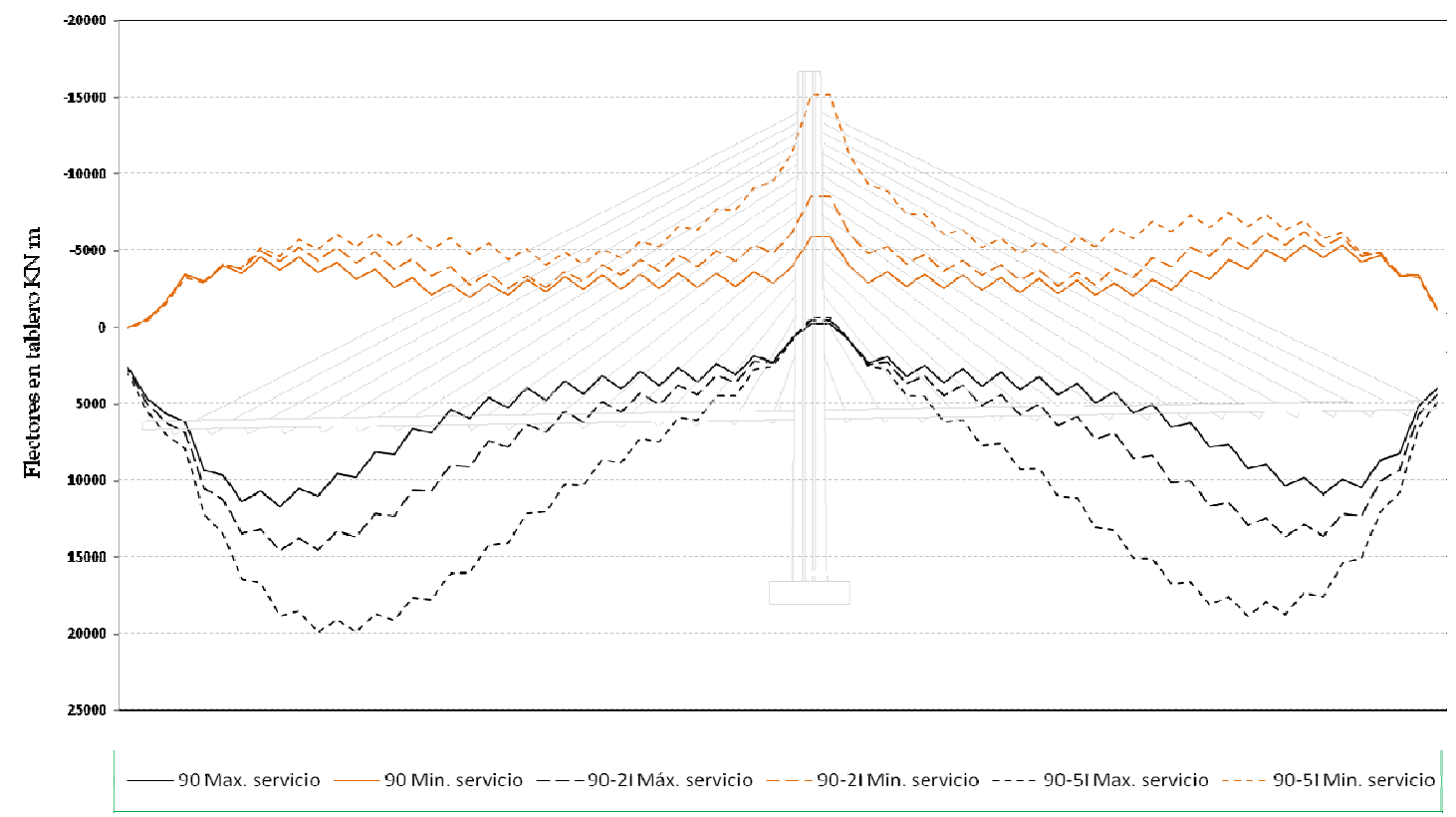

Figura 171. Comparativa de las envolventes de flectores en el tablero para las fases de servicio de los modelos 90, 90-2l y 90-5I. . 
En las dos gráficas anteriores (Figura 170 y Figura 171) se puede observar que, tanto en construcción como en servicio, el aumento de la rigidez del tablero conlleva mayores esfuerzos máximos y menores esfuerzos mínimos, es decir que las envolventes de flectores son mayores en valor absoluto conforme aumenta la rigidez del tablero. Hay que hacer notar que estos gráficos son muy similares a los presentados por Walther en los estudios paramétricos que incluyó en su texto [103].

En el siguiente gráfico (Figura 172) se analiza la evolución del flector en 4 nudos de la estructura, en función de la inercia del tablero, pudiendo observarse que el aumento de esfuerzos en el tablero es mucho más acusado que la variación de axiles en los tirantes anteriormente representados (Figura 168).

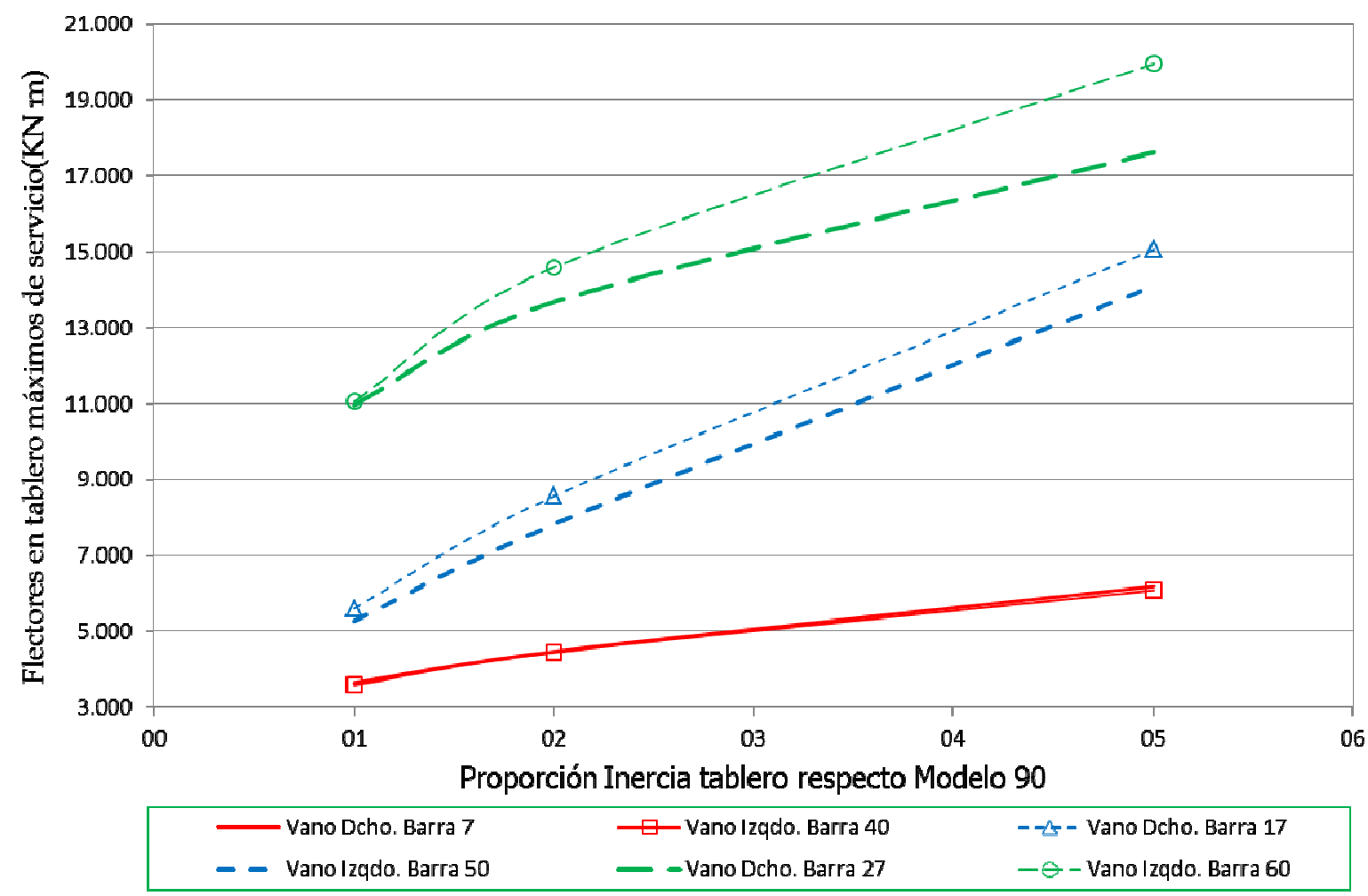

Figura 172. Evolución de las flectores en las barras $7,17,27,40,50$ y 60 del tablero para los máximos de servicio en función de la inercia del tablero. Resultados en nudo frontal.

En el gráfico anterior se observa nuevamente que todas las curvas son crecientes, no existiendo en general una proporcionalidad entre el aumento de la inercia y la respuesta del tablero frente a las sobrecargas de servicio.

Finalmente quedan por incluir los resultados de los flectores en el pilono, que se presentan en la siguiente gráfica (Figura 173). En concreto se han representado sólo los resultados para la 
situación de servicio, que se ha considerado la más significativa para caracterizar la influencia de la modificación de la inercia del tablero en el comportamiento del pilono.

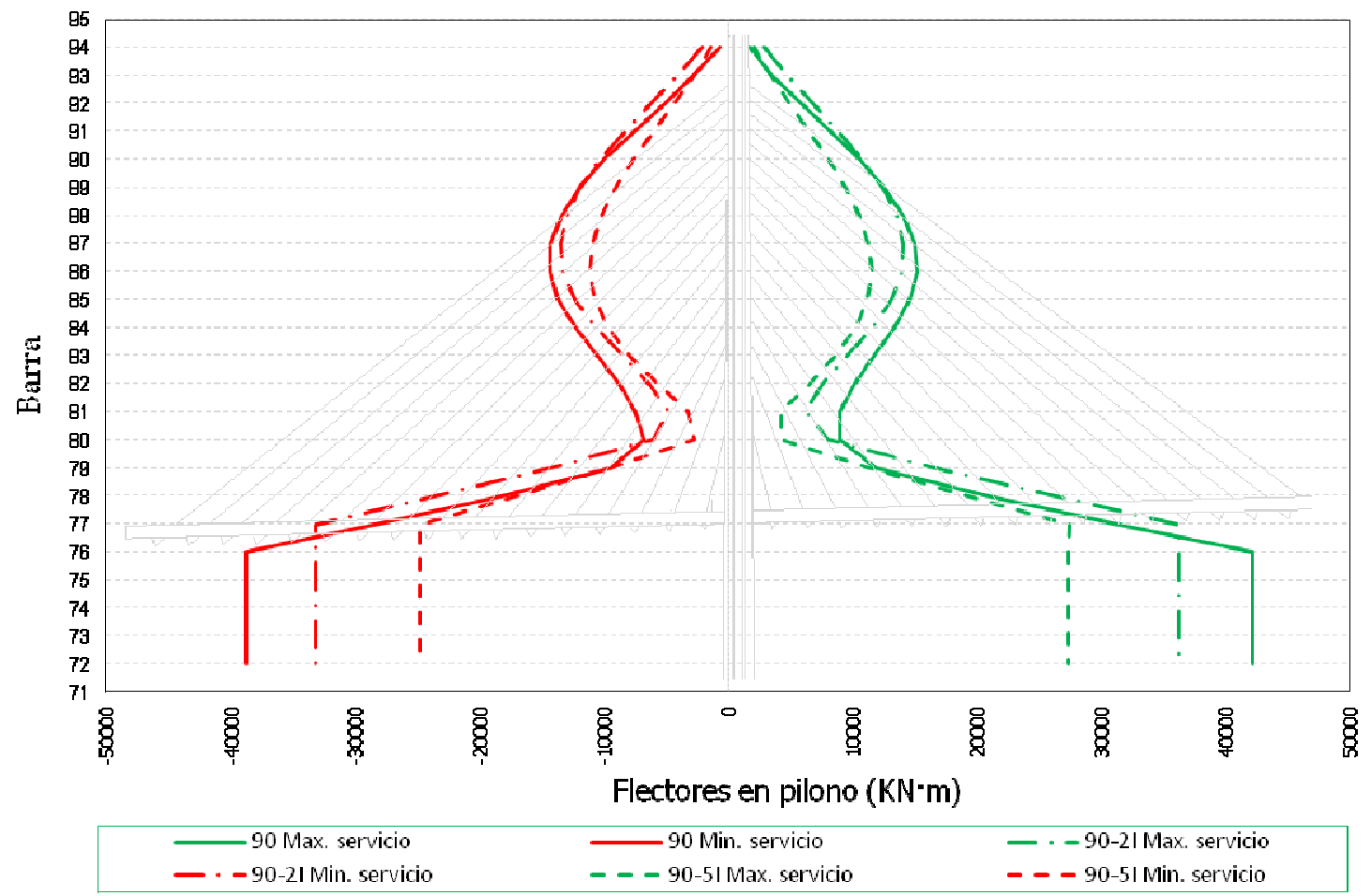

Figura 173. Comparación de las envolventes de flectores en el pilono para la situación de servicio en los modelos $90,90-21$ y $90-51$.

En los resultados anteriores se observa que conforme se aumenta rigidez del tablero, al reducirse la eficacia relativa del atirantamiento respecto de la capacidad a flexión del tablero, y por lo tanto al absorber los cables menos carga, los efectos de desequilibrio sobre el pilono se van reduciendo de cuantía, generándose así por lo tanto flexiones de menor valor sobre éste. 


\subsection{RESULTADOS OBTENIDOS.}

De los dos modelos teóricos anteriormente mencionados se ha pasado a analizar la influencia de los errores aleatorios de montaje sólo para el caso del Modelo 90-51, dado que es el que supone una mayor diferencia respecto del caso del Modelo $\mathbf{9 0}$ original, y por lo tanto será aquel donde la influencia de la modificación de la inercia del tablero sobre los resultados se pueda interpretar con mayor claridad.

De esta forma, se ha procedido a introducir en este caso errores en la instalación de los cables utilizando sólo el método de las fuerzas, generándose para ello 3 muestras de 100 elementos cada una. Con dichos valores se ha procedido a realizar el proceso de montaje según el proceso constructivo ya señalado en los capítulos anteriores.

Así, para el caso del Modelo $\mathbf{9 0 - 5 I}$ se procede a realizar entonces $3 \times 100$ procesos de cálculo del montaje de la estructura, de forma similar a como se ha realizado en los cálculos desarrollados en los anteriores capítulos.

Igualmente se ha utilizado la definición del valor característico ya reseñada, y se han definido las mismas situaciones de cálculo que en el capítulo anterior (situación final, situaciones de construcción y situaciones de servicio).

Se adjuntan a continuación los resultados más significativos de todos estos cálculos. 


\subsubsection{Resultados en tirantes.}

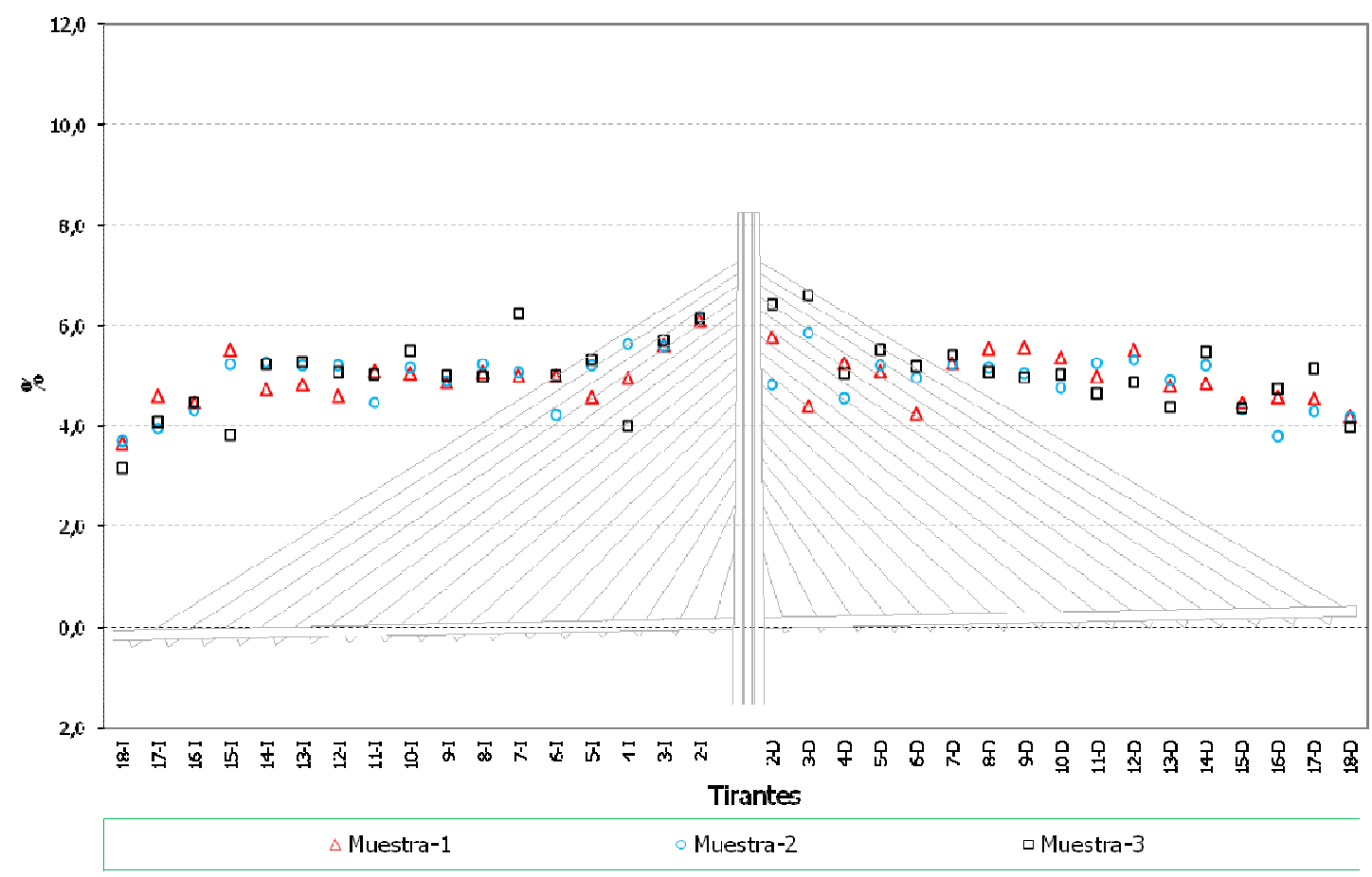

Figura 174. Axiles finales en los tirantes. Diferencia en \% de los valores caracteristicos superiores respecto de los teóricos.

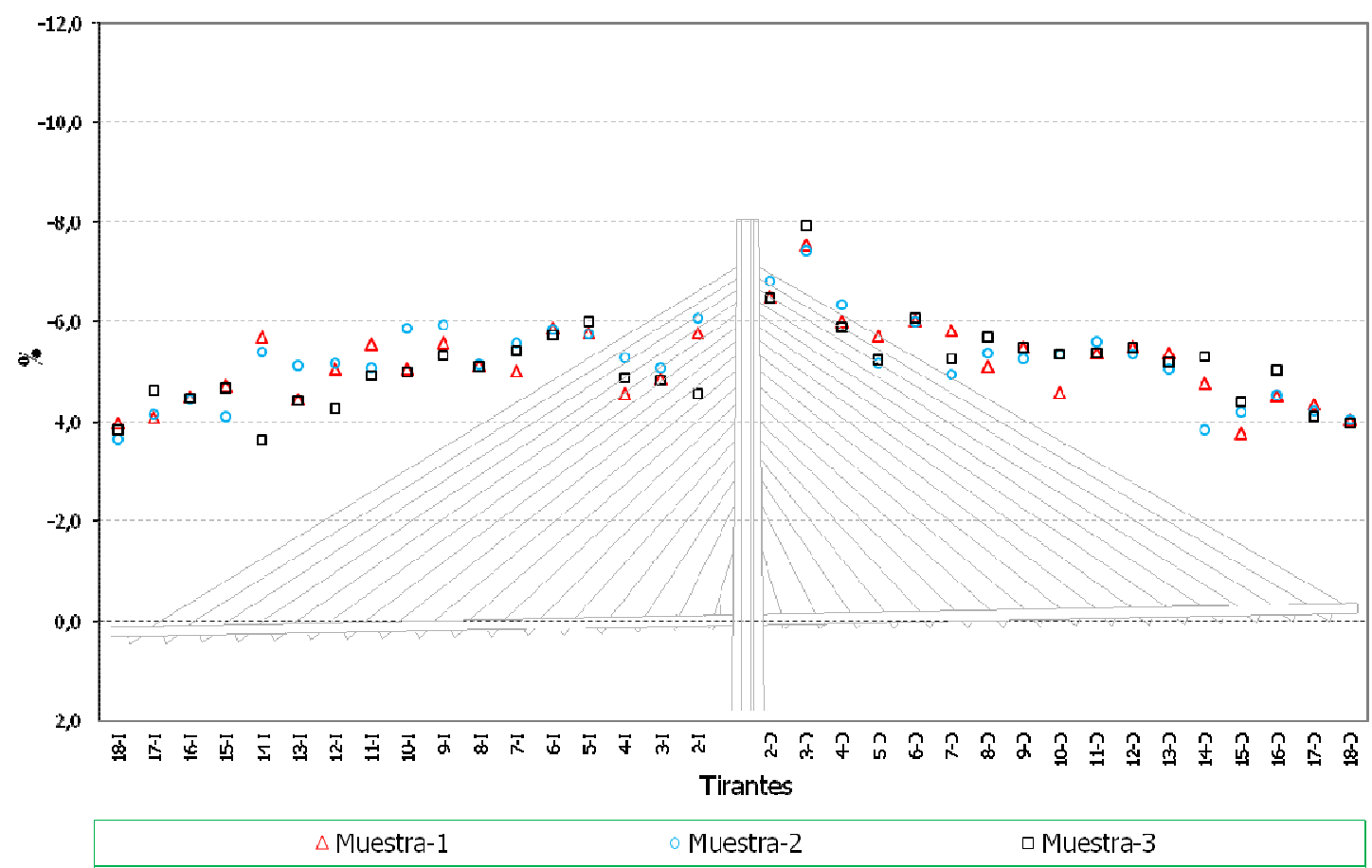

Figura 175. Axiles finales en los tirantes. Diferencia en \% de los valores característicos inferiores respecto de los teóricos 


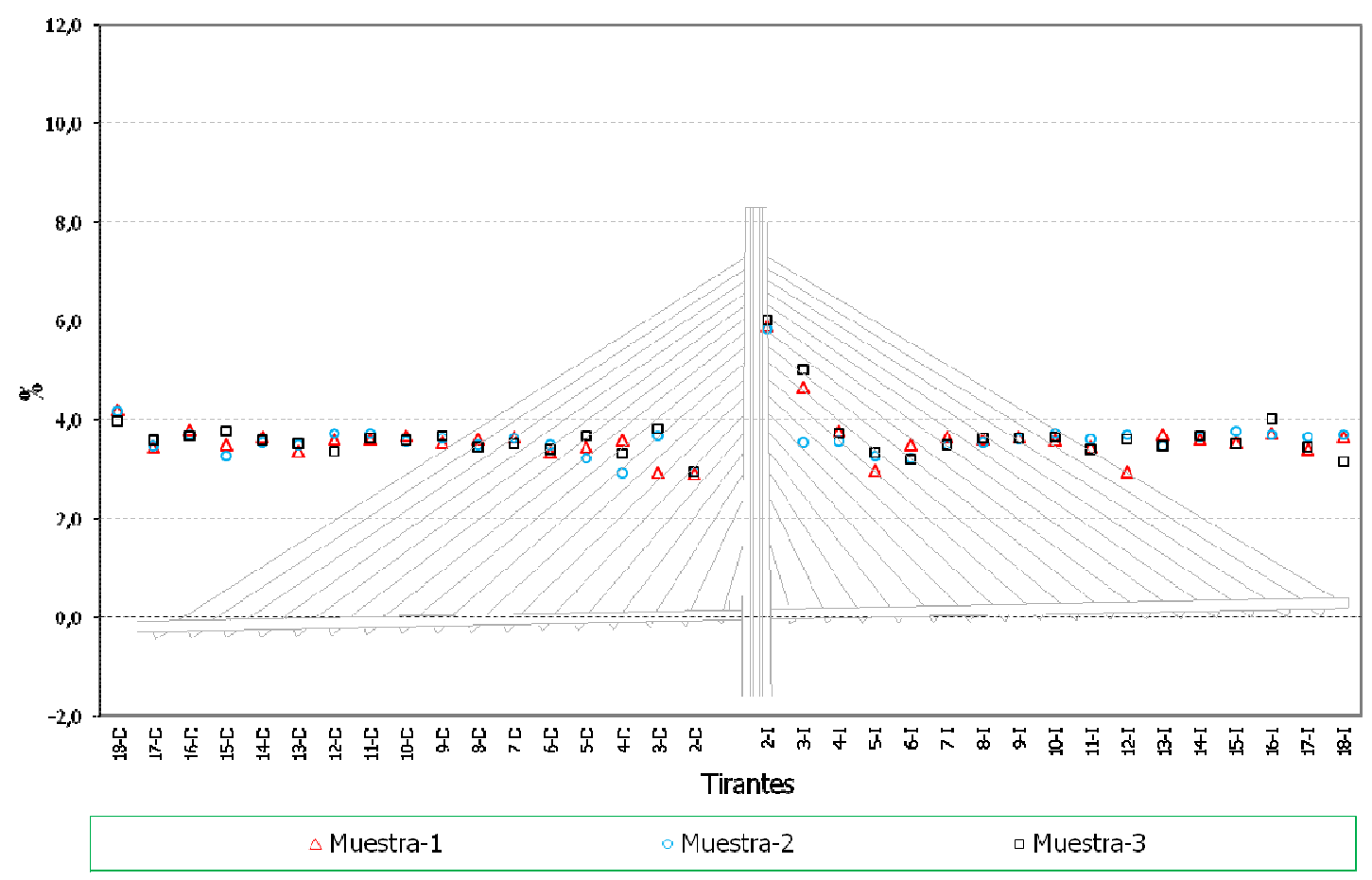

Figura 176. Axiles en los tirantes máximos de construcción. Diferencia en \% de los valores característicos superiores respecto de los teóricos.

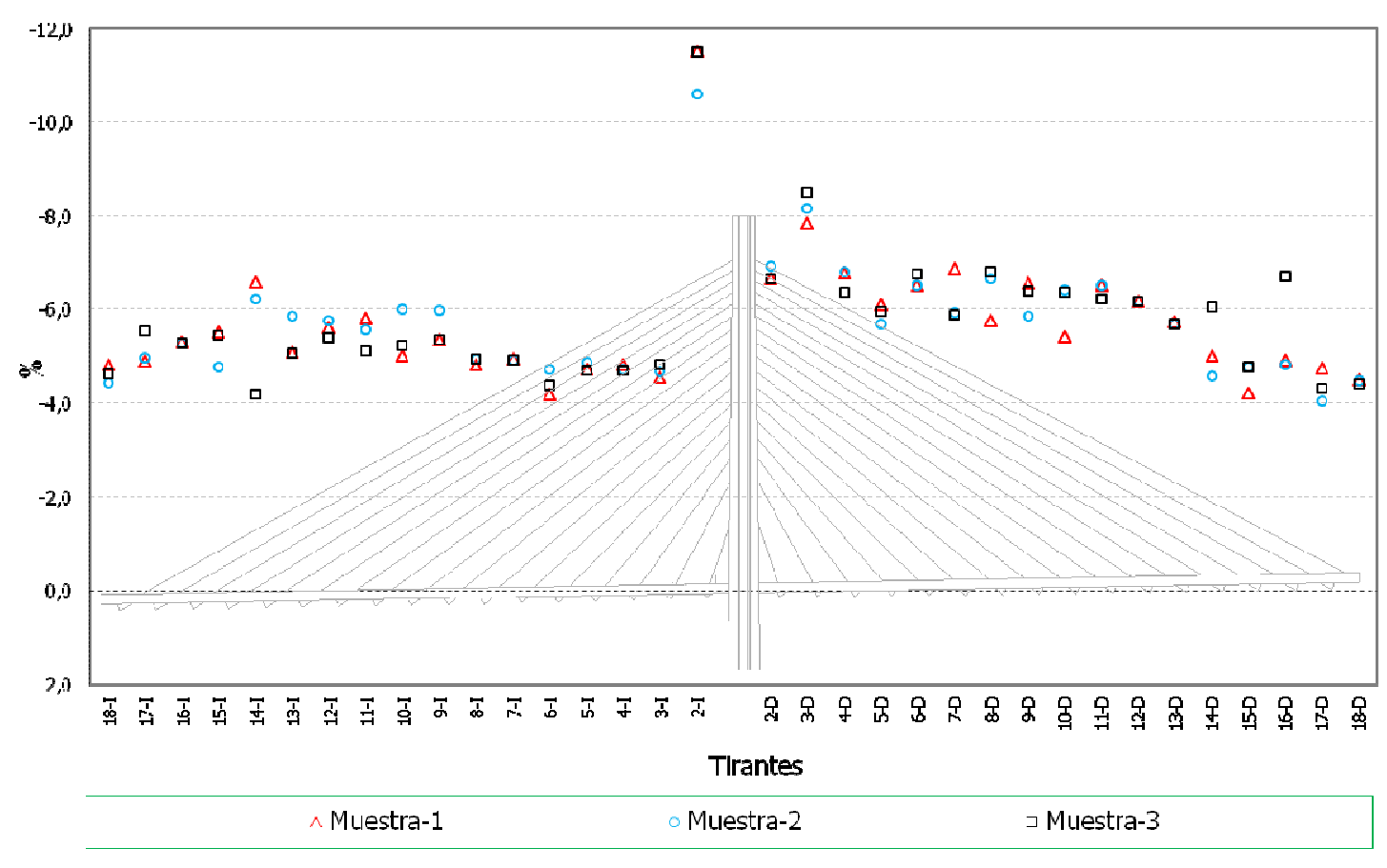

Figura 177. Axiles en los tirantes mínimos de construcción. Diferencia en \% de los valores característicos inferiores respecto de los teóricos. 


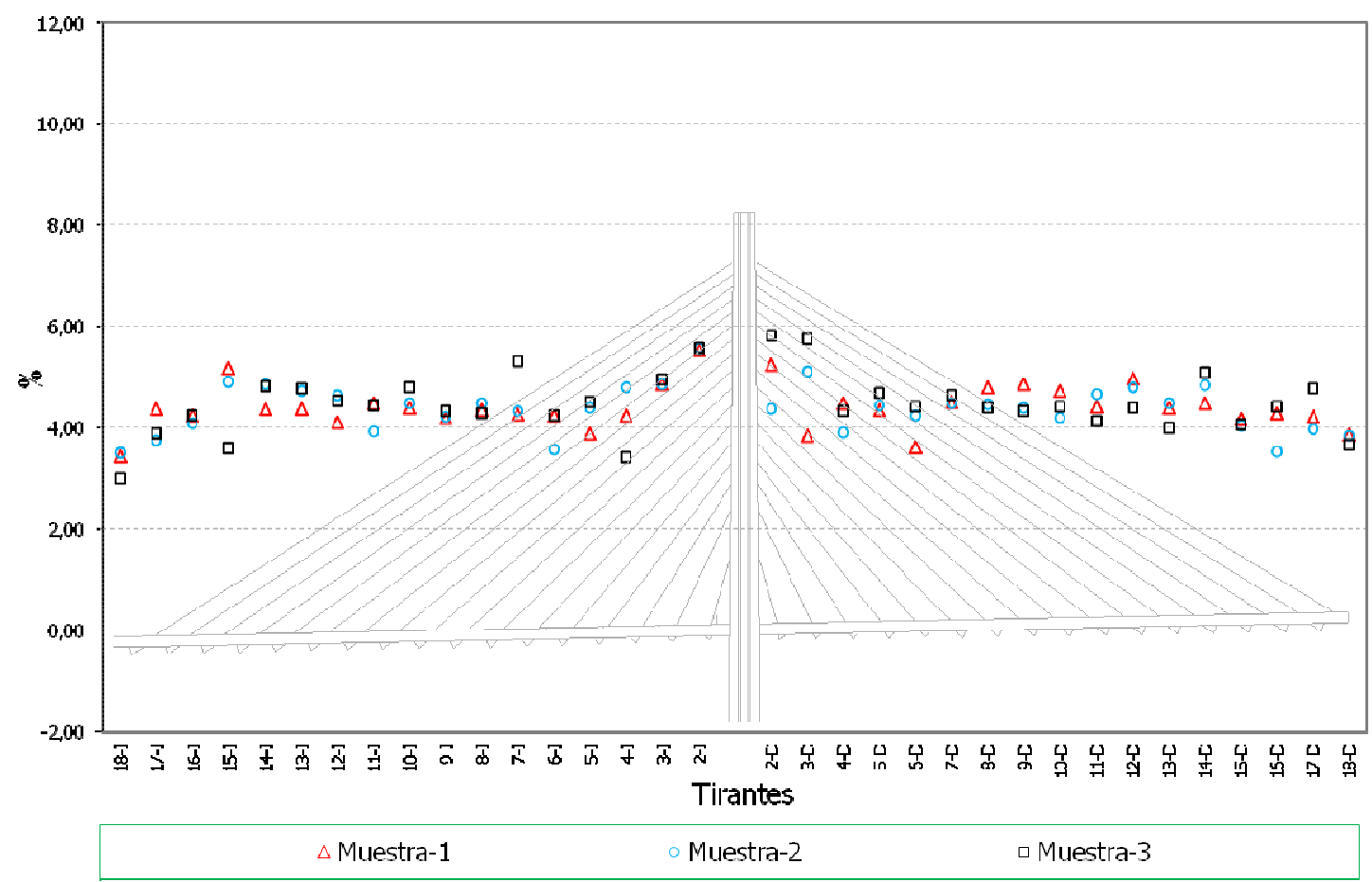

Figura 178. Axiles en los tirantes máximos de servicio. Diferencia en \% de los valores característicos superiores respecto de los teóricos.

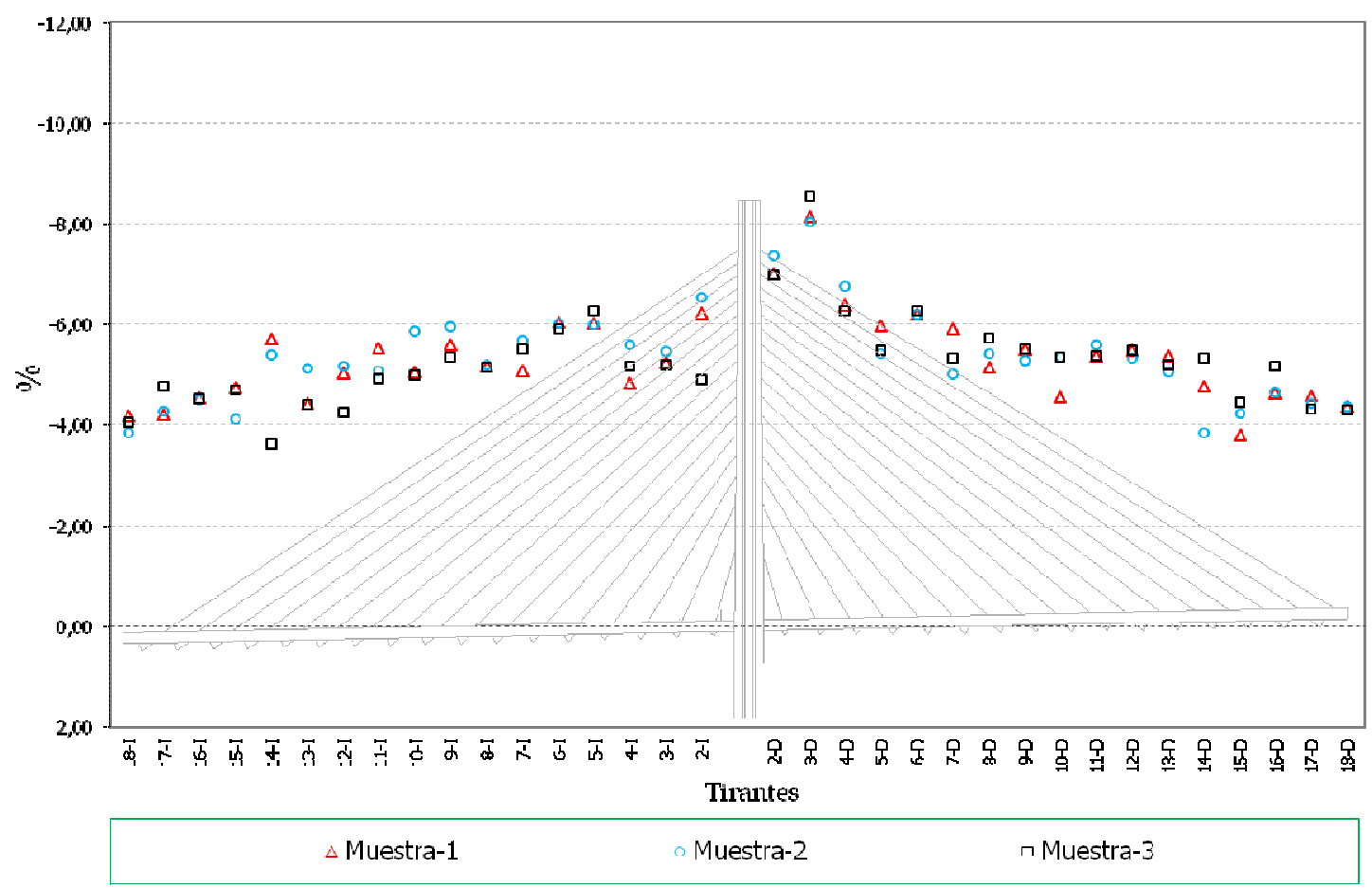

Figura 179. Axiles en los tirantes mínimos de servicio. Diferencia en \% de los valores característicos inferiores respecto de los teóricos. 


\subsubsection{Comparativa de resultados en tirantes.}

Se procede en este apartado a presentar los valores obtenidos en los axiles de los tirantes en los diferentes modelos calculados hasta este punto del estudio (Modelo 90, Modelo 90M, Modelo 180 y Modelo 90-5I), de tal manera que se pueda comparar entre todos ellos los diferentes resultados obtenidos para así tener una visión global de estos resultados. De esta forma se incluyen a continuación los gráficos correspondientes a los obtenidos en cada modelo para los axiles finales, de construcción y en servicio.

Señalar que para clarificar la presentación de estos resultados, los valores obtenidos de cada modelo son el valor medio de los resultados de cada una de las 3 muestras calculadas en cada modelo de cálculo estudiado.

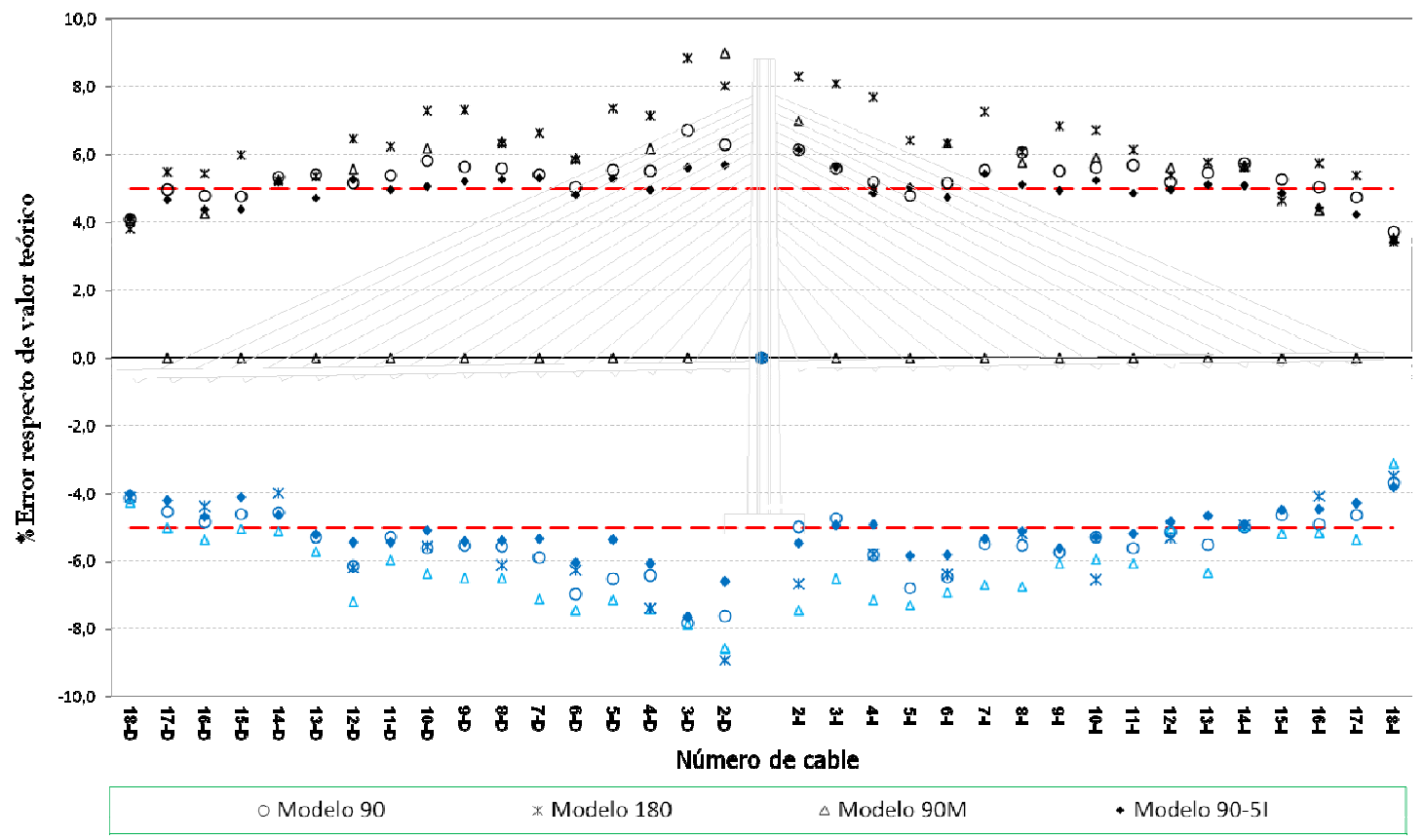

Figura 180. Axiles finales en los tirantes. Diferencia en \% de los valores característicos respecto de los teóricos en los modelos 90, 90M, 180 y 90-5I. 


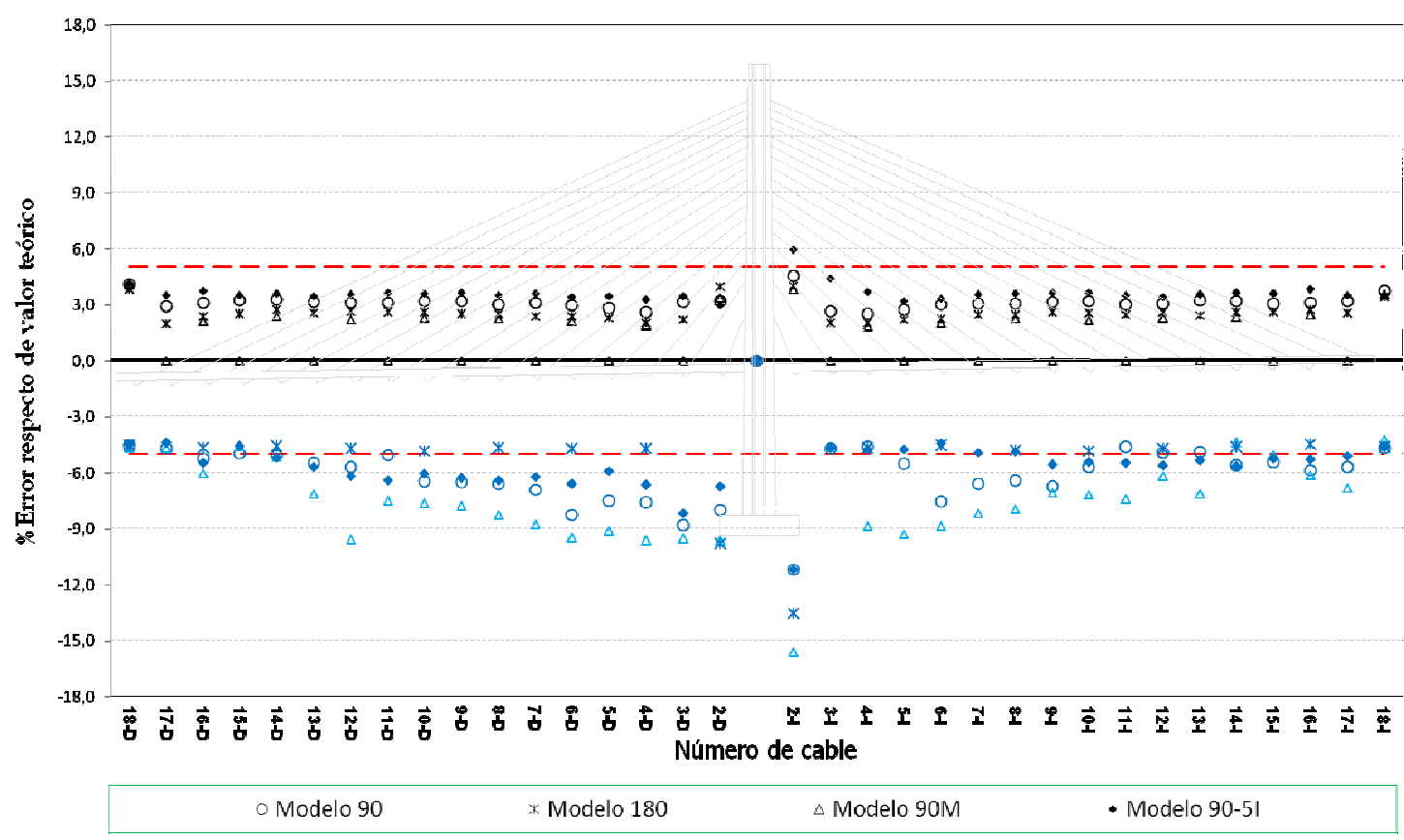

Figura 181. Axiles en fases de construcción en los tirantes. Diferencia en \% de los valores característicos respecto de los teóricos en los modelos 90, 90M, 180 y 90-5I.

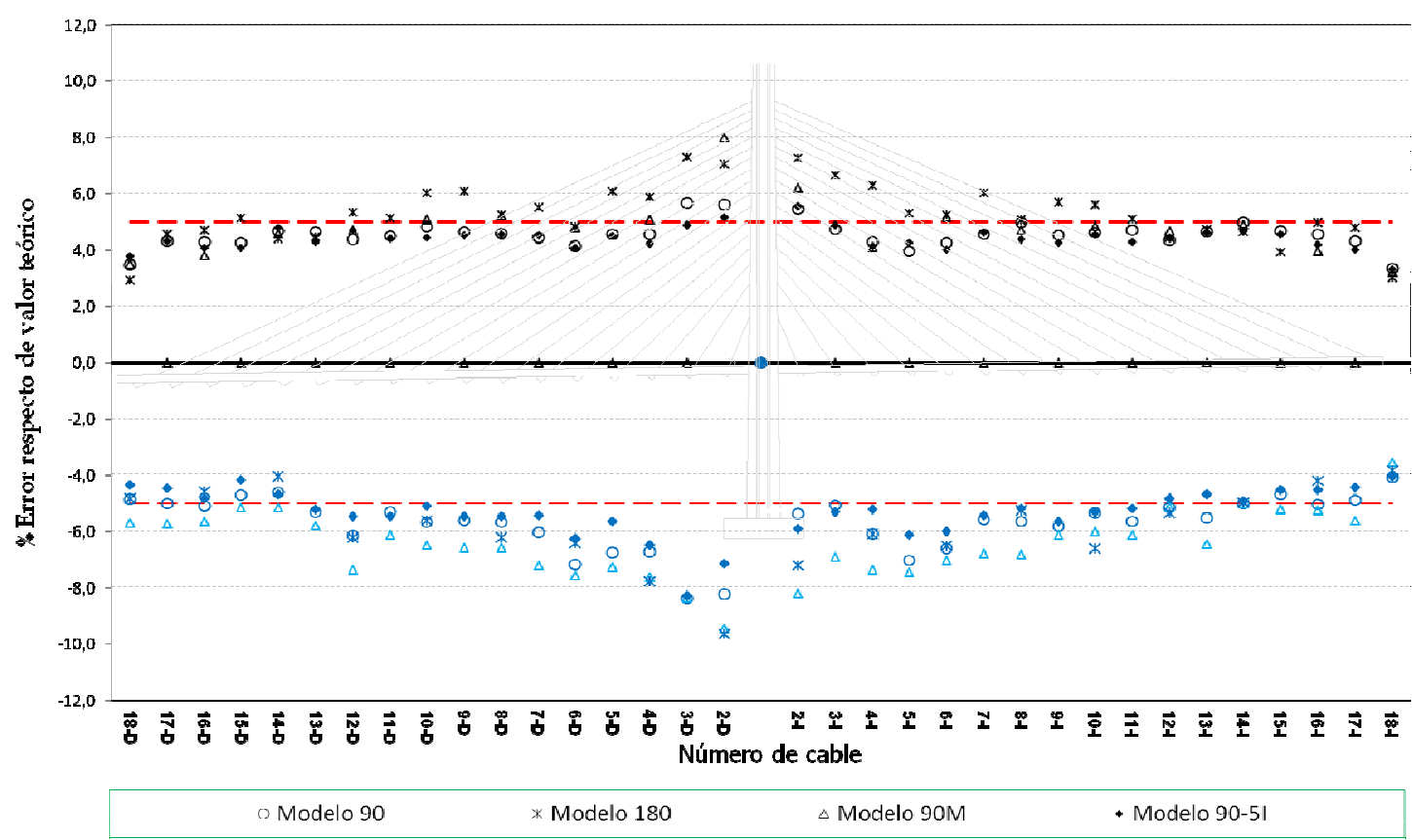

Figura 182. Axiles en situaciones de servicio en los tirantes. Diferencia en \% de los valores característicos respecto de los teóricos en los modelos 90, 90M, 180 y 90-5I. 


\subsubsection{Resultados en tablero.}

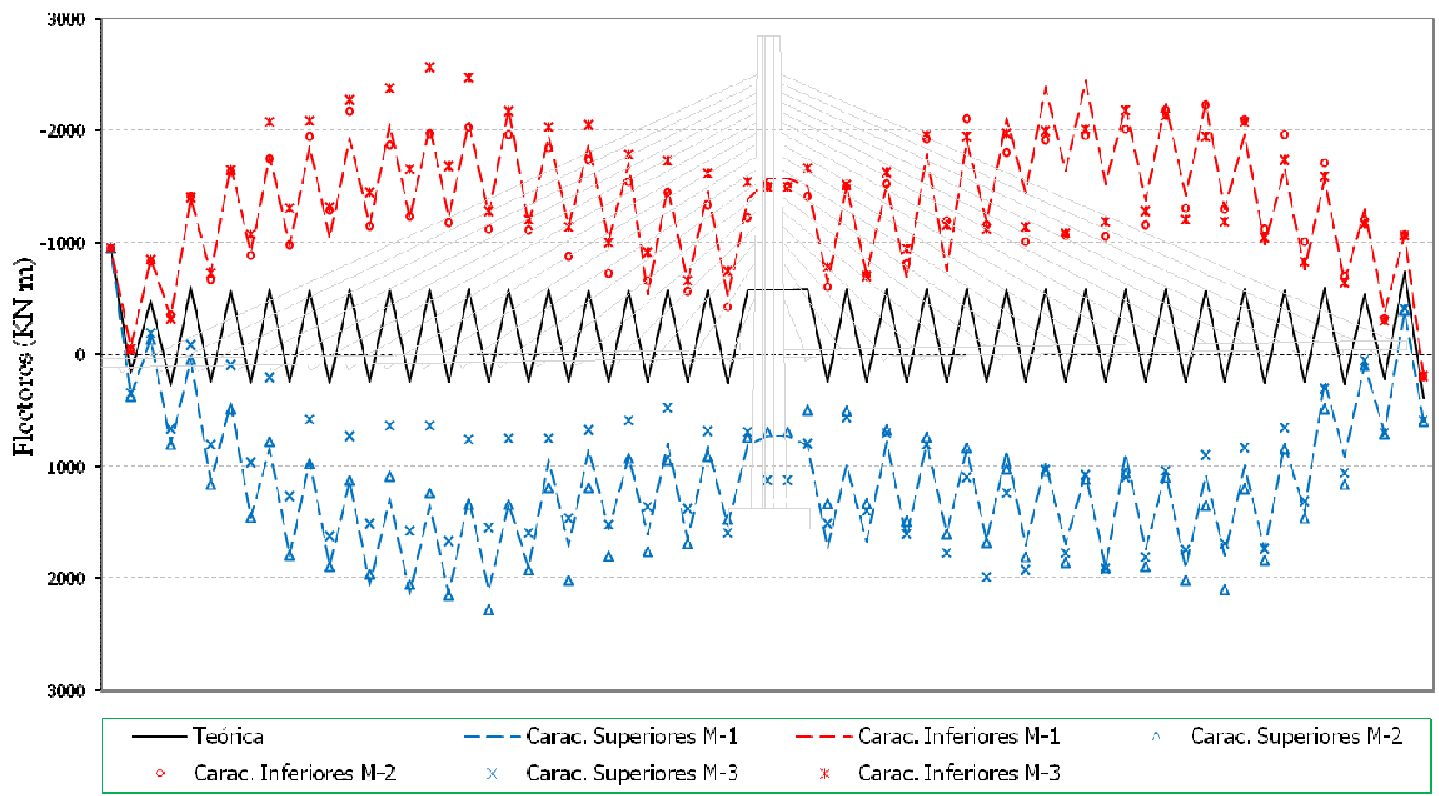

Figura 183. Flectores en tablero en situación final. Valores característicos superiores e inferiores.

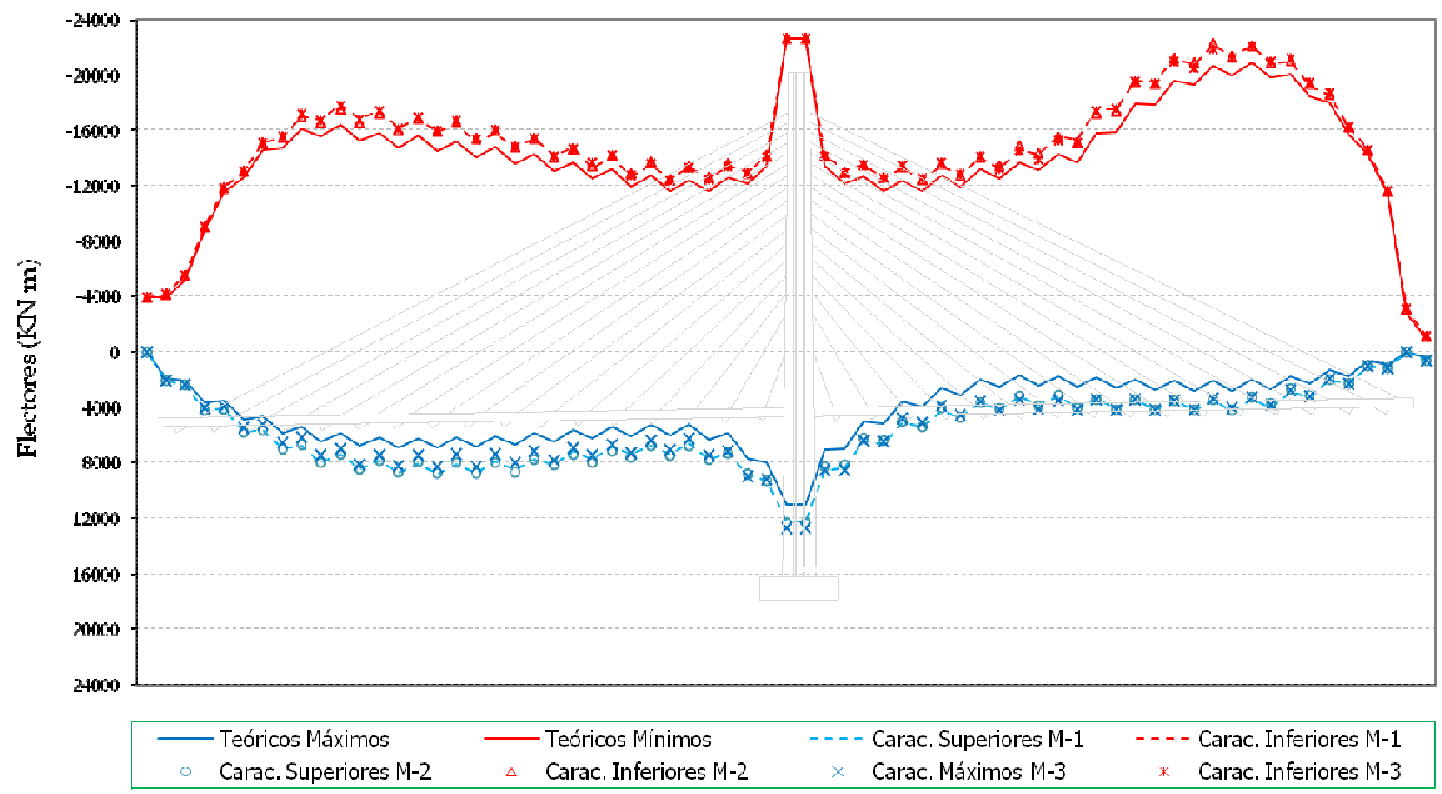

Figura 184. Flectores en tablero en fases de construcción. Valores característicos superiores e inferiores. 


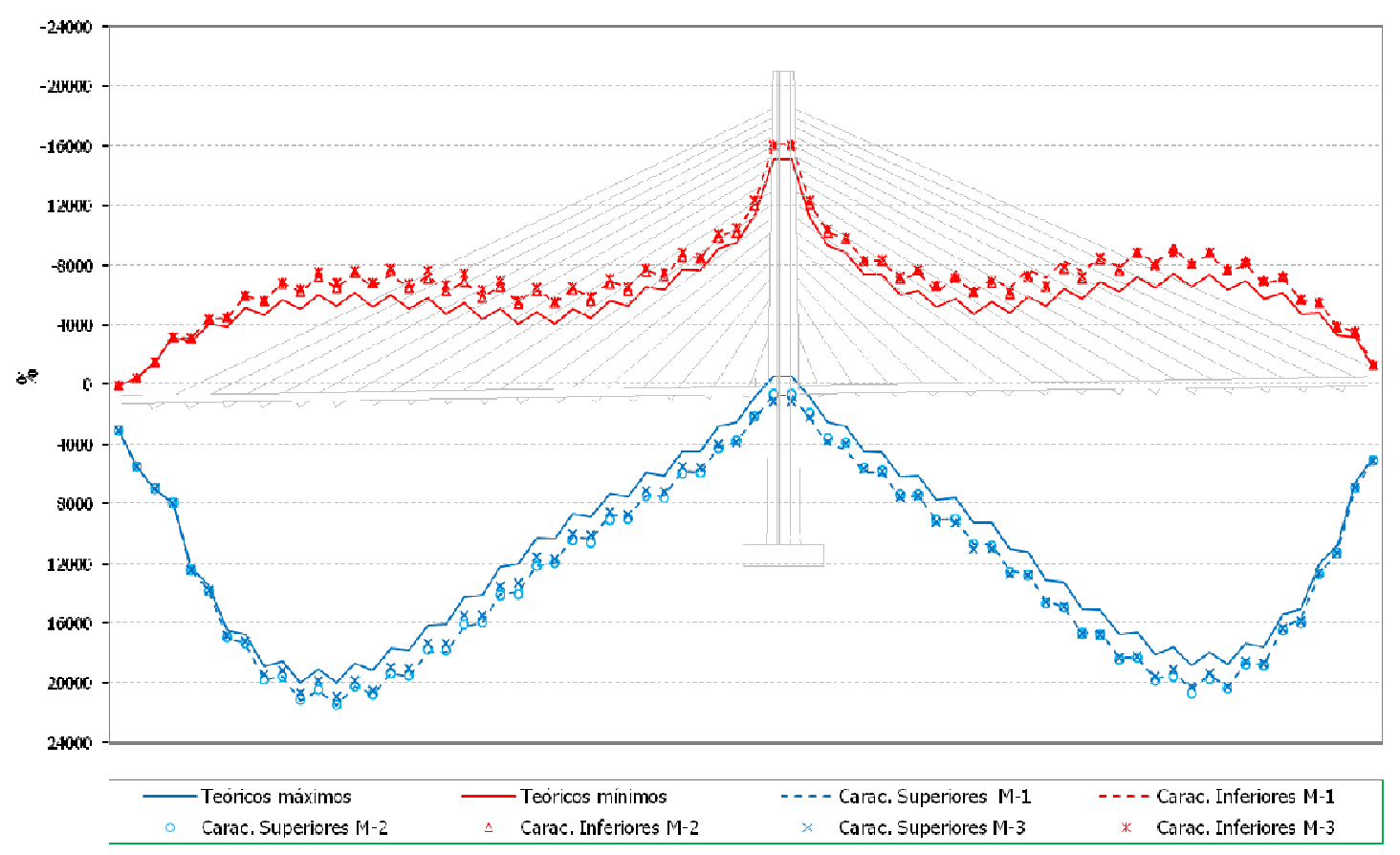

Figura 185. Flectores en tablero en fases de servicio. Valores característicos superiores e inferiores.

\subsubsection{Resultados en pilono.}

Se incluyen en este apartado los resultados considerados más significativos en este elemento, que en concreto son la envolvente de flectores mínimos en fase de construcción y la envolvente de máximos en servicio. 


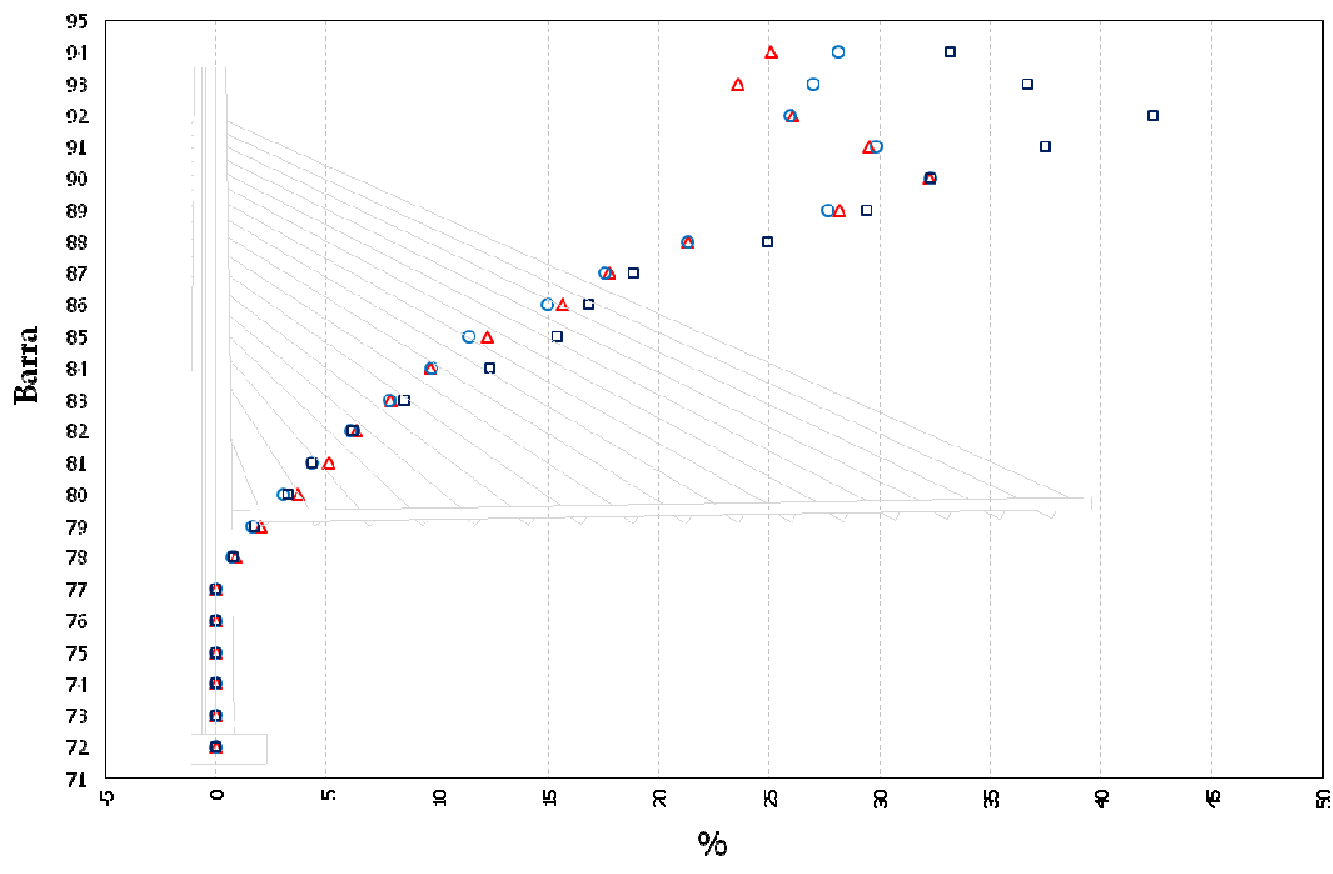

$\Delta$ Muestra-1 $\quad$ Muestra-2 $\quad$ Muestra-3

Figura 186. Flectores en pilono mínimos en fases de construcción. Diferencias en \% de los valores característicos inferiores respecto de los teóricos.

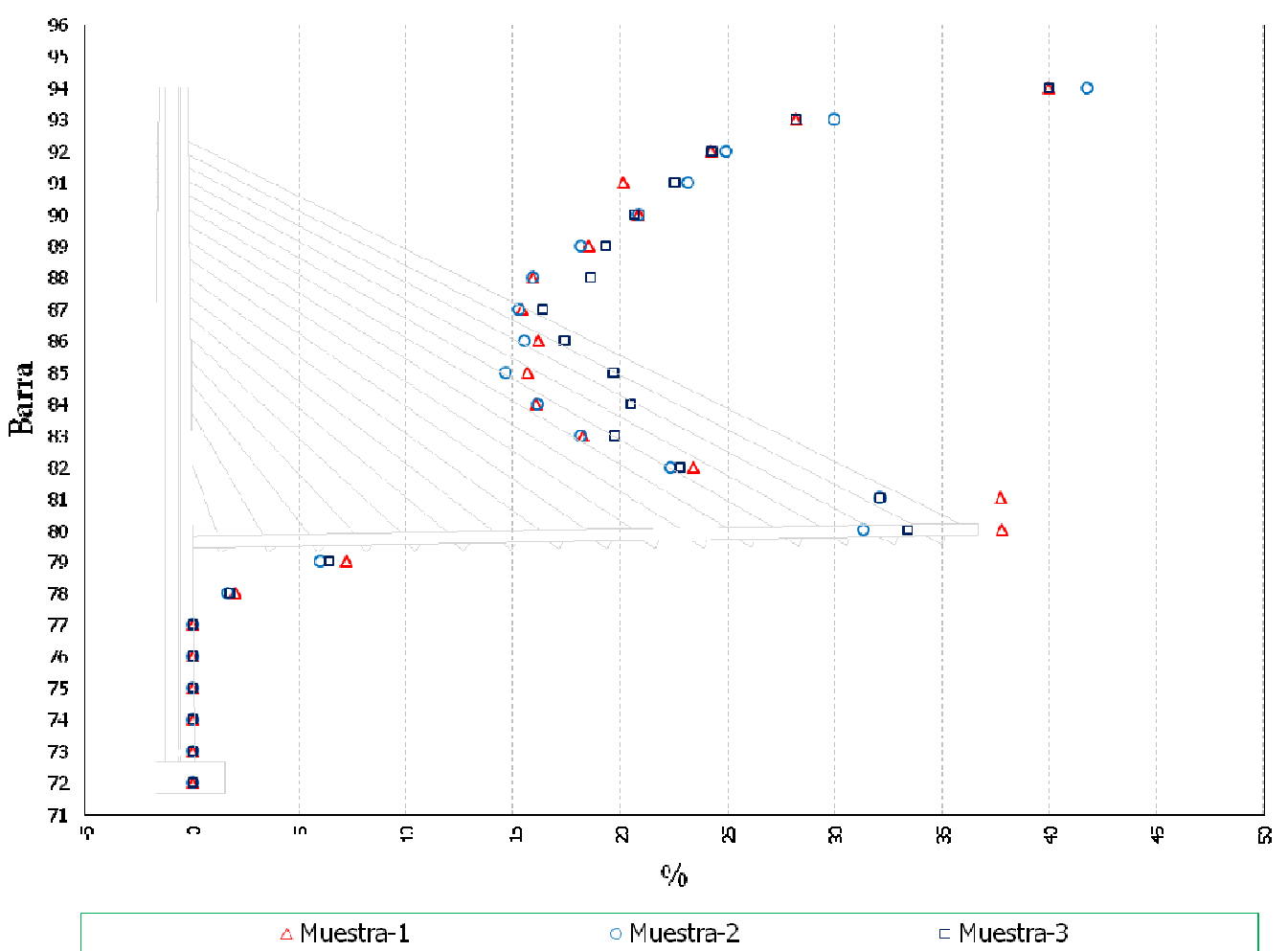

Figura 187. Flectores en pilono máximos en fases de servicio. Diferencias en \% de los valores característicos superiores respecto de los teóricos. 


\subsection{ANALISIS DE LOS RESULTADOS OBTENIDOS.}

Se procede a continuación a analizar los resultados obtenidos en este capítulo tanto en los tirantes como en el tablero y el pilono.

\subsubsection{Resultados en tirantes.}

- $\quad$ Para el caso de los axiles finales se observa que presentan unas desviaciones medias del orden del 4 al 6\%, llegando a valores máximos de hasta un $8 \%$ en los primeros cables colocados. Sí se observa que la cuantía de los errores disminuye a lo largo del desarrollo del tablero, conforme nos alejamos del pilono. La cuantía de los errores obtenidos resultan relativamente pequeñas y en general menores que las obtenidas en el resto de modelos analizados en este estudio.

- Los axiles máximos de construcción vienen condicionados por el peso de las dovelas, igual que en el resto de modelos, siendo los resultados obtenidos en este caso muy poco dependientes de la introducción de errores aleatorios. En el caso de los mínimos de construcción se observan errores de cuantía similar a los obtenidos en el Modelo $\mathbf{9 0 ,}$ lo que significa que a su vez son menores que los obtenidos en el Modelo 180.

- En servicio los resultados son muy similares a los obtenidos con el Modelo 90 con errores con cuantías en torno al 4-6\%, tanto en el caso de los valores máximos como de los mínimos.

\subsubsection{Comparación de resultados en tirantes.}

El análisis de la comparativa de los resultados obtenidos en axiles en tirantes en las distintas estructuras estudiadas permite señalar las siguientes conclusiones:

- La primera es que en promedio, en todas las estructuras, los errores finales en tirantes quedan en el rango del 5-8\%, es decir relativamente cercanos a los establecidos como valores límite por el documento de ACHE [2] del 5\%.

- Que en general, en todas las situaciones analizadas, los mayores errores se producen en los primeros cables y los menores en los últimos cables instalados, existiendo en todos los casos una cierta simetría de los resultados respecto del pilono central.

- Que la cuantía de errores en las situaciones finales y de servicio son similares, no siendo así en el caso de las fases de construcción, donde para el caso de los máximos de construcción se obtienen siempre valores muy reducidos, dado que sobre ese valor 
tiene una influencia el peso de las dovelas ejecutadas a continuación del tirante analizado, pesos que están perfectamente determinados, por lo que el efecto aleatorio tiene una menor importancia sobre dicho resultado final.

- Que las mayores desviaciones se producen en el Modelo 180, para el caso de los axiles máximos y del Modelo 90M para el caso de los axiles mínimos, siendo el modelo en el que se producen menores errores el Modelo 90-5I. Es decir, que las cuantías de errores en tirantes está relacionado con la rigidez y luz del tablero, o lo que es lo mismo, que a mayor capacidad del tablero para resistir flexiones menor es la cuantía de los errores generados.

\subsubsection{Resultados en tablero.}

- Los resultados en flectores finales quedan muy amplificados respecto del valor teórico, con desviaciones superiores a las aparecidas en el Modelo 90.

- Respecto de los errores generados en las fases de construcción y servicio, los porcentajes de error alcanzados son bastante más pequeños, siendo de nuevo en este caso de valor similar a lo obtenido para el Modelo 90.

\subsubsection{Resultados en pilono.}

- En primer lugar que, como en el resto de estructuras analizadas en este estudio, los flectores generados en el tramo del pilono por debajo del tablero no se ven afectados por la introducción de errores aleatorios en el montaje de los tirantes.

- Que los valores mínimos de construcción muestran un comportamiento con desviaciones claramente crecientes en porcentaje con la altura, con valores similares a los del Modelo 90. Como ya se ha indicado anteriormente esta cuestión se justifica por el hecho de que los valores de los flectores en la parte superior del pilono son muy pequeños y por lo tanto cualquier variación introducida sobre ellos significará porcentualmente un valor muy alto.

- Es en el caso de los valores de las desviaciones para la situación de los máximos de servicio donde se producen las mayores diferencias con lo aparecido con el Modelo 90, ya que en este caso se alcanzan valores de hasta un 30-40\% de errores respecto de los valores teóricos. 


\subsection{RESULTADOS EN DEFORMACIONES.}

Como complemento a los análisis realizados en los epígrafes 4.8 y 6.6, se adjuntan en este epígrafe los resultados en deformaciones correspondientes a la introducción de errores aleatorios para el caso de la estructura objeto de este capítulo Modelo 90-51. Se han estudiado de nuevo las fases que se consideran más representativas del proceso constructivo, analizándose la deformada del tablero completo correspondiente a la máxima y mínima deformación característica. Al igual que en los casos anteriores, para cada una de las fases estudiadas se ha analizado el nudo extremo de la dovela tesada, tomándose esos valores como referencia para la obtención de las deformadas que se incluyen a continuación (Figura 188 a Figura 191).

En concreto se han elegido 4 fases del proceso correspondientes a la instalación de 2 cables simétricos cortos (cables 7 Izquierda y 7 Derecha) y a la colocación de los 2 cables simétricos más largos (cables 18 Izquierda y 18 Derecha). Se pretende también así poder valorar qué cuantía de deformaciones verticales suponen los errores aleatorios en las fases iniciales, cuando la deformabilidad del tablero no es aun elevada, y en las fases finales, cuando el tablero será muy flexible y por lo tanto sensible a la aplicación de estas fuerzas erróneas.

Los valores representados lo han sido analizando una sola de las muestras generadas anteriormente.

De esta forma, las fases analizadas y los nudos correspondientes utilizados como referencia de los resultados son:

- Fase 24 - Tesado del cable 7 Izquierdo. Nudo de referencia 39.

- Fase 26 - Tesado del cable 7 Derecho. Nudo de referencia 38.

- Fase 68 - Tesado del cable 18 Izquierdo. Nudo de referencia 95.

- Fase 70 - Tesado del cable 18 Derecho. Nudo de referencia 91.

Se pasa a continuación a presentar estos resultados: 


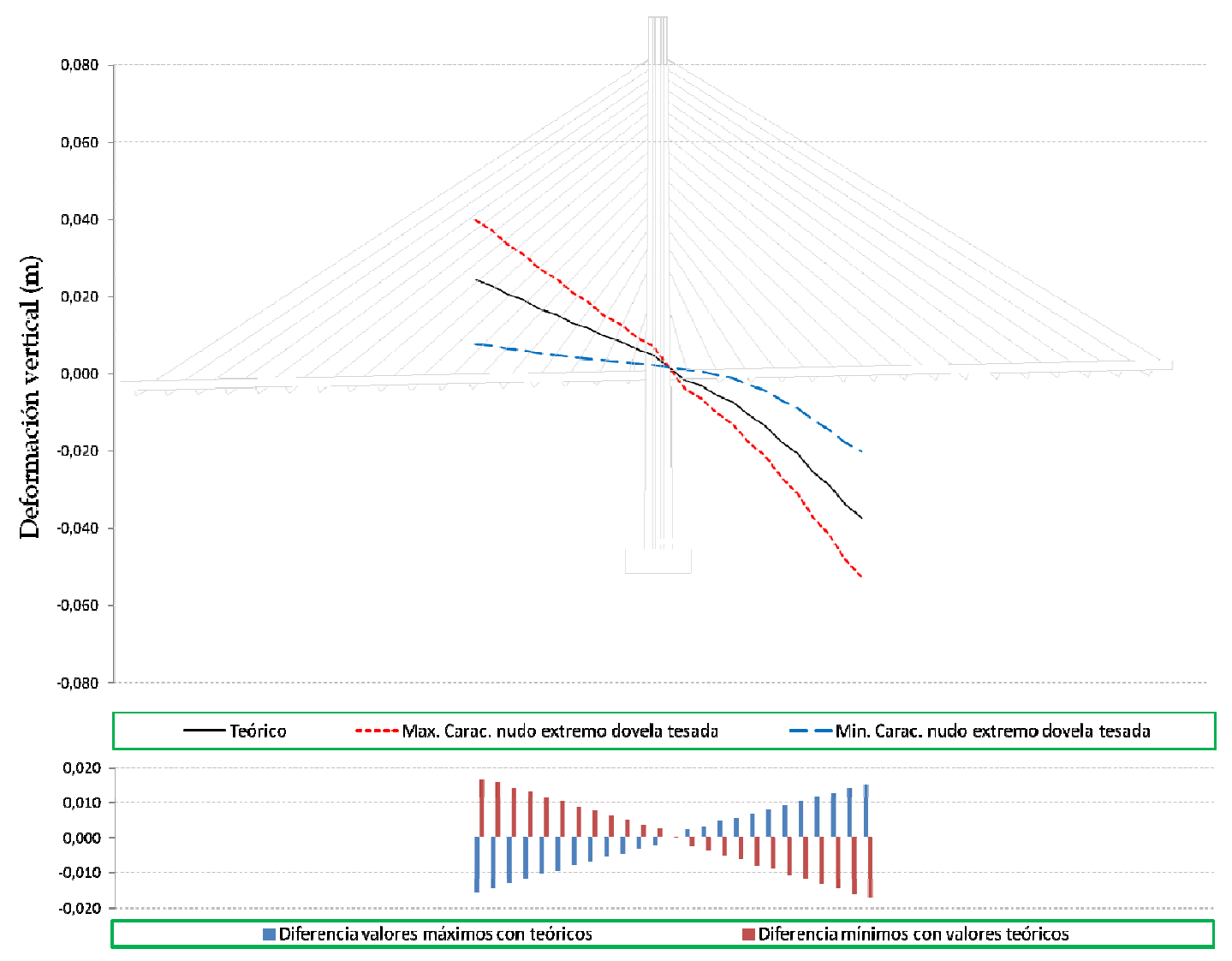

Figura 188. Deformaciones verticales del tablero para el caso de la fase 24 del proceso constructivo. Resultados correspondientes a la deformada obtenida a partir del valor característico del nudo 39 extremo de dovela tesada con el tirante 7 Izquierdo. 


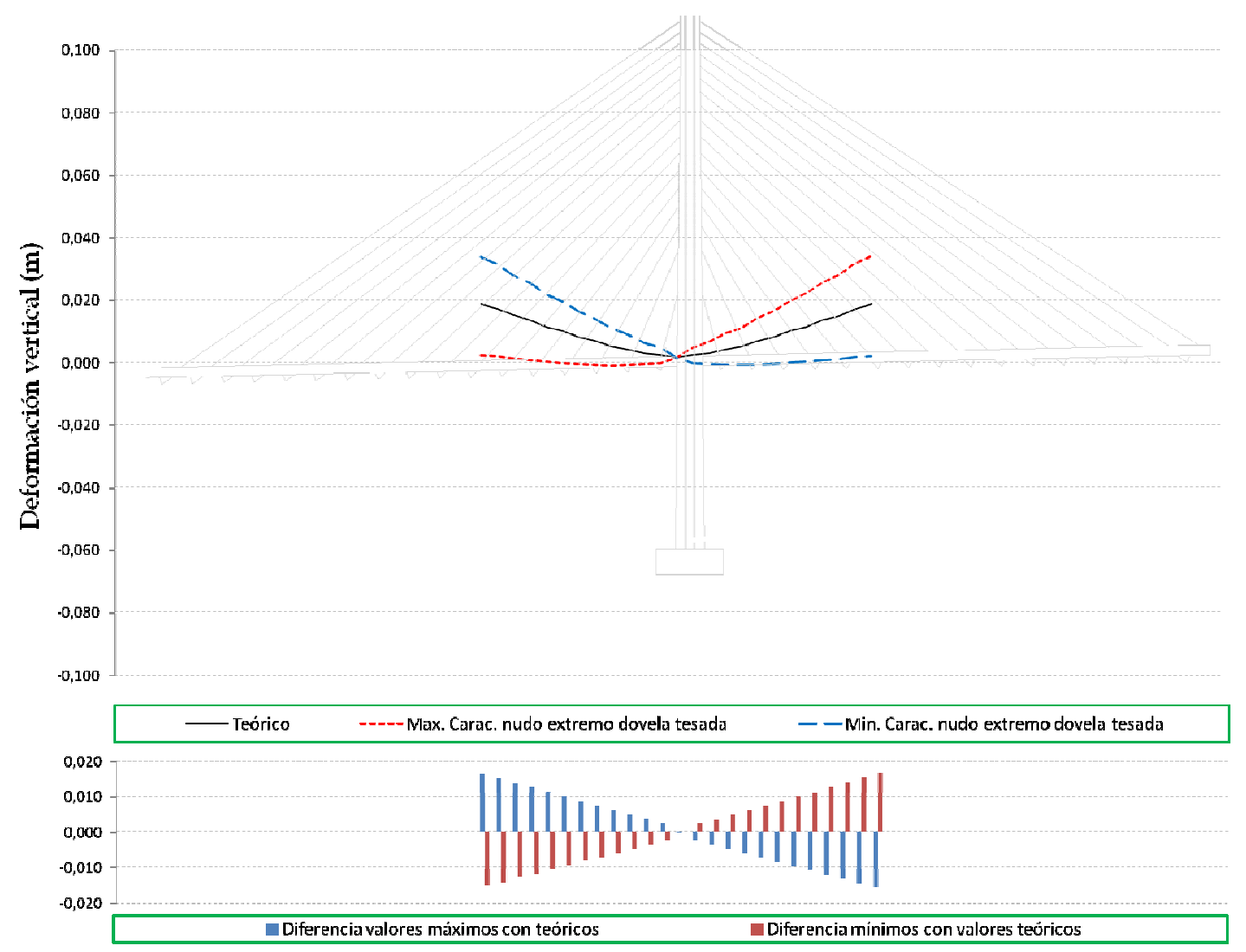

Figura 189. Deformaciones verticales del tablero para el caso de la fase 26 del proceso constructivo. Resultados correspondientes a la deformada obtenida a partir del valor característico del nudo 38 extremo de dovela tesada con el tirante 7 Derecho. 


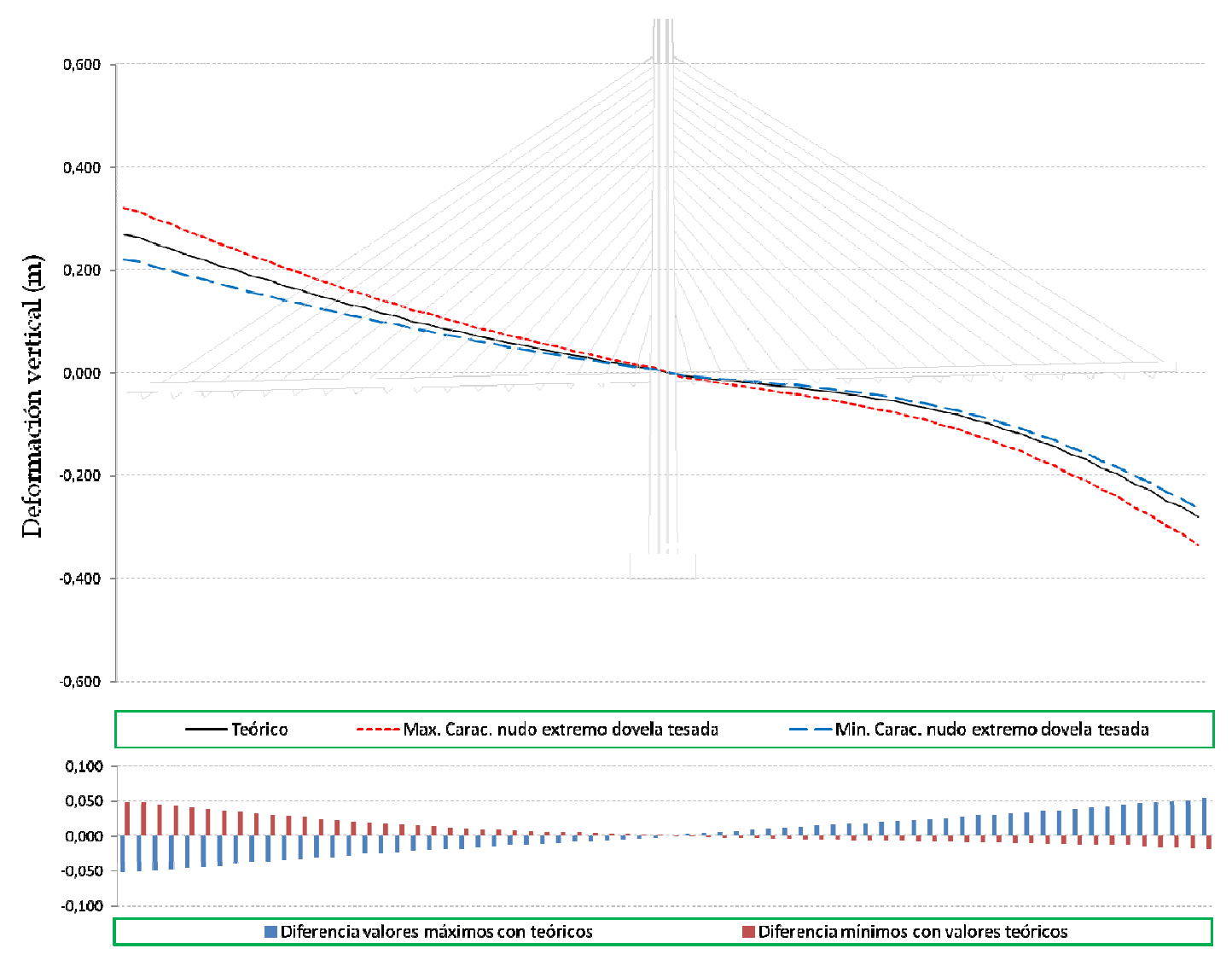

Figura 190. Deformaciones verticales del tablero para el caso de la fase 68 del proceso constructivo. Resultados correspondientes a la deformada obtenida a partir del valor característico del nudo 95 extremo de dovela tesada con el tirante 18 Izquierdo. 


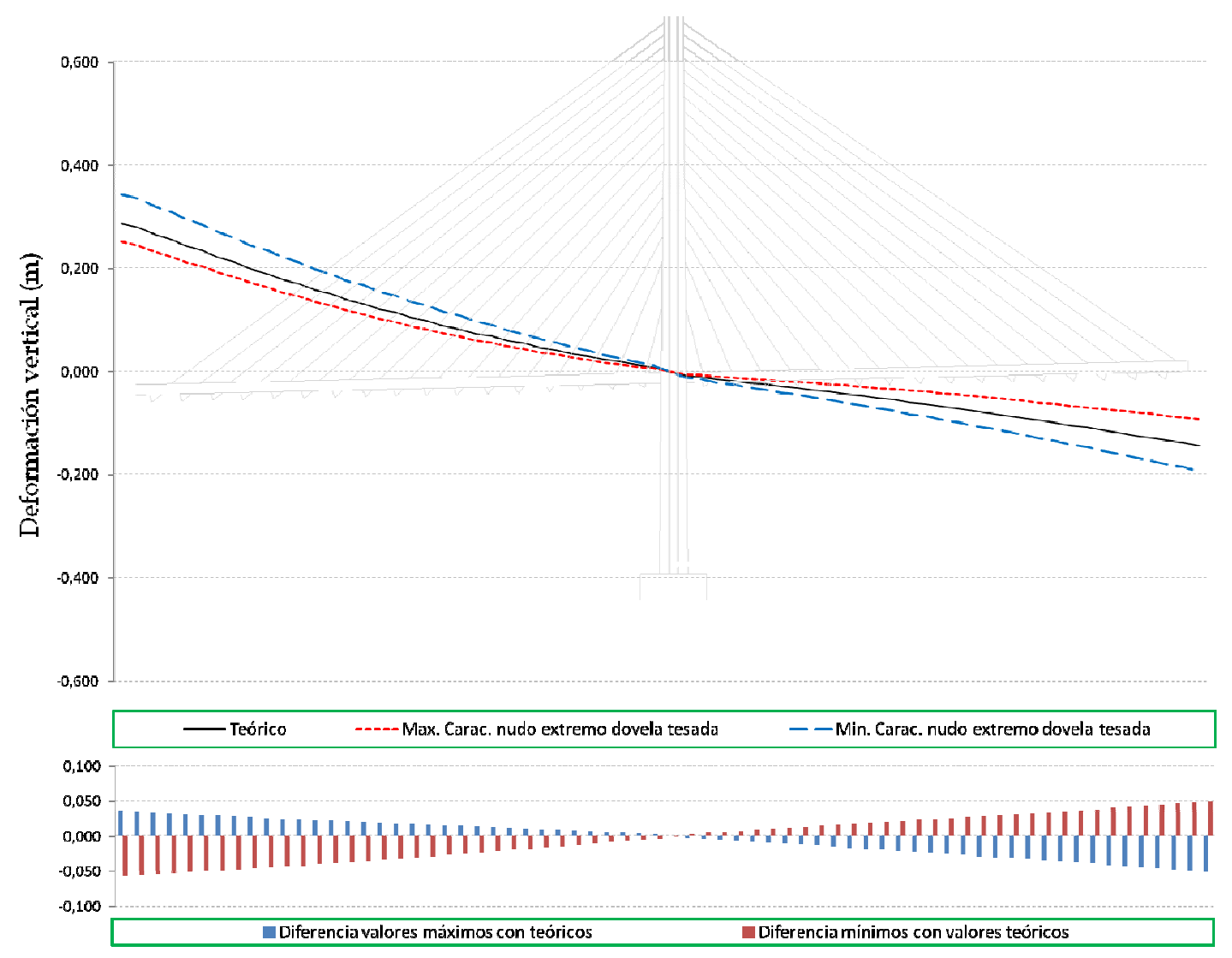

Figura 191. Deformaciones verticales del tablero para el caso de la fase 70 del proceso constructivo. Resultados correspondientes a la deformada obtenida a partir del valor característico del nudo 91 extremo de dovela tesada con el tirante 18 Derecho.

De todos los resultados reflejados en las gráficas de deformadas anteriores se puede concluir que:

- $\quad$ Se sigue observando en estos casos una rotación del tablero alrededor del punto de apoyo del tablero en el pilono como sólido rígido.

- Al igual que en el Modelo 90, las deformaciones en las primeras fases del proceso de construcción del tablero, al ser de cuantía relativamente reducida difícilmente pueden ayudar a detectar la presencia de errores en el proceso constructivo, más aun cuando lo que se analizaría sería la diferencia con los valores teóricos, diferencia que sería la que alertaría de la desviación respecto de la situación correcta de dicho proceso. Esos diferencias en las primeras fases quedan en valores del orden de menos de $2^{\prime} 0 \mathrm{~cm}$, 
valores en los que será muy difícil distinguir que parte es debida a la variación de temperatura, que parte a la modificación del módulo de deformación del hormigón respecto del valor teórico y que parte realmente al proceso erróneo de montaje de los cables.

- En estas primeras fases las deformaciones observadas son incluso menores que en el caso del Modelo 90, lo cual es lógico al haber aumentado la inercia del tablero, y en consecuencia por lo tanto la rigidez de éste.

- Que en las fases finales las deformaciones alcanzadas llegan a ser del orden de 20-30 $\mathrm{cm}$, algo menores también que en el Modelo 90, y las diferencias con los valores teóricos del orden de $5 \mathrm{~cm}$. Con este rango de deformaciones sí empezaría a ser posible realizar el seguimiento del proceso constructivo sin excesivas dificultades.

En definitiva, se puede concluir de nuevo que, el seguimiento del proceso constructivo mediante el control topográfico de la rasante del tablero implicará la necesidad de una precisión mucho más alta para las fases iniciales que para las fases finales. Incluso podría decirse que, en el caso de tableros relativamente rígidos, como el caso analizado del Modelo 90-51, con dicho seguimiento resultará muy alcanzar el objetivo de detectar errores en el tesado de los cables. 


\section{CAPITULO 8.}

\section{FORMATOS DE SEGURIDAD.}

\subsection{INTRODUCCION.}

Se desarrolla en este capítulo el otro objetivo que se ha buscado lograr con esta investigación, que como se indicó es el estudio de los formatos de seguridad a aplicar a los esfuerzos axiles existentes en los cables de un puente atirantado.

Tal y como ya se adelantó, el axil de un tirante en la situación final con el puente en servicio estará conformado por dos factores: uno primero dependiente de la fuerza aplicada al cable en el momento de su tesado (axil activo) y otro dependiente de la actuación de dicho cable como elemento pasivo de la estructura (axil pasivo), y por lo tanto dependiente del resto de cargas aplicadas sobre la estructura (pesos propios y cargas muertas).

La idea a desarrollar en este capítulo es entonces valorar en qué medida se pueden aplicar los formatos de seguridad al esfuerzo del tirante de forma global o de forma diferenciada para cada uno de los términos que lo componen.

Como complemento para el anterior análisis disponemos de los resultados obtenidos en los capítulos anteriores, donde se ha desarrollado un estudio completo de la influencia de la aleatoriedad que se genera sobre los axiles de montaje de los cables al realizar una puesta en carga de los tirantes no acorde con el valor teórico, siendo por lo tanto introducido en este punto la aleatoriedad en los cálculos.

De esta forma, con los resultados anteriores contamos con una cuantificación de en qué medida el efecto de un axil o deformación de montaje de los cables afecta al conjunto de la estructura, habiéndose realizado dicho estudio para distintas estructuras, de tal manera que esa cuantificación se pueda poner en relación a los parámetros diferenciadores de las estructuras analizadas.

Por otra parte, en los capítulos anteriores también se ha ido desarrollando un estudio, en este caso sobre el cálculo teórico, para conocer en que en medida se puede valorar la parte 
activa y pasiva del axil de un cable, comparándose dichos resultados con los obtenidos en el estudio paramétrico incluido en el apartado 3.3.

Igualmente el control geométrico del tablero estará en conjunción con este análisis, ya que es este mismo control el que introducirá un cierto nivel de seguridad sobre la estructura, ya que los puentes atirantados cuentan con su elevada flexibilidad como factor que permite la evaluación de las acciones actuantes sobre la estructura de forma indirecta mediante el seguimiento de las deformaciones verticales del tablero.

Conocidos estos aspectos y valorando las disposiciones que se indican hoy en día en las distintas normativas de referencia se puede proponer un tratamiento específico, como conclusión de todo el análisis anterior, para el caso de puentes atirantados con tablero de hormigón, luces pequeñas o medias y ejecutados mediante voladizos desde el pilono.

De la misma forma se pretenderá analizar las implicaciones a nivel de seguridad en rotura, y de control geométrico en servicio del axil realmente aplicado en los tirantes. Este análisis se deberá realizar incluyendo todas las fases de construcción ya que, como ya se ha señalado a lo largo de este documento, es en las fases de construcción donde se pueden producir los esfuerzos dimensionantes.

\subsection{EFECTO DE LA PUESTA EN CARGA ERRONEA DE UN TIRANTE.}

De los capítulos anteriores se puede deducir que, para todos los casos analizados, que la variabilidad, o el proceso erróneo de puesta en carga de un tirante, tiene un efecto sobre los propios axiles de los tirantes que se puede resumir en la Tabla 9, en la Figura 192 y en la Figura 193. Estas han sido elaboradas para el caso de introducción de errores en fuerzas, aunque de igual forma podría plantearse para el caso de la introducción de errores en deformaciones, obteniéndose resultados muy similares como se ha visto en los capítulos anteriores.

De estos datos se deduce que:

$\checkmark \quad$ La cuantía de error no es homogénea en todos los cables de una estructura para cada situación en concreto calculada, siendo mayor en los cables más cortos, es decir en los que se colocan en primer lugar..

$\checkmark \quad$ Que la cuantía del error medio queda en el intervalo del 5 al $7 \%$ respecto del axil teórico, salvo en el caso del Modelo 180, donde claramente las desviaciones son mayores, lo que implica que la luz de la estructura tendrá influencia en la cuantía de los errores finalmente introducidos por los cables. 


\begin{tabular}{|c|c|c|c|c|c|c|c|}
\hline \multicolumn{8}{|c|}{ Desviación máxima respecto del valor teórico del axil del tirante } \\
\hline \multirow{3}{*}{ Estructura } & \multirow{3}{*}{ Cable } & \multicolumn{6}{|c|}{ Situación } \\
\hline & & \multicolumn{2}{|c|}{ Final } & \multicolumn{2}{|c|}{ Construcción } & \multicolumn{2}{|c|}{ Servicio } \\
\hline & & Carac. sup. & Carac. inf. & Carac. sup. & Carac.inf. & Carac. sup. & Carac.inf. \\
\hline \multirow{6}{*}{ MODELO 90} & $3 \mathrm{D}$ & 6.7 & 7.8 & 3.1 & 8.8 & 5.7 & 8.4 \\
\hline & 31 & 5.6 & 4.8 & 2.6 & 4.7 & 4.7 & 5.0 \\
\hline & $8 \mathrm{D}$ & 5.6 & 5.6 & 3.0 & 6.6 & 4.6 & 5.7 \\
\hline & 81 & 6.1 & 6.4 & 3.0 & 6.4 & 5.0 & 5.6 \\
\hline & $18 \mathrm{D}$ & 4.1 & 4.2 & 4.1 & 4.5 & 3.5 & 4.8 \\
\hline & 181 & 3.7 & 3.7 & 3.7 & 4.7 & 3.4 & 4.1 \\
\hline \multirow{6}{*}{ MODELO 90M } & $2 \mathrm{D}$ & 6.1 & 7.4 & 1.9 & 4.7 & 5.1 & 7.8 \\
\hline & 21 & 5.0 & 5.8 & 1.9 & 4.7 & 4.1 & 6.1 \\
\hline & $8 \mathrm{D}$ & 6.4 & 6.1 & 2.2 & 4.7 & 5.2 & 6.2 \\
\hline & 81 & 5.8 & 5.2 & 2.2 & 4.8 & 4.7 & 5.3 \\
\hline & $18 \mathrm{D}$ & 4.1 & 4.1 & 4.2 & 4.5 & 3.6 & 4.8 \\
\hline & 181 & 3.6 & 3.5 & 3.6 & 4.6 & 3.2 & 3.8 \\
\hline \multirow{6}{*}{ MODELO 180} & $3 \mathrm{D}$ & 8.8 & 7.9 & 2.2 & 9.6 & 7.3 & 8.3 \\
\hline & 31 & 8.1 & 6.5 & 2.0 & 4.6 & 6.7 & 6.9 \\
\hline & $8 \mathrm{D}$ & 6.3 & 6.5 & 2.5 & 8.3 & 5.3 & 6.6 \\
\hline & 81 & 6.1 & 6.8 & 2.5 & 8.0 & 5.1 & 6.8 \\
\hline & $18 \mathrm{D}$ & 3.8 & 4.3 & 3.8 & 4.6 & 3.0 & 5.7 \\
\hline & 181 & 3.4 & 3.1 & 3.4 & 4.3 & 3.0 & 3.5 \\
\hline \multirow{6}{*}{ MODELO 90-5I } & $3 \mathrm{D}$ & 5.6 & 7.6 & 3.5 & 8.2 & 4.9 & 8.3 \\
\hline & 31 & 5.6 & 4.9 & 4.4 & 4.7 & 4.9 & 5.3 \\
\hline & $8 \mathrm{D}$ & 5.3 & 5.4 & 3.5 & 6.4 & 4.5 & 5.5 \\
\hline & 81 & 5.1 & 5.1 & 3.6 & 4.9 & 4.4 & 5.2 \\
\hline & $18 \mathrm{D}$ & 4.1 & 4.1 & 4.1 & 4.4 & 3.8 & 4.3 \\
\hline & 181 & 3.5 & 3.8 & 3.5 & 4.6 & 3.3 & 1 \\
\hline
\end{tabular}

Tabla 9. Desviación del axil característico (valor medio de los valores característicos obtenidos en las 3 muestras calculadas) en \% respecto del axil teórico.

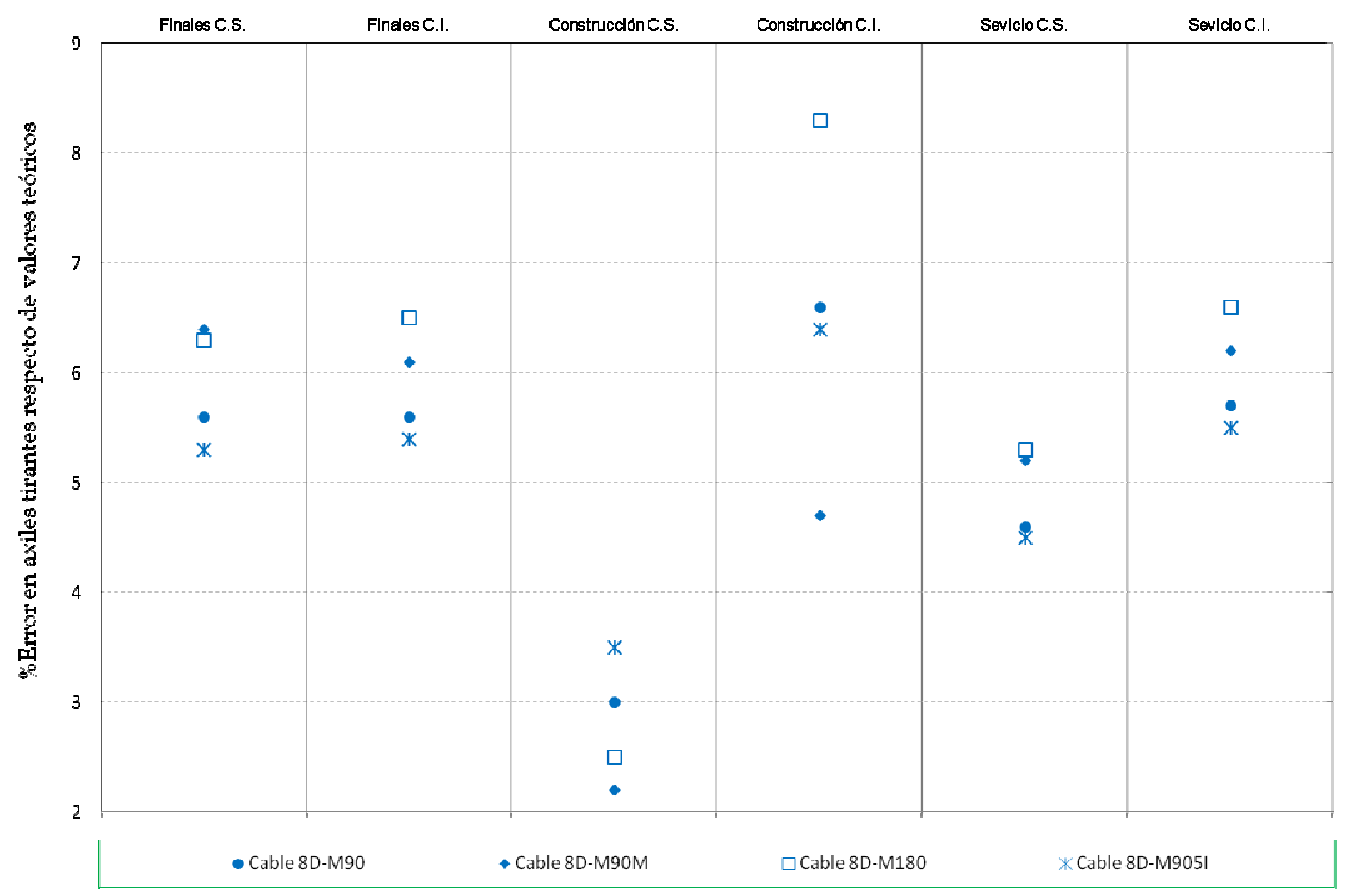

Figura 192. Porcentaje de error (valor medio de las 3 muestras calculadas de items aleatorios) respecto de los valores teóricos de los axiles. Modelos 90, 90M, 180 y 90-5I. Cable 8D. 


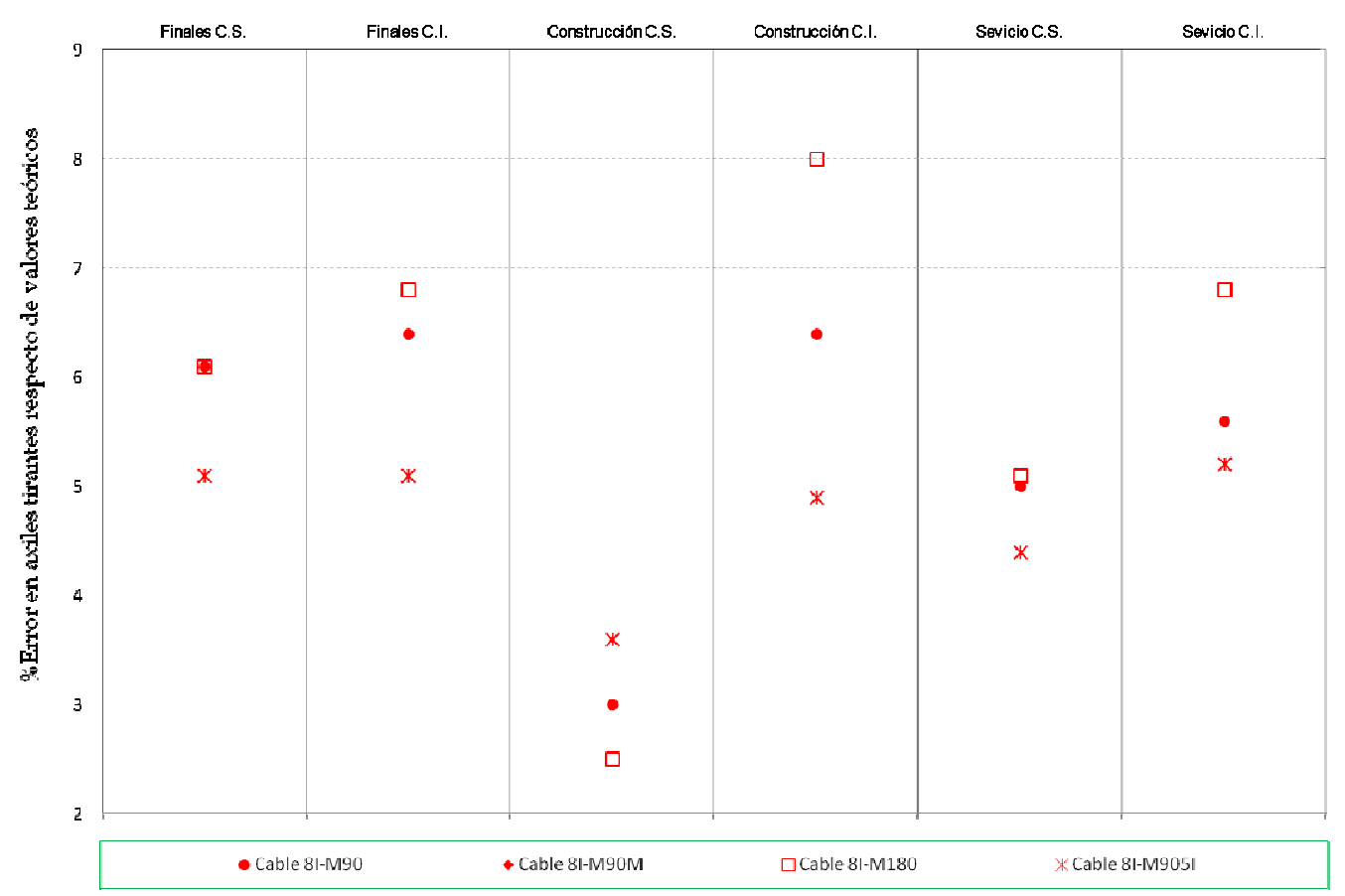

Figura 193. Porcentaje de error (valor medio de las 3 muestras calculadas de items aleatorios) respecto de los valores teóricos de los axiles. Modelos 90, 90M, 180 y 90-5I. Cable 81.

En conclusión se podría señalar que la propia arbitrariedad en el axil de los tirantes, debida a los procesos erróneos de ejecución, tienen en general una repercusión relativamente acotada siempre que se realice un control en obra que permita limitar el valor de los errores introducidos. Pero también hay que señalar que dicha repercusión será más importante en los cables iniciales y en las estructuras de mayor luz, luego el control geométrico deberá tener unas tolerancias menores en estos casos para conseguir que cumpla su función de acotación de las desviaciones respecto del proceso teórico de construcción.

\subsection{EFECTO DE LA VARIABILIDAD DE LAS CARGAS PERMANENTES.}

Cualquier desviación, respecto del valor teórico calculado inicialmente, de las cargas permanentes que actúan sobre la estructura producirá unos efectos similares a los que se han analizado respecto del axil de montaje de los tirantes. Así, a nivel de proyecto, en función del rango de variación estimado, se establecerán unos coeficientes de seguridad, función del nivel de fiabilidad o probabilidad de fallo exigida a la estructura. Estos coeficientes de seguridad deberán aplicarse para realizar las comprobaciones en servicio y últimas correspondientes. 
Es aquí importante distinguir un aspecto que puede ser definitorio en adelante, como es el momento en que se produce la variación de la carga en relación al instante de la puesta en carga de los tirantes.

En este sentido, se puede pensar en analizar el efecto de la variación de los pesos propios respecto de los teóricos previstos, como por ejemplo ocurrió en el Viaducto sobre el río Iregua [78], donde se presentaron desviaciones en los pesos propios del tablero de hasta un $12 \%$. En aquel caso hay que señalar que el tipo de ejecución planteada para ese elemento, mediante su cimbrado completo sobre apoyos provisionales, llevó a que esta desviación en las cargas sólo pudiera ser detectada en el momento en que se pusieron en carga los tirantes, por lo que la desviación tuvo que ser asumida íntegramente por un ajuste del axil a aplicar a los cables.

Este incremento se considerará cubierto, a nivel de las verificaciones en rotura, por los coeficientes de seguridad a aplicar a las cargas permanentes según la normativa vigente [51], [52] y [33], pero tendrá sus implicaciones a nivel de las verificaciones en servicio y del control geométrico de la estructura que deberán ajustarse durante la propia ejecución, como se hizo en aquel caso.

Si la ejecución del tablero mediante dovelas es simultánea a la puesta en carga de los tirantes, como en los casos estudiados en esta investigación, dichos errores en los volúmenes de hormigón deberían ser detectados y por lo tanto corregidos o ajustados durante la propia ejecución, siendo razonable pensar que debería eliminarse, o por lo menos reducirse, la causa de este error. Es decir, que la ejecución por avance en voladizo implica un control de los pesos aplicados simultáneo a la colocación de los tirantes que no se dispone si el tablero se ejecuta sobre apoyos provisionales. Esta cuestión nos llevaría a plantear este aspecto, en relación a la variabilidad de las cargas de peso propio, de forma diferenciada dependiendo del método constructivo empleado.

Podría ocurrir en el caso de la ejecución del tablero por dovelas, siendo éstas ejecutadas a su vez en varias fases, que las variaciones en los pesos de las fases finales de ejecución se produjera cuando los cables ya han sido instalados, con lo que en este caso el axil de los tirantes se vería incrementado en esa proporción. Por otra parte, ante esta situación la configuración geométrica del tablero delatará su discrepancia con la solución teórica

En el caso de las cargas muertas su actuación siempre será posterior a la aplicación de las fuerzas de tesado sobre los tirantes, por lo que su variabilidad si deberá ser asumida por los cables, pero también es cierto que normalmente el valor de las cargas muertas es sensiblemente inferior al de los pesos propios en este tipo de estructuras y más en concreto en el caso de puentes para tráfico no ferroviario. 
Por último hay que comentar que en los casos en que el incremento del peso propio se produce después del tesado podrá ser necesario realizar un proceso de retesado de los cables, con lo que el sistema proyectado deberá contar con esa capacidad.

\subsection{CUANTIFICACION DE LOS FACTORES DEL AXIL DE UN TIRANTE.}

A partir de los estudios desarrollados en los capítulos anteriores se pueden llegar a conocer para los distintos casos estudiados los porcentajes de axil pasivo (dependiente de las cargas permanentes) y axil activo que se han obtenido en cada caso calculado. Todos estos valores se resumen en la Tabla 10.

\begin{tabular}{|c|r|r|r|r|}
\hline \multicolumn{5}{|c|}{ \%Axil pasivo respecto del axil final del tirante } \\
\hline Cable & Modelo 90 & Modelo 90M & Modelo 180 & Modelo 90-5I \\
\hline $4 \mathrm{D}$ & 7.0 & 23.0 & 24.0 & 0.0 \\
\hline $4 \mathrm{I}$ & 25.0 & 61.0 & 28.0 & 32.0 \\
\hline $8 \mathrm{D}$ & 13.0 & 29.0 & 25.0 & 8.0 \\
\hline 81 & 13.0 & 29.0 & 25.0 & 11.0 \\
\hline $18 \mathrm{D}$ & 9.0 & 9.0 & 8.0 & 9.0 \\
\hline 181 & 21.0 & 24.0 & 28.0 & 17.0 \\
\hline
\end{tabular}

Tabla 10. Porcentaje del axil pasivo respecto del axil final en los cables. Modelos 90, 90M, 180 y 90-5I.

De estos valores se pueden deducir varias cuestiones:

$\checkmark \quad$ En primer lugar que el porcentaje de axil pasivo es constante en los cables centrales pero presenta singularidades importantes en el caso de los cables extremos.

$\checkmark \quad$ En segundo lugar que a mayor rigidez del tablero menor será la componente pasiva de los tirantes, luego este aspecto deberá considerarse en adelante.

$\checkmark \quad$ Por último que, salvo en algún caso particular, los axiles pasivos no representan más allá de un $30 \%$ respecto del axil final del tirante.

$\checkmark \quad$ Que en los cables de retenida (18D) el porcentaje de axil pasivo es muy inferior al resto de casos evaluados.

Hay que indicar que estos valores se refieren al caso del axil pasivo respecto del axil final, pero en las situaciones de construcción, donde se alcanzará el mayor valor del axil calculado cuando se instale la dovela adyacente, los porcentajes de axil pasivo serán muy superiores, dado que el efecto del peso de este elemento es directo sobre el cable. De esta última 
consideración derivaría entonces que el porcentaje de axil pasivo de un cable en concreto, de una estructura determinada será a su vez variable en función de la fase del proceso constructivo que se analice.

\subsection{CONTROL GEOMETRICO DE LA EJECUCION.}

El principal método para la corrección de las desviaciones, producidas durante el proceso de construcción del tablero y de montaje de los cables, será la realización de un control geométrico de las deformaciones verticales del tablero, basándonos en la flexibilidad de este tipo de estructuras frente a la aplicación de cargas, sobre todo en las fases avanzadas del proceso constructivo.

Hay que tener en cuenta que la corrección geométrica de la rasante de un tablero en la situación final es, en la mayoría de los casos muy difícil, de lograr, aunque se realice un retesado final de los cables, por lo que la corrección geométrica de la rasante del tablero deberá realizarse durante el proceso constructivo, encajando la geometría del tablero respecto de los valores teóricos, bien mediante el ajuste de la posición del carro de avance a la geometría de la última dovela ejecutada, si las desviaciones detectadas no son muy elevadas, bien mediante el recálculo de los axiles a aplicar a los cables utilizando los resultados en deformaciones que la monitorización del tablero estaría aportando.

El control geométrico tal y como propone el SETRA[102] se deberá realizar, sobre todo en el caso de tableros de hormigón, utilizando una metodología que permita evitar las irregularidades de la superficie del tablero. Por este motivo se propone la definición de una línea de referencia asociada a las dovelas ya ejecutadas, de tal forma que conocido el valor de las desviaciones de la superficie del tablero se pueda calcular la variación de flecha producida en relación a la referencia absoluta que define la rasante definitiva del puente. Esta cuestión queda reflejada en el siguiente esquema tomado de la publicación de Marchetti y Lecinq [73].

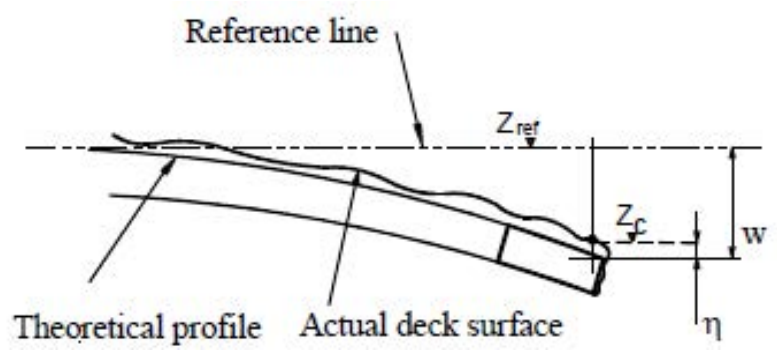

Figura 194. Procedimiento de control geométrico utilizando una referencia absoluta y otra relativa a las dovelas ejecutadas [73]. 
Hay que señalar que, en cualquier caso, en relación al control de la geometría del tablero será muy importante distinguir el origen de las desviaciones de las deformaciones verticales, que se estén produciendo sobre el tablero, respecto de lo teóricamente calculado. Esas desviaciones podrán estar originadas por la actuación tanto de una carga térmica como por alguna carga provisional no contemplada originalmente, como a una mala evaluación de las cargas permanentes o incluso a una incorrecta determinación del módulo de deformación del hormigón.

Teóricamente si los cables se instalan utilizando el método de las deformaciones, basado en la definición de la longitud en vacío de los cables, tanto los efectos de la temperatura ambiente, como de los gradientes térmicos o de las cargas provisionales aplicadas en la estructura se podrían evitar, al ser independiente de estos factores el parámetro utilizado para la puesta en tensión de los cables. Pero también como ya se ha comentado, este método exige como contrapartida un control geométrico muy preciso de la posición de los anclajes de los cables, aspecto que hace de muy difícil aplicación este método en el caso de los puentes con tablero de hormigón.

Por este motivo, dado que lo más habitual en puentes con tablero de hormigón será la aplicación de fuerzas de tesado al colocar los cables, será imprescindible, antes de proceder a realizar ningún tipo de corrección, conocer el origen de las desviaciones detectadas.

Una forma de evitar este inconveniente será utilizar el concepto ya comentado en 2.3.2 del uso de la denominada tensión de referencia, valor intrínseco a la estructura que nos permite conocida la posición relativa de los anclajes de los tirantes en una fase respecto de la teórica de partida evitar la influencia de la presencia de cargas no consideradas en los cálculos y los efectos de la temperatura. Este método de control nos permitirá además poder evitar el cálculo de todas las fases anteriores del proceso y calcular sólo la fase a analizar con el consiguiente ahorro de tiempo de cálculo. Es decir, que este método de control será muy adecuado para su desarrollo como asistencia al proceso constructivo y como complemento al control geométrico del tablero.

El otro aspecto que dificultará el control geométrico del tablero será la flexibilidad de la estructura, es decir el orden de magnitud de las deformaciones a producirse. Está claro que en puentes de grandes luces, en las fases avanzadas de construcción, las deformaciones verticales a producirse en el tablero serán muy altas y por lo tanto las diferencias que se puedan producir respecto del comportamiento teórico serán fácilmente medibles y detectables. Pero el problema puede aparecer cuando la estructura no sea tan flexible, como puede ocurrir en el caso de puentes de menores luces o bien en el caso de las fases iniciales del proceso constructivo. En estos casos el control geométrico deberá ser mucho más 
exigente para alcanzar la misma precisión que en los casos de estructuras flexibles. Aparentemente en estos casos parecería entonces que el problema del ajuste de rasante del tablero será menor, ya que la rigidez de la estructura limitaría las desviaciones máximas que se pudieran producir, pero por contra esta pequeña flexibilidad de la estructura en estos casos impedirá detectar desviaciones generadas por los errores en el proceso de montaje de los tirantes.

Es decir que el control geométrico deberá ser mucho más preciso en estructuras de pequeñas luces si se pretende poder detectar los axiles erróneos, o por el contrario se deberá asumir que en puentes con tablero de hormigón de luces cortas o medias los errores introducidos en el desarrollo de este estudio serán aplicados y no corregidos, por lo que en los formatos de seguridad a plantear para los axiles de pretensado de los cables se debe valorar la cuantía de dichos errores tal y como se han obtenido en los capítulos anteriores.

\subsection{ANALISIS DE LA NORMATIVA VIGENTE.}

Para proceder a valorar cómo se debe considerar los esfuerzos en los tirantes en relación a los formatos de seguridad, se procede a continuación a realizar un repaso de los planteamientos de la normativa actual.

\subsubsection{Instrucciones de acciones en puentes: IAP11 e IAPFO7.}

Según se indica en las normativas vigentes de acciones sobre puentes de carretera y ferrocarril se distingue, para el caso de las acciones de pretensado, dos casos diferentes:

- Pretensado tipo $P_{1}$ : Aquel que se materializa mediante elementos dispuestos dentro del canto de la sección, ya sea dentro de la sección de hormigón (pretensado convencional), como si se sitúa fuera de la sección de hormigón (pretensado exterior).

- Pretensado tipo $P_{2}$ : Aquel que se materializa mediante elementos situados fuera del canto de la sección, como es el caso de los tirantes. La normativa indica que su valor depende directamente del valor de las cargas permanentes, y que se debe asegurar un correcto proceso de ejecución, de tal manera que se garantice que, tanto los pesos propios, como las tensiones aplicadas en los cables, queden dentro de un rango de variación pequeño.

A partir de estas definiciones, la Normativa indica que se deberán considerar los siguientes coeficientes de seguridad para estas acciones en las verificaciones de los distintos ELU's y ELS's: 


\begin{tabular}{|c|c|c|c|c|}
\hline Coef. Seguridad $Y_{G}$ & \multicolumn{2}{|c|}{ ELU } & \multicolumn{2}{c|}{ ELS } \\
\hline Efecto & Favorable & Desfavorable & Favorable & Desfavorable \\
\hline Pretensado $P_{2}$ & 1 '00 & 1 1'35 & 1 1'00 & $1^{\prime} 00$ \\
\hline
\end{tabular}

Tabla 11. Coeficientes de seguridad propuestos en la IAP11 e IAPF07 para la acción de los tirantes.

A la vista de estos valores, se confirma que el planteamiento indicado por la normativa es el considerar las acciones debidas al pretensado $\mathrm{P}_{2}$ como directamente dependientes de las cargas permanentes y por lo tanto, se deberán ver afectados por los mismos coeficientes de seguridad utilizados para estas últimas.

Se deberán además realizar dos comprobaciones adicionales, utilizándose finalmente la más desfavorable de las tres anteriores:

- Caso con las cargas permanentes contrarrestadas mayoradas por un coeficiente de 1'05, y el pretensado por 0'95.

- Caso del pretensado mayorado por 1'05 y las cargas permanentes contrarrestadas por un coeficiente de 0'95.

Se plantea, eso si, la salvedad de que estos dos últimos no se aplicarán en los casos en los que las variaciones anteriores sean detectables por el control geométrico, como es el caso de tableros flexibles.

\subsubsection{Instrucción de hormigón estructural (EHEO8) y Código Modelo CEB-FIP (2010).}

Tanto la EHE08 [1] como el CM-2010 [26] no son estrictamente de aplicación para las estructuras estudiadas, pero en este último documento se indica que las cargas permanentes deberán definirse mediante un valor máximo y un valor mínimo, que se utilizarán en los cálculos, pero señala a su vez que si las diferencias entre ambas no son superiores al $5 \%$ se podrá adoptar en los cálculos un único valor coincidente con el valor medio. Por lo que implícitamente, al considerar las acciones de pretensado como acciones permanentes de valor no constante, este podría ser el rango de variación permitido a las tensiones. 
Esta hipótesis con lo señalado a lo largo de esta investigación en relación a la tolerancia admisible en los axiles de los tirantes, habiéndose adoptado un valor límite para la desviación a introducir respecto del valor teórico de un $5 \%$.

\subsubsection{Eurocódigos.}

Tanto el documento de "Bases de cálculo "[33] (en concreto el anexo 2 relativo a la " Aplicación a puentes "), como el documento de "Diseño de estructuras de cables de alta resistencia"[34] se hace mención al tratamiento a dar a las acciones de pretensado de los tirantes de las estructuras de cables, indicando que se deberán tratar de forma conjunta con las cargas permanentes.

Establece también una diferenciación entre las fases provisionales de montaje y las fases definitivas de cálculo, señalando diferentes coeficientes de seguridad en ambas:

\begin{tabular}{|c|c|c|c|c|c|c|}
\hline Coef. Seg. $Y_{G}$ & \multicolumn{2}{|c|}{ ELU } & \multicolumn{2}{c|}{ ELS } & \multicolumn{2}{c|}{ ELU provisional } \\
\hline Efecto & Fav. & Desfav. & Fav. & Desfav. & Fav. & Desfav. \\
\hline$C P+$ Pret. & $1^{\prime} 00$ & $1^{\prime} 35$ & $1^{\prime} 00$ & $1^{\prime} 00$ & $1^{\prime} 00$ & $1^{\prime} 20$ \\
\hline
\end{tabular}

Tabla 12. Coeficientes de seguridad propuestos en el EC-1 para la acción de los tirantes.

En base al cuadro anterior, se utilizarán los mismos coeficientes de seguridad para ambas acciones en todas las situaciones, salvo en el caso de que durante las fases provisionales el efecto del pretensado se pueda considerar como favorable, caso en el que se deberá utilizar un coeficiente $\gamma_{\mathrm{p}}$ para el pretensado de valor l'0.

\subsubsection{Manual de tirantes. ACHE 2007.}

Se señala en este documento la diferenciación entre el comportamiento activo y pasivo de los tirantes, indicando el diferente origen de cada uno, y la necesidad de evaluar ambos efectos de diferente manera debido a su distinto origen. 
Se señala además que la consideración adoptada en las normativas de acciones IAP98 ${ }^{6}$ de mayorar el pretensado por el mismo coeficiente que el de las cargas permanentes quedará del lado de la inseguridad en los casos en los que las estructuras sean relativamente rígidas.

\subsubsection{Haubans. Recommandation de la commission interministérielle de la précontrainte. SETRA 2001.}

Se indica en este documento la diferenciación entre las componentes activas y pasivas de las tensiones de los cables, así como se comenta que el control geométrico deberá ser capaz de detectar errores sistemáticos en la geometría ejecutada, que implicarán el recálculo de todo el proceso. En el caso de generación de errores geométricos puntuales señala la necesidad de corregirlo mediante el ajuste de la geometría de las fases siguientes del tablero, recomendando no corregir dichos errores mediante variaciones en las tensiones de montaje originales en los cables.

Se indica también que las tensiones de los cables no se deben desviar en más de un $2 \%$ de las teóricas, o bien un 0'01\% de la longitud total del cable si se miden deformaciones durante el proceso de tesado. También establece la necesidad de que se deben poder asumir variaciones en las cargas permanentes de hasta un $5 \%$.

Por último, en lo relativo a los formatos de seguridad empleados, remite a la aplicación de los mismos que se establecen en el Eúrocódigo señalado anteriormente.

\subsection{REVISION DE ESTOS PLANTEAMIENTOS.}

Una vez conocido el planteamiento normativo vigente en relación a los formatos de seguridad a aplicar a los axiles de los tirantes, se procede a detallar en este epígrafe los diferentes análisis de esta problemática realizadas por varios investigadores, donde podemos observar que los enfoques tienen alguna línea común sobre la que deberemos profundizar.

Según lo señalado por autores como Virlogeux [101], del Pozo [29] y Ruiz Terán [92], a partir del estudio de la puesta en carga de los tirantes en este tipo de estructuras y de la definición de axil pasivo y activo de un tirante queda claro que existe una parte del valor de las tensiones finales en los cables que depende de la magnitud de las cargas permanentes, y que por lo tanto se debería mayorar por los mismos coeficientes de seguridad empleados para el caso de estas cargas para así realizar un planteamiento formal coherente.

\footnotetext{
${ }^{6}$ Se señala en este documento la referencia a la norma IAP98 que estaba aún en vigor en el momento de redctar este documento. Hay que señalar que tanto la IAP98, cómo las vigentes IAPF07 e IAP11 realizan un planteamiento similar en relación al aspecto considerado.
} 
El problema se encuentra en el tratamiento de la parte activa de las tensiones finales en los cables, ya que llegados a este punto es donde encontraremos las discrepancias en su análisis.

Todos los autores antes indicados aceptan que el tratamiento del conjunto de las fuerzas de tesado de los cables por los mismos coeficientes de seguridad que las cargas permanentes no es correcto, y que incluso puede llevar a situaciones del lado de la inseguridad.

Tanto en [101] como en [92], se propone que sea el criterio del proyectista el que fije estos coeficientes de seguridad, indicando Ruiz-Terán que se debería aplicar un valor de 1'00 para el coeficiente $\gamma_{G}$.

En [92] se indica además, que se puede analizar el esquema de seguridad según dos casos posibles: primero que se produzca un incremento de las cargas permanentes antes del tesado de los tirantes, o que éste se produzca después del tesado.

Utilizando la terminología planteada por esta autora, se define como:

$$
\begin{aligned}
& \text { To - Carga en los tirantes en la fase de estructura terminada. } \\
& T_{a} \text { - Carga actuante en los tirantes debida a su tesado. } \\
& T_{p} \text { - Carga en los tirantes debido a la acción de las cargas permanentes. }
\end{aligned}
$$

Se tendrá así en el primer caso, con incremento de las cargas permanentes antes del tesado, que se habrá incrementado la componente pasiva de la acción de los tirantes hasta un valor de:

$$
\text { [Ec8.1.] } \quad T_{p} \cdot 1 ' 35
$$

y al mantenerse el valor de la tensión final en los tirantes To, la carga introducida en ellos se habrá quedado escasa en una proporción:

$$
\text { [Ec8.2.] } \quad \mathrm{Ta}^{\prime}=\mathrm{T}_{\mathrm{a}}-0^{\prime} 35 \cdot \mathrm{T}_{\mathrm{p}}
$$

En este caso, si se está realizando un control adecuado de la geometría del tablero, se podrá corregir este defecto en las tensiones aumentando el axil activo a aplicar a los tirantes.

En el caso en el que el incremento de las cargas permanentes se produzca después de haber tesado los tirantes, como podría ser el caso de la aplicación de una carga muerta mayor a la considerada en proyecto o bien en el caso de la ejecución de la sección transversal en dos fases, los esfuerzos en éstos resultan de valor:

$$
\text { [Ec8.3.] } \quad T_{0}=1 ' 00 \cdot T_{a}+1 ' 35 \cdot T_{p}
$$


Como la IAP11 preconiza la mayoración según los mismos coeficientes indicados para la carga permanente resultaría que las tensiones en los tirantes serían:

$$
\text { [EC8.4.] } \quad T_{o}=1^{\prime} 35 \cdot T_{a}+1,35 \cdot T_{p}
$$

Lo que supone que, cuando en los esfuerzos de cualquier parte de la estructura el efecto de la carga en los tirantes sea de signo contrario al debido a las cargas permanentes, se está del lado de la inseguridad, ya que la parte activa de la componente de los tirantes no podrá aumentar aunque lo haga el peso propio o las cargas muertas.

Es decir que Ruiz-Terán señala que el formato de seguridad propuesto por la normativa puede llevar a situaciones del lado de la inseguridad si el incremento de las acciones permanentes se produce después de haber tesado los tirantes, pero que en el caso contrario el propio control geométrico del tablero hará corregir esta situaciones.

Comentar también que Ruiz-Terán señala muy acertadamente la incoherencia que supone de multiplicar los pesos propios por un coeficiente de 1'35, cuando el propio proceso constructivo, en su caso asociado a puentes extradosados pero perfectamente aplicable al estudiado en esta investigación, implica la realización de un control geométrico del tablero que en definitiva sirve también para calibrar de forma muy ajustada los pesos del tablero.

Por otra parte del Pozo, en la referencia [29], añade que el tratamiento de las fuerzas de tesado por el mismo coeficiente de seguridad que el aplicado a las cargas permanentes, será tanto más desacertado cuanto mayor sea la rigidez del tablero, es decir cuanto mayor sea la componente activa de las tensiones de los tirantes. Por lo que indica que para puentes de luces pequeñas o medias es cuando este planteamiento llevará a mayores errores. Este autor propone finalmente la utilización de dos coeficientes de seguridad diferentes para cada componente de la tensión en los cables, indicando un formato de seguridad que se resume en la siguiente tabla:

\begin{tabular}{|c|c|c|c|c|}
\hline Coef. Seguridad $Y_{G}$ & \multicolumn{2}{|c|}{ ELU } & \multicolumn{2}{c|}{ ELS } \\
\hline Efecto & Favorable & Desfavorable & Favorable & Desfavorable \\
\hline Parte activa & $1^{\prime} 00$ & $1^{\prime} 00$ & 0 & $1^{\prime} 05$ \\
\hline Parte pasiva & $1^{\prime} 00$ & $1^{\prime} 35$ & $1^{\prime} 00$ & $1^{\prime} 00$ \\
\hline
\end{tabular}

Tabla 13. Coeficientes de seguridad propuestos por del Pozo en [29]. 
Por su parte en cambio C. Menn [77] propone mayorar, tal y como indica el Eurocódigo [33], las acciones del pretensado de los tirantes por el mismo coeficiente con el que se multipliquen las cargas permanentes, ya que señala que, de no hacerlo sí, los valores de cálculo de los esfuerzos en los diferentes elementos pueden diferir en gran medida de los planteados en las situaciones de servicio, e incluso ser de signo opuesto. Es decir que si se mayoran las cargas permanentes por un coeficiente de seguridad de 1'35 puede que los momentos de cálculo en el tablero obtenidos como:

$$
\text { [Ec8.5.] } \quad M_{d}=1^{\prime} 35 \cdot M_{G}+M p r e t
$$

resultaran incluso de signo opuesto a los obtenidos para la situación de servicio, por lo que podría resultar que se dispusiera la armadura de forma no acorde a los esfuerzos realmente actuantes sobre el tablero.

Por último hay que comentar las indicaciones realizadas por Ploch [83] en su tesis doctoral planteó que los coeficientes de seguridad a emplear para el pretensado de los cables situados fuera de la sección de hormigón (tirantes o cables de pretensado exterior) siguieran lo plasmado en la siguiente tabla:

\begin{tabular}{|c|c|c|}
\hline \multicolumn{2}{|c|}{$\begin{array}{c}\text { Coeficientes de seguridad del pretensado de los cables situados } \\
\text { fuera de la sección de hormigón }\end{array}$} \\
\hline Tipo estructura / Efecto & Favorable & Desfavorable \\
\hline Estructuras muy flexibles & $1^{\prime} 1$ & $1^{\prime} 2$ \\
\hline Resto & $1^{\prime} 0$ & $1^{\prime} 2$ \\
\hline
\end{tabular}

Tabla 14. Coeficientes de seguridad propuestos por Ploch en [83].

\subsection{CONCLUSIONES DERIVADAS DE ESTE ESTUDIO.}

A partir de lo señalado en los distintos epígrafes de este capítulo se pueden realizar las siguientes puntualizaciones

\subsubsection{Cuantificación del efecto del esfuerzo de tesado de los cables.}

De lo señalado anteriormente y de los resultados obtenidos a lo largo de esta investigación, para las estructuras con la tipología reseñada, se puede concluir que el efecto de la introducción de esfuerzos activos en los cables distintos de los teóricos tienen unas cuantías, 
en general, relativamente acotadas, por lo que el criterio de mayorar la parte activa del axil de un tirante por un coeficiente similar al del pretensado convencional es razonable.

Como particularidades a este razonamiento habrá que señalar que podrán existir cables singulares debido a la existencia de fases del proceso constructivo que necesiten analizarse de forma particular.

Igualmente hay que recordar que este planteamiento es tanto más válido cuanto mayor sea la rigidez del tablero en proporción a la del sistema de atirantamiento, por lo que conforme aumente la luz de la estructura más necesario será la reconsideración de esta cuestión.

\subsubsection{Determinación del axil pasivo y activo.}

Relativas a la determinación de las componentes activa y pasiva del axil de un tirante:

- En primer lugar, y tal y como se señaló en el epígrafe 3.3 que la componente pasiva de un tirante depende de la rigidez relativa del atirantamiento respecto de la del tablero, y a su vez esta relación depende de varios factores con distinto origen como puede ser la tensión de trabajo de los tirantes, la separación entre anclajes o la inercia del tablero. Es más esta dependencia no es lineal en ninguno de los casos y muestra en algún caso un comportamiento muy asintótico.

- Que por el motivo anterior, en los casos de estructuras de luces medias o cortas será muy difícil distinguir entre qué estructuras son flexibles y cuáles no, o en qué medida son más o menos flexibles.

- Por otra parte, si consideramos la carga aplicada en el tirante en el momento de su tesado como la parte activa del axil del tirante, en consonancia con lo que ocurre en un cable de pretensado convencional, los porcentajes del axil pasivo en los casos analizados en los epígrafes 4.3, 5.2, 6.2, y 0 varían entre un 15 y un $25 \%$ del axil en situación final. Es decir que en las estructuras analizadas la flexibilidad de la estructura no es tan alta como la que considera el formato de seguridad de la normativa, tal y como adelantaba del Pozo [29].

- Que si hiciéramos la comparación entre el axil de montaje y los máximos axiles de construcción, los porcentajes de axil pasivo se observaría que variarían a lo largo del tablero, es decir que en una misma estructura se pueden presentar tirantes con distintas componentes del axil pasivo.

- Es más, existen casos puntuales en todas las estructuras analizadas, bien por el proceso constructivo seguido, bien por ser los cables extremos de un puente ligeramente 
asimétrico, que presentan valores muy alejados del porcentaje de axil pasivo que presentan el resto de cables, como ocurre en todos los casos en el cable 18D de retenida, el cual tiene un axil final que es prácticamente igual al axil de montaje, es decir que su componente pasiva puede llegar a ser muy pequeña.

En definitiva se puede señalar que el análisis de que parte del axil de un tirante procede de la aplicación de las cargas permanentes es complicado, ya que dependerá del cable en concreto en estudio así como del juego de rigideces establecido entre el tablero y los tirantes. Es decir que cualquier consideración que se haga en relación a esta distinción de las componentes del esfuerzo de los tirantes deberá realizarse teniendo en cuenta todas las particularidades antes comentadas y no de forma general.

\subsubsection{Influencia del método de puesta en carga de los tirantes.}

Adicionalmente, respecto de las conclusiones del epígrafe anterior conviene hacer una puntualización, basada en el siguiente razonamiento:

Si distinguimos los dos casos posibles de control del proceso de tesado de los cables, control geométrico de los alargamientos de los cables o control manométrico de las tensiones aplicadas en éstos, tendríamos, para la primera alternativa, el siguiente esquema del proceso a realizar si analizamos la misma estructura representada en la figura 1, siendo entonces:
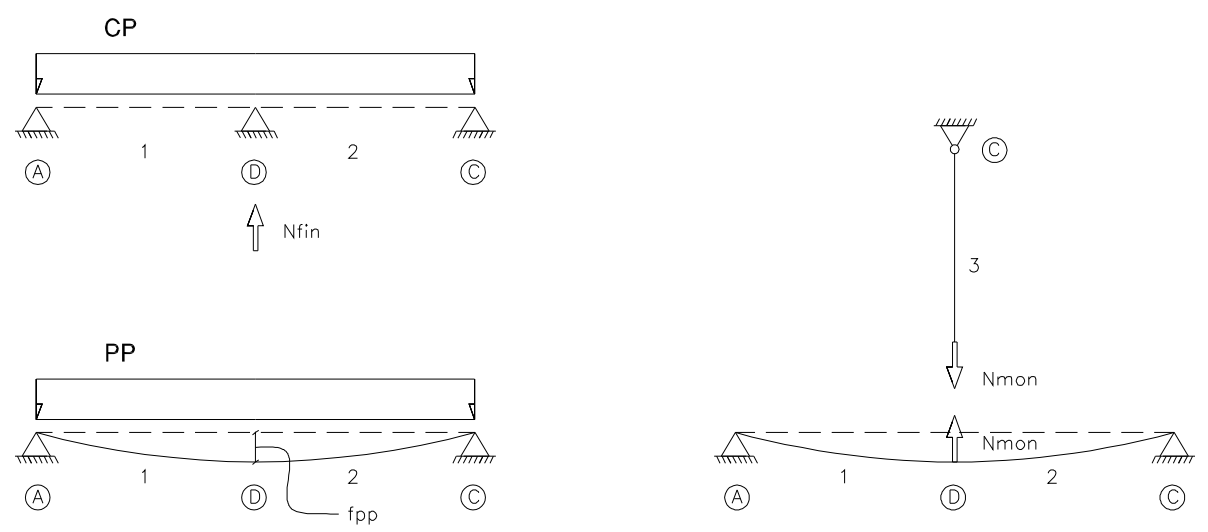

Figura 195. Método de control en deformaciones.

Con las siguientes definiciones de las variables empleadas:

$\delta_{\text {pred }} \rightarrow$ Predeformación del cable para que la reacción que introduzca sobre el tablero coincida con la correspondiente a la situación de viga continua frente a carga permanente.

$f_{p p} \rightarrow$ Flecha del tablero para el caso de la actuación del peso propio.

$\delta_{\text {tirante }} \rightarrow$ Alargamiento del tirante al aplicar el esfuerzo de montaje del tirante, $N_{m o n}$. 


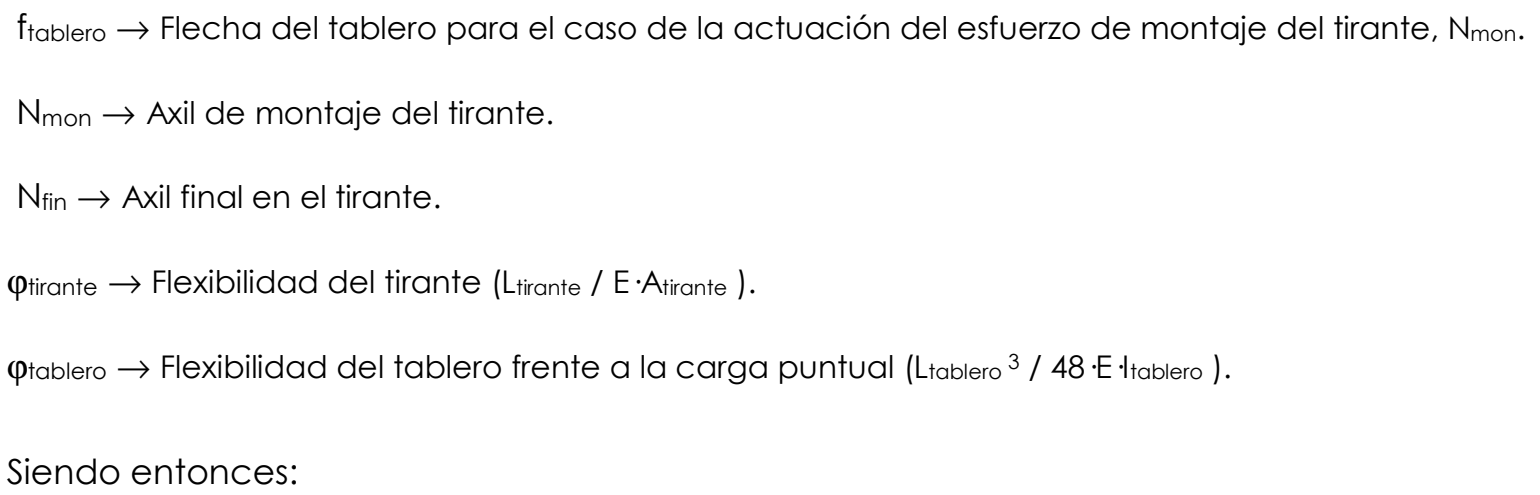

$[E C 8.6.] \quad \delta_{\text {pred }}=N_{\text {fin }} \cdot \varphi$ tirante

[Ec8.7.] $\quad \delta_{\text {tirante }}=\mathrm{N}_{\text {mon }} \cdot \varphi_{\text {tirante }}$

[Ec8.8.] $\quad f_{\text {tablero }}=\mathrm{N}_{\text {mon }} \cdot$ tablero

debiéndose verificar el cierre del hueco existente entre tablero y cable lo que significa que:

[Ec8.9.] $\quad f_{p p}+\delta_{\text {pred }}=\delta_{\text {tirante }}+f_{\text {tablero }}$

o lo que es lo mismo:

$[E c 8.10.] \quad f_{p p}+\delta_{\text {pred }}=N_{m o n} \cdot \varphi$ tirante $+N_{\text {mon }} \cdot$ Ptablero

resultando entonces que:

[EC8.11.] $\quad N_{\text {mon }}=\frac{\delta_{\text {pred }}+f_{p p}}{\varphi_{\text {tirante }}+\varphi_{\text {tablero }}}$

Se observa que en este caso de puesta en tensión del tirante por control de longitudes, si se produce un incremento en el peso propio previo al tesado, sólo uno de los dos sumandos que componen el esfuerzo del tirante se vería afectado, $\mathrm{f}_{\mathrm{pp}}$. Sería entonces necesario que, al detectar la diferencia de flechas debidas a este incremento, se tuviera la certeza de que realmente se debieran a dicha causa, y no a otras como las diferencias entre el módulo de deformación real del hormigón y el utilizado en los cálculos, el efecto de la temperatura, etc.

En ese caso, rehaciendo el cálculo, se afectaría la predeformación del cable $\delta_{\text {pred, }}$, en la proporción debida, con lo que el esfuerzo de montaje acabaría afectado en la misma proporción, tal y como propone la IAP-11.

Por otro lado, si se realiza un control en tensiones, ni siquiera se compensará un aumento del hueco generado por un aumento del peso propio, término $f_{p p}$, ya que lo que se ejecutará en obra es la introducción de una tensión de montaje $N_{\text {mon }}$ sobre la estructura, no cubriendo en este caso ninguna variación sobre el planteamiento teórico inicial. 
La decisión de variar $\delta_{\text {pred }}$ O variar $N_{\text {mon }}$ dependerá en cualquier caso del sistema utilizado en el control de la ejecución y de su precisión, por lo que esta variable influirá de manera determinante sobre la seguridad de la estructura.

Recordar de nuevo que una forma de evitar este problema en el caso de la colocación de los tirantes mediante fuerzas puede ser el uso de la tensión de referencia como parámetro intrínseco de la estructura.

\subsubsection{Validez del control geométrico del tablero.}

Tal y como se ha venido detallando anteriormente, el control geométrico del tablero permitirá realizar un seguimiento de forma indirecta del proceso de tesado de los cables e indirectamente de los niveles de esfuerzos alcanzados en la estructura.

Pero este proceso de control deberá ajustarse en su intensidad y precisión tanto en función de la flexibilidad de la estructura como en función de la fase del proceso constructivo que se esté evaluando. Es decir que el planteamiento a seguir para que el control geométrico sea realmente eficaz será el realizarlo de forma más exigente en las primeras fases del proceso constructivo de las tipologías ejecutadas por avance en voladizo y a su vez intensificarlo también en puentes de luces pequeñas.

Por otra parte, si el control geométrico llevado a cabo es el adecuado según los criterios antes señalados, el control de los pesos aplicados sobre la estructura será muy exacto y por lo tanto no tendrá sentido, o por lo menos no se ajustará a la realidad, el planteamiento de mayorar dichas acciones pensando en su variabilidad. Es decir que amplificar estos pesos, cuando han quedado perfectamente calibrados y acotados, no parece coherente.

\subsubsection{Propuesta de formato de seguridad para el caso analizado.}

En definitiva, las ideas obtenidas en este capítulo serían:

- Que la determinación de las componentes activa y pasiva de un tirante es complicada en la mayor parte de los casos, ya que dentro de una misma estructura se pueden dar componentes pasivas de diferente cuantía entre los distintos cables.

- Que en puentes de luces cortas o medias pueden resultar las componentes pasivas de pequeña cuantía en relación al axil activo.

- Que en algunos casos la consideración de la mayoración del axil de pretensado por el mismo coeficiente que las cargas permanentes puede dar lugar a situaciones del lado de la inseguridad. 
- Que el control geométrico permite una calibración muy aproximada de las cargas permanentes aplicadas sobre la estructura.

- Que el efecto de la aleatoriedad de los tirantes está relativamente acotado.

Por todo ello se concluye que, en el caso de puentes atirantados con tablero de hormigón luces medias y ejecución mediante voladizos sucesivos desde el pilono, puede ser razonable considerar la aplicación de los coeficientes de seguridad del pretensado convencional para el caso del axil de los tirantes, ya que los resultados obtenidos a lo largo de esta investigación avalarían dichos coeficientes, dado que el margen de error que se presenta en los resultados en axiles de las distintas estructuras calculadas quedan en orden del 5-7\%.

Sí habría que prestar atención especial a los cables singulares que se ha comentado anteriormente, como los que por el proceso constructivo adoptado, en nuestro caso los primeros instalados y el cable $18 \mathrm{l}$ que soporta la dovela del vano izquierdo asimétrica. 


\section{CAPITULO 9.}

\section{CONCLUSIONES Y FUTURAS LINEAS DE INVESTIGACION.}

\subsection{INTRODUCCION.}

Una vez desarrollada todo el cuerpo de la investigación, se procede en este apartado de la tesis a detallar los aspectos novedosos introducidos en este estudio, a desglosar las conclusiones particulares de cada uno de los estudios anteriores capítulos, a redactar unas conclusiones generales obtenidas a partir de los diferentes aspectos analizados, así como finalmente a proponer una serie de futuras líneas de investigación como ampliación de esta tesis.

\subsection{APORTACIONES NOVEDOSAS DE LA INVESTIGACION.}

Dentro del conjunto de esta investigación se pueden destacar como aspectos innovadores de ésta los siguientes:

- $\quad$ En primer lugar hay que reseñar el método de simulación de errores aleatorios durante la instalación de los cables, el cual aporta una forma de implementar un proceso real de instalación de los tirantes, con todas sus implicaciones respecto de la desviación del proceso teórico.

Este método además se ha optimizado para que no suponga un tiempo excesivo de cálculo pero a la vez se respeten los condicionantes impuestos inicialmente:

$\checkmark \quad$ Que los valores erróneos simulados siguen una distribución normal, al considerarse que provienen de la suma de efectos independientes unos de otros, resultando así por el "Teorema central del Límite" que la variable aleatoria que representa el efecto conjunto de todos ellos tendrá una distribución aproximadamente normal.

$\checkmark$ Que a su vez estas muestras cumplen las limitaciones de errores máximos establecidos en la normativa, es decir que se está suponiendo que por encima de 
estas limitaciones deberá disponerse en obra de un control de ejecución que permita detectar y eliminar errores de superior cuantía y errores sistemáticos.

$\checkmark \quad$ Que las desviaciones típicas adoptadas en cada caso se justifican como aquellas que producen una máxima dispersión en los resultados, compatible con alcanzar la convergencia al aplicar el algoritmo de verificación de la normalidad de las muestras de Anderson-Darling en un tiempo razonable.

$\checkmark$ Que el tamaño de las muestras es el adecuado como para dar por suficientemente aproximados los resultados, sin incurrir en un costo en tiempo de cálculo demasiado elevado.

En definitiva se puede señalar que el método implementado en esta investigación es un procedimiento original que permite la introducción en los cálculos de aquellos errores generados durante la ejecución real de un puente atirantado y que será imposible eliminar por los controles de

obra.

- Por otra parte, el estudio paramétrico desarrollado sobre diferentes puentes atirantados, y en relación a la influencia de un proceso constructivo real sobre ellos, se puede considerar un aspecto innovador, ya que aúna la comparación del comportamiento de diferentes estructuras con el estudio de los efectos aleatorios debidos a un proceso constructivo real.

Como se ha comentado en el capítulo 2, existen numerosas referencias donde se realizan estudios paramétricos de estas estructuras e igualmente también se han desarrollado varias investigaciones en relación a la aleatoriedad de ciertos parámetros de la estructura a calcular, pero estas últimas siempre lo han sido orientadas a la determinación teórica de los axiles de montaje de los cables o al análisis en fiabilidad de la estructura. Pero hay destacar que no se ha encontrado ninguna investigación donde se hayan conjugado ambos aspectos de forma conjunta para su análisis como se ha hecho en este texto.

\subsection{CONCLUSIONES PARTICULARES DEL ESTUDIO.}

Se proceden a continuación a compendiar las conclusiones de los distintos aspectos analizados en esta investigación, siguiendo el mismo orden en que se han ido mostrando en esta tesis. 


\subsubsection{ANALISIS DE LOS FACTORES QUE COMPONEN EL AXIL DEL TIRANTE.}

Del estudio paramétrico, llevado a cabo sobre el modelo simplificado con un sólo tirante, desarrollado para conocer los factores que influyen en la conformación del axil pasivo de un tirante se puede indicar:

- Que en todos los casos analizados en el epígrafe 3.3, el axil pasivo supone un porcentaje reducido del axil total de los tirantes (Ver Tabla 10 de resumen en página 248).

- Que el aumento de la separación entre anclajes genera el efecto más acusado de aumento del porcentaje de la parte pasiva respecto del axil total en el tirante de todos los casos analizados (Figura 43). Aunque separaciones del orden de 5 a $9^{\prime} 0 \mathrm{~m}$, que resultarían razonables en el caso de la ejecución del tablero mediante voladizos sucesivos desde el pilono, implicarán porcentajes de error de cuantías menores del 10\%.

- Que el aumento de la longitud del cable (Figura 44) y el aumento de la rigidez del tablero (Figura 49), conllevan una disminución del porcentaje de la parte pasiva respecto del axil total del tirante, dado que estos aspectos suponen una pérdida de eficacia del sistema de atirantamiento respecto de la rigidez flexión del tablero.

- Que el aumento de la tensión en el tirante implica un decremento de la parte pasiva del axil del tirante, dado que dicho aumento de la tensión deriva de una menor área del tirante colocado y por lo tanto una menor rigidez y eficacia del sistema de atirantamiento (Figura 48).

- Que el efecto de la variación de la carga muerta es pequeño en comparación con el del resto de parámetros analizados.

- En definitiva se puede indicar que, conforme disminuye la rigidez del tablero frente al sistema de atirantamiento, mayor es el porcentaje de axil pasivo en el cable, es decir que conforme aumenta la eficacia del atirantamiento aumenta a su vez la capacidad de absorber esfuerzos por parte del tirante después de ejecutado el tesado lesfuerzo pasivo).

Hay que recalcar además que todos los anteriores aspectos señalados se han visto corroborados por los resultados obtenidos en las diferentes estructuras calculadas a lo largo de esta investigación (ver Tabla 10). 


\subsubsection{ANALISIS DE LOS METODOS EN FUERZAS Y DEFORMACIONES.}

El primer estudio realizado sobre un modelo de puente atirantado lo ha sido para conocer qué influencia tiene el método de colocación de los cables sobre los resultados obtenidos al final del proceso constructivo.

Por este motivo, hay que señalar que en primer lugar se analizó si la repercusión de los valores límite para el error en fuerzas de tesado, establecido por las recomendaciones de ACHE[2], en comparación con el producido por el error máximo en el caso de la colocación de los cables mediante la medición de deformaciones, marcado por el SETRA[102], eran similares. De este análisis resultó que en ambos casos se llegaban a limitaciones con el mismo orden de magnitud (Ver tablas 3, 6 y 7). Sí hay que señalar que existen casos particulares de cables en los que se presentaban discrepancias en los resultados obtenidos usando ambos métodos, debido a sus especiales características y configuración, debido al proceso constructivo, como se puede observar en la Figura 67.

Por otra parte, del estudio realizado sobre la primera estructura calculada, Modelo $\mathbf{9 0}$, se puede concluir, respecto de los resultados en los tirantes que:

- Los errores en los axiles (Figura 68 a Figura 73 y Figura 79 a Figura 84) alcanzan valores relativamente acotados tanto en las situaciones de servicio como en las de construcción y finales. Ver siguiente tabla:

\begin{tabular}{|c|c|r|r|r|r|r|r|}
\hline \multirow{2}{*}{$\begin{array}{c}\text { Comparativa de la cuantía de error en } \\
\text { axiles de cables producida en función del } \\
\text { método de colocación de los tirantes }\end{array}$} & \multicolumn{2}{|c|}{ Axiles finales } & \multicolumn{2}{|c|}{ Axiles de construcción } & \multicolumn{2}{c|}{ Axiles en servicio } \\
\cline { 2 - 8 } & \multicolumn{1}{|c|}{ Máximos } & Mínimos & Máximos & Mínimos & Máximos & Mínimos \\
\hline \multirow{2}{*}{ Errores medios } & Met. F's & 5.5 & 6.0 & 3.0 & 6.5 & 4.5 & 6.0 \\
\cline { 2 - 8 } & Met. Deformac. & 3.8 & 3.6 & 2.5 & 4.5 & 3.5 & 3.8 \\
\hline \multirow{2}{*}{ Errores máximos } & Met. F's & 8.0 & 9.0 & 5.0 & 11.0 & 6.5 & 10.0 \\
\cline { 2 - 8 } & Met. Deformac. & 4.5 & 4.5 & 4.0 & 9.0 & 4.0 & 4.8 \\
\hline
\end{tabular}

Tabla 15. Comparativa de errores generados en función del método de colocación de los tirantes.

- En el caso de las envolventes de axiles en las fases de construcción, se observa que el axil máximo queda muy condicionado por el peso de las dovelas y por lo tanto, el efecto aleatorio introducido en los cables no tiene apenas significación en este caso. (Ver tabla anterior).

- Que los errores en la mayor parte de los casos estudiados son mayores en los primeros cables instalados. Esta cuestión se justifica por el hecho de que existe una cierta acumulación de errores en los tirantes ya colocados y además porque en ellos el efecto 
de la menor longitud del cable supone una mayor repercusión de las imperfecciones en la ejecución simuladas.

- Respecto del caso de instalación con errores en la posición de los anclajes se observa que existe un clarísimo aumento de la desviación de los resultados en los primeros cables colocados (Figura 90 a Figura 95). Esto implicará que, para conseguir limitar la cuantía de los errores generados por igual en toda la estructura, el control en la posición de los anclajes debe ser mucho más exigente en el caso de los cables cortos que en los cables más largos.

Por otra parte en el caso de los resultados en flectores del tablero:

- Se observa en todos los casos analizados, que existe una gran influencia en la situación final del proceso constructivo, el cual queda relativamente alejado de la situación objetivo de la viga continua (Figura 74, Figura 85 y Figura 96).

- En cambio, de cara a las envolventes que verdaderamente dimensionarían el tablero, mínimos de construcción y máximos de servicio (Figura 75 a Figura 76, Figura 86 a Figura 87), las diferencias, respecto de los valores teóricos, obtenidas con la introducción de errores de montaje resultan del orden del 5-7\% para los flectores mínimos y del orden de hasta un 20-30\% en el caso de los máximos de servicio.

Por último, en el caso de los flectores en el pilono (Figura 77 a Figura 78, Figura 88 a Figura 89) se puede indicar que:

- En primer lugar, los flectores en las barras del pilono situados por debajo del tablero no se ven afectados por los errores de montaje de los cables, dada la conexión existente entre ambos elementos.

- En las barras situadas por encima del tablero las diferencias aumentan de forma sustancial con la altura del pilono, alcanzando porcentajes en torno al $40 \%$ de error para los flectores mínimos en fases de construcción en las barras superiores, y de hasta un $20 \%$ los flectores mínimos de servicio. Estos valores tan altos en porcentaje son debidos a que los valores teóricos en dicha zona son muy pequeños.

Adicionalmente, a la vista de los resultados obtenidos utilizando tanto el método de colocación de los cables en fuerzas como en deformaciones, se puede concluir que:

- $\quad$ El método que introduce errores en fuerzas de tesado produce en general mayores desviaciones que el caso de los errores en deformaciones. 
- La introducción de errores en el replanteo de los anclajes provoca esfuerzos en los tirantes con mucha mayor desviación. Es decir, que estos errores invalidan la aplicación del método en deformaciones, salvo que se realice un control muy preciso del replanteo de dichos puntos durante todo el proceso constructivo.

Por último, del análisis de las deformaciones verticales del tablero (Figura 101 a Figura 104) producidas por los procesos erróneos de montaje de los tirantes, se puede indicar que:

- En las primeras fases del proceso constructivo será difícil detectar los errores en el montaje de los cables, mediante el seguimiento topográfico del alzado del tablero construido, ya que diferencias de pequeña cuantía con los resultados teóricos como las indicadas se pueden producir muy fácilmente por otras causas, además de por un proceso de montaje erróneo.

- En cambio en las fases más avanzadas del proceso constructivo este control geométrico puede servir para detectar los errores de ejecución. En definitiva se puede concluir que para que el seguimiento topográfico de del tablero permita detectar los errores de ejecución en la instalación de los tirantes, éste debe ser mucho más preciso en las fases iniciales que en las fases finales.

\subsubsection{ANALISIS DE LA INFLUENCIA DEL NUMERO DE CABLES}

Del análisis de la estructura Modelo 90M (Figura 110 a Figura 131) se puede concluir que:

- Que, en general, las desviaciones obtenidas son similares a las aparecidas en el Modelo 90 (Ver Tabla 9), por lo que parece que no hay acumulación de los efectos de la aleatoriedad en función del número de cables de la estructura, dado que es la propia aleatoriedad la que suaviza y compensa los errores introducidos en los diferentes cables, independientemente del número de éstos que disponga la estructura.

- $\quad$ Sí sigue siendo apreciable el efecto sobre los axiles de los cables que presentan alguna singularidad debido al proceso constructivo, siendo en este caso el efecto más acusado que en el Modelo 90, dado que los tirantes de esta estructura tienen individualmente en proporción una mayor influencia sobre el comportamiento resistente de la estructura, al ser menor su número.

- Que de nuevo en el tablero y pilono los efectos producidos por la generación de errores aleatorios, utilizando tanto el método en fuerzas como el método en deformaciones, quedan en porcentajes relativamente acotados. 


\subsubsection{ANALISIS DE LA INFLUENCIA DE LA LUZ DE LA ESTRUCTURA.}

En primer lugar sobre los axiles de los tirantes se puede indicar que:

- Los axiles finales (Figura 138 a Figura 139) alcanzan errores de hasta el 10\% respecto de los valores teóricos utilizando el método en fuerzas, produciéndose una distribución de errores con mayores cuantías en los primeros cables instalados. Si se aplica el método en deformaciones (Figura 149 a Figura 150) los errores obtenidos son menores, quedando en un orden de magnitud de menos del 4'5\%.

- Los axiles de construcción muestran de nuevo un comportamiento diferenciado de los valores máximos y mínimos. Los mínimos alcanzan valores mayores que en el resto de estructuras analizadas, con desviaciones de hasta un 16\% (Figura 141) utilizando el método en fuerzas y de hasta un $8 \%$ (Figura 152) utilizando el método en deformaciones.

- Los axiles de servicio (Figura 142 a Figura 143 y Figura 153 a Figura 154) muestran una acumulación de errores en los primeros tirantes, con valores del mismo orden que en el caso de los Modelos 90 y $90 \mathrm{M}$, de un $8 \%$ con el método en fuerzas y del $4 \%$ con el método en deformaciones.

Por otra parte en el caso de los resultados en flectores del tablero y pilono:

- En las situaciones de construcción (Figura 145, Figura 147, Figura 156 y Figura 158) las diferencias con los valores teóricos son reducidas, salvo en el caso de los flectores en el pilono al aplicar el método en fuerzas, por los motivos antes señalados.

- En las situaciones de servicio (Figura 146, Figura 148, Figura 157 y Figura 159) las diferencias son algo mayores que en construcción respecto de los valores teóricos, aunque la magnitud de estas desviaciones siguen siendo, en general, reducidas. En el caso de los mínimos de construcción, los errores más acusados se producen de nuevo en la parte superior del pilono.

Así, en este estudio se deduce de nuevo que los errores generados con el método en fuerzas son mayores que al aplicar el método en deformaciones, ya que estas diferencias entre métodos de colocación de cables son más acusadas que en los capítulos anteriores.

Del estudio en deformaciones realizado en este capítulo (Figura 160 a Figura 163) se deduce que, lógicamente, en este caso la estructura es mucho más flexible que las anteriores, motivo por el que se deduce que el control geométrico podrá más fácilmente detectar y corregir errores conforme aumenta la luz de la estructura. 


\subsubsection{ANALISIS DE LA INFLUENCIA DE LA INERCIA DEL TABLERO.}

El análisis de la comparativa de los resultados obtenidos en axiles en tirantes, aplicando el método en fuerzas en las distintas estructuras estudiadas, permite señalar las siguientes conclusiones:

- Que los errores finales en tirantes (Figura 174 y Figura 175) quedan de nuevo en valores limitados en el rango del 5-10\%.

- Que, en general en todas las situaciones analizadas de construcción y servicio (Figura 176 a Figura 179), los mayores errores se producen en los primeros cables y los menores en los últimos cables instalados, existiendo en todos los casos una cierta simetría de los resultados respecto del pilono central.

- Que los mayores errores se producen en el Modelo 180 dentro de todos los analizados (Figura 180 a Figura 182), siendo el modelo en el que se producen menores errores el Modelo 90-5I. Es decir, que las cuantías de errores en tirantes está relacionado con la rigidez y luz del tablero o, lo que es lo mismo, que a mayor capacidad del tablero para resistir flexiones menor es la cuantía de los errores generados en los tirantes.

Respecto de los resultados en el tablero:

- Que los errores generados en las fases de construcción y servicio (Figura 184 y Figura 185), alcanzan porcentajes bastante pequeños, siendo de nuevo en este caso de valor similar a lo obtenido para el Modelo 90.

y por último en el pilono (Figura 186 y Figura 187) se observa que:

- Que los flectores generados en el tramo del pilono por debajo del tablero no se ven afectados por la introducción de errores aleatorios.

- Los valores mínimos de construcción muestran un comportamiento con desviaciones claramente crecientes en porcentaje con la altura, con valores similares a los del Modelo 90.

- $\quad$ En el caso de los valores de las desviaciones para la situación de los máximos de servicio es donde se producen mayores diferencias con lo aparecido en el Modelo 90. 
Del análisis en deformaciones (Figura 188 a Figura 191) se deduce que la flexibilidad del tablero es aun más reducida que en el caso del Modelo 90, por lo que las conclusiones extraídas respecto de aquel caso incluso se ven hora acrecentadas.

\subsubsection{ANALISIS DE LOS FORMATOS DE SEGURIDAD.}

Del estudio realizado en el CAPITULO 8 en relación a los formatos de seguridad y del control geométrico de la ejecución se puede indicar que:

- Que la componente pasiva de un tirante depende de muy distintos factores y situaciones, resultando así que su determinación no es directa.

- Que en los casos de estructuras de luces medias o cortas será muy difícil distinguir que estructuras son flexibles y cuáles no, o en qué medida son más o menos flexibles con las implicaciones en el reparto de esfuerzos entre tablero y tirantes que esto conlleva.

- Que en las estructuras analizadas, la flexibilidad de éstas no es tan alta como la que considera el formato de seguridad de la normativa.

- Que el porcentaje de axil pasivo en cada cable varía a lo largo del tablero, es decir que en una misma estructura se pueden presentar tirantes con distintas porcentajes del axil pasivo. Es más, que existen casos puntuales en todas las estructuras analizadas, donde el comportamiento de algún tirante aislado difiere del resto de tirantes de la estructura.

- Que en definitiva, dados todos los anteriores factores, y dado que en algunos casos la consideración de la mayoración del axil de pretensado por el mismo coeficiente que las cargas permanentes puede dar lugar a situaciones del lado de la inseguridad, no parece adecuado el planteamiento efectuado en la normativa en relación a este aspecto.

- Se propone así para el caso de puentes atirantados con tablero de hormigón, ejecutados por voladizos desde el pilono y con luces cortas o medias aplicar a las fuerzas de los cables unos coeficientes de seguridad similares a los del pretensado convencional. 


\subsection{CONCLUSIONES GENERALES.}

Como resumen de todo lo anteriormente expuesto, se pueden deducir las siguientes conclusiones generales a todos los estudios realizados:

- De los dos métodos de colocación de tirantes, en fuerzas y en deformaciones, el método en deformaciones introduce menos desviaciones en los resultados respecto de los resultados teóricos que el método en fuerzas.

- El método en deformaciones cuenta con el inconveniente de necesitar un control geométrico de la posición de los anclajes muy riguroso, de tal forma que esos errores queden en el orden de menos de $1 \mathrm{~cm}$, por lo que su aplicación al caso de puentes atirantados con tablero de hormigón es complicada.

- El método en fuerzas por otra parte necesita por su parte de un control muy intenso de las cargas aplicadas en el momento del tesado de los cables, tanto de la posible aplicación de cargas provisionales como de los efectos de la temperatura. Una alternativa en este caso puede ser el uso del método de la tensión de referencia, el cual es especialmente adecuado para el análisis y seguimiento del proceso de ejecución de la estructura.

- Que en cualquier caso, aplicando el método en fuerzas, si se realiza un seguimiento adecuado del proceso de tesado, no admitiendo errores más allá de los límites marcados por la normativa del 5\% respecto del valor teórico, se producirán errores en los resultados obtenidos, en relación a los teóricos de referencia calculados, que no supondrán grandes desviaciones (<10\%).

- Que, eso sí, habrá que prestar una especial atención a los cables que tienen un comportamiento singular dentro del esquema general de atirantamiento de la estructura, como pueden ser los primeros cables colocados y los cables extremos y de retenida.

- Que del estudio de las estructuras analizadas, se deduce que el número de tirantes de la estructura no supone una relación directa con la cuantía de los errores generados, luego no existe un fenómeno de acumulación de errores, función de dicho número de cables.

- Que igualmente los errores generados tanto en el caso de variación de la luz del tablero como de la modificación de la inercia del tablero son de cuantía relativamente reducida, apareciendo las mayores desviaciones en el caso del tablero de mayor luz y 
las menores en el caso del tablero más rígido y luz corta. Es decir, que cuanto mayor es la capacidad del tablero, menor es el efecto de la aleatoriedad de los errores en el tesado de los tirantes.

- Que el seguimiento topográfico de la nivelación del tablero, en el caso de los puentes de mayores luces puede servir como instrumento para la detección y corrección de los errores en el proceso de montaje de los cables, pero que este aspecto es más complicado de llevar a la práctica en puentes con tableros más rígidos o en las primeras fases de construcción.

- Que el planteamiento normativo de considerar la acción del tesado de los cables como una fuerza dependiente íntegramente del valor de las cargas permanentes aplicadas, y en consecuencia su mayoración por los mismos coeficientes de seguridad que los establecidos para aquellas, puede llevar a situaciones del lado de la inseguridad en aquellos puentes atirantados con tableros más rígidos.

- Que, de los datos obtenidos de esta investigación se deduce que, los puentes atirantados con tablero de hormigón de luces cortas o medias, están lejos del planteamiento adoptado en la normativa de considerarlos como estructuras muy flexibles, y por lo tanto los axiles de los cables no son dependientes por completo de las carga permanentes tal y como se establece en dichos documentos.

- Que adicionalmente, dicho axil pasivo en una misma estructura puede presentarse con porcentajes diferentes respecto del axil total, dependiendo del cable analizado, lvego el planteamiento de mayorar de forma independiente las dos componentes del axil total de un tirante, axil pasivo y axil activo, por coeficientes de seguridad diferentes debería hacerse de forma individualizada para el caso de cada tirante en particular.

- Que la determinación del valor del axil pasivo de un tirante depende de varios factores y no sigue un comportamiento proporcional en función de la variación de los parámetros analizados como son la separación entre anclajes, la longitud del cable, la inercia del tablero, etc.

Que en definitiva, dada la dificultad para establecer a priori el valor del axil pasivo de un tirante y dadas las anteriores cuestiones en relación a los formatos de seguridad empleados para la mayoración del axil de pretensado de los cables, debería valorarse, más aún en el caso de los puentes de luces cortas o con tableros muy rígidos, la adopción de unos coeficientes de seguridad similares a los del pretensado convencional. 


\subsection{FUTURAS LINEAS DE INVESTIGACION.}

Dada las restricciones que se indicaron al comienzo de este documento que se habían planteado para reducir y limitar el alcance de este estudio, claramente se pueden, en primer lugar, indicar las siguientes líneas de ampliación de este trabajo:

- Aplicación de los procesos aleatorios al caso de puentes con tablero metálico, en los que las especiales características de éstos, de entrada, casi con seguridad, implicarían un cálculo de los axiles de montaje diferente al planteado en este documento. Otro aspecto que aportaría un elemento diferenciador respecto de este estudio, sería la evaluación de las componentes de los axiles pasiva y activa de los cables, ya que al ser el peso propio del tablero mucho menor, sería de esperar que la componente pasiva del tirante igualmente también lo fuera.

- Aplicación de los mismos procesos de montaje erróneos al caso de un puente de grandes luces, ya que esta investigación se ha limitado a estudiar estructuras con tableros con una luz máxima de $180 \mathrm{~m}$, o lo que es lo mismo, a un puente de doble pilono con luz principal de $360 \mathrm{~m}$. En estos casos además empezará a ser necesaria la consideración de los efectos de segundo orden que se han obviado en esta investigación, dadas las dimensiones de las estructuras analizadas.

- Integración de estos procesos con el tratamiento de efectos reológicos, los cuales afectarán con seguridad al estudio en deformaciones realizado en esta investigación.

- Aplicación de estos mismos planteamientos al caso de una estructura claramente asimétrica, donde el diferente funcionamiento del atirantamiento respecto de los analizados en este estudio, pudiera dar lugar a resultados distintos a los obtenidos en esta tesis.

- Sería también interesante analizar todos los planteamientos aquí recogidos para el caso de que la ejecución del tablero se realice cimbrada sobre el terreno y no mediante voladizos sucesivos.

- Igualmente sería interesante valorar en qué medida se diferenciarían los resultados obtenidos en el caso de un puente de ferrocarril respecto de los aquí estudiados para el caso de un puente de carreteras.

- Evaluación de la influencia del comportamiento claramente tridemensional de un puente con dos planos de atirantamiento, pensando en que la introducción de errores 
en el montaje no sólo tenga una influencia en el plano de atirantamiento sino sobre la estructura completa tridimensional.

Por otra parte, sería también una interesante línea de ampliación de este trabajo el enlazar los resultados, obtenidos a partir de la aleatoriedad de los procesos de montaje, con planteamientos relacionados con la fiabilidad de la estructura, de tal forma que se pudieran justificar analíticamente la adopción de unos determinados coeficientes de seguridad. 
ANEXOS 


\section{ANEXOS.}


ANEXOS 


\section{A.1. CARACTERISTICAS DE LOS MODELOS DE CALCULO EMPLEADOS.}

1) DEFINICION DEL MODELO DE CALCULO: MODELO 90.

\section{COORDENADAS DE LOS NUDOS}

\begin{tabular}{|c|c|c|}
\hline Nudo & 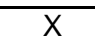 & $\bar{Y}$ \\
\hline 1 & 0.00 & -1.50 \\
\hline 2 & 0.00 & 0.00 \\
\hline 3 & 0.00 & 5.38 \\
\hline 4 & 0.00 & 10.75 \\
\hline 5 & 0.00 & 16.13 \\
\hline 6 & 0.00 & 21.49 \\
\hline 7 & 0.00 & 21.50 \\
\hline 8 & 0.00 & 25.80 \\
\hline 9 & 0.00 & 30.10 \\
\hline 10 & 0.00 & 34.40 \\
\hline 11 & 5.00 & 21.50 \\
\hline 12 & -5.00 & 21.50 \\
\hline 13 & 7.00 & 21.50 \\
\hline 14 & -7.00 & 21.50 \\
\hline 15 & 0.00 & 37.30 \\
\hline 16 & 10.00 & 21.50 \\
\hline 17 & -10.00 & 21.50 \\
\hline 18 & 12.00 & 21.50 \\
\hline 19 & -12.00 & 21.50 \\
\hline 20 & 0.00 & 39.30 \\
\hline 21 & 15.00 & 21.50 \\
\hline 22 & -15.00 & 21.50 \\
\hline 23 & 17.00 & 21.50 \\
\hline 24 & -17.00 & 21.50 \\
\hline 25 & 0.00 & 41.15 \\
\hline 26 & 20.00 & 21.50 \\
\hline 27 & -20.00 & 21.50 \\
\hline 28 & 22.00 & 21.50 \\
\hline 29 & -22.00 & 21.50 \\
\hline 30 & 0.00 & 43.00 \\
\hline 31 & 25.00 & 21.50 \\
\hline 32 & -25.00 & 21.50 \\
\hline 33 & 27.00 & 21.50 \\
\hline 34 & -27.00 & 21.50 \\
\hline
\end{tabular}

\begin{tabular}{|c|c|c|}
\hline Nudo & $\mathrm{X}$ & $\bar{Y}$ \\
\hline 35 & 0.00 & 44.85 \\
\hline 36 & 30.00 & 21.50 \\
\hline 37 & -30.00 & 21.50 \\
\hline 38 & 32.00 & 21.50 \\
\hline 39 & -32.00 & 21.50 \\
\hline 40 & 0.00 & 46.70 \\
\hline 41 & 35.00 & 21.50 \\
\hline 42 & -35.00 & 21.50 \\
\hline 43 & 37.00 & 21.50 \\
\hline 44 & -37.00 & 21.50 \\
\hline 45 & 0.00 & 48.50 \\
\hline 46 & 40.00 & 21.50 \\
\hline 47 & -40.00 & 21.50 \\
\hline 48 & 42.00 & 21.50 \\
\hline 49 & -42.00 & 21.50 \\
\hline 50 & 0.00 & 50.25 \\
\hline 51 & 45.00 & 21.50 \\
\hline 52 & -45.00 & 21.50 \\
\hline 53 & 47.00 & 21.50 \\
\hline 54 & -47.00 & 21.50 \\
\hline 55 & 0.00 & 51.90 \\
\hline 56 & 50.00 & 21.50 \\
\hline 57 & -50.00 & 21.50 \\
\hline 58 & 52.00 & 21.50 \\
\hline 59 & -52.00 & 21.50 \\
\hline 60 & 0.00 & 53.45 \\
\hline 61 & 55.00 & 21.50 \\
\hline 62 & -55.00 & 21.50 \\
\hline 63 & 57.00 & 21.50 \\
\hline 64 & -57.00 & 21.50 \\
\hline 65 & 0.00 & 54.90 \\
\hline 66 & 60.00 & 21.50 \\
\hline 67 & -60.00 & 21.50 \\
\hline 68 & 62.00 & 21.50 \\
\hline
\end{tabular}

\begin{tabular}{|c|c|c|}
\hline Nudo & $X$ & $\bar{Y}$ \\
\hline 69 & -62.00 & 21.50 \\
\hline 70 & 0.00 & 56.27 \\
\hline 71 & 65.00 & 21.50 \\
\hline 72 & -65.00 & 21.50 \\
\hline 73 & 67.00 & 21.50 \\
\hline 74 & -67.00 & 21.50 \\
\hline 75 & 0.00 & 57.60 \\
\hline 76 & 70.00 & 21.50 \\
\hline 77 & -70.00 & 21.50 \\
\hline 78 & 72.00 & 21.50 \\
\hline 79 & -72.00 & 21.50 \\
\hline 80 & 0.00 & 58.90 \\
\hline 81 & 75.00 & 21.50 \\
\hline 82 & -75.00 & 21.50 \\
\hline 83 & 77.00 & 21.50 \\
\hline 84 & -77.00 & 21.50 \\
\hline 85 & 0.00 & 60.15 \\
\hline 86 & 80.00 & 21.50 \\
\hline 87 & -80.00 & 21.50 \\
\hline 88 & 82.00 & 21.50 \\
\hline 89 & -82.00 & 21.50 \\
\hline 90 & 0.00 & 61.40 \\
\hline 91 & 85.00 & 21.50 \\
\hline 92 & -85.00 & 21.50 \\
\hline 93 & 0.00 & 64.00 \\
\hline 94 & 85.00 & 21.40 \\
\hline 95 & -86.50 & 21.50 \\
\hline 96 & 85.00 & 11.50 \\
\hline 97 & -88.00 & 21.50 \\
\hline 98 & -89.50 & 21.50 \\
\hline 99 & -91.00 & 21.50 \\
\hline 100 & -92.00 & 21.50 \\
\hline 101 & -91.00 & 21.40 \\
\hline 102 & -91.00 & -0.50 \\
\hline
\end{tabular}


CONDICIONES DE CONTORNO

\begin{tabular}{|c|c|c|c|}
\hline Nudo & Coac.-X & Coac.-Y & Giro \\
\hline 1 & 1 & 1 & 1 \\
\hline 96 & 1 & 1 & 1 \\
\hline 102 & 1 & 1 & 1 \\
\hline
\end{tabular}

CARACTERISTICAS MECÁNICAS DE LAS BARRAS

\begin{tabular}{|c|c|c|c|}
\hline Tipo & Area & Inercia & Mod.Elast. \\
\hline 1 & 9.998 & 1.053 & 30900000 \\
\hline 2 & 154 & 1552.8 & 29800000 \\
\hline 3 & 15.874 & 34.249 & 29800000 \\
\hline 4 & 15.542 & 31.55 & 29800000 \\
\hline 5 & 15.21 & 28.993 & 29800000 \\
\hline 6 & 14.877 & 26.564 & 29800000 \\
\hline 7 & 14.579 & 24.49 & 29800000 \\
\hline 8 & 14.313 & 22.731 & 29800000 \\
\hline 9 & 14.047 & 21.054 & 29800000 \\
\hline 10 & 19.97 & 22.51 & 29800000 \\
\hline 11 & 16.67 & 10.76 & 29800000 \\
\hline 12 & 16.18 & 9.62 & 29800000 \\
\hline 13 & 15.67 & 8.47 & 29800000 \\
\hline 14 & 15.5 & 8.19 & 29800000 \\
\hline 15 & 13.22 & 4.08 & 29800000 \\
\hline 16 & 13.17 & 4.08 & 29800000 \\
\hline 17 & 13.12 & 4.08 & 29800000 \\
\hline 18 & 12.76 & 3.65 & 29800000 \\
\hline 19 & 12.59 & 3.5 & 29800000 \\
\hline 20 & 12.51 & 3.45 & 29800000 \\
\hline 21 & 12.44 & 3.4 & 29800000 \\
\hline 22 & 12.4 & 3.4 & 29800000 \\
\hline 23 & 12.36 & 3.4 & 29800000 \\
\hline 24 & 12.33 & 3.4 & 29800000 \\
\hline 25 & 12.18 & 3.26 & 29800000 \\
\hline 26 & 14.66 & 8.29 & 29800000 \\
\hline 27 & 0 & 0 & 195000000 \\
\hline & & & \\
\hline
\end{tabular}

\begin{tabular}{|c|c|c|c|}
\hline Tipo & Area & Inercia & Mod.Elast. \\
\hline 28 & 0.003574 & 0 & 195000000 \\
\hline 29 & 0.003944 & 0 & 195000000 \\
\hline 30 & 0.004358 & 0 & 195000000 \\
\hline 31 & 0.004755 & 0 & 195000000 \\
\hline 32 & 0.00511 & 0 & 195000000 \\
\hline 33 & 0.005425 & 0 & 195000000 \\
\hline 34 & 0.005703 & 0 & 195000000 \\
\hline 35 & 0.005956 & 0 & 195000000 \\
\hline 36 & 0.006189 & 0 & 195000000 \\
\hline 37 & 0.006414 & 0 & 195000000 \\
\hline 38 & 0.006633 & 0 & 195000000 \\
\hline 39 & 0.006635 & 0 & 195000000 \\
\hline 40 & 0.006855 & 0 & 195000000 \\
\hline 41 & 0.006848 & 0 & 195000000 \\
\hline 42 & 0.007048 & 0 & 195000000 \\
\hline 43 & 0.007075 & 0 & 195000000 \\
\hline 44 & 0.007335 & 0 & 195000000 \\
\hline 45 & 0.007229 & 0 & 195000000 \\
\hline 46 & 0.007218 & 0 & 195000000 \\
\hline 47 & 0.007624 & 0 & 195000000 \\
\hline 48 & 0.008613 & 0 & 195000000 \\
\hline 49 & 0.007059 & 0 & 195000000 \\
\hline 50 & 0.008612 & 0 & 195000000 \\
\hline 51 & 0.009809 & 0 & 195000000 \\
\hline 52 & 0.6 & 0 & 1000000 \\
\hline 53 & 54.4 & 72.5 & 27300000 \\
\hline 54 & 3.85 & 0.39 & 27300000 \\
\hline 55 & 0 & 25 & 29800000 \\
\hline & & & \\
\hline
\end{tabular}

\section{TOPOLOGIA DE LAS BARRAS}

\begin{tabular}{|c|c|c|c|c|}
\hline Barra & $\begin{array}{c}\text { Nudo } \\
\text { Dorsal }\end{array}$ & $\begin{array}{c}\text { Nudo } \\
\text { Frontal }\end{array}$ & Tipo & Conex. \\
\hline 1 & 7 & 11 & 1 & 0 \\
\hline 2 & 11 & 13 & 1 & 0 \\
\hline 3 & 13 & 16 & 1 & 0 \\
\hline 4 & 16 & 18 & 1 & 0 \\
\hline 5 & 18 & 21 & 1 & 0 \\
\hline 6 & 21 & 23 & 1 & 0 \\
\hline 7 & 23 & 26 & 1 & 0 \\
\hline 8 & 26 & 28 & 1 & 0 \\
\hline 9 & 28 & 31 & 1 & 0 \\
\hline 10 & 31 & 33 & 1 & 0 \\
\hline 11 & 33 & 36 & 1 & 0 \\
\hline 12 & 36 & 38 & 1 & 0 \\
\hline 13 & 38 & 41 & 1 & 0 \\
\hline 14 & 41 & 43 & 1 & 0 \\
\hline 15 & 43 & 46 & 1 & 0 \\
\hline 16 & 46 & 48 & 1 & 0 \\
\hline 17 & 48 & 51 & 1 & 0 \\
\hline 18 & 51 & 53 & 1 & 0 \\
\hline 19 & 53 & 56 & 1 & 0 \\
\hline 20 & 56 & 58 & 1 & 0 \\
\hline 21 & 58 & 61 & 1 & 0 \\
\hline 22 & 61 & 63 & 1 & 0 \\
\hline 23 & 63 & 66 & 1 & 0 \\
\hline
\end{tabular}

\begin{tabular}{|c|c|c|c|c|}
\hline Barra & $\begin{array}{c}\text { Nudo } \\
\text { Dorsal }\end{array}$ & $\begin{array}{c}\text { Nudo } \\
\text { Frontal }\end{array}$ & Tipo & Conex. \\
\hline 46 & 39 & 42 & 1 & 0 \\
\hline 47 & 42 & 44 & 1 & 0 \\
\hline 48 & 44 & 47 & 1 & 0 \\
\hline 49 & 47 & 49 & 1 & 0 \\
\hline 50 & 49 & 52 & 1 & 0 \\
\hline 51 & 52 & 54 & 1 & 0 \\
\hline 52 & 54 & 57 & 1 & 0 \\
\hline 53 & 57 & 59 & 1 & 0 \\
\hline 54 & 59 & 62 & 1 & 0 \\
\hline 55 & 62 & 64 & 1 & 0 \\
\hline 56 & 64 & 67 & 1 & 0 \\
\hline 57 & 67 & 69 & 1 & 0 \\
\hline 58 & 69 & 72 & 1 & 0 \\
\hline 59 & 72 & 74 & 1 & 0 \\
\hline 60 & 74 & 77 & 1 & 0 \\
\hline 61 & 77 & 79 & 1 & 0 \\
\hline 62 & 79 & 82 & 1 & 0 \\
\hline 63 & 82 & 84 & 1 & 0 \\
\hline 64 & 84 & 87 & 1 & 0 \\
\hline 65 & 87 & 89 & 1 & 0 \\
\hline 66 & 89 & 92 & 1 & 0 \\
\hline 67 & 92 & 95 & 1 & 0 \\
\hline 68 & 95 & 97 & 1 & 0 \\
\hline
\end{tabular}

\begin{tabular}{|c|c|c|c|c|}
\hline Barra & $\begin{array}{c}\text { Nudo } \\
\text { Dorsal }\end{array}$ & $\begin{array}{c}\text { Nudo } \\
\text { Frontal }\end{array}$ & Tipo & Conex. \\
\hline 91 & 65 & 70 & 21 & 0 \\
\hline 92 & 70 & 75 & 22 & 0 \\
\hline 93 & 75 & 80 & 23 & 0 \\
\hline 94 & 80 & 85 & 24 & 0 \\
\hline 95 & 85 & 90 & 25 & 0 \\
\hline 96 & 90 & 93 & 26 & 0 \\
\hline 97 & 7 & 10 & 27 & 0 \\
\hline 98 & 10 & 11 & 28 & 0 \\
\hline 99 & 10 & 12 & 28 & 0 \\
\hline 100 & 15 & 16 & 29 & 0 \\
\hline 101 & 15 & 17 & 29 & 0 \\
\hline 102 & 20 & 21 & 30 & 0 \\
\hline 103 & 20 & 22 & 30 & 0 \\
\hline 104 & 25 & 26 & 31 & 0 \\
\hline 105 & 25 & 27 & 31 & 0 \\
\hline 106 & 30 & 31 & 32 & 0 \\
\hline 107 & 30 & 32 & 32 & 0 \\
\hline 108 & 35 & 36 & 33 & 0 \\
\hline 109 & 35 & 37 & 33 & 0 \\
\hline 110 & 40 & 41 & 34 & 0 \\
\hline 111 & 40 & 42 & 34 & 0 \\
\hline 112 & 45 & 46 & 35 & 0 \\
\hline 113 & 45 & 47 & 35 & 0 \\
\hline
\end{tabular}



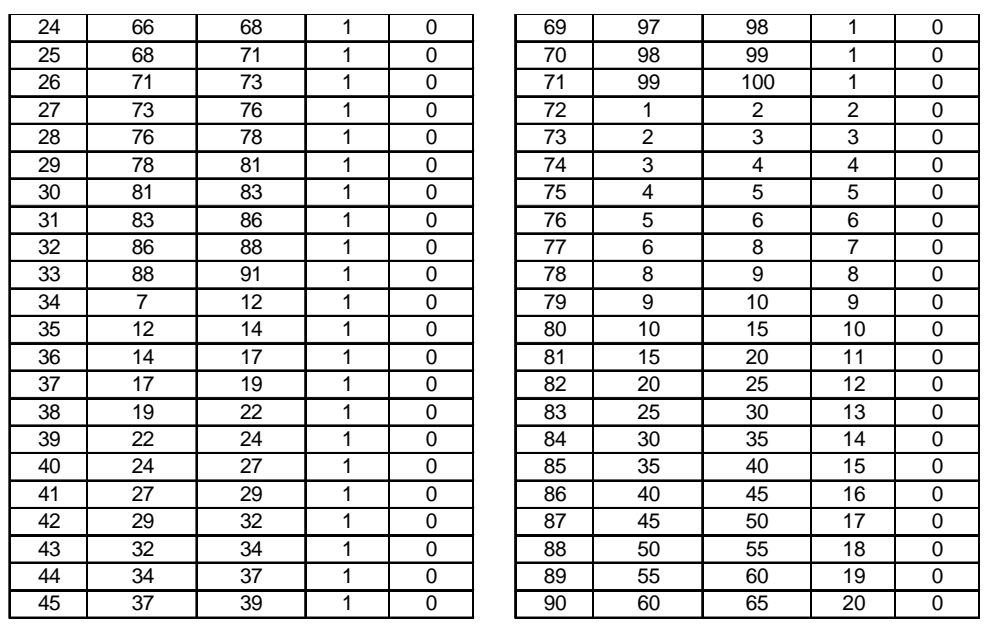

\begin{tabular}{|c|c|c|c|c|}
\hline 114 & 50 & 51 & 36 & 0 \\
\hline 115 & 50 & 52 & 36 & 0 \\
\hline 116 & 55 & 56 & 37 & 0 \\
\hline 117 & 55 & 57 & 37 & 0 \\
\hline 118 & 60 & 61 & 38 & 0 \\
\hline 119 & 60 & 62 & 39 & 0 \\
\hline 120 & 65 & 66 & 40 & 0 \\
\hline 121 & 65 & 67 & 41 & 0 \\
\hline 122 & 70 & 71 & 42 & 0 \\
\hline 123 & 70 & 72 & 43 & 0 \\
\hline 124 & 75 & 76 & 44 & 0 \\
\hline 125 & 75 & 77 & 45 & 0 \\
\hline 126 & 80 & 81 & 46 & 0 \\
\hline 127 & 80 & 82 & 47 & 0 \\
\hline 128 & 85 & 86 & 48 & 0 \\
\hline 129 & 85 & 87 & 49 & 0 \\
\hline 130 & 90 & 91 & 50 & 0 \\
\hline 131 & 90 & 92 & 51 & 0 \\
\hline 132 & 99 & 101 & 52 & 0 \\
\hline 133 & 101 & 102 & 54 & 0 \\
\hline 134 & 91 & 94 & 52 & 0 \\
\hline 135 & 94 & 96 & 53 & 0 \\
\hline 136 & 6 & 7 & 6 & 2 \\
\hline
\end{tabular}

COORDENADAS DE LOS NUDOS

\begin{tabular}{|c|c|c|}
\hline Nudo & $X$ & $\bar{Y}$ \\
\hline 1 & 0.00 & -1.50 \\
\hline 2 & 0.00 & 0.00 \\
\hline 3 & 0.00 & 5.38 \\
\hline 4 & 0.00 & 10.75 \\
\hline 5 & 0.00 & 16.13 \\
\hline 6 & 0.00 & 21.49 \\
\hline 7 & 0.00 & 21.50 \\
\hline 8 & 0.00 & 25.80 \\
\hline 9 & 0.00 & 30.10 \\
\hline 10 & 0.00 & 34.40 \\
\hline 11 & 5.00 & 21.50 \\
\hline 12 & -5.00 & 21.50 \\
\hline 13 & 7.00 & 21.50 \\
\hline 14 & -7.00 & 21.50 \\
\hline 15 & 0.00 & 37.30 \\
\hline 16 & 10.00 & 21.50 \\
\hline 17 & -10.00 & 21.50 \\
\hline 18 & 12.00 & 21.50 \\
\hline 19 & -12.00 & 21.50 \\
\hline 20 & 0.00 & 39.30 \\
\hline 21 & 15.00 & 21.50 \\
\hline 22 & -15.00 & 21.50 \\
\hline 23 & 17.00 & 21.50 \\
\hline 24 & -17.00 & 21.50 \\
\hline 25 & 0.00 & 41.15 \\
\hline 26 & 20.00 & 21.50 \\
\hline 27 & -20.00 & 21.50 \\
\hline 28 & 22.00 & 21.50 \\
\hline 29 & -22.00 & 21.50 \\
\hline 30 & 0.00 & 43.00 \\
\hline 31 & 25.00 & 21.50 \\
\hline 32 & -25.00 & 21.50 \\
\hline 33 & 27.00 & 21.50 \\
\hline 34 & -27.00 & 21.50 \\
\hline
\end{tabular}

\begin{tabular}{|c|c|c|}
\hline Nudo & $x$ & $Y$ \\
\hline 35 & 0.00 & 44.85 \\
\hline 36 & 30.00 & 21.50 \\
\hline 37 & -30.00 & 21.50 \\
\hline 38 & 32.00 & 21.50 \\
\hline 39 & -32.00 & 21.50 \\
\hline 40 & 0.00 & 46.70 \\
\hline 41 & 35.00 & 21.50 \\
\hline 42 & -35.00 & 21.50 \\
\hline 43 & 37.00 & 21.50 \\
\hline 44 & -37.00 & 21.50 \\
\hline 45 & 0.00 & 48.50 \\
\hline 46 & 40.00 & 21.50 \\
\hline 47 & -40.00 & 21.50 \\
\hline 48 & 42.00 & 21.50 \\
\hline 49 & -42.00 & 21.50 \\
\hline 50 & 0.00 & 50.25 \\
\hline 51 & 45.00 & 21.50 \\
\hline 52 & -45.00 & 21.50 \\
\hline 53 & 47.00 & 21.50 \\
\hline 54 & -47.00 & 21.50 \\
\hline 55 & 0.00 & 51.90 \\
\hline 56 & 50.00 & 21.50 \\
\hline 57 & -50.00 & 21.50 \\
\hline 58 & 52.00 & 21.50 \\
\hline 59 & -52.00 & 21.50 \\
\hline 60 & 0.00 & 53.45 \\
\hline 61 & 55.00 & 21.50 \\
\hline 62 & -55.00 & 21.50 \\
\hline 63 & 57.00 & 21.50 \\
\hline 64 & -57.00 & 21.50 \\
\hline 65 & 0.00 & 54.90 \\
\hline 66 & 60.00 & 21.50 \\
\hline 67 & -60.00 & 21.50 \\
\hline 68 & 62.00 & 21.50 \\
\hline
\end{tabular}

\begin{tabular}{|c|c|c|}
\hline Nudo & $\mathrm{X}$ & $\bar{Y}$ \\
\hline 69 & -62.00 & 21.50 \\
\hline 70 & 0.00 & 56.27 \\
\hline 71 & 65.00 & 21.50 \\
\hline 72 & -65.00 & 21.50 \\
\hline 73 & 67.00 & 21.50 \\
\hline 74 & -67.00 & 21.50 \\
\hline 75 & 0.00 & 57.60 \\
\hline 76 & 70.00 & 21.50 \\
\hline 77 & -70.00 & 21.50 \\
\hline 78 & 72.00 & 21.50 \\
\hline 79 & -72.00 & 21.50 \\
\hline 80 & 0.00 & 58.90 \\
\hline 81 & 75.00 & 21.50 \\
\hline 82 & -75.00 & 21.50 \\
\hline 83 & 77.00 & 21.50 \\
\hline 84 & -77.00 & 21.50 \\
\hline 85 & 0.00 & 60.15 \\
\hline 86 & 80.00 & 21.50 \\
\hline 87 & -80.00 & 21.50 \\
\hline 88 & 82.00 & 21.50 \\
\hline 89 & -82.00 & 21.50 \\
\hline 90 & 0.00 & 61.40 \\
\hline 91 & 85.00 & 21.50 \\
\hline 92 & -85.00 & 21.50 \\
\hline 93 & 0.00 & 64.00 \\
\hline 94 & 85.00 & 21.40 \\
\hline 95 & -86.50 & 21.50 \\
\hline 96 & 85.00 & 11.50 \\
\hline 97 & -88.00 & 21.50 \\
\hline 98 & -89.50 & 21.50 \\
\hline 99 & -91.00 & 21.50 \\
\hline 100 & -92.00 & 21.50 \\
\hline 101 & -91.00 & 21.40 \\
\hline 102 & -91.00 & -0.50 \\
\hline
\end{tabular}


CONDICIONES DE CONTORNO

\begin{tabular}{|c|c|c|c|}
\hline Nudo & Coac.-X & Coac.-Y & Giro \\
\hline 1 & 1 & 1 & 1 \\
\hline 96 & 1 & 1 & 1 \\
\hline 102 & 1 & 1 & 1 \\
\hline
\end{tabular}

CARACTERISTICAS MECÁNICAS DE LAS BARRAS

\begin{tabular}{|c|c|c|c|}
\hline Tipo & Area & Inercia & Mod.Elast. \\
\hline 1 & 9.998 & 1.053 & 30900000 \\
\hline 2 & 154 & 1552.8 & 29800000 \\
\hline 3 & 15.874 & 34.249 & 29800000 \\
\hline 4 & 15.542 & 31.55 & 29800000 \\
\hline 5 & 15.21 & 28.993 & 29800000 \\
\hline 6 & 14.877 & 26.564 & 29800000 \\
\hline 7 & 14.579 & 24.49 & 29800000 \\
\hline 8 & 14.313 & 22.731 & 29800000 \\
\hline 9 & 14.047 & 21.054 & 29800000 \\
\hline 10 & 19.97 & 22.51 & 29800000 \\
\hline 11 & 16.67 & 10.76 & 29800000 \\
\hline 12 & 16.18 & 9.62 & 29800000 \\
\hline 13 & 15.67 & 8.47 & 29800000 \\
\hline 14 & 15.5 & 8.19 & 29800000 \\
\hline 15 & 13.22 & 4.08 & 29800000 \\
\hline 16 & 13.17 & 4.08 & 29800000 \\
\hline 17 & 13.12 & 4.08 & 29800000 \\
\hline 18 & 12.76 & 3.65 & 29800000 \\
\hline 19 & 12.59 & 3.5 & 29800000 \\
\hline 20 & 12.51 & 3.45 & 29800000 \\
\hline 21 & 12.44 & 3.4 & 29800000 \\
\hline 22 & 12.4 & 3.4 & 29800000 \\
\hline 23 & 12.36 & 3.4 & 29800000 \\
\hline 24 & 12.33 & 3.4 & 29800000 \\
\hline 25 & 12.18 & 3.26 & 29800000 \\
\hline 26 & 14.66 & 8.29 & 29800000 \\
\hline 27 & 0 & 0 & 195000000 \\
\hline & & & \\
\hline
\end{tabular}

\begin{tabular}{|c|c|c|c|}
\hline Tipo & Area & Inercia & Mod.Elast. \\
\hline 28 & 0.005943 & 0 & 195000000 \\
\hline 29 & 0.00865 & 0 & 195000000 \\
\hline 30 & 0.009777 & 0 & 195000000 \\
\hline 31 & 0.009779 & 0 & 195000000 \\
\hline 32 & 0.011029 & 0 & 195000000 \\
\hline 33 & 0.011023 & 0 & 195000000 \\
\hline 34 & 0.0119 & 0 & 195000000 \\
\hline 35 & 0.011924 & 0 & 195000000 \\
\hline 36 & 0.012915 & 0 & 195000000 \\
\hline 37 & 0.012819 & 0 & 195000000 \\
\hline 38 & 0.013154 & 0 & 195000000 \\
\hline 39 & 0.013532 & 0 & 195000000 \\
\hline 40 & 0.016259 & 0 & 195000000 \\
\hline 41 & 0.014765 & 0 & 195000000 \\
\hline 42 & 0.012342 & 0 & 195000000 \\
\hline 43 & 0.013465 & 0 & 195000000 \\
\hline 44 & 0.6 & 0 & 1000000 \\
\hline 45 & 54.4 & 72.5 & 27300000 \\
\hline 46 & 3.85 & 0.39 & 27300000 \\
\hline 47 & 0 & 25 & 29800000 \\
\hline 48 & 0.00001 & 0 & 195000000 \\
\hline
\end{tabular}

TOPOLOGIA DE LAS BARRAS

\begin{tabular}{|c|c|c|c|c|}
\hline Barra & $\begin{array}{c}\text { Nudo } \\
\text { Dorsal }\end{array}$ & $\begin{array}{c}\text { Nudo } \\
\text { Frontal }\end{array}$ & Tipo & Conex. \\
\hline 1 & 7 & 11 & 1 & 0 \\
\hline 2 & 11 & 13 & 1 & 0 \\
\hline 3 & 13 & 16 & 1 & 0 \\
\hline 4 & 16 & 18 & 1 & 0 \\
\hline 5 & 18 & 21 & 1 & 0 \\
\hline 6 & 21 & 23 & 1 & 0 \\
\hline 7 & 23 & 26 & 1 & 0 \\
\hline 8 & 26 & 28 & 1 & 0 \\
\hline 9 & 28 & 31 & 1 & 0 \\
\hline 10 & 31 & 33 & 1 & 0 \\
\hline 11 & 33 & 36 & 1 & 0 \\
\hline 12 & 36 & 38 & 1 & 0 \\
\hline
\end{tabular}

\begin{tabular}{|c|c|c|c|c|}
\hline Barra & $\begin{array}{c}\text { Nudo } \\
\text { Dorsal }\end{array}$ & $\begin{array}{c}\text { Nudo } \\
\text { Frontal }\end{array}$ & Tipo & Conex. \\
\hline 46 & 39 & 42 & 1 & 0 \\
\hline 47 & 42 & 44 & 1 & 0 \\
\hline 48 & 44 & 47 & 1 & 0 \\
\hline 49 & 47 & 49 & 1 & 0 \\
\hline 50 & 49 & 52 & 1 & 0 \\
\hline 51 & 52 & 54 & 1 & 0 \\
\hline 52 & 54 & 57 & 1 & 0 \\
\hline 53 & 57 & 59 & 1 & 0 \\
\hline 54 & 59 & 62 & 1 & 0 \\
\hline 55 & 62 & 64 & 1 & 0 \\
\hline 56 & 64 & 67 & 1 & 0 \\
\hline 57 & 67 & 69 & 1 & 0
\end{tabular}

\begin{tabular}{|c|c|c|c|c|}
\hline Barra & $\begin{array}{c}\text { Nudo } \\
\text { Dorsal }\end{array}$ & $\begin{array}{c}\text { Nudo } \\
\text { Frontal }\end{array}$ & Tipo & Conex. \\
\hline 91 & 65 & 70 & 21 & 0 \\
\hline 92 & 70 & 75 & 22 & 0 \\
\hline 93 & 75 & 80 & 23 & 0 \\
\hline 94 & 80 & 85 & 24 & 0 \\
\hline 95 & 85 & 90 & 25 & 0 \\
\hline 96 & 90 & 93 & 26 & 0 \\
\hline 97 & 7 & 10 & 27 & 0 \\
\hline 98 & 10 & 11 & 28 & 0 \\
\hline 99 & 10 & 12 & 28 & 0 \\
\hline 100 & 15 & 16 & 27 & 0 \\
\hline 101 & 15 & 17 & 27 & 0 \\
\hline 102 & 20 & 21 & 29 & 0 \\
\hline
\end{tabular}




\begin{tabular}{|c|c|c|c|c|}
\hline 12 & 36 & 38 & 1 & $\overline{0}$ \\
\hline 13 & 38 & 41 & 1 & 0 \\
\hline 14 & 41 & 43 & 1 & 0 \\
\hline 15 & 43 & 46 & 1 & 0 \\
\hline 16 & 46 & 48 & 1 & 0 \\
\hline 17 & 48 & 51 & 1 & 0 \\
\hline 18 & 51 & 53 & 1 & $\overline{0}$ \\
\hline 19 & 53 & 56 & 1 & 0 \\
\hline 20 & 56 & 58 & 1 & 0 \\
\hline 21 & 58 & 61 & 1 & 0 \\
\hline 22 & 61 & 63 & 1 & 0 \\
\hline 23 & 63 & 66 & 1 & 0 \\
\hline 24 & 66 & 68 & 1 & $\overline{0}$ \\
\hline 25 & 68 & 71 & 1 & 0 \\
\hline 26 & 71 & 73 & 1 & 0 \\
\hline 27 & 73 & 76 & 1 & 0 \\
\hline 28 & 76 & 78 & 1 & 0 \\
\hline 29 & 78 & 81 & 1 & 0 \\
\hline 30 & 81 & 83 & 1 & 0 \\
\hline 31 & 83 & 86 & 1 & 0 \\
\hline 32 & 86 & 88 & 1 & 0 \\
\hline 33 & 88 & 91 & 1 & 0 \\
\hline 34 & 7 & 12 & 1 & 0 \\
\hline 35 & 12 & 14 & 1 & $\overline{0}$ \\
\hline 36 & 14 & 17 & 1 & 0 \\
\hline 37 & 17 & 19 & 1 & 0 \\
\hline 38 & 19 & 22 & 1 & 0 \\
\hline 39 & 22 & 24 & 1 & 0 \\
\hline 40 & 24 & 27 & 1 & 0 \\
\hline 41 & 27 & 29 & 1 & $\overline{0}$ \\
\hline 42 & 29 & 32 & 1 & 0 \\
\hline 43 & 32 & 34 & 1 & 0 \\
\hline 44 & 34 & 37 & 1 & 0 \\
\hline 45 & 37 & 39 & 1 & 0 \\
\hline
\end{tabular}

\begin{tabular}{|c|c|c|c|c|}
\hline 57 & 67 & 69 & 1 & 0 \\
\hline 58 & 69 & 72 & 1 & 0 \\
\hline 59 & 72 & 74 & 1 & 0 \\
\hline 60 & 74 & 77 & 1 & 0 \\
\hline 61 & 77 & 79 & 1 & 0 \\
\hline 62 & 79 & 82 & 1 & 0 \\
\hline 63 & 82 & 84 & 1 & 0 \\
\hline 64 & 84 & 87 & 1 & 0 \\
\hline 65 & 87 & 89 & 1 & 0 \\
\hline 66 & 89 & 92 & 1 & 0 \\
\hline 67 & 92 & 95 & 1 & 0 \\
\hline 68 & 95 & 97 & 1 & 0 \\
\hline 69 & 97 & 98 & 1 & 0 \\
\hline 70 & 98 & 99 & 1 & 0 \\
\hline 71 & 99 & 100 & 1 & 0 \\
\hline 72 & 1 & 2 & 2 & 0 \\
\hline 73 & 2 & 3 & 3 & 0 \\
\hline 74 & 3 & 4 & 4 & 0 \\
\hline 75 & 4 & 5 & 5 & 0 \\
\hline 76 & 5 & 6 & 6 & 0 \\
\hline 77 & 6 & 8 & 7 & 0 \\
\hline 78 & 8 & 9 & 8 & 0 \\
\hline 79 & 9 & 10 & 9 & 0 \\
\hline 80 & 10 & 15 & 10 & 0 \\
\hline 81 & 15 & 20 & 11 & 0 \\
\hline 82 & 20 & 25 & 12 & 0 \\
\hline 83 & 25 & 30 & 13 & 0 \\
\hline 84 & 30 & 35 & 14 & 0 \\
\hline 85 & 35 & 40 & 15 & 0 \\
\hline 86 & 40 & 45 & 16 & 0 \\
\hline 87 & 45 & 50 & 17 & 0 \\
\hline 88 & 50 & 55 & 18 & 0 \\
\hline 89 & 55 & 60 & 19 & 0 \\
\hline 90 & 60 & 65 & 20 & 0 \\
\hline
\end{tabular}

\begin{tabular}{|c|c|c|c|c|}
\hline 102 & 20 & 21 & 29 & 0 \\
\hline 103 & 20 & 22 & 29 & 0 \\
\hline 104 & 25 & 26 & 27 & 0 \\
\hline 105 & 25 & 27 & 27 & 0 \\
\hline 106 & 30 & 31 & 30 & 0 \\
\hline 107 & 30 & 32 & 31 & 0 \\
\hline 108 & 35 & 36 & 27 & 0 \\
\hline 109 & 35 & 37 & 27 & 0 \\
\hline 110 & 40 & 41 & 32 & 0 \\
\hline 111 & 40 & 42 & 33 & 0 \\
\hline 112 & 45 & 46 & 27 & 0 \\
\hline 113 & 45 & 47 & 27 & 0 \\
\hline 114 & 50 & 51 & 34 & 0 \\
\hline 115 & 50 & 52 & 35 & 0 \\
\hline 116 & 55 & 56 & 27 & 0 \\
\hline 117 & 55 & 57 & 27 & 0 \\
\hline 118 & 60 & 61 & 36 & 0 \\
\hline 119 & 60 & 62 & 37 & 0 \\
\hline 120 & 65 & 66 & 27 & 0 \\
\hline 121 & 65 & 67 & 27 & 0 \\
\hline 122 & 70 & 71 & 38 & 0 \\
\hline 123 & 70 & 72 & 39 & 0 \\
\hline 124 & 75 & 76 & 27 & 0 \\
\hline 125 & 75 & 77 & 27 & 0 \\
\hline 126 & 80 & 81 & 40 & 0 \\
\hline 127 & 80 & 82 & 41 & 0 \\
\hline 128 & 85 & 86 & 27 & 0 \\
\hline 129 & 85 & 87 & 27 & 0 \\
\hline 130 & 90 & 91 & 42 & 0 \\
\hline 131 & 90 & 92 & 43 & 0 \\
\hline 132 & 99 & 101 & 44 & 0 \\
\hline 133 & 101 & 102 & 46 & 0 \\
\hline 134 & 91 & 94 & 44 & 0 \\
\hline 135 & 94 & 96 & 45 & 0 \\
\hline 136 & 6 & 7 & 6 & 2 \\
\hline
\end{tabular}


3) DEFINICION DEL MODELO DE CALCULO: MODELO 180.

\section{COORDENADAS DE LOS NUDOS}

\begin{tabular}{|c|c|c|}
\hline Nudo & $\bar{X}$ & $\bar{Y}$ \\
\hline 1 & 0.00 & $\begin{array}{l}-1.50 \\
\end{array}$ \\
\hline 2 & 0.00 & 0.00 \\
\hline 3 & 0.00 & 5.38 \\
\hline 4 & 0.00 & 10.75 \\
\hline 5 & 0.00 & 16.13 \\
\hline 6 & 0.00 & 21.49 \\
\hline 7 & 0.00 & 21.50 \\
\hline 8 & 0.00 & 25.80 \\
\hline 9 & 0.00 & 30.10 \\
\hline 10 & 0.00 & 34.45 \\
\hline 11 & 5.00 & 21.50 \\
\hline 12 & -5.00 & 21.50 \\
\hline 13 & 9.00 & 21.50 \\
\hline 14 & -9.00 & 21.50 \\
\hline 15 & 0.00 & 39.37 \\
\hline 16 & 15.00 & 21.50 \\
\hline 17 & -15.00 & 21.50 \\
\hline 18 & 19.00 & 21.50 \\
\hline 19 & -19.00 & 21.50 \\
\hline 20 & 0.00 & 43.07 \\
\hline 21 & 25.00 & 21.50 \\
\hline 22 & -25.00 & 21.50 \\
\hline 23 & 29.00 & 21.50 \\
\hline 24 & -29.00 & 21.50 \\
\hline 25 & 0.00 & 46.77 \\
\hline 26 & 35.00 & 21.50 \\
\hline 27 & -35.00 & 21.50 \\
\hline 28 & 39.00 & 21.50 \\
\hline 29 & -39.00 & 21.50 \\
\hline 30 & 0.00 & 50.32 \\
\hline 31 & 45.00 & 21.50 \\
\hline 32 & -45.00 & 21.50 \\
\hline 33 & 49.00 & 21.50 \\
\hline 34 & -49.00 & 21.50 \\
\hline
\end{tabular}

\begin{tabular}{|c|c|c|}
\hline Nudo & $\bar{X}$ & $\bar{Y}$ \\
\hline 35 & 0.00 & 53.52 \\
\hline 36 & 55.00 & 21.50 \\
\hline 37 & -55.00 & 21.50 \\
\hline 38 & 59.00 & 21.50 \\
\hline 39 & -59.00 & 21.50 \\
\hline 40 & 0.00 & 56.34 \\
\hline 41 & 65.00 & 21.50 \\
\hline 42 & -65.00 & 21.50 \\
\hline 43 & 69.00 & 21.50 \\
\hline 44 & -69.00 & 21.50 \\
\hline 45 & 0.00 & 58.97 \\
\hline 46 & 75.00 & 21.50 \\
\hline 47 & -75.00 & 21.50 \\
\hline 48 & 79.00 & 21.50 \\
\hline 49 & -79.00 & 21.50 \\
\hline 50 & 0.00 & 61.47 \\
\hline 51 & 85.00 & 21.50 \\
\hline 52 & -85.00 & 21.50 \\
\hline 53 & 89.00 & 21.50 \\
\hline 54 & -89.00 & 21.50 \\
\hline 55 & 0.00 & 63.97 \\
\hline 56 & 95.00 & 21.50 \\
\hline 57 & -95.00 & 21.50 \\
\hline 58 & 99.00 & 21.50 \\
\hline 59 & -99.00 & 21.50 \\
\hline 60 & 0.00 & 66.47 \\
\hline 61 & 105.00 & 21.50 \\
\hline 62 & -105.00 & 21.50 \\
\hline 63 & 109.00 & 21.50 \\
\hline 64 & -109.00 & 21.50 \\
\hline 65 & 0.00 & 68.97 \\
\hline 66 & 115.00 & 21.50 \\
\hline 67 & -115.00 & 21.50 \\
\hline 68 & 119.00 & 21.50 \\
\hline
\end{tabular}

\begin{tabular}{|c|c|c|}
\hline Nudo & $\mathrm{X}$ & $\bar{Y}$ \\
\hline 69 & -119.00 & 21.50 \\
\hline 70 & 0.00 & 71.47 \\
\hline 71 & 125.00 & 21.50 \\
\hline 72 & -125.00 & 21.50 \\
\hline 73 & 129.00 & 21.50 \\
\hline 74 & -129.00 & 21.50 \\
\hline 75 & 0.00 & 73.97 \\
\hline 76 & 135.00 & 21.50 \\
\hline 77 & -135.00 & 21.50 \\
\hline 78 & 139.00 & 21.50 \\
\hline 79 & -139.00 & 21.50 \\
\hline 80 & 0.00 & 76.47 \\
\hline 81 & 145.00 & 21.50 \\
\hline 82 & -145.00 & 21.50 \\
\hline 83 & 149.00 & 21.50 \\
\hline 84 & -149.00 & 21.50 \\
\hline 85 & 0.00 & 78.97 \\
\hline 86 & 155.00 & 21.50 \\
\hline 87 & -155.00 & 21.50 \\
\hline 88 & 159.00 & 21.50 \\
\hline 89 & -159.00 & 21.50 \\
\hline 90 & 0.00 & 81.47 \\
\hline 91 & 165.00 & 21.50 \\
\hline 92 & -165.00 & 21.50 \\
\hline 93 & 0.00 & 83.97 \\
\hline 94 & 165.00 & 21.40 \\
\hline 95 & -168.00 & 21.50 \\
\hline 96 & 165.00 & 11.50 \\
\hline 97 & -171.00 & 21.50 \\
\hline 98 & -174.00 & 21.50 \\
\hline 99 & -177.00 & 21.50 \\
\hline 100 & -178.00 & 21.50 \\
\hline 101 & -177.00 & 21.40 \\
\hline 102 & -177.00 & -0.50 \\
\hline
\end{tabular}

\section{CONDICIONES DE CONTORNO}

\begin{tabular}{|c|c|c|c|}
\hline Nudo & Coac.-X & Coac.-Y & Giro \\
\hline 1 & 1 & 1 & 1 \\
\hline 96 & 1 & 1 & 1 \\
\hline 102 & 1 & 1 & 1 \\
\hline
\end{tabular}


CARACTERISTICAS MECÁNICAS DE LAS BARRAS

\begin{tabular}{|c|c|c|c|}
\hline Tipo & Area & Inercia & Mod.Elast. \\
\hline 1 & 9.998 & 1.053 & 30900000 \\
\hline 2 & 20.6362 & 59.93575 & 29800000 \\
\hline 3 & 20.2046 & 55.2125 & 29800000 \\
\hline 4 & 19.773 & 50.73775 & 29800000 \\
\hline 5 & 19.3401 & 46.487 & 29800000 \\
\hline 6 & 18.9527 & 42.8575 & 29800000 \\
\hline 7 & 18.6069 & 39.77925 & 29800000 \\
\hline 8 & 18.2611 & 36.8445 & 29800000 \\
\hline 9 & 25.961 & 39.3925 & 29800000 \\
\hline 10 & 21.671 & 18.83 & 29800000 \\
\hline 11 & 21.034 & 16.835 & 29800000 \\
\hline 12 & 20.371 & 14.8225 & 29800000 \\
\hline 13 & 20.15 & 14.3325 & 29800000 \\
\hline 14 & 17.186 & 7.14 & 29800000 \\
\hline 15 & 17.121 & 7.14 & 29800000 \\
\hline 16 & 17.056 & 7.14 & 29800000 \\
\hline 17 & 16.588 & 6.3875 & 29800000 \\
\hline 18 & 16.367 & 6.125 & 29800000 \\
\hline 19 & 16.263 & 6.0375 & 29800000 \\
\hline 20 & 16.172 & 5.95 & 29800000 \\
\hline 21 & 16.12 & 5.95 & 29800000 \\
\hline 22 & 16.068 & 5.95 & 29800000 \\
\hline 23 & 16.029 & 5.95 & 29800000 \\
\hline 24 & 15.834 & 5.705 & 29800000 \\
\hline 25 & 19.058 & 14.5075 & 29800000 \\
\hline 26 & 14.66 & 8.29 & 29800000 \\
\hline 27 & 0 & 0 & 195000000 \\
\hline
\end{tabular}

\begin{tabular}{|c|c|c|c|}
\hline Tipo & Area & Inercia & Mod.Elast. \\
\hline 28 & 0.00594 & 0 & 195000000 \\
\hline 29 & 0.008636 & 0 & 195000000 \\
\hline 30 & 0.009761 & 0 & 195000000 \\
\hline 31 & 0.011002 & 0 & 195000000 \\
\hline 32 & 0.01191 & 0 & 195000000 \\
\hline 33 & 0.012775 & 0 & 195000000 \\
\hline 34 & 0.013603 & 0 & 195000000 \\
\hline 35 & 0.01438 & 0 & 195000000 \\
\hline 36 & 0.015102 & 0 & 195000000 \\
\hline 37 & 0.015748 & 0 & 195000000 \\
\hline 38 & 0.016321 & 0 & 195000000 \\
\hline 39 & 0.016326 & 0 & 195000000 \\
\hline 40 & 0.016855 & 0 & 195000000 \\
\hline 41 & 0.016836 & 0 & 195000000 \\
\hline 42 & 0.01727 & 0 & 195000000 \\
\hline 43 & 0.017343 & 0 & 195000000 \\
\hline 44 & 0.017905 & 0 & 195000000 \\
\hline 45 & 0.017625 & 0 & 195000000 \\
\hline 46 & 0.017503 & 0 & 195000000 \\
\hline 47 & 0.018569 & 0 & 195000000 \\
\hline 48 & 0.020871 & 0 & 195000000 \\
\hline 49 & 0.016816 & 0 & 195000000 \\
\hline 50 & 0.021129 & 0 & 195000000 \\
\hline 51 & 0.024228 & 0 & 195000000 \\
\hline 52 & 0.6 & 0 & 1000000 \\
\hline 53 & 54.4 & 72.5 & 27300000 \\
\hline 54 & 3.85 & 0.39 & 27300000 \\
\hline 55 & 0 & 25 & 29800000 \\
\hline 56 & 0.001 & 0 & 1000 \\
\hline & & & \\
\hline
\end{tabular}

\section{TOPOLOGIA DE LAS BARRAS}

\begin{tabular}{|c|c|c|c|c|}
\hline Barra & $\begin{array}{c}\text { Nudo } \\
\text { Dorsal }\end{array}$ & $\begin{array}{c}\text { Nudo } \\
\text { Frontal }\end{array}$ & Tipo & Conex. \\
\hline 1 & 7 & 11 & 1 & 0 \\
\hline 2 & 11 & 13 & 1 & 0 \\
\hline 3 & 13 & 16 & 1 & 0 \\
\hline 4 & 16 & 18 & 1 & 0 \\
\hline 5 & 18 & 21 & 1 & 0 \\
\hline 6 & 21 & 23 & 1 & 0 \\
\hline 7 & 23 & 26 & 1 & 0 \\
\hline 8 & 26 & 28 & 1 & 0 \\
\hline 9 & 28 & 31 & 1 & 0 \\
\hline 10 & 31 & 33 & 1 & 0 \\
\hline 11 & 33 & 36 & 1 & 0 \\
\hline 12 & 36 & 38 & 1 & 0 \\
\hline 13 & 38 & 41 & 1 & 0 \\
\hline 14 & 41 & 43 & 1 & 0 \\
\hline 15 & 43 & 46 & 1 & 0 \\
\hline 16 & 46 & 48 & 1 & 0 \\
\hline 17 & 48 & 51 & 1 & 0 \\
\hline
\end{tabular}

\begin{tabular}{|c|c|c|c|c|}
\hline Barra & $\begin{array}{c}\text { Nudo } \\
\text { Dorsal }\end{array}$ & $\begin{array}{c}\text { Nudo } \\
\text { Frontal }\end{array}$ & Tipo & Conex. \\
\hline 46 & 39 & 42 & 1 & 0 \\
\hline 47 & 42 & 44 & 1 & 0 \\
\hline 48 & 44 & 47 & 1 & 0 \\
\hline 49 & 47 & 49 & 1 & 0 \\
\hline 50 & 49 & 52 & 1 & 0 \\
\hline 51 & 52 & 54 & 1 & 0 \\
\hline 52 & 54 & 57 & 1 & 0 \\
\hline 53 & 57 & 59 & 1 & 0 \\
\hline 54 & 59 & 62 & 1 & 0 \\
\hline 55 & 62 & 64 & 1 & 0 \\
\hline 56 & 64 & 67 & 1 & 0 \\
\hline 57 & 67 & 69 & 1 & 0 \\
\hline 58 & 69 & 72 & 1 & 0 \\
\hline 59 & 72 & 74 & 1 & 0 \\
\hline 60 & 74 & 77 & 1 & 0 \\
\hline 61 & 77 & 79 & 1 & 0 \\
\hline 62 & 79 & 82 & 1 & 0 \\
\hline
\end{tabular}

\begin{tabular}{|c|c|c|c|c|}
\hline Barra & $\begin{array}{c}\text { Nudo } \\
\text { Dorsal }\end{array}$ & $\begin{array}{c}\text { Nudo } \\
\text { Frontal }\end{array}$ & Tipo & Conex. \\
\hline 91 & 65 & 70 & 21 & 0 \\
\hline 92 & 70 & 75 & 22 & 0 \\
\hline 93 & 75 & 80 & 23 & 0 \\
\hline 94 & 80 & 85 & 24 & 0 \\
\hline 95 & 85 & 90 & 25 & 0 \\
\hline 96 & 90 & 93 & 26 & 0 \\
\hline 97 & 7 & 10 & 27 & 0 \\
\hline 98 & 10 & 11 & 28 & 0 \\
\hline 99 & 10 & 12 & 28 & 0 \\
\hline 100 & 15 & 16 & 29 & 0 \\
\hline 101 & 15 & 17 & 29 & 0 \\
\hline 102 & 20 & 21 & 30 & 0 \\
\hline 103 & 20 & 22 & 30 & 0 \\
\hline 104 & 25 & 26 & 31 & 0 \\
\hline 105 & 25 & 27 & 31 & 0 \\
\hline 106 & 30 & 31 & 32 & 0 \\
\hline 107 & 30 & 32 & 32 & 0 \\
\hline
\end{tabular}




\begin{tabular}{|l|l|l|l|l|}
\hline 18 & 51 & 53 & 1 & 0 \\
\hline 19 & 53 & 56 & 1 & 0 \\
\hline 20 & 56 & 58 & 1 & 0 \\
\hline 21 & 58 & 61 & 1 & 0 \\
\hline 22 & 61 & 63 & 1 & 0 \\
\hline 23 & 63 & 66 & 1 & 0 \\
\hline 24 & 66 & 68 & 1 & 0 \\
\hline 25 & 68 & 71 & 1 & 0 \\
\hline 26 & 71 & 73 & 1 & 0 \\
\hline 27 & 73 & 76 & 1 & 0 \\
\hline 28 & 76 & 78 & 1 & 0 \\
\hline 29 & 78 & 81 & 1 & 0 \\
\hline 30 & 81 & 83 & 1 & 0 \\
\hline 31 & 83 & 86 & 1 & 0 \\
\hline 32 & 86 & 88 & 1 & 0 \\
\hline 33 & 88 & 91 & 1 & 0 \\
\hline 34 & 7 & 12 & 1 & 0 \\
\hline 35 & 12 & 14 & 1 & 0 \\
\hline 36 & 14 & 17 & 1 & 0 \\
\hline 37 & 17 & 19 & 1 & 0 \\
\hline 38 & 19 & 22 & 1 & 0 \\
\hline 39 & 22 & 24 & 1 & 0 \\
\hline 40 & 24 & 27 & 1 & 0 \\
\hline 41 & 27 & 29 & 1 & 0 \\
\hline 42 & 29 & 32 & 1 & 0 \\
\hline 43 & 32 & 34 & 1 & 0 \\
\hline 44 & 34 & 37 & 1 & 0 \\
\hline 45 & 37 & 39 & 1 & 0 \\
\hline
\end{tabular}

\begin{tabular}{|c|c|c|c|c|}
\hline 63 & 82 & 84 & 1 & 0 \\
\hline 64 & 84 & 87 & 1 & 0 \\
\hline 65 & 87 & 89 & 1 & 0 \\
\hline 66 & 89 & 92 & 1 & 0 \\
\hline 67 & 92 & 95 & 1 & 0 \\
\hline 68 & 95 & 97 & 1 & 0 \\
\hline 69 & 97 & 98 & 1 & 0 \\
\hline 70 & 98 & 99 & 1 & 0 \\
\hline 71 & 99 & 100 & 1 & 0 \\
\hline 72 & 1 & 2 & 2 & 0 \\
\hline 73 & 2 & 3 & 3 & 0 \\
\hline 74 & 3 & 4 & 4 & 0 \\
\hline 75 & 4 & 5 & 5 & 0 \\
\hline 76 & 5 & 6 & 6 & 0 \\
\hline 77 & 6 & 8 & 7 & 0 \\
\hline 78 & 8 & 9 & 8 & 0 \\
\hline 79 & 9 & 10 & 9 & 0 \\
\hline 80 & 10 & 15 & 10 & 0 \\
\hline 81 & 15 & 20 & 11 & 0 \\
\hline 82 & 20 & 25 & 12 & 0 \\
\hline 83 & 25 & 30 & 13 & 0 \\
\hline 84 & 30 & 35 & 14 & 0 \\
\hline 85 & 35 & 40 & 15 & 0 \\
\hline 86 & 40 & 45 & 16 & 0 \\
\hline 87 & 45 & 50 & 17 & 0 \\
\hline 88 & 50 & 55 & 18 & 0 \\
\hline 89 & 55 & 60 & 19 & 0 \\
\hline 90 & 60 & 65 & 20 & 0 \\
\hline
\end{tabular}

\begin{tabular}{|c|c|c|c|c|}
\hline 108 & 35 & 36 & 33 & 0 \\
\hline 109 & 35 & 37 & 33 & $\overline{0}$ \\
\hline 110 & 40 & 41 & 34 & 0 \\
\hline 111 & 40 & 42 & 34 & 0 \\
\hline 112 & 45 & 46 & 35 & 0 \\
\hline 113 & 45 & 47 & 35 & 0 \\
\hline 114 & 50 & 51 & 36 & 0 \\
\hline 115 & 50 & 52 & 36 & 0 \\
\hline 116 & 55 & 56 & 37 & 0 \\
\hline 117 & 55 & 57 & 37 & 0 \\
\hline 118 & 60 & 61 & 38 & 0 \\
\hline 119 & 60 & 62 & 39 & 0 \\
\hline 120 & 65 & 66 & 40 & 0 \\
\hline 121 & 65 & 67 & 41 & 0 \\
\hline 122 & 70 & 71 & 42 & 0 \\
\hline 123 & 70 & 72 & 43 & 0 \\
\hline 124 & 75 & 76 & 44 & 0 \\
\hline 125 & 75 & 77 & 45 & 0 \\
\hline 126 & 80 & 81 & 46 & 0 \\
\hline 127 & 80 & 82 & 47 & 0 \\
\hline 128 & 85 & 86 & 48 & 0 \\
\hline 129 & 85 & 87 & 49 & 0 \\
\hline 130 & 90 & 91 & 50 & 0 \\
\hline 131 & 90 & 92 & 51 & 0 \\
\hline 132 & 99 & 101 & 52 & $\overline{0}$ \\
\hline 133 & 101 & 102 & 54 & 0 \\
\hline 134 & 91 & 94 & 52 & 0 \\
\hline 135 & 94 & 96 & 53 & 0 \\
\hline 136 & 6 & 7 & 6 & 2 \\
\hline
\end{tabular}

COORDENADAS DE LOS NUDOS

\begin{tabular}{|c|c|c|}
\hline Nudo & $\mathrm{X}$ & $\mathrm{Y}$ \\
\hline 1 & 0.00 & -1.50 \\
\hline 2 & 0.00 & 0.00 \\
\hline 3 & 0.00 & 5.38 \\
\hline 4 & 0.00 & 10.75 \\
\hline 5 & 0.00 & 16.13 \\
\hline 6 & 0.00 & 21.49 \\
\hline 7 & 0.00 & 21.50 \\
\hline 8 & 0.00 & 25.80 \\
\hline 9 & 0.00 & 30.10 \\
\hline 10 & 0.00 & 34.40 \\
\hline 11 & 5.00 & 21.50 \\
\hline 12 & -5.00 & 21.50 \\
\hline 13 & 7.00 & 21.50 \\
\hline 14 & -7.00 & 21.50 \\
\hline 15 & 0.00 & 37.30 \\
\hline 16 & 10.00 & 21.50 \\
\hline 17 & -10.00 & 21.50 \\
\hline
\end{tabular}

\begin{tabular}{|c|c|c|}
\hline Nudo & $\mathrm{X}$ & $\mathrm{Y}$ \\
\hline 35 & 0.00 & 44.85 \\
\hline 36 & 30.00 & 21.50 \\
\hline 37 & -30.00 & 21.50 \\
\hline 38 & 32.00 & 21.50 \\
\hline 39 & -32.00 & 21.50 \\
\hline 40 & 0.00 & 46.70 \\
\hline 41 & 35.00 & 21.50 \\
\hline 42 & -35.00 & 21.50 \\
\hline 43 & 37.00 & 21.50 \\
\hline 44 & -37.00 & 21.50 \\
\hline 45 & 0.00 & 48.50 \\
\hline 46 & 40.00 & 21.50 \\
\hline 47 & -40.00 & 21.50 \\
\hline 48 & 42.00 & 21.50 \\
\hline 49 & -42.00 & 21.50 \\
\hline 50 & 0.00 & 50.25 \\
\hline 51 & 45.00 & 21.50 \\
\hline
\end{tabular}

\begin{tabular}{|c|c|c|}
\hline Nudo & $\mathrm{X}$ & $\mathrm{Y}$ \\
\hline 69 & -62.00 & 21.50 \\
\hline 70 & 0.00 & 56.27 \\
\hline 71 & 65.00 & 21.50 \\
\hline 72 & -65.00 & 21.50 \\
\hline 73 & 67.00 & 21.50 \\
\hline 74 & -67.00 & 21.50 \\
\hline 75 & 0.00 & 57.60 \\
\hline 76 & 70.00 & 21.50 \\
\hline 77 & -70.00 & 21.50 \\
\hline 78 & 72.00 & 21.50 \\
\hline 79 & -72.00 & 21.50 \\
\hline 80 & 0.00 & 58.90 \\
\hline 81 & 75.00 & 21.50 \\
\hline 82 & -75.00 & 21.50 \\
\hline 83 & 77.00 & 21.50 \\
\hline 84 & -77.00 & 21.50 \\
\hline 85 & 0.00 & 60.15 \\
\hline
\end{tabular}




\begin{tabular}{|c|c|c|}
\hline 18 & 12.00 & 21.50 \\
\hline 19 & -12.00 & 21.50 \\
\hline 20 & 0.00 & 39.30 \\
\hline 21 & 15.00 & 21.50 \\
\hline 22 & -15.00 & 21.50 \\
\hline 23 & 17.00 & 21.50 \\
\hline 24 & -17.00 & 21.50 \\
\hline 25 & 0.00 & 41.15 \\
\hline 26 & 20.00 & 21.50 \\
\hline 27 & -20.00 & 21.50 \\
\hline 28 & 22.00 & 21.50 \\
\hline 29 & -22.00 & 21.50 \\
\hline 30 & 0.00 & 43.00 \\
\hline 31 & 25.00 & 21.50 \\
\hline 32 & -25.00 & 21.50 \\
\hline 33 & 27.00 & 21.50 \\
\hline 34 & -27.00 & 21.50 \\
\hline
\end{tabular}

\begin{tabular}{|c|c|c|}
\hline 52 & -45.00 & 21.50 \\
\hline 53 & 47.00 & 21.50 \\
\hline 54 & -47.00 & 21.50 \\
\hline 55 & 0.00 & 51.90 \\
\hline 56 & 50.00 & 21.50 \\
\hline 57 & -50.00 & 21.50 \\
\hline 58 & 52.00 & 21.50 \\
\hline 59 & -52.00 & 21.50 \\
\hline 60 & 0.00 & 53.45 \\
\hline 61 & 55.00 & 21.50 \\
\hline 62 & -55.00 & 21.50 \\
\hline 63 & 57.00 & 21.50 \\
\hline 64 & -57.00 & 21.50 \\
\hline 65 & 0.00 & 54.90 \\
\hline 66 & 60.00 & 21.50 \\
\hline 67 & -60.00 & 21.50 \\
\hline 68 & 62.00 & 21.50 \\
\hline
\end{tabular}

\begin{tabular}{|c|c|c|}
\hline 86 & 80.00 & 21.50 \\
\hline 87 & -80.00 & 21.50 \\
\hline 88 & 82.00 & 21.50 \\
\hline 89 & -82.00 & 21.50 \\
\hline 90 & 0.00 & 61.40 \\
\hline 91 & 85.00 & 21.50 \\
\hline 92 & -85.00 & 21.50 \\
\hline 93 & 0.00 & 64.00 \\
\hline 94 & 85.00 & 21.40 \\
\hline 95 & -86.50 & 21.50 \\
\hline 96 & 85.00 & 11.50 \\
\hline 97 & -88.00 & 21.50 \\
\hline 98 & -89.50 & 21.50 \\
\hline 99 & -91.00 & 21.50 \\
\hline 100 & -92.00 & 21.50 \\
\hline 101 & -91.00 & 21.40 \\
\hline 102 & -91.00 & -0.50 \\
\hline
\end{tabular}

\section{CONDICIONES DE CONTORNO}

\begin{tabular}{|c|c|c|c|}
\hline Nudo & Coac.-X & Coac.-Y & Giro \\
\hline 1 & 1 & 1 & 1 \\
\hline 96 & 1 & 1 & 1 \\
\hline 102 & 1 & 1 & 1 \\
\hline
\end{tabular}

\section{CARACTERISTICAS MECÁNICAS DE LAS BARRAS}

\begin{tabular}{|c|c|c|c|}
\hline Tipo & Area & Inercia & Mod.Elast. \\
\hline 1 & 9.998 & 5.265 & 30900000 \\
\hline 2 & 154 & 1552.8 & 29800000 \\
\hline 3 & 15.874 & 34.249 & 29800000 \\
\hline 4 & 15.542 & 31.55 & 29800000 \\
\hline 5 & 15.21 & 28.993 & 29800000 \\
\hline 6 & 14.877 & 26.564 & 29800000 \\
\hline 7 & 14.579 & 24.49 & 29800000 \\
\hline 8 & 14.313 & 22.731 & 29800000 \\
\hline 9 & 14.047 & 21.054 & 29800000 \\
\hline 10 & 19.97 & 22.51 & 29800000 \\
\hline 11 & 16.67 & 10.76 & 29800000 \\
\hline 12 & 16.18 & 9.62 & 29800000 \\
\hline 13 & 15.67 & 8.47 & 29800000 \\
\hline 14 & 15.5 & 8.19 & 29800000 \\
\hline 15 & 13.22 & 4.08 & 29800000 \\
\hline 16 & 13.17 & 4.08 & 29800000 \\
\hline 17 & 13.12 & 4.08 & 29800000 \\
\hline 18 & 12.76 & 3.65 & 29800000 \\
\hline 19 & 12.59 & 3.5 & 29800000 \\
\hline 20 & 12.51 & 3.45 & 29800000 \\
\hline 21 & 12.44 & 3.4 & 29800000 \\
\hline 22 & 12.4 & 3.4 & 29800000 \\
\hline 23 & 12.36 & 3.4 & 29800000 \\
\hline 24 & 12.33 & 3.4 & 29800000 \\
\hline 25 & 12.18 & 3.26 & 29800000 \\
\hline 26 & 14.66 & 8.29 & 29800000 \\
\hline 27 & 0 & 0 & 195000000 \\
\hline
\end{tabular}

\begin{tabular}{|c|c|c|c|}
\hline Tiрo & Area & Inercia & Mod.Elast. \\
\hline 28 & 0.003574 & 0 & 195000000 \\
\hline 29 & 0.003944 & 0 & 19500000 \\
\hline 30 & 0.004358 & 0 & 19500000 \\
\hline 31 & 0.004755 & 0 & 19500000 \\
\hline 32 & 0.00511 & 0 & 19500000 \\
\hline 33 & 0.005425 & 0 & 19500000 \\
\hline 34 & 0.005703 & 0 & 195000000 \\
\hline 35 & 0.005956 & 0 & 195000000 \\
\hline 36 & 0.006189 & 0 & 195000000 \\
\hline 37 & 0.006414 & 0 & 195000000 \\
\hline 38 & 0.006633 & 0 & 195000000 \\
\hline 39 & 0.006635 & 0 & 195000000 \\
\hline 40 & 0.006855 & 0 & 195000000 \\
\hline 41 & 0.006848 & 0 & 195000000 \\
\hline 42 & 0.007048 & 0 & 195000000 \\
\hline 43 & 0.007075 & 0 & 19500000 \\
\hline 44 & 0.007335 & 0 & 19500000 \\
\hline 45 & 0.007229 & 0 & 195000000 \\
\hline 46 & 0.007218 & 0 & 19500000 \\
\hline 47 & 0.007624 & 0 & 19500000 \\
\hline 48 & 0.008613 & 0 & 19500000 \\
\hline 49 & 0.007059 & 0 & 19500000 \\
\hline 50 & 0.008612 & 0 & 19500000 \\
\hline 51 & 0.009809 & 0 & 19500000 \\
\hline 52 & 0.6 & 0 & 1000000 \\
\hline 53 & 54.4 & 72.5 & 27300000 \\
\hline 54 & 3.85 & 0.39 & 27300000 \\
\hline 55 & 0 & 25 & 29800000 \\
\hline & & & \\
\hline
\end{tabular}


TOPOLOGIA DE LAS BARRAS

\begin{tabular}{|c|c|c|c|c|}
\hline Barra & $\begin{array}{c}\text { Nudo } \\
\text { Dorsal }\end{array}$ & $\begin{array}{l}\text { Nudo } \\
\text { Frontal }\end{array}$ & Tіро & Conex. \\
\hline 1 & 7 & 11 & 1 & 0 \\
\hline 2 & 11 & 13 & 1 & 0 \\
\hline 3 & 13 & 16 & 1 & 0 \\
\hline 4 & 16 & 18 & 1 & 0 \\
\hline 5 & 18 & 21 & 1 & 0 \\
\hline 6 & 21 & 23 & 1 & 0 \\
\hline 7 & 23 & 26 & 1 & 0 \\
\hline 8 & 26 & 28 & 1 & 0 \\
\hline 9 & 28 & 31 & 1 & 0 \\
\hline 10 & 31 & 33 & 1 & 0 \\
\hline 11 & 33 & 36 & 1 & 0 \\
\hline 12 & 36 & 38 & 1 & 0 \\
\hline 13 & 38 & 41 & 1 & 0 \\
\hline 14 & 41 & 43 & 1 & 0 \\
\hline 15 & 43 & 46 & 1 & 0 \\
\hline 16 & 46 & 48 & 1 & 0 \\
\hline 17 & 48 & 51 & 1 & 0 \\
\hline 18 & 51 & 53 & 1 & 0 \\
\hline 19 & 53 & 56 & 1 & 0 \\
\hline 20 & 56 & 58 & 1 & 0 \\
\hline 21 & 58 & 61 & 1 & 0 \\
\hline 22 & 61 & 63 & 1 & 0 \\
\hline 23 & 63 & 66 & 1 & 0 \\
\hline 24 & 66 & 68 & 1 & 0 \\
\hline 25 & 68 & 71 & 1 & 0 \\
\hline 26 & 71 & 73 & 1 & 0 \\
\hline 27 & 73 & 76 & 1 & 0 \\
\hline 28 & 76 & 78 & 1 & 0 \\
\hline 29 & 78 & 81 & 1 & 0 \\
\hline 30 & 81 & 83 & 1 & 0 \\
\hline 31 & 83 & 86 & 1 & 0 \\
\hline 32 & 86 & 88 & 1 & 0 \\
\hline 33 & 88 & 91 & 1 & 0 \\
\hline 34 & 7 & 12 & 1 & 0 \\
\hline 35 & 12 & 14 & 1 & 0 \\
\hline 36 & 14 & 17 & 1 & 0 \\
\hline 37 & 17 & 19 & 1 & 0 \\
\hline 38 & 19 & 22 & 1 & 0 \\
\hline 39 & 22 & 24 & 1 & 0 \\
\hline 40 & 24 & 27 & 1 & 0 \\
\hline 41 & 27 & 29 & 1 & 0 \\
\hline 42 & 29 & 32 & 1 & 0 \\
\hline 43 & 32 & 34 & 1 & 0 \\
\hline 44 & 34 & 37 & 1 & 0 \\
\hline 45 & 37 & 39 & 1 & 0 \\
\hline
\end{tabular}

\begin{tabular}{|c|c|c|c|c|}
\hline Barra & $\begin{array}{c}\text { Nudo } \\
\text { Dorsal }\end{array}$ & $\begin{array}{c}\text { Nudo } \\
\text { Frontal }\end{array}$ & Tipo & Conex. \\
\hline 46 & 39 & 42 & 1 & 0 \\
\hline 47 & 42 & 44 & 1 & 0 \\
\hline 48 & 44 & 47 & 1 & 0 \\
\hline 49 & 47 & 49 & 1 & 0 \\
\hline 50 & 49 & 52 & 1 & 0 \\
\hline 51 & 52 & 54 & 1 & 0 \\
\hline 52 & 54 & 57 & 1 & 0 \\
\hline 53 & 57 & 59 & 1 & 0 \\
\hline 54 & 59 & 62 & 1 & 0 \\
\hline 55 & 62 & 64 & 1 & 0 \\
\hline 56 & 64 & 67 & 1 & 0 \\
\hline 57 & 67 & 69 & 1 & 0 \\
\hline 58 & 69 & 72 & 1 & 0 \\
\hline 59 & 72 & 74 & 1 & 0 \\
\hline 60 & 74 & 77 & 1 & 0 \\
\hline 61 & 77 & 79 & 1 & 0 \\
\hline 62 & 79 & 82 & 1 & 0 \\
\hline 63 & 82 & 84 & 1 & 0 \\
\hline 64 & 84 & 87 & 1 & 0 \\
\hline 65 & 87 & 89 & 1 & 0 \\
\hline 66 & 89 & 92 & 1 & 0 \\
\hline 67 & 92 & 95 & 1 & 0 \\
\hline 68 & 95 & 97 & 1 & 0 \\
\hline 69 & 97 & 98 & 1 & 0 \\
\hline 70 & 98 & 99 & 1 & 0 \\
\hline 71 & 99 & 100 & 1 & 0 \\
\hline 72 & 1 & 2 & 2 & 0 \\
\hline 73 & 2 & 3 & 3 & 0 \\
\hline 74 & 3 & 4 & 4 & 0 \\
\hline 75 & 4 & 5 & 5 & 0 \\
\hline 76 & 5 & 6 & 6 & 0 \\
\hline 77 & 6 & 8 & 7 & 0 \\
\hline 78 & 8 & 9 & 8 & 0 \\
\hline 79 & 9 & 10 & 9 & 0 \\
\hline 80 & 10 & 15 & 10 & 0 \\
\hline 81 & 15 & 20 & 11 & 0 \\
\hline 82 & 20 & 25 & 12 & 0 \\
\hline 83 & 25 & 30 & 13 & 0 \\
\hline 84 & 30 & 35 & 14 & 0 \\
\hline 85 & 35 & 40 & 15 & 0 \\
\hline 86 & 40 & 45 & 16 & 0 \\
\hline 87 & 45 & 50 & 17 & 0 \\
\hline 88 & 50 & 55 & 18 & 0 \\
\hline 89 & 55 & 60 & 19 & 0 \\
\hline 90 & 60 & 65 & 20 & 0 \\
\hline & & & & \\
\hline
\end{tabular}

\begin{tabular}{|c|c|c|c|c|}
\hline Barra & $\begin{array}{c}\text { Nudo } \\
\text { Dorsal }\end{array}$ & $\begin{array}{l}\text { Nudo } \\
\text { Frontal }\end{array}$ & Tipo & Conex. \\
\hline 91 & 65 & 70 & 21 & 0 \\
\hline 92 & 70 & 75 & 22 & 0 \\
\hline 93 & 75 & 80 & 23 & 0 \\
\hline 94 & 80 & 85 & 24 & 0 \\
\hline 95 & 85 & 90 & 25 & 0 \\
\hline 96 & 90 & 93 & 26 & 0 \\
\hline 97 & 7 & 10 & 27 & 0 \\
\hline 98 & 10 & 11 & 28 & 0 \\
\hline 99 & 10 & 12 & 28 & 0 \\
\hline 100 & 15 & 16 & 29 & 0 \\
\hline 101 & 15 & 17 & 29 & 0 \\
\hline 102 & 20 & 21 & 30 & 0 \\
\hline 103 & 20 & 22 & 30 & 0 \\
\hline 104 & 25 & 26 & 31 & 0 \\
\hline 105 & 25 & 27 & 31 & 0 \\
\hline 106 & 30 & 31 & 32 & 0 \\
\hline 107 & 30 & 32 & 32 & 0 \\
\hline 108 & 35 & 36 & 33 & 0 \\
\hline 109 & 35 & 37 & 33 & 0 \\
\hline 110 & 40 & 41 & 34 & 0 \\
\hline 111 & 40 & 42 & 34 & 0 \\
\hline 112 & 45 & 46 & 35 & 0 \\
\hline 113 & 45 & 47 & 35 & 0 \\
\hline 114 & 50 & 51 & 36 & 0 \\
\hline 115 & 50 & 52 & 36 & 0 \\
\hline 116 & 55 & 56 & 37 & 0 \\
\hline 117 & 55 & 57 & 37 & 0 \\
\hline 118 & 60 & 61 & 38 & 0 \\
\hline 119 & 60 & 62 & 39 & 0 \\
\hline 120 & 65 & 66 & 40 & 0 \\
\hline 121 & 65 & 67 & 41 & 0 \\
\hline 122 & 70 & 71 & 42 & 0 \\
\hline 123 & 70 & 72 & 43 & 0 \\
\hline 124 & 75 & 76 & 44 & 0 \\
\hline 125 & 75 & 77 & 45 & 0 \\
\hline 126 & 80 & 81 & 46 & 0 \\
\hline 127 & 80 & 82 & 47 & 0 \\
\hline 128 & 85 & 86 & 48 & 0 \\
\hline 129 & 85 & 87 & 49 & 0 \\
\hline 130 & 90 & 91 & 50 & 0 \\
\hline 131 & 90 & 92 & 51 & 0 \\
\hline 132 & 99 & 101 & 52 & 0 \\
\hline 133 & 101 & 102 & 54 & 0 \\
\hline 134 & 91 & 94 & 52 & 0 \\
\hline 135 & 94 & 96 & 53 & 0 \\
\hline 136 & 6 & 7 & 6 & 2 \\
\hline
\end{tabular}


A.2. ARCHIVOS LOGICOS PARA DEFINICION DEL PROCESO CONSTRUCTIVO.

1) ARCHIVO PARA DESMONTAJE MODELO 90 EN FUERZAS.

Fase-1

- Colocación esfuerzos viga continua

L00 Base.dat

Coord.dat

Coacc. dat

Tipobarr.dat

Topologi.dat

1 Estado0.prn

Fase-2

Quitar C.M.

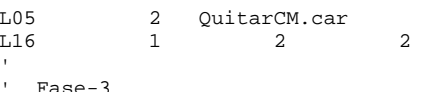

Fase-3

Quitar Dovela18I

Barra-71

L05 3 QuitarPPBarra71.car

L16 $23 \quad 3 \quad 3$

$\begin{array}{llll}\text { LEB } & \text { Barra-132 } & & \\ & 132 & 3 & 4\end{array}$

L05 5 QuitarPPBarra70.car

$\begin{array}{llll}\text { L05 } & 5 & \text { Quita } \\ \text { L16 } & 4 & 5 & 5\end{array}$

16 45 Barra- 69

L05 6 QuitarPPBarra69.car

L16 566

L05 7 QuitarPPBarra68.car

L16 677

EB Quitar barra 13

$\begin{array}{llll}\text { L16 } & 8 & 8 & 3\end{array}$

Fase-4

Quitar Tirant18D

$130^{\text {Barra-130 }}$

Quitar Dovela17D

Barra-33

L05 5 QuitarPPDovela17D.car

$\begin{array}{llll}16 & 4 & 5 & 5\end{array}$

L16

Fase-6

Quitar Tirant18

Barra-131

131

Quitar Dovela17I

Barras 67 y 66

L05 7 QuitarPPDovela17I.car

L16 676

Fase-7

Quitar Cargas de los dos carros de avance

- correspondientes a las dovelas 17I y 17D

L05 7 QuitarDosCarrosDovelas17.car

16 - 77

Fase-8

Quitar Tirant17D

' Barra-128

LEB

128

Quitar Dovela16D

Barras 32 y 31

L05 9 QuitarPPDovela16D.car

L16 $8 \begin{array}{lll}8 & 9 & 9\end{array}$

Fase-10

Quitar Tirant17I

Barra-129

10

Quitar Dovela16I

Barras 65 y 64

05 11 QuitarPPDovela16I.car

L16 $10 \quad 11 \quad 10$

Fase-11

- Ouitar Cargas de los dos carros de avance

correspondientes a las dovelas 16I y 16D

L05 11 QuitarDosCarrosDovelas16.car

L16 $1011 \quad 11$ 
' Fase-12

; Quitar Tirant16D

LEB $\quad \begin{array}{lll}\text { Barra-126 } & & \\ 126 & 11 & 12\end{array}$

- Quitar Dovela15D

' Barras 30 y 29

L05 13 QuitarPPDovela15D.car

L16 $12 \quad 13 \quad 13$

Fase-14

Quitar Tirant16I

$\begin{array}{lcll} & \text { Barra-127 } & & \\ \text { LEB } & 127 & 13 & 14\end{array}$

' Quitar Dovela15I

Barras 63 y 62

0515 QuitarPPDovela15I.car

$\begin{array}{llll}\text { L16 } & 14 & 15 & 14\end{array}$

Fase-15

Quitar Cargas de los dos carros de avance

correspondientes a las dovelas 15I y 15D

L05 15 QuitarDosCarrosDovelas15.car

L16 $14 \quad 1515$

Fase-16

Quitar Tirant15D

$\begin{array}{llll}\text { 'LEB } & \text { Barra-124 } & & \\ \text { ' } & 124 & 15 & 16\end{array}$

Quitar Dovela14D

Barras 28 y 27

L05 17 QuitarPPDovela14D.car

$\begin{array}{llll}\text { L16 } & 16 & 17 & 17\end{array}$

Fase-18

Quitar Tirant15I

LEB $\quad$ Barra-125 125

' Quitar Dovela14I

Barras 61 y 60

L05 19 QuitarPPDovela14I.car

$\begin{array}{llll}\text { L16 } & 18 & 19 & 18\end{array}$

- Fase-19

Quitar Cargas de los dos carros de avance - correspondientes a las dovelas 14I y 14D L05 19 QuitarDosCarrosDovelas14.car

L16 $18 \quad 1919$

Fase-20

Quitar Tirant14D

$\begin{array}{lcll} & \text { Barra-122 } & & \\ \text { LEB } & 122 & 19 & 20\end{array}$

' Quitar Dovela13D

Barras 26 y 25

L05 21 QuitarPPDovela13D.car

$\begin{array}{llll}\text { L16 } & 20 & 21 & 21\end{array}$

Fase-22

Quitar Tirant14I

BeB Barra-123 123

' Quitar Dovela13I

Barras 59 y 58

L05 23 QuitarPPDovela13I.car

L16 $22 \quad 23 \quad 22$

' Fase-23

Quitar Cargas de los dos carros de avance

correspondientes a las dovelas 13I y 13D

L05 23 QuitarDosCarrosDovelas13.car

L16 $22 \quad 23 \quad 23$

, Fase-24

Fase-24
Quitar Tirant13D

- Quitar Tirant13D

LEB 120

$23 \quad 24$

Quitar Dovela12D

Barras 24 y 23

L05 25 QuitarPPDovela12D.car

L16 $24 \quad 2525$

Fase-26

Quitar Tirant13I

Barra-121

$\begin{array}{llll} & 121 & 25 & 26\end{array}$

Quitar Dovela12I

rras 57 y 56

05 27 QuitarPPDovela12I.car

L16 $26 \quad 27 \quad 26$

' Fase-27

' Quitar Cargas de los dos carros de avance

' Correspondientes a las dovelas $12 \mathrm{I}$ y 12D

L05 27 QuitarDosCarrosDovelas12.car

$\begin{array}{lll}10 & 26 & 27 \\ \text { L16 }\end{array}$ 
' Fase-28

' Quitar Tirant12D

$\begin{array}{llll} & \text { Barra-118 } & & \\ \text { LEB } & 118 & 27\end{array}$

' Quitar Dovela11D

Barras 22 y 21

L05 29 QuitarPPDovela11D.car

$\begin{array}{llll}\text { L16 } & 28 & 29 & 29\end{array}$

Fase-30

'Quitar Tirant12I

$\begin{array}{lll}\text { Barra-119 } & 29 & \\ 119 & 29\end{array}$

' Quitar Dovela11I

, Quitar Dovela11I

L05 31 QuitarPPDovela11I.car

$\begin{array}{llll}\text { L16 } & 30 & 31 & 30\end{array}$

Fase-31

Quitar Cargas de los dos carros de avance

correspondientes a las dovelas 11I y 11D

L05 31 QuitarDosCarrosDovelas11.car

L16 $30 \quad 3131$

Fase-32

' Quitar Tirant11D

Barra-116

$\begin{array}{llll} & 116 & 31 & 32\end{array}$

Quitar Dovela10D

Barras 20 y 1

L05 33 QuitarPPDovela10D.car

$\begin{array}{llll}116 & 32 & 33 & 33\end{array}$

Fase-34

'Quitar Tirant11I

$\begin{array}{lcll} & \text { Barra-117 } & & \\ \text { LEB } & 117 & 33 & 34\end{array}$

' Quitar Dovela10I

Barras 53 y 52

L05 35 QuitarPPDovela10I.car

L16 $34 \quad 35 \quad 34$

' Fase-35

- Quitar Cargas de los dos carros de avance ' correspondientes a las dovelas 10I y 10D L05 35 QuitarDosCarrosDovelas10.car

$\begin{array}{llll}\text { L1 } & 34 & 35 & 35\end{array}$

Fase-36

Quitar Tirant10D

$\begin{array}{llll}\text { LEB } & \text { Barra-114 } & & \\ \text { LE } & 114 & 35\end{array}$

' Quitar Dovela9d

Barras 18 y 17

L05 37 QuitarPPDovela09D.car

L16 $36 \quad 37 \quad 37$

Fase-38

Quitar Tirant10I

Barra-115

LEB 115

$37 \quad 38$

Quitar Dovela9I

Barras 51 y 50

L05 39 QuitarPPDovela09I.car

L16 $38 \quad 3938$

Fase-39

Quitar Cargas de los dos carros de avance

correspondientes a las dovelas 9I y 9D

L05 39 QuitarDosCarrosDovelas09.car

L16 $38 \quad 3939$

Fase-40

Quitar Tirant9D

- Quitar Tirant9D

LEB 112

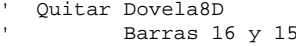

L05 41 QuitarPPDovela08D.car

L16 $40 \quad 41 \quad 41$

Fase-42

Quitar Tirant9I

Barra-113

$\begin{array}{lll}\text { LEB } & 113 & 41\end{array}$

' Quitar Dovela8I

Barras 49 y 48

L05 43 QuitarPPDovela08I.car

L16 $42 \quad 43 \quad 42$

' Fase-43

- Quitar Cargas de los dos carros de avance

- correspondientes a las dovelas 8I y 8D

L05 43 QuitarDosCarrosDovelas08. car 


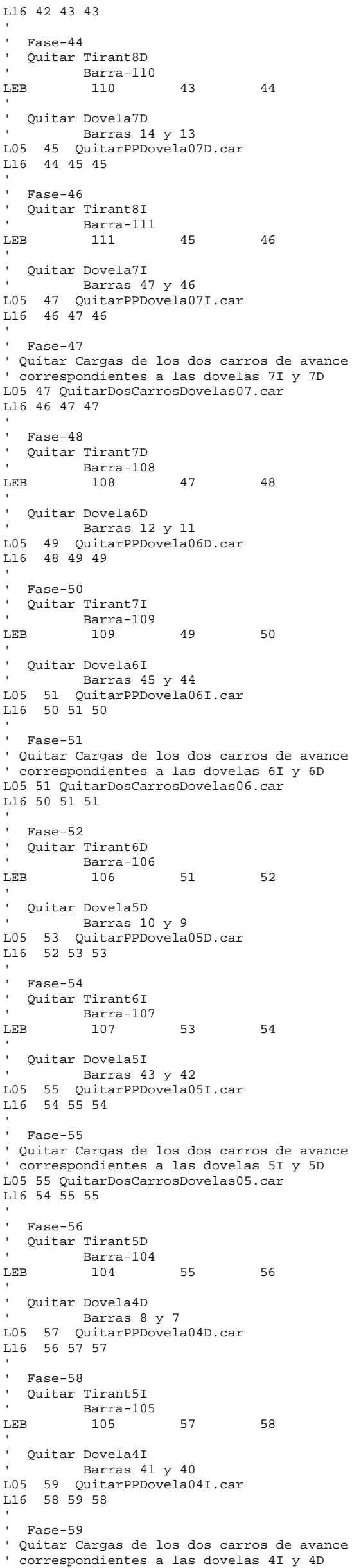




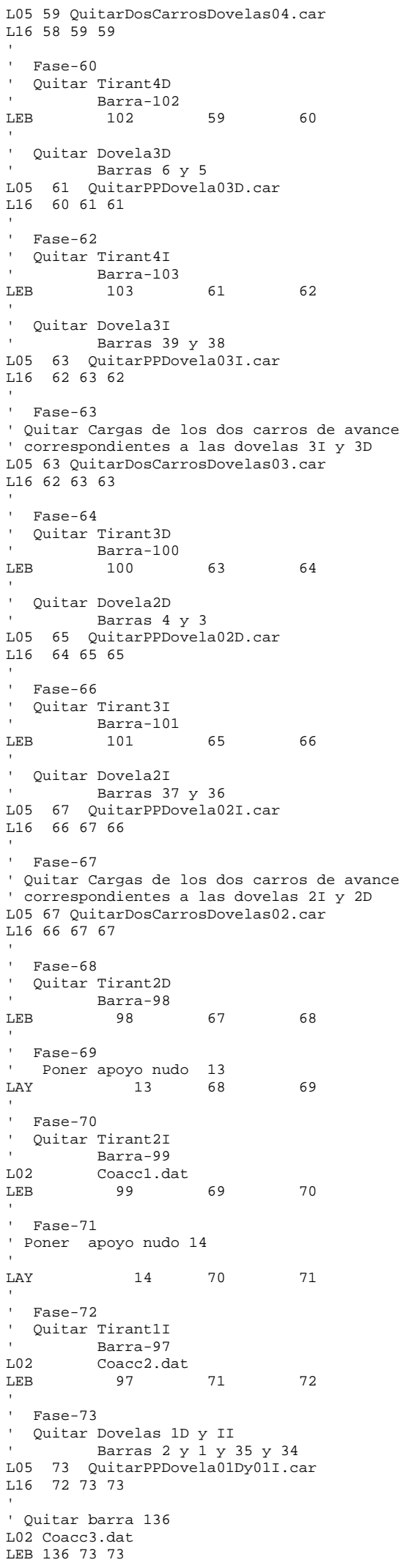




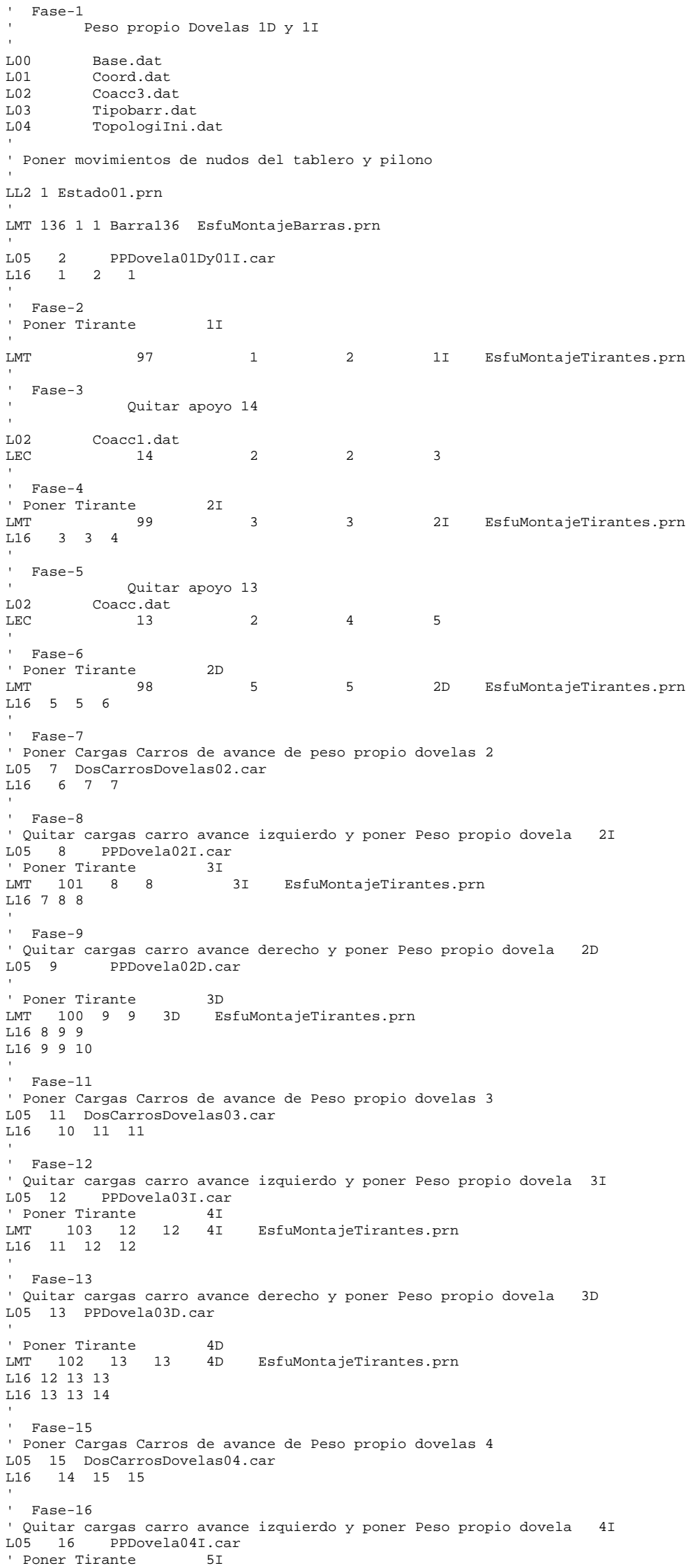




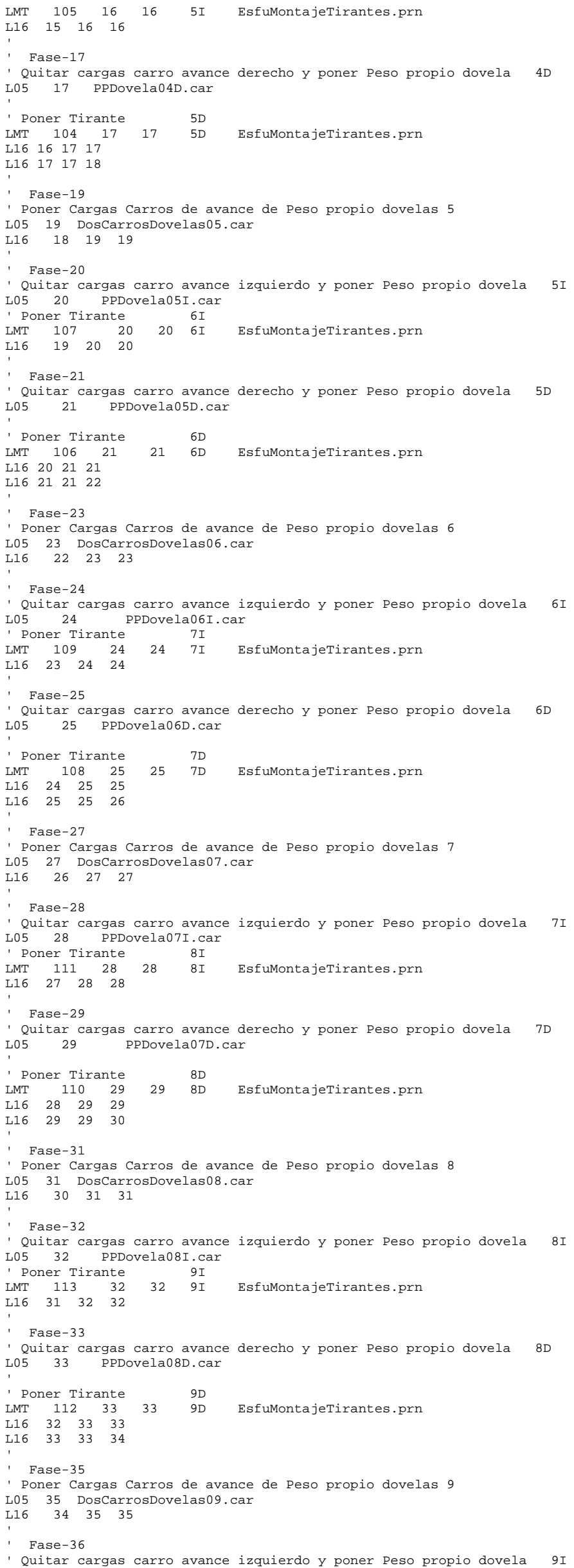




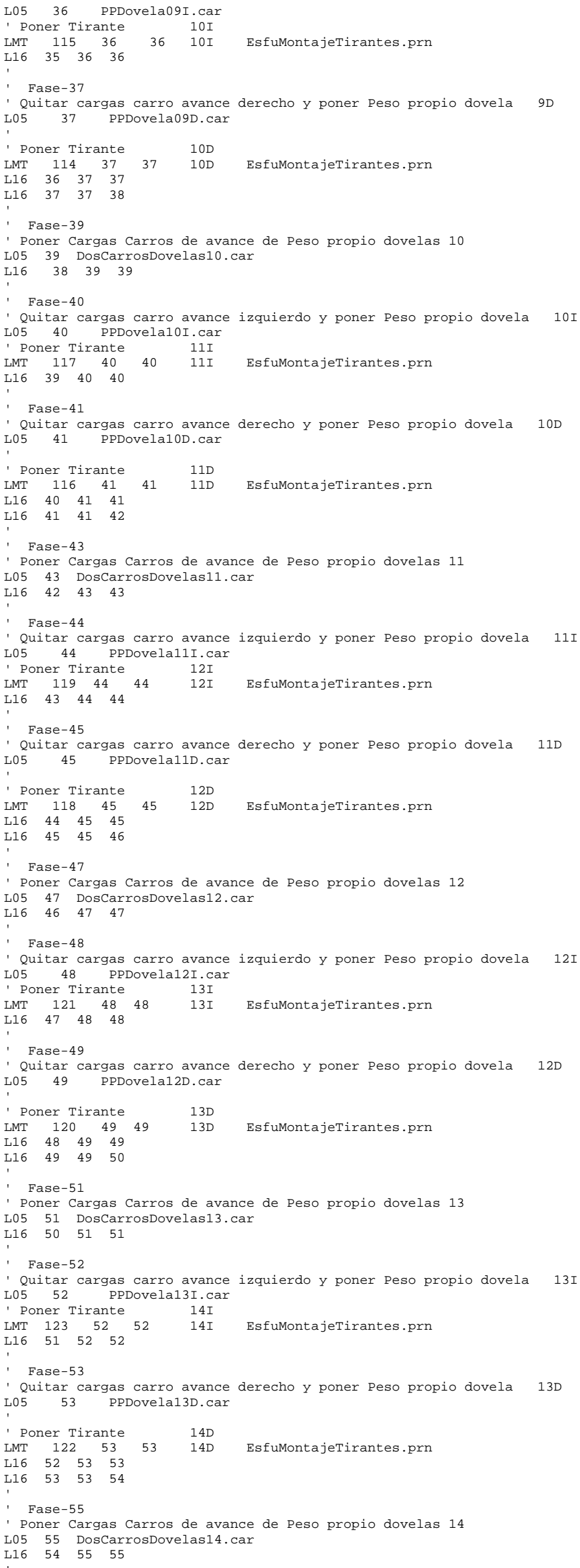




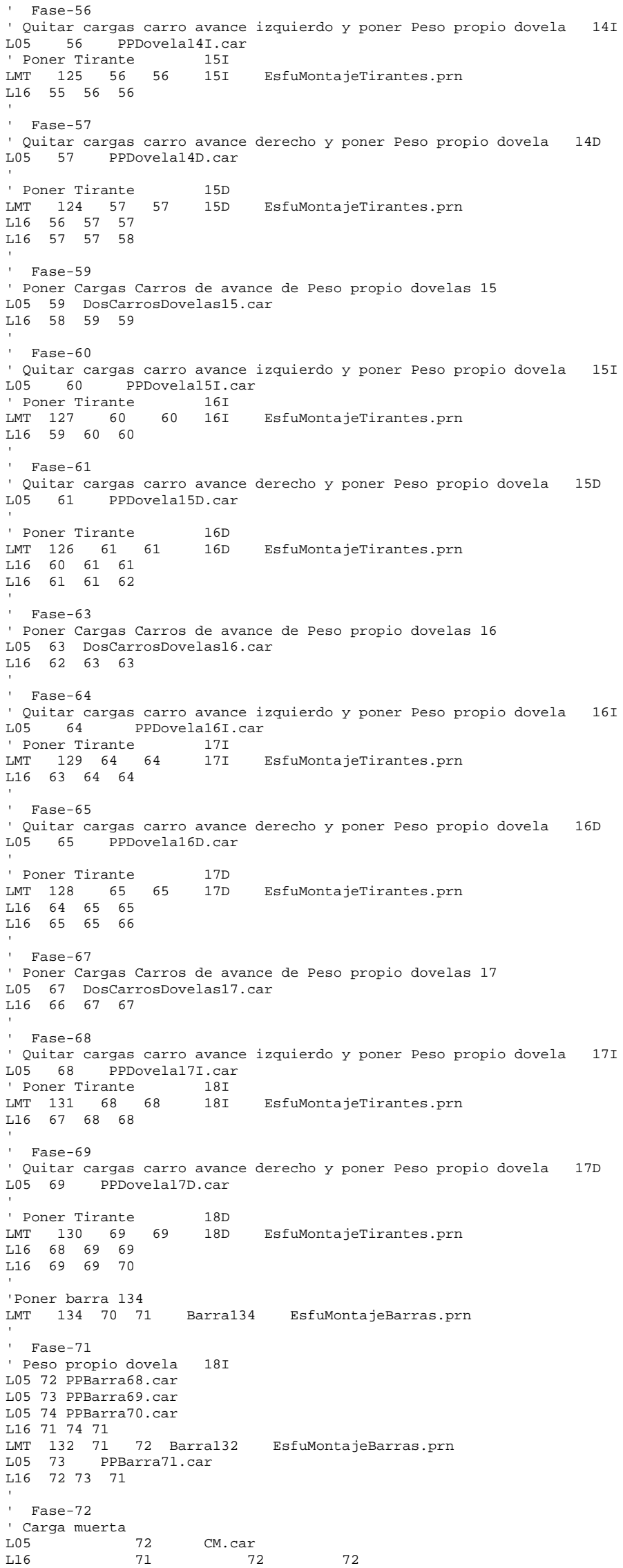


3) ARCHIVO PARA MONTAJE DEL MODELO 90 EN DEFORMACIONES.

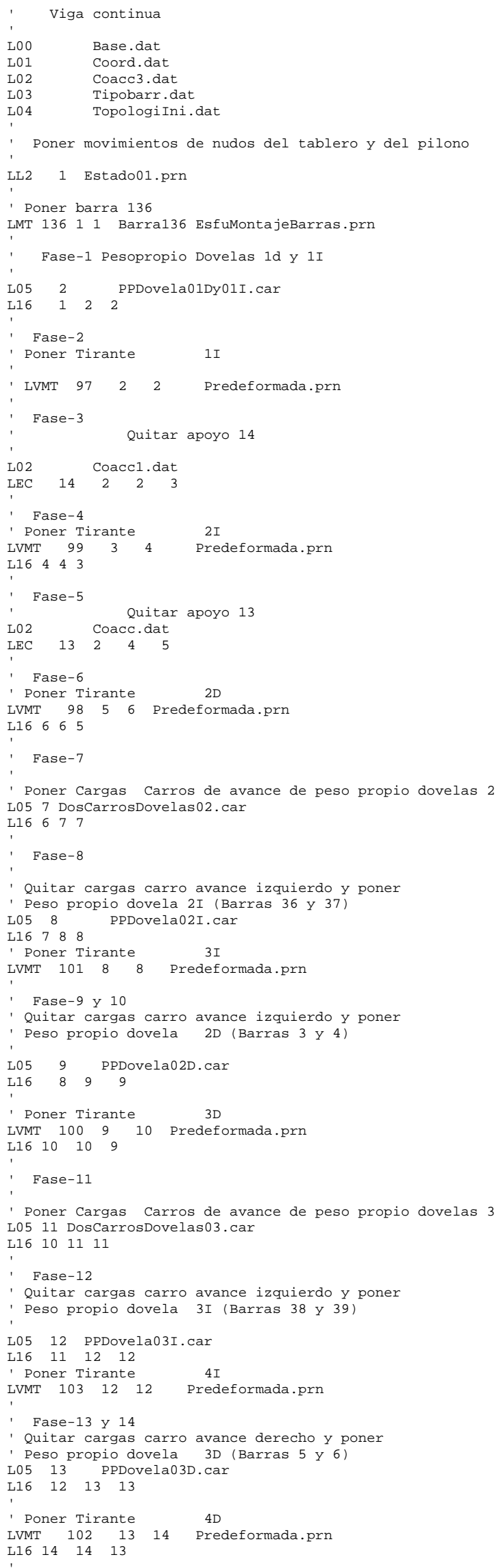


' Fase-15

- Poner Cargas Carros de avance de peso propio dovelas 4 0515 DosCarrosDovelas04.car

$1614 \quad 15 \quad 15$

Fase-16

Quitar cargas carro avance izquierdo y poner

Peso propio dovela 4I (Barras 40 y 41)

L05 16 PPDovela04I.car

L16 $15 \quad 16 \quad 16$

' Poner Tirante 5I

LVMT $105 \quad 16 \quad 16$ Predeformada.prn

' Fase-17 y 18

Quitar cargas carro avance derecho y poner

- Quitar cargas carro avance derecho y

05 17 PPDovela04D.car

$\begin{array}{llll}\text { L16 } & 16 & 17 & 17\end{array}$

' Poner Tirante 5D

LVMT $\quad \begin{array}{llll}104 & 17 & 18 & \text { Predeformada.prn }\end{array}$

$\begin{array}{llll}\text { L16 } & 18 & 18 & 17\end{array}$

Fase-19

Poner Cargas Carros de avance de peso propio dovelas 5 L05 19 DosCarrosDovelas05.car

L16 $18 \quad 1919$

Fase-20

' Quitar cargas carro avance izquierdo y poner

Peso propio dovela 5I (Barras 42 y 43)

L05 20 PPDovela05I.car

$\begin{array}{llll}\text { L16 } & 19 & 20 & 20\end{array}$

I Poner Tirante

LVMT $\begin{array}{llll}107 & 20 & 20 & \text { Predeformada.prn }\end{array}$

' Fase-21 y 22

Quitar cargas carro avance derecho y poner

- Peso propio dovela 5D (Barras 9 y 10)

L05 21 PPDovela05D.car

$\begin{array}{llll}\text { L16 } & 20 & 21 & 21\end{array}$

' Poner Tirante

LVMT $\begin{array}{llll}106 & 21 & 22 & \text { Predeformada.prn }\end{array}$

L16 $22 \quad 22 \quad 21$

, Fase-23

Poner Cargas Carros de avance de peso propio dovelas 6 L05 23 DosCarrosDovelas06.car

L16 $22 \quad 2323$

Fase-24

Quitar cargas carro avance izquierdo y poner

Peso propio dovela 6I (Barras 44 y 45)

L05 24 PPDovela06I.car

$\begin{array}{llll}\text { L16 } & 23 & 24 & 24\end{array}$

Poner Tirante $7 I$

LVMT $109 \quad 24 \quad 24 \quad$ Predeformada.prn

, Fase-25 y 26

' Quitar cargas carro avance derecho y poner

Peso propio dovela 6D (Barras 11 y 12)

L05 25 PPDovela06D.car

$\begin{array}{llll}\text { L16 } & 24 & 25 & 25\end{array}$

Poner Tirante 4047

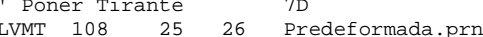

$16 \quad 26 \quad 26 \quad 25$

$11626 \quad 26 \quad 25$

' Fase-27

Poner Cargas Carros de avance de peso propio dovelas 7 L05 27 DosCarrosDovelas07.car

L16 $26 \quad 27 \quad 27$

' Fase-28

Quitar cargas carro avance izquierdo y poner

- Peso propio dovela 7 I (Barras 46 y 47)

L05 28 PPDovela07I.car

$\begin{array}{llll}\text { L16 } & 27 & 28 & 28\end{array}$

- Poner Iirante 81

LVMT $\begin{array}{llll}111 & 28 & 28 & \text { Predeformada.prn }\end{array}$

- Fase-29 y 30

- Quitar cargas carro avance derecho y poner

Peso propio dovela 7D (Barras 13 y 14)

L05 29 PPDovela07D.car

$\begin{array}{llll}\text { L16 } & 28 & 29 & 29\end{array}$

, Poner Tirante

$\begin{array}{llll}29 & 30 & \text { Predeformada.prn }\end{array}$ 


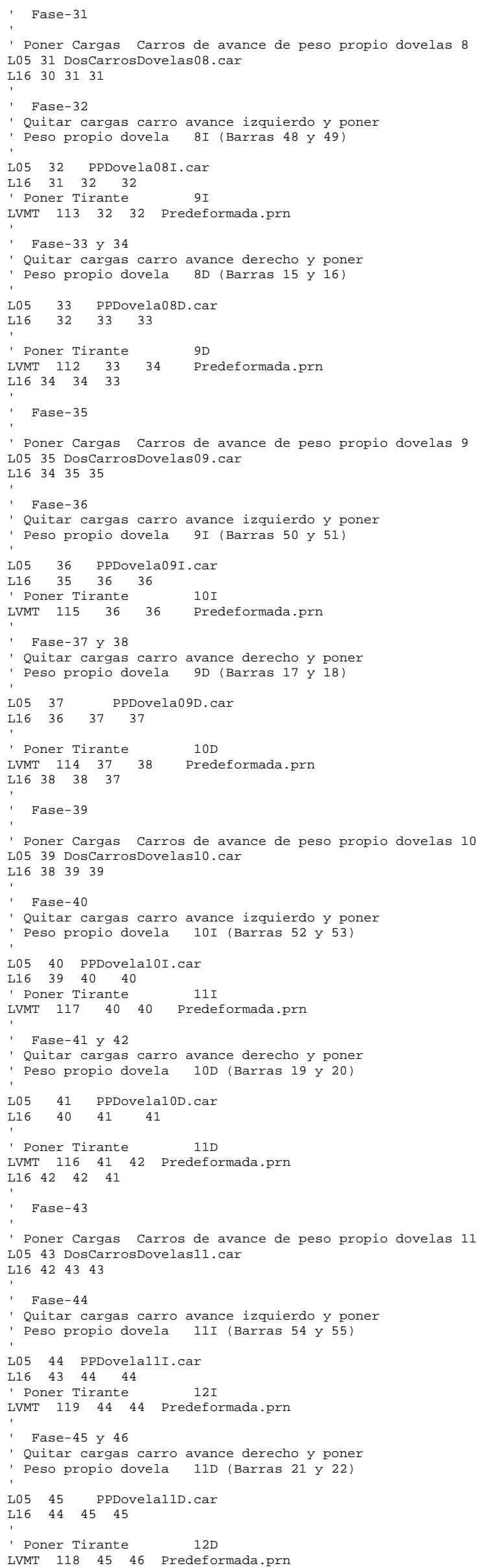




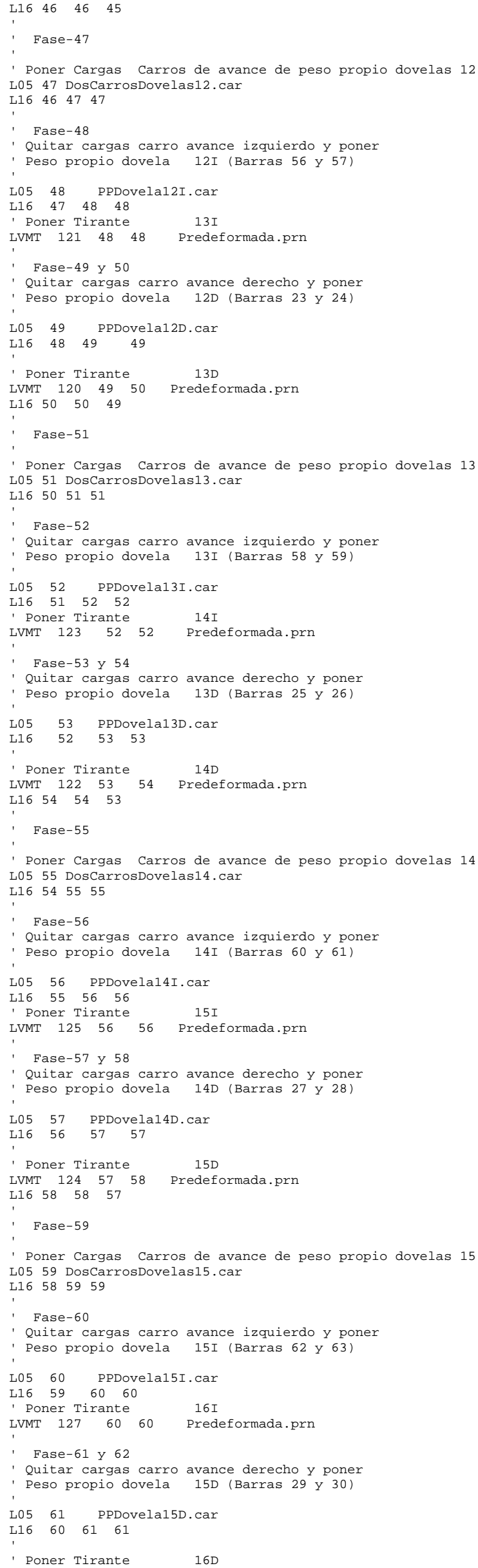


LVMT $126 \quad 61 \quad 62$ Predeformada.prn

L16 $62 \quad 62 \quad 61$

Fase-63

- Poner Cargas Carros de avance de peso propio dovelas 16 L05 63 DosCarrosDovelas16.car

L16 $62 \quad 63 \quad 63$

- Fase-64

Ouitar cargas carro avance izquierdo y poner

- Peso propio dovela 16I (Barras 64 y 65)

L05 64 PPDovela16I.car

$\begin{array}{llll}\text { L05 } & 64 & & \text { PPDovela16I.car } \\ \text { L16 } & 63 & 64 & 64\end{array}$

1 Poner Tirante $17 \mathrm{I}$

LVMT $129 \quad 64 \quad 64$ Predeformada.prn

' Fase-65 y 66

Quitar cargas carro avance derecho y poner

Peso propio dovela 16D (Barras 31 y 32)

L05 65 PPDovela16D.car

L16 $64 \quad 65 \quad 65$

' Poner Tirante 17D

$\begin{array}{lllll}\text { LVMT } & 128 & 65 & 66 & \text { Predeformada.prn }\end{array}$

L16 $66 \quad 66 \quad 65$

Fase-67

Poner Cargas Carros de avance de peso propio dovelas 17 L05 67 DosCarrosDovelas17.car

L16 $66 \quad 67 \quad 67$

Fase-68

- Quitar cargas carro avance izquierdo y poner

Peso propio dovela 17I (Barras 66 y 67)

L05 68 PPDovela17I car

$\begin{array}{lllll}\text { L16 } & 67 & 68 & 68\end{array}$

1 Poner Tirante $18 \mathrm{I}$

LVMT $13168 \quad 68$ Predeformada.prn

Fase-69 y 70

Quitar cargas carro avance derecho y poner

- Peso propio dovela 17D (Barra 33)

L05 69 PPDovela17D.car

L16 $68 \quad 69 \quad 69$

1 Poner Tirante 18D

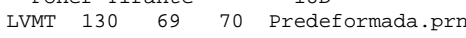

' Poner barra 134

LMT 1347071 Barra134 EsfuMontajeBarras.prn

Fase-71

Peso propio dovela $18 I$

L05 72 PPBarra68.car

L05 73 PPBarra69.car

L05 74 PPBarra70.car

$\begin{array}{llll}\text { L16 } & 71 & 74 & 71\end{array}$

LMT 1327172 Barra132 EsfuMontajeBarras.prn

L05 73 PPBarra71 car

$\begin{array}{llll}\text { L16 } & 72 & 73 & 71\end{array}$

1 Fase-72

- Carga muerta

L05 72 CM.car

$\begin{array}{lll}\text { L16 } & 71 & 72\end{array}$ 
Las ordenes lógicas especiales utilizadas son:

Private Sub OrdenLMT(NB As Long, Hn1 As Integer, Hn2 As Integer, Nombre1\$, Nombre2\$)

' Orden para introducir los esfuerzos de montaje de un tirante. Realiza los siguientes pasos:

' 1) Lee en el archivo "Nombre2\$" el axil que le corresponde al tirante a introducir,

' la barra "nb".

' 2) A partir de ese axil genera el archivo de cargas:

"MontarTirante" \& Nombrel\$ \& ".car", en el que se introducen sobre los nudos

extremos de la barra "nb" las cargas equivalentes a dicho axil

' 3) Calcula la hipótesis de carga correspondiente a dicho archivo de cargas,

almacenándola en la posición "hn1+1"

- 4) Suma la hipótesis de carga "hn1" con "hn1+1" y el resultado lo almacena en "hn2"

' 5) Suma directamente a los esfuerzos que tenga la barra "nb" en la hipótesis "hn2"

el axil leído en el fichero "nombre2\$" en el paso $1^{\circ}$.

' 6) Activa la barra "nb" que debe estar previamente desactivada

Private Sub OrdenLEC(Nn1 As Long, il As Integer, Hn1 As Integer, Hn2 As Integer)

' Orden que permite eliminar una coacción de un nudo, calculando una hipótesis de

la reacción

carga en la que se aplica una carga en dicho nudo igual y contraria a coacción.

existente en dicho nudo en una hipótesis previa en la que existe dicha

- $\mathrm{Nn} 1=\mathrm{n}^{\circ}$ del nudo que tiene la coacción impuesta que se quiere eliminar

' $i 1$ = indicador de la coacción que se quiere eliminar:

' =1 elimina coacción al Mov-X

' $=2$ elimina coacción al Mov-Y

' =3 elimina coacción al Giro

' $h n 1=n^{\circ}$ de la hipótesis previa en la que existe la coacción que se

' quiere eliminar

' hn2 $=\mathrm{n}^{\circ}$ de la hipótesis donde se almacena el resultado del cálculo de eliminar

la coacción

' Se genera un archivo de cargas auxiliar que contiene la carga en el nudo $\mathrm{Nn} 1$ 
' igual a -Reacción existente. El nombre de este archivo de carga es

' "EliminaCoaccion(MovX o MovY o Giro) Nudo"Nn1".car"

Private Sub OrdenLEB (nb1 As Long, Hn1 As Integer, Hn2 As Integer)

- Orden que permite eliminar la barra Nb1, generando un archivo con unas cargas

' en sus nudos dorsal y frontal iguales y contrarias a los esfuerzos que tiene

' dicha barra en la hipótesis Hn1. Calcula la nueva hipótesis correspondiente a

' ese archivo de cargas guardándola como hipótesis n Hn1+1. Graba en esta hipótesis

' Hn1+1 como esfuerzos de la barra nb1 los iguales y contrarios a los existentes en

' la hipótesis Hn1. A continuación suma la hipótesis Hn1 con la Hn1+1 y el resultado

' lo guarda en Hn2.

Private Sub OrdenLAY(Nn1 As Integer, Hh1 As Integer, Hh2 As Integer)

- Orden para calcular una hipótesis de carga que anula el

' movimiento Y del nudo Nn1 en la hipótesis Hh1. La hipótesis resultante

' se graba en Hh2

\section{Ordenes Lógicas para el cálculo normal de la estructura:}

L00 = Lectura de los datos generales de la estructura:

$\mathrm{n}^{\circ}$ nudos, $\mathrm{n}^{\circ}$ de barras, $\mathrm{n}^{\circ}$ de nudos coaccionados, $\mathrm{n}^{\circ}$ de tipos de barras

L01 = Lectura de las coordenadas de los nudos

L02 = Lectura de los nudos coaccionados con sus coacciones

L03 = Lectura de las características mecánicas de los diferentes tipos de barras

L04 = Lectura de la topología de la estructura (Barras)

L05 = Cálculo de las hipótesis de cargas existentes en el archivo cuyo nombre se pasa 
ANEXOS

como $2^{\circ}$ parámetro a la subrutina, grabándolas a partir de la posición que se

indica en el primer parámetro pasado.

L16 = Suma todas las hipótesis de carga comprendidas entre la indicada con el primer

parámetro hasta la indicada con el $2^{\circ}$ parámetro y el resultado lo almacena en la

posición señalada por el tercer parámetro.

LL2 = Graba en los archivos de hipótesis de carga, en la posición indicada con el primer

parámetro, el título de la hipótesis, los movimientos, esfuerzos y reacciones,

almacenados en el archivo cuyo nombre se pasa a la subrutina como $2^{\circ}$ parámetro. 


\section{A.3. ALGORITMOS PROGRAMADOS.}

\section{SUBRUTINA DE CALCULO DE COEFICIENTES ALEATORIOS}

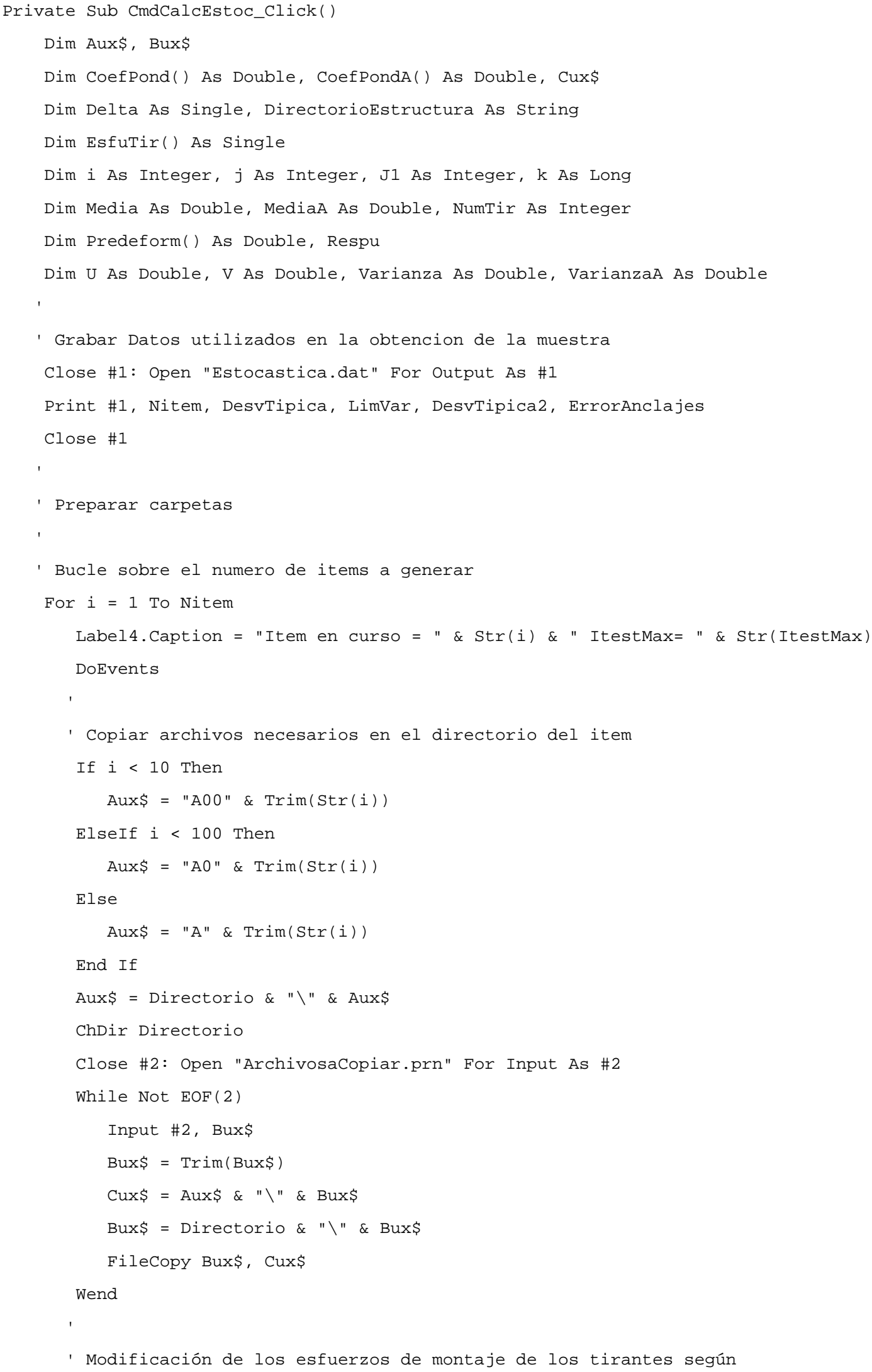


- el método indicado

If Metod $=1$ Or Metod $=2$ Or Metod $=100$ Then

ChDir Aux\$

Close \#2: Open "EsfuMontajeTirantes.prn" For Input As \#2

Input \#2, NumTir

ReDim EsfuTir(3, NumTir), CoefPond(NumTir)

' Cálculo de los coeficientes de ponderacion de los tirantes

CalculocoefPond NumTir, CoefPond()

For $j=1$ To NumTir

Input \#2, EsfuTir $(1, j), \operatorname{EsfuTir}(2, j)$

EsfuTir $(3, j)=\operatorname{EsfuTir}(2, j) * \operatorname{CoefPond}(j)$

Next j

Close \#2: Open "EsfuMontajeTirantes.prn" For Output As \#2

Close \#3: Open "DeltasAplicadas.prn" For Output As \#3

Print \#2, NumTir

Media $=0:$ Varianza $=0$

For $j=1$ To NumTir

Print \#2, FormateB(EsfuTir $(1, j), \quad$ \#\#\#\#\#\#");

Print \#2, FormateB(EsfuTir $(3, j)$, "\#\#\#\#\#\#\#\#.\#0")

Print \#3, FormateB(EsfuTir $(1, j)$, "\#\#\#\#\#0");

Print \#3, FormateB(EsfuTir $(2, j)$, "\#\#\#\#\#\#\#\#.\#0");

Print \#3, FormateB(EsfuTir (3, j), "\#\#\#\#\#\#\#\#0.\#0");

Print \#3, FormateB (CoefPond (j), "\#\#\#0.\#\#\#0")

Media $=$ Media + CoefPond $(j) /$ NumTir

Next j

For $j=1$ To NumTir

Varianza $=\operatorname{Varianza}+($ CoefPond $(j)-$ Media $) \wedge 2 /$ NumTir

Next j

Print \#3, " Media = "; FormateB (Media, "\#\#0.\#\#\#\#\#0")

Print \#3, "Varianza = "; FormateB(Varianza, "\#\#0.\#\#\#\#\#0")

Close \#2, \#3

ElseIf Metod $=3$ Then

ChDir Aux\$

Close \#2: Open "PreDeformada.prn" For Input As \#2

Input \#2, NumTir

ReDim Predeform(7, NumTir), CoefPond(NumTir), CoefPondA(NumTir)

For $j=1$ To NumTir

Input \#2, Predeform (1, j)

For $k=2$ To 4

Input \#2, Predeform $(k, j)$

Next k

Next j

' Cálculo de los coeficientes de ponderacion de los tirantes

CalculoCoefPondLong NumTir, Predeform(), CoefPond(), CoefPondA() 


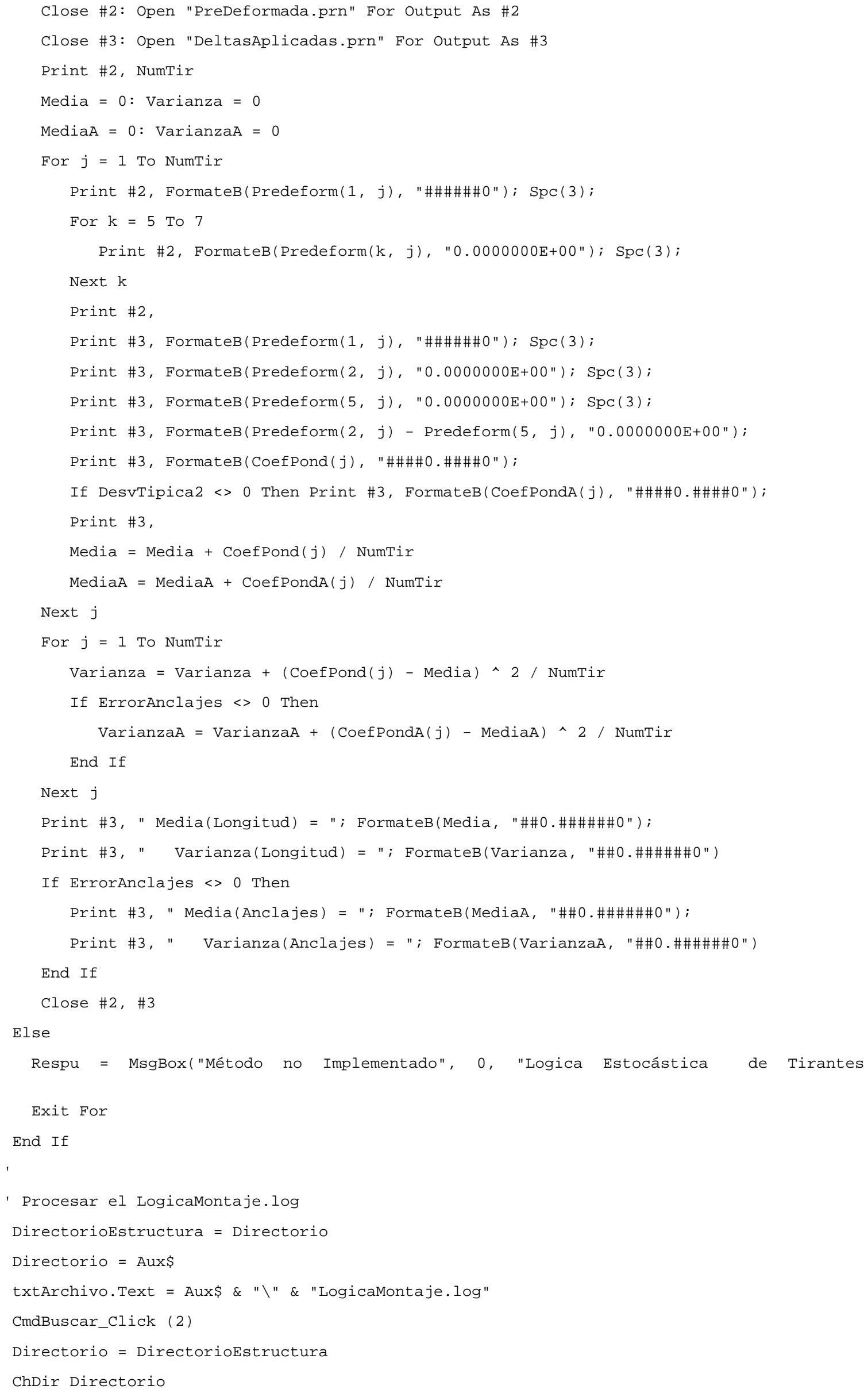


ANEXOS

If ItestMax < Itest Then ItestMax = Itest

Next i

Label4. Caption = "Item en curso = " \& Str(Nitem) \& " ItestMax= " \& Str(ItestMax) DoEvents

CmdBuscar (1). SetFocus

End Sub 


\section{SUBRUTINA GENERACION COEFICIENTES PONDERACION ESFUERZOS DE TIRANTES}

Private Sub CalculocoefPond(NumTir As Integer, CoefPond() As Double)

' Determinar los coeficientes de ponderación de los tirantes en cada item

Dim AD As Double, AD1 As Double

Dim Bw() As Double, Bw1() As Double, CV As Double

Dim i As Integer, $j$ As Integer, J1 As Integer

Dim Un As Double, Vn As Double, Wn As Double, Xn As Double

ReDim Bw(NumTir), Bw1 (NumTir)

Itest $=0$

10 Labe13. Caption $=\operatorname{Str}$ (Itest)

DoEvents

For $i=1$ To NumTir

Randomize

If Metod $=1$ Then ' Distribucion Uniforme Limitado a $1 \pm$ Desv.Tipica

CoefPond $(i)=1+(-1+2 *$ Rnd $) *$ DesvTipica

ElseIf Metod $=100$ Then

- Método de Box-Muller

100

$\mathrm{Un}=1$ - Rnd: Randomize: $\mathrm{Vn}=$ Rnd

, Delimitado

' If Abs((1 / Sqr (-2 * Log(Un)) / Sin(2 * pi *Vn)) * DesvTipica) > DesvTipica

Then GoTo 100

CoefPond $(i)=1+(1 / \operatorname{Sqr}(-2 * \log (U n)) / \operatorname{Sin}(2 * \mathrm{pi}$ * Vn $))$ * DesvTipica

ElseIf Metod $=2$ or Metod $=3$ Then

' Método del limite central o media de 12 valores aleatorios

$\mathrm{Un}=0$

For J1 $=1$ To 12

Randomize

$\mathrm{Un}=\mathrm{Un}+\mathrm{Rnd}$

Next J1

CoefPond $(i)=($ Un -6$) *$ DesvTipica +1

If Abs (1 - CoefPond(i)) > LimVar Then GoTo 120

' If Abs(1 - CoefPond(i)) > DesvTipica Then GoTo 120

End If

Labe13. Caption = "Tirante-" \& i \& "Coef.Ponder.=" \& CoefPond(i) Next i

- Comprobar para la distribucion normal que la muestra es normal

' utilizando el test de Anderson-Darling

If Metod $=2$ Or Metod $=100$ Then

For $i=1$ To NumTir

$\mathrm{Bw}(i)=$ CoefPond $(i)$

' Printer.Print BW(i)

Next i 
- Ordenar la muestra de menor a mayor

For $i=1$ To NumTir -1

For $j=i+1$ To NumTir

If $\mathrm{Bw}(i)>\mathrm{Bw}(j)$ Then Swap Bw(i), Bw(j)

Next j

Next $i$

- For $i=1$ To NumTir: Printer.Print BW(i): Next $i$

- Cálculo de los valores de la funcion de distribucion correspondientes a

' los Bw(i)

$\mathrm{Un}=0: \mathrm{Vn}=0$

For $i=1$ To NumTir

Labe13. Caption = "Itest=" \& Str(Itest) \& " $i=" \&$ Str(i)

DoEvents

$\mathrm{Xn}=(\mathrm{Bw}(\mathrm{i})-1) /$ DesvTipica

If $\mathrm{Xn}<=-5.7$ Then

$\mathrm{Bw} 1(\mathrm{i})=-34.2 * 10 \wedge-9 / \mathrm{Xn}$

ElseIf $\mathrm{Xn}>=5.7$ Then

Bw1(i) $=1-34.2 * 10 \wedge-9 / \mathrm{Xn}$

Else

If Un $=0$ Then Un $=-5.7: \mathrm{Vn}=-34.2 * 10 \wedge-9: \mathrm{Wn}=0.000001$

While Un $<=\mathrm{Xn}$

$\mathrm{Vn}=\mathrm{Vn}+1 / 2.506628275 * \operatorname{Exp}(-(\mathrm{Un} \wedge 2) / 2) * \mathrm{Wn}$

$\mathrm{Un}=\mathrm{Un}+\mathrm{Wn}$

Wend

$\mathrm{Bw} 1(\mathrm{i})=\mathrm{Vn}$

End If

' Printer.Print i; BW(i); BW1(i)

Next i

$\mathrm{AD}=-$ NumTir

For $i=1$ To NumTir

$\mathrm{AD} 1=(1-2 \star i) / \operatorname{NumTir} *(\log (\mathrm{Bw} 1(i))+\log (1-\operatorname{Bw1}(\operatorname{NumTir}+1-i)))$

$\mathrm{AD}=\mathrm{AD}+\mathrm{AD} 1$

Next i

- Prueba de rechazo con confianza del 95\%

$\mathrm{CV}=0.752 /(1+0.75 /$ NumTir $+2.25 /$ NumTir^ 2)

If $\mathrm{AD}>\mathrm{CV}$ Then

Itest $=$ Itest $+1:$

If Itest < 3000 Then GoTo 10 Else Stop

End If

End If

Labe13. Caption $=" "$

DoEvents

End Sub 


\section{SUBRUTINA GENERACION COEFICIENTES PONDERACION LONGITUDES DE TIRANTES}

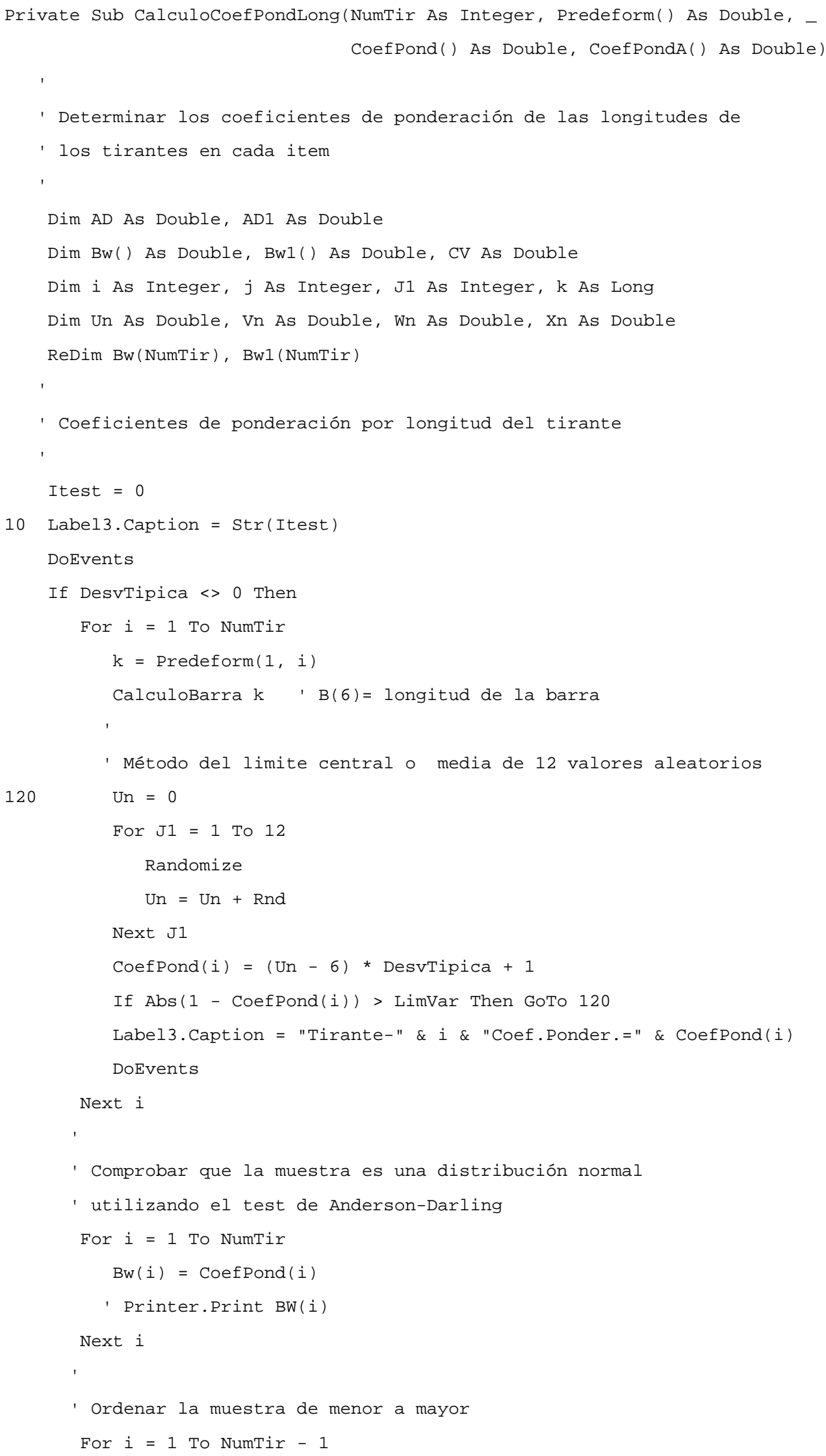


For $j=i+1$ To NumTir

If $\mathrm{Bw}(i)>\mathrm{Bw}(j)$ Then Swap $\mathrm{Bw}(i), \mathrm{Bw}(j)$

Next j

Next i

- Cálculo de los valores de la funcion de distribucion correspondientes a

' los Bw(i)

$\mathrm{Un}=0: \mathrm{Vn}=0$

For $i=1$ To NumTir

Label3. Caption = "Itest=" \& Str(Itest) \& " $i="$ \& Str(i)

Label3.BackColor $=\& H 8080 \mathrm{FF}$

DoEvents

$\mathrm{Xn}=(\mathrm{Bw}(\mathrm{i})-1) /$ DesvTipica

If $\mathrm{Xn}<=-5.7$ Then

$\mathrm{Bw} 1(\mathrm{i})=-34.2 * 10 \wedge-9 / \mathrm{Xn}$

ElseIf $\mathrm{Xn}>=5.7$ Then

$\mathrm{Bw} 1(\mathrm{i})=1-34.2 * 10 \wedge-9 / \mathrm{Xn}$

Else

If $\mathrm{Un}=0$ Then $\mathrm{Un}=-5.7: \mathrm{Vn}=-34.2 * 10 \wedge-9: \mathrm{Wn}=0.000001$

While Un $<=\mathrm{Xn}$

$\mathrm{Vn}=\mathrm{Vn}+1 / 2.506628275 * \operatorname{Exp}(-(\mathrm{Un} \wedge 2) / 2) \star \mathrm{Wn}$

$\mathrm{Un}=\mathrm{Un}+\mathrm{Wn}$

Wend

$\mathrm{Bw} 1(\mathrm{i})=\mathrm{Vn}$

End If

' Printer.Print i; BW(i); Bw1(i)

Next i

$\mathrm{AD}=-$ NumTir

For $i=1$ To NumTir

$\mathrm{AD} 1=(1-2 \star i) / \operatorname{NumTir} *(\log (\operatorname{Bw} 1(i))+\log (1-\operatorname{Bw1}(\operatorname{NumTir}+1-i)))$

$\mathrm{AD}=\mathrm{AD}+\mathrm{AD} 1$

Next i

' Prueba de rechazo con confianza del 95\%

$\mathrm{CV}=0.752 /(1+0.75 /$ NumTir $+2.25 /$ NumTir^ 2)

If $\mathrm{AD}>\mathrm{CV}$ Then

Itest $=$ Itest $+1:$

If Itest < 1000 Then GoTo 10 Else Stop

End If

Labe13. Caption = " "

DoEvents

End If

- Coeficientes de ponderación por error posicion de anclajes

If DesvTipica $2<>0$ Then 
b10: Labe13. Caption = Str(Itest)

DoEvents

For $i=1$ To NumTir

$\mathrm{k}=\operatorname{Predeform}(1, \mathrm{i})$

CalculoBarra $\mathrm{k}$, $\mathrm{B}(6)=$ longitud de la barra

- Método del limite central o media de 12 valores aleatorios

b120: $\quad$ Un $=0$

For J1 $=1$ To 12

Randomize

$\mathrm{Un}=\mathrm{Un}+\mathrm{Rnd}$

Next J1

CoefPondA $(i)=($ Un -6$) *$ DesvTipica2 +1

If Abs (1 - CoefPondA(i)) * B(6) > ErrorAnclajes Then GoTo b120

Label3.Caption = "Tirante-" \& $i \&$ "Coef.Ponder.=" \& CoefPondA(i)

DoEvents

Next i

- Comprobar que la muestra es una distribución normal

' utilizando el test de Anderson-Darling

For $i=1$ To NumTir

$\mathrm{BW}(\mathrm{i})=$ CoefPondA $(i)$

' Printer.Print BW(i)

Next i

' Ordenar la muestra de menor a mayor

For $i=1$ To NumTir -1

For $j=i+1$ To NumTir

If $\mathrm{Bw}(i)>\mathrm{Bw}(j)$ Then Swap Bw(i), Bw(j)

Next $j$

Next i

- Cálculo de los valores de la función de distribución correspondientes a

' los Bw(i)

$\mathrm{Un}=0: \mathrm{Vn}=0$

For $i=1$ To NumTir

Labe13. Caption $=$ "Itest $A=" \& \operatorname{Str}($ Itest) \& " $i="$ \& Str(i)

Label3. BackColor $=\&$ H80FFFF

DoEvents

$\mathrm{Xn}=(\mathrm{Bw}(\mathrm{i})-1) /$ DesvTipica2

If $\mathrm{Xn}<=-5.7$ Then

$\mathrm{Bw} 1(\mathrm{i})=-34.2 * 10 \wedge-9 / \mathrm{Xn}$

ElseIf $\mathrm{Xn}>=5.7$ Then

$\mathrm{Bw} 1(\mathrm{i})=1-34.2 * 10 \wedge-9 / \mathrm{Xn}$

Else

If $\mathrm{Un}=0$ Then $\mathrm{Un}=-5.7: \mathrm{Vn}=-34.2 \star 10 \wedge-9: \mathrm{Wn}=0.000001$ 


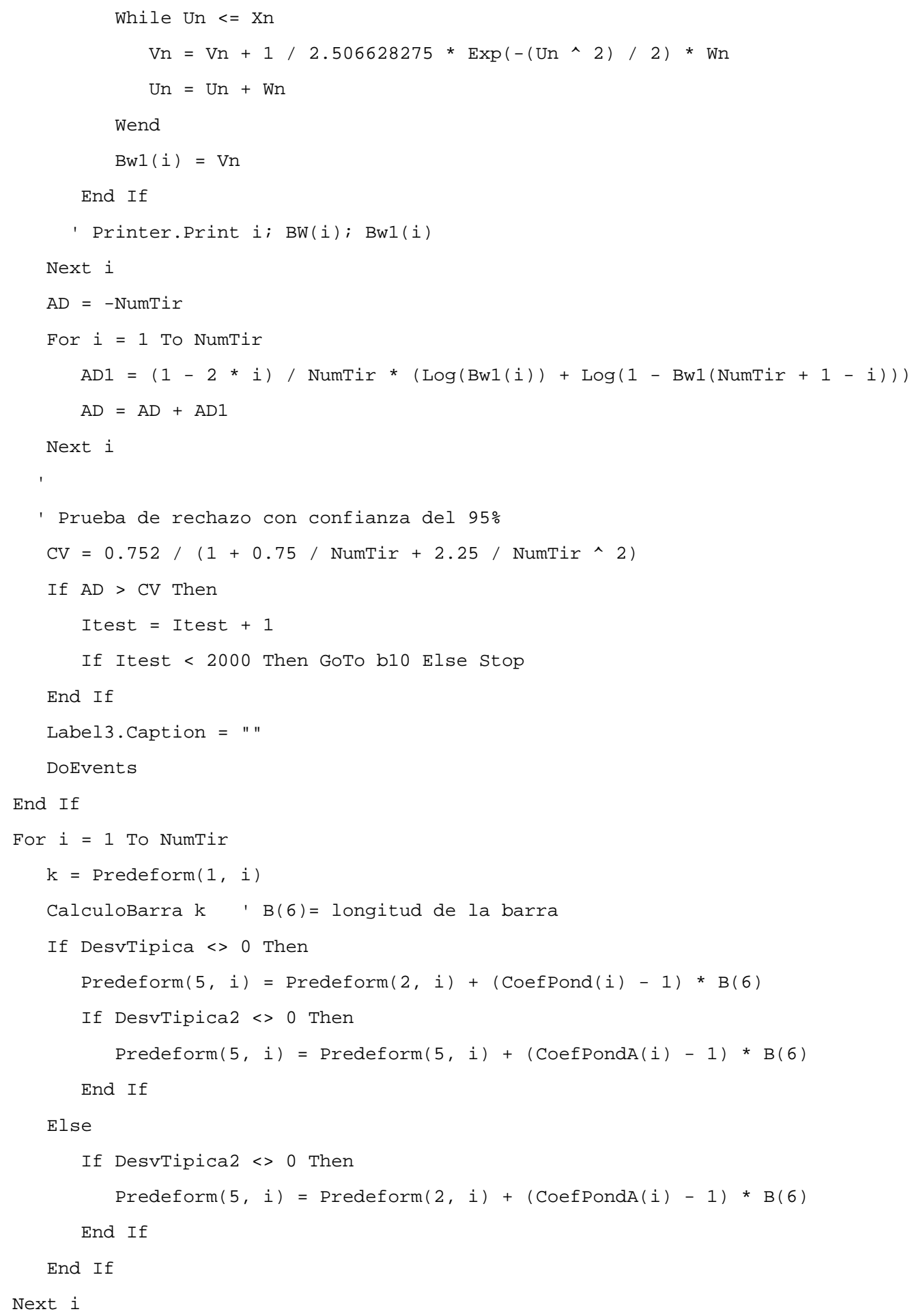




\section{BIBLIOGRAFIA.}

[1] "Instrucción de hormigón estructural EHE-08". Ministerio de Fomento. 2008.

[2] "Manual de tirantes". Recomendaciones y manuales técnicos. E-12. ACHE 2007.

[3] Agrawal, T.P., "Cable-stayed bridges - Parametric study". Journal of Bridge Engineering, Vol 2 No2. 1997.

[4] Alvarez, S.J. "Estadística aplicada teoría y problemas". Universidad Complutense de Madrid. 2000.

[5] Anderson, T.W. \& Darling, D.A. "A test of godness of fit". Journal of the American Statistical Association. 49(268). 1954.

[6] Aparicio, A. "Acciones y coeficientes de seguridad". Ponencia del curso impartido en la E.T.S. de I.C.C.P. de Madrid. 1990, sobre Puentes atirantados.

[7] Arenas, J.J. "El tirante ". Ponencia del curso de Puentes atirantados impartido en la E.T.S. de I.C.C.P. de Madrid. 1990.

[8] Arenas, J.J. y Pantaleón M. "El Viaducto de la Arena, en la autopista del Cantábrico". Hormigón y Acero n¹96. 1995.

[9] Astiz, M.A. "Análisis no lineal de puentes atirantados". Ponencia del curso de Puentes atirantados impartido en la E.T.S. de I.C.C.P. de Madrid. 1990.

[10] Astiz, M.A. "El sistema de auscultación del Puente de Waterford en Irlanda". V congreso de ACHE. Madrid 2014.

[11] Astiz, M.A. y otros. "El puente atirantado sobre el río Suir en de Waterford". Hormigón y Acero $n^{\circ} 62.2011$.

[12] Baldomir A., Hernández S., Nieto F. y Jurado J.A. "Cable optimization of long span cablestayed bridge in La Coruña". Advances in Engineering software 41. 2010.

[13] Behin, Z. \& Murray, D. "A subestructure frontal technique for cantilever erection analysis of cable-stayed bridges". Computer\&Structures, 42(2). 1992.

[14] Biondini, F., Bontempi, F. Malera, P.G. y Martinez y Cabrera, F. "Reliability assesment of cable-stayed bridges". IABSE Conference on cable supported bridges. Seul. 2001. 
BIBLIOGRAFIA.

[15] Blaiotta, J. y Delieutrz, P. "Teorema central del límite". Universidad de Buenos Aires, Facultad de Ciencias exactas. 2004.

[16] Bokan, H., Jancic, D. and Heiden, M. "Optimization of the tensioning schedule for cablestayed bridges using dynamic software". IABSE Symposium. Budapest. 2006.

[17] Bokan, H., Janjic, D. and Heiden, M. "Optimization of the cable tensioning in cablestayed ". Journal of Bridge Engineering. 8(3). 2003

[18] Boucher, C.G. \& Bourgound, U. "A fast and efficent response surface approach for structural reliability problems". Structural safety. Vol 7. 1990.

[19] Bruneau, M. "Evaluation of system reliability methods for cable-stayed bridge design". Journal of Structural Engineering. Vol 118. 1992.

[20] Cluley, N. C. and Shepherd, R., "Analysis of concrete cable-stayed bridges for creep, shrinkage and relaxation effects". Computer\&Structures Vol 58, n² pp 337-350. 1995.

[21] Chen, D.W., Au, F.T.K., Tham, L.G. and Lee, P.K.K. "Determination of initial cable forces in prestressed concreted cable-stayed bridges for given design deck profiles using the force equilibrium method". Computer\&Structures. 74. 2000.

[22] Chen, S. \& Chen, Z. "Reliability of construction control of cable stayed-bridges". Bridge engineering. Vol. 164. 2011.

[23] Cheng, J. "Determination of cable forces in cable-stayed bridges constructed under parametric uncertainty". Engineering Computaions. Vol 27. 2010.

[24] Cheng, J. \& Xiao, R. "Serviciability reliability analysis of cable-stayed bridges". Structural Engineering and Mechanics. Vol 20. 2005.

[25] Cheng, J. Xiao \& R, Jiang, J. "Probabilistic determination of initial cable forces of cablestayed bridges under dead loads". Structural Engineering and Mechanics Vol. 17 No 2. 2004.

[26] Código Modelo CEB-FIP 2010.

[27] Cramer, H. "Teoría de probabilidades y aplicaciones". Ed. Aguilar. 1968.

[28] de Miranda, F. "Steel and composite long span bridge stiffness". Ponencia del curso impartido en la E.T.S. de I.C.C.P. de Madrid. 1990, sobre Puentes atirantados.

[29] del Pozo, F. "Puentes atirantados. Modelización". Jornada Técnica anual sobre "Ultimas tendencias y realizaciones de puentes atirantados". Madrid. Mayo 2007.

[30] del Pozo, F., Arrieta, J.M., García, J.L., Celemín, J. y Madrid, A. "Viaducto sobre el río Iregua en la circunvalación Sur de Logroño". Ponencia del II Congreso de ACHE. 2002. 
BIBLIOGRAFIA.

[31] El Araby, El Shenawy, "Form finding for cable-stayed and extradosed bridges". Tesis leida en la Universidad Técnica de Berlín. 2013.

[32] Ernst, H.J. "Der E-modul von Seilen unter Berücksichtigung des Durchhanges". Der Bauingenieur. 40(2). 1965.

[33] Eurocódigo EC-1. "Bases de cálculo en estructuras". UNE-EN-1990/2003./A:2010..

[34] Eurocódigo EC3-7. "Estructuras de acero. Cables de alta resistencia". UNE-EN-1993-1-11. 2015.

[35] Fernández Troyano, L. " Construcción de puentes atirantados ". Ponencia del curso impartido en la E.T.S. de I.C.C.P. de Madrid. 1990, sobre Puentes atirantados.

[36] Fernández Troyano, L. "El proyecto del puente sobre el río Lérez en Pontevedra". Revista de Obras Públicas n³378. 1998.

[37] Fernández Troyano, L. "Tierra sobre el agua. Vol 2". Colegio de Ingenieros de Caminos. Colección, ciencia, Humanidades e Ingeniería n55. Ed 2. 2004.

[38] Fernández Troyano, L., Fernández Muñoz, L. y Ayuso Calle, G., "Puente atirantado sobre el río Besaya". Ponencia de las II Jornadas sobre la vida de los puentes. San Sebastián 2006.

[39] Fernández Troyano, L., Fernández Muñoz, L. y Ayuso Calle, G. "Viaducto de Arbizelai". Ponencia del V Congreso de ACHE. Barcelona 2011.

[40] Fernández Troyano, L. y otros. "Viaducto atirantado de Bucaramanga. Colombia". Ponencia del VI Congreso de ACHE. Madrid 2014.

[41] Fernández-Ordoñez, J.A. "Control y seguimiento del montaje del puente atirantado Fernando Reig, sobre el río Barxell, en Alcoy". Hormigón y Acero nº170. 1989.

[42] Fernández-Ordoñez, J.A. Martinez Calzón, J. Millanes, F., Ortíz, A y Ventura J.M. "Puente Fernando Reig sobre el barranco Barxell en Alcoy". Hormigón y acero 161. 1986.

[43] Floriano, A.M. "Instrumentación del Puente Fernando Reig sobre el río Barxell". Hormigón y Acero $n^{\circ} 169.1988$.

[44] Furukawa, K. \& Yamada, Y. "Studies on optimization of cable prestressing for cablestayed bridges". International Conference on Cable-stayed bridges. 1987.

[45] Gabrow, M.J. "Construction stage analysis of cable-stayed bridges". Thessis submitted to the Faculty of the Technical University of Hamburg. 2004.

[46] Gimsing, N.J. "Cable supported bridges". John Wiley\&Sons Ltd. 1997.

[47] Goodyear, D. "Elements in the design and construction of cable-stayed bridges". OTEC Columbus Ohio). 2002. 
BIBLIOGRAFIA.

[48] Guzmán, L. y otros. "Construction and geometric control of the Baluarte Cable-Stayed bridge". 37th IABSE Symposium. Madrid 2014.

[49] Hassan, M.M. "Optimization of cable-stayed bridges using finite element genetic algorithm, and B-spline combined technique". Engineering Structures. (49). 2013.

[50] Hassan, M.M., Nassef, A.O. and El Damatty, A.A. "Determination of optimum posttensioning cable fores of cable-stayed bridges". Engineering Structures. (44). 2012.

[51] IAP11 "Instrucción de acciones en puentes de carreteras". Ministerio de Fomento. 2011.

[52] IAPF07. "Instrucción sobre las acciones a aplicar en puentes de ferrocarril". Ministerio de Fomento. 2007.

[53] Imai, K. \& Frangopol, D.M. "System reliability of suspension bridges". Structural safety. Vol 24. 2002.

[54] Janjic, D., Pircher, M. and Pircher, H. "The unit load method. Some recent aplications". Advances in steel structures Vol II. 2002.

[55] Kasuga, A., Arai, H., Breen, J.E. and Furukawa, K. "Optimum cable-force adjustements in concrete cable-stayed bridges". Journal of structural Engineering. Vol 121. 1995.

[56] Lazar, B.E. \& Troitsky, M.S. y Douglas, M.C. "Load balancing analysis of cable-stayed bridges". ASCE Journal of Structural Engineering, 92(8). 1972.

[57] Leonhardt, F. "Cable stayed bridges with prestressed concrete". PCI Journal. 1986.

[58] Liu, M. \& Wang, P. "Initial shapes of cable stayed bridges during construction by cantilever methods - Numerical simulation and validation of the Kao Ping Hsi Bridge". Intech. 2015.

[59] Lozano-Galant, J.A. "Construction control of cable-stayed bridges". Tesis doctoral dirigida por Jose Turmo, Ignacio Payá y Enrique Castillo Ron. U.P.C. Junio 2013.

[60] Lozano-Galant, J.A. y Turmo, J. "An algorithm for simulation of concrete cable-stayed bridges built on temporary supports and considring time dependets effects".

Engineereing Structures. (79). 2014.

[61] Lozano-Galant, J.A., Payá-Zaforteza, I., Xu, D. y Turmo, J. "Analysis of the construction process of cable-stayed bridges built on temporary supports". Engineering Structures. (40). 2012.

[62] Lozano-Galant, J.A., Payá-Zaforteza, I., Xu, D. y Turmo, J. "Forward algorithm for the construction control of cable-stayed bridges built on temporary supports". Engineering Structures. (40). 2012. 
BIBLIOGRAFIA.

[63] Lozano-Galant, J.A., Xu, D. Payá-Zaforteza, I. y Turmo, J. "Direct simulation of the tensioning process of cable-stayed bridges". Computer\&Structures. (121). 2013.

[64] Lute, V. Upadhyay, A. y Singh, K. K., "Genetic algorithms - Based optimization of cable stayed bridges". journal of software engineering and appications Vol 4 pp 571-578. 2011.

[65] Manterola J. et al. "Bridge over the Cadiz Bay". IABSE Symposioum Report. Vol 102. 2014.

[66] Manterola J. y otros. "El puente sobre la Bahía de Cádiz". Ponencia del V Congreso de ACHE. Madrid 2014.

[67] Manterola, J y Fernández Troyano, L. "Cálculo de puentes atirantados". apuntes del curso desarrollado en la E.T.S.I. de Caminos, C. y P. en Junio de 1990.

[68] Manterola, J y Fernández Troyano, L. "El puente de Fernández Casado sobre el embalse de Barrios de Luna". Hormigón y Acero n¹51. Madrid 1984.

[69] Manterola, J. "Puentes, apuntes para su diseño, cálculo y construcción". Colegio de Ingenieros de Caminos C. y P. $1^{a}$ Edición. 2006.

[70] Manterola, J. "Tendencias actuales en la construcción de puentes atirantados". Hormigón y Acero n 163. 1986.

[71] Manterola, J. y Fernández Troyano, L. "Puente atirantado, sobre el río Nalón, en Sama de Langreo". Hormigón y Acero n¹74. 1990.

[72] Manterola, J., Montero López, J., Muñoz-Rojas, J. y Fernández Alonso, S. "Puente Príncipe de Viana sobre el río Segre en Lleida". Ponencia del V Congreso de ACHE. Barcelona 2011.

[73] Marchetti, M \& Lecinq, B. "Stay adjustement from design perspective to on site practice". Proceedings of IABSE Conference.

[74] Marchetti, M et al. "Adjustement of the Rion-Antirion cable-stayed bridge. An innovative mutidisciplinary response to a construction challenge". Symposioum on engineering surveys for construction works and structural engineering. Nottingham (GB). 2004.

[75] Marí, R. "Fluencia, retracción y temperatura". Ponencia del curso de Puentes atirantados impartido en la E.T.S. de I.C.C.P. de Madrid. 1990.

[76] Martí, R. y Rubacabado, R. "El puente atirantado Ben-Ahin en Bélgica". Hormigón y Acero $\mathrm{n}^{\circ} 169.1988$.

[77] Menn, C. "Prestressed concreted bridges". 1ª Ed. Paul Graveau. 1990.

[78] Millanes, F., Pascual, J. y Diaz de Argote, J.I. "Una metodología para el control de la puesta en tensión de los tirantes del puente sobre el río Iregua en Logroño". II Congreso de ACHE. 2002. 
BIBLIOGRAFIA.

[79] Negrao, J.H.J.O y Simoes, L.M.C. "Optimization of cable stayed bridges with threedimensional modelling". Computer\&Structures 64. 1997.

[80] Ontañón, G. "Puente del Centenario sobre la dársena del Guadalquivir en Sevilla". Hormigón y Acero n¹95. 1995.

[81] Park, J.B., Park, J.I. and Cho, J. "Estimation of error factors in concrete cable-stayed structures with sensitivity of creep". Journal of Structural Engineering. 2011.

[82] Pedrozzi, P. "A software tool for the analysis, design and monitoring of cable-stayed bridges". Tesis leída en el Instituto Técnológico de Zurich en 2004.

[83] Ploch, J. "Zur definition und zum Sicherheitskonzept der vorspannung". Institut für Leichtbau Entwerfen und kostruieren. ILEK. Universidad de Stutgart. 2004.

[84] Podolny, W. \& Scalzy, J. "Construction and design of cable-stayed bridges". John Wiley\&Sons. 1976.

[85] Poyatos, J.L. y González, J.I. "IV Puente sobre el río Guadiana en Badajoz". Hormigón y Acero $n^{\circ} 194.1994$.

[86] PTI Guide specification "Recommendations for stay cable design, testing and installation". Post Tensioning Institute. Phoenix 2001.

[87] Puy, J. "Algoritmos numéricos en Pascal". E.T.S.I de Caminos. 2aEdición. 1985.

[88] Quintana Ytza, M.F. "Métodos constructivos de puentes atirantados. Estudio de la distribución de fuerzas en los tirantes". Ph.D. Universidad Politécnica de Sao Paulo. 2009.

[89] Reddy, P., Ghaboussy, J. and Hawkins, N.M. "Simulation of construction of cable stayed bridges". Journal of bridge engineering. Noviembre 1999.

[90] Rodado, J. "Comportamiento y optimización de puentes atirantados continuos". Tesis doctoral dirigida por J. Manterola. U.P.M. 1999.

[91] Rubio Peirotén, D. "Métodos de definición de cargas en tirantes en puentes atirantados". Proyecto fin de master. UPM 2010.

[92] Ruiz Terán, A. "Puentes atirantados no convencionales. Comportamiento estructural y criterios de diseño ". Tesis Doctoral dirigida por A.C. Aparicio. U.P. Cantabria 2005.

[93] Schlaich, M. "Erection of cable stayed bridges having composite decks with precasts concrete slabs". Journal of civil engineering. Sep.-Oct. 2001

[94] Scotti A. "Long term behaviour of cable stayed bridges". Tesis Master. Politecnica de Milán. 2003.

[95] Simoes, L.M.C. \& Negrao, J.H.J.O. "Optimization of cable-stayed bridges with box girder decks". Advances in Engineering software 31. 2000. 
BIBLIOGRAFIA.

[96] Simoes, L.M.C., Martins, A. M. B. Monteiro, S. R. S., "Discrete optimum design of cablestayed bridges". 8th World congress on Structural and multidisciplinary optimization". Lisboa 2009.

[97] Strassky, J. "Stress ribbon and cable supported bridges". Ed. Thomas Telford. 2005.

[98] Sung, H. H. "A study on safety assessment of cable-stayed bridges based on stochastic finite element analysis and reliability analysis". KSCE Journal of civil engineering 2011. $15(2): 305-315$.

[99] Svensson, H. "Cable-stayed bridges. 40 years of experience worldwide". Ernst\&Sohn. 2012.

[100] Virlogeux M. "Recent evolution of cable stayed bridges". Engineering structures. 1999.

[101] Virlogeux, M. "Erection of cable-stay bridges. The control of the desired geometry " Conference AFPC sur le ponts suspendus et à haubans. Deauville. France. 1994.

[102] Virlogeux, M. et al. "Haubans. Recommandations de la commission interministérielle de la precontrainte" SETRA. Nov. 2001.

[103] Walther, R. y otros. "Cable stayed bridges". Ed. Thomas Thelford. 2a Ed. 1999.

[104] Wang, P.H. \& Yang, C.G. "Parametric studies on cable stayed bridges". Computer\&Structures. Vol 60. 1996.

[105] Wang, P.H., Tang, T.Y. and Zheng, H.N. "Analysis of cable-stayed-bridges during construction by cantilever methods". Computer\&Structures. 82. 2004.

[106] Wang, PH., Tseng, T.C. and Yang, C.G. "Initial shape of cable stayed bridges". Computer\&Structures. 46(6). 1993.

[107] Zadeh, O. S., "Comparison between three types of cable stayed bridges using structural optimization". Thessis submitted to the University of Ontario. 2012.

[108] Zhang, T \& Wu, Z. "Dead load analysis of cable-stayed bridges". International conference on intelligent building and management. Singapur. 2011. 
BIBLIOGRAFIA. 